\title{
Development of Knowledge Economy Framework in the Life Science Sector in the Kingdom of Saudi Arabia
}

\author{
Alharbi, Omar
}

How to cite:

Alharbi, Omar (2018) Development of Knowledge Economy Framework in the Life Science Sector in the Kingdom of Saudi Arabia. Doctoral thesis, Swansea University.

http://cronfa.swan.ac.uk/Record/cronfa43855

Use policy:

This item is brought to you by Swansea University. Any person downloading material is agreeing to abide by the terms of the repository licence: copies of full text items may be used or reproduced in any format or medium, without prior permission for personal research or study, educational or non-commercial purposes only. The copyright for any work remains with the original author unless otherwise specified. The full-text must not be sold in any format or medium without the formal permission of the copyright holder. Permission for multiple reproductions should be obtained from the original author.

Authors are personally responsible for adhering to copyright and publisher restrictions when uploading content to the repository.

Please link to the metadata record in the Swansea University repository, Cronfa (link given in the citation reference above.)

http://www.swansea.ac.uk/library/researchsupport/ris-support/ 


\section{Development of Knowledge Economy Framework in The Life Science Sector in The Kingdom of Saudi Arabia}

\section{Omar Hamdan Alharbi}

Submitted to Swansea University in fulfilment of the requirements for the Degree of Doctor of Philosophy

\section{SWANSEA UNIVERSITY \\ SCHOOL OF MANAGEMENT}

2018 


\section{DECLARATION AND STATEMENT}

This work has not previously been accepted in substance for any degree and is not being concurrently submitted in candidature for and degree.

Singed. (Candidate)

Date.

\section{STATEMEN 1}

This thesis is the result of my own investigations, except where otherwise stated. Where corrections services have been used, the extent and nature of the correction is clearly a footnote (s).

Other sources are acknowledged by footnotes giving explicit references. A bibliography is appended.

Singed. (Candidate).

Date.

\section{STATEMENT 2}

I hereby give consent for my thesis, if accepted, to be available for photocopying and inter-library loan, and for the title and summary to be made available to outside organisations.

Signed.

(Candidate).

Date. 


\section{TABLE OF CONTENTS}

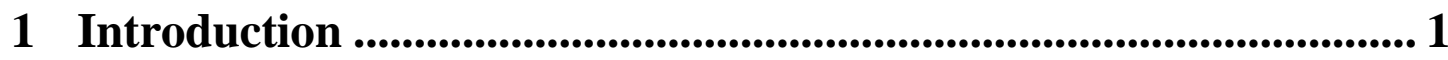

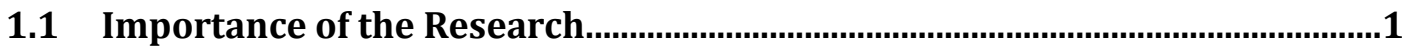

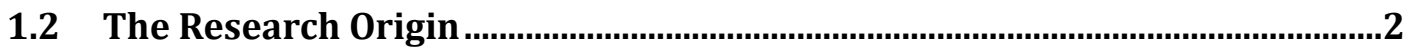

1.2.1 Personal and professional context …………………………………………..... 2

1.2.2 Government orientation ............................................................................................ 4

1.2.3 Literature review.............................................................................................. 4

1.2.4 Research aims, objectives, and questions......................................................... 5

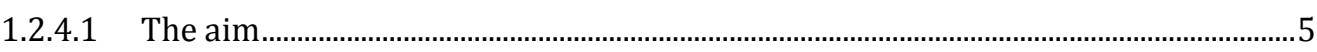

1.2.4.2 The objectives ..........................................................................................................6

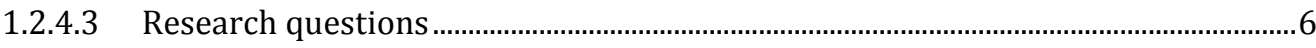

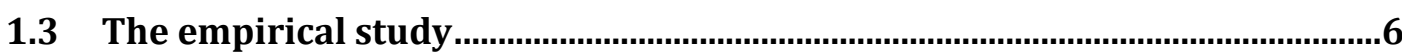

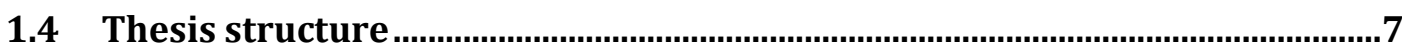

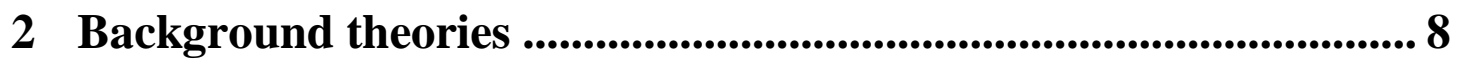

2.1 Systems Theory

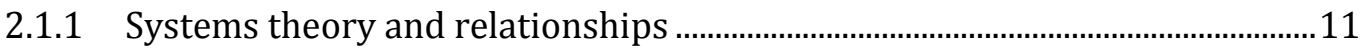

2.1.2 System transparency.......................................................................................13

2.1.3 Viable system model (VSM) …………………...................................................15

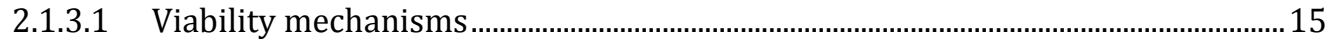

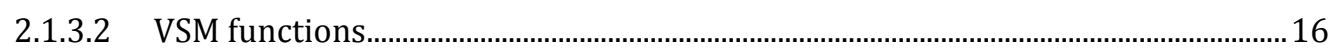

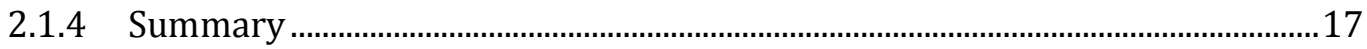

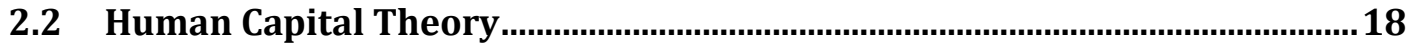

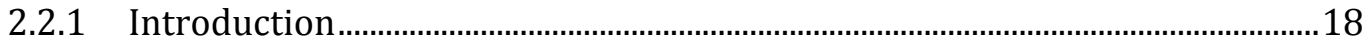

2.2.2 Investment in human capital ............................................................................19

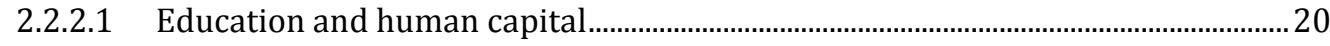

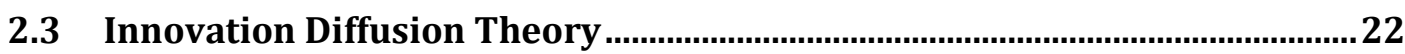

2.3.1 Knowledge transfer ...........................................................................................22

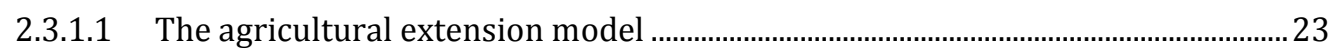

2.3.1.2 Knowledge transfer mechanisms ...........................................................................2

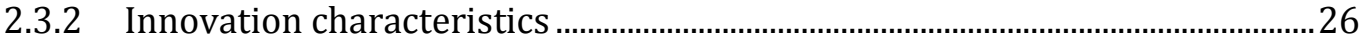

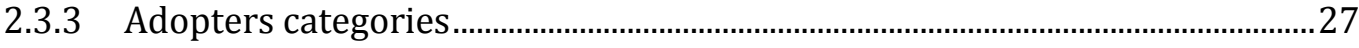

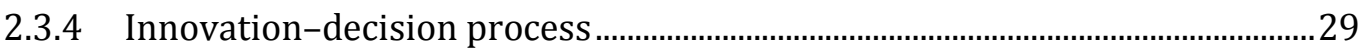

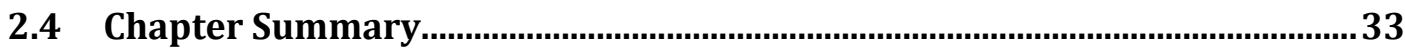

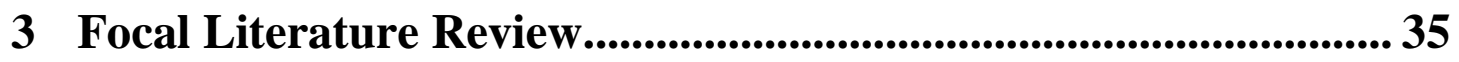

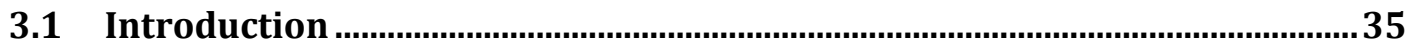




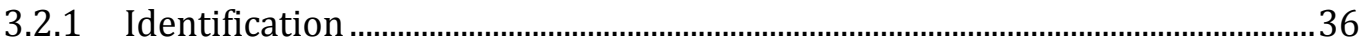

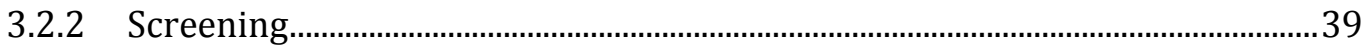

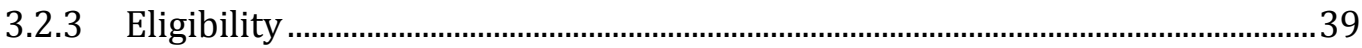

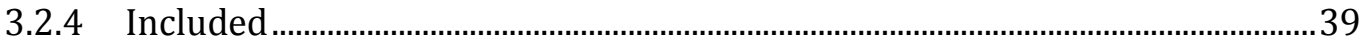

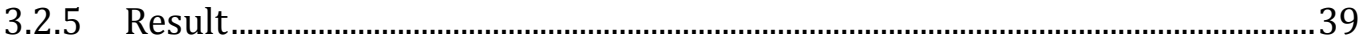

3.3 Economic Development …........................................................................

3.3.1 Capabilities and economic development ............................................................. 41

3.3.2 Clusters and economic development .................................................................. 42

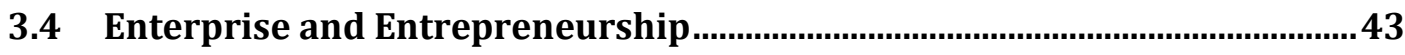

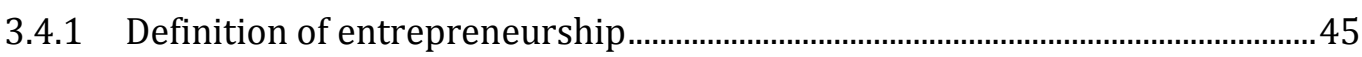

3.4.2 Entrepreneurial competencies ……………….................................................. 47

3.4.3 Micro and SME Businesses ....................................................................................... 48

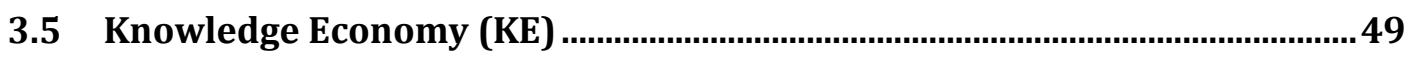

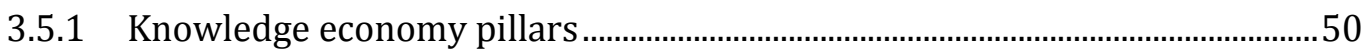

3.5.1.1 Economic and institutional regime........................................................................... 51

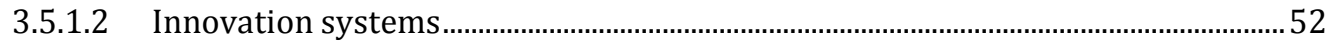

3.5.1.2.1 Knowledge-Intensive Business Services (KIBS) ………………......................... 54

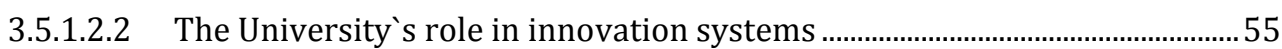

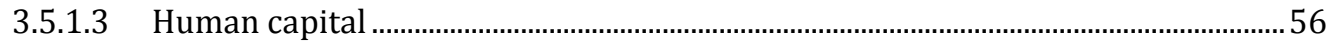

3.5.1.4 Information and Communication Technology Infrastructure (ICT)......................58

3.6 The role of SMEs in the Knowledge Economy...............................................63

3.6.1 The definition of SMEs..........................................................................................63

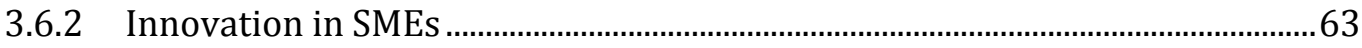

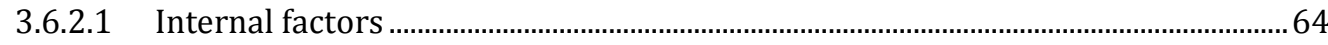

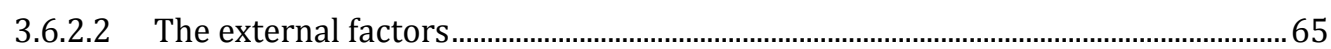

3.6.2.3 Absorptive capacity and knowledge management...................................................66

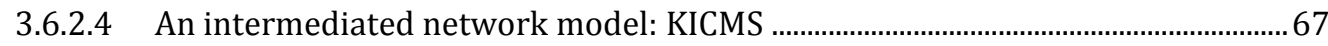

3.6.2.5 Entrepreneurial orientation in SMEs........................................................................... 67

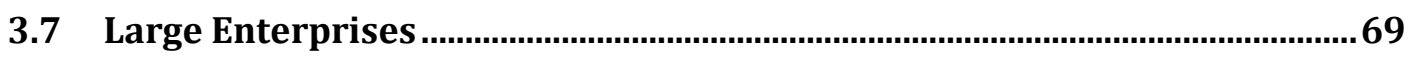

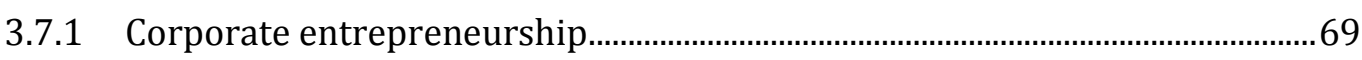

3.7.2 Entrepreneurship Strategy .....................................................................................

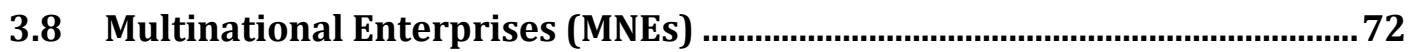

3.8.1 MNEs and dynamic capabilities (DCs) …………………………………...........

3.8.2 Foreign direct investment (FDI) ............................................................................

3.8.2.1 Definition and historic perspective …………………………………………….......

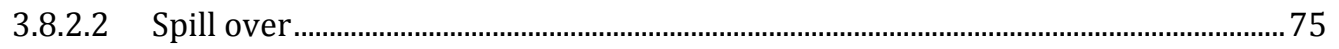


3.8.2.3 Prerequisites for FDI spill over and growth ....................................................... 77

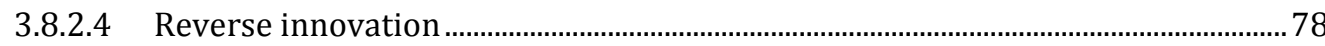

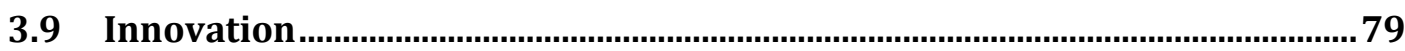

3.9.1 Different discipline perspectives around innovation .........................................80

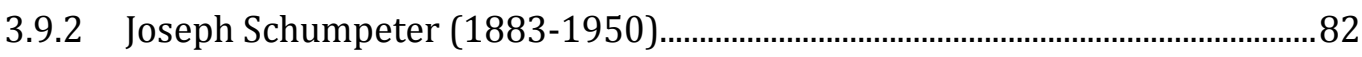

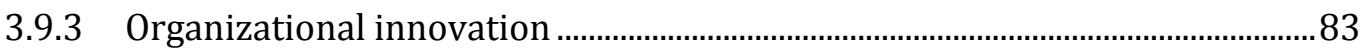

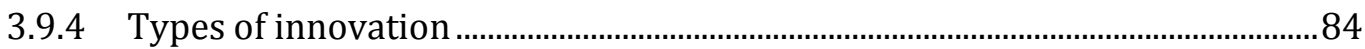

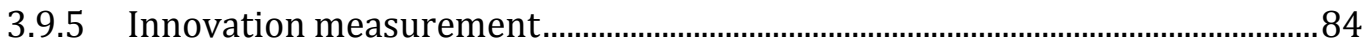

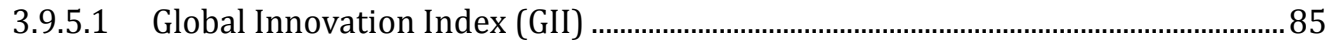

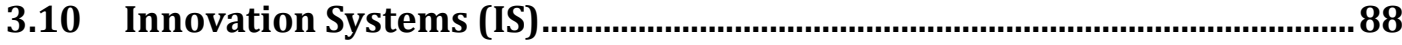

3.10.1 National Innovation System (NIS) definition ..................................................8

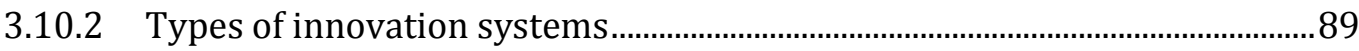

3.10.3 NIS preconditions and capabilities ......................................................................90

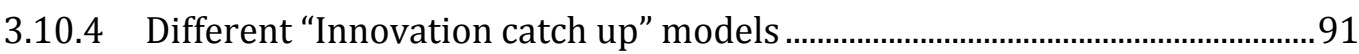

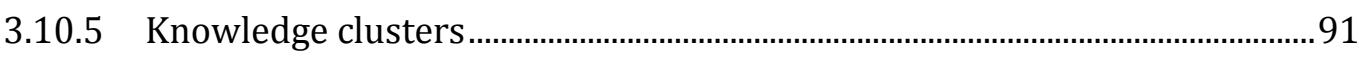

3.10.5.1 Cluster development and evolution ......................................................................... 92

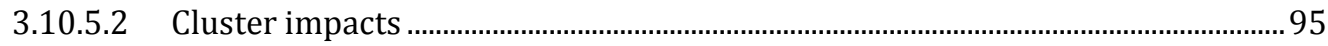

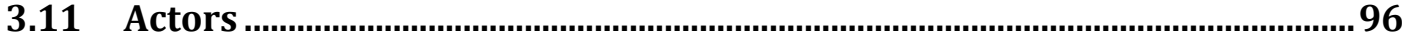

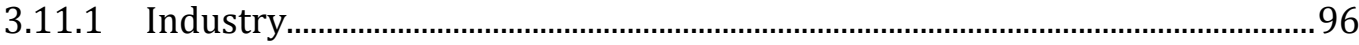

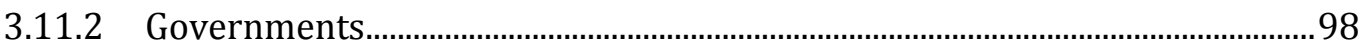

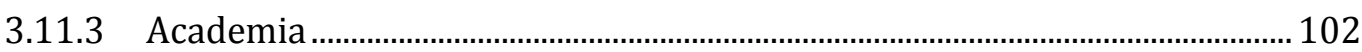

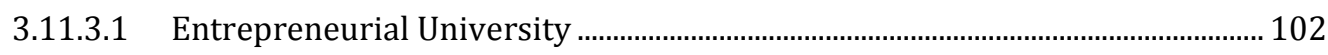

3.12 Theoretical model …...................................................................................... 104

4 Research Design ................................................................................................ 107

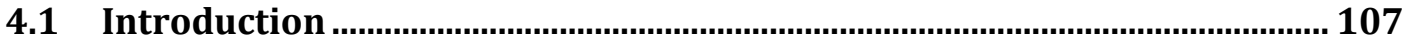

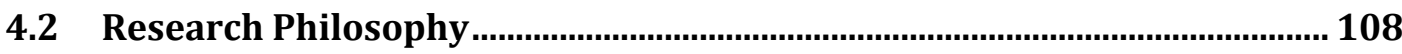

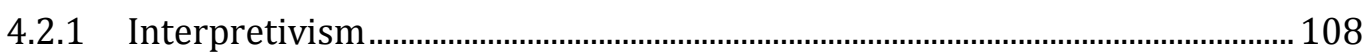

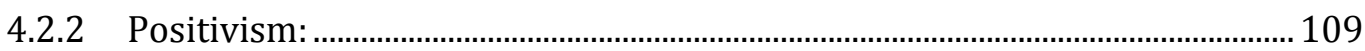

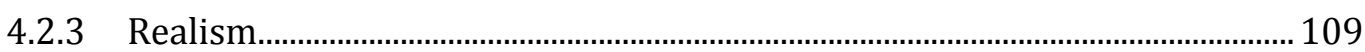

4.2.4 The Study`s Research Philosophy (Critical Realism) ......................................... 110

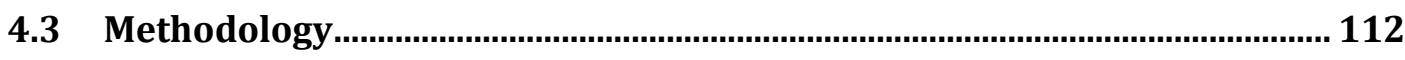

4.3.1 Grounded theory .................................................................................................... 114

4.4 Phases of the Research ..................................................................................... 117

4.4.1 First phase ................................................................................................................ 117

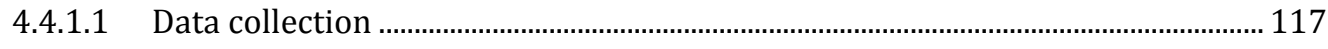

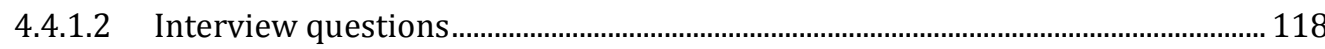


4.4.1.3 Data collection process ................................................................................................. 121

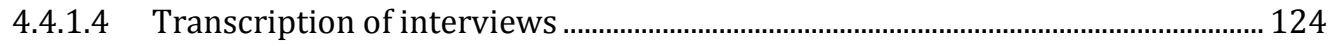

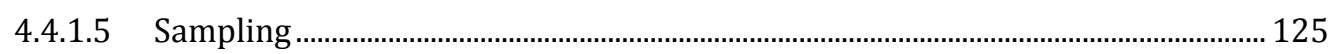

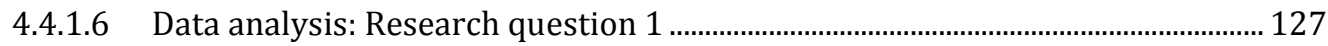

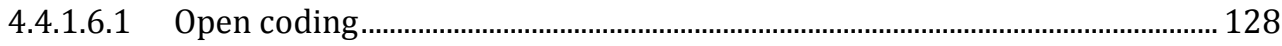

4.4.1.6.2 Axial coding .................................................................................................... 128

4.4.1.7 Data analysis: Research question 2 ............................................................................. 129

4.4.1.8 Data analysis: LS innovation system: Research question 3 ............................... 129

4.4.2 Second Phase .............................................................................................................. 129

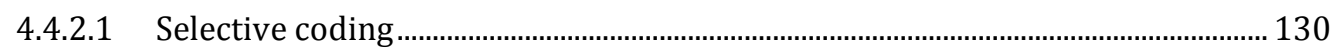

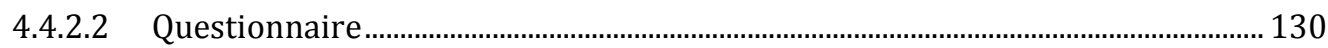

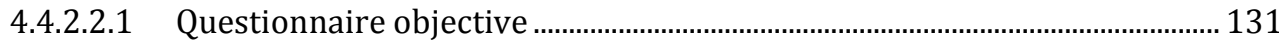

4.4.2.2.2 Questionnaire design ..................................................................................... 132

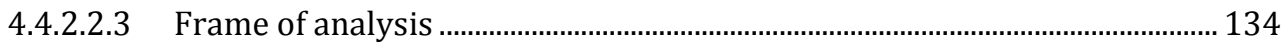

4.4.2.2.4 Questionnaire validity and reliability .................................................................. 135

4.5 Research Reliability and Validity ................................................................... 135

4.6 Revision Team (RT) ....................................................................................... 138

4.7 Research Ethics and limitations................................................................ 138

5 The KSA and Context ......................................................................... 140

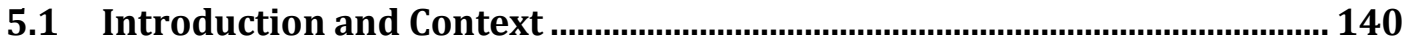

5.2 Kingdom of Saudi Arabia (KSA) ................................................................. 141

5.2.1 Saudi Arabia and its Influence on the World Landscape................................. 141

5.2.2 Saudi Arabia Establishing: Historical Perspective ............................................ 142

5.2.2.1 First Saudi state, 1744-1818 …......................................................................................... 142

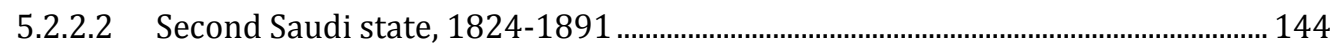

5.2.2.3 Third Saudi state (kingdom of Saudi Arabia) 1902-1953 .................................... 144

5.2.3 Civilization and Culture Change ........................................................................ 147

5.2.4 The Economic Diversification Strategy of the KSA ......................................... 147

5.2.5 Saudi Economic Development.............................................................................. 150

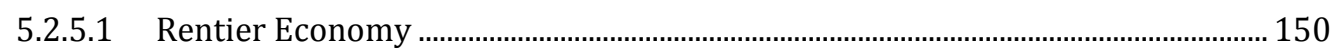

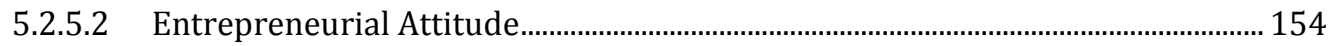

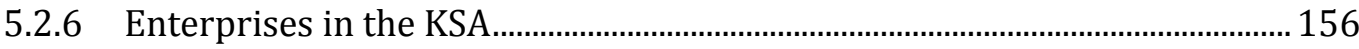

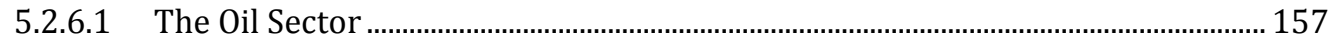

5.2.6.2 The trade and services sector ................................................................................ 157

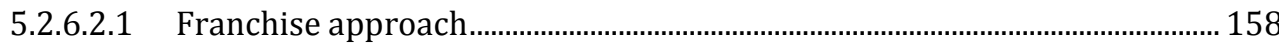

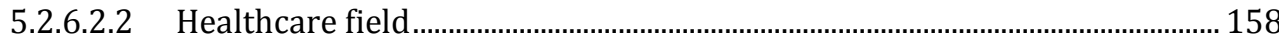

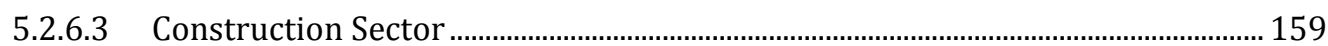

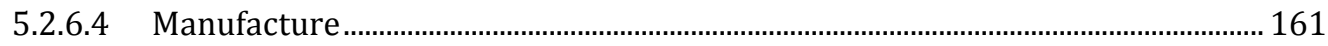




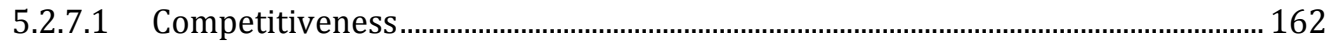

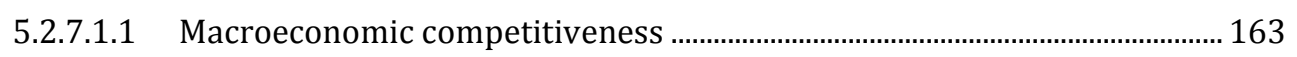

5.2.7.1.2 Microeconomic competitiveness .........................................................................164

5.2.7.2 KSA Competitiveness ............................................................................................... 165

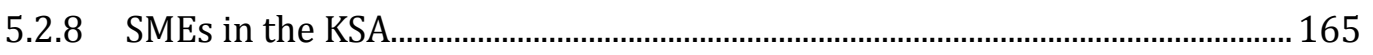

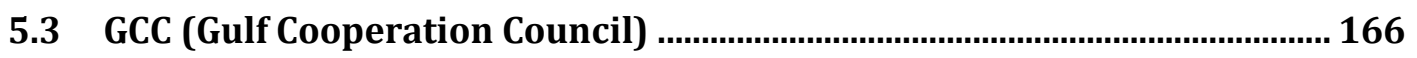

5.3.1 GCC Economic Structure.................................................................................. 167

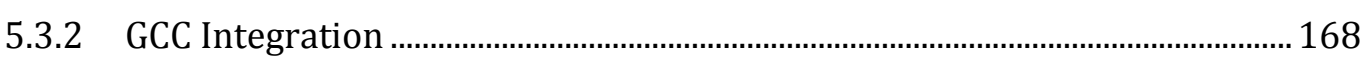

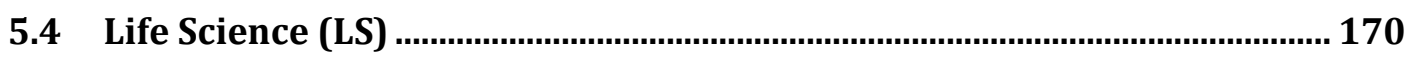

5.4.1 Biotechnology as a Promising Sector ............................................................ 170

5.4.1.1 Biotechnology Industry Dynamics ............................................................................. 171

5.4.1.2 Challenges facing developing LS innovation system ............................................ 174

5.4.1.3 Fundamental Forces That Shape the LS Industry .............................................174

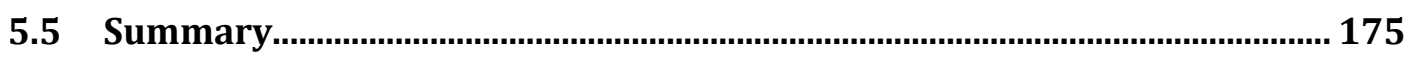

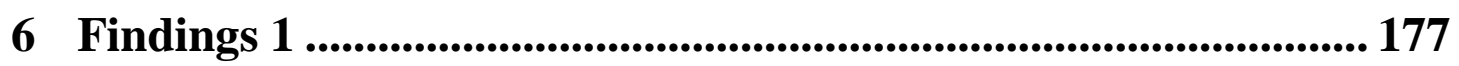

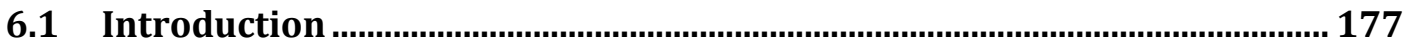

6.2 Open coding

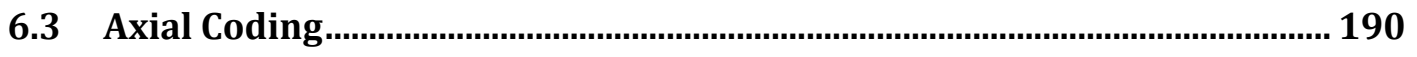

6.4 Answer for Research Question 1...................................................................... 192

6.4.1 Developing Human Capital ................................................................................. 192

6.4.1.1 The pool of scientists is not so big (A 31\%, G 18\%, P 0\%) ................................... 192

6.4.1.2 Shortage of scientific graduates (A 0\%, G 9\%, P 14\%) ......................................... 193

6.4.1.3 Lack of high managerial and technical skills (A 31\%, G 18\%, P 29\%) ............... 194

6.4.1.4 Engaging more medical doctors in research (A 0\%, G 9\%, P 0\%) ....................... 194

6.4.1.5 Exposing for international effect (A 38\%, G 64\%, P 14\%)..................................... 195

6.4.1.6 Raising education system quality (A 46\%, G 18\%, P 42\%) .................................. 196

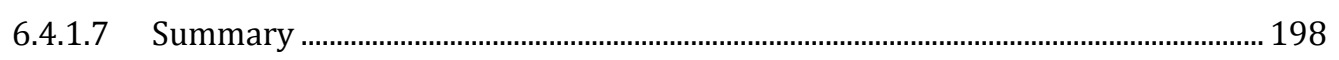

6.4.2 Developing World-Class Research Infrastructure........................................... 199

6.4.2.1 Research capabilities are weak (A 8\%, G 18\%, P 42\%) ......................................... 199

6.4.2.2 Research infrastructure is weak (A 31\%, G 36\%, P 29\%)................................... 199

6.4.2.3 Developing strong research centres (A 54\%, G 27\%, P 0\%) ................................ 200

6.4.2.4 Developing cluster based on strengths and priorities (A 77\%, G 45\%, P 14\%) 201

6.4.2.5 Developing research entrepreneurial universities (A 77\%, G 9\%, P 0\%) ....... 201

6.4.2.6 Transferring foreign knowledge (A 31\%, G 9\%, P 42\%) ....................................... 203

6.4.2.7 Developing collaboration with foreign parties (A $15 \%$, G 36\%, P 0\%) ............ 203 
6.4.2.8 Developing an online platform (A 23\%, G 9\%, P 14\%) ….................................... 205

6.4.2.9 Developing LS specialties (A 62\%, G 27\%, P 42\%) ................................................. 206

6.4.2.10 Summary .................................................................................................................. 208

6.4.3 Developing Entrepreneurial and Efficient Funding System .......................... 210

6.4.3.1 Risk-averse financial system (A 23\%, G 45\%, P 0\%) ............................................ 210

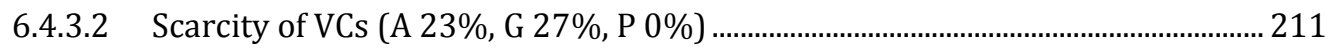

6.4.3.3 More funding for R\&D (A 38\%, G 36\%, P 42\%) ................................................... 211

6.4.3.4 Developing seed funds (A 54\%, G 27\%, P 0) …….................................................... 213

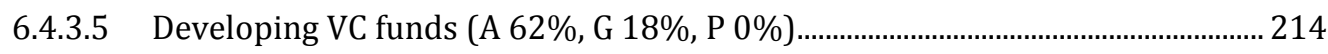

6.4.3.6 Raising funding efficiency (A 62\%, G 27\%, P 28\%) …........................................... 215

6.4.3.7 Developing angel investors (A 15\%, G 0\%, P 0\%) ……........................................ 216

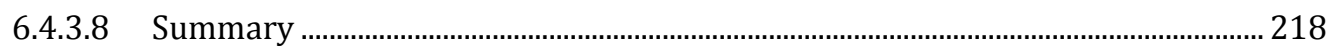

6.4.4 Developing Supportive Regulations and Enabling Policy ................................ 219

6.4.4.1 Raising the quality of institutions (A 38\%, G 36\%, P 29\%) ................................... 219

6.4.4.2 Building social capital (A 31\%, G 36\%, P 0\%) .......................................................... 220

6.4.4.3 Building on strengths and priorities (A 54\%, G 36\%, P 14\%)............................ 220

6.4.4.4 Supporting entrepreneurship culture (A 54\%, G 55\%, P 14\%) ......................... 221

6.4.4.5 Developing government-led project (A 54\%, G 36\%, P 42\%) ............................. 223

6.4.4.6 Developing enabling policy for R\&D, and spin-off (A 70\%, G 55\%, P 14\%) .... 224

6.4.4.7 Improving industry infrastructure and business environment (A 54\%, G 18\%, P 42\%) 226

6.4.4.8 Improving living standards and relaxing cultural barriers (A 15\%, G 11\%, P 14\%) 227

6.4.4.9 Developing supportive staffing regulations (A 15\%, G 18\%, P 0\%)................... 228

6.4.4.10 Developing a KE coordinating organization (A 31\%, G 9\%, P 0\%) ................. 229

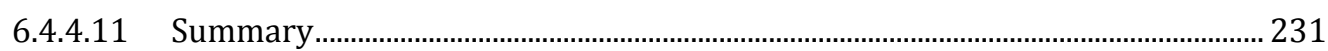

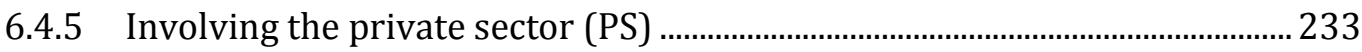

6.4.5.1 Lack of academia-PS collaboration (A 46\%, G 27\%, P 29\%) ............................... 233

6.4.5.2 Lack of innovation strategy and attitude in the PS (A 38\%, G 55\%, P 57\%) ... 234

6.4.5.3 Developing academia-PS strong connections (A 62\%, G 36\%, P 0\%) .............. 235

6.4.5.4 Funding programmes for academia-PS collaboration (A 70\%, G 36\%, P 57\%) 236

6.4.5.5 Involving all actors in developing policy (A 38\%, G 9\%, P 0\%) .......................... 238

6.4.5.6 Transferring foreign knowledge (A 54\%, G 27\%, P 29\%) ..................................... 238

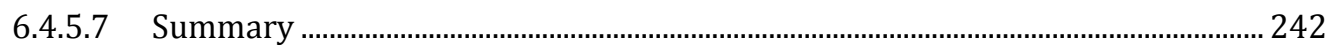

7 Findings 2 ...................................................................................................... 244

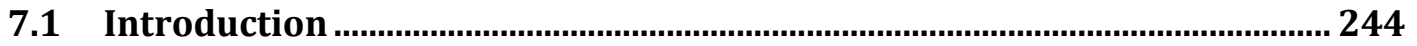

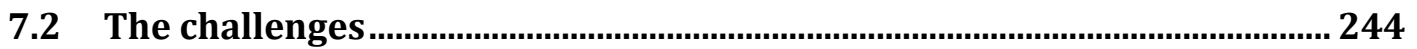


7.2.1 No attractive life standard, and restrictive cultural barriers (16\%)............ 245

7.2.2 No leadership (10\%) ....................................................................................... 245

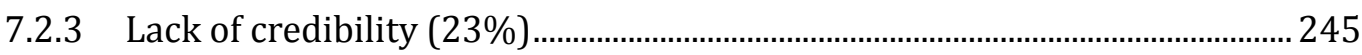

7.2.4 Risk-averse behaviour (20\%) ………………............................................. 246

7.2.5 Lack of innovation strategy and attitude in PS (39\%) ................................... 246

7.2.6 Lack of connections and Academia-PS collaboration (42\%)......................... 246

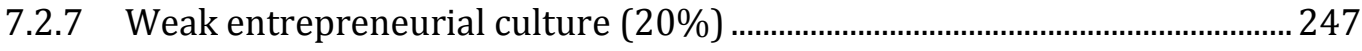

7.2.8 Weak research infrastructure (39\%) ............................................................... 247

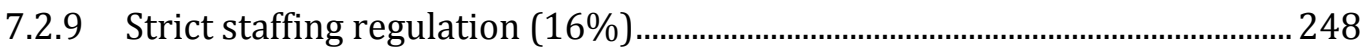

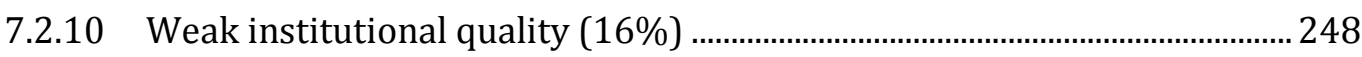

7.2.11 Shortage of medical doctor researchers (3\%) ............................................. 248

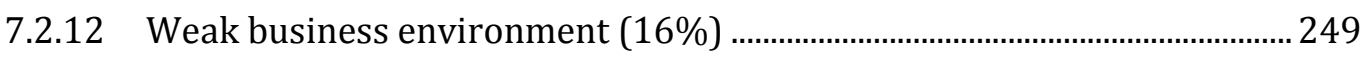

7.2.13 No collective work in developing policy (3\%) ............................................. 249

7.2.14 Lack of awareness of the requirements for a KE (10\%) ..............................249

7.2.15 Weak collaboration and networking culture (13\%) ..................................... 250

7.2.16 Lack of enabling policy for R\&D and spin-offs (10\%) .................................. 250

7.2.17 Lack of high managerial and technical skills (13\%)..................................... 250

7.2.18 Summary ………………............................................................................. 251

8 Findings 3 .................................................................................................... 255

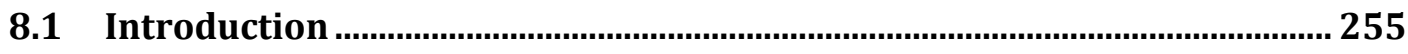

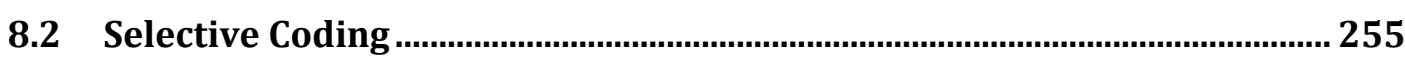

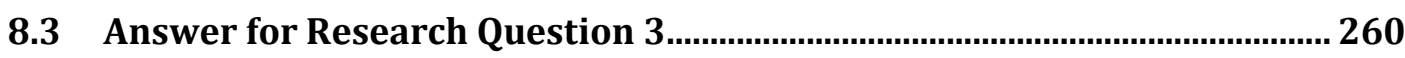

8.3.1 LS model (themes level) ................................................................................. 260

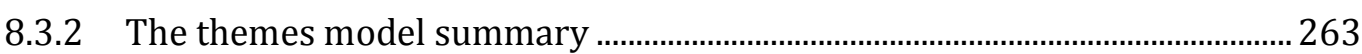

8.3.3 The code model summary ................................................................................... 269

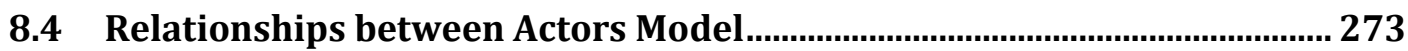

8.4.1 Saudi LS Innovation Model (SALSIM) ………………….................................... 276

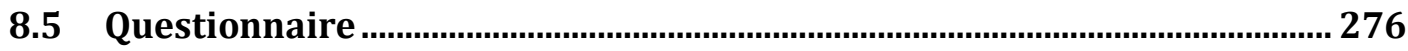

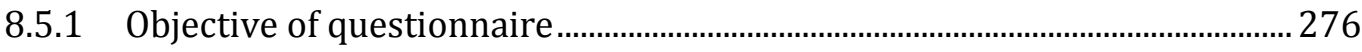

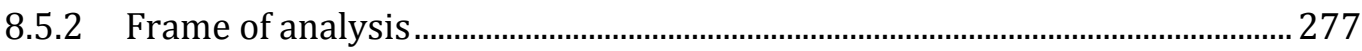

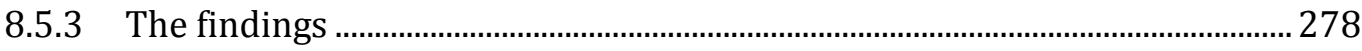

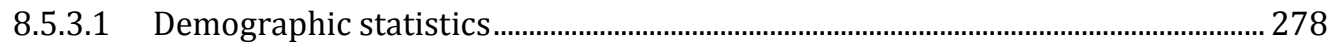

8.5.3.2 Frequency analysis (Mode Test).......................................................................... 280

8.5.3.2.1 Private sector-life science (PS-LS)..................................................................... 280

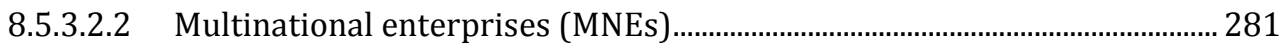

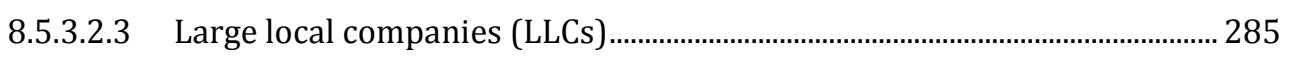




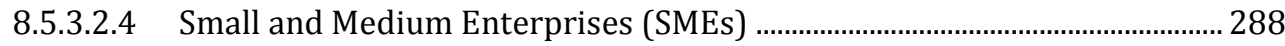

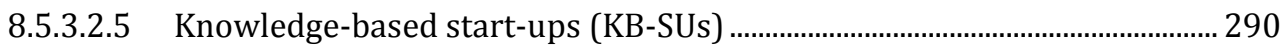

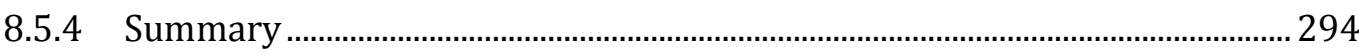

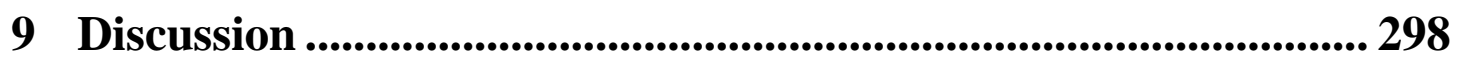

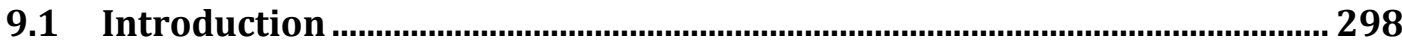

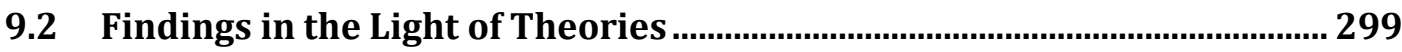

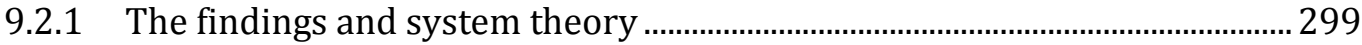

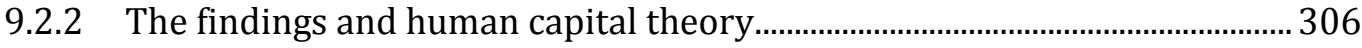

9.2.3 The findings and the innovation diffusion theory ...........................................308

9.2.4 The Saudi LS Innovation Model against other models in the literature ..... 312

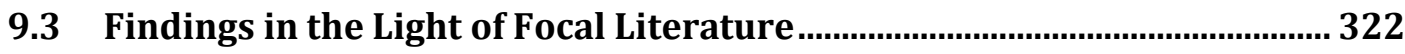

9.3.1 Knowledge economy requirements ...................................................................... 322

9.3.1.1 Economic and institutional regime............................................................................. 322

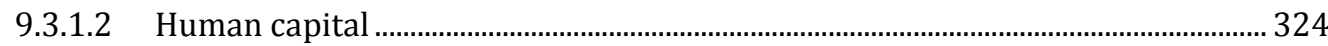

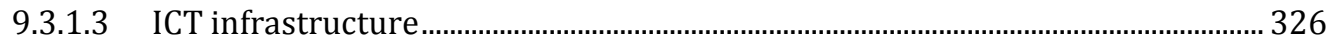

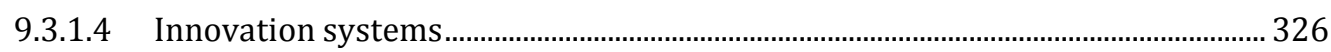

9.3.2 Questionnaire findings against first study findings .........................................329

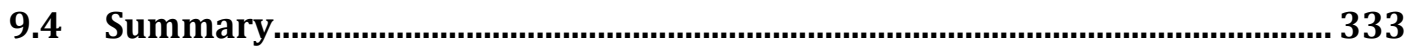

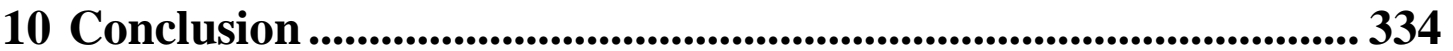

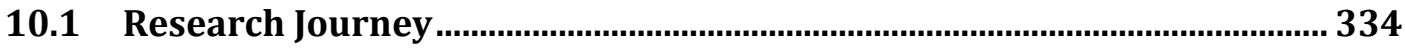

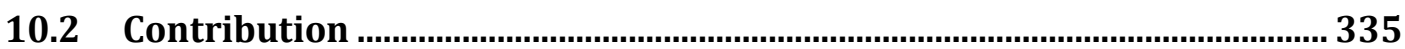

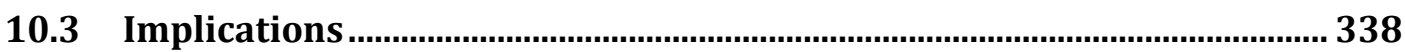

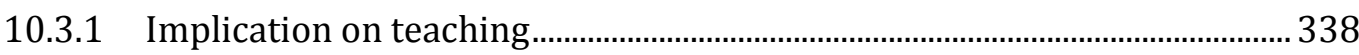

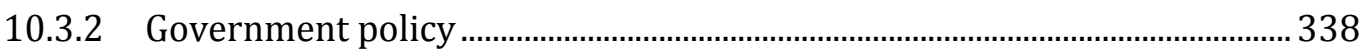

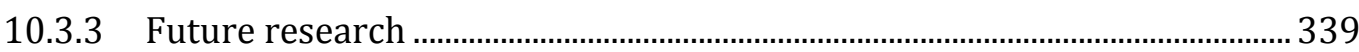

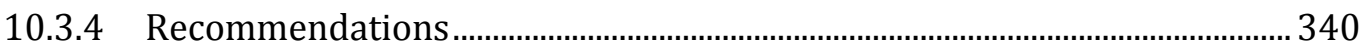

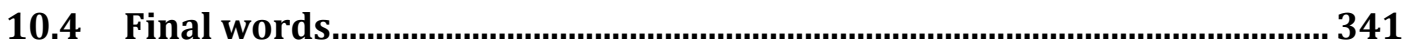

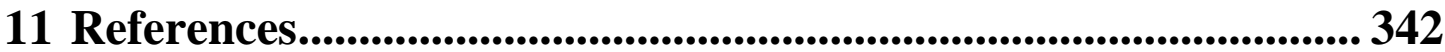

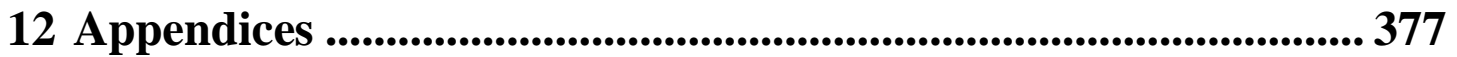

12.1 Appendix 1: Interview questions (Pilot study) ……..................................... 377

12.2 Appendix 2: Interview questions (Main Sample) ..................................... 378

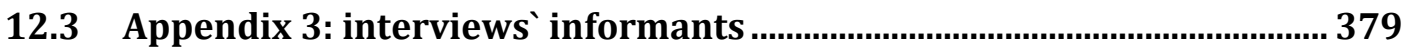

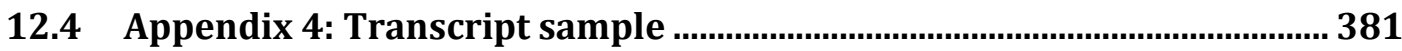

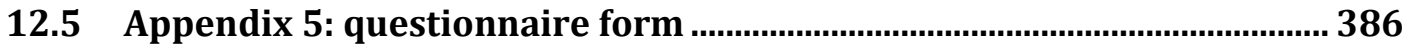




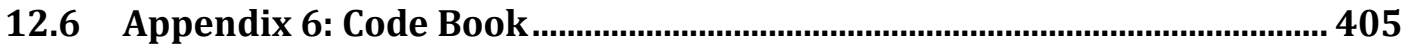




\section{LIST OF TABLES}

Table 2-1: Key features of system theory. 9

Table 2-2: Key themes of human capital theory. 21

Table 2-3: Centralized and decentralized knowledge transfer paradigms. Source: Adapted to diffusion of innovation systems (Rogers 1983).

Table 2-4: Key concepts related to the innovation diffusion theory. 32

Table 3-1: Focal literature review map. 39

Table 3-2: Dimensions of knowledge activities and the indicators of inputs and outputs. 62

Table 4-1: Pilot study interviews questions. 119

Table 4-2: Interview questions for the main sample 120

Table 4-3: informants' detail. Source: The researcher. 122

Table 4-4: Validity procedures with respect to the two governors perspectives. 136

Table 5-1: Distribution of Saudi government expenditures on different sectors. 154

Table 5-2: Structure of real GDP over the three sectors (governmemnt, private, Oil). 154

Table 6-1: Open codes and their properties. 179

Table 6-2: Code distributions against different perspectives; except for the overall perspective, the percentages are based on number of participants for each respective sector, not the whole sample saize. $N=$ number of participants. Overall $N=31$, Academia $N=13$, government $N=11$, priate sector $N=7$. The codes ordered in ascending order for the overall perspective values. 183

Table 6-3: Axial codes and their properties (open codes). Source: The researcher.

Table 8-1: Quantitative perspectives for categories ' relevant keywords. This table details and compares the relevant keywords appended to each of the five themes (categories) to show which theme has the more prominence and importance in the collected data. About 9 keywords were found related to the private sector, and their frequency achieved 592 times, overcaming all the other keywords related to the other categories (developing human capital, developing world-class research infrastructure, developing supportive regulations and enabling policy, and developing entrepreneurial funding and financial system).

Table 8-2: Properties of categories as identified by their related open codes. The selective code is highlighted in column four. Source: The researcher.

Table 8-3: Measurement codes.

Table 8-4: Gender details. 278

Table 8-5: Sectors details. 278

Table 8-6: Job details.

Table 8-7: Nationality details.

Table 8-8: Country of residence details.

Table 8-9: Age details. 280

Table 8-10: PS mode. 280 
Table 8-11: PS frequencies result details. 281

Table 8-12: MNEs`internal encouraging variables modes. 282

Table 8-13: MNEs' internal discouraging variables mode. 282

Table 8-14: Breakdown of MNEs`internal discouraging variables frequencies. 283

Table 8-15: MNEs' external encouraging variables modes. 283

Table 8-16:MNEs' external hindering variables modes. 284

Table 8-17: LLCs' internal encouraging variables modes. 285

Table 8-18: LLCs' internal hindering variables modes. 286

Table 8-19: LLCs 'external encouraging variables modes. 287

Table 8-20: LLCs' external hindering variables modes. 287

Table 8-21: SMEs`internal encouraging variables modes. 288

Table 8-22: SMEs' internal hindering variables modes. 289

Table 8-23: SMEs' external encouraging variables modes. 289

Table 8-24: SMEs' external hindering variables modes. 290

Table 8-25: KB-SUs' internal encouraging variables modes. 291

Table 8-26: KB-SUs' internal hindering variables modes. 292

Table 8-27: KB-SUs' external encouraging variables. 293

Table 8-28: KB-SUs' external hindering variables modes. 293

Table 9-1: System concepts in the findings and their implications. 305

Table 9-2: Aspects of different innovation models from different countries with compared with Saudi context. Source: The researcher (2017). 314

Table 9-3: Encouraging and hindering variables for MNEs. Source: The researcher. 330

Table 9-4: Encouraging and hindering variables for LLCs. Source: The researcher 330

Table 9-5: Encouraging and hindering variables for SMEs. Source: The researcher. 331

Table 9-6: Encouraging and hindering variables for KB-SUs. Source: The researcher. 331

Table 9-7: Encouraging and hindering variables for the private sector. Source: The researcher._ 332 


\section{LIST OF FIGUERS}

Figure 2-1: Adaptor classification with respect to innovativeness. 28

Figure 2-2: Innovation-decision process (Source: Diffusion of Innovation, fifth edition, 2003) ........... 30

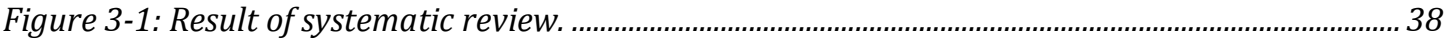

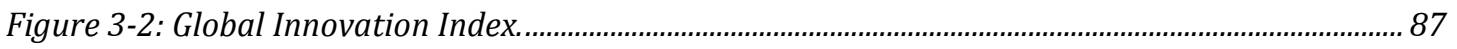

Figure 3-3: Model for theoretical innovation system. Source: The researcher......................................105

Figure 4-1: Research procee onion. Source: Adapted from Saunders et al. (2011) ..............................107

Figure 4-2: Ontological and epistemological ideologies of the study. Source: The researcher...........111

Figure 4-3: Qualitative data analysis components. Source: Adpated to Huberman \& Miles (1994) 113

Figure 4-4: Axial coding paradigm. Source: Adapted from (Corbin \& Strauss, 1990).........................116

Figure 4-5: Types of questionaire (Study approach in bold type). Source: (Saunders et al., 2011) ..133

Figure 5-1: First Saudi state map .............................................................................................................143

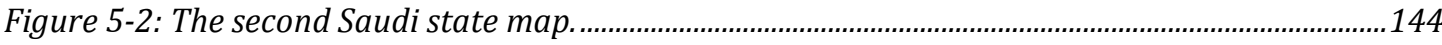

Figure 5-3: The current (third) Saudi state (the Kingdom of Saudi Arabia) map ...............................146

Figure 5-4: Saudi government's sources of income. Source: GISreportonline.com ...............................148

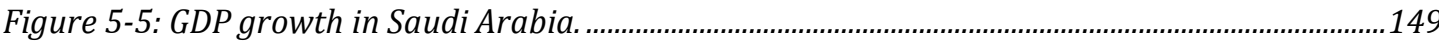

Figure 5-6: Economic activities distribution in Saudi economy (share of GDP). ...................................156

Figure 5-7: Construction sub-sectors. Source: Adapted to:(Middle East, 2011) ...................................160

Figure 6-1: Overall perspective against codes (the numbers represent the percentage of informants in favour of the code). Source: The researcher. 185

Figure 6-2: differnt perspectives ( $A=$ =Academai, G=Government, $P S=$ Private sector) against codes (the numbers represent the percentage of informants in favour of the code). Source: The researcher.

Figure 6-3: Figure 6 3: Academia perspective against codes (the number represents the percentage of informants in favour of the code). Source: The researcher.

Figure 6-4: Governmemnt perspective against codes (the numbers represent the percentages of informants in favour of the code). Source: The researcher. 188

Figure 6-5: Private sector perspective against codes (the numbers represent the percentage of informants in favour of the code). Source: The researcher.

Figure 6-6: Axial coding paradigm. Source: Corbin \& Straus, 1990).

Figure 6-7: This model represents the "developing human capital" theme and its properties. 197

Figure 6-8: This model shows "developing world-class research infrastructure" theme and its properties. Source: the researcher.

Figure 6-9: model represents the "entreprenurial funding and effecient system" theme and its properties. Source: The researcher.

Figure 6-10: Model for developing supportive regulations and enabling policy.Source: the researcher. 
Figure 6-11: Model represents the theme "involving the private sector". Source: The researcher...241 Figure 7-1: Quantitative perspective for challenges facing progressing LS in the KSA. Vertical axis represents participants ' number. Horizontal axia represents challenges. Source: The researcher..254 Figure 8-1: Quantitative perspectives for catgories ' relevant keywords. Source: The researcher....258 Figure 8-2: The themes model. Developing supportive and enabling policy is viewed as the initial step that influencse (and influenced by) the other four themes. Source: the researcher.

Figure 8-3: All the codes (Vertical Axil) ordered in intensity descending order according to participants' point of view. Source: The researcher.

Figure 8-4: The codes model. This model shows the prominent relationships among the key codes). Source: The researcher.

Figure 8-5: Relationships among the actors model.

Figure 8-6: Saudi Life Science Innovation Model (SALSIM). The figure shows the emperically derived model that was developed from the raw grounded theory research, which represents the actual practice of innovation in the Kingdom of Saudi Arabia (the LS Innovation System). Source: The researcher.

Figure 8-7: Current Saudi innovation system (Dotted lines mean the relationships are very weak or do not exist)

Figure 8-8: current innovation system, and the proposed Viable Saudi LS Innovation System (VSLSIS)

Figure 10-1: current innovation system, and the proposed Viable Saudi LS Innovation System (VSLSIS) 


\section{ABBREVATIONS}

ASEAN: The Association of Southeast Asian Nations

FDI: Foreign Direct Investment

GCC: Gulf Cooperation Council

GDP: Gross Domestic Production

GII: Global Innovation Index

GPT: General Purpose Technology

ICT: Information Communication Technology

IMF: International Monetary Fund

IS: Innovation system

KAM: Knowledge Assessment Methodology

KB-SUs: Knowledge Based Start-ups

KE: Knowledge Economy

KEI: Knowledge Economy Index

KSA: Kingdom of Saudi Arabia

LLCs: Large Local Companies

LS: Life Science

MNEs: Multi National Enterprises

NIS: National Innovation Systems

OECD: The Organisation for Economic Co-operation and Development

PS: Private Sector

RCs: Research centres

RT: Revision Team

SALSIM: Saudi Life Science Innovation Model

SKEO: Saudi Knowledge Economy Organization

SMEs: Small and Medium Enterprises

TFP: Total Factor Productivity

UNCTAD: the United Nations Centre on Transnational Corporations

VSLSIS: Viable Saudi Life Science Innovation Systems Model

WBI: World Bank Institute

WIPO: The world Intellectual Property Organisation 


\section{ACKNOWLEGEMENT}

Nothing can take place without the desire and help of Allah. Mighty thanks and praises to my God.

\section{Personal}

I would like to thanks my great mother Salhah Alharbi, as the first supporting and inspiring person in my life, I ask Allah to save her, and bestow his mercy on her.

Also I want to thank my big brother Dakheel and all my family members for standing by me along the $\mathrm{PhD}$ journey.

\section{Professional}

I would like to thank my first supervisor, Professor Marc Clement for his encouragement and caring from the beginning of this study.

His inspiring personal leadership characteristics, and his significant contribution to establishing the life science hub at Swansea University have enabled me to learn a lot from my experiences of them.

I would like deeply to thank the second supervisor Professor Nicholas Rich for agreeing to join the supervision team and for his key role and support in my completion of my study.

Also I would like to thank the third supervisor Dr Daniele Doneddu and the other supervision team members: Dr. Gareth Davies and Sian Roderick for their inputs and hints in the regular tutorials. Thanks to Mrs Jennifer Childs for help in proofreading. Lastly, I want to thank my friend Dr Farhana Tegwen Malik for her support and encouragement. 


\begin{abstract}
Natural resources-based income economies (Rentier economies) often suffer from a weak productive manufacturing base and low revenue and tax generation from these products and services. The prices of natural resources, such as oil and gas, change and fluctuate over time which poses a risk to national income and impedes the development of longer-term diversification/innovation strategies. It is these conditions which provide the context of this research into the current national policies to develop the "high value added" life sciences sector in the Kingdom of Saudi Arabia (KSA). This study adopts a grounded theory approach to the national innovation systems for the life sciences sector in the KSA. Little is known about the models employed and the current state of system development for the country and whether it varies from other such models. An initial literature review to frame the study was conducted on Innovation models to form a conceptual framework (India, Singapore, Taiwan, and South Korea). Field research was conducted and later a second systematic literature review was used to build theory and test the similarities/differences with the emerging KSA model (created by political, social, economic and industrial contingencies). The guiding research questions were:

- What are the key requirements in developing knowledge economy (KE) in the life science (LS) sector in KSA?

- What are the challenges in developing knowledge economy (KE) in LS in the KSA?

- What model of innovation system best fits the Saudi context?
\end{abstract}

Semi-structured in depth interviews (purposive sample) were conducted with senior stakeholders in the life science sector (government, private sector, and academia, ministry of health) to develop new theory. Through a three-stage open coding process including axial coding and selective coding, the findings revealed the challenges for developing an innovative LS system in Saudi Arabia. A questionnaire was developed to triangulate the earlier findings. The study finds that the KSA model differs from existing Innovation Models (India, Singapore, South Korea, and Taiwan) and creates a new insight into innovation in an Islamic context. It is recommended that this model be tested against similar contexts like Arabic Gulf countries (GCC) in future work. 


\section{Introduction}

The purpose of this chapter is to establish the importance and the context of this study. The chapter will present the origins of the research and how it has evolved, explaining how the aims, objectives, and research questions have been developed before exploring the structure of the thesis and how this grounded approach was conducted.

\subsection{Importance of the Research}

Moving from a rentier to a knowledge-based economy is a very critical transitional moment in the history of any country (Gallarotti, 2013). The importance of this study stems from realizing the challenges faced by the Kingdom of Saudi Arabia that has relied on natural resources as a main source of income for decades. This economic system has established many complicated conditions in terms of economic structures, government efficiency and socio-economy matters (Science \& York, 2014). The weakness of the current national system of economic value generation, which is dependent upon oil extraction, questions whether the necessary structures for innovation exploitation have yet to be built (Alshumaimri, Aldridge, \& Audretsch, 2010; Khursani, Bazuhair, \& Khan, 2011). Such weaknesses could include the structures and actors engagement necessary for collaboration, trust, and networking to build an entrepreneurial culture and generate value.

The current implication of a model that has been built on a rentier economy means that the Saudi government must develop such structures and systems from scratch. In effect the Kingdom of Saudi Arabia is a developing knowledge economy and this presents a research gap as most studies are restricted to mature manufacturing economies. However, few studies have addressed how an entire economy can move from a rentier economy to one that is based on high added value exploited from knowledge management. In effect the government can no longer afford to continue to exploit oil revenues and must invest in the development of a knowledge-based system of revenue generation. The shift is based on the writings of Michael Porter (1990) and the competitive advantage of nations. His ideas rely upon the movement of traditional 
industry to that of knowledge-based productivity. In his view no mature economy can survive without making this transition. It is in this context that this study is set. The study seeks to understand, in a dynamic environment, how an entire economy is moving from a traditional to a modern approach. It seeks to explore the main elements and actors that operate within the Kingdom of Saudi Arabia and how these relationships are establishing or changing the manner in which the economy works.

Saudi Arabia, as a benchmark for the remainder of the GCC countries, presents a unique insight into innovation and this study is of high importance for many other GCC countries which have yet to make the transition (often from oil or agricultural systems). The movement of the Saudi economy, from an oil based to a knowledge based system, will set and potentially develop a new theoretical model for the way in which countries transition.

\subsection{The Research Origin}

The section will explain how the research began and the primary factors that contributed in shaping this contemporary study. The study was inspired and motivated by two factors:

1- Personal and professional motivations

2- Government orientation and a lack of guidance in the academic and professional literatures

These factors will now be explained.

\subsubsection{Personal and professional context}

The author has been employed by the Ministry of Energy, Industry and Minerals, for around 15 years in the manufacturing licenses department. He has therefore acquired a good insight and experience regarding the national 'current state' and life sciences industry dynamics in the KSA. He found himself facing many questions that were of an academic nature - in terms of how a country can engage in innovation and whether a Saudi model would be different to the major models adopted in the literature. Currently, the KSA life science industry is dominated by imitation and production 
activities but there are concerns that it is insufficiently innovative despite Saudi Arabia being the biggest health care market in the Middle East (demand side). The growth rate of public spending between 1999 and 2005 was about 7.2\% (Ram, 2014) which is a level of government spending that is unsustainable. Furthermore, the KSA has high levels of country-specific health conditions (diabetes etc.).

In this research the author can be described as a passive participant. He has extensive knowledge of the manufacturing sector and life science organizations in the country of Saudi Arabia, and that is reflected on developing his contextual knowledge and experience. The declaration of the researcher as a 'passive participant' is based on the definitions below:

- Non-participatory, where there is no contact with the population or field of study

- Passive participation, where the researcher is watching what is going on and acts as a bystander.

- Moderate participation, where the researcher balances participation and objectivity

- Active participation, where the researcher is a member of the group by behaving the same as the population in terms of skills, actions, and customs.

- Complete participation, where the researcher is completely integrated or is a member of the population or field of study (Spradley, 1980; Spradley, 2016).

Such a position is conducive to academic works of a grounded nature where knowledge can be generated by seeing the phenomena then "making sense" of it through a systematic literature review after completing extensive fieldwork (and reaching a point of saturation). It is believed, by academia and the media, that the industry of KSA has no innovative entrepreneurial attitude and this has been unchallenged until this study (Kayed \& Kabir Hassan, 2011). For a long time, the quality and intensity of research and development has been, it is argued by the researcher, underestimated and ignored as a field of study (for industry in general and especially in the life science sector). To some, R\&D is considered unimportant and in general the perception of these kinds of "knowledge activities" are very risky, costly, and uncertain (Kayed \& Hassan, 2011a). In effect, "business" would prefer to avoid these risks of investing in innovation and prefer instead to imitate developed and 
existing products (product, services, processes) with lower risk yet better profit margins and returns. This kind of perspective and attitude dominates managerial and board of directors' views ( Farmer 1959; Kayed \& Kabir Hassan 2011; Khan 2013).

The business view is but one perspective of a national system and the author deliberately sought to understand and attend meetings with people in government institutions as well as private sector organizations to gain a clear insight into the phenomena and the nature of relationships/co-ordination that existed between key stakeholders (known as actors). These actors included the University system itself which is a recipient and developer of innovation for any nation.

\subsubsection{Government orientation}

Beside government's role in motivating and supporting the researcher to undertake the study, government's orientation and policies support the importance of studies of the knowledge economy in general. The rentier economy of the KSA (Beblawi, 1987; Hertog, 2010; 'Rentier State and Shi' a Islam in the Iranian Revolution Author ( s ): Theda Skocpol', 2014) has created serious economic troubles for the government, and undermined the long-term strategies and planning due to the high fluctuation of oil prices over the medium term (more details in chapter 5). As such, this chapter in the history of the KSA is critical as it will potentially rebalance the economy and create a value added sector to support the movement away from purely oil revenues to a sustainable knowledge economy. Regarding this fact, and to address this problem, the Saudi government started to think about developing a knowledge-based economy and diversifying the economy to mitigate its economic vulnerability; this effort is captured in the "Developing vision 2030" document (Mckinsey global, 2015). This national vision has provided further motivation for the author to undertake this kind of research and to contribute to theory building in the KSA knowledge economy and life sciences sector in particular.

\subsubsection{Literature review}

The literature review was critical in helping the author define an initial gap in the body of academic knowledge and develop initial research aims, objectives, and 
questions. It should be noted that this study employs a grounded theory approach and such a review is used only to frame the study and allow field research to begin. Unlike traditional studies, a grounded approach relies upon a much more detailed post hoc literature review to "make sense" of real world/work experiences and to develop an empirically grounded conceptual framework.

According to Cooper (1988a) reviewing academic publications provides a clear taxonomy-based literature review and is a source for developing a fitting methodology to conduct the research (Cooper, 1988b). Amongst many themes and theories, the author reviewed theories such as systems theory, innovation diffusion theory, and human capital theory; topics such as knowledge economy, innovation, innovation systems, life science sector, micro, small and medium enterprises (SMEs), triple helix systems and clusters; case studies about countries transitioned to knowledge-based economies (like South Korea, Singapore and Taiwan), as well as context related sources.

The review constituted an initial conceptual and theoretical framework to guide the field research and form a foundation for this study. It deepened the author`s quality of knowledge and enabled him to define the research aims, objectives, and research questions. To assist with the operationalization of the research, a pilot study was undertaken through in-depth semi-structured interviews, targeting four participants based on their background (purposive sampling of experts). The pilot investigation outputs were insightful and allowed a much clearer and more focused approach to the field research in the KSA during the further phases of the research.

\subsubsection{Research aims, objectives, and questions}

The research aims and objectives that have been developed for this study are:

\subsubsection{The aim}

The aim of this research is to investigate the current state of management practice and progress towards a knowledge-based economy framework in the life science sector in the Kingdom of Saudi Arabia. The thesis will provide practical and applied insights 
into the meaningful areas for economic development in the country. Three objectives were identified to help in reaching this aim, which are as follows:

\subsubsection{The objectives}

- To explore the current system design and engagement of actors in the life science sector of the KSA.

○ To identify shortcomings and challenges in the design of the life sciences sector in the KSA

○ To capture and identify the key requirements for building the life science sector and the potential evolution (future challenges) of the developing LS innovation system that could fit Saudi context.

\subsubsection{Research questions}

From the research objectives three research questions emerged that were supported by the initial literature review:

RQ1: What are the key requirements for developing knowledge economy (KE) in the life science (LS) sector in KSA?

RQ2: What are the challenges in developing a KE in LS in the KSA?

RQ3: What model of LS innovation system best fits the Saudi context?

\subsection{The empirical study}

As not much is known about the Saudi national innovation system, a grounded theory methodology was used to collect and analyse data. To answer the research questions, semi-structured interviews were undertaken with experts from relevant industrial and academic backgrounds and people involved in the life science industry from different sectors (government, academia, and private sectors).

The researcher then conducted coding of the data collected and presented qualitative and quantitative perspectives that addressed the national requirements and the challenges that the combined actors would face in developing the LS sector in Saudi 
Arabia. Based on those, the requirements and challenges, a model for a Saudi LS Innovation System was developed.

To enhance the validity of the study and provide both greater confirmation of the study findings and a deeper more focussed exploration, a questionnaire was developed (triangulation method). The method was designed to give a more quantitative perspective and to explore inhibitors/encouraging variables concerning involving private sectors in the innovation system.

\subsection{Thesis structure}

This thesis is divided into eight chapters: the first chapter is an introduction, the second chapter explores the background theories that have been perceived to have important relevance to the study, the third chapter concerns the focal literature review, the fourth chapter details the research design, the fifth deals with the KSA and context of this study, the sixth describes the findings of collected data, the seventh chapter presents analysis of the findings, and final chapter sets out the conclusions. 


\section{Background theories}

The purpose of this chapter is to present the background theories that underpin this study and provide a means to make an academic contribution. The researcher undertook an initial literature review to help frame the subject and an extensive systematic literature review (post field research) to relate his findings to the extant literature concerning the knowledge economy and context of the life science sector. As such, these chapters will present a logical argument to the reader and they have been written to create a narrative or story from the findings to support the researcher's contribution. The actual methodology and design will be discussed later but to assist the reader, the literature reviews will be presented now.

This chapter will present the theories which underpin the background to the study, which include systems theory, human capital theory, and innovation diffusion theory. As it will be shown, these theories have underpinned many previous Western studies and are considered by the researcher to be the most relevant theories for a study of this nature.

These theories were chosen (amongst other competing theories) for the reasons that the objective of the study is to investigate the LS innovation system in the KSA (a developing country). As such it is important to review systems theory as it has been inspiring and relevant in developing systems, especially in the developing countries where the systematic structure is not very common. Systems theory is at the heart of most studies of national business sectors and of how they can be researched and 'sense made' as to how the parts of the national system work together.

Developing an innovation system requires highly skilled people (for producing technologies, developing innovations, building new start-ups, and doing many other high technical and managerial jobs) and therefore the researcher reviewed the human capital theory. The last theory, innovation diffusion theory has two stages; the first concerns transferring knowledge from the source-academia or any other sources- to 
end in developing products or services, and the second stage concerns in transferring the products (innovations) to the end users.

Based on this proven link and their utility to frame this study, the researcher will present a review of these three theories. The next sections will review each of these background theories in turn.

\subsection{Systems Theory}

According to Kast \& Rosenzweig (1985) a system is "an entity in its own right, with unique properties understandable only in terms of the whole, especially in the face of a more traditional reductionist or mechanistic focus on the separate parts and a more simplistic notion of how these parts fit". As such systems can be organisations or indeed sectors in a national economy. A system has some features that distinguish it from a non-system; the following table presents these features:

Table 2-1: Key features of system theory.

\begin{tabular}{|c|c|c|c|}
\hline $\begin{array}{l}\text { Systems } \\
\text { Feature }\end{array}$ & Definition & Relevance to this Study & Key Authors \\
\hline Viable system & $\begin{array}{l}\text { The capacity for a system and } \\
\text { subsystems to solve problems } \\
\text { and be adaptable and have the } \\
\text { capacity to respond and survive } \\
\text { any significant changes } \\
\text { occurring in the environment, } \\
\text { and to sustain growth. }\end{array}$ & $\begin{array}{l}\text { The innovation landscape } \\
\text { has a global dimension and } \\
\text { features continuous change, } \\
\text { so the capacity to respond to } \\
\text { change is crucial. }\end{array}$ & $\begin{array}{l}\text { (Beer, 1989; Raul } \\
\text { Espejo, 1990). }\end{array}$ \\
\hline $\begin{array}{l}\text { Input-Process- } \\
\text { Output (IPO) }\end{array}$ & $\begin{array}{l}\text { The inputs of universities, } \\
\text { firms, government, banking. } \\
\text { Process includes developing } \\
\text { innovation, investing in people, } \\
\text { planning and feed-forward, } \\
\text { feedback, and collaboration. } \\
\text { Outputs include products, } \\
\text { services, firms, and interactions } \\
\text { with environment, and } \\
\text { adaptation. }\end{array}$ & $\begin{array}{l}\text { IPO is the very essence of } \\
\text { the innovation system, } \\
\text { where the system members } \\
\text { work as a team to produce } \\
\text { value. }\end{array}$ & $\begin{array}{l}\text { Huczynski \& } \\
\text { Buchanan (2010) }\end{array}$ \\
\hline
\end{tabular}




\begin{tabular}{|c|c|c|c|}
\hline $\begin{array}{l}\text { Feed-forward } \\
\text { processes }\end{array}$ & $\begin{array}{l}\text { The collective planning of } \\
\text { system members and its policies } \\
\text { for future assignment of } \\
\text { resources to meet the future } \\
\text { predicted demands of the } \\
\text { environment. }\end{array}$ & $\begin{array}{l}\text { It is important to understand } \\
\text { local policies for system and } \\
\text { the wider innovation } \\
\text { environment dynamics to } \\
\text { adjust accordingly. }\end{array}$ & $\begin{array}{l}\text { Kluger \& Nir } \\
\text { (2010) }\end{array}$ \\
\hline $\begin{array}{l}\text { Broader system } \\
\text { relationships \& } \\
\text { the Internal Fit }\end{array}$ & $\begin{array}{l}\text { The integration and } \\
\text { collaboration of members } \\
\text { especially researchers and } \\
\text { policy makers to satisfy } \\
\text { collective interests within an } \\
\text { IPO process stage within and } \\
\text { across the boundaries of } \\
\text { organizations. }\end{array}$ & $\begin{array}{l}\text { The study of the } \\
\text { organisation as a system } \\
\text { should include various } \\
\text { stakeholder perspectives. }\end{array}$ & $\begin{array}{l}\text { Delery \& Gupta } \\
\text { (2016) }\end{array}$ \\
\hline $\begin{array}{l}\text { Feedback } \\
\text { measurement } \\
\text { processes }\end{array}$ & $\begin{array}{l}\text { Internal skills to manage the } \\
\text { flow and react to deviations in } \\
\text { measured performance. }\end{array}$ & $\begin{array}{l}\text { It is important to assess the } \\
\text { measures adopted and } \\
\text { provide feedback of } \\
\text { performance to staff. }\end{array}$ & $\begin{array}{l}\text { Kast \& } \\
\text { Rosenzweig } \\
\text { (1985) }\end{array}$ \\
\hline $\begin{array}{l}\text { Learning } \\
\text { processes and } \\
\text { capabilities }\end{array}$ & $\begin{array}{l}\text { The ability of the system to } \\
\text { respond to environment } \\
\text { disruptions and use feed- } \\
\text { forward and feedback } \\
\text { information flows to improve } \\
\text { performance over time through } \\
\text { continuous learning and } \\
\text { training. }\end{array}$ & $\begin{array}{l}\text { The mechanisms engaged } \\
\text { by employers to support } \\
\text { staff to learn and improve } \\
\text { are a critical aspect of this } \\
\text { study and the adaptive } \\
\text { capabilities of healthcare } \\
\text { organisations and their staff } \\
\text { (performance management). }\end{array}$ & $\begin{array}{l}\text { Senge (1990; } \\
\text { Senge (2006) }\end{array}$ \\
\hline
\end{tabular}

\section{Source: The Researcher}

Furthermore, systems can be characterized at an international, national and organizational level:

○ International level: the environment that surrounds the national systems that operates in, and made up of all countries.

○ National level: at the national level lies the national system, which is made up of many national key actors such as government institutions, universities, and industry etc.

- Organisational level: this level is made of units and sub-systems inside an organisation. 
Since the International level can be considered as the surrounding environment, this study is interested in national systems and organisational systems.

National systems are made up of organisations, and according to Emery (1969), "Human organisations are living systems and should be analysed accordingly. Management is concerned with the control of social systems, technologies, and markets therefore living systems are essentially 'open' systems, not 'closed' systems." So, in this respect the life science innovation system behaves like any other innovation system (Dunlop John, 1958). Therefore, like in any country, it is acceptable to argue that organisations and the life science sector may be perceived as an open system (with inputs such as universities, banking, government strategies interventions, and firms, etc.) as well as being a process which transfers knowledge through collaborative research and innovation into the workplace and is enveloped in feedforward planning systems by the government, industry and academia (inputprocess-output).

Any system will also adapt and will incorporate learning over time, therefore it will evolve; organisations must change to meet these new circumstances and the operating environment. In terms of the life science sector, feedback is very important and outputs from the system are likely to generate new learning, new products and services therefore reinforcing the link between governments, industry and academia.

An open system interacts with its environment locally and globally to sustain growth and success. The availability of good building blocks is important, however, in order to have an effective system, interactions and synergies among all components need to be established, to optimize emergent property ${ }^{1}$ and outcome by virtue of organic unity (Checkland \& Poulter, 2010).

\subsubsection{Systems theory and relationships}

Individuals develop many kinds of relationships: adversarial, competitive, trust and collaboration. However, collaboration and trust are essential for innovation in a

\footnotetext{
${ }^{1}$ What is said to be a system must have some properties as a single whole, so-called emergent properties
} 
system at least initially and in developing economies (such as the life sciences sector in the KSA).

The organisational system as a controller of its relevant environment can only respond to a relatively small number of possible states in this environment, but uses agents in this environment to respond indirectly to all the others. It is this capacity to collaborate with others and support action in the shared environment that allows the organisational system to deal with a much larger variety than otherwise would be the case (Raúl Espejo, 2003). As such, it is likely that any actor in the KSA will deliberately seek to collaborate with others to lower risks and to exploit value from the life science processes that lead to profitable innovations.

According to the triple helix perspective, the interactions between the three key actors (government, academia, private sector) can create new combinations of resources and knowledge that enhance innovation practice (Hausmann et al., 2014). Governmentprivate sector-academia relations are key component of any national or even multinational innovation plan. The collaboration between industry and institutions of fundamental research reflected on technologies studies and policies (Etzkowitz, 2003). The complex interactions between the system actors allow each actor to assume the other roles in a way that facilitates the innovation process (Leydesdorff \& Etzkowitz, 1998). These cross-boundary relationships among actors requires building and maintaining trust to avoid any probability for conflicts (Ferraro \& Borroi, 1998). So, the collaborative nature of relationships in the national system and among the actors is very important and trust, especially at the early stage of development of national system such as the Kingdom of Saudi Arabia, is critical. The reason why collaborative relationships are needed is because the system is not optimised and therefore each actor must engage in a form of trust with each other in order to optimise the system and develop the necessary policies, structures and practices (Checkland, 2000; Raul Espejo, 1990).

There is a lack of studies regarding the relationships between actors within national and organisational systems of developing countries (Gu, 1999), and the resulting nonoptimised system show a gap in the body of knowledge concerning systems theory, the life sciences sector and developing countries. 
Optimisation of the system is likely to require the application of specific policies, structures, practices that maintain continuous learning and an adaptive behaviour that responds to the changing environment to help in developing the required competitive advantages. The sector itself (all actors) must be aware of and react to these environmental signals if adaptation is to happen and gains (win/win for all actors) are to be exploited and a system developed in an effective manner.

The great success of any system is strongly affected by the level of interactions and harmony among system elements, so the aim is to optimize the parts of the system, in turn optimizing the whole system (Checkland \& Poulter, 2010). This discussion further highlights the importance of adopting a systems theory approach to tackle this research consisting of many subsystems in different sectors such as academia, industry, and government, if a life science innovation system is to be created.

\subsubsection{System transparency}

Spreading the awareness about the potential benefits of adopting systemic approaches is presented in the academic literature as very important. System leadership in organisations and often in developing countries rarely uses sophisticated management tools and instead founds decision on the leaders`experiences and intuition. Such experience is often shaped by interactions with other actors and conducted without awareness of models that can help in solving problems, improving outcomes, and dealing with different disruptive external variables. In short practical issues to exploit knowledge are not grounded on a knowledge and implementation of a dominant model for every economy.

According to Forrester (1994) there are three major benefits of a system approach when studying a phenomenon such as the life sciences sector and actors in a developing country:

- "Systems thinking can alert the public to systems as the cause of puzzling pathologies in business and social activities, but the wise person will not use such superficial knowledge as a basis for corrective action”.

- "Systems thinking can serve a constructive role as a door opener to system dynamics and to serious work toward understanding systems" 
- "On the other hand, unquestioning and superficial enthusiasm for systems thinking may lead some people into trouble. Some people attain enough revealing insights from systems thinking that they feel the need for nothing else. Such people are in danger of finding that systems thinking does not help in solving their problems, or worse, that they take ill advised actions that make matters worse".

An environment that surrounds system actors that is complex, unfolding and where organisations respond to complexity to co-create organisational and sector structures is often underpinned by collective action and shared models of the future with a common purpose and relationship values (hence collaboration for mutual gains). To achieve this level of transformational change in a sector of actors and organisations, transparency is needed so that stakeholders can optimise the organisational qualities that result in better performance of the sector (Raúl Espejo, 2003).

From 2.1.1 and 2.1.2 above it can be perceived that a national system of knowledge has features including the following:

o the mutual recognition of each other`s actors efforts towards a collective goal;

o developing collaboration and trust among system actors to share risks and gains;

○ transparency so that the intentions and actions of actors/stakeholders are known (including signalling of actions that will be taken - such as investments in the sector or ways of reducing complexity and risk); and,

○ interacting with the environment for mutual learning (information processing) so that actors can contribute and shape the environment for future success.

During this conceptualization stage, of the KSA life sciences sector, a specific framework or system methodology was designed to help guide this research and for the researcher to identify the most salient system models that allow the sector to be studies as a Viable System Model (VSM). 


\subsubsection{Viable system model (VSM)}

The term "viable system model" used for research diagnosis and improvement/learning/redesign was inspired by the human nervous system structure (Beer, 1984). The British pioneer in cybernetics, Stafford Beer described this model in three of his books Brain of the firm (1972), The Heart of Enterprise (1979), and Diagnosing The System for Organizations (1985). According to Umpleby (2007), this model was developed based on the theory of adaptive behaviour and the law of requisite variety from Ross Ashby in his book An Introduction to Cybernetics (William Ross Ashby, 1956). Requisite variety is simply the level of organization complexity and ability to respond to the changing environment; from this definition, the variety of responses should be at least equal to the variety of disruptions (Ross Ashby, 1991; Beer, 1989).

Historically organization structures are Hierarchal, and this structure prevents an organization from surviving the changes in the environment that surrounds it and from working effectively. Rejection of this perspective gave rise to the viable systems model, which is embedded by the concept of structural recursion which postulates autonomous units and systems that have the capacity to solve their own problems by developing policy and regulation by implementing activities (Raul Espejo, 1990). In this sense, the researcher believed that a complex and uncertain environment in the KSA life sciences sector would result in greater transparency, greater relationship building and flatter (less hierarchal) structures (to improve the speed of decisionmaking in response to a quickly changing environment).

In the next section two mechanisms to provide viability for systems are presented.

\subsubsection{Viability mechanisms}

To make a system viable, two key mechanisms should be in place and activated. The first one is to secure the cohesion among organization members where the individual and collective interests are not necessarily the same but aligned. The second one is to provide the capacity for organization systems and units to be adaptable and to have the capacity to respond to any significant change in the environment (Raul Espejo, 1990). 
VSM is an adaptive system, which considers the management of variety as a key feature. The system features five functions which provide it with the necessary conditions for viability (Beer, 1985; Raúl Espejo \& Gill, 1997).

\subsubsection{VSM functions}

For VSM in the KSA life sciences sector, five key conditions must be met with the system to function effectively. The five key management functions are: implementation, coordination, control, intelligence and policy:

- Implementation: implementing the primary activities, which are the activity that an organization provides (its products or services) to the value chain ${ }^{2}$ of the organizations of key actors in the environment. Primary activities are complex in nature and therefore there is a need to unfold this complexity and to find out the organizational processes that make this a viable system.

- Co-ordination: through this task it should be assured that there is no conflict or confusion for implementing the primary activities, otherwise process bottlenecks, failed production planning and other problems may occur. Examples: developing schedules, timetable etc.

- Control: concerns developing two-ways communications between system levels, negotiating resources, evaluating performance and providing accountability reports for the upper level

- Intelligence: this includes the mechanisms for adaptation and capacity for change with respect to the change that might take place in the environment and the surroundings; this capacity should be in all sub-systems of the organization. Research and development, marketing and forward planning are example of the intelligence activities.

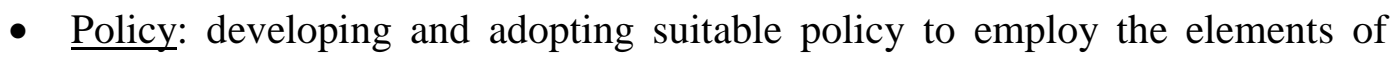
cohesion (interest in inside organization and the "now"), and elements of intelligence (interest in looking outside and explore the future) (Beer, 1985; Raúl Espejo \& Gill, 1997).

\footnotetext{
${ }^{2}$ Definition: "the productive (i.e. value added) activities that lead to and support the end use of a set of related products or services, including lead firm(s)".

Metrics: "the bundles of activities that various actors do, or do not, engage in" (Sturgeon, 2001).
} 
The above five system principles reinforce mutual working and good relationship development and were considered by the researcher as a means to help frame the model resulting from the research. After the field research, great utility was found in looking at the KSA life sciences sector as a dependent population of actors with a common intent yet the findings would later show major issues with the sector and its function as a system in the KSA. The author argues that, from the previous statements, it would be possible to review the KSA LS sector and the actors of the national system using this approach and to use VSM to found the author's model and contribution to the academic literatures.

The research supports the view that "...VSM is an extremely fast and precise tool for both diagnosing weaknesses in existing organizations and designing new organizational structures. It can and has been used successfully on organizations of all types and all sizes from the small team to the nation state" (Hoverstadt \& Bowling, 2002). However, in spite of many consultants using the VSM for diagnosing the weaknesses of organizations to improve their efficiency, few organizations have applied it as a formal structure. Other methodologies such as system dynamics can be used together with the VSM (Umpleby, 2007). To the best of the researcher`s knowledge this is the first application of VSM to the study of the life sciences sector, previous Asian models have hinted at, but not explored the system design itself. Thus, grounding the study in this manner allows a contribution to be made to the study of innovation systems which have tended to focus only on the views of the government as a key actor (but has excluded universities and private manufacturing businesses).

\subsubsection{Summary}

It is believed that in developing countries, the existence of systematic structure, aligned behaviour and actor engagement in a system is weak and that it is reflected in poor collective and individual performance of actors (rather than collaboration to improve the entire system or sector upon which every actor is dependent). Developing a systemic structure is considered a crucial step for innovation systems (often cited by previous studies that have focused on the role of the government) as it enables many functions, such as: implementation, co-ordination, control, intelligence and policy 
development, to take place, in turn leading to raising efficiency and performance, and providing viability conditions (central planning).

The interaction of a system with the surrounding environment provides learning and new knowledge that would lead to the development of a mature system, which can later contribute to shaping the environment as systems and structures mature, become more formalised and align with national policies and investments.

\subsection{Human Capital Theory}

\subsubsection{Introduction}

Developing a system requires a continuous learning process and the building of the required variety of organisations so that success will lead to the survival and improved efficiency/effectiveness of each actor. This in turn requires different and complex high capabilities, necessary to run the system to the required quality levels; this implies the selection, retention and development of scarce skills - human capital. In this manner the researcher found it of a great use to view the life science system from the perspective of human capital and value development.

A system, such as an LS innovation system, requires the availability of high skilled people within each key actor (government, academia, industry), to perform relevant highly sophisticated activities in order to exploit innovations and create a virtuous cycle of benefits. From this point of view, reviewing the human capital theory is perceived as important and very relevant to this study.

In the 1950 s emerging theory presented the view that human capital was a product which had the potential to lead to greater returns on investment for any organisation. Previously production factors were perceived as including labour, land and physical assets (Becker, 1993) but knowledge of workers has been clearly linked to higher system performance - especially for mature economies. Based on these traditional production factors, relevant studies could not explain the total contributing factors for economic growth in the USA, and that study found a gap which was later explained by the role of human capital (Schultz, 1961). 
At this point it is helpful to define what is meant by the term human capital theory. "Human capital is the knowledge and skills that people acquire through education and training being a form of capital, and that this capital is a product of deliberate investment that yields returns" according to Schultz (1961). It is also argued "human capital is a form of investment by individuals in education up to the point where the returns in extra income are equal to the costs of participating in education. Returns are both private to the individual in the form of additional income, and to the general society in the form of greater productivity provided by the educated" (Becker, 1962, 1975). And from later economists "human capital is acquired human capabilities that are durable traits yielding some positive effects upon performance in socially valued activities" (David \& Lopez, 2001).

From these embedded definition and principles, which treat human capabilities as assets with values like other conventional production inputs, investing in this new form of emerging capital is an interesting subject area as it has impacts on system performance and has been linked to economic growth. In the next section the relevant aspects of investing in human capital will be explored.

\subsubsection{Investment in human capital}

As mentioned in the previous section, production factors and the role of human capital were identified as key components within innovation systems. However, these human assets should also be employed effectively, in order to impact profitably the individuals, organizations and society (Schultz, 1961). Schultz considers expenditure on health and education and the cost of internal migration as a kind of investment in human capital, and as individuals invest in themselves the opportunities and remunerative income increase. This view has deep-seated references in the history; according to (Marshall, 2009) "The most valuable of all capital is that invested in human beings". So, production inputs have human and non-human capitals, and investment in human capital is positively linked to economic growth, which has lucrative returns on individuals, and society. When we compare globally the South (poor countries) to the North (rich countries) or the West (rich countries) we find a big difference in terms of average of earnings in both the West and the North, which can be explained by the differences in investment in health and education in the 
South, compared with the North and West. The rate of increase in the USA income overtook the rate of increase in the conventional production factors, and that can only be attributed to the contribution of human capital generating greater levels of added value to the economy (Schultz, 1961). So investment in education is an important factor for raising population production capacity, which means an educated population has more productive people (Olaniyan \& Okemakinde, 2008). For the KSA such investment would be necessary to exploit the benefits of a life sciences system of innovation and revenues (including taxes).

\subsubsection{Education and human capital}

The investment in human capital comes in five forms: education or schooling, on job training, internal migration, health, and study programmes (Schultz, 1961). However, "education and training are the most important investment in human capital" (Becker, 1994). According to human capital theory, key capabilities and assets, such as knowledge, skills and problem solving, can be provided by education and positively impact income and productivity. However, earnings are linked basically to the traits of people and level of productivity they have, so such individuals can benefit more from schooling. Firms are usually looking for the quality of performance at work, with no high special regard for credentials or certificates (Becker, 1994). This view posits an increased need to understand the criteria and issues around the educational process investment and the investment in human capital, especially when countries such as some developing countries spend a lot on education without producing matching returns.

Investment in human capital should have three positive implications: people assimilate the accumulation of knowledge created by past generation; people build on that knowledge to develop new knowledge and ideas in the form of products, services or production methods; and finally, people should be prompted to generate totally new knowledge in form of ideas, products, services or processes (Babalola, 2003).

Based on the promising outcomes that human capital theory assumes, this investment is justified and requires huge public expenditures on education whether in developed or developing countries. However, for significant economic value related to the investment in education, the equilibrium position should be sought so there is no unnecessary investment, which could ultimately result in unemployment, and on the 
other hand that shortage of human capital is avoided, to prevent limiting the economic growth (Babalola, 2003). Also, the education cannot work in isolation, which means that other structural reforms should be put in place (Fägerlind \& Saha, 2016). So, economic gains, particularly in developing countries, where the quality of education is low and also political intervening factors affect the education system (Bronchi, 2003; Crepaz \& Moser, 2004), would not solely be a result solely of investment in education.

There are many examples where nations have managed to achieve significant economic growth and development because of prudent and substantial investment in education, such as South Korea, Singapore, and Taiwan - what are known as the “Asian tigers" (Wong, 1999). To ensure the effective economic value of investment in education, strategies should be aligned and developed with respect to specific local advantages and a country's priorities.

Table 2-2: Key themes of human capital theory.

\begin{tabular}{|c|c|c|}
\hline Theory themes & Importance to innovation system & Key Author \\
\hline Human capital & $\begin{array}{l}\text { Treating human knowledge as an asset and } \\
\text { an important factor in the inputs of } \\
\text { production equation based on the concept } \\
\text { of knowledge economy. }\end{array}$ & ( Schultz, 1961). \\
\hline $\begin{array}{l}\text { Investment in } \\
\text { human capital }\end{array}$ & $\begin{array}{l}\text { The investment in human capital comes in } \\
\text { five forms: education or schooling, on job } \\
\text { training, internal migration, health, and } \\
\text { study programmes }\end{array}$ & $\begin{array}{l}\text { (Schultz, 1961; Becker, } \\
\text { 1962). }\end{array}$ \\
\hline $\begin{array}{l}\text { Quality of education } \\
\text { system }\end{array}$ & $\begin{array}{l}\text { Economic gains linked to the quality of } \\
\text { education system. }\end{array}$ & $\begin{array}{l}\text { (Bronchi, 2003; Crepaz \& } \\
\text { Moser, 2004). }\end{array}$ \\
\hline
\end{tabular}

Source: The researcher.

In summary, education and the building of human capital are connected to knowledge transfer and innovation and diffusion concepts. It is also true that any system will not adapt and improve performance unless skills are upgraded and enhanced to add value to sectors such as the life sciences especially in developing economy systems. The next section will review the importance of innovation diffusion theory. 


\subsection{Innovation Diffusion Theory}

The ultimate objective of innovation is to make an economic impact and return on invested capital from knowledge transfer and marketing of innovation. Studying patterns and structures that enable innovation diffusion is therefore crucial for the success of innovations and of any system which involves high levels of human capital investment. The inherent characteristic of innovation is its "newness" which is a certain form of uncertainty and tends to attract early adopter companies who seek to be first to market and exploit innovations from a system. In his seminal book Diffusion of Innovations (Rogers, 1983), Rogers proposed the theory of innovation diffusion, which addresses the adopters' categories and the characteristics of innovation that affect the rate of adoption. Rogers ' model is one of the most wellknown and popular concerned with the adoption behaviour for new innovations (Sherry \& Gibson, 2002). This model has been applied as a theoretical framework in many research disciplines such as economics, politics, communications, public health, history and technology adoption (Dooley, 1999; Stuart, 2000). Although Rogers' model has been widely accepted it is a business view and tends to ignore the role of universities and governments. The latter actors are necessary for a systems approach and for the necessary development of a sector. The model is useful though in taking another non-government approach to the subject of innovation - and supports the multiple perspectives taken by this study - which has, as an objective, the need for a multiple stakeholder/actor view of an emerging system within a developing economy. Before going into detail about innovation diffusion it is very important to introduce a closely related concept: that of knowledge transfer.

\subsubsection{Knowledge transfer}

According to Singley \& Anderson (1989), knowledge transfer is concerned with R\&D activities and the transferring of the output to the end users. Developing innovation usually originates from $\mathrm{R} \& \mathrm{D}$ activities that diffuse the innovation to potential adopters (Rogers, 2007). The reflection of knowledge transfer results from a change in performance and knowledge of the recipient, and the creation or transfer of knowledge is a key thing for developing a competitive advantage. Knowledge transfer in organizations "is the process through which one unit (e.g., group, department, or division) is affected by the experience of another" (Argote \& Ingram, 2000). 
Knowledge is perceived as transferred when a recipient understands the intricacies and implications attached to the knowledge so it can then be applied (Argote, 2012; Darr \& Kurtzberg, 2000). Another definition for knowledge transfer is provided by Szulanski (1996): “dyadic exchanges of organizational knowledge between a source and a recipient unit in which the identity of the recipient matters". At the individuals level, Singley \& Anderson (1989) define knowledge transfer as "how knowledge acquired in one situation applies (or fails to apply) to another". In developed or developing countries, knowledge transfer activities are seen as one of the government responsibilities whereas adoption is seen as a business responsibility (Rogers, 2007).

\subsubsection{The agricultural extension model}

All knowledge transfer models were inspired by the agricultural extension model (industrialisation after rentier farming) as this was historically the first to be theorised and subsequently influenced the thinking around this area of study. There are eight components that comprise the agricultural extension model:

1. Critical mass: this represents the smallest amount of innovation that has commercialization potential,

2. Market orientation researches: promoted by policy that provides incentives and a motivation system for researchers,

3. High degree of involvement: users have a significant role in developing policy that guides research orientation, and in feeding back to the research system,

4. Structural linkages among the research utilization system`s elements, working like an organic unit,

5. A high degree of customer contact,

6. The sustaining of social aspects and linkages across the system units,

7. Knowledge transfer as a complete system, instead of being a component in the research system, and, a

8. Proactive system: the system is designed to shape the environment around the system not the opposite, so it is prepared for any changes in the industry.

These eight components are flexible and can be adapted when extended to other knowledge transfer systems, but in order to obtain a successful knowledge transfer system the foundations should be set based on the needs of users rather than those of the researcher, and the goal of transferring knowledge should be very clear at the 
outset (Rogers, 2007). There are two paradigms on knowledge transfer: centralized and decentralized, but in reality there is no complete centralized or decentralized knowledge transfer, but a mix of them in dynamic equilibrium. The next table shows the differences between the two paradigms. 
Table 2-3: Centralized and decentralized knowledge transfer paradigms. Source: Adapted to diffusion of innovation systems (Rogers 1983).

\begin{tabular}{|c|c|c|}
\hline Diffusion characteristics & Centralized diffusion systems & $\begin{array}{l}\text { Decentralized diffusion } \\
\text { systems }\end{array}$ \\
\hline $\begin{array}{l}\text { Decision making: degree of } \\
\text { centralization. }\end{array}$ & $\begin{array}{l}\text { Controlled by national } \\
\text { government and technical } \\
\text { experts. }\end{array}$ & $\begin{array}{l}\text { Shared decision among the } \\
\text { diffusion system actors }\end{array}$ \\
\hline Diffusion direction & $\begin{array}{l}\text { Vertical diffusion from experts } \\
\text { to customers. }\end{array}$ & $\begin{array}{l}\text { Horizontal collaborative } \\
\text { networks. }\end{array}$ \\
\hline Sources of innovation. & $\begin{array}{l}\text { Based on R\&D conducted by } \\
\text { technical experts. }\end{array}$ & From non-experts, like users. \\
\hline Innovation diffusion decision. & $\begin{array}{l}\text { Made by top management } \\
\text { and technical experts. }\end{array}$ & $\begin{array}{l}\text { Based on clients' needs and } \\
\text { evaluations of the innovations. }\end{array}$ \\
\hline $\begin{array}{l}\text { To what extent clients' needs } \\
\text { are important in driving the } \\
\text { diffusion process? }\end{array}$ & $\begin{array}{l}\text { An innovation-centred } \\
\text { strategy: technology-push, by } \\
\text { creating need for the } \\
\text { innovations }\end{array}$ & $\begin{array}{l}\text { A problem-centred strategy: } \\
\text { technology-pull, based on local } \\
\text { needs and problems. }\end{array}$ \\
\hline Amount of re-invention? & $\begin{array}{l}\text { A low degree of local re- } \\
\text { invention of the innovations by } \\
\text { adopters }\end{array}$ & $\begin{array}{l}\text { A high degree of local re- } \\
\text { invention by adopters. }\end{array}$ \\
\hline
\end{tabular}

Again the table reinforces the need for collaboration (vertical and horizontal) as well as dependency between actors in the system and a high reliance on the exploitation of expert knowledge (high human capital). These features firmly convinced the researcher that these lenses and approaches were suitable means for studying the complex and uncertain life science systems in operation in the KSA.

\subsubsection{Knowledge transfer mechanisms}

In some countries, such as emerging economies, where there is a lack of local capability to survive global competitiveness, local firms are looking to acquire those capabilities and valuable skills from multi-national companies (MNCs) and to transfer knowledge through developing joint ventures (Lane, Salk, \& Lyles, 2001; Tsang, 2002). Transferring knowledge can occur by developing strategic alliances or building joint ventures (Powell, Koput, \& Smith-Doerr, 1996); acquisitions and mergers also can help fill the knowledge gap (Haunschild \& Miner, 1997). Other 
mechanisms for knowledge transfer have been described, such as training, communication, and labour mobility (Argote, Ingram, Levine, \& Moreland, 2000).

Networks help organizations to access markets, resources, technologies or knowledge. Enduring and robust relationships among organizations result in exchanging knowledge (Inkpen \& Tsang, 2005). Networking comes in three types: within an organization, between organizations in different or the same geographies (strategic alliances), or within a cluster. However, there are some factors that effectively influence knowledge transfer within networks; those factors and conditions include mainly absorptive capacity and social capital. Absorptive capacity helps the recipient to assimilate and effectively understand transferred knowledge and to build on it. Social capital provides motivations and increased willingness for collaboration and transfer of knowledge (Reagans \& McEvily, 2003). R\&D helps significantly in building absorptive capacity (Cohen \& Levinthal, 1990). According to Minbaeva et al. (2003) and Martin \& Salomon (2003) social aspects play a significant role in transferring knowledge, especially in turbulent environments (mainly through the medium of collaboration). Social aspects are supposed to attach to commercial activities to develop relational embeddedness (Uzzi \& Lancaster, 2003). So it is really important to pay serious attention and to exert special efforts to develop strong ties, trust, shared systems and values to facilitate and enhance knowledge transfer processes between foreign parent $\mathrm{MNC}$ and the joint venture (Dhanaraj, Lyles, Steensma, \& Tihanyi, 2004).

\subsubsection{Innovation characteristics}

There are some characteristics of an innovation which affect the innovation decision process and adoption. "Individuals' perceptions of these characteristics predict the rate of adoption of innovations" (Rogers, 2010). According to Rogers in his model an innovation has five attributes that influence adopter`s decision and diffusion process

- Relative advantage: "the degree to which an innovation is perceived as being better than the idea it supersedes" (Rogers, 2010). He believes that this characteristic is the most influential on the rate of adoption. Relative advantage includes the innovation aspects, cost, and social status (Sahin, 2006). 
- Compatibility: "compatibility is the degree to which an innovation is perceived as consistent with the existing values, past experiences, and needs of potential adopters" (Rogers, 2010). So as compatibility increases the rate of adoption increases. Here we should think about the context in terms of needs and values to see if potential innovations meet these determinants.

- Complexity: "the degree to which an innovation is perceived as relatively difficult to understand and use" (Rogers, 2010). If complexity increases the rate of adoption decreases, so there is a negative correlation.

- Trialability: "trialability is the degree to which an innovation may be experimented with on a limited basis" (Rogers, 2010). Trialability gives a chance to potential adopters to test innovations and reduce the level of uncertainty, and that helps in raising the rate of adoption.

- Observability: this characteristic also been defined by Rogers (2010) as "the degree to which the results of an innovation are visible to others" this concept also been confirmed by (Parisot, 1997); for technology diffusion a role model can help in the adoption process as a key motivational element.

According to Rogers (2010) innovation that increases the levels of relative advantage (simplicity, trialability, compatibility, and observability) will have a better rate of adoption. To achieve this increased rate of adoption, an economic sector such as the life sciences within the KSA would need to have collaborative relationships and a stock of innovations emanating from business or academia in order to support such knowledge transfer. The most prized of all such transfers would be the movement of academic knowledge into the exploitation of life science products for businesses resulting in new funds for more $R \& D$ or academic research. In this manner the model supports an active role for university staff in the dissemination process and as catalysts for innovation.

\subsubsection{Adopters categories}

After presenting the factors that influencing the adoption decision we come to the adopters`classifications with respect to their order in time of adoption. According to (Rogers, 2010), the adopters` categories are "the classifications of members of a social system on the basis of innovativeness"; he also defined innovativeness as "the 
degree to which an individual or other unit of adoption is relatively earlier in adopting new ideas than other members of a system". This innovativeness was considered as the classification tool for innovation adoption among individuals. Based on this, Rogers proposed the following figure (Figure 2-1) to express normal distribution for the categories of adaptors. However, Rogers`categories assume that all products follow the same normal distribution patterns. This assumption has been disputed by Peterson (1973) as he said new products follow non normal distribution patterns. Also he disputes the size of categories as they adopted a fixed percentage, for example $2.5 \%$ for innovators, $13.5 \%$ for early adaptors, etc. but there is no empirical evidence that could confirms that.

Moreover, according to the Bass innovation diffusion model the adopters`categories are not the same for all innovations and the grouping process of categories is unique for every innovation (Bass, 1969). However, the interpersonal interactions behaviour and its effect on the diffusion process that was proposed by Rogers is used by many innovation diffusion models including the Bass model (Mahajan et al., 1990).

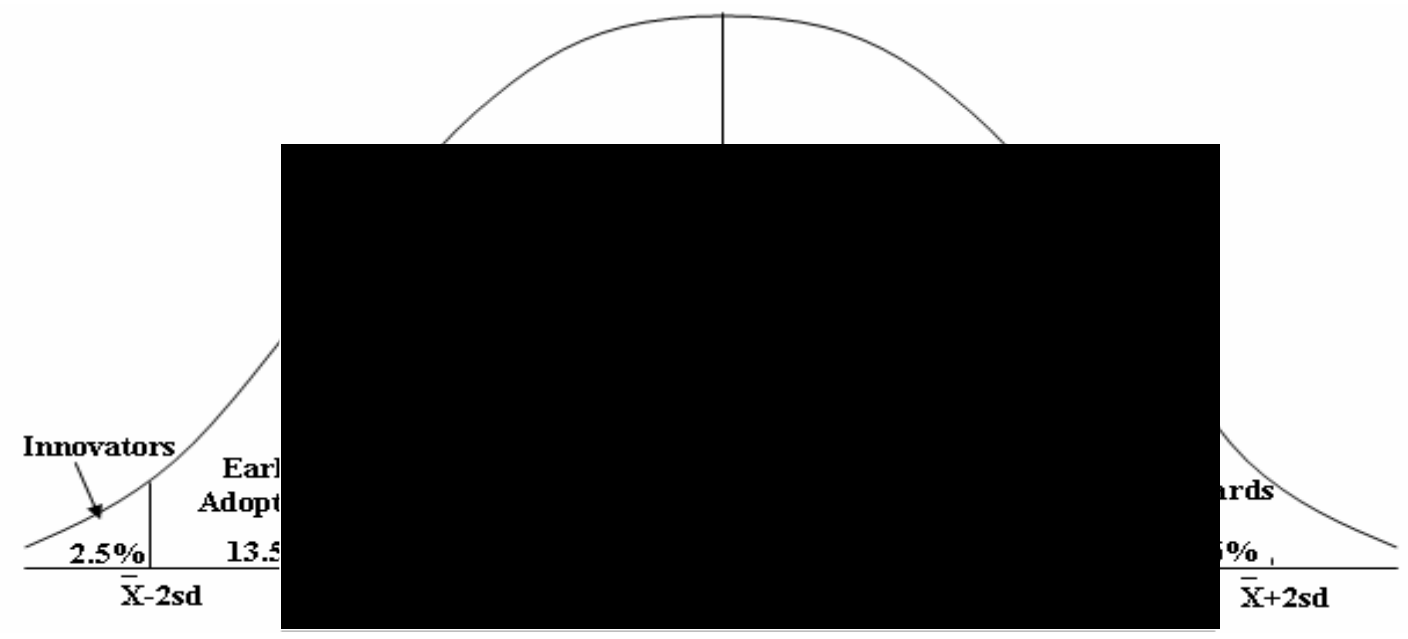

Figure 2-1: Adaptor classification with respect to innovativeness.

Source: diffusion of innovation, fifth edition, 2003)

According to Rogers (2010) the adopters have different characteristics: innovators are venturesome, early adopters are opinion leaders or role models and the more influential in the social system, early majority are deliberate, late majority are sceptical about the benefit of innovation, and lastly the laggards are traditional and 
take a long time to make sure about the value of an innovation and wish to know the impressions and experience of other people who have used it.

\subsubsection{Innovation-decision process}

Rogers (2010) proposes that diffusion is "the process in which an innovation is communicated thorough certain channels over time among the members of a social system". This definition contains four elements that constitute the diffusion of innovation: innovation, communication channels, time, and social systems. The adoption process go through a process called innovation-decision which been defined by (Rogers, 2010) as "an information-seeking and information-processing activity, where an individual is motivated to reduce uncertainty about advantages and disadvantages of an innovation". As shown in the next figure, he mentions five steps for the innovation-decision process, shown in time-based order. 
COMMUNICATION CHANI

PRIOR

CONDITIONS

1. Previous practice

2. Felt needs/problem

3 . Innovativeness

4. Norms of the socia systems
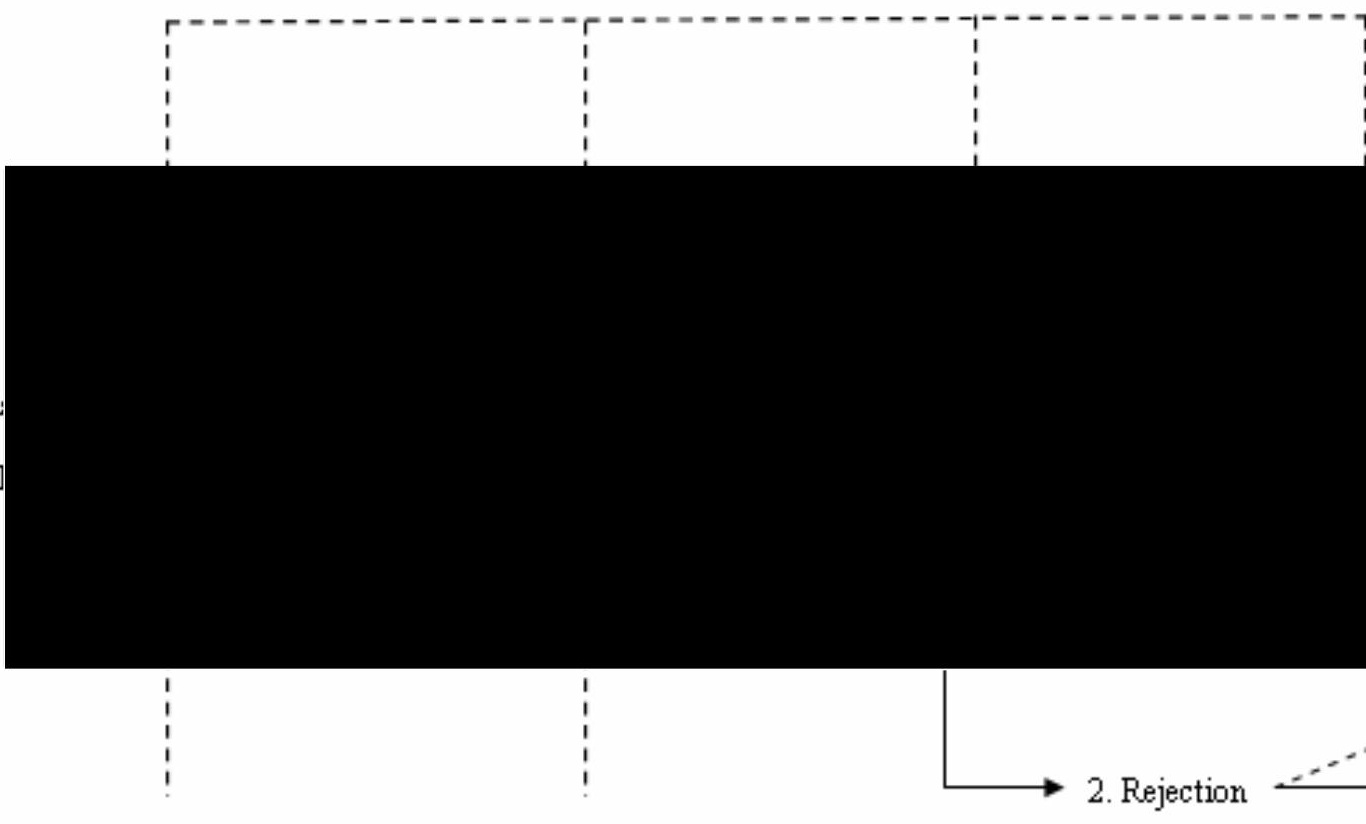

Characteristics of

Perceived Characteristics

the Decision-

of the Innovation

Making Unit

1. Relative advantage

1. Socioeconomic

2. Compatibility

characteristics

3. Complexity

2. Personality variables

4. Trialability

3. Communication behavior

$$
\text { behavior }
$$

Figure 2-2: Innovation-decision process (Source: Diffusion of Innovation, fifth edition, 2003) 
- Knowledge stage: this stage is a more cognitive stage, when the individual is seeking knowledge about the existence of an innovation (awareness knowledge), how the innovation works (how knowledge: according to Rogers this knowledge is very important in the innovation decision process), and what the purpose is or why he uses it (principles knowledge). Having all these different kinds of knowledge does not necessarily lead to adopting the innovation, which depends also on the individuals`attitudes which influence their opinions and decisions (Sahin, 2006).

- Persuasion stage: in this stage the individual starts to reduce uncertainty and constitutes and shapes his opinion, affected by his own knowledge and effect of the knowledge of others. "While information about a new innovation is usually available from outside experts and scientific evaluations, teachers usually seek it from trusted friends and colleagues whose subjective opinions of a new innovations are most convincing"(Sherry, 1997)

○ Decision stage: at this stage the individual takes decision to adopt or reject. However, techniques like partial trials or vicarious trials help in adopting innovation (Sahin, 2006). In some cases or contexts such as Eastern countries where there is a collectivistic culture, the order of the innovation-decision process change from knowledge-persuasion-decision to knowledge-decision persuasion, because of the effect of collective opinion (Rogers, 2010).

- Implementation stage: at this stage the innovation is put into practice, and there is should be a kind of technical support and assistance that help in reducing uncertainty. Innovation-decision will end when "the innovation losses its distinctive quality as separate identity, of the new idea disappears" (Rogers, 2010). Innovations that are more open to reinvention are more rapidly adopted; reinvention means can be modified to suit different applications and uses, such as the computers (Rogers, 2010).

- Confirmation stage: this the last stage in the innovation-decision process. The adopter should look for supporting messages that confirm his decision; otherwise conflicting messages may reverse his decision. So at this stage the adopter`s attitude and supporting messages affect the continuity of adoption (Rogers, 2010). 
Therefore, in summary, the various stages of knowledge transfer which result in an actual product (the second stage of innovation) suggest that these stages need to be formalised and involve all actors: the government to support such a system, academia to offer innovations and an engaged private sector keen to exploit the innovations and make a return. The next table gives ideas about the key concepts related to the innovation diffusion theory.

Table 2-4: Key concepts related to the innovation diffusion theory.

\begin{tabular}{|l|l|}
\hline Themes & Explanation \\
\hline Innovation diffusion & $\begin{array}{l}\text { The process through which an innovation is communicated } \\
\text { thorough certain channels over time among the members of a } \\
\text { social system (Rogers, 2010). }\end{array}$ \\
\hline Innovation characteristics & $\begin{array}{l}\text { Individuals' perceptions of these characteristics predict the rate of } \\
\text { adoption of innovations (Rogers, 2010). }\end{array}$ \\
\hline Relative advantage & $\begin{array}{l}\text { The degree to which an innovation is perceived as being better } \\
\text { than the idea it supersedes (Rogers, 2010). }\end{array}$ \\
\hline Compatibility & $\begin{array}{l}\text { The degree to which an innovation is perceived as consistent with } \\
\text { the existing values, past experiences, and needs of potential } \\
\text { adopters (Rogers, 2010). }\end{array}$ \\
\hline Complexity & $\begin{array}{l}\text { The degree to which an innovation is perceived as relatively } \\
\text { difficult to understand and use (Rogers, 2010). }\end{array}$ \\
\hline Trialability & $\begin{array}{l}\text { The degree to which an innovation may be experimented with on a } \\
\text { limited basis (Rogers, 2010). }\end{array}$ \\
\hline Observability & $\begin{array}{l}\text { The degree to which the results of an innovation are visible to } \\
\text { others (Rogers, 2010). } \\
\text { advantages and disadvantages of an innovation (Rogers, 2010). }\end{array}$ \\
\hline An information-seeking and information-processing activity, \\
\hline
\end{tabular}

\section{Source: The researcher}

Finally, it is believed that these theories, namely system theory, human capital theory and innovation diffusion theory represent the theoretical framework and the underlying threshold pillars for this research, they have been discussed in not too much detail as that would be outside the scope of the study. It is believed that this brief review is enough and satisfies the objective for this chapter. 


\subsection{Chapter Summary}

In this chapter, three relevant theories have been reviewed: systems theory, human capital theory and innovation diffusion theory. These theories constitute the theoretical foundation for this study. This study concerns the development of a life science innovation system in the Kingdom of Saudi Arabia; this system is supposed to have many organizations and members such as universities, firms, and government working and collaborating to align their different interests and achieve collective aims. For that reason there is a strong rationale to adopt a systems theory in developing the Saudi LS innovation system, to consider collective work relationships, sharing of decision-making and how different perspectives (actors) are aligned so that human capacity can be exploited and continuous learning can occur. As such the system will learn and improve the efficiency with which innovations are exploited enabling the system to respond to the changing environment and to exploit innovation the infrastructure and landscape. Only by this form of mutuality, transparency and value exploitation will a systems approach yield economic value.

With respect to the concept of systems adaptation and "continuous learning", system theory intersects with the human capital theory as the innovation system would not work effectively without high quality and highly skilled people - both researchers and managers, in turn developing new innovations; therefore the investment in human capital is an inevitable requirement for developing innovation systems. Following on from the development of innovations it is necessary to study its origination and diffusion, so that suitable strategies can be well perceived and developed. The researcher is of course aware that, due to the abundance of theories and theoretical approaches, there are other theories that could have some relevance and contribute, to some extent, to the theoretical foundation for this thesis; however it is perceived that, in light of the aforementioned considerations, the three theories reviewed in this chapter (system, human capital, and innovation diffusion theory) satisfy the theoretical basis for this thesis. 
Table 2.5: Key Issues for the three theories

\begin{tabular}{|c|c|}
\hline Key Issue for an effective system & Theory \\
\hline $\begin{array}{l}\text { Actors must be aligned to achieve the same intended results and to } \\
\text { optimize the flow of innovation in a system }\end{array}$ & Systems Theory \\
\hline $\begin{array}{l}\text { Transparency must exist to show what each actor is intending so that } \\
\text { investments can be made in the most effective manner to stimulate } \\
\text { innovation. }\end{array}$ & Systems Theory \\
\hline $\begin{array}{l}\text { Collaboration is favored above competition or adversarial } \\
\text { relationships to share risks and gain synergies from working together. }\end{array}$ & Systems Theory \\
\hline $\begin{array}{l}\text { Communication between national actors (including attracting new } \\
\text { global investors) is very important for improving the national system } \\
\text { and responding to environmental change. }\end{array}$ & Systems Theory \\
\hline $\begin{array}{l}\text { There must be a free flow of ideas from a developed scientific } \\
\text { academic base and low rigidities in the labour market and in other } \\
\text { government policies that would restrict flexibilities. }\end{array}$ & $\begin{array}{l}\text { Systems Theory \& Human } \\
\text { Capital Theory }\end{array}$ \\
\hline $\begin{array}{l}\text { Building capabilities is crucial for developing innovation system } \\
\text { (availability and quality of human resources) }\end{array}$ & Human Capital Theory \\
\hline $\begin{array}{l}\text { Human beings are valuable assets, and investment in them is very } \\
\text { profitable. }\end{array}$ & Human Capital Theory \\
\hline Continuous learning is crucial for system sustainability and adaptation & Human Capital Theory \\
\hline $\begin{array}{l}\text { Innovation should come from both ways: academia to industry and } \\
\text { vice versa including labour mobility between actor organisations. }\end{array}$ & $\begin{array}{l}\text { Innovation Diffusion } \\
\text { Theory }\end{array}$ \\
\hline $\begin{array}{l}\text { The academic system must have formalized processes that disseminate } \\
\text { innovation and attract research funding. }\end{array}$ & $\begin{array}{l}\text { Innovation Diffusion } \\
\text { Theory }\end{array}$ \\
\hline $\begin{array}{l}\text { Considering advantages and priorities of the context very important } \\
\text { for success of innovation. }\end{array}$ & $\begin{array}{l}\text { Innovation Diffusion } \\
\text { Theory }\end{array}$ \\
\hline $\begin{array}{l}\text { There are two key innovation diffusion strategies: innovation push, } \\
\text { and market pull }\end{array}$ & $\begin{array}{l}\text { Innovation Diffusion } \\
\text { Theory }\end{array}$ \\
\hline
\end{tabular}

Source: The researcher. 


\section{Focal Literature Review}

\subsection{Introduction}

This study concerns a developing knowledge economy and investigation of the framework (in the life science sector in the kingdom of Saudi Arabia KSA) that supports an innovation diffusion (process) for national prosperity. This section of the thesis will present the focal systematic literature review and explore the themes relevant to the topic. It must again be noted that - for methodological reasons - the main literature review followed the field research to enable a grounded approach to be undertaken. The grounded approach is better suited to addressing contemporary phenomena where little is known and for research subjects where significant gaps in the literature exist. This chapter will provide the conceptual framework to help the reader understand the empirical study findings concerning the current state of innovation and the model adopted in the Saudi context. In this chapter many themes will be covered that significantly relate to the research scope and include economic development, enterprise management, innovation and innovation systems, knowledge clusters, foreign direct investment (FDI), knowledge economy (KE) actors, KE measurements and indices, KE pillars, and entrepreneurship.

\subsection{Literature Review Strategy: Systematic Review}

"A systematic review is a review of a clearly formulated question that uses systematic and explicit methods to identify, select, and critically appraise relevant research, and to collect and analyse data from the studies that are included in the review" (Moher, Liberati, Tetzlaff, Altman, \& Group, 2009). The student engaged in a widespread search of library and online databases using key word searches to generate as many publications as possible concerning the subject under study (Scholar/ Web Science). The purpose of this literature review is to explore current "best practice" from the literature in the area of the "knowledge economy" and how it is structured/operates. The literature review will then explore the context of the life science sector and relate the study findings to key arguments in the field. The researcher adopted a narrative 
data approach (no Meta-Analysis) to review all publications and academic sources of evidence. The qualitative data, used for theory building by the researcher, will be presented later in this section of the thesis. The researcher focussed mainly on qualitative and context rich academic studies rather than quantitative studies (where issues of smoothing and data manipulation lack contextual relevance). This chapter will present the key themes that unite the field research and the extant academic body of knowledge (a process of review, extraction and synthesis). In 1996, the QUOROM Statement (Quality Of Reporting Of Meta-analysis) was renamed the PRIZMA Statement (Preferred Reporting Items for Systematic Reviews and Meta-analysis). The PRIZMA approach is a method for conducting a quality systematic literature review and was accepted by the researcher as a means of framing the literature in a way that allowed key themes to develop and to address key conceptual and practical outcomes for businesses as a result of the grounded field research (Moher et al., 2009). According to PRIZMA 2009 techniques the process will go through four key steps: Identification; Screening; Eligibility, and Included (Moher et al., 2009).

\subsubsection{Identification}

From this initial step of identification, the researcher developed many keywords and synonyms including the use of Truncation and limit terms (AND, OR, NOT). Those keywords and synonyms included:

- Knowledge economy, knowledge-based economy, knowledge society, knowledge production, knowledge transfer, innovation, innovation systems, regional innovation systems, national innovation systems, clusters, knowledge clusters, dynamic clusters, knowledge management, human capital, social capital, networking, collaboration.

- Entrepreneurship, entrepreneurial university, entrepreneurial state, science parks, incubators, venture capital, seed funding, financial systems, triple helix, Information\& communication technology, knowledge exchange, open innovation, innovation, life science, biotechnology, innovation initiative, developing countries, knowledge diffusion, knowledge commercialization, intellectual property,

- Economic development, socio-economic development, history of KSA, socioeconomic development in KSA, GCC countries, SMEs, SMEs in KSA, 
multinational companies, foreign direct investment, foreign direct investment impact, reverse foreign direct investment, high-knowledge intensive industries, spill overs, spill over effects, education systems, Saudi culture, SMEs` innovation strategy, innovation models, innovative strategy, spin-offs, spinouts, joint venture, acquisition, partnership, industrial organization, global value chains.

The researcher used search engines and databases such as Scholar and Web Science to generate 1205 papers. Only papers written in the English language (420 generated via Scholar, and 785 via Web Science) were included. After checking the duplications 74 papers were excluded, resulting in 1131 papers. These papers were reviewed and summarised by the researcher. 
Figure 3-1: Result of systematic review.

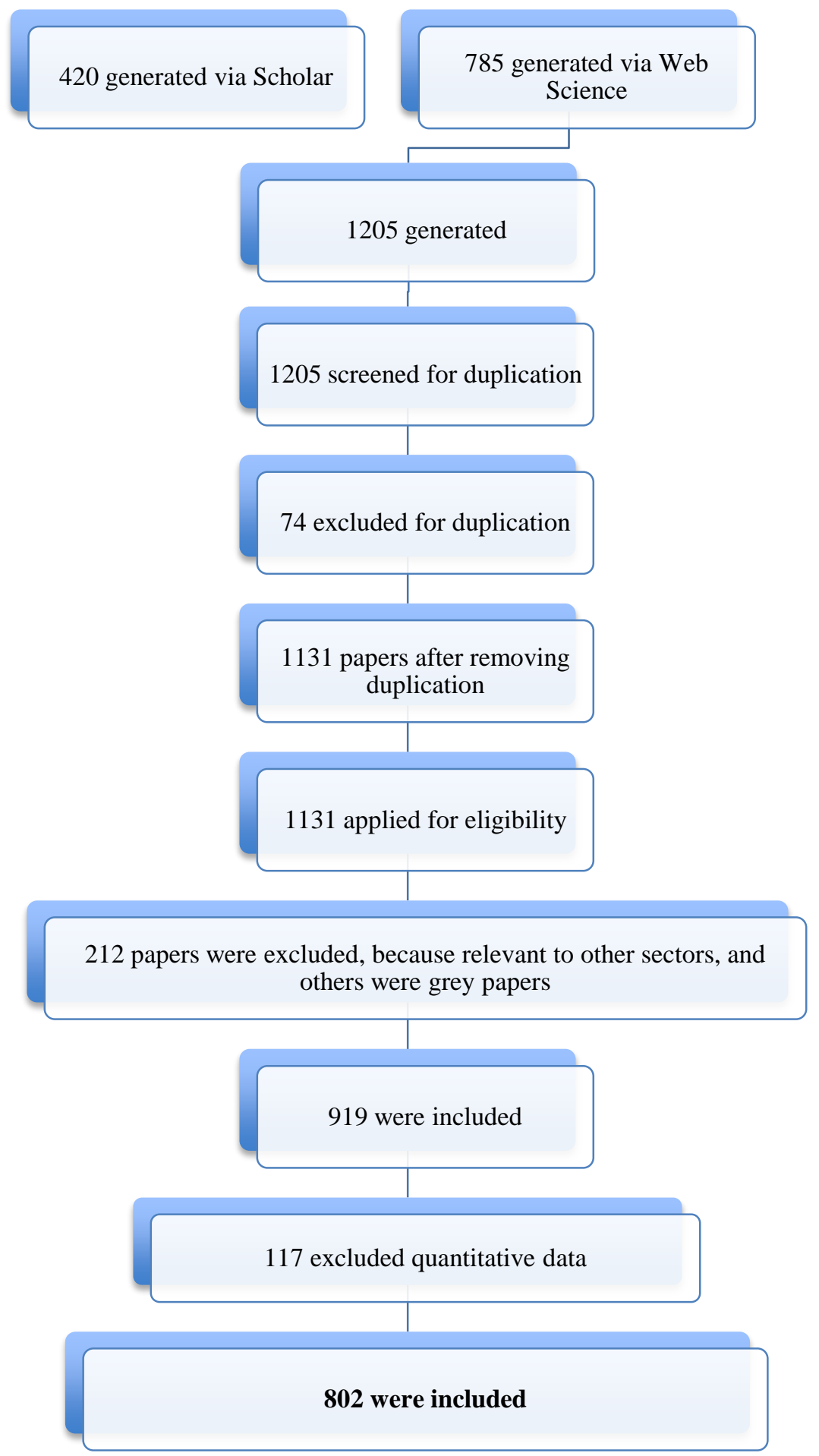

Source: The researcher. 


\subsubsection{Screening}

During this step in the systematic process, the researcher applied a review process for the titles and abstracts and another 212 papers were excluded as they were found to be irrelevant (ultimately 919 papers were reviewed). The researcher applied criteria for inclusion and exclusion relying on excluding "grey" papers (considered biased or less academically rigorous). Papers from other sectors that were deemed irrelevant to the life Science sector were excluded (for example energy, Information \& communication industry, electronics sectors).

\subsubsection{Eligibility}

Having the final population of papers, the researcher conducted full text reading and annotation of each paper and a further 54 were excluded having been reviewed. The resultant 865 papers were deemed highly relevant to this study.

\subsubsection{Included}

At this stage 802 qualitative papers were included and no quantitative papers were included.

\subsubsection{Result}

After this stage the researcher developed sub-topics that constituted the focal literature review map (see the next table).

Table 3-1: Focal literature review map.

\begin{tabular}{|ll|}
\hline Introduction & \\
\hline \multirow{2}{*}{$\begin{array}{ll}\text { Literature review strategy: } \\
\text { Systematic Review }\end{array}$} & Identification \\
\cline { 2 - 2 } & Screening \\
\cline { 2 - 2 } & Eligibility \\
\cline { 2 - 2 } & Included \\
\hline Economic development & Result \\
\hline Enterprises and Entrepreneurship & Capabilities and economic development \\
\cline { 2 - 2 } & Clusters and economic development \\
\hline
\end{tabular}




\begin{tabular}{|c|c|c|}
\hline & \multicolumn{2}{|c|}{ Entrepreneurial competencies } \\
\hline & \multicolumn{2}{|l|}{ Micro and SMEs } \\
\hline \multirow{4}{*}{ Knowledge Economy } & \multirow{4}{*}{$\begin{array}{l}\text { Knowledge economy } \\
\text { pillars }\end{array}$} & Economic and institutional regime \\
\hline & & Innovation systems \\
\hline & & Human capital \\
\hline & & ICT \\
\hline \multirow{6}{*}{ The role of SMEs in KE } & \multicolumn{2}{|l|}{ The definition of SMEs } \\
\hline & \multirow{5}{*}{ Innovation in SMEs } & Internal factors \\
\hline & & External factors \\
\hline & & $\begin{array}{l}\text { Absorptive capacity and knowledge } \\
\text { management }\end{array}$ \\
\hline & & $\begin{array}{l}\text { An intermediated network model: } \\
\text { KICMS }\end{array}$ \\
\hline & & Entrepreneurial orientation in SMEs \\
\hline \multirow{2}{*}{ Large Enterprises } & \multicolumn{2}{|l|}{ Corporate entrepreneurship } \\
\hline & \multicolumn{2}{|l|}{ Entrepreneurship strategy } \\
\hline \multirow{5}{*}{ Multinational Enterprises } & \multicolumn{2}{|c|}{ MNEs and dynamic in capabilities (DCs) } \\
\hline & & Definition and historic perspective \\
\hline & & Spill over \\
\hline & $(\mathrm{FDI})$ & $\begin{array}{l}\text { Prerequisites for FDI spill over and } \\
\text { growth }\end{array}$ \\
\hline & & Reverse innovation \\
\hline \multirow{5}{*}{ Innovation } & \multicolumn{2}{|c|}{ Different discipline perspectives around innovation } \\
\hline & \multicolumn{2}{|c|}{ Joseph Schumpeter (1883-1950). } \\
\hline & \multicolumn{2}{|l|}{ Organizational innovation } \\
\hline & \multicolumn{2}{|l|}{ Types of innovation } \\
\hline & Innovation measurement & Global innovation index \\
\hline \multirow{6}{*}{ Innovation Systems } & \multicolumn{2}{|l|}{ Innovation system definition } \\
\hline & \multicolumn{2}{|l|}{ Types of innovation systems } \\
\hline & \multicolumn{2}{|c|}{ NIS preconditions and capabilities } \\
\hline & \multicolumn{2}{|c|}{ Different innovation catch up models } \\
\hline & \multirow{2}{*}{ Knowledge clusters } & Cluster development and evolution \\
\hline & & Clusters impacts \\
\hline \multirow{3}{*}{ Actors } & \multicolumn{2}{|l|}{ Industry } \\
\hline & \multicolumn{2}{|l|}{ Governments } \\
\hline & Academia & Entrepreneurial university \\
\hline \multicolumn{3}{|l|}{ Theoretical Model } \\
\hline
\end{tabular}

Source: The researcher

The next section will explore the themes that were identified in the systematic literature review. 


\subsection{Economic Development}

The subject of economic development underpins any national system and according to Porter (2000) "economic development seeks to achieve long-term sustainable development in a nation's standard of living, adjusted for purchasing power parity" The concept of economic development differs from economic growth which is represented by the annual GDP rate. Economic development is therefore a broader concept that includes job creation and the well-being of people and their lifestyle (Toma, Grigore, \& Marinescu, 2014). Economic development is concerned with structural transformation in the services and manufacturing of a nation - in terms of technology advancement and modernity (Naudé 2008, p. 18). It relates to how economic growth is reflected in improving society well-being, which can be measured by GDP per capita, productivity, employment, and GDP growth (Naudé 2011, p. 304). So, the economic activities have a social dimension and positive impact on economic development. Existing academic literature confirms that knowledge and technology are key driving factors for economic growth and development (Howitt, 1992; Lucas, 1998; Romer, 1990). For this study, the researcher has explored the system that supports economic development as an outcome of actors and current practices.

\subsubsection{Capabilities and economic development}

Economic development requires many capabilities and factors to exist within a specific geography. The differences between countries in the level of development are linked to differences in technology (Gerschenkron, 1962) and technological capabilities are the driving force for development. Consequently developing a technological capability is a crucial step towards development (Romer, 1990) and, in this context, countries looking to catch-up in technologies, developing institutional instruments or organisations that have the capabilities to figure out the opportunities and efficiently use the resources is crucial. From a systems perspective these organisations and processes need to be operated within a favourable and collaborative government-industry relationship. The social capabilities, technological and managerial capabilities, effective and supportive financial systems, and instrumental government are confirmed as an important for the catching-up process (Gerschenkron, 
1962). As seen previously, it is important that a skilled workforce is available to exploit any new technological innovation.

Kim defined the technological capabilities as the process of assimilation, adoption, use and improvement of the existing technology (Kim 1997). Technological capabilities include innovation capability, production capability, and investment capability. In general, commercial capabilities that enable commercial development and exploitation of knowledge would all help in the way of catching-up development (Fagerberg \& Srholec, 2008). The national technological capability can be improved by domestic efforts, international trade of high technology, or foreign investment (Lall, 1992). Among the capabilities, the following four are seen as critically important in the process of catching-up technology: innovation system, governance, political system, and economic openness (Fagerberg \& Srholec, 2008).

The quality of governance of the national system is of critical importance for economic development (Fagerberg \& Srholec, 2008). Kim confirmed that countries which achieved "catch-up" parity with mature economies paid serious attention to their innovation systems (Kim, 1997). The evidence of which political system generates more innovation than others is quite weak. For example, China and Vietnam achieved significant economic development in spite of their non-democratic

political systems. As such, a monarchy-led state such as the Kingdom of Saudi Arabia would not be disadvantaged by being so as long as the necessary infrastructure is in place to enhance innovation and technology development. In terms of openness, this is dependent upon the social absorptive capability, which means that poor countries with low absorptive capability would not benefit from foreign investment like richer countries (Fagerberg \& Srholec, 2008).

\subsubsection{Clusters and economic development}

A "cluster" is a geographic industry location where interrelated entities work in competitive and complementary ways, involving companies, upstream organizations, downstream, and other agencies and institutions that support and provide a healthy business environment (Porter, 2000). Within clusters, all actors work in both competitive and complementary ways, and the companies' thinking and strategies should consider development and competitiveness to necessitate cooperation and 
involvement of the actors beyond their walls with competitors, suppliers, and customers (in this manner the explicit recognition of dependency on each other and the synergy of a systems approach). The competition/collaboration in the proximate location stimulates innovation and improves productivity, which provides a competitive advantage to the industry within a cluster.

This form of economic development aims to improve the standard of people's lives which productivity can achieve through improving efficiencies, reducing the costs of production and pricing products to be profitable. The competitiveness within clusters stimulates innovation and the capacity to deliver new innovative products with respect to the level of sophistication, demand conditions and expectations that customers have, and consequently economic development will be enhanced by this economic mechanism (Porter, 2000). Due to the location of the university system, and as universities are immobile assets and actors, the literature suggested that other actors would co-locate or invest in research in order to exploit innovation commercially. The later KSA field research of this study indicates that this did not and is unlikely to happen to form a local eco-system and thus the national system will remain the most important unit of analysis.

\subsection{Enterprise and Entrepreneurship}

Policy makers and government officials view entrepreneurship as a key driver for supporting economic development and good practice that could deliver economic growth per capita and the creation of jobs (Shane, 2007). Regarding this, economists shifted their attention from big companies as the traditional means for economic development, to a focus on SMEs as a start point for entrepreneurs' activities. Entrepreneurship and its impact on economic development comes through its outcomes which include new ventures, job creation, and new services and products (Toma et al., 2014). Schumpeter sees the entrepreneurship and its implied innovation coming in five ways: new products or service, new production method, new source of supply, new market, and new organizations (Schumpeter, 1911). "The entrepreneur is to be seen as responding to opportunities rather than creating them; as capturing profit opportunities rather than generating them" (Kizner, 1973). Entrepreneurship is an individual and creative activity that can see opportunity and move up the resources 
from one level of productivity to another, and serves as a driving force for change (Toma et al., 2014). In the context of explaining the relation between entrepreneurship and economic development, the US Small Business Administration stated: "the crucial barometer of economic freedom and well-being is the continued creation of new and small firms in all sectors of the economy by all segments of society" (Administration, 1998). New industrial and economic theories consider entrepreneurship as directly connected to economic growth because of the role it plays in stimulating and disseminating change (Toma et al., 2014).

There are three ways that embody the role of entrepreneurship in supporting economic development: firstly, creating competition in the business environment by increasing the number of enterprises: this competition also leads to creating knowledge-based new ideas and in turn supports economic development. Secondly, entrepreneurship encourages spill over and knowledge transfer that are conducive to endogenous growth through new start-ups. Thirdly, it creates diversity in enterprises within any location which benefits economic growth (Burns, 2007). However, the impact of entrepreneurship on economic development is less effective in the earlier stages of economic development than in later stages (Naudé, 2013). The effectiveness of entrepreneurship depends on a number of factors including cultural traditions of entrepreneurship, entrepreneurial education, market opportunities, institutional support and government policies. People with no tradition of entrepreneurship, or lacking entrepreneurial education, might find it difficult to practise or make a success of business entrepreneurship (Toma et al., 2014). The required capabilities, linked to economic development, are presented in the next section. In the following sections the concept of entrepreneurship will be contextualised using three different sizes of firm (micro and SMEs, large companies, MNEs). 


\subsubsection{Definition of entrepreneurship}

"Entrepreneurship is the manifest ability and willingness of individuals, on their own, in teams, within and outside existing organizations, to perceive and create new economic opportunities (new products, new production methods, new organizational schemes and new product market combinations) and to introduce their ideas in the market in the face of uncertainty and other obstacles, by making decisions on location, form and the use of resources and institutions" (Thurik \& Wennekers, 2004; Wennekers \& Thurik, 1999).

The shift to the supply side of the economy as a new model can be explained by the role and importance of entrepreneurship in an economy (Wennekers \& Thurik, 1999). The increased number of SMEs in 1980s and the unemployment problem in Western countries stimulated political and economic interest in entrepreneurship and the important role of SMEs as a source of growth, innovation and job creation. The role of entrepreneurship is not only to utilize the existing technology/innovations profitability (neo-classical economy theory) but also to stimulate competitiveness and exploit the potential opportunities via new ideas (supply-side economy theory).

According to Schumpeter, innovation is at the centre of any entrepreneurial activity or venture (Schumpeter, 1961). He identified five criteria for an entrepreneurial venture, namely: introducing a new product or service, introducing a new production method, having a new market, having a new source of supply, restructuring the industry. Entrepreneurial behaviour can extend to macro level to include industries, cities, regions and nations. The entrepreneurs can be categorized in three classes: Schumpeterian entrepreneurs, entrepreneurs, and managerial business owners. Schumpeterian entrepreneurs usually exist in SMEs; they own and manage their own innovative business, which are supposed to be creatively destructive of the existing industry structures, then they develop into managerial business owners to start again with new ventures.

Entrepreneurs work on behalf of their employer and risk their position and reputation to develop new commercial initiatives within the large enterprise. They may start their own business via spin-off and become Schumpeterian entrepreneurs. The last one, called Managerial business owners, constitutes the majority of the SMEs, their 
enterprises lack innovation, instead doing business like shopkeepers, franchisees and so on (Wennekers \& Thurik, 1999). In the West, during the 1970s and 1980s, the rate of SME start-ups increased and economic activity moved from large to small firms, which reflected on their contribution significantly in employment (Wennekers \& Thurik, 1999). For example, the share of the large American 500 firms (so-called 500 Fortune) in employment dropped from $20 \%$ in 1970 to $8.5 \%$ in 1996 (Carlsson, 1992, 1999). Also in Europe between 1988 and 1998 the growth of employment in SMEs overtook that of the largest firms (EIM, 1997).

This shift toward smallness can be explained or attributed to many reasons: the increase of uncertainty and of fragmentation in the market, and an increase in global competition (Carlsson, 1992). Another explanation has been suggested by Piore (1984) who claimed the instability in markets led to the dismissal of mass production and economies of scale (in favour of lower scale businesses and economies of scope for manufacturers). In their paper "Small Business Economics", Brock \& Evans (1989) contended that this phenomenon of smallness was economy wide and not confined to manufacturing. They also added four other reasons behind the smallness shift process. The first explanation concerns the labour markets and the increase of labour supply in parallel to a comparative net decrease in wages and increase in the educational level of workers. The second reason was attributed to the changes in customer tastes and the sophistication of demand. Third is the relaxation and overhaul of entry regulations to encourage competition. Lastly, they considered the period of creative destruction as a reason.

The 'scale' shifting process was also considered necessary to transfer to a knowledgebased economy (Audretsch \& Thurik, 1998) although Acs (1992) upholds the important role of increased SME numbers and impacts, resulting in economic change, their role in entrepreneurial activities, innovative activities, stimulating the industries' development, and their role in generating significant share in the new jobs. From the literature, the existing authors show a bias towards innovation generated by SME businesses. Such a lack of a co-ordinating structure to access and understand SMEs poses many difficulties for policy makers and those civil servants required to support SME development. In the absence of regional clusters this task is harder still as there is no means of collectivising such businesses. 


\subsubsection{Entrepreneurial competencies}

Entrepreneurs are supposed to have common characteristics and personal traits, such as creativity, initiative, commitment, self-confidence, risk taking, need for autonomy, independence and achievement, great energy, internal locus control, and perseverance (Schmitt-Rodermund, 2004). According to Holland (1997) the entrepreneurial personality thrives in an environment that has competition, involves initiative, dominance, self-confidence and capabilities in managerial, sales, and business issues, and rewards the ability to convince, manipulate and direct others,. He assumes two influencing sources, the first being the traits that one is born with, while the second source is the feeding of this personality by cultivating and enhancing the inborn traits via parental support and other encouragement that can lead to enforcing and shaping the potential entrepreneurial personality.

According to Sánchez (2011) competencies are the sum of related skills, knowledge, attitude and characteristics that impact the individual behaviour, performance and work. These features can be measured, and improved via training and development. Also Woollacott (2009) defines the competencies as "the knowledge, skills, abilities, attitudes, and other characteristics that enable a person to perform skilfully (i.e., to make sound decisions and take effective action) in complex and uncertain situations such as professional work, civic engagement, and personal life". The competencies include three main features namely; skills, characteristics, knowledge (Mojab, Zaefarian, \& Azizi, 2011). In terms of entrepreneurial competencies, which are important in delivering sustainable success, the traits of the entrepreneur should cover attitude, knowledge, beliefs, values, social, managerial and technical experience, characteristics, abilities, wisdom, and lastly his/her mental and behavioural tendencies. At the task level, the entrepreneur is assumed to have the competencies to perform the following tasks:

- Exploring, recognizing, and exploiting the potential opportunities within the markets.

- The ability to effectively communicate, discuss, convince the stakeholders, competitors and other partners in the business environment

○ The ability to build collaborative networks. 
○ The ability to understand entrepreneurship and its embedded uncertainty and rapidly changing business environment.

- The ability to develop learning and entrepreneurial organization, business development management, and flexible strategic orientation (Onstenk, 2003).

The competencies are different in terms of the ability to bring about development and in the extent of time needed, for example competencies that are relevant to the personal characteristics are difficult to develop and take a long time, while ones that are related to knowledge, skills and attitude can be learned and developed in a short time (Mojab et al., 2011). Entrepreneurial education is significant for students in universities, for instance Harvard Business School began to teach students entrepreneurial competencies in 1947 (Ilie, 2014). With regards to the literature, there is clear support for an active university system in any economy and for the generation of knowledge to support SME start up exploitation. In this manner a supportive government policy and leadership as an actor must be supported by an equally proactive university system that has the formalised processes to develop entrepreneurs and SME businesses.

\subsubsection{Micro and SME Businesses}

There are many relevant and inherent challenges that face SMEs, especially their access to the financial system, and the status of their limited resources, which may affect and impede their adopting a new technology, and innovation. These conditions entail developing a clear definition to apply special measures that help in mitigating and overcoming these challenges, and improve the business environment, promote entrepreneurship, investments and growth for SMEs. From this point, identifying a clear and agreed definition becomes important and necessary. According to European Commission (2005) "The category of micro, small and medium-sized enterprises (SMEs) is made up of enterprises which employ fewer than 250 persons and which have an annual turnover not exceeding 50 million euro, and/or an annual balance sheet total not exceeding 43 million euro". 
SMEs have a significant role in economic development, especially in the manufacturing sector. They contribute to up to $90 \%$ of the enterprises in the world and from 50 to $60 \%$ of the employment. SMEs have a key role in the development process for many reasons: firstly, SMEs are more labour-intensive, which helps in absorbing the huge numbers of unemployed people in the developing countries. SMEs help in supporting fair distribution of wealth among people and poverty alleviation. Secondly, the links between small and large enterprises support the resilience and dynamics of the economic system and help in absorbing the productive resources at all economic levels. Thirdly, the wide geographical distribution of SMEs reduces the disparity in the economy and fills the gap between urban and rural areas, and develops the entrepreneurial spirit and skills (Hobohm, 2000). SMEs are important to both developing and developed economies (Szirmai, Naudé, \& Goedhuys, 2011); however SMEs find more support in the developed countries than in developing countries. For example, loans made to SMEs constitute 13\% of GDP in the developed economies, while they constitute only $3 \%$ of GDP in the developing ones (Set, 2011).

The implications of an effective innovation system design for SME businesses is that they must be attractive to investors if the finances are to be available in a risky sector of an economy. From a systems perspective the financial actors must therefore support SME development and this will only occur if the government policies and infrastructure (together with the universities) promote SME development.

\subsection{Knowledge Economy (KE)}

There many definitions of the term 'knowledge economy': for example, the knowledge-based economy (or knowledge economy used exchangeable) is an economy where the knowledge is acquired, generated, published and disseminated, and used effectively to promote economic development and growth (Chen, Dahlman, $\&$ Dc, 2005). The World Bank says "The application of knowledge is now recognized to be one of the key sources of growth in the global economy. The term Knowledge Economy (KE) has been coined to reflect this increased importance of knowledge. A knowledge economy is one where organizations and people acquire, create, disseminate, and use knowledge more effectively for greater economic and social development" (World Bank, 2016). 
Another international organization (the European Commission) has its own definition: "The term "knowledge economy" is commonly used to describe economic activity that relies not on "natural" resources (like land or minerals) but on intellectual resources such as know-how and expertise. A key concept of the knowledge economy is that knowledge and education (also referred to as "human capital") can be treated as a commercial asset or as educational and intellectual products and services that can be exported for a high value return. It is obvious that the knowledge economy is rather more important for those regions whose natural resources are scarce" (Communities, 2008).

The Organization for Economic Co-operation and Development (OECD), which is constituted of 34 countries (the majority of them western and developed economies) is concerned with developing policy and recommendations for economic development. It defines the knowledge economy as: "Economy which is characterized by the recognition of knowledge as a driver of productivity and economic growth, leading to a new focus on the role of information, technology and learning in economic performance" (OECD, 1996).

All the above definitions share quite similar concepts, which focus on knowledge as a key input for the economic activities in the context of knowledge based economy. In spite of the concept of knowledge economy became popular by the virtue of Peter Drucker and his seminal book The Age of Discontinuity- the concept having firstly been posed by Fritz Machlup, but the credit for being known as the father of scientific management was given to Fredrick Winslow (Peter, 1969). In the following section the framework or pillars that constitute the knowledge economy are discussed in detail.

\subsubsection{Knowledge economy pillars}

The World Bank, through its World Bank Institution`s Knowledge for Development programme, offers four pillars (economic and institutional regime, human capital, ICT infrastructure, and innovation systems) as key components that illustrate the status of a country as a knowledge economy. These pillars actually test the innovation system and measure the knowledge accumulation in a country. Countries that use them will 
develop appropriate policies that help build the capacity of a country to assimilate, create, share and use knowledge in the transition to the knowledge economy (World Bank, 2012). The following sections will detail the four pillars and their relation to developing a knowledge economy.

\subsubsection{Economic and institutional regime}

The economic and institutional regime is concerned with providing suitable regulations and incentives that have the potential to enable effective use of existing knowledge and the creation of new knowledge. These regulations or incentives are supposed to enhance the economy's capacity to respond and adapt to competitive and changing business conditions and to address opportunities, and to develop the abilities to increase productivity and develop new innovative activities. The economic and institutional regime is a central factor to the economy's ability to use knowledge effectively, and is also critical to the functioning of the other three pillars of the knowledge economy. The influence of the economic and institutional regime covers many areas such as the product market, financial market, labour market, knowledge market, and industrial restructuring and entrepreneurship. The incentives and regulations should lead to supporting and facilitating practices and matters such as competition between foreign and domestic firms, customer protection, corporate governance, venture capital and equity, transparency, labour mobility, intellectual property rights, valuation of intangible assets, entrepreneurship and SMEs (Thomas \& Carl, 2001).

According to Kaminski (2013), incentives that facilitate entrepreneurship within an economy deliver more utilization of the other three pillars, and the level of economic development is positively correlated to the economic governance quality. The existence of innovation-supportive institutions, which support the best use and

utilization of the resources and production factors, is high in countries with high economic development. The effective economic regime has the rules and incentives that create a supportive and motivating environment for entrepreneurs to develop innovative activities (Baumol, Litan, \& Schramm, 2007). On the other hand, economic regimes which build barriers to entry and doing business, will impede competition, not protecting intellectual property rights, imposing more tax burdens 
and obstructing entrepreneurship, hampering innovation and the development of new products and services (Kaminski, 2013). The political conditions and structure have a significant effect on the sustainability of economic growth, and it is suggested that only democratic arrangements support the sustainability of economic growth (Acemoglu, Robinson, \& Woren, 2012). Financial systems are another important element of economic and institutional regime; because of their influence on the rate of savings, decisions on investments and technical innovation, they have a significant impact on the diffusion of knowledge, knowledge economy and economic growth.

By facilitating access to external financing, the knowledge economy and economic growth will be enhanced (Levine, 2005). In the US experience, venture capital works as a main driver and important factor behind the success of innovative enterprises, start-up companies, and the knowledge economy (Stiglitz, 1999; Thomas \& Carl, 2001). Developing intellectual property rights protection motivates knowledge creation, however excessive protection and very strong relevant regulations might impede the pace of innovation through increasing the price of putting the knowledge ingredient into innovation process (Stiglitz, 1999).

\subsubsection{Innovation systems}

In his seminal book Japan: a New National Innovation System, Christopher Freeman was the first author to mention the concept of a National Innovation System (Niosi, 2011a). The emergence of National Innovation systems began in the mid of 1980s, with debates taking place in Europe's Industrial Policy (Freeman, 1987). The OECD was one of the first international organizations which started to use the NIS concept as an integral part of their analytical perspective (Lundvall, Johnson, Andersen, \& Dalum, 2002). A National Innovation System is defined as “... a set of institutions whose interactions determine the innovative performance of national firms" (Nelson, 1993). Nelson also emphasizes that the core of an innovation system is the policy of having science, technology, and innovation that support R\&D (derived from Government policies). Lundvall defines the National Innovation System as "constituted by elements and relationships, which interact in production, diffusion and use of new and economically useful knowledge" (Lundvall, 1992). These elements include institutions such as universities and public research centres, firms, 
government regulatory systems, educational system, and financial institutions (Godin, 2009). Again these actors confirm the importance of this research study and the relationships that both exist currently and are being developed in the Kingdom of Saudi Arabia and the life science sector.

Smith (the author of the OECD methodological manual on measuring innovation) also stresses the concept of interactions between actors: "the overall innovation performance of an economy depends not so much on how specific formal institutions (firms, research institutions, universities, etc.) perform, but on how they interact with each other" (Smith, 1995). Freeman also defines the national innovation system as "the network of institutions in the public and private sectors whose activities and interactions initiate, import, modify and diffuse new technologies" (Freeman, 1987) and Metcalfe sees NIS as "the set of distinct institutions which jointly and individually contribute to the development and diffusion of new technologies and which provides the framework within which governments form and implement policies to influence the innovation process. As such it is a system of interconnected institutions to create, store and transfer the knowledge, skills and artefacts, which define new technologies" (Metcalfe, 1995). The World Bank Institute, which developed the knowledge economy framework and its embedded four pillars and considered the NIS as one of the four pillars, defines NIS as: "An efficient innovation system comprising firms, science and research centres, universities, think tanks, consultants and other organizations that can interact and tap into the growing stock of global knowledge; assimilate and adapt it to local needs; and use it to create new knowledge and technology".

The production and innovation processes within a knowledge economy need effective linkages, effective use of resources, and motivating incentive structures which can respond and come up with changes in technology, market and social conditions, and involve complementarity among the innovation systems' actors (Thomas \& Carl, 2001). However, what can be concluded is that the concept of the National Innovation System pays great attention to the interactions between all actors and the innovative output of these interactions and collaborations (OECD, 1997). The literature strongly 
supports a national system of innovation as a transforming mechanism to move an economy to one of knowledge based trading and this requires significant and prolonged interactions between actors to co-ordinate and support an innovation system. The implication is that any study of national innovation must adopt a multistakeholder perspective when assessing the interactions and quality of interaction between organisations. High dependency relations between stakeholders make this form of study, rare in the field of knowledge economy, highly important to test how developing countries are using knowledge-based assets.

\subsection{Knowledge-Intensive Business Services (KIBS)}

According to Muller, KIBS (service providers) can be defined as consultancy firms providing high-added-value services or activities to other firms (Muller, 1999). These activities or services come into two categories, the first one includes traditional professional activities such as marketing, advertising, design, management, financial services, etc. the second category involves more new technology-based activities such as software, technological training, computer networks, design involving new technology, R\&D services, technical engineering etc. (Miles et al., 1995).

KIBS interact with SMEs in such a way as to lead to enhancing their innovation capacities. KIBS, through this mechanism, effectively contribute by generating and diffusing knowledge within an economy (Muller \& Zenker, 2001). Also (Corrocher \& Cusmano, 2014) assure us that KIBS are main actors in innovation systems, and these embedded intangible factors are a key source of competitiveness. These kind of firms, which engage in activities like market research, consultancy, engineering, and technical and design services, are expected to perform a significant role in regional innovation systems, creating interactive learning and increasing the innovation system's connectivity and networks. KIBS evolved from merely providers of information to nodes of knowledge-related networks and innovation systems (Muller \& Doloreux, 2007). MNEs help in creating markets and demand for this kind of activity provided by KIBS (Jacobs, van Rietbergen, Atzema, van Grunsven, \& van Dongen, 2014). 


\subsection{The University`s role in innovation systems}

According to many authors (Charles, 2006; Chatterton \& Goddard, 2000; Shaw \& Allison, 1999) the national university subsystem plays a main role in the development process of a region, and especially the development of the knowledge-based economy. The universities contribute crucially in building the region's competitive advantage (Goddard \& Chatterton, 1999), and the expected role of universities in producing knowledge and knowledge transfer effectively help a region in retaining talented and human capital, and in providing high added-value activities (Richard, 2005). The role of universities in knowledge transfer comes in three key forms of contribution, namely commoditized knowledge (commercialization), human capital development, and social capital building (Charles, 2006). (Etzkowitz, 2003) also stressed their role in technology transfer and spin-off firms. As civic institutions, universities can significantly influence the cultural and political factors of socioeconomic development (Charles, 2006).

Regarding these three elements (knowledge transfer - treating knowledge as commodity, human capital, and social capital) universities constitute a key element in regional innovation systems (Braczyk, Cooke, \& Heidenreich, 1998). Cooke (2004) also stressed the crucial importance of social capital formation, and its role in facilitating networking and knowledge diffusion, without which the innovations systems actors will be idle and learning processes will be hindered. He also asserts the great importance of the existence of universities and research intermediaries (public or private) for the effectiveness and health of innovation systems. In his perspective, the regional innovation systems approach is an interactive system that relies on knowledge transfer among involved actors. Triple Helix is a prominent mechanism and combination, where universities, government, and industry relationships work in a way that facilitates knowledge production, diffusion, and commercialization. Public innovation agencies are involved and necessary to supporting innovation systems. However there is no 'one size fits all' solution, and the context is considered to determine the suitable innovation systems that can work properly and effectively (Cooke, 2004). The latter argument means that a system of innovation is contingent upon the country concerned. Thus, a context-rich study of actors is required here as

globally dominant models do not exist and, moreover, the Kingdom of Saudi Arabia 
is unlike most of the countries - particularly Asian ones - that have dominated historic studies of knowledge economies.

\subsubsection{Human capital}

Human capital has been defined as the collection of investments in education, training, health, and migration which enhance the productivity of individuals in the labour market (Becker, 1962; Kiker, 1966). Other definitions describe human capital as the collection of knowledge, skills, and innate abilities that people gain and develop through their lifetime (Laroche, Mérette, Ruggeri, \& Merette, 1999). The shift to a knowledge-based economy, which was driven by technological change and market globalization, put human capital with other themes at the centre of policymakers`attention and concerns (Laroche, Mérette, Ruggeri, et al., 1999).

Human capital has five aspects which are significantly influenced by the national education system, which affect the individual's path to a higher education,, namely: Cultural capital, which is concerned with the cultural background and family traits such as language, community, neighbourhoods, and ethnic ties; Economic and educational capital, correlating with the educational attainment and the economic status of the family; Social capital, or the behavioural knowledge that concerns the understanding of the society's manners and its institutions, best use of opportunities, and effectively dealing with and navigating growing bureaucracies; Cognitive capital, concerned with the different intelligence forms and their distribution, and finally Aspiration capital, which emphasizes that ambition has a major effect in shaping the social capital and cognitive capital, and is itself influenced by the environment factors and personal traits (Douglass, 2010).

Many studies confirm the strong relationship between economic growth and investments in human capital (Azariadis \& Drazen, 1990; Lucas, 1998; Romer, 1986; Solow, 1956), and the significant role of the existence of high quality human resources in attracting foreign direct investment (Koike, 1996). In the developing countries, human capital contributes significantly to raising the level of income through the capacity of absorption and imitation of international technology (Ruggeri, et al., 1999). Developing educated and entrepreneurial people who are able to create, 
share, and effectively use knowledge and skills, is a key element in developing a knowledge economy (Thomas \& Carl, 2001). To develop a knowledge economy, education and training is vital for human resources development, enhancing abilities for research and knowledge generation, and up-skilling worker competencies (Peters, 2001). According to Drucker "In the knowledge society into which we are moving, individuals are central. Knowledge is not impersonal like money. Knowledge does not reside in a book, a databank, or software program. Knowledge is always embodied in person; carried by a person; created, augmented, or improved by a person; applied by a person; taught and passed on by a person; used or misused by a person. The shift to the knowledge society therefore puts the person in the centre" (Drucker, 1994).

Production of knowledge is a key contributor to the competitiveness of a nation (Porter, 1990, 2011). To pursue competitive advantage, knowledge workers must be positioned at the centre of attention as they represent a key contributor and factor (Reich, 1991). Education results in delivering engineers and scientists who have a role of producing knowledge through research activities. This process leads to the formation of human capital, which affects the accumulation of knowledge and increases in productivity (Peters, 2001). However, the kinds of education and knowledge that should be pursued include many forms of cognitive abilities and skills such as developing the ability to integrate informal and formal learning, "know how" and "know what", the ability to search, access, choose and evaluate knowledge, the ability to work and learn in a team, the ability to deal with uncertainty and unpredictable problems and situations, the ability to produce and transfer knowledge, and finally the ability to deal with multiple careers (learning how to marketing yourself, selecting the relevant training and education, and re-designing yourself) (Hargreaves, 2000).

Creative people are key drivers and crucial for growth and development, they work like points of attraction for economic activities, which results in developing new innovative businesses in a region. Also a social climate which includes tolerance and openness to new ideas and new people is the perfect environment to attract and 
encourage people to work (Richard, 2004). In concentrations of human capital (universities, high tech firms and agglomerations, research centres) technology advancement and development is determined by human capital`s role (Jacobs, 1986). In the USA, between 1948 and 1984, the amount of investment in human capital was four fold that in physical capital, while the value of human capital was more than eleven times its physical counterpart (Jorgenson \& Fraumeni, 1989).

Lundvall also supports this concept that revolves around the key role of human capital in development: "Human capital plays a decisive role, and the capacity to learn matters more than the level of knowledge. While secondary school certificates were the trump cards of industrialization, higher degrees are those of the knowledge economy. Lifelong training is essential" (Lundvall, 2002). The ability to attain and develop knowledge is critical for people and entities to integrate with the global economy (Development, 2001). For an economy to prosper, local industries need to be globally traded; one-third of the industries should be traded globally - through global investment or export orientation; this approach requires more local innovative pace to maintain effective integration and keep up with the international dynamics of technological changes and market trends, consequently, this requires continuing learning and human capital development (Walshok, 2012).

\subsubsection{Information and Communication Technology Infrastructure (ICT)}

The purpose of providing an effective information and communication infrastructure is to enable a successful means of communicating and disseminating knowledge and of fast information processing (Thomas \& Carl, 2001). Globalization has implications for economic and social networks, which require effective information and communication technologies infrastructure to ease and facilitate access to information or knowledge. This provision of effective access and its reflection in knowledge production helps in bridging the knowledge gap which exists between nations. Such knowledge production has the potential to develop into innovation, which has become a vital part of survival in global competition and open markets (Lor \& Britz, 2007).

The information-based economy relies completely on the availability of effective ICT infrastructure, but this "soft" infrastructure will not work properly without physical 
infrastructure such as airports, roads, harbours, warehouses, etc., which make the delivery process for physical products effectively achievable. Besides enabling access to the ideas of others, ICTs enable people to contribute to global information-based socio-economic activities. According to (Polder, Leeuwen, Mohnen, \& Raymond, 2009), at the firms' level, ICTs in service sectors are key drivers for all kinds of innovation (product, process, and organizational innovation), while they have less important in the manufacturing sector, in spite of their positive effects.

Many studies from different national contexts (Black \& Lynch 2001; Bresnahan et al. 1999; Bugamelli \& Pagano 2004; Castiglione 2010) found a positive relation between ICT investment and the increase of productivity at the firm level. However, ICTs would not work effectively alone, but require interaction with some complementarities such as human capital, R\&D, and organizational innovation (Black \& Lynch, 2001; Bresnahan et al., 1999). ICT works as an enabler for innovation and technology development, and productivity enhancement takes place in industries that produce and intensively use ICT (Cardona, Kretschmer, \& Strobel, 2013). ICT is, like electricity, considered as a general-purpose technology (GPT), which has great impact on the whole economy through affecting and changing how household life and business is conducted (Jovanovic \& Rousseau, 2005). According to (Bresnahan \& Trajtenberg, 1995), GPT is characterized by three features or criteria:

- Pervasiveness: broad range of applicability

- Improvement: keeps lowering cost within the firms using and producing it because of the scope for improvement and experimentation

○ Innovation spawning: helps with invention and producing new products and processes within the firms using and producing it.

According to Forman \& Zeebroeck (2012) the main role of ICT is in storing and sending knowledge and information, and through facilitating communication. ICT eases efficient exchange and collaboration and this leads to or helps in creating new knowledge. This role of ICT supports the concept that considers ICT as enabling technology (Jovanovic \& Rousseau, 2005). ICT makes a significant contribution to supporting and facilitating the practices of and requirements for open innovation such 
as collaboration, working across boundaries and network-centred innovation practices (Christensen \& Maskell, 2003; Pavitt, 2003; Tapscott, 1996). The outstanding economic growth achieved by Japan (and other Asian countries) was attributed, in part, to having better access to the market information ( $\mathrm{Vu}, 2011)$. The easy access to information enhanced labour skills, increased customer sophistication, and increased basic education levels (Quah, 2002). However, while investment in ICT has shown significant returns in the developed countries, results demonstrate no significant returns in the developing countries (Dewan \& Kraemer, 2000). But (Vu, 2011) sets out many benefits of ICT that lead to economic growth, such as the reduction of the cost of invention, reducing the costs of learning and communication related to innovation activities, reducing $R \& D$ costs and facilitating outsourcing, enhancing the knowledge and learning of the labour force - which increases productivity -, enhancing the quality of governance, and enhancing innovation imitation. However, he proposed that some priorities be adopted for optimizing ICT benefits, such as reforming the education system to prepare people to make better use of ICT, providing internet-based services, improving the availability of information on technology and on the market, encouraging innovation and collaboration, and supporting the quality of decision making. In their study on Italian SMEs, (Colombo, Croce, \& Grilli, 2013) stress this idea, and they emphasize that the adopting of ICT and its effect on productivity is dependent upon contingent factors such as applying advanced communication applications in services, and on customer management and the supply chain in manufacturing; and that this must be associated with organizational and strategic change to the way firms do business. According to Wiseman \& Anderson (2012) ICT has an important role and eases the role of the education system in developing students' skills to make best use of ICT in order to acquire, create and disseminate knowledge, which in the end feeds the national innovation system. This potential impact is constrained by some conditions that are concerned with community norms, culture, and values.

Regarding the above pillars, WBI developed two indexes, the first one called the Knowledge Index (KI) which covers three pillars (indexes) and every pillar represented by three variables: Education index (average years of schooling, secondary enrolment, tertiary enrolment); Innovation index (royalty payments and 
receipts, patents count, journal and articles); and ICT index (number of telephones/1000 people, number of computers/1000 people, internet users/10,000 people). The second index is called the Knowledge Economy Index (KEI); in addition to the three pillars mentioned above, this index includes the economic and institutional regime and covers three different variables: tariff and nontariff barriers, quality of regulation and rule of law (Institute, 2011a). Basically, knowledge assessment methodology (KAM) uses 148 variables that represent and cover all aspects of the four pillars, but as simplified representing process; KAM developed six different methods to display the results indexes, and some methods like scorecards, adopted 12 variables only to show the result. The methods are: basic scorecard, custom scorecard, tables of all countries on all pillars, comparison over time, barchart comparison of up to 20 countries, and a coloured world map (Institute, 2011b). However, following investigations of KAM, suggestions for its improvement have been made. According to Afzal \& Lawrey (2012), KAM does not enable a country to emulate another to improve its position, given that the ranking methodology does not clarify strengths or weaknesses in terms of knowledge acquisition, production, distribution and utilization. They also emphasize that focussing on the selected variables of KAM does not explain how a country acquires, produces, distributes and utilizes knowledge.

Four dimensions, namely knowledge acquisition, production, distribution and utilization should be measured to show the efficiency of a country's performance. This enables easy targeting and emulation of the specific dimension, and the identification of the extent to which it has succeeded in conveying knowledge inputs to outputs (Afzal \& Lawrey, 2012a). Afzal \& Lawrey (2012b) suggest Data Envelopment Analysis (DEA) as a well-known software-programming tool in measuring the efficiency of Decision Making Units (country in our case); this software program calculates the result by dividing the outputs by the inputs to give the efficiency of a specific dimension, and hence any country can spot and target any other country which shows strength in any dimension and then emulate its behaviour to improve its own weakness. The dimensions and their input and output variables are illustrated in the next table: 
Table 3-2: Dimensions of knowledge activities and the indicators of inputs and outputs.

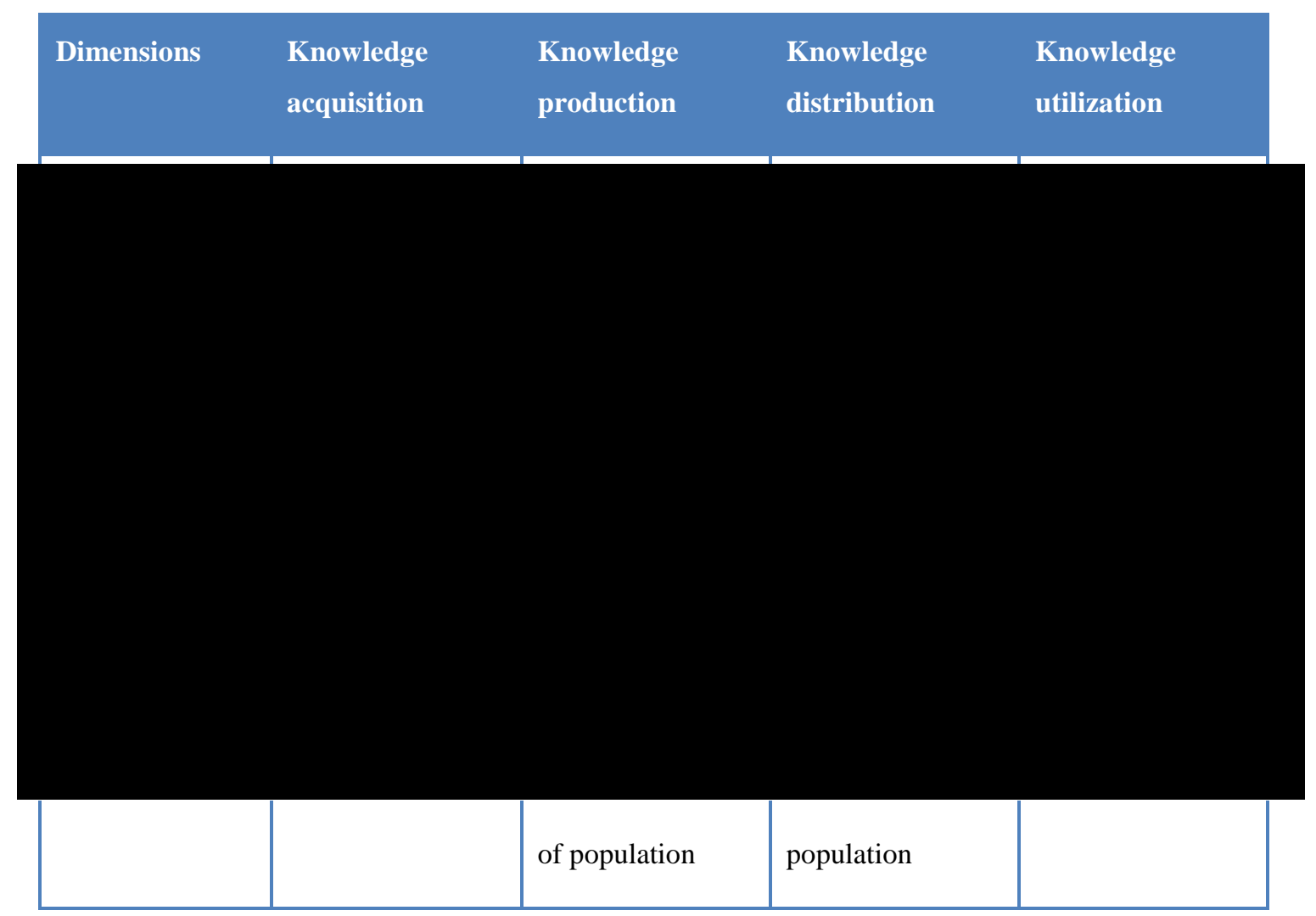

Source: The World Bank Institute (2012)

The four pillars present general guidelines for governments for help in developing a knowledge-based economy, and these pillars and methods represent benchmarking tools for governments to measure their status against, evaluate their progress, and identify and overcome any gaps or voids. The pillars offer a primary idea about the key areas of study and requirements for developing a knowledge economy, however every context has its own needs and characteristics and that should be taken into account (as confirmed by this study's findings). 


\subsection{The role of SMEs in the Knowledge Economy}

Knowledge activities and innovation can take place in any organization regardless of its size, however SMEs have some unique features and special requirements and require a tailored policy for support. The next two sections will review these matters.

\subsubsection{The definition of SMEs}

For SMEs, the relevant and inherent thresholds and difficulties such as access to financial sources and the status of their limited resources, which may affect and impede their adopting a new technology, or involving in innovation process mean that having clear definitions of these sorts of business is vital. Such definitions enable the development of special measures that can be of help in mitigating and overcoming limits, improve the business environment, promote entrepreneurship, investments and growth for SMEs. Therefore, identifying an agreed and clear definition becomes important and necessary.

According to the European Commission (2005) "The category of micro, small and medium-sized enterprises (SMEs) is made up of enterprises which employ fewer than 250 persons and which have an annual turnover not exceeding 50 million euro, and/or an annual balance sheet total not exceeding 43 million euro". Actually, not all countries in the world use the same definition, but they are quite similar. According to the OCED (2005) "Small and medium-sized enterprises (SMEs) are non-subsidiary, independent firms which employ fewer than a given number of employees. This number varies across countries. The most frequent upper limit designating an SME is 250 employees, as in the European Union. However, some countries set the limit at 200 employees, while the United States considers SMEs to include firms with fewer than 500 employees. Small firms are generally those with fewer than 50 employees, while micro-enterprises have at most 10, or in some cases 5, workers".

\subsubsection{Innovation in SMEs}

SMEs have the potential for flexibility (to apply manufacturing systems that deliver short life-cycle products) and innovation. The competitive and changing global 
business environment requires SMEs to develop their capabilities, promote and sustain their competitive advantages and deliver innovations; otherwise they would not be able to grow or survive (Gray, 2006). The entrepreneurial SMEs are the main source of competitive innovations that feed the knowledge economy (Gray, 2006). However the potential of innovation in SMEs revolves around a small portion of this sector, those firms whom have the entrepreneurial attitude and willingness to compete and grow, and who are in the focus of attention of policy makers (Gray, 2002; Harding, Hart, Jones-Evans, \& Levie, 2002). Regarding their vulnerability to uncertainty and limited resources, SMEs should be innovative to have a competitive advantage (Keskin, 2006). The innovativeness is a key means for SMEs to perform the role of an engine for economic growth and employment (Radas \& Božić, 2009). According to (Chesbrough, 2003), SMEs' contribution to the innovation landscape has increased and become prominent.

To stimulate innovation in SMEs there are antecedent factors which must be considered as having a significant effect on the delivery of innovativeness; some internal and others external The internal ones concern the characteristics and policies of SMEs, while external ones relate to the opportunities that can be explored and exploited in their environment (Keizer, Dijkstra, \& Halman, 2002).

\subsubsection{Internal factors}

Internal factors cover many areas, according to many authors (Hoffman, Parejo, Bessant, \& Perren, 1998; LeBlanc, Nash, Gallagher, Gonda, \& Kakizaki, 1997): highly qualified managers or founders, strong leadership, and the existence of qualified engineers and scientists are all important as internal innovative variables. Hoffman added other variables including the extent of marketing involvement in product planning, technology competence and strategy, and marketing and commercialization efforts as being key to effective knowledge exploitation. According to Freel (1999) there are some key skills that should exist in SMEs to help in delivering innovations; these include marketing and technical skills, and managerial competencies. Additional to Research \& Development (R\&D), a high skilled workforce, high degree of human capital and the presence of engineers and scientists are key variables in delivering economic knowledge (Audretsch, 2003). 
Project management structures must also be considered as a main factor (Larson, Gobeli, \& Gray, 1991). Other factors such as planning for the future and technology policy were mentioned by (Docter \& Stokman, 1988) and (Oerlemans, Meeus, \& Boekema, 1998). Clear strategies that stimulate risk taking behaviour and creativity were seen as important factors in enhancing the innovativeness in SMEs (Birchall et al., 1996).

Unsurprisingly, in most developing countries, the weakness of human resources constitutes one of the key challenges and barriers for developing a knowledge economy and economic development in general. In this study, significance was given to the issues of developing human capital. As new ideas mainly emerge from university systems, as do those for progressing innovation, SMEs must feature those kinds of skills to assimilate knowledge, as well as the technical and managerial competences that help in developing innovation and commercializing new ideas.

\subsubsection{The external factors}

Other important variables that impact on innovation come in three groups: collaboration with other firms, linkages with knowledge centres such as universities, research centres, think tanks, and professional consultants; utilizing financial support through funds that finance R\&D projects, and the bureaucracy (company laws, access to finance and regulatory framework etc.) that make it easier for SMEs to do business and innovate effectively (Keizer et al., 2002). The USA in the early 1980s developed a programme to promote competitiveness and generate highly innovative firms; this was called the Small Business Innovation Research Program (SBIR), Between 1992 and 1996 there was a 15\% increase in employment in Silicon Valley, and the mean income was 50\% greater than that for the rest of the USA (Audretsch, 2003). Collaboration with other organizations can be deeper, taking the shape of strategic alliances to overcome the nature of the changing global business environment, which considers the development of technology as a competitive weapon, especially in short life-cycle situations, and the rapid diffusion of technology (Cooke \& Wills, 1999; Forrest, 1990). The current nature of the business environment, and the conditions surrounding the SME sector have contributed to the increasing practice of the open innovation model in order to overcome the relevant difficulties and challenges (Van de Vrande, De Jong, Vanhaverbeke, \& De Rochemont, 2009). However, these strong ties can create barriers and companies can forgo opportunities and alternatives by 
overly committing to a partnership, to the exclusion of other ties to innovative organizations (Jones \& Tilley, 2003).

Compared to the conventional production model and its input variables, which include: labour, capital, and land, the knowledge based economy model considers knowledge as a key input to that production function as markets become changeable and the demand increases for short life cycle products and highly technological ones. The comparative advantages that featured in the traditional production paradigm have shifted towards seeking knowledge activities and developing competitive advantages to sustain economic growth and industry sustainability.

\subsubsection{Absorptive capacity and knowledge management}

This term 'absorptive capacity' concerns the precondition of a firm that helps in receiving knowledge and effectively using it. Firms which have the capabilities to manage internal and external knowledge are proactive and more able to produce innovations and promote their competitiveness (Gray, 2006). According to Zahra \& George (2002) firms which are innovative have effective knowledge management over four areas: acquisition and assimilation (potential absorptive capacity), transportation and exploitation (realized absorptive capacity). The absorptive capacity represents the existing resources, explicit and tacit knowledge, culture, management capabilities, and internal routines. The lack of these capacities creates difficulties and challenges to the relevant firms. By improving and promoting absorptive capacity, SMEs can utilize spill over resources and open innovation mechanisms to develop a new innovative ideas (Cohen \& Levinthal, 1990).

Sharing knowledge and effective knowledge management through constant open communication within the firm lead to innovative processes and to creating new knowledge. The effective ICT system plays a key role in facilitating knowledge management via building networks and virtual clusters that help in knowledge exchange and support a specific spill over mechanism within the clusters. To enhance the firm`s absorptive capacity resources, capabilities and routines should be updated. Also the entrepreneurial and management competences of the manager or owner should be maintained. Their levels in education, employee development, and their 
orientation to growth and propensity to innovate reflect the differences between SMEs in their absorptive capacity and their entrepreneurial attitude, and consequently their growth and performance (Gray, 2006).

Authors agree that an effective ICT system is an enabler for innovation and knowledge exchange but it is merely an enabler. It offers no quality of exchange as this results from knowledge management and absorptive capacity in the firms involved in a national innovation system (which for the most part since the 1980s and around the world is dominated by SME business development). The literature is less clear on how small businesses can gain such capacity when they employ low numbers of staff. As such, SMEs alone do not create a good and holistic view of any national system despite their economic and employment potential for a country, hence the need to incorporate larger companies in any such study.

\subsubsection{An intermediated network model: KICMS}

Based on a survey of innovative Korean firms designed to identify the needs among SMEs to enhance and support innovation activities and build collaborative networks, South Korea established an organization called KICMS to facilitate contact and collaboration between firms. KICMS managed to build a huge database on the capabilities of the firms, which makes the Organization a centre to hold collaborative agreements between firms and an easily accessed source for finding missed innovative capabilities. By 2007, 4415 firms had joined the organization as members. This model has helped the collaboration and networking process and enables the open innovation approach to enhance innovations generated among SMEs (Lee, Park, Yoon, \& Park, 2010).

\subsubsection{Entrepreneurial orientation in SMEs}

Referring to the Schumpeterian view which considers innovation to be at the centre of entrepreneurial activity, Paper et al. (2011) suggest that SMEs are more innovative than large firms, and can compete with large firms by adopting niche strategies that embody flexibility, exploring and responding to customers' needs, and delivering high product quality. According to many authors (Hamel, 1996; Miller, 1983; Sathe \& Drucker, 2003) the performance of entrepreneurial firms, which create, define, discover and exploit opportunities, is better than their rivals and a step ahead of them. Their entrepreneurial and marketing orientation are strategically complementary to 
each other. This orientation relies on adopting the behaviour of detecting competitors' strategy and markets, and analysing the relevant information to respond timely and innovatively to the customers' needs and expectations, and to the changing business environment (Huang \& Wang, 2011). This orientation embodies innovation willingness and requires learning orientation to ease and facilitate the innovation performance (Baker \& Sinkula, 2009). According to Lumpkin \& Dess (1996) the entrepreneurial SME has three key dimensions namely; innovativeness, proactivity, and risk-taking. The entrepreneurial orientation and consequent learning orientation help SMEs to compete with large firms in sustaining competitive advantage through continuous learning and innovation (Huang \& Wang, 2011). With regard to the uncertain and shifting environment of business, entrepreneurship is a key practice, which SMEs adopt to develop innovations and deliver new products (Avlonitis \& Salavou, 2007). 


\subsection{Large Enterprises}

To establish a balanced view of an economy in transition, it is important to review the system from the large company perspective (where resources are greater than SMEs and many businesses engage in global knowledge transfer and investments). However, large companies have more structural design, and the decision making process takes longer than in SMEs; the nature of this structure design and decision making process reflects on the entrepreneurial performance of large companies; more details of this will be given in the following sections.

\subsubsection{Corporate entrepreneurship}

Under the conditions of bureaucracy, complexity in processes, weight of size, inertia, and hierarchy, large companies lose their agility, proactivity, risk-taking and innovation. Corporate Entrepreneurship and its embedded features can bring the large companies to reintroduce the missing role(s) and that can help them in achieving growth and sustaining competitive advantage (Thornberry, 2001). Control systems and mind-sets in large firms do not usually fit with innovative and entrepreneurial behaviour. SMEs are more efficient in entrepreneurial activities. The lacking of entrepreneurial energy, weakness in reallocating resources with enough speed, and the high cost of non-traditional technology create difficulties for large firms in the exploitation of entrepreneurial opportunities, and usually their $R \& D$ investment focuses on incremental innovations instead of radical ones (Hoskisson \& Busenitz, 2002), and they find it inherently difficult to cope with radical and disruptive inventions (Christiansen, 1997). That said, irrespective of size, entrepreneurship can be practised in any organization. However, global competition and business conditions of uncertainty and technological change mean that large companies need to adopt entrepreneurial strategies to survive and sustain growth (Morris, Kuratko, \& Covin, 2010).

According to Lassen et al. (2006) companies that adopt an entrepreneurial orientation have significant influence on radical innovation. Corporate entrepreneurship activities can be carried out through business units, teams or any form of decentralization process like for example, stimulating spin-offs. This practice could be seen as 
mimicking smallness - entrepreneurs or corporate entrepreneurs implementing their entrepreneurial actions by mimicking smallness in the form of joint ventures, business units or subsidiaries (Wennekers \& Thurik, 1999). However, in terms of the context effect (Amsden, 2011) suggests that large privately-owned enterprises in the developing countries are more innovative than multinational companies. Hoskisson \& Busenitz (2002) identify three kinds of model for corporate entrepreneurship, namely, internal venturing, joint venture, and acquisition; these models should be developed and adapted with regard to two key dimensions, namely market uncertainty, and learning distance. They suggest that adopting an acquisition approach would be more suitable in the conditions of low market uncertainty (to what extent the market will accept the new product or service) and a high level of learning distance (how close the firm's current capabilities are to the required capabilities necessary for realizing and exploiting entrepreneurial opportunity and developing a new product). Joint ventures are supposed to be effective in situations of high market uncertainty and high learning distance. Finally in the model is internal venturing - which is recommended as an approach when the case is characterized by high uncertainty and the potential innovation is related to the current firm`s capabilities, which represent low learning distance.

However, Thornberry (2001) sees corporate entrepreneurship from four dimensions, namely corporate venturing, entrepreneurship, organizational transformation, and industry rule-breaking. Corporate venturing means starting new business, which depends on the core competence of the firm but using it in a new way, by entrepreneurship, company enhancing and spreading the attitude of entrepreneurship behaviour among its employees to explore new ideas and develop spin-offs projects. Organizational transformation involves a new arrangement, innovation or combination of resources, which lead to creating sustainable value. Finally, industry rule-breaking requires changing the structure or the game rules, for example Toyota, by developing low cost and high quality automobiles, forced the American and European competitors to adopt the same approach, also Amazon and its model in ecommerce changed the way that books are sold.

Authors see large companies as needing to exhibit the agile small company characteristics in order to exploit knowledge. Large companies have resources in 
people and finances to invest and work with partners (governments and academia) and they have a greater ability to undertake riskier ventures. As such they form a major part of any national system and can rapidly bring knowledge to a country or bring products to market in a much more effective mode than SMEs. Larger companies also tend to be at the head of supply chains and are influential in marketing innovation; they are reliant on SMEs that are further down their supply chain. In addition, large companies may outsource to SMEs and there is a dependency relationship between large and small businesses as well as a dependency with the government in terms of policies to enable and support innovation.

\subsubsection{Entrepreneurship Strategy}

Entrepreneurial strategy is considered as a way to develop a firm`s capabilities and a tool for sustaining competitive advantage (Cooper, Markman, \& Niss, 2000). According to Ireland et al. (2009) "corporate strategy ...[is] ... a vision-directed, organization-wide reliance on entrepreneurial behaviour that purposefully and continuously rejuvenates the organization and shapes the scope of its operations through the recognition and exploitation of entrepreneurial opportunity". The authors developed a corporate strategy model with three core dimensions:

Corporate entrepreneurship strategy antecedents

○ Organization`s members entrepreneurial cognitions (attitude, beliefs, and values)

○ External environment conditions (intensity of competition, product marketfragmentation and product market-emergence, change in technology)

Corporate entrepreneurship strategy elements (these elements are essential for company which wants to have entrepreneurial strategy)

- Organizational architecture (structure, resources and capabilities, reward system, culture)

○ Entrepreneurial strategic vision

- Entrepreneurial behaviour (recognition of and exploiting opportunities)

Consequences of applying entrepreneurial strategy

- Competitive capability 
- Strategic repositioning

The authors' formula is significant as it features in all knowledge economy models that have been developed and the dimensions used allow the KSA life sciences current system status to be assessed in terms of how large companies (who often do not need to borrow money like SMEs) get involved with a national system and influence it. Hence the need to include these forms of informants in order to gain a holistic insight into what supports or prevents meaningful progress in the KSA.

\subsection{Multinational Enterprises (MNEs)}

MNEs usually have superior resources in terms of managerial, technical and financial capabilities; these features make countries, especially developing countries, welcoming of their investments and motivate the development of an attractive infrastructure and regulations that could attract them. Usually they have an entrepreneurial strategy and attitude, however carrying out knowledge activities is linked to the context, and the external and internal enabling factors. Such a context and reaction to inward investment is therefore significant to countries like the KSA.

\subsubsection{MNEs and dynamic capabilities (DCs)}

According to Velasquez \& Velazquez (2002) an MNE is 'a company that maintains manufacturing, marketing, service, or administrative operations in many different host countries'. Also it has been defined as a firm that has overseas managed operations and resources dispersed over more than one country to generate income (Jones, 1996). Historically, Hymer is considered as the founder of the MNE theory, as he suggested the establishment of MNEs and foreign direct investment FDI is motivated by monopoly reasons, enabling the firms in the developed markets to be globally dominant (Dunning \& Rugman, 1985). However, Pitelis \& Teece (2010) see, despite cost minimizing being a factor behind MNE existence, that cross-border market co-creation, ecosystem co-creation, dynamic and entrepreneurial capabilities, and value creation are the essence of and constitute the theory of MNEs. They also confirm that MNEs impact technology transfer and lead host countries to encourage inward FDI by improving their competitive capabilities via access to knowledge. 
Knowledge and capabilities are key requirements for MNEs which can scale and employ them globally to create additional value (Teece, 1977). In this respect the motivation of MNEs is to extract value and they will locate in geographies that are conducive for this, which implies that developing countries must either offer knowledge resources for exploitation or offer favourable financial incentives/tax regimes.

Those capabilities and resources that are non-imitable and difficult to trade are called dynamic capabilities and lead to achieving the sustainable competitive advantage, which enable firms to create knowledge, respond to competition and changing customer needs. Also these dynamic capabilities are relevant to the knowledge-based economy and the semi-globalized markets. Because of several factors and reasons such as production factors mobility, innovation sources becoming more global, and the expanded global trade and investment; competition increased, on a global scale, over the production factors (capital, skilled labour, technology) (Pitelis \& Teece 2010).

According to Augier \& Teece (2009) these dynamic capabilities (DCs) are related to the ability to market and source globally, to the possession of the business vision and insights required to create new business models that can be globally scaled, to the need for astute investment tools to identify, address and create new technologies and markets, and the capacity to estimate uncertainty. These capabilities are assumed to exist in entrepreneurial management and are rooted in organization processes and routines. Also these DCs include so-called managerial orchestration, which is concerned with the continuous monitoring and tracing of changing global opportunities to capture new ideas and implement them promptly (Teece, 2007). The substitute innovations are usually threatened by imitation and that puts a significant importance on to complementary innovations (co-specialized intangible assets), which by their nature are global in scope (cross-border) (Pitelis \& Teece, 2010). Regarding this, Augier \& Teece (2007) see the ability to identify, develop and leverage cospecialized assets globally as a key dynamic capability (DC). MNEs, with the embedded DCs, effectuate solving market failure through cross-border markets, 
ecosystem and cluster co-creation (Pitelis \& Teece, 2010), where market co-creation is relevant in the semi-globalized knowledge-based economy (Teece, 2007).

The current situation of dispersed technologies over the world means that reliance on in-house $R \& D$ as the only way to competitive advantage no longer accepted, which entails firms adopting internal and external approaches to develop new products and services that match customers' values and expectations (Augier \& Teece, 2007). With regard to the internalisation, inclusion of contractual/transaction reduction cost (comparative advantage) perspective, which is a particular aspect of MNE theory, neglects other aspects of MNE theory, such as the dynamic capabilities-based entrepreneurial MNE theory. This new theory considers entrepreneurial management and leadership incorporated into the dynamic capabilities, and explains how strategy and dynamic capabilities develop and sustain competitive advantage in different global environments (Teece, 2014). Regarding the new structures of worldwide dispersed knowledge, the knowledge-based theory confirms that MNEs have become knowledge systems and need to sense and explore any useful combinations of knowledge where they are in the world to develop new products or services.

This approach requires specific managerial skills and capabilities to facilitate the exploring and exploiting processes (Foss \& Santos, 2011). Among the managerial skills and with respect to the nature of different cultures and values relevant to the concept of sharing knowledge between the MNEs employees, MNEs should develop organizational cultures to mitigate the differences of culture values among employees, and this issue should be taken into account in the staffing process (Sandhu \& Ching, 2014).

\subsubsection{Foreign direct investment (FDI)}

Some countries have benefited from FDI while some have not. To benefit from foreign direct investment for the sake of innovation and knowledge transfer requires numerous items to be in place to ensure that knowledge transfer happens. The next sections explore these conditions in detail. 


\subsubsection{Definition and historic perspective}

The United Nations Centre on Transnational Corporations (UNCTAD) defines FDI as "an investment involving a long-term relationship and reflecting a lasting interest in and control of an enterprise resident in an economy other than that of the investor. FDI inflows are capital provided by a foreign direct investor to an FDI enterprise".

The International Monetary Fund (IMF) defines net FDI inflows as the net investment inflows to own $10 \%$ or more of voting stock in enterprise locates in deferent country of investor. The US department of commerce considers an investment as foreign direct investment if the foreign investor owns $10 \%$ or more of a local firm`s equity. The investment via purchasing firm`s securities and bonds without having any controlling or decision over the firm is called portfolio investment (Alfaro, 2014).

Firms or individuals are motivated to invest abroad through attractive factors such as accessing special inputs (labour, raw materials), accessing a new market directly, increasing efficiency, and keeping competitors from getting strategic assets (Desai, 2009). However, capital moving abroad is related to capital cost and returns on equity, when capital moves from low capital returns rate countries to high capital returns rate ones, which is known as the cost-of-capital theory. There is evidence that investors usually do not bring all their capital with them and instead use local financing mechanisms (Alfaro, 2014). This suggestion is argued against by Hymer, who contradicts this financial approach, believing that the cost of capital is not the reason behind the foreign investment and suggesting that certain assets (patents, know-how, technology, managerial skills) are worth more under foreign control rather than local, and that this enables the firm to compete in unfamiliar environments and to outperform competitors (Hymer, 1976).

\subsubsection{Spill over}

Foreign direct investment embodies capital, know-how, and technology transfer. Knowledge spill over from foreign firms is supposed to improve the productivity of local firms; this takes place as a result of various reasons or mechanisms: the introduction of new products or services, observing foreign firms, labour movements from foreign firms to local ones, such workers being supposed to have higher 
knowledge and skills due to the perceived superiority of foreign firms, which would be expected to reflect on the productivity of the new local employers. Other spill over forms can take place through a spin-offs approach, when employees from foreign firms develop their own ideas and business and exploit the knowledge, experience and skills which they have gained from foreign firms (Alfaro, 2014).

MNEs produce spill over impact and technology transfer, which leads host countries to encourage inward FDI by improving their competitive capabilities (Pitelis \& Teece, 2010). Spill over, and enhancing productivity and wages within the economy, would be achieved through building active forward and backward linkages among the local firms and foreign ones. So, involving local firms in the value chain is critical to help in the spill over process and knowledge transfer (Rodriguez-Clare, 1996). Otherwise, foreign firms would work in isolated enclaves and import their inputs from abroad without involving local firms in their value chain (which would negatively impact on local firms and the economy), and might force the local firms to exit the market (Markusen \& Venables, 1999). This exit comes as a result of the reallocation of resources in the market factors from less-productive local firms to high-productive foreign firms (Melitz, Helpman, \& Yeaple, 2004; Melitz, 2003). However, that reallocation could force and encourage the local firms to upgrade their capabilities and compete with foreign firms (Bao \& Chen, 2013).

Knowledge transfer and any other positive effects of the FDI are debatable issues. The potential for any knowledge transfer, raising productivity, and positive effects relies on the host country's status and conditions in terms of social capability, firms' capacity and technological congruence (Pessoa, 2007). It is dependent on local firms' awareness, capability to react and performance (Meyer \& Sinani, 2009). There is no strong evidence that FDI has a positive impact on economic growth or productivity in the developing countries (Alfaro, Chanda, Kalemli-Ozcan, \& Sayek, 2004; Borensztein, De Gregorio, \& Lee, 1998; Carkovic \& Levine, 2002). The spill over from FDI at the horizontal level (because of the worries of technological leakage to competitors) cannot be assured, but vertical spill overs might take place to improve productivity with suppliers (Alfaro, 2014). This vertical spill over also has been 
investigated by Javorcik (2004) in his study of Lithuania, and Blalock \& Gertler (2008 and 2009) concerning the Indonesian manufacturing sector. Other studies in Romania (Javorcik \& Spatareanu, 2011), Colombia (Kugler, 2006), and Ireland (Görg, Hanley, \& Strobl, 2011) confirm that these inter-industry externalities and the vertical positive effects exist.

\subsubsection{Prerequisites for FDI spill over and growth}

The conditions and fitting policies for obtaining positive FDI effects reside at the macro and micro economic level. At the macro level, the labour-market policies and the ease of entry and exit of firms are correlated to the positive impact of FDI (Harrison \& Rodríguez-Clare, 2009). Outward-oriented trade policies enhance the growth caused by FDI (Balasubramanyam, Salisu, \& Sapsford, 1996). The competitive environment is seen as important and conducive to the growth via manufacturing FDI (Moran, Alfaro, \& Javorcik, 2007). According to some authors (Borensztein et al., 1998; Xu, 2000) the minimum human capital threshold in host countries is critical to knowledge transfer and growth caused by FDI; they find that FDI is more productive than local investment in the case of the provision and availability of human capital thresholds, and they argue that most developing countries have a shortage in this area, which makes them miss opportunities to benefit from technology transfer via FDI. Other macro aspects concern the local financial sector and its relation to growth caused by FDI. The high professional financial markets have the capabilities to reduce transaction costs and manage their financing processes better by far in terms of reallocating funds to more productive enterprises with the potential of high returns (Alfaro, 2014). The supposed growth comes mainly through the gains in the total factor productivity (TFP) beside the accumulation of the physical and human capital, when less-productive firms exit, and market reallocation supports the more-productive firms (Alfaro, Kalemli-Ozcan, \& Sayek, 2008).

As such, the aggregate productivity increases in the host country via two mechanisms, the first through the spill over from FDI, and the second from market reallocation, which increases the average productivity of the country as a result of the exit and crowding out of the less productive firms. This could hinder domestic entrepreneurship, which necessitates improving domestic market conditions such as 
credit access, labour supply, and eliminating any regulatory barriers to the reallocation (Alfaro, 2014; Alfaro \& Chen, 2013). On the other side, at the micro level, in addition to the macro factors illustrated in human capital and high quality financial markets, forward, backward and intra-industry linkages are critical to utilizing the benefits of the FDI spill over (Bruno \& Campos, 2013). The spill over, and enhancing productivity and wages within the economy, would be achieved through building active forward and backward linkages among the local firms and foreign ones. So, involving local firms in the value chain is quite significant as an aid to the spill over process and knowledge transfer (Rodriguez-Clare, 1996). It is important that, through the interaction between foreign and indigenous labour, the nature of different cultures and values relevant to the concept of sharing knowledge between the MNE's employees is understood; MNEs should develop organizational culture which can mitigate the differences of culture values among employees, and this issue should be taken into account in the staffing process (Sandhu \& Ching, 2014). In this manner a compatible corporate culture and local national cultures should be developed so that innovation can flow freely to and beyond the MNE.

\subsubsection{Reverse innovation}

According to Govindarajan \& Ramamurti (2011) reverse innovation refers to the diffusion of innovations, and new products and services from emerging countries such as China and India to the developed countries. The current situation of MNEs is changing as the companies from emerging economies such as China and India start to take a share of global market places, and compete with those from developed countries (Kothari, Kotabe, \& Murphy, 2013). By 2009, the share of the USA and Japan overseas stock investment decreased while Chinese and Indian MNEs increased (Ramamurti, 2012).

The MNEs from emerging countries have overcome the conditions of a weak business infrastructure and environment, and developed capabilities which later they managed to expand into the developed markets and compete. These firms developed over three stages, namely 'licking dirt to carve out the way'; 'taking off with speed and strength', and 'around the world with excellence' (Kothari et al., 2013). Through these stages the firms developed capabilities to beat the shortcomings in the business environment, and subsequently to succeed domestically, in the second stage and after building good financial positions they started to explore the developed markets to find 
suitable partners or make acquisitions to enhance their presence. By the final and third stage the firms managed to develop their own brands and move up in the value chain by undertaking high added value activities. Basically, these firms benefited from low cost high skilled people, and invested in R\&D to develop new innovation and enhance their absorptive capacity. They applied strategies along two paths, the first path working on utilizing the niche markets which were ignored by their rivals (demandside theory), the second path by investing in research and developing new products and services (supply-side theory) (Kothari et al., 2013).

These MNEs from emerging economies exploited the low cost features of skilled and unskilled people to export competitive products (in terms of price) at the pre globally expansion and also to become dominant domestically. Later, in the stage of global expansion and working as multinational firms, they exploited the low cost labour feature to export intermediate components to their subsidiaries and to gain intangible assets such as patents and innovative products. The low cost labour enabled them to accelerate their multinational growth and acquisition and assimilation of western technology. By acquisition and asset-seeking processes, these firms managed to catch up with new technology and bring technology transfer to their headquarters in the home countries, especially medium-technology goods in some industries such as pharmaceuticals, software, electronic consumer goods, and some segments in mechanical engineering (Andreff \& Balcet, 2013).

In summary, innovation and labour is a concern. Cheaper labour that lacks technical skill does not support a national system of dissemination and exploitation for a national life sciences system. High quality and highly skilled academics and industrialists are, as many authors identify, key to generating high levels of value added and higher levels of FDI.

\subsection{Innovation}

Innovation is an indicator of a modern and sophisticated society, and a source utilized to solve problems (Godin, 2008b). Schumpeter defined innovation as: doing something new, or even in different way for the already existing things (Schumpeter, 1947). 


\subsubsection{Different discipline perspectives around innovation}

Sociologists developed their own first theory of innovation, thanks to the French sociologist Gabriel Trade in the late nineteenth century (De Tarde, 1903). Trade studied social change, which covered: economic regime, industry, arts, constitution, law, language and grammar. In discussing social changes or evolution, he used a range of terms, including: initiative, discovery, ingenuity, creation, novelty, invention, originality and imagination. His theory was triplex, since it consisted of three elements: invention, opposition and imitation. According to this theory, imitation originates from different inventions; at the same time, the new inventions and the old ones may create opposition or may originate competition. The success of an invention and its subsequent diffusion through imitation relies on social factors and on the competition between the existing and subsequent new inventions. To Trade, invention is society-driven and comes from socially influenced individuals. He also saw society as having fundamentally imitative characteristics (Godin, 2008b). However, sociologists were also criticized at that time, as they were concerned with imitation as a socialization movement which ignored the creativity dimension (Berk \& Galvan, 2009). The sociologists further developed and evolved their theories about innovation, and from 1920 stopped viewing invention and imitation as contrasting concepts, and started to see them as stages of a whole sequential process, thus originating the so called linear innovation model, one where invention is followed by imitation (Godin, 2008b).

In the US, Ogburn and Gilfillan were amongst the first sociologists to develop this model (McGee, 1995). According to them, inventions lead to social or cultural change in terms of behaviour and social organizations (Godin, 2008b). According to Ogburn, using technology is a significant element in any people's culture (Ogburn, 1922). He also believed that the increasing lag between the material culture (technology) and culture that relies on adaptation (adaptive culture) is attributed to shortcomings in social adaptation, which require more adjustment and planning (Godin, 2008b). 
Technological inventions are social processes rather than individual ones affected by social forces, such as democracy, cultural heritage (cumulative process over time rather a one-step action), and they are organized systematic processes beginning from research laboratories. Technological inventions are a combination of the previous technology and ideas, and some elements include: design, method, material, capital, management, and science (Gilfillan, 1935). Economists see innovation as a commercialized technological invention process (Godin, 2008b). Economists see change in the economy as caused by technology that leads to economic growth. The concept of production, work, and economic growth lies in the centre of their concerns and early economic theories. Karl Marx was one of those who believed this (Rosenberg, 1976). Marx saw that changes and development of production techniques lead to modern industry. Machines lead to social changes, as they create a capitalgoods sector, and enhance productivity in the other economic sectors (Benoit Godin, 2008b). Historically, the first economists who paid interest in the technological innovation (technological change) were the mainstream economists (Godin, 2008b). The historical debate about the issue of the effect of mechanization on employment re-emerged after the Great Depression, where the economic theory discussed whether mechanization would be labour-saving, capital saving, or neutral (Pigou, 1924; Robinson, 1938). The Cambridge economist J. Robinson revived this discussion in the 1960s under the name of induced innovation; it was the start of extensive use of the term innovation in economic literature. In the 1930s productivity measurement became an indicator of the use of technology as some public organizations such as the US National Bureau of Economic Research, and the US Bureau of Labour Statistics started to publish statistics, then production function developed to be a measurement tool (Godin, 2008b). This function, which was proposed in the late 1920s, linked the output of the production process to the input (labour and capital) (Cobb \& Douglas, 1928).

The production function states that there are a variety of techniques, which can optimize the output of the available input. The movement in the production function curve illustrates technological change where the capital substitutes for labour. In the late 1950s, the mainstream economists started to link R\&D to productivity, and analyse the contribution of $\mathrm{R} \& \mathrm{D}$ to performance, productivity and economic growth 
and, generally, to industrial development. By the early 1940s, the economic historian Maclaurin used the technological change term to mean the development and commercializing of new products instead of using it to mean only the technical process in production. Later, in the early 1950s, Maclaurin started to use both terms, technological change, and technological innovation, which is reflected in the later literature (Godin, 2008b).

\subsubsection{Joseph Schumpeter (1883-1950)}

Schumpeter was one of the forerunner economists who integrated innovation and considered the role of innovation for an evolving economy. He suggested that technological change came from a trilogy: invention; innovation; and diffusion (Mahdjoubi, 1997). Schumpeter`s trilogy divided technological change into three connected stages, starting with the invention process that produces new ideas, then innovation concerned with the process of producing a new marketable product or service, and the last stage of diffusion (in this stage the potential products or services penetrate the market).

The impact of the diffusion process can be measured by exploring the economic impact, caused by the new technology (Stoneman, 1995). Schumpeter used innovation as a specific stage in the technological change process. However, innovation had been used widely to mean the whole technological change process in shorthand way. According to Schumpeter`s trilogy, basic research is linked to the invention stage while applied research and development is linked to the innovation stage (Stoneman, 1995). In his book Creative Response In Economic History, Schumpeter defined innovation as: doing something new, or even in a different way for the already existing things (Schumpeter, 1947). Schumpeter described the entrepreneurial function as restructuring the production pattern by: exploiting inventions or untried technology to deliver a new product or service or an old one in a different manner; opening a new market or new source of supply, or restructuring the industry (Schumpeter, 2013). He believed that competition between firms shifted from price competition to technological competition through a new product or improved process (Mahdjoubi, 1997). 
To Schumpeter, innovation is responsible for creative destruction as a key feature of capitalism, by restructuring the current industry structure via consistent novelty and changing process (Schumpeter, 1928). Schumpeter is credited as the first economist on technological innovation. He developed some ideas about technological innovation as connected to business cycles (Benoît Godin, 2008b). However, according to Maclaurin, Schumpeter never developed a theory of technological innovation (Maclaurin, 1953). The first theorist on technological innovation is Maclaurin, who undertook economic study in industrial research and the commercialization of inventions (Godin, 2008a). From the early 1940s onward, Maclaurin dedicated himself to studying technological change. Maclaurin was concerned with the factors and conditions that are responsible for and would lead to technological development. Maclaurin explained how the technological change process happens, and broke down the technological change process into sequential steps starting from basic research to diffusion, and then technological innovation defined as commercialized innovation (Godin, 2008b).

\subsubsection{Organizational innovation}

By the beginning of the 1920s, following technological innovation and commercial innovation, other kinds of innovation started to develop called political innovation. These concerned governmental agencies and schools influenced economic activity (McVoy, 1940). The previous kinds of innovation were focused on individuals, but later the concept of organizational innovation was developed by the schools of management and business.

The study of innovative organizational behaviour evolved through concerns regarding organizational structure and style of management (Burns \& Stalker, 1961). Industrialists and managers paid attention to research activities management following the First World War (Mees, 1920). They started to understand investment in technological innovation as an efficiency measure, in terms of returns of investment in $R \& D$ that would enhance firms' performance in terms of market share, profit, and productivity. Organizational environment, conditions and incentives that 
lead to productivity and creativity were of significant interest to researchers (Godin, 2008b).

\subsubsection{Types of innovation}

In the past, an organization worked inside its boundaries (inward focus) and applied a "closed" innovation model, where only its own capabilities were utilized. This concept has changed, and it is no longer seen as the best way of innovating. No organization can have all the necessary capabilities within its own boundaries, so collaboration and bridging with others is very important to integrate with others and gain new ideas, so the open innovation model was developed, based on collaboration outside the boundaries of the organization.

The process of open innovation has three forms: "outside-in" process: in this process a company enhances its knowledge by building integration with external parties such as customers, suppliers, and other external organizations. "Inside-out" process: through this kind of practice, a company gains profit by creating ideas and selling IP to the market. The last form is called: the "coupled process", in this process a company builds cooperation, an alliance or joint venture with a complementary partner (Enkel, Gassmann, \& Chesbrough, 2009). The best known example of open innovation practice is Xerox PARC (Smith \& Alexander, 1988), when some twenty four spin-off firms from 1979 to 1998 departed Xerox to build new business relying on Xerox`s technologies (Chesbrough, 2003). According to Chesbrough, "Not all the smart people work for us. We need to work with smart people inside and outside our company" (Chesbrough, 2003). Again these authors confirm the need for an effective innovation process at an organisation (regardless of its size) if exploitation of innovation is to be achieved. For developing countries this means that innovation and collaboration must be practised and that industry must bring - as an input - an effective process of commercialisation.

\subsubsection{Innovation measurement}

The measurement of technological innovation began in the academic literature in 1910 by counting patents and reached a peak in the 1950s, according to the economist Schmookler (1966). The patent counting process, however, does not cover all 
technological innovation processes, given it was concerned only with invention steps; this led to the emerging of another measurement of $R \& D$ expenditures which gave a weighting of two-thirds to the D of "Development" (of technology) compared to the R of "Research" (Godin, 2006). The US National Science Foundation began applying surveys via this practice, which later spread over other countries and became known as the OECD Frascati Manual (Benoit Godin, 2013). In 1976, Charpie report was published by the US Department of Commerce; it was the first governmental survey about technological innovation (Charpie \& De Simone, 1967). This report showed that $R \& D$ does not represent the main source of technological innovation (Godin, 2008b). By 1992 the OECD developed a methodological tool to measure the innovation process which was called the Oslo Manual (de Oslo, 1992). This manual enabled countries to build statistics about companies' innovation activities, and relied on the same definition of innovation as technological innovation conducive to commercialised innovation that was adopted by Charpie report (Godin, 2008b).

\subsubsection{Global Innovation Index (GII)}

The first edition of the GII was issued in 2008 by three partners, namely: Johnson Cornel University, INSEAD, and the World Intellectual Property Organization (WIPO). The GII consists of two indexes, one to measure the overall GII by calculating the average of input and output elements, and the other to measure the efficiency of innovation by dividing output average by the input average. The enablers or the innovation input sub-indexes include the following pillars:

○ Institutions

○ Human capital and resources

- Infrastructure

○ Market sophistication

- Business sophistication

While the innovation output sub-indexes consist of:

o Knowledge and technology outputs

o Creative outputs (see Figure 3-2). 
Every pillar of the above ones has its own sub-pillars, and every sub-pillar has its own indicators, the total number of indicators being 84 . The average of indicators gives the sub-pillar, and the average of sub-pillars gives the results of the pillar (Dynamics, 2013). 


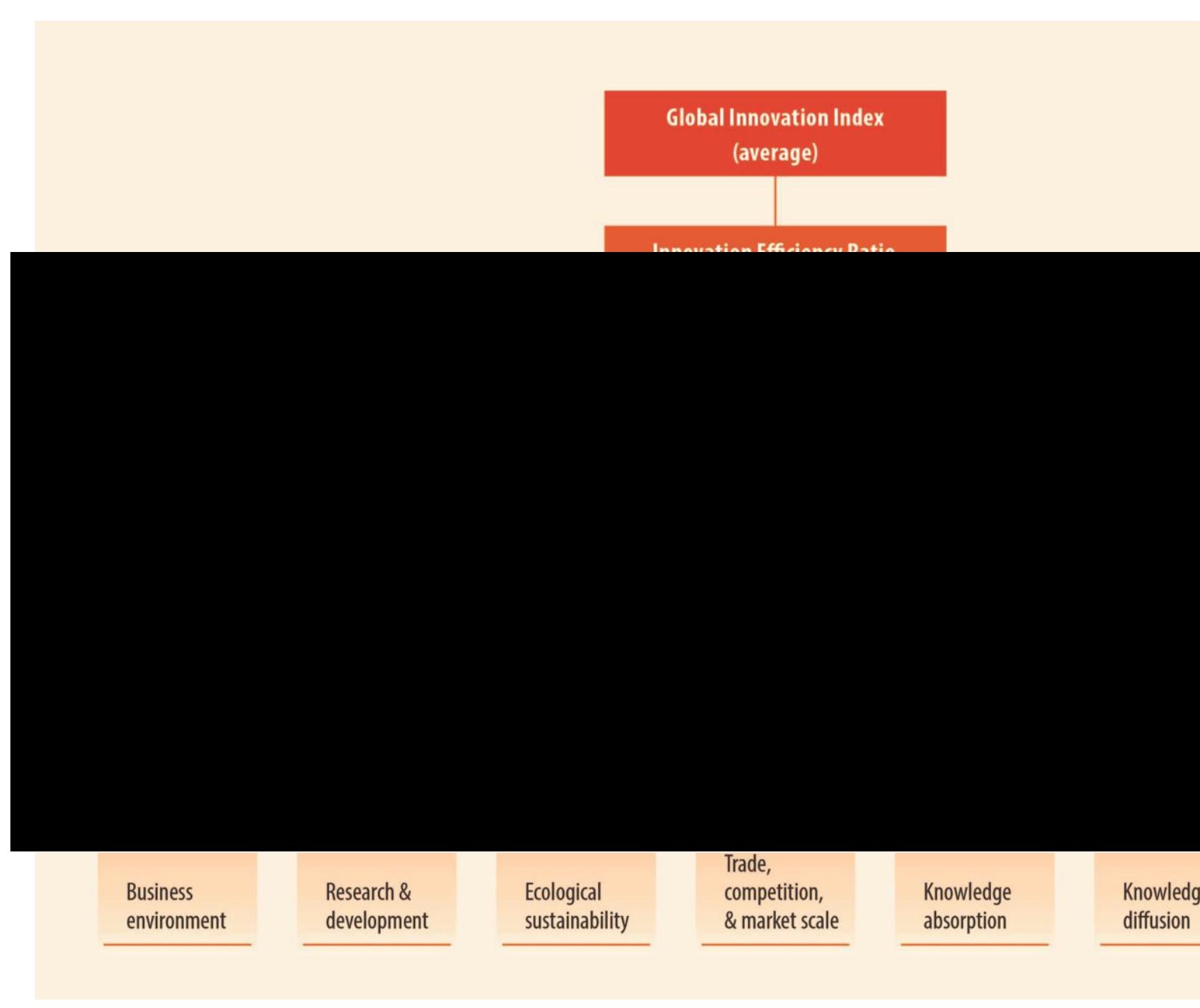

Figure 3-2: Global Innovation Index.

Source: Global Innovation Index (2017). 


\subsection{Innovation Systems (IS)}

There are different types and definitions of innovation systems, and many requirements and conditions for developing innovation systems; all these things are detailed in the following sections.

\subsubsection{National Innovation System (NIS) definition}

In his seminal book the Japan: a New National System of Innovation, Christopher Freeman is the first who to mention the concept of a National Innovation System (Niosi, 2011a). The emergence of National Innovation Systems began in the mid-1980s when the debates took place about European Industrial Policy (Freeman, 1987). The OECD was one of the first International organizations which started to use the NIS concept as an integral part of their analytical perspective (Lundvall et al., 2002).

A National Innovation System "is a set of institutions whose interactions determine the innovative performance of national firms" (Nelson, 1993). Nelson also emphasizes that the core of the innovation system is the policy of science, technology, and innovation that supports R\&D. Lundvall considers the National Innovation System as "constituted by elements and relationships, which interact in production, diffusion and use of new, and economically useful, knowledge" (Lundvall, 1992). The elements include institutions such as universities and public research centres, firms, government regulatory systems, the educational system, and financial institutions (Godin, 2009). Smith (the author of OECD methodological manual on measuring innovation) also stresses the concept of interactions between actors: "the overall innovation performance of an economy depends not so much on how specific formal institutions (firms, research institutions, universities, etc.) perform but on how they interact with each other" (Smith, 1995). Freeman defines the national innovation system as "the network of institutions in the public and private sectors whose activities and interactions initiate, import, modify and diffuse new technologies" (Freeman, 1987). Metcalfe sees NIS as "the set of distinct institutions which jointly and individually contribute to the development and diffusion of new 
technologies and which provides the framework within which governments form and implement policies to influence the innovation process. As such it is a system of interconnected institutions to create, store and transfer the knowledge, skills and artefacts, which define new technologies" (Metcalfe, 1995). However, what can be concluded is that the concept of the National Innovation System pays great attention to and revolves around the interactions between all actors and the innovative output of these interactions and collaborations (OECD, 1997).

\subsubsection{Types of innovation systems}

Building national, regional or sectoral innovation systems requires very effective system structures that work with high transparency and efficiency which can develop and implement policies and incentives to facilitate knowledge flow among all actors and create competitiveness in the business sector. So this meritocratic bureaucracy is a crucial feature for developing countries, while developed ones already have it (Bellows, 1985; Rauch \& Evans, 2000). In spite of the markets having an important role in creating innovative situations in the private sector organization and routines, the role of the government is key; this includes developing innovation policy, funding technical innovation, supporting technical and academic research and education (Niosi, 2011a).

Building innovation systems must take into account contextual factors, which means that 'copying and pasting' other policies would not work well and would not result in innovation and economic development. National scope covers the regional and sectoral systems, but some industries and regions have their own specific characteristics and conditions which should be taken into account when developing policies (Malerba \& Nelson, 2011; Niosi, 2011a). Innovation systems and the quality of governance therefore have an important impact on the economic development of any geography and sector (Fagerberg \& Srholec, 2008). 


\subsubsection{NIS preconditions and capabilities}

Developing countries seek to "catch up" to close the technological gap with leading countries. In his study of the performance of European countries in their endeavours to catch up with Great Britain, Gerschenkron identifies developing new institutional instruments or capabilities such as high quality organizations to identify promising options, and mobilizing the required resources to exploit potential opportunities as key factors for success (Gerschenkron, 1962). Moses Abramowitz saw the term "social capital" as an important factor in developing a 'catch-up' process. In his understanding, social capital includes: supportive, stable, and effective government; technical and managerial competences; efficient and professional financial institutions, and the spread of social values such as honesty and trust (Abramovitz, 1994). According to Shin, the factors and preconditions of catch-up are historic in nature and differ from one period of time to another (Shin, 1996). The work of Abramowitz and Gerschenkorn are studies of a European and American base (Fagerberg \& Srholec, 2008). In his analysis of the Korean catch up process, Kim introduces "technological capabilities" and defines it as "the ability to make technological knowledge in efforts to assimilate, use, adapt and change existing technologies" (Kim, 1997). He sees this concept as having three aspects: production capability, investment capability and innovation capability, with those capabilities moving up in rank and quality as the catch-up process progresses and ascends the technological ladder.

A national technological capability therefore has three aspects: skills, which include managerial and technical competences; the national technical efforts (including R\&D efforts, patents, and technical personnel); and the ability to mobilize financial resources and use them efficiently. Local technological efforts are not enough, and the use of foreign technology, which can be imported via machinery trade or foreign direct investment, is important (Lall, 1992). These features are key to creating a bond that unites any system such as the innovation system in the KSA life sciences sector and these important features are tested by this study. 


\subsubsection{Different "Innovation catch up" models}

East Asian countries (South Korea, Taiwan, Singapore) had similar common factors: heavy investment in developing human capital, stability in the political situation, wise macroeconomic policies, export-orientation, and high saving rate government supportive policies (Page \& Campos, 1993). However, these three new industrialized economies (NIEs) had different technological capability development strategies (Kim, 1997). They adopted three different national innovation systems models, which embedded different mixes of firm strategies, government intervention roles, and innovation network structures (Wong, 1999). Regarding the different conditions for the firms of NIEs, which were looking to catch up with those from advanced countries while working on sustaining their competitive situation, these entailed different national innovation systems and different industrial organizations (Kim, 1997; Mathews \& Cho, 1996). The three countries applied three different models: for example, Korea relied on the agglomeration of large firms, while Taiwan depended on SMEs to achieve its strategies in the catch-up process, and Singapore relied heavily on the MNCs.

NIEs did not engage directly in developing innovations of new products or services like the developed economies, but relied firstly on developing process and production capabilities, and then used their activities within the global value chains to move their targets and expand their activities within the value chain ladder (Wong, 1999). This suggests that there is a sequence to the development of any national innovation system, with the Koreans showing a bias for large indigenous industry as the catalyst and first mover to create a system.

\subsubsection{Knowledge clusters}

It is believed that geographic proximity has a significant positive impact on innovation by easing knowledge "spill over" and using collaboration between cluster members to create an effective ecosystem. The cluster concept was developed by Michael Porter. The cluster is a geographic area which contains a group of firms and institutions in a specific 
field, interacting and connected in a complementary and collaborative manner (Porter, 1998). "A knowledge cluster is a local innovation system organized around universities, research institutions and firms which intend to drive innovations and create new industries" (Evers, 2008). This concept came to be at the heart of economic competitiveness theory, and the competitiveness of a nation is strongly connected to the existence of such clusters in specific industries and to the extent of their strength. Economic growth can be influenced via the clusters approach through three key channels: stimulating the formation of new businesses and expanding clusters; increasing productivity among firms; and identifying the pace and direction of innovation (Huggins, 2008). The clusters work on gathering the distributed and fragmented local strengths in a specific location, which leads to creating high added value (Roelandt \& den Hertog, 1998). According to Fleming \& Marx (2006) this requires a high level of openness for new ideas to be exchanged and combinations of collaborative business environment between the actors within clusters or which are involved in the innovation process. This behaviour will ease "spill over" and help develop technology. The networking culture is a key factor in the knowledge transfer process and the proximity feature of the cluster eases the interaction and face to face mechanism among all actors (Bathelt, Malmberg, \& Maskell, 2004; Sorenson, Rivkin, \& Fleming, 2006). Knowledge clusters have become the driving force for prosperity in a nation, and achieving competitive advantage through innovation is the key objective which clusters look for. Within knowledge clusters, the knowledge workers (individual, firms, universities, and any knowledge creating centre) exchange knowledge and sustain their collective interests to produce new ideas and innovation and consequently facilitate this objective (Romer, 1990).

\subsubsection{Cluster development and evolution}

Developing knowledge clusters requires integration, organization and optimization for the exploitation of production factors (raw materials, labour, and capital). This facilitates collaboration and spill over of knowledge - as a key raw material - in a highly networked business culture and infrastructure. The strong ties and the face-to-face interactions that constitute the highly networked business culture among actors within a specific geographic proximity facilitate the exchange of complex knowledge (Bathelt et al., 2004; Sorenson et al., 2006). The technological knowledge "spill over" is strongly related to 
proximity conditions because non-codified tacit knowledge requires close interactions and contacts to be facilitated between organisations (Lund, 1986). Dynamic interactions between actors has not been the subject of extensive past research but it has been found to underpin successful clusters (Lawson \& Lorenz, 1999; Sexenian, 1994). However, with regard to the kind of knowledge and its sources, and the absorptive capacity of firms, networking can be extended beyond the boundaries of clusters and can have an international spatial dimension (Cohen \& Levinthal, 1990; Drejer \& Vinding, 2007).

Knowledge clusters adapt, shifting their behaviour from being reliant only on internal interactions and local connectivity to connecting globally and developing an international network to enable effective knowledge flow (through global value chain channels). Developing a 'soft infrastructure' (of networking, collaboration, a global supply chain) is also perceived as critical and more important than the hard infrastructure for cluster success. The implication for the KSA is that social and formal business structures must be grown to facilitate these forms of exchange and value exploitation - however the literature is unclear as to whether the Government must lead this activity or if it will occur organically as businesses (large and small) share the same geography or value chain and seek to trade together.

Thought-leading knowledge clusters are, authors suggest, working on widening their scope through consolidation with other neighbouring clusters or by developing international networks (Huggins, 2008). According to Glaeser et al. (1991) developing successful external networks is an outcome of successful local networking and collaboration. Through networks firms may downsize, outsourcing most of the none core activities, which enables them to become more specialized and to focus their resources around their core activity, in order to develop a competitive advantage (Gulati, 1999). However, networking and interaction among actors does not happen spontaneously, and entails cluster administration for developing specific protocols and effective governance that facilitates the "spill over" processes (Bocquet \& Mothe, 2010). There is no specific set of policies which precisely address cluster success, and because of the differences between industries, locations, and operating conditions, replicating other clusters would 
not work, which means that the context must be taken into account and significantly respected (Cortright, 2006). This has significant bearing on the KSA and developing insight into how best to localise clustering of businesses and supporting government infrastructures. The lack of protocols is important in the context of the KSA, where the innovation system is being stimulated and will, from its current state, evolve to command a higher priority in the economy of the country.

The evolution of knowledge clusters encompasses four stages: genesis, development, growth, and renewal or demise (Huggins, 2008). The existence of institutional triggers such as universities or research centres, which would work as a magnet for talent, is a crucial factor which is highly important for the creation of knowledge clusters (Harrison, Cooper, \& Mason, 2004). The key importance of the existence of the institutional triggers resides in the social networks and effective linkages which can be assumed to have already been developed. Social capital is a key element in demonstrating the positive outcomes of interactions and relations among cluster actors. This capital, which embeds social values, beliefs and norms, is crucial to the effectiveness of collaboration and business culture (Garnsey \& Heffernan, 2005). At the development stage, the intuitional triggers spin off knowledge that enables collaborative entrepreneurs to start up their enterprises and build new networks between them and the institutions.

The growth stage of knowledge clusters is the most critical and should attract venture capital organizations, knowledge-based enterprises, and other actors in business and professional activities. Again the literature is unclear concerning the role of the government in stimulating such a scenario for business.

At this stage relationships start to be reshaped into the form of alliances and stronger ties, besides extending their networks across cluster boundaries. Knowledge clusters are similar to products in terms of their life cycle. The final stage or what is called the renewal (or demise) stage is very important, with the life cycle of the cluster relying on 
its actions and dynamics at this point. To survive and grow, the cluster needs to achieve creative destruction by introducing new products and creating new markets, otherwise it will die. This step is dependent on the continual upgrading of human capital capabilities, thus renewing networks, and the development of new modes of interaction (Huggins, 2008). What is important also is the diversification of cluster sectors, which minimize risk and reduce the vulnerability of knowledge clusters at times of economic downturn (Gittell \& Sohl, 2005). The existence of large companies acting as cluster champions helps to provide resources to the cluster in terms of accessing and sharing financial, technical, and managerial capabilities. These companies have high quality marketing and R\&D facilities, and highly skilled workers, which would assist in attracting other companies and raising innovation quality and the potential of the cluster's success (Dynamics, 2013).

\subsubsection{Cluster impacts}

Clusters enable higher added-value by benefiting from the concentration of potential local strengths, as compared to the case of fragmented firms over different geographical areas (Roelandt \& den Hertog, 1998). Clusters impact economic growth through three means, namely: motivating the formation of new enterprises (entrepreneurship), increasing firms' productivity and governing the pace and direction of innovation. Clusters are a physical demonstration of a knowledge-based economy. By exchanging and transferring knowledge among cluster actors, leading to the production of new ideas, and in respect of the importance and crucial role of innovation to achieving competitive advantage, knowledge clusters become key drivers for prosperity (Huggins, 2008). This mechanism of transferring and exchanging knowledge is important in generating new ideas and enhancing the collective interests of cluster actors (Romer, 1990). There is a positive effect from strong clusters on the development and formation of new enterprises (entrepreneurship) and their survival, where related industries and complementarities exist (Delgado, Porter, \& Stern, 2010; Rocha, 2004). However, the expected technological development within clusters requires a high level of openness to new combinations or new ideas by both firms and individuals in order to facilitate product development and commercialization (Fleming \& Marx, 2006). Firms no longer compete within a specific geographical location on the basis of the cost of inputs, or special 
endowments, as these can be overcome or mitigated via outsourcing some activities elsewhere. Competition has instead moved to the field of productivity enhancement and the effective optimization of inputs through developing competitive advantage, mainly achieved through innovation. Due to various factors, such as the existence of sophisticated buyers, interactions and networks within the cluster, knowledge creation centres and others, the clusters have come to represent centres for innovation (Porter, 1998).

\subsection{Actors}

Existing initiatives and actors' roles give insight, but the context must be carefully considered, otherwise copying others' strategies or initiatives would not help in different contexts or conditions. The next sections will explore different system actors (industry, governments and academia).

\subsubsection{Industry}

Technology is spread within an economy by firms (industry), and "catch-up" motivations in order to reach parity with developed countries (industry-government interactions). Such a strategy relies on absorbing pre-existing foreign technology to bridge local gaps in technology rather than through developing an entirely new invention. This means that the speed and acceleration mechanisms that technology diffuses through the economy are very important (Goedhuys \& Veugelers, 2012). According to many authors (Bercovitz \& Feldman, 2007; Brem \& Voigt, 2009; Peeters \& de la Potterie, 2006), innovation strategies can be developed in respect of different dimensions, as follows:

- Exploitation capabilities versus exploration

○ Technology push strategy versus market pull strategy

○ Outsourcing capabilities against internal orientation

- Process against product innovation.

The Indian biotechnology companies are examples of companies which have managed to compete with multinational enterprises (MNEs) in the local market despite the MNEs' 
strengths in terms of financial and technological resources. Indian firms have displayed high quality performance in exploiting and exploring practices. They have managed to leverage external knowledge resources and to develop internal innovation activities at the same time. They have worked on linking innovation strategies to their competitive strategies (Krishnan \& Jha, 2011). Biocon, a large Indian pharmaceutical company, worked on strategies which rely on exploiting their core competences and exploring potential areas of opportunity that require special capabilities, and then developing collaborations with external partners to transfer knowledge, making a joint venture and then a complete acquisition of the joint venture. This company also follows a partnership strategy with key actors in global industry and also small biotechnology firms spun out from universities, and have relied heavily on its external sources, either by acquisition or alliance, to enhance its internal capabilities. The affordability aspect was emphasized to ensure ordinary people's ability to access its drugs without too great a financial burden. With regard to Indian market conditions and the market demand for diabetes drugs, the company has adopted a market-pull strategy and product innovation. In addition to research and manufacturing facilities, the company has expanded its position within the value chain by setting up facilities for clinical trials activities (Krishnan \& Jha, 2011).

In a study of manufacturing firms in Brazil by Goedhuys \& Veugelers (2012), the combination of make and buy strategies proved to be the most effective to fit the Brazilian context and that of other developing countries. However, organizational culture is considered to be one of the most important determinant factors supporting innovative orientation. Moreover, a hierarchical structure promotes an imitation orientation, while an adhocracy structure brings about an innovative orientation (Naranjo-Valencia, JiménezJiménez, \& Sanz-Valle, 2011).

To access international tangible and intangible resources, and fill gaps in their capabilities which are difficult to fill through normal market transactions, Indian firms have practised an acquisition strategy (Gubbi, Aulakh, Ray, Sarkar, \& Chittoor, 2010). Strategic choice has been emphasized by many leading authors (Capron, Dussauge, \& Mitchell, 1998; Ethiraj \& Levinthal, 2004) in enabling emerging companies (which have the 
complementary assets required for achieving strategic objectives and solving complex problems) to engage with each other. Internal growth is time consuming and path dependent, while acquisitions may shorten the time needed to develop high quality capabilities for strategic renewal (Nelson, 2005). In their study on the Taiwanese biotechnology industry, Su et al. (2009) confirm the concept of advantage and its relation to local comparative strengths; the Taiwanese biotechnology companies managed to outperform others and to excel by virtue of their advantage in chip-making and precision engineering, which enabled them to succeed in producing diagnostic kit. The results of that study also shed light on the impact of internal capabilities ( $R \& D$, marketing, manufacturing) and external partnership. They find that R\&D capabilities have a positive effect on the development of new products or processes when a firm develops partnerships with universities and public research institutions. Marketing capabilities are connected to customer partnership and the feedback loop generated supports innovativeness in a product. Through marketing capabilities, the supplier positively interacts with and impacts manufacturing capabilities, thus enhancing process innovativeness. According to Teece (1986) and Dosi (1982) the existing internal capabilities within a firm impede innovativeness. Leveraging the existing capabilities may be an effective strategy in the short term; but, for long-term innovative performance, a firm needs to develop partnerships to avoid shortcomings in the exploration of new capabilities (Leonard - Barton, 1992; Levinthal \& March, 1993). This concept is also confirmed by other authors (Baum, Calabrese, \& Silverman, 2000; Powell et al., 1996; Shan, Walker, \& Kogut, 1994) who support the idea that external partnerships enhance the innovation performance of a firm by providing opportunities for exploiting new knowledge. By their scientific discoveries, universities and public research institutions traditionally have strong collaboration with biotechnology firms in developing new products (Rothaermel \& Deeds, 2006).

\subsubsection{Governments}

The role of government is central to system design and operation, especially in developing countries. Besides other tasks, building the required research infrastructure is 
the responsibility of the government, and as such, any national development project must be government-led. According to Ahn \& York (2011), enabling initial local advantageous factors is very important for developing a successful biotechnology industry. Thus, developing a distinctive infrastructure with critical capabilities, and facilitating the bi-directional flow of capital and technology, are crucial governmental roles.

Government should evaluate the resources required (skilled labour, national endowments, capital, alliances, networks) and compare them with the existing ones. This would lead to a reduction in the academic knowledge gap within the national context and culture, so that policies can be developed to fit the context (a systems approach).

Another very important model comes from Malaysia, where the government took important initial initiatives to support the biotechnology industry development, including:

- Providing sustainable research funding over the R\&D cycle times;

○ Encouraging accessibility to patients for clinical studies;

- Attracting superstar scientists and world class talents to carry out their research, and acting as a magnet for post-graduate and graduate students through the provision of financial incentives and academic positions;

- Attracting foreign investment via incentive schemes;

- Encouraging immigration and repatriation of talented people;

- Allocating \$1billion to implement the Ninth Malaysian Plan dedicated to biotechnology development (2006-2010);

○ Establishing National Biotech Parks;

○ Developing the Third Industrial Master plan;

- With regard to the natural endowments of palm oil and other agricultural traditional strengths, considering biotechnology as the priority sector for sustainability and enhancing productivity;

- Establishing the Biotech Corporation for the purpose of coordinating the biotechnology industry and supporting the alliances among both local and international firms; 
- Establishing a Malaysian Life Science Fund to anchor venture capital, and providing tax incentives (Ahn \& York, 2011).

Again these findings support the 'government-led' first stage of any systems design and the importance of a proactive government presence in setting up the system.

Singapore is another Asian country that adopted a strategy to develop the biotechnology sector as a key sector that would enhance its economy's growth; this was done in the 1980s (Finegold et al. 2004a). By developing a "business friendly environment", worldclass infrastructure, financial incentives, and high quality human capital, Singapore had a good and successful experience with foreign direct investment in ICT, electronics, and petrochemicals (Wong, 2002; Wong, 2001). For this, Singapore relied on a multinational company (MNC) approach as a key contributor and driver for developing its biotechnology sector. Its initiative to developing the biotechnology sector and the embedded huge investment, entailed a strong governmental intervention to act as a driver for the initiative (Finegold et al. 2004b).

The government undertook many actions in support of this initiative. For instance, to support the basic research and building technological infrastructure, it:

- established the Institute of Molecular and Cellular Science in 1987 at the National University of Singapore;

- established other research institutes (Bioinformatics Centre, the Genome Institute of Singapore, the Bioprocessing Technology Centre, and Institute of Bioengineering and Nanotechnology), all between 1996 and 2002.

- developed financial grants (project grants as seed funding, programme grants for more extensive research, grants for strengthening the core capabilities and competencies of research centres) to support basic research.

Another key step taken was to attract the foreign investment via developing venture capital offices in the US, aimed at investing in dedicated US biotechnology firms and thus to have a presence on a global scale, to build networks among American firms and to 
promote Singapore among American venture capitalists. Later these venture capitalists would bring some of their investments to Singapore and contribute to start-up alliances. To maintain both sustainability and impetus, Singapore allotted $\$ 1$ billion to build three bioscience funds, namely: Life Science Investment, Pharm Bio Growth Fund, and Singapore Bio Innovations, which enabled new start-ups in Singapore and joint ventures based in Singapore, and attracted MNCs to invest in Singapore via financial incentives. World class research centres also attracted MNCs to Singapore (Saywell, 2001).

To create the required highly skilled workforce Singapore developed short-, medium- and long-term plans. To build immediate credibility as nascent player in the biotechnology field, it presented financial incentives for international scientist stars to bring their laboratories and research teams to Singapore and to work as magnets to attract young scientists. Given the modest experience of Singaporean companies in this new field, the government encouraged them to recruit scientific and managerial leaders from foreign firms to help in developing new start-ups. Through the short/medium-term plan the government sent talented students to study at the top foreign research universities and then to return to Singapore. Lastly, via the long-term strategy, the government encouraged local universities to build alliances with top international research universities and encourage them to have a presence in Singapore, in order to help in developing the skills required for the future (Finegold et al. 2004b).

To provide a supportive and attractive life science ecosystem to assist the growth of the biotechnology industry, Singapore developed Tusa Biomedical Park and Biopolis near the National University of Singapore (effectively establishing clusters); this park also included a vivarium to accommodate mice for pre-clinical trials. Besides providing a high quality of life, the government developed necessary services such as a lawyers' patent office. To develop the relevant services for legal, ethical and social issues, the government established the Bioethics Advisory Committee (Kong, 2003). In contrast to the status of Intellectual Property protection systems (IP) in some of the Asian countries (China, India) that of Singapore is strong. In this context Singapore established the 
Exploit Technologies body to commercialize the intellectual output of national research institutes.

However, its management of IP is more centralized than, and works counter to the one in the USA, which operates according to the Bayh-Dole Act, giving the universities more freedom to commercialize government-funded research. Such centralization can stifle creativity as it enhances governmental involvement (Vig, 2003). In terms of risk-taking Singaporeans seem to lack the entrepreneurial attitude and that impedes entrepreneurship (Finegold et al., 2004).

This lack of entrepreneurial attitude is reflected in the behaviour of the research scientists, who often fail to spin out their research outputs. Given the government-driven, top-down approach, companies working out of this context find difficulties to have financial resources as their governmental counterpart. Collaborations within and between companies, research centres and industry suffer, and these problems constitute impediments and shortcomings in the Singaporean context (Stein, 2003).

\subsubsection{Academia}

Academia is a very important and vital actor in the innovation process and knowledge transfer, as the functions of universities have evolved to include entrepreneurship alongside the well-known functions of research and education.

\subsubsection{Entrepreneurial University}

Within the context of the Knowledge Economy, scientific knowledge constitutes a key input in innovation and production theory (Mansfield \& Lee, 1996; Mowery \& Sampat, 2005), and this development has become a third mission for universities in additional to the previous traditional two (education and research). The third mission has shaped the new role of the universities in commercializing knowledge, knowledge transfer, and engagement with the activities of society in general and the business sector in particular (Etzkowitz, 1998; Hansen, Dirckinck-Holmfeld, Gregersen, \& Lehmann, 2008; Lehmann, Christensen, Thrane, \& Jørgensen, 2009; Martin, 2003). Also Leydesdorff (1995) added that increasing enrolment of people on higher education course has 
enhanced the university's role in the innovation process. The emergence of disciplines such as biotechnology, and the effects of funding reductions, globalization and the nature of innovation has been seen as a process involving different actors; all this helped in the emergence of what has been called the entrepreneurial university (Rasmussen, Moen, \& Gulbrandsen, 2006). Furthermore, with respect to its ownership of intellectual property rights (IPR); the university in its new role goes beyond cooperation with industry, to include some entrepreneurial activities such as spin-offs, licensing and developing science parks and incubators within the university campus (Etzkowitz, Webster, Gebhardt, \& Terra, 2000; Rasmussen et al., 2006; Wong, Ho, \& Singh, 2007). In the context of the knowledge economy, the university works as a talent collector, providing significant infrastructure for developing the capabilities required for companies' prosperity and survival (Rorida, 1999). However, this new role has been criticized as detrimental to the traditional missions (education and research), and to academic freedom (Nelson, 2004). Etzkowitz (1998) identified concerns related to the short time-scope of research and the potential tensions related to conflicts of interest and the difficulty of maintaining impartiality in the entrepreneurial university. Some of the initiatives that were directed to the commercialization of knowledge produced in the universities were driven by the government (top-down), whereas others were driven by individuals and departments in the universities (bottom-up) (Goldfarb \& Henrekson, 2003). In addition to organizational arrangements, these kinds of activities (knowledge commercializing) require the development and promotion of an entrepreneurship culture and behaviour among university staff and students (Henrekson \& Rosenberg, 2001; Klofsten \& JonesEvans, 2000). Klofsten \& Jones-Evans (2000) propose three actions that should be taken for spreading entrepreneurial culture within a university: building and maintaining the culture of enterprise across the whole of the university; developing particular programmes for those wishing to start enterprises; and developing specific courses on entrepreneurship.

At this point in the systematic literature review, the system actors have been identified and their roles clarified in terms of the system. It is clear that the KSA needs these actors to create and sustain its innovation policies and to rejuvenate a rentier economy. At this 
point, and following the grounded field research, the researcher has developed a conceptual model which will be reviewed later in the findings and analysis but is presented here to assist the reader. It is noted that the model is drawn from many decades of published works and an initial 1200+ papers that were subjected to PRISMA.

\subsection{Theoretical model}

The review of focal literature and theories resulted in the development of a theoretical model for an innovation system that drew inspiration from different contexts. The next figure presents the theoretical model: 


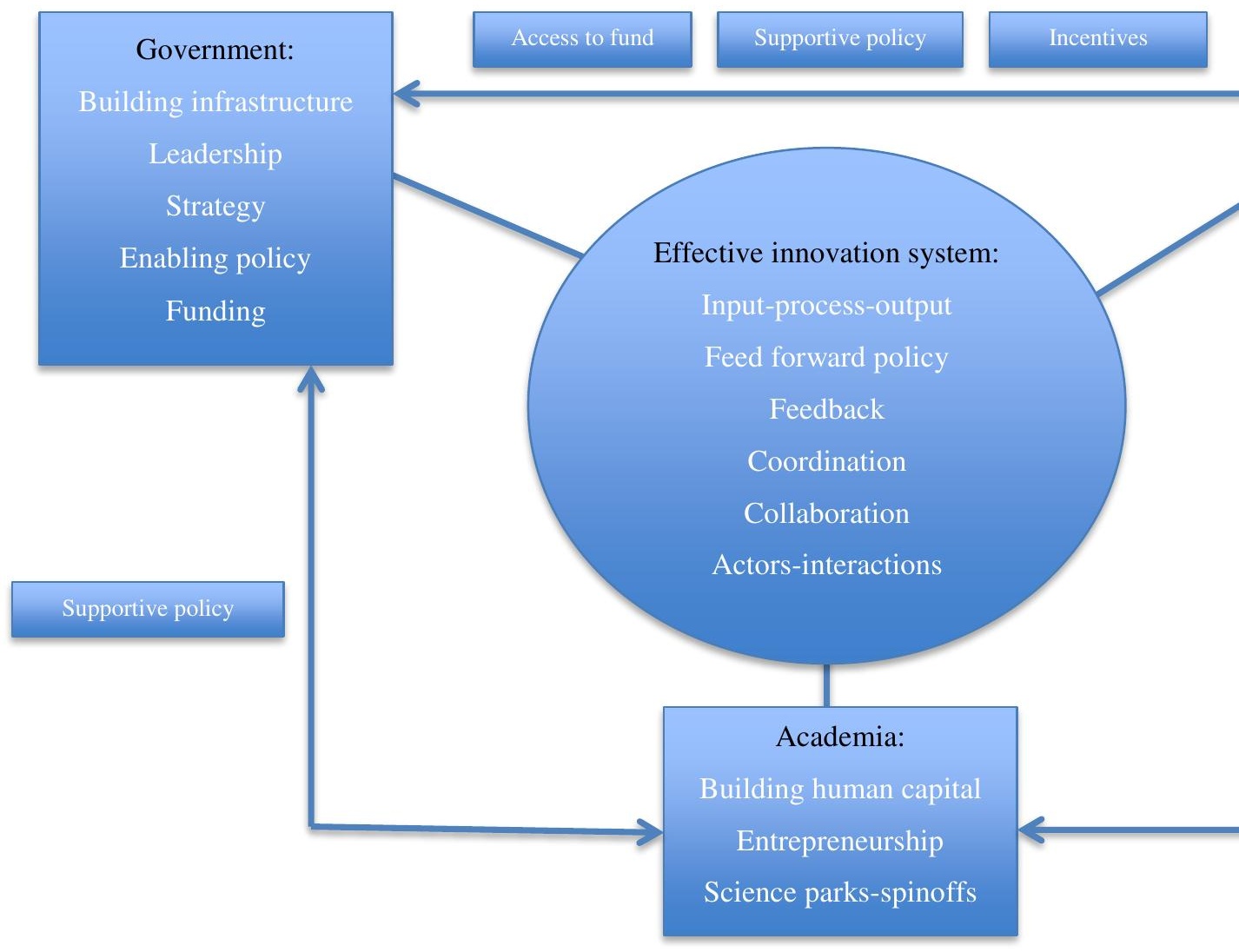

Figure 3-3: Model for theoretical innovation system. Source: The researcher 
The above theoretical model represents the interactions and systematic behaviour that connect the key actors, namely government, academia, and industry. Every party should have internal and external roles for he system to be effective and efficient.

For the government's part, it is expected to undertake the more risky and costly tasks of building infrastructure, funding, developing enabling and supportive policy, taking on the leadership role and involving all the other key actors. By providing the incentives and easing access to money, government can encourage and help industry to do its part. In turn, industry should invest in $\mathrm{R} \& \mathrm{D}$ and work on transferring foreign knowledge by developing partnerships, joint ventures, licensing technologies, or making acquisitions. Industry should collaborate with academia to solve problems and transfer knowledge.

In turn, academia should develop the human capital able to produce and manage quality knowledge, develop entrepreneurial attitudes, develop science parks, and spin-offs to exploit the knowledge it produces. Academia needs government regulations and policy that can assist it in performing its new additional role.

These practices should be governed within a system that engages all parties in developing and undertaking all the necessary processes and assuring coordination and collaboration, and that helps in developing a feedforward policy, and feedback for the entire system. 


\section{Research Design}

\subsection{Introduction}

This chapter will present and defend the philosophy of research and the research design that underpins this study. The aim, objectives, and research questions that bind this research have been identified in section 1.2.4. This chapter will present and defend the design methodology from an ontological, epistemological and methodological perspective. The chapter will follow the research onion that was designed by Saunders et al. (2011). The onion is shown below:

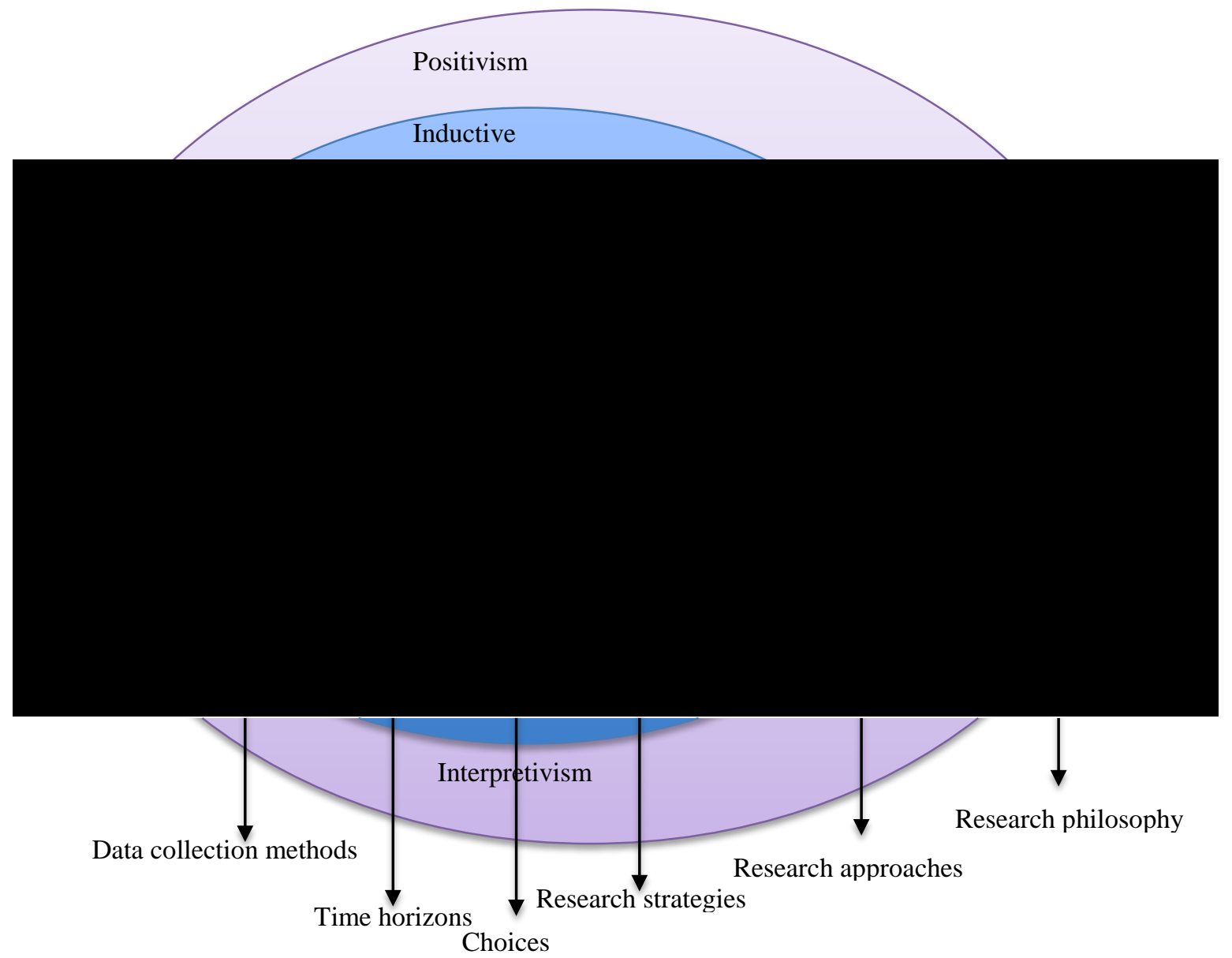

Figure 4-1: Research procee onion. Source: Adapted from Saunders et al. (2011) 
The layers of the "onion" give insight, guidelines and a pathway in many aspects of the research design process: the philosophy of research, the research approach adopted, the research stategy, the time line and the appropriate data-collection methods/limitations. These will now be explored.

\subsection{Research Philosophy}

According to Saunders et al. (2011), Ontology is "the researcher's view of the nature of reality or being" whereas Epistemology is "the researcher's view regarding what constitutes acceptable knowledge" and both need to be understood by a researcher as they influence what is accepted and valid knowledge.

The key ontological question is whether social organizations should be considered as social constructions extracted from the social actors within them, or whether they should be considered objective entities where reality is external to its social actors (Bryman, 2012) and can be measured or experimented with. In other words, social organizations are either viewed from a constructivist or objectivist perspective respectively (Bryman, 2012). Based on the paradigms presented in Figure 4-1, three research philosophy traditions exist (Interpretivism, Positivism, Realism) are detailed below:

\subsubsection{Interpretivism}

Interpretivism considers reality from multiple perspectives and as a relative concept (Hudson \& Ozanne, 1988). The knowledge is subjective not objective, and gaining this knowledge requires social construction accounts and a mutual understanding between informants and the researcher to make sense of the perception of reality (Carson, Gilmore, Perry, \& Gronhaug, 2001). In effect reality does not exist beyond the perceptions and thoughts of the individual and it cannot be captured by numbers and statistics. For Interpretivism, the researcher starts by building the background to the research context, allowing room for redesign of the research because of the unpredictable nature of social situations and the perception of reality. So, it is important for any researcher to understand motives and other subjective matters in relation to their time and 
context (Hudson \& Ozanne, 1988). Such individual biases are important to this form of highly contextual research, especially where multiple actors share a common environment.

\subsubsection{Positivism:}

Positivists prefer "working with an observable social reality and that the end product of such research can be law-like generalisations similar to those produced by the physical and natural scientists” (Remenyi, Williams, Money, \& Swartz, 1998). The positivists believe that "reality" is univocally objective and bears no relation to the researcher's beliefs or perspectives (Hudson \& Ozanne, 1988). In this manner, positivists distance themselves from the subject of their study to remain 'scientific' and treat evidence as only legitimate when it describes a whole population. Positivists apply a structural approach and keep themselves separate from participants in order to preserve neutral emotions maintain distinctions between personal experiences and observations, as well between as feeling and reason. They use mathematical and statistical procedures to seek a single objective reality (Carson et al., 2001). Positivists also believe that human behaviour can be explained in relation to the causes that precede action, they believe that the researcher and subjects are independent and they do not influence each other and that established statistical tests can maintain this objectivity (Hudson \& Ozanne, 1988).

\subsubsection{Realism}

Realists occupy a middle ground in philosophical terms and exist between the two extremes. The social world of business and management is complex to the extent that the application of positivist philosophy would lose the insights of its inherent social complexity. Also new models would not be discovered, as every positivist seeks to test those already in existence. In this manner, complexity and context as influences are reduced to a series of law-like generalisations such as physical sciences (Saunders et al., 2011). The positivist will also smooth away outlier actors or elements of the real world that do not fit the a priori model. The development of such a model could not be 
conducted for this study as little research evidence, with calibrated concepts and questions or data on the population size can be established for the KSA.

Realism is a research philosophy located between positivism and Interpretivism, and considers the truth of reality through the experiences that we have. Reality is considered to be independent of our minds. Realism, like positivism, suggests a scientific way of knowledge development (Saunders et al., 2011) and comes in two forms: direct realism and critical realism. Direct realism argues that what we sense and experience is the only accurate account of worldly reality. The second form of realism is critical realism which considers our experiences as sensations and images of realities, not realities themselves. However, the direct realist argues that, to consider reality as truth, we need to experience things from different perspectives and angles and to gather sufficient information (Saunders et al., 2011). Such an approach has proved useful for this study, combining as it does multiple stakeholders who are, from their own perspectives, making sense of a complex life science system in the KSA.

\subsubsection{The Study`s Research Philosophy (Critical Realism)}

For a researcher, it is important to determine which research philosophy to adopt in order to explain what knowledge is considered to be valid, which methodology has been applied, and how data has been collected (Creswell, Hanson, Plano, \& Morales, 2007; Saunders et al., 2011). The researcher chose critical realism because of a lack of previous studies in this sector and also because the research questions call for a qualitative and context-rich understanding of the phenomena. The situation involves multiple actors, each with their own motivations and potential interests and actions within a system. Critical realists apply multi-level studies at the level of individuals, people and organisations to explore the social structures that underpin a phenomenon.

As critical realism considers the social world as constantly changing, it supports the purpose of business and management research as it explores the reasons for phenomena and for adaptive system change (Saunders et al., 2011). The use of this epistemological framework is consistent with other studies in this area, such as those on industrial 
relations. The next figure shows the ontological and epistemological ideologies of this study:

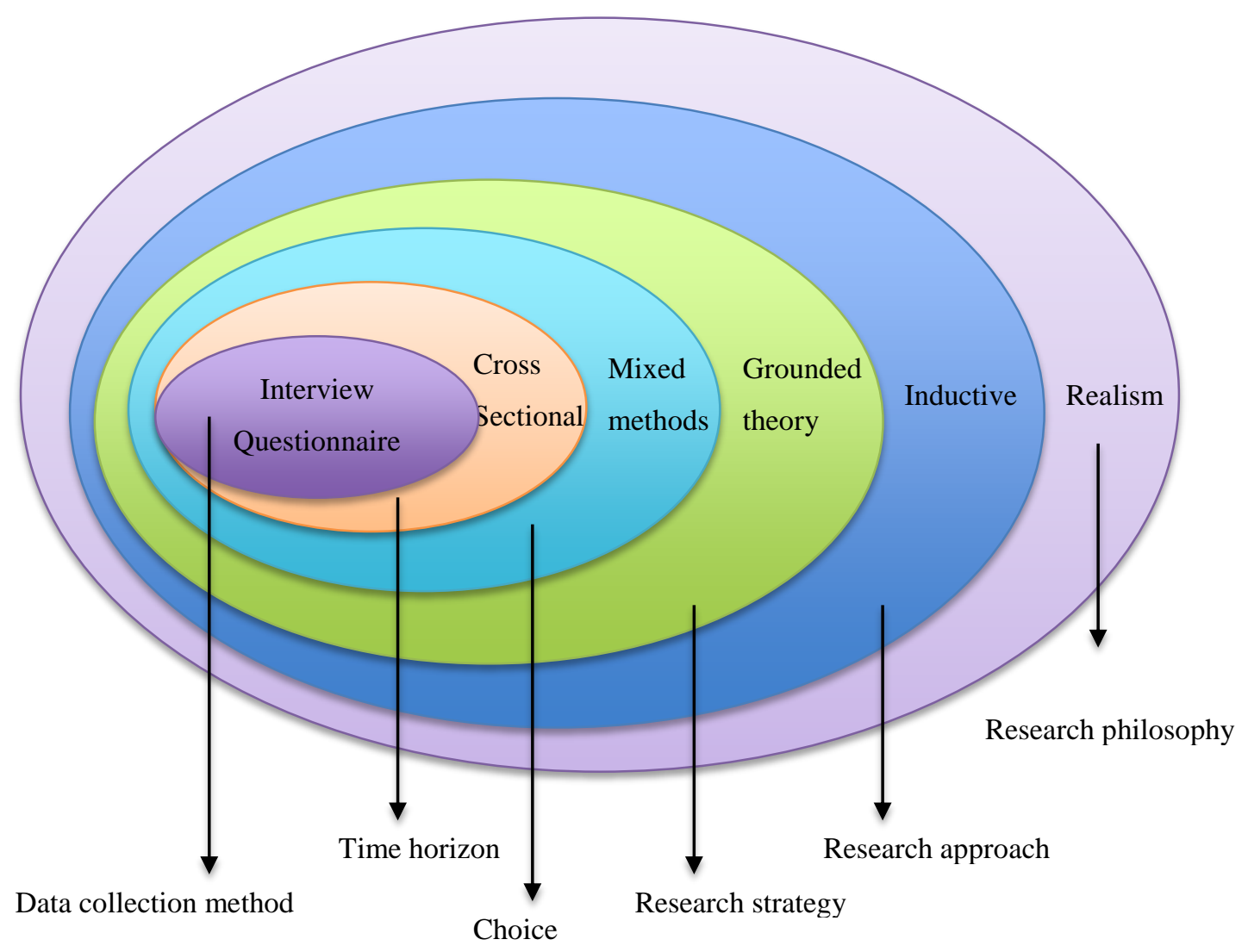

Figure 4-2: Ontological and epistemological ideologies of the study. Source: The researcher.

The social world is what concerns business and management research. According to Bhaskar (2010), understanding the social world is linked to and depends on understanding the social conditions and structures that produce the phenomena that we try to investigate. This means that what we see is not the whole picture, and we can identify the missed parts of the picture through practical and theoretical processes. Regarding all these considerations, this study has adopted critical realism philosophy. The researcher would describe the epistemological approach to this study as positioned between realism and Interpretivism due to the multiple stakeholder views needed to make sense of a complex and messy real world life sciences system where no previous research of this kind has been conducted. 


\subsection{Methodology}

According to Creswell (2013), qualitative approach research is used for theory building rather than testing and includes five approaches, namely grounded theory, case study, narrative, ethnography, and phenomenology research:

Grounded theory: in this approach the researcher tries to develop a general theory grounded on the views of informants of action, process, or interaction. The approach does not promote a deep and systematic literature review before entering the field of study this is to prevent the researcher from gaining bias as a result of the literature. An unbiased researcher seeks to enter the field of study and then - afterwards - return to the literature in order to ground or make sense of the findings. This approach requires multiple steps of data collection and categorizing for information. This strategy features two processes, the constant comparison of data with emerging themes and concepts, and theoretical sampling of different perspectives to optimize the differences and similarities of information (Corbin \& Strauss, 1990).

Case study: the researcher investigates in depth an event, an activity, a programme, or one or more individuals. The case(s) are limited by activity and time, and the researcher applies a range of different methods for data collection and validation/triangulation (Stake, 1995).

Narrative Accounts: a strategy where the investigator studies the lives of people by asking one or more individuals to tell stories about their lives. The investigator then retells the information in a narrative chronology. Finally, the investigator combines views of his life with the informants' lives in a collaborative narrative (Clandinin \& Connelly, 2000).

Ethnography: the researcher collects primarily observational information through studying a cultural group in its natural setting during a prolonged period of time, either overtly or covertly (Creswell, 1998). 
Phenomenology: based on the informants' inputs, the researcher tries to generate and extract the core of human experiences around the phenomena being studied. This requires studying a small number of subjects during a prolonged involvement to understand the relationships of meanings and to develop patterns (Moustakas, 1994).

All these approaches have their own different focus, and methods for data collection and analysis. All are valid approaches and therefore their appropriateness is contingent on the subject of study. According to Miles \& Huberman (1994), qualitative data analysis has three components concurrent and interacting through the analysis: data reduction, data display, and data drawing and verifying Figure 4-3.

There are many different techniques for analysing qualitative research, and these are often overlapping, interconnected and complementary. The diversity in qualitative data analysis techniques means there is no single right way to perform analysis, as this is dependent upon the research purpose, and the analysis technique should be considered and integrated with the research process from the beginning (Punch \& Oancea, 2014). This diversification is positive, but scholarly robustness is a key issue. According to Coffey \& Atkinson (1996) "what links all the approaches is a central concern with transforming and interpreting qualitative data - in a rigorous and scholarly way- in order to capture the complexities of the social worlds we seek to explain". The following diagram shows the interactive model that represents the three components of data analysis developed by Miles and Huberman.

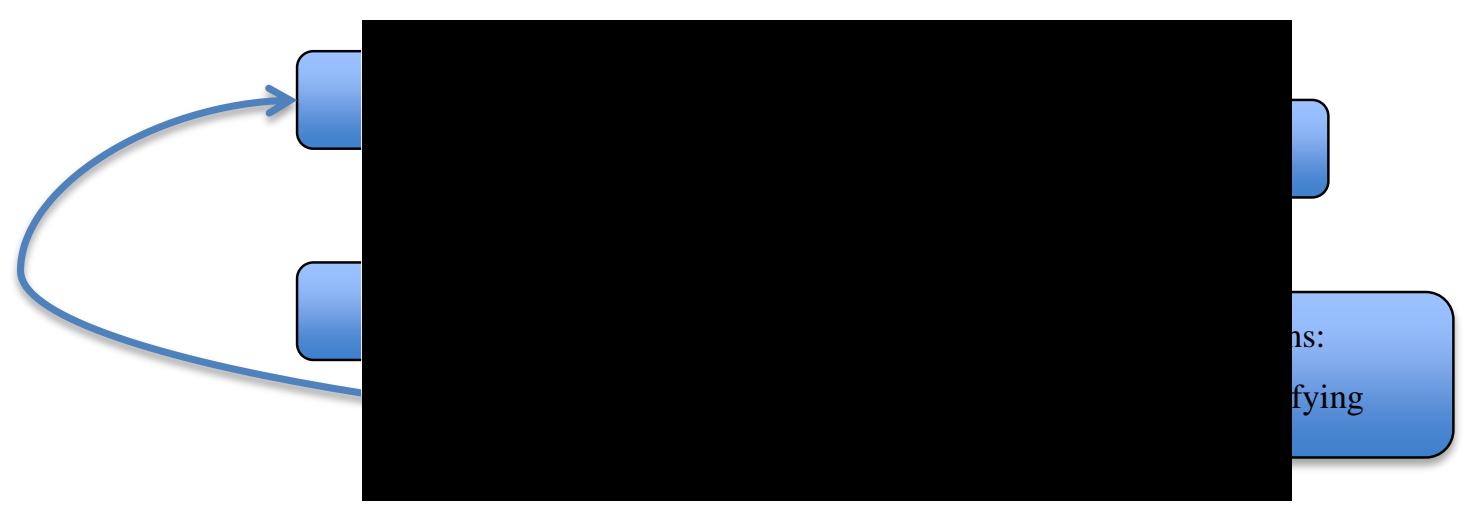

Figure 4-3: Qualitative data analysis components. Source: Adpated to Huberman \& Miles (1994) 
Most organisational research in the field of management studies are case based when developing theory, but the immaturity of the life sciences systems in the KSA means that this was considered inappropriate by the researcher as an exclusive method, because greater context was required. Having conducted a thorough review of methods, the benefits of a case approach were combined with the overall superiority of a grounded approach to theory building. That is to say that actors in the life sciences system are often organisations and therefore could be treated as cases but, rather than conduct research with many managers from each organisation, the decision was taken to maximise the number of representatives of organisations rather than drawing them from any given organisation as this suited the objectives of the study more effectively. In this respect the researcher uses grounded theory development as his main approach and applied methodology to address the research questions and to generate valid knowledge through theory building.

\subsubsection{Grounded theory}

A qualitative methodology can be used to extract indirect information by filtering indepth interviews through the interviewees`opinions (Creswell, 1998). The qualitative approach is effective in answering questions about the complex nature of mainly human behaviour, by understanding and describing the behaviour or phenomena via the participants`opinions (Leedy \& Ormrod, 2005). The content and process of grounded theory (a qualitative approach) was reviewed by the researcher and found to have the most utility for this study. The grounded theory approach requires abstention from a structured and detailed literature review in favour of a general review that allows the researcher to enter the field and build theory from the ground up (a small scoping literature review is conducted initially to help verify the main gaps and issues in the subject under study). The grounded theory approach is most suited to environments where little is known about the phenomena and this is the case with the knowledge economy, especially in the Kingdom of Saudi Arabia.

The researcher also believed that current literature was biased more towards American and European models of innovation management than to any that might exist in the GCC 
countries. Few studies have taken place in this area and therefore existing models might not hold true in such a context. A grounded theory approach is also relevant to studies where the system is evolving and can be studied as a dynamic process of change. The grounded theory approach was thus regarded as offering the best means of capturing insights into the newly developing knowledge-based economy in the Kingdom of Saudi Arabia. The researcher duly adopted this approach as a means of framing and housing his study.

The researcher chose to develop a new model grounded on new collected data instead of testing an existing one (without calibrated and accepted questions for testing, this approach would have been inappropriate to achieve the objectives of this study). It might also limit the study's thinking. An initial review of the literature revealed some innovation models, for example, from western countries and other Asian countries such as Singapore, India, South Korea, and Taiwan. However, these models had been applied in very different contexts, in terms of political, economic, and culture and social conditions. By examining these, the key issues of other researchers could be understood without biasing the present study. The final reason for seeking to develop a new model is that there is no LS innovation model in the GCC, and also the LS is untraditional to Saudi context. Beside all these factors, and having regard to the epistemological and ontological considerations (constructivism and Interpretivism, respectively); the need to gain in-depth insight into participant actions and reactions, by constructing mutual understanding and knowledge against the phenomenon under study, and maintaining the subjective perspective (Carter \& Little, 2007; Guba \& Lincoln, 1982), grounded theory was chosen as the most suitable to address the research questions. Grounded theory helps in “understanding how participants make meaning of a situation or phenomenon" and how "this meaning is mediated through the researcher as instrument, the strategy is inductive, and the outcome is richly descriptive" (Merriam 2002,P.6), and it is mainly used in qualitative studies (Glaser \& Strauss, 1967). Grounded theory uses data to produce concepts and then to develop theories from the concepts rather than testing specific theories against the gathered data. "Grounded theory methods consist of systematic, yet flexible guidelines for collecting and analysing qualitative data to construct theories 
'grounded' in the data themselves. The guidelines offer a set of general principles and heuristic devices rather than formulaic rules" (Charmaz 2006, P.2).

Historically, grounded theory was developed by Barney Glaser and Anselm Strauss (Corbin \& Strauss, 1990; Glaser \& Strauss, 1967) as an inductive approach against the prevailing thought at that time which was the deductive approach. They adopted this methodology so theories can emerge from reduction of real data and later they developed this approach which has become two schools of thought; Glaser school (Glaser, 1992), and Strauss school (Corbin \& Strauss, 1990). The researcher believes that for conducting quality and credible research, it is very important to develop and build a general background to the subject under investigation. This helps the researcher to hold in-depth discussions during interviews. As such this study has adopted the Strauss school which operates in three stages, namely open coding, axial coding, and selective coding (Böhm, 2004).

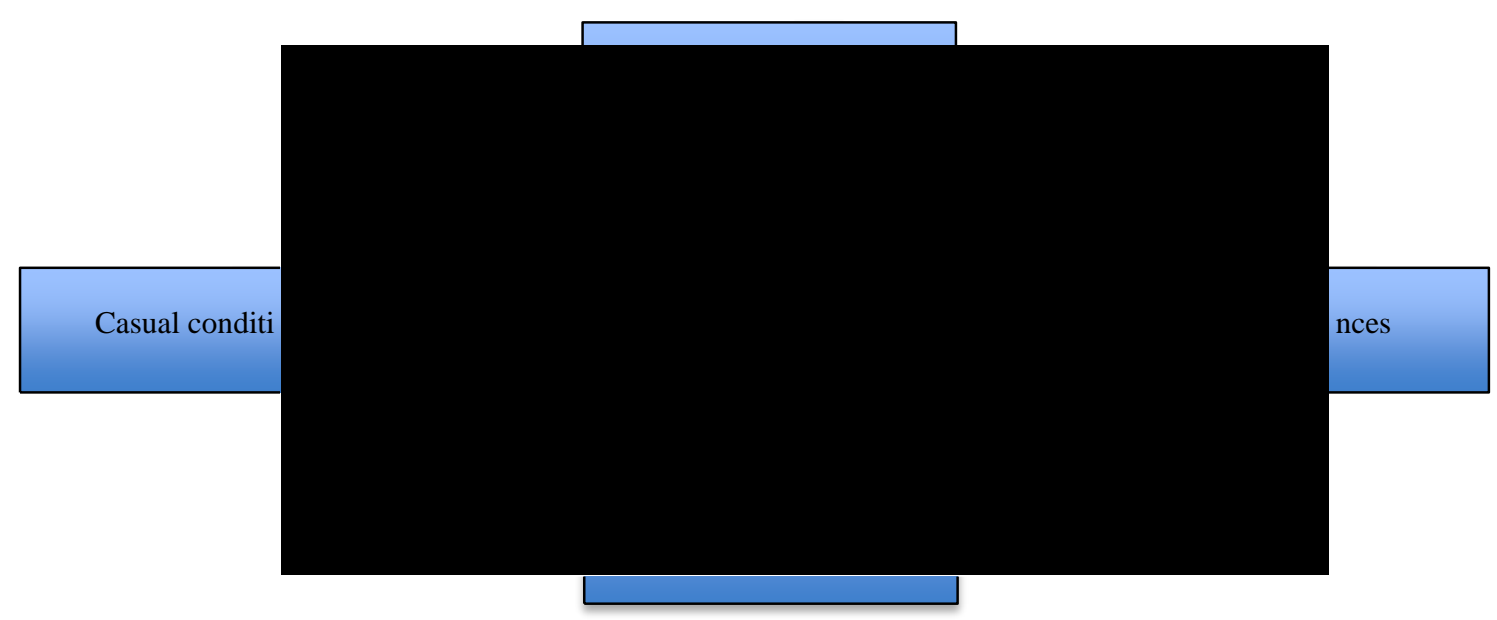

Figure 4-4: Axial coding paradigm. Source: Adapted from (Corbin \& Strauss, 1990) 
Corbin Strauss (Corbin \& Strauss, 1990; \& Strauss, 2008) developed coding paradigm (Figure 4-4) which uses theoretical or conceptual frameworks to work like lenses for the novice researcher to aid data reduction and organisation. This was a major advantage for this study. On the other side, the Glaser School sees this paradigm as an act of forcing data and an abstinence from any literature review (no matter how brief) before entering the field (Böhm, 2004; Fram, 2013). The latter was considered unacceptable by the researcher as this risked a lack of knowledge of what to look at and which aspects of management would be most fruitful. In this respect the researcher undertook a very light literature review to identify the current debates in the field of study, but not in such depth as to bias the study. The result of the initial literature review was a "loose" conceptual model of the ideas which needed to be explored. The model was then revisited after the field research to create the contribution of the study.

\subsection{Phases of the Research}

This study was conducted over two phases, the first conducted through semi-structured in- depth interviews, and the second through a questionnaire based on the findings of the first phase.

\subsubsection{First phase}

Throughout the first phase the researcher conducted semi-structured interviews with senior stakeholders of the LS ecosystem.

\subsubsection{Data collection}

Grounded theory was used to collect and analyse data to answer the research questions through identifying the requirements and challenges for reviewing a developing LS innovation system. It was hoped that these inputs (requirements and challenges) would result in a review of the developing model that represents the Saudi life science innovation system. For the data collection process, a semi-structured and in depth interview method was adopted (to gain most insight into contextual issues and the 
perceptions of the informants). The next four sections will offer a more detailed account of this process.

\subsubsection{Interview questions}

Interviews take many forms, from fully structured to completely informal. Some means of structuring is necessary to focus any conversation or informant engagement. Fully structured interviews were rejected as they would lead to bias, which does not support a grounded approach. As such, semi-structured interviews based around themes were selected as most appropriate to the context. The semi-structured interview questions aimed to describe the situation and the perspective of the informant, and to explore ideas for building a knowledge economy in the life science sector (see appendices). The researcher started the interviews by presenting an introduction to the study and definitions of some jargon such as knowledge economy and life science etc.; this was to make sure that the participants properly understood the questions. The pilot study interview questions are presented in Table 4-1.

These questions were evaluated and analysed (as a test of the quality of questions used) which led to developing new modified interview questions. The new questions consisted of 5 key questions and 20 secondary questions, but the nature of interviews and discussion opened the door for many questions through the interviews to trace and cover any topic or idea that might emerge. 


\section{Table 4-1: Pilot study interviews questions.}

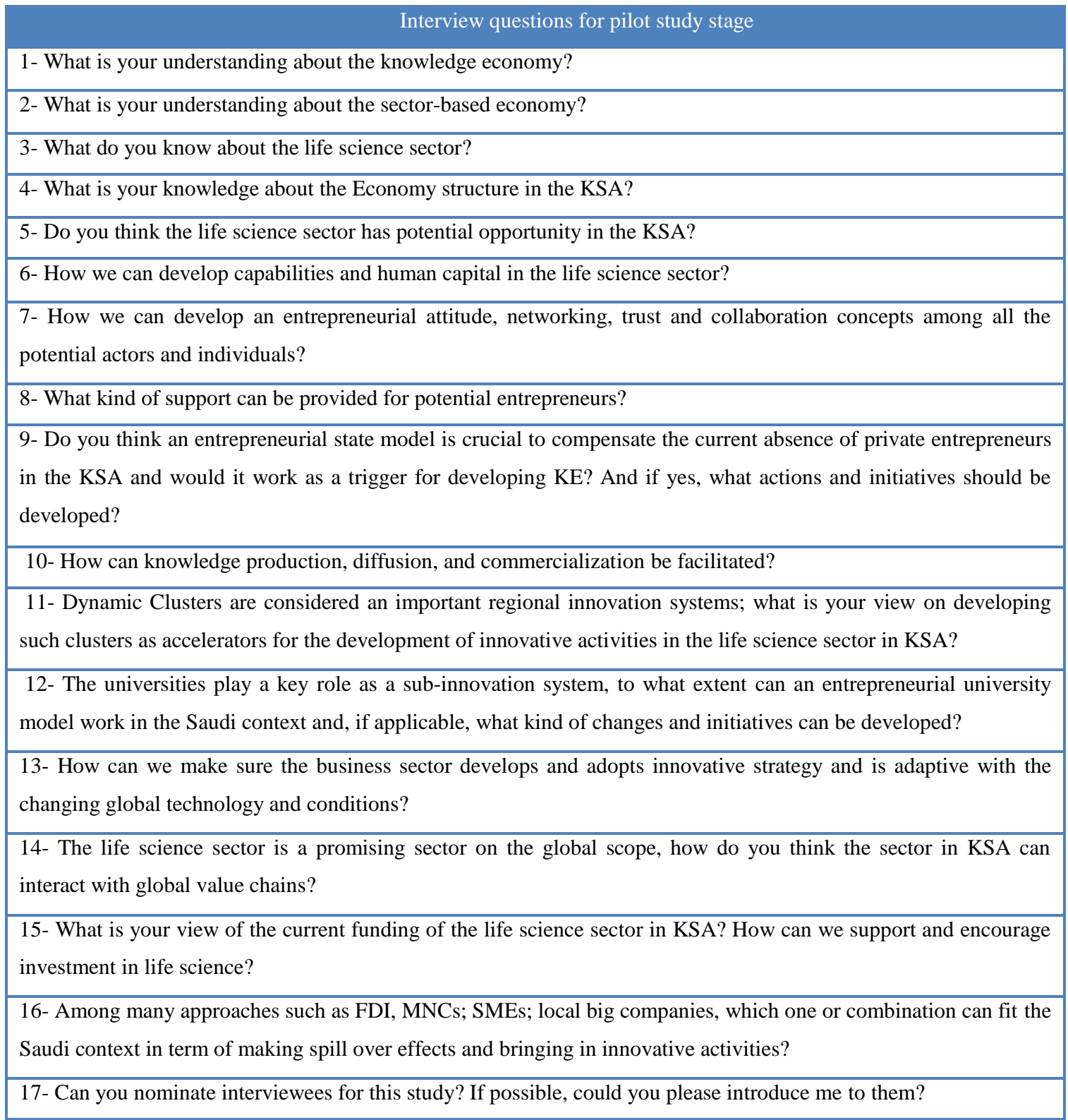

\section{Source: the researcher}

Table 4-2 includes the interview questions used for the second stage (the first stage was the pilot stage which tested the robustness of the questions to be used) main sample (27 participants). 
Table 4-2: Interview questions for the main sample

\begin{tabular}{|c|c|c|c|c|c|}
\hline $\mathbf{R . Q}$. & Key themes & Sub-themes & Master questions & & \\
\hline $\begin{array}{l}\text { What are the key } \\
\text { requirements of } \\
\text { the LS sector in } \\
\text { KSA? }\end{array}$ & $\begin{array}{l}\text { Infrastructure } \\
\text { High skilled people } \\
\text { Funding }\end{array}$ & $\begin{array}{l}\text { General infrastructure } \\
\text { Scientific infrastructure } \\
\text { High life standard } \\
\text { ecosystem } \\
\text { Scientific capabilities } \\
\text { Managerial capabilities } \\
\text { Brain recycling } \\
\text { Education system } \\
\text { Venture capital market } \\
\text { Seed funding }\end{array}$ & $\begin{array}{l}\text { 1) Could you tell me a little } \\
\text { about your role in relation } \\
\text { to LS and GGC? } \\
\text { 2) What is your knowledge } \\
\text { about the Economy } \\
\text { structure in KSA? } \\
\text { 3) What are the key } \\
\text { components to develop } \\
\text { the LS sector in KSA? }\end{array}$ & $\begin{array}{l}\text { 1) } \\
\text { 2) } \\
\text { 3) } \\
\text { 4) } \\
\text { 5) } \\
\text { 6) } \\
\text { 7) } \\
\text { 8) } \\
\text { 9) }\end{array}$ & $\begin{array}{l}\text { What is your un } \\
\text { What is your } \\
\text { economy? } \\
\text { What do you kn } \\
\text { What is your k } \\
\text { KSA? } \\
\text { To what extent } \\
\text { opportunities in } \\
\text { How can we dev } \\
\text { How can we cre } \\
\text { What kind of in } \\
\text { sector? } \\
\text { To what extent } \\
\text { innovative ecosy }\end{array}$ \\
\hline $\begin{array}{l}\text { What are the } \\
\text { challenges in } \\
\text { developing a KE in } \\
\text { LS in KSA? }\end{array}$ & $\begin{array}{l}\text { Cultural aspects } \\
\text { Social capital } \\
\text { Priorities } \\
\text { identification } \\
\text { Global vision } \\
\text { Government } \\
\text { commitment }\end{array}$ & $\begin{array}{l}\text { Entrepreneurship } \\
\text { Attitude and values } \\
\text { Collaboration } \\
\text { Networking } \\
\text { Global value chains } \\
\text { interaction }\end{array}$ & $\begin{array}{l}\text { 4) What are the challenges/ } \\
\text { barriers to progressing LS } \\
\text { in KSA? }\end{array}$ & $\begin{array}{l}\text { 10) } \\
\text { 11) } \\
\text { 12) } \\
\text { 13) } \\
\text { 14) }\end{array}$ & $\begin{array}{l}\text { How can we } \\
\text { entrepreneurial } \\
\text { How can we sul } \\
\text { How extensiv } \\
\text { involvement be } \\
\text { To what exten } \\
\text { success in the L } \\
\text { How can we int }\end{array}$ \\
\hline $\begin{array}{l}\text { What model of } \\
\text { innovation system } \\
\text { would best fit the } \\
\text { Saudi context? }\end{array}$ & $\begin{array}{l}\text { University role } \\
\text { Knowledge transfer } \\
\text { Commercial } \\
\text { orientation-based } \\
\text { researches } \\
\text { Industry innovative } \\
\text { strategy } \\
\text { Government role }\end{array}$ & $\begin{array}{l}\text { Entrepreneurial university } \\
\text { Science parks } \\
\text { Incubators } \\
\text { Spin-offs } \\
\text { Entrepreneurial state } \\
\text { Clusters } \\
\text { Friendly Business } \\
\text { Regulations } \\
\text { Incentives }\end{array}$ & $\begin{array}{l}\text { 5o what extent can } \\
\text { clusters perform an } \\
\text { accelerator role for } \\
\text { developing the LS sector? }\end{array}$ & $\begin{array}{l}\text { 15) } \\
\text { 16) } \\
\text { 17) } \\
\text { 18) } \\
\text { 19) } \\
\text { 20) }\end{array}$ & $\begin{array}{l}\text { What is the pote } \\
\text { centres in devel } \\
\text { How can we fac } \\
\text { To what extent } \\
\text { developing the I } \\
\text { How can we inc } \\
\text { How can we } \\
\text { universities and } \\
\text { What initiative } \\
\text { knowledge exch }\end{array}$ \\
\hline
\end{tabular}

Source: The researcher 


\subsubsection{Data collection process}

In Saudi Arabia knowledge-based life science is an emerging sector and there are few formalised structures or salient achievements relevant to investigate/uncover the actual situation of the system and its interactions. The author therefore chose to interview a range of stakeholders who could help provide critical insights and relevant data through the use of semi-structured interviews. This approach aligns with Creswell's view that qualitative methodology can be used in extracting indirect information by filtering in-depth interviews through the interviewees opinions (Creswell, 1998). In effect, the chosen methodology was validated by the Creswell (1998) model.

The researcher began with a selective sample of expert informants from Wales for the pilot study. He believes that experts face similar challenges around the world and this stage would therefore allow the robustness of the methods to be tested effectively before engaging in research in the KSA. The pilot sample participants were part of the life science ecosystem and had rich backgrounds in the area of study; some of them had been to Saudi Arabia to take part in conferences, and another already worked in a neighbouring country to Saudi Arabia which has quite similar conditions (see sample section 4.4.1.5). This first stage took more than a month and involved informants with a minimum of 5 years of experience in the life sciences sector. Later the researcher moved to Saudi Arabia to pursue interviews following reflection on and modification of the questions of the pilot stage.

Saudi Arabia is a large, geographically dispersed country which has over 2 million $\mathrm{Km}^{2}$ of landmass. The interview process in Saudi Arabia took a further nine months, and the researcher drove more than 7000 Kilometres to conduct interviews (27 interviews). Accessing very senior people in Saudi Arabia is not an easy task and takes a long time. The participants were distributed over many cities. To ensure a high level of representativeness and high reliability, the researcher was careful to assure that the interview was conducted in a suitable setting and only with very representative people from government, academia, and the private sector. These individuals held strategic and executive levels of responsibility for their respective organizations. See the following table. 
Table 4-3: informants` detail. Source: The researcher.

\begin{tabular}{|c|c|c|c|c|}
\hline Informant $^{3}$ & Location & Position & Subsector & Interaction \\
\hline $\mathrm{A} 1^{4}$ & Swansea & $\mathrm{PhD}$ & Academia & $\begin{array}{l}\text { Face to face } \\
\text { Interview }\end{array}$ \\
\hline A2 & Cardiff & Full Professor & Academia & $\begin{array}{l}\text { Face to face } \\
\text { Interview }\end{array}$ \\
\hline A3 & Cardiff & Full professor & Academia & $\begin{array}{l}\text { Face to face } \\
\text { Interview }\end{array}$ \\
\hline G1 & Cardiff & PhD student & Arabic gulf government & $\begin{array}{l}\text { Face to face } \\
\text { Interview }\end{array}$ \\
\hline $\mathrm{G} 2$ & Saudi Arabia & Deputy director-research professor & PRC & $\begin{array}{l}\text { Face to face } \\
\text { Interview }\end{array}$ \\
\hline G3 & Saudi Arabia & Director-PhD & PRC & $\begin{array}{l}\text { Face to face } \\
\text { Interview }\end{array}$ \\
\hline G4 & Saudi Arabia & Manager & PRC-incubator & $\begin{array}{l}\text { Face to face } \\
\text { Interview }\end{array}$ \\
\hline A4 & Saudi Arabia & Director-PhD & Academia (VC) & $\begin{array}{l}\text { Face to face } \\
\text { Interview }\end{array}$ \\
\hline G5 & Saudi Arabia & Assistant manger & Government (fund) & $\begin{array}{l}\text { Face to face } \\
\text { Interview }\end{array}$ \\
\hline G6 & Saudi Arabia & Senior chief consultant-MD & PRC & $\begin{array}{l}\text { Face to face } \\
\text { Interview }\end{array}$ \\
\hline A5 & Saudi Arabia & Dean & Academia & $\begin{array}{l}\text { Face to face } \\
\text { Interview }\end{array}$ \\
\hline A6 & Saudi Arabia & CEO- $\mathrm{PhD}$ & Academia- science park & $\begin{array}{l}\text { Face to face } \\
\text { Interview }\end{array}$ \\
\hline A7 & Saudi Arabia & Director of science park & Academia-private & $\begin{array}{l}\text { Face to face } \\
\text { Interview }\end{array}$ \\
\hline A8 & Saudi Arabia & Manager- PhD & Academia-private & $\begin{array}{l}\text { Face to face } \\
\text { Interview }\end{array}$ \\
\hline A9 & Saudi Arabia & Assistant professor-researcher & Academia-private & $\begin{array}{l}\text { Face to face } \\
\text { Interview }\end{array}$ \\
\hline A10 & Saudi Arabia & Full Prof. Dean-researcher & Academia & $\begin{array}{l}\text { Face to face } \\
\text { Interview }\end{array}$ \\
\hline G7 & Saudi Arabia & Researcher-MD & Ministry of Health & $\begin{array}{l}\text { Face to face } \\
\text { Interview }\end{array}$ \\
\hline G8 & Saudi Arabia & Planning and policy advisor-PhD & Government & $\begin{array}{l}\text { Face to face } \\
\text { Interview }\end{array}$ \\
\hline
\end{tabular}

${ }^{3} \mathrm{~A}=$ Academia, $\mathrm{G}=$ Government, $\mathrm{P}=$ Private sector.

${ }^{4}$ The first four informants represent the interview sample in the pilot study. 


\begin{tabular}{|c|c|c|c|c|}
\hline G9 & Saudi Arabia & Operation manager & PRC-VC & $\begin{array}{l}\text { Face to face } \\
\text { Interview }\end{array}$ \\
\hline G10 & Saudi Arabia & Vice president & PRC & $\begin{array}{l}\text { Face to face } \\
\text { Interview }\end{array}$ \\
\hline G11 & Saudi Arabia & Minister advisor-PhD & Government & $\begin{array}{l}\text { Face to face } \\
\text { Interview }\end{array}$ \\
\hline A11 & Saudi Arabia & Full professor-Researcher & Academia & $\begin{array}{l}\text { Face to face } \\
\text { Interview }\end{array}$ \\
\hline A12 & Saudi Arabia & Director of innovation centre-PhD & Academia & $\begin{array}{l}\text { Face to face } \\
\text { Interview }\end{array}$ \\
\hline A13 & Saudi Arabia & Director prototyping centre-PhD & Academia & $\begin{array}{l}\text { Face to face } \\
\text { Interview }\end{array}$ \\
\hline G12 & Saudi Arabia & Deputy director & Government-fund & $\begin{array}{l}\text { Face to face } \\
\text { Interview }\end{array}$ \\
\hline P1 & Saudi Arabia & CEO & Private & $\begin{array}{l}\text { Face to face } \\
\text { Interview }\end{array}$ \\
\hline $\mathrm{P} 2$ & Saudi Arabia & Business development manager & Private & $\begin{array}{l}\text { Face to face } \\
\text { Interview }\end{array}$ \\
\hline P3 & Saudi Arabia & General manger & Private & $\begin{array}{l}\text { Face to face } \\
\text { Interview }\end{array}$ \\
\hline $\mathrm{P} 4$ & Saudi Arabia & CEO & Private & $\begin{array}{l}\text { Face to face } \\
\text { Interview }\end{array}$ \\
\hline P5 & Saudi Arabia & Production manager in $\mathrm{MNO}$. & Private & $\begin{array}{l}\text { Face to face } \\
\text { Interview }\end{array}$ \\
\hline P6 & Saudi Arabia & CEO & Private & $\begin{array}{l}\text { Face to face } \\
\text { Interview }\end{array}$ \\
\hline
\end{tabular}

The researcher found the interview process and protocol very interesting as it uncovered an emerging story as knowledge about the phenomena unfolded. Each next 
interviewee was determined with respect to the emerging concepts as required by theoretical sampling, the number of interviewees being related to the representativeness and consistency of concepts that could deliver theories, which began to emerge from the simultaneous process of gathering and analysing data (Corbin \& Strauss, 1990). For the sake of reducing bias as far as possible the researcher used data triangulation by interviewing participants from different subsectors; this strategy increased the validity of the data. According to Lindlof \& Taylor (2010) data triangulation is a good technique for limiting bias, and enhancing data validity. Such an approach was duly accepted by the researcher.

For the sake of awareness and focus during interviews (and to maintain the consistency of the discussion) the researcher did not use field notes and instead used, with full informant permission, a high-quality digital voice-recording device for the interviews. All recordings were uploaded to a portable hard drive that was passwordsecured and all informants were given a code known only to the researcher. Then later, within 24 hours, the data was prepared and in a verbatim manner transcribed (164 pages were transcribed for all interviews). The interviews were then coded according to grounded theory methodology, which ensured that the essence of the interviews was captured effectively. Later the interviews were analysed and the process of research continued to a questionnaire stage to close any knowledge gaps. Following this the researcher reflected on the findings and analysis before presenting recommendations for future work in the final thesis.

\subsubsection{Transcription of interviews}

Interview recordings were transcribed by the researcher in Microsoft Word $2011 \mathrm{Mac}$ and literal descriptions of the interviews were captured and coded resulting in 164 pages of transcription and more than 400 hours of transcription time.

The next section shows a sample of the transcription of one of the interviews:

Could you tell me a little bit about your role in relation to LS?

Firstly, I will give brief about my background: I graduated from King Saud University In the department of biochemistry, and Master from Oregon University in Biochemistry, and PhD from Kent University in Biochemistry and Biotechnology. I am interested in Bioprocessing and exactly in Metabolic engineering in animal cells, 
because the Pharmaceutical companies mostly, depend on animal cells to produce Protein for treatment. There is interface between academia and industry. In term of admin I am currently the manager of the National Centre for Biotechnology, which is one of five centres under the title of environmental and life science institute.

To what extent are you happy about the progression and achievement of the LS? At the level of region, I think we have good progression.

At the context of knowledge economy, to what extent have your achievements made an economic impact?

Actually, when you talk about revenue, it is take long time; you need firstly, to build basic knowledge. The most profitable and producing revenue in the pharmaceutical sector, and I think currently, there is no one doing this in KSA, because the infrastructure and ecosystem not yet ready.

For more please see appendix 12.4 .

\subsubsection{Sampling}

The researcher embarked on the interviews without a specific sample size. Such an approach is positivistic and relies upon an effective knowledge of the whole population. For a theory builder and systems researcher this would have been a foolhardy activity. Instead grounded theory relies upon reaching a knowledge saturation point. The point is arrived at when the concepts and themes of interviews repeat themselves to the extent that each new interview adds no more to the knowledge of the researcher. This point was reached by interview number 21 and increased as the process continued. Interviews continued to 31 , four of them being from the Welsh life science ecosystem and people with background in developing competitive regions, and the other 27 from the Saudi Arabian life science ecosystem, distributed over many cities and some sub-sectors (government, industry, academia and health services). All were senior people (CEOs, professors, consultants, professionals, researchers, high officers and medical research doctors). They were an elite and expert informants sample (see appendix 12.3). A very representative 
population was chosen, the number of interviewees being related to the representativeness and consistency of concepts that can deliver theories, which begin to emerge from the simultaneous process of gathering and analysing data (Corbin \& Strauss, 1990).

According to Hunterl et al. (2011), "Sample size is deemed to be satisfactory only when the key concepts that have been identified from the collected data have reached saturation point, in other words, when no new data emerges". When the generated notes, themes and patterns become similar and no new ideas come up that means that saturation point been reached (Corbin \& Strauss, 1994). The researcher conformed to and supported this approach to research and data management.

As guidance for sample size, any research that tries to investigate the commonalities among a homogeneous population should engage with 12 in-depth interviews. However this sample size was not considered enough in the case of this heterogeneous population with wide-ranging research questions (Guest, Bunce, \& Johnson, 2006). According to Creswell (2007), it is expected that 25 to 30 interviews should be undertaken for a general study and this quality assurance level was achieved by the researcher.

At the beginning, the informants were identified with respect to their background and engagement in the life science ecosystem. The approach to sampling conforms with the advice of Corbin and Strauss (1990) who state "Sampling in grounded theory proceeds not in terms of drawing samples of specific groups of individual, units of time, and so on, but in terms of concepts, their properties, dimensions, and variations. When a project begins, the researcher brings to it some idea of the phenomenon he or she wants to study. Based on this knowledge, groups of individuals, an organization, or community representative of that phenomenon can be selected for the study" (Corbin \& Strauss, 1990). Informants were drawn from the three key actors of the life sciences system in general (government, academia and industry).

The researcher conducted all interviews in face-to-face meetings and this allowed for more discussion and deeper understanding of the meanings, motives and experiences behind the opinions, and the phenomena under study. The approach was highly effective and led to many interesting insights. For the purpose of enhancing the 
reliability of data, the researcher paid great attention to undertaking the interviews in convenient conditions and suitable settings for discussion, and this was one of the reasons for extending the interview process over more than six months. The interviews with the informants also allowed them (due to the location of the interviews) to retrieve secondary information to support their views where it was available (such as company reports etc.).

After every interview the snowballing technique was used to identify the next participant, with regard to expert informant involvement. Such an approach is common to social scientific studies of this nature and allows concepts to emerge from data (theoretical sampling) and also has a major benefit to the grounded researcher (as collecting data and analysis happen in parallel). This approach is reinforced by Drucker et al who argue "Theoretical sampling is often distinguished from selective sampling. Whereas theoretical sampling is guided by emerging theory, selective sampling is the identification of populations and settings prior to data collection... Sampling in grounded theory is thus sequential, beginning with selective sampling and moving into theoretical sampling when concepts begin to emerge. Researchers must decide when to shift from selective to theoretical sampling" (Draucker, Martsolf, Ross, \& Rusk, 2007).

\subsubsection{Data analysis: Research question 1}

What are the key requirements for developing the knowledge economy in the LS sector in the KSA?

Grounded theory methodology has been used to answer this question. "The procedures of grounded theory are designed to develop a well-integrated set of concepts that provide a thorough theoretical explanation of social phenomena under study. A grounded theory should explain as well as describe. It may also implicitly give some degree of predictability, but only with regard to specific conditions" (Corbin \& Strauss, 1990).

For answering this question two coding processes were implemented (open coding and axial coding) on the data collected through the pilot and main study which resulted in numbers of categories that represented the key requirements for 
developing LS in Saudi Arabia. In compliance with good practice "Data collection and analysis are interrelated process. In grounded theory, the analysis begins as soon as the first bit of data is collected. In contrast, it is not uncommon for some qualitative researchers to collect much of their data prior to beginning systematic analysis" (Corbin \& Strauss, 1990).

The pilot study involved four participants from the UK; the analysis of this part of study was relevant and has been added to the main study. In the main study 27 participants were involved and all of them were from Saudi Arabia. The whole study coding analysis (pilot and main) resulted in generating 38 open codes and five categories (See Table 6-3).

\subsection{Open coding}

Open coding was selected as most appropriate and sympathetic approach for grounded theory and began by identifying incidents, events and themes. A constant comparison process was performed to group those incidents and themes into codes as a method of data reduction based on the properties of this data. Coding is a labelling process, and this process is directed by two tasks: making comparisons, and asking questions. The first requires making constant comparisons of different pieces of data to develop categories. The second requires posing questions such as: what is this piece of data an example of? Or, what does this piece of data stand for, or represent? Or, what category or property of a category does this piece of data indicate? (Punch \& Oancea, 2014) Open coding resulted in generating 38 open codes for the total (see Table 6-1).

\subsection{Axial coding}

These open codes were part of a constant comparison process based on the axial coding paradigm. Open codes and properties show the interconnections among them and were scaled by the researcher into upper level themes (representing the categories of the data analysis - see Table 6-3) of which five categories and their properties were identified. These constituted the key requirements for developing the life science sector in Saudi Arabia and answered the research question 1. 


\subsubsection{Data analysis: Research question 2}

What are the challenges in developing a KE in the LS sector in Saudi Arabia?

For this research question the answer was extracted and coded from interview question responses. The main question used for this was "what are the challenges/barriers to progressing LS in the KSA?" but other responses were used where relevant. The data was coded in open coding to identify the challenges and barriers in more detail. A quantitative approach was also developed to present quantitative insight about the challenges and barriers captured in the previous qualitative perspective (see Figure 7-1).

\subsubsection{Data analysis: LS innovation system: Research question 3}

The theoretical coding, which took roughly six months, led to identifying a basic social process, as a process changes over time and would resolve the problem of the phenomena under investigation. "Conceptual elaboration concludes when the relationships among individually elaborated concepts emerge through the identification and use of appropriate theoretical codes to achieve an integrated theoretical framework for the overall grounded theory. Theoretical codes conceptualize how the substantive codes may relate to each other as hypotheses to be integrated into the theory. They help the analyst maintain the conceptual level in writing about concepts and their interrelations" (Holton, 2010). This basic process constitutes all aspects of the life science innovation system that have been developed (see Figure 8-8).

\subsubsection{Second Phase}

The first phase was a qualitative study of experts, but the second phase was designed to iteratively test the findings of the first phase using a population of senior, high profile informants from the UK and KSA system (29 participants). The second phase began by carrying out selective coding to identify what the data implied and what was the core of the themes around which the story and phenomena revolved. Then later, and based on identifying the selective code, the researcher developed a questionnaire 
to explore the variables that could encourage or hinder involving private sector in the innovation system (the selective code).

\subsubsection{Selective coding}

Through a qualitative perspective by going through the collected data, this selective code emerged as the main phenomenon where all other categories connected and interacted in important relationships; this category captures the story of the data. A quantitative perspective was also applied, by using the keywords relevant to all core categories, to enhance the quality of identifying the selective code (see Table 8-2).

\subsubsection{Questionnaire}

As used within the survey strategy for Phase 2, a questionnaire is one of the most popular and common techniques for data collection. As the responses of all participants emerge from the same questions, a questionnaire provides an efficient way to collect data (Saunders et al., 2011). Before embarking on a questionnaire, it is very important to understand the underpinning organizations and culture of the context, otherwise mistakes can occur. Conducting in-depth and semi-structured interviews to understand and uncover these cultural and organisational aspects is one of the techniques that works effectively and is fully aligned with realist research of a phased nature (Saunders et al., 2011). Using questionnaire in qualitative research is significantly supported by (Miles, Huberman, \& Saldana, 2013).

For a questionnaire to work most effectively, it is argued that the questions should be standardised, understood and interpreted in the same way by all participants. A questionnaire does not work well with exploratory research questions (Robson, 2002), but is a better fit for descriptive and explanatory research questions. Exploratory research requires open-ended questions, whilst descriptive investigation uses opinion and attitude questions, and questions on organizational practices, and that enable description of the variability in phenomena. Explanatory concerns cause and effect relationships between factors (Gill \& Johnson, 2010). The questionnaire was designed as a descriptive explorative method to meet the needs of this phase of the research. 


\subsection{Questionnaire objective}

This study is descriptive and explorative and all variables were developed to satisfy the research objectives and answer the research questions. All variables were extracted from the first phase in this study 4.4.1 (in-depth semi-structured interviews` transcript), and also from the literature review (See appendix 12.6). There are three types of variables: opinion, attribute and behaviour. Opinion variables are designed to measure the respondent's opinion about whether something is true or wrong. Attributes variables concern participants' characteristics such as age, marital status, gender, occupation etc., whilst behaviour variables are engaged in measuring the respondents' experiences in the past, something they are doing now or will do later (Dillman, 2007). This study contains attribute variables (demographic questions), and all the rest are opinion variables (rating questions). Rating questions usually use the Likert-style where the participant answers as to what extent he or she agrees or disagrees with a statement (Saunders et al., 2011).

In this study, based on the selective coding for section 0 which produced involving the private sector as a selective code, and also for more confirmative and credible investigation, a triangulation method was used by developing a questionnaire to explore the strategies or variables that would encourage/discourage the different private sub-sectors (namely: Multinational enterprises (MNEs), local large companies (LLCs), small and medium-sized enterprises (SMEs), and knowledge-based start-ups (KB-SUs)) to be involved in knowledge-creation activities.

Triangulation is critically important for a qualitative and realist study of this nature. "Triangulation is not aimed merely at validation but at deepening and widening one's understanding, and tends to support interdisciplinary research rather than a strongly bounded discipline of sociology or anthropology" (Yeasmin \& Rahman, 2012). "By combining multiple observers, theories, methods, and empirical materials, researchers can hope to overcome the weakness or intrinsic biases and the problems that come from single-method, single-observer, single-theory studies. Often the purpose of triangulation in specific contexts is to obtain confirmation of findings through convergence of different perspectives. The point at which the perspectives 
converge is seen to represent reality" (Jakob, 2001). These research quality conditions were built into the study and the researcher used various reflective periods to deliberately focus on the methodological issues and findings to support processes of validation and triangulation with secondary information and other evidence sources.

\subsection{Questionnaire design}

The response rates, reliability, and validity of collected data are significantly affected by the questionnaire design. So, to improve these elements (response rate, reliability, validity), some steps should be taken:

- Careful design of the questions;

○ Making the questionnaire layout pleasing and clear;

- Clarifying the questionnaire's purpose;

○ Undertaking a pilot test;

○ Planning the questionnaire's administration carefully (Saunders et al., 2011).

It is difficult to make sure that the specific person you want to answer the questionnaire is the one who did. The type of questionnaire plays a significant role, and an internet-mediated questionnaire offers more control and a greater chance of the questionnaire being answered by the targeted person (Witmer, Colman, \& Katzman, 1999). See (Figure 4-5) for questionnaire types.

In this study, the researcher took pre-emptive steps to "design in" quality within the questionnaire:

- The questionnaire developed by the researcher was reviewed and revised many times by the revision team's members, individually and collectively, to examine the wording of the questions and make sure that they were linked strongly to the questionnaire's objectives and to answering the research questions. The questionnaire's clarity, appearance, structure, and layout were also reviewed, and the questionnaire's purpose clarified.

- A pilot study been undertaken to examine the quality of the questionnaire in all aspects. 
- An Internet mediated questionnaire was used to enhance the rating response, and to raise the probability of the questionnaire being answered by the targeted participant (See Figure 4-5).

○ A representative sample (expert sample) from all stakeholders sectors was targeted.

Survey Monkey was used as a medium to access the questionnaire and this was sent out as an Internet link by email (internet-mediated questionnaire) to 36 senior stakeholders of the LS sector (four of them in the UK and the rest from Saudi Arabia). About $80 \%$ (29 respondents) of them answered the questionnaire, three of the respondents were from the UK and the rest were from the KSA. According to Saunders et al. (2011) targeting representative sample is crucial for be able to generalise about the total population. Coding book and a questionnaire copy are included in the appendices.

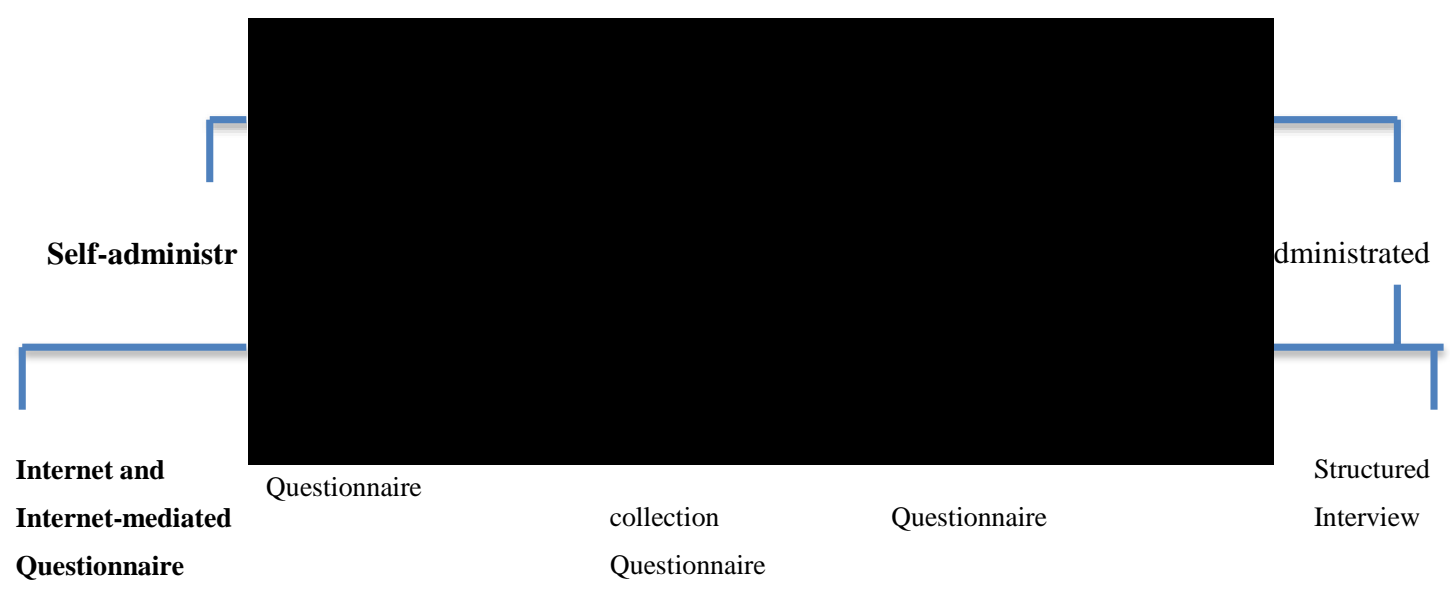

Figure 4-5: Types of questionaire (Study approach in bold type). Source: (Saunders et al., 2011)

Besides the demographic questions-attribute variables (gender, sector, position, nationality, country of residence, age), the questionnaire also included 88 ordinal variables - opinion and attitude variables - distributed unequally into 17 questions (four questions for every sub-sector and one question about the private sector in general and to what extent it is important to involve it in developing a knowledge 
economy (KE) in the life science (LS) sector in the KSA). These variables and strategies were mostly inspired by the first study findings in the qualitative part of this research, and also were inspired by the literature. According to Saunders et al. (2011) it is important that investigative questions and the data collected is related to the literature that has been reviewed and any preliminary study carried out. The latter advice was duly accepted by the researcher.

\subsection{Frame of analysis}

The Likert scale was developed by Likert (1932) to measure attitudes. This kind of scale usually uses 5- or 7-point ordinal scale, and the informants choose the degree to which they agree or disagree with a statement. The differences between responses are not necessarily the same (Jamieson, 2004; Sullivan \& Artino Jr, 2013). Usually, parametric tests are used for interval variables, and non-parametric tests are used for ordinal variables. The use of parametric or non-parametric tests is a very controversial issue among experts; some argue that ordinal variables cannot be treated like interval variables, whilst others posited that the two should be treated the same. These conditions: adequate sample size (at least 5-10 per group), and data are normally or nearly normally distributed (Knapp, 1990; Sullivan \& Artino Jr, 2013).

To measure the central tendency for ordinal variables, a mode or median test should be applied (Clegg 1982), mean and standard deviation are inappropriate for ordinal variables (Blaikie, 2003; Clegg, 1982). Descriptive data [as the case in this study] is used to measure a central tendency, the mode is the only test that can interpret the data sensibly (Saunders et al., 2011). In this study, with respect to the question types (ordinal questions) and the objective of the investigation (to identify the strategies or variables that would encourage/discourage or hinder the different private sub-sectors), only descriptive statistics were implemented to measure the central tendency of the participants by implementing a mode test to identify which internal or external variables would encourage or hinder the involving of the private sub-sector in knowledge activities in the LS sector in the KSA. Additional information about frequencies for the demographic questions was presented. 


\subsection{Questionnaire validity and reliability}

A valid questionnaire will lead to collecting accurate data, and a reliable one means that the data were collected consistently. The questionnaire's structure and layout, the quality of the pilot study and the design of the questions all affect significantly the response rate and the validity and reliability of the collected data (Saunders et al., 2011). Regarding questions and answers, and their relation to validity and reliability, Foddy (1994) argues that "the question must be understood by the respondent in the way intended by the researcher and the answer given by the respondent must be understood by the researcher in the way intended by the respondent".

The issuing and analysis of the questionnaire concluded the second phase of the research process, allowing the key research objectives to be realized and research questions to be answered.

\subsection{Research Reliability and Validity}

A study is considered to be reliable if, when repeating the same study within the same context, using the same methods and the same informants, the same result would be obtained (Shenton, 2004). The researcher has offered significant and transparent information about the process design in the belief that this would be the case if it were repeated. The researcher devised the research questions and the interview questions to help achieve a high level of reliability and validity. The researcher began the research by undertaking pilot (test) interviews with high senior stakeholders. The main purpose of this pilot study was to make sure that the interview questions were clear and relevant and could therefore answer the research questions. After finishing the pilot study, the interview questions were modified to overcome any shortcomings or ambiguities that appeared during the pilot study; this made significant improvements to ensuring research validity. The reliability is also a core issue; to ensure a high level of reliability, the researcher stressed the need to interview only representative people with a significant involvement and background in the research subject.

The cultural context of Saudi Arabia means that accessing 27 very senior informants and assuring that interviews could take place in convenient settings for these individuals were not easy tasks. Because of this the data collection in Saudi Arabia 
took more than six months, to make sure that the data collected were of high quality and reliable.

According to Schwandt (1997), validity is defined as "how accurately the account represents participants' realities of the social phenomena and is credible to them". So, seeking validity is the aim in order to establish the credibility of the research (Creswell \& Miller, 2000).

There are several procedures that are used to enhance validity for qualitative researches. These include: triangulation, peer review, member checking, external audit, and thick description. However, two perspectives should govern the choice of applying validity procedures: the lens chosen to validate the research, and researchers' paradigm assumptions. This lens can be the researcher`s own lens by which the time needed for collecting data can be evaluated, together with whether the data has reached saturation of themes and categories. The participants are the second lens that can be used by the researcher, ensuring that their inputs represent the realities perceived by them. External reviewers are the third lens that can be used to help in establishing validity (Creswell \& Miller, 2000). The paradigm assumptions include Postpositivist, constructivist, and the choice of critical influence researchers (Guba \& Lincoln, 1994). The following table details the relations between the two determinants' perspectives and the validity procedures. These nine validity procedures are not exhaustive, but they represent the most common in the qualitative literature (Creswell \& Miller, 2000).

Table 4-4: Validity procedures with respect to the two governors perspectives.

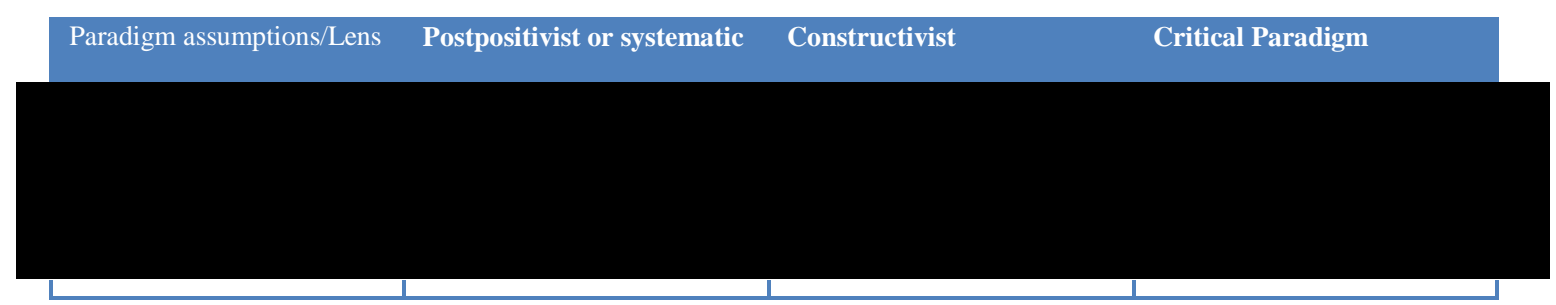

Source: Adapted from (Creswell \& Miller, 2000).

Thick description has been used as a validation procedure so that a reader can see the context and environment of the research and its process. Thick description is the validity procedure that helps in building credibility in the research by presenting very 
detailed descriptions of the settings, the participants and the themes. This procedure provides readers with a rich image of the feelings and experiences by contextualizing the people and sites involved in the study. Thus, the credibility can be built through the lens of the readers who are transported into a detailed setting and situation. Also, thick description gives the readers the ability to see to what extent the findings could be applied to other similar contexts (Creswell \& Miller, 2000).

The nature of this study provides another validity procedure, as there is a close collaboration between the researcher and peers at the University, which is a feature of critical paradigm. A peer debriefing procedure was applied, as the researcher belongs to a $\mathrm{PhD}$ group with interests in the life sciences sector (globally) and often presented to his supervision team with regular feedback at every step of the research process. According to Lincoln \& Guba (1985), peer review provides support and feedback, challenges the researcher`s assumptions, and asks questions about interpretations and methods.

According to Creswell \& Miller (2000) "Triangulation is a validity procedure where researchers search for convergence among multiple and different sources of information to form themes or categories in a study". By conducting two phases of research, and having constant comparison throughout the study between informants distributed over three sectors (academia, government, and private sector), triangulation is inherent in this study by design.

According to (Lincoln \& Guba, 1985) "since there can be no validity without reliability, a demonstration of the former [validity] is sufficient to establish the latter [reliability]."

Reliability is a consequence and result of validity (Patton, 1990).

According to Miles et al. (2013), biased sources (that might decrease the research validity) include:

○ The holistic fallacy: this happens by imposing pattern on events which is not really there.

- Elite bias: this takes place when overweighting data from elite people and underrepresenting data from lower status participants.

○ Personal bias: any personal agenda will harm the trustworthiness of the study. 
○ "Going native": this happens when the researcher loses his/her perspective and becomes immersed in the explanations and perceptions of local informants.

Recalling all these kinds and sources of bias in the mind of the researcher in this study was very helpful in developing valid findings and designing out potential issues such as a powerful single informant dominating the development of themes during the initial phases of the research (e.g. using multiple experts who were all treated equally in terms of status and influence).

\subsection{Revision Team (RT)}

Additional to the author, the research was coordinated and monitored in a systematic way managed by an experienced team which has very significant expertise in the academia and life science sector, and has been involved for a long time in the life science ecosystem in Wales. The revision team held regular weekly tutorials to discuss any relevant issues and evaluate any step or progress in the research. Inputs from presenting the research to a peer group were critically important for the progress of the research and took place over the entire research process: from defining the literature review and conceptual framework to research design, research aims, objectives, questions, data collection and analysis.

Additional to the cumulative work in the factors mentioned above, this close caring, intervention, and collective behaviour of the revision team underpinned the progress of the research and provided expertise and support in developing the research aims, objectives, and questions. It was also helpful in mitigating any kind of bias from the author and enhancing credibility.

\subsection{Research Ethics and limitations}

For ethical matters, the researcher emailed all participants a consent letter to sign and return before holding the interviews. This consent letter gave the participants the right to withdraw their inputs at any time. Participants registered using anonymous characters, and all data was used only for this research purpose and not shared with any third party. These processes conform to the good practice established by the 
Swansea University. The term ethics "refer[s] to the appropriateness of [a researcher`s] behaviour in relation to the rights of those who became the subject of [his/her research], or are affected by [the researcher]".

Participants can be affected or harmed directly or indirectly, by mentioning their names, positions, or the names of their organization (Bryman, 2015; Saunders et al., 2011). In this study, all participants' names or any information might lead to their recognition were anonymized.

Research ethics were fully respected and understood to the extent that ensured all participants' rights to not be harmed in any way (including the right to withdraw and to review all written materials about the informant). The ethical issues were approved by the Swansea University and also comply with the guidelines of the British Psychological Association.

The limitations of this research include that the majority of private sector representatives in this study were drawn from the pharmaceutical sector, due to the nature of the industry landscape in the KSA. Also, the number of participants was not equal (Academia (13), Government (12), Private sector (6). While it would have been achievable to make equal numbers of sectors participants, the priority was to assure reliability and quality of the data collected that would reflect the research objectives. Finally, the research was conducted during a period of change in the Saudi economy as opposed to an established and buoyant economy. 


\section{The KSA and Context}

\subsection{Introduction and Context}

This chapter presents an overview of two key contextual elements: Saudi Arabia and the life science sector. In the first contextual element the researcher explores some political, social and economic aspects of the Kingdom of Saudi Arabia. The second presents the life science sector and its importance in global industry.

The innovation-based biopharmaceutical sector contributes significantly to economic growth on a global scale, particularly in developed countries. In particular in the USA, where the sector is substantial, bio-pharma, for example, has an important role in job creation as well as generating tax income for the government. This, in parallel, results in enhanced wellness and improves the standard of living of individuals and citizens (Greffe, 2016; PhRMA, 2011).

Saudi Arabia has the biggest healthcare market in the Middle East and the annual growth rate of public spending on healthcare between 1999 and 2005 was about $7.2 \%$ (Ram, 2014). Such a high growth rate (in health services) prompted the Saudi government to develop this sector, especially to solve and manage "local-based" diseases and health issues. The country also has the potential opportunity to tap into some special advantages of its location by accessing a migrant market of millions of Muslim pilgrims and visitors (who continuously visit Makkah and Madinah - the two most holy cities of the Islamic faith).

These conditions stimulated the Saudi government to exploit the growing demand and address the need for human capital and capabilities. However, due to the relatively underdeveloped indigenous capability, the government took the strategic decision to accelerate the development of a skills base through harnessing foreign institutions' education capabilities. It therefore sent thousands of students and employees to attend 
research institutes in developed countries to study and conduct research in order to support the domestic demand for knowledge in specific areas ${ }^{5}$.

\subsection{Kingdom of Saudi Arabia (KSA)}

The Kingdom of Saudi Arabia (sometimes called Saudi Arabia) is an Arabic country located in the Middle East. It comprises a huge land area $\left(2\right.$ million $\left.\mathrm{Km}^{2}\right)$ on the Arabic peninsula, see Figure 5-3. In this section six relevant topics will be explored: Saudi Arabia and its influences on the Islamic World; the establishment of Saudi Arabia: a historical perspective; the national civilization and culture change; Saudi economic development; membership of the gulf cooperation countries (GCC), and enterprise management in Saudi Arabia.

\subsubsection{Saudi Arabia and its Influence on the World Landscape}

Saudi Arabia is both the spiritual and the economic centre of the Middle East and Islamic countries. It is home to Makkah (the holy city located in the western province of Saudi Arabia), the place which more than one and a half billion people (from Morocco to Indonesia) kneel down and face when they pray five times each day. The huge national wealth, based upon natural resources, and the two holy cities (Makkah and Madinah) mean that the country has significant influence on the Islamic world. Economically, Saudi Arabia has $16 \%$ of the world`s oil reserves and, in critical times, its interventions are of especial help in mitigating oil price fluctuations and protecting the world economy from any potential harm. For instance, in 2003 the USA invaded Iraq, a situation which had negative implications for the oil price and consequently for the world economy. Saudi Arabia intervened by increasing the amount of oil in the world markets, which stabilized the oil price within an affordable and accepted range (Weston, 2011). Saudi Arabia is also a member of the G20 which includes the biggest 20 economies in the world.

\footnotetext{
${ }^{5}$ It should be noted that the researcher is sponsored by the Ministry of Industry (his employer) but that no ministry officials were involved with influencing this research beyond identifying the sector as of strategic importance to the diversification of the country beyond rentier oil dominance.
} 


\subsubsection{Saudi Arabia Establishing: Historical Perspective}

Saudi Arabia is a nascent country; the first Saudi state was established in 1744 and has evolved over three significant stages. The first stage, started in 1744, was based on an alliance between Mohammed bin Saud (the Royal family) and Mohammed bin Abdalwahab (the religious leader). The second stage occurred in 1824, following a period of anarchy and subsequent to the invasion of Saudi by the Egyptian Army. The third stage began in 1902 with the return of the ruling family from exile (following on from their defeat in 1891 at the hand of a competing family) - see Weston (2011). The following sections describe each of these stages in detail.

\subsubsection{First Saudi state, 1744-1818}

The first Saudi state began through an alliance between Mohammed bin Saud and Mohammed bin Abdalwahab, who became, respectively, ruler and religious support. This military and spiritual co-operation is still considered one of the cornerstones of the royal family`s legitimacy. Via this alliance, Mohammed bin Saud (by his forces) and Mohammad bin Abdalwahab (as a preacher) managed to rule most of the Arabic peninsula (Weston, 2011). See Figure 5-1(source unknown).

During this period Wahabism began as a movement against superstition, idolatry and magic. The coalition adopted the Unitarian movement (Wahabism) as their doctrine. The disciples of Mohammad bin Abdalwahab prefer to be called Unitarians (by emphasizing their belief of the oneness of God) instead of Wahabists, as they are also commonly termed by western writers (Bligh, 1985). The main aim of this alliance was to establish a state to apply Islamic law across the Arabic peninsula. The alliance took place in 1744 at Alderiyah, which was regarded as the capital city of Al Saud (near to Riyadh, the current capital city). At that time the dominance of the Al Saud family was limited to the Najd area (the central territory of Saudi Arabia today). In 1765 Mohammed bin Saud died but the alliance persisted between his son Abdul-Aziz bin Mohammed bin Saud and Mohammed bin Abdalwahab, who became a close advisor to Abdul-Aziz. By 1792 the rule of al Saud extended from the southern Syrian border in the north to the Dawasin Valley in southern Arabia, and from the Hejaz border in the west to the shores from Arabian Gulf in the east. 


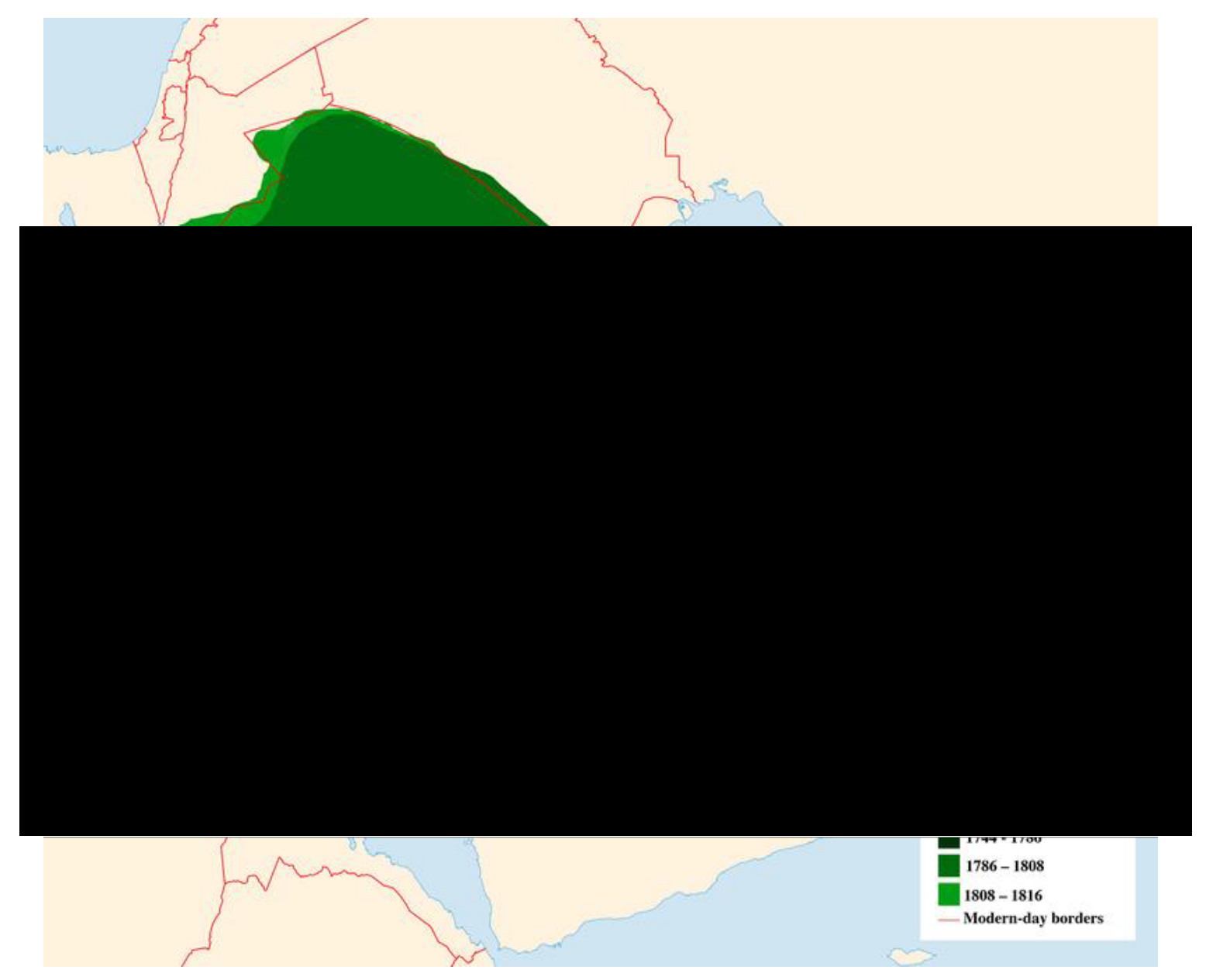

Figure 5-1: First Saudi state map.

By 1803 Ibn Saud had defeated Ghalib Al Sharif, the ruler of the Hejaz (the Al Sharif family is descended from the Prophet Mohamed, peace be upon him) and occupied the Hejaz. By 1814 Ibn Saud ruled most of the Arabian Peninsula. Ottoman Sultan (the Turkish ruler) found the expansion and the evolving strength of Arabia to be a threat. Consequently, he commanded Mohammed Ali Basha, his viceroy in Egypt, to reinstate Ottoman rule over Makkah. The forces, which were led by Ibrahim Basha, managed to besiege Alderiyah, and Abdullah bin Saud bin Abdul-Aziz bin Mohammed bin Saud surrendered to Ibrahim Basha, the commander of the campaign, bringing the first Saudi state to an end (Kechichian, 1986). 


\subsubsection{Second Saudi state, 1824-1891}

Following on from a six-year period of absence of rule, during which tribal law applied, the Saudi state Figure 5-2 was re-established in 1824 by Turki bin Abdullah, who confirmed the interdependence between Islam and the country's development and identity. Turki bin Abdullah was well known as an exceptionally brave individual, a quality he is said to have proved to possess especially in the battlefield. Turki was assassinated in 1834 and was succeeded by his son Faisal who was later sent to prison in Cairo by Mohammed Ali. Faisal managed to escape from jail in 1843, restored his rule on Najd (the centre territory of Saudi Arabia) and freed the access to the holy cities by chasing out marauders. As a result of three factors, namely: the opening of the Suez Canal; a feud between Faisal's sons and the prominence of the house of Al Rashid against the house of Al Saud, Ottoman domination returned to the Arabic peninsula. The rule of Al Rashid in Najd forced Abdurrahman, the youngest son of Faisal, to go into exile to Kuwait (Kechichian, 1986). This event ended the Second Saudi State.

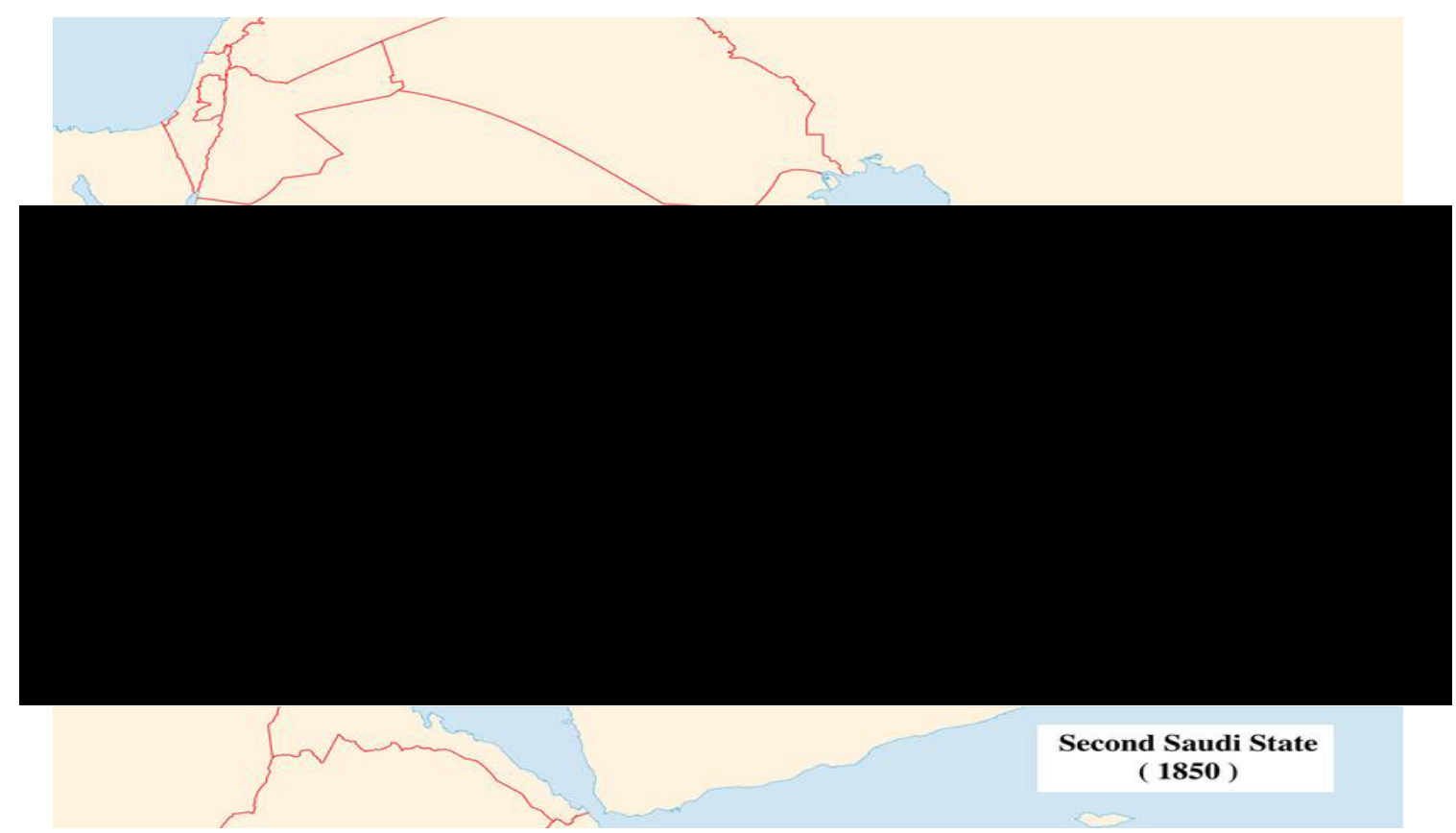

Figure 5-2: The second Saudi state map.

\subsubsection{Third Saudi state (kingdom of Saudi Arabia) 1902-1953}

Al Rashid captured Riyadh in 1892 after defeating Al Saud. As a result, Abdul-Aziz bin Abdurrahman bin Faisal Al Saud (whilst in exile in Kuwait) started to plan to 
restore Saudi rule over Arabia. The plan centred around establishing two hundred settlements to accommodate the people of the tribes so that they could practise activities such as military, agricultural and missionary work (Kechichian, 1986). After ten years, in January 1902, Abdul-Aziz's forces defeated Al Rashid and restored Riyadh and the rule on Najd, which marks the beginning of establishing the third Saudi state (Bligh, 1985). A planning phase was implemented, giving rise to the first settlement (Hijra), founded in 1912. From these Hijras Abdul-Aziz initiated the Ikhwan movement as a main part of his forces. The Ikhwan movement participated in all battles and efforts, ultimately uniting the whole of modern Saudi Arabia. In 1913 Abdul-Aziz managed to conquer Alhasa (the eastern part of Saudi Arabia). In 1921 he conquered Hail (north of Riyadh), the capital of the Al Rashid House. He then embarked on the difficult mission of occupying Asir (south of Saudi Arabia) and Hejaz between 1924-1925. Before 1921, Abdul-Aziz was given the religious title of Imam which he changed to that of sultan and, after his occupation of Hejaz and domination over all Saudi Arabia, announced himself as king of Saudi Arabia in 1932.

After Abdul-Aziz had successfully occupied Hejaz, disputes began to take place between him and the Ikhwan movement. The latter rebelled against Abdul-Aziz's rule by attacking the borders of Saudi Arabia and some pilgrims' caravans, causing serious embarrassment to Abdul-Aziz with neighbouring countries. The rebellion ended decisively in March 1929 with the battle of Sabila, when Abdul-Aziz managed to attract the support of the majority of Harb and Qahtan tribes and factions of the Mutayer and Utayba tribes which had seceded from their leaders (Kostiner, 1985).

Oil was first discovered in 1938 (in large quantities) and this initiated the modernization of the country. According to Saudi tradition, any decision that affects society should be approved by the Ulama (religious scientists). Ulama's decision making is heavily affected by traditions, as well as their confidence in being able to explain modernizations - and in general any new incumbent processes, in the light of traditional cultural roots. This routinely leads to intense and protracted debates in order for the Ulama to be satisfied with the potential decision. As an example, the king put considerable effort into persuading the Ulma to introduce the telephone; in 
another occasion, King Faisal's decision to permit television broadcasts was met with initial resistance (Kechichian, 1986).

Governmental structures were put in place and, in parallel, the Ministry of Foreign Affairs was first established in 1930 (followed by the ministries of Finance and Defence in 1937 and 1944 respectively). After the Second World War the income from oil steeply increased from $\$ 10.4 \mathrm{~m}$ in 1946 to reach $\$ 56.7$ million in 1950 . In 1951 Abdul-Aziz separated the Ministry of the Interior from the Ministry of Finance in order to distribute governmental responsibilities among members of the royal family. In 1953, shortly before Abdul-Aziz's death, the Council of Ministers was formed.

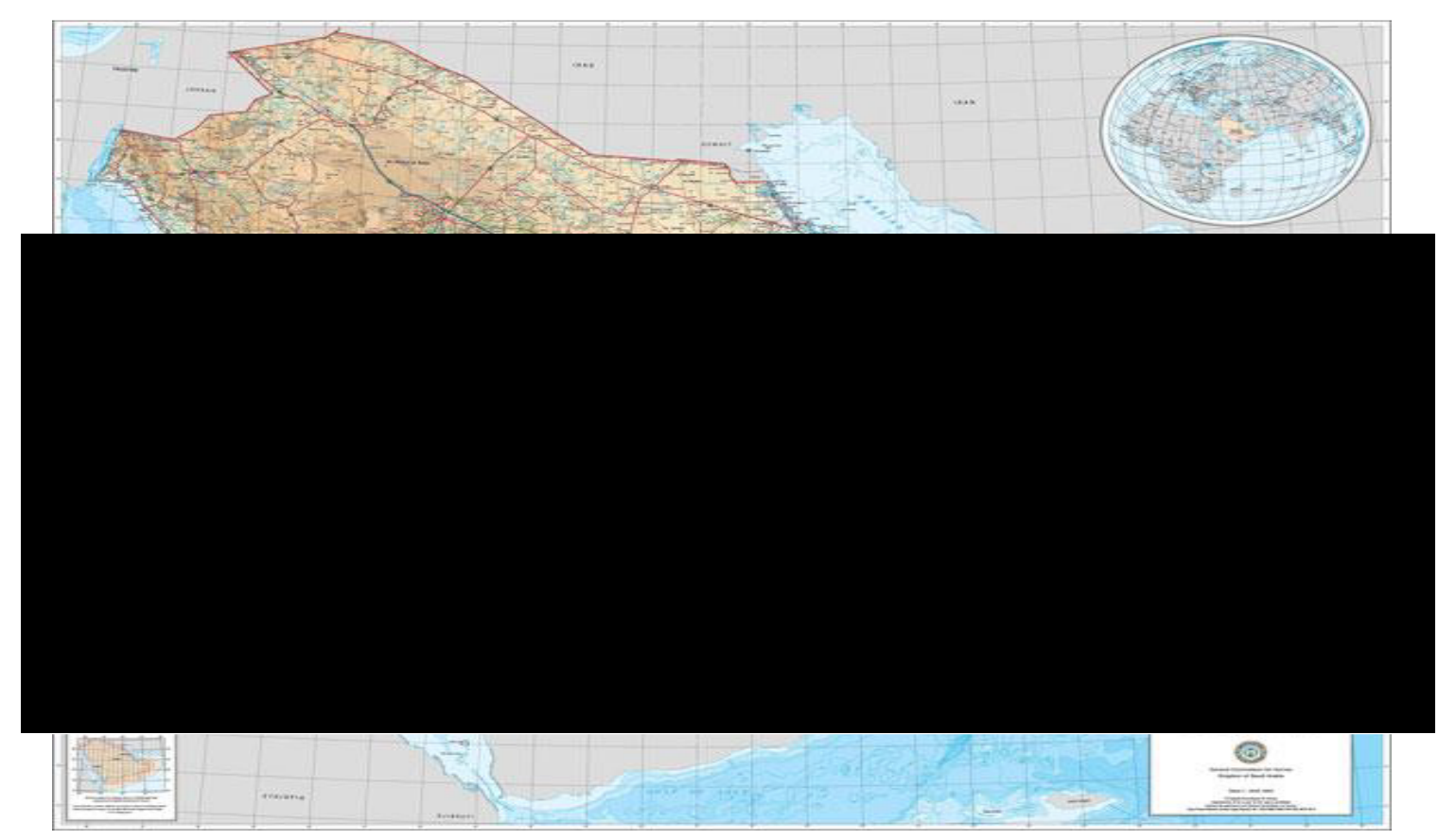

Figure 5-3: The current (third) Saudi state (the Kingdom of Saudi Arabia) map.

Source: General Commission for Survey, the Kingdom of Saudi Arabia 


\subsubsection{Civilization and Culture Change}

According to Huntington (1996) civilizations include religion, language, political, descent, history, customs, values and institutions, all of which shape the identity of a nation. National identity, in turn, consists of the forms of disintegration, cohesion, and conflict within a nation. The differences in culture within civilizations lead to differences in political and economic development. While culture shapes human behaviour, social processes, and social institutions within a specific civilization, it would not be able to have the same effect outside its civilization. Western culture came about through the power gained by the west over non-western societies, not by the superiority of its values or religion. Thus, power is important and necessary to the diffusion of culture between societies. The inverse of this perspective is also true, with non-western societies being able to reinstate their own culture and values after growing their power (Huntington, 1996). Other arguments propose that relationships among the components of culture are not firm and are subject to change in relation to social conditions. Only part of cultural values can prevail in society at any specific time. Identity`s determinants change from having a religious basis, to ethnicity, to nationality, depending on the conditions. The change in the basis of identity may also lead to changes in political attitudes.

Change within a culture is considered as a crucial resolution, when people and social leaders perceive that the current culture does not fit their needs and ambitions, and instead shift toward an accepted and satisfactory change. Thus, predicting any potential change is linked to the types of issues that prevail in public debate in society (Moaddel, 2010).

\subsubsection{The Economic Diversification Strategy of the KSA}

Saudi Arabia is a country which is categorized as a "rentier country" and rentier economy. As such the majority of the government income comes from natural resources, in this case crude oil (Beblawi, 1987; Hertog, 2010; Skocpol, 2014). This deviation or distortion in the economy's structure (from that of most developed countries) has created serious economic issues for the Saudi government, and meant that the country's long-term strategies and plans could no longer be sustained by high 
oil prices. Given the exposure of national income to the oil prices, which fluctuate widely, alternative forms of value and revenue generation were sought. The government strategy implies investment in sectors that currently do not exist or are fledgling in nature (such as the life sciences sector).

With regard to this fact, and to address this problem, the Saudi government has begun to transition to a knowledge-based economy and diversify the economy so as to mitigate the national exposure to economic vulnerability and oil dependency. Currently, the Saudi government has very ambitious strategies framed under its 2030 vision for the country. This vision identifies how the nation will address the economic pitfalls of continued oil dependency and this study hopes to contribute to this national agenda (in essence this research complements and matches the objectives of the 2030 vision).

The next two figures show the effect of oil prices on Saudi government income and gross domestic product (GDP) growth.

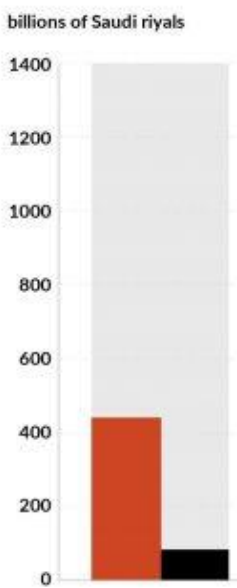

2009

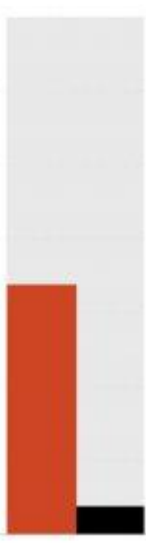

2010

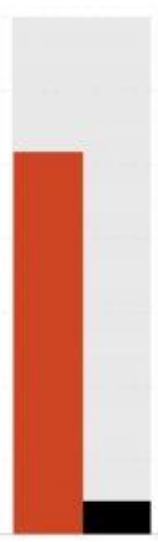

2011

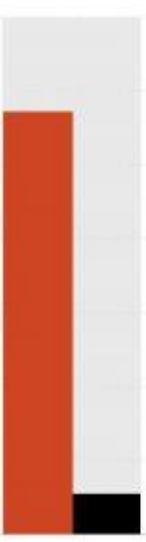

2012

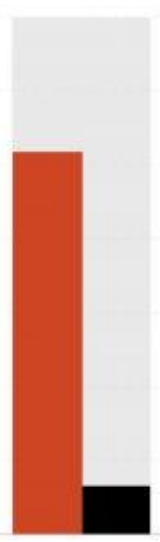

2013

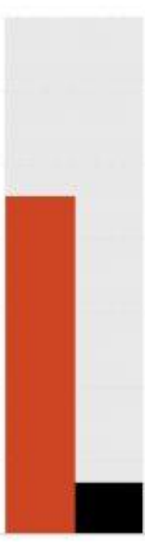

2014

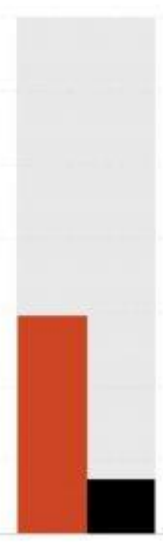

2015

Gis

Figure 5-4: Saudi government`s sources of income. Source: GISreportonline.com

The figure above shows a high fluctuating Saudi government income from oil (in the red columns) and how such revenue dominates Saudi government income in 
comparison to other sources of income (the black columns). The exposure to commodity price movements creates a signifcant vulnerability in the Saudi national government income. The 2030 strategy therefore highlights diversification and the development of new income streams (to increase the national income from the 'black columns').

The figure below also reinforces this point and clearly illustrates the impact of the changes in oil prices on the GDP (gross domestic product) of Saudi Arabia. For example, in 2010 GDP growth was less than 5\%, but the next year (2011) the annual growth in GDP was approximately $10 \%$, due directly to the sudden boom in oil prices.

\section{EconomicSlowdown}

The drop in oil is forcing Saudi Arabia to look for other sources of income

\section{GDP Growth}

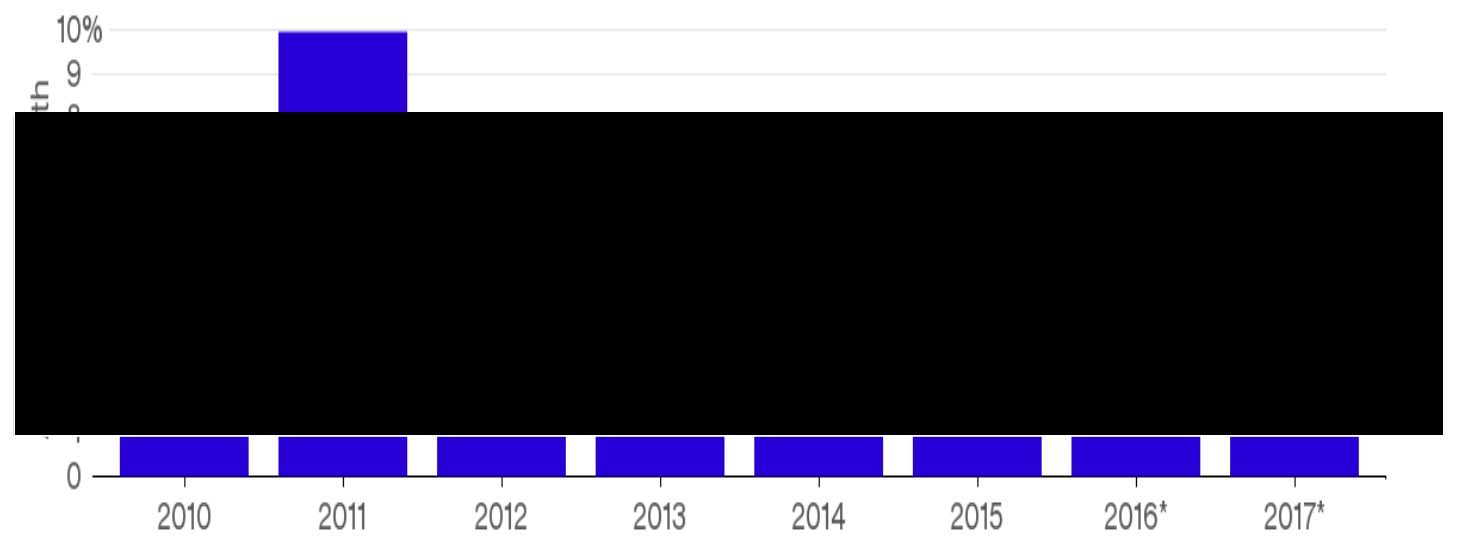

Source: Bloomberg data

"Median forecast of 12 economists in a Bloomberg survey

Bloomberg $\llbracket$

Figure 5-5: GDP growth in Saudi Arabia.

High fluctuating market prices and fluctuating government income influences the economic growth rate of the country and is a serious national problem, creating instability in developing and adopting long term strategies. This is because of the high level of uncertainty regarding government spending in the economy (income to finance national programmes and expenditures). Therefore balancing the economy to 
avoid these distortions is important in order to avoid serious economic and financial crises that could harm the Saudi kingdom socially, economically and politically.

\subsubsection{Saudi Economic Development}

The concept of the rentier state means that most national income comes from external rents, such as oil and mineral exporter countries (Ross, 2001). This definition requires further exploration, for example, a rentier state exists where its income comes from foreign governments, individuals or concerns (Mahdavy, 1970). This definition also has been refined to state that the rentier state is the state where the rents come from external actors and accrue directly to the government, with only a few being able to control and manage this wealth by distributing it amongst the majority (Beblawi, 1987).

\subsubsection{Rentier Economy}

The Saudi government developed successive five-year national development plans (which began in 1970 and are still in use). The first three plans focused on physical and social infrastructure development (Alshahrani \& Alsadiq, 2014). In the agriculture sector the government established the agriculture fund and presented generous subsidies to support mainly cereals products, which enabled the sector to be self-sufficient, and also to export any surplus production. In the industrial sector, the government established two huge industrial cities (Jubail and Yanbu) devoted mainly to the petrochemical industries, where the government had the main investments in this kind of high capital-intensive industry: for instance SABIC is one of the biggest petrochemical companies in the world (Niblock, 2008). About 26\% of the assured oil reserves in the world are located in Saudi Arabia, which has led Saudi Arabia to be the biggest exporter of oil in the world (Kayed \& Hassan, 2011b). Regarding this situation, about $90 \%$ of the Saudi government's income comes from oil rents, which constitute $35 \%$ of the GDP and $88 \%$ of export earnings (Alshahrani \& Alsadiq, 2014).

The other context which is very important to Saudi status and positioning both economically and politically results from its hosting of the two holy mosques (more than 11 million pilgrims a year visit the holy places) and these provide the Saudi 
government with another stream of income from religious tourism (Kayed \& Hassan, 2011b).

The Saudi government has benefited from two boom periods of oil price rises. The first was between 1973 and 1982, and the second between 2003 and 2014. Between 1982 and 2002 the government experienced quite a difficult financial situation due to a decline in the oil price which reflected on the economic growth and created a deficit in the government budget, although the huge foreign assets which the government had managed to build helped in covering this; that experience provided indications that the government could not succeed in building suitable economic conditions which would maintain sustainable economic growth (Niblock, 2008). The petrochemical industries faced difficulties because the government could no longer support them; also international markets adopted protective tariff procedures (before Saudi Arabia became a World Trade Organization member).

Given the fact that Saudi Arabia has a desert climate, irrigation for agriculture caused depletion of the underground water reservoirs, badly affecting the environment, and the government reduced its attention and support to the agricultural sector. Meanwhile, over the three consecutive five-year plans between 1985 and 2000, average economic growth was $1.2 \%$ per annum while the population growth was $4 \%$, which caused a significant drop in economic growth (Gross Domestic Product, GDP) per capita from $\$ 22,000$ in 1982 to $\$ 7,500$ by the end of 1990 . This economic deterioration was due to an over-reliance on oil revenues, which during that time had reduced significantly (due to price falls) (Niblock, 2008). Economic reforms were severe, yet vital, a crucial corrective to the overreliance on oil rents and a necessary encouragement to economic diversification.

By 2005, Saudi Arabia had joined the World Trade Organization (WTO), which necessitated significant economic reforms. The membership embodied significant potential benefits to Saudi Arabia by helping it gain international markets with no discrimination protection procedures and helping the Saudi petrochemical industry to access international markets. Amongst the new economic reforms were Foreign Investment Laws, which enacted policies to encourage foreign investment (for 
example for the first time the regulations permitted foreign investors to have $100 \%$ ownership of their enterprises - see Alshahrani \& Alsadiq (2014). Also new intellectual property protection procedures were applied, and effective tariff reductions introduced, which enhanced the products market in terms of prices and quality. However, this economic behaviour and the five-year development plans could not address the growing problem of unemployment and the employment culture of Saudi individuals who have a preference for public sector jobs (Kayed \& Hassan, 2011b). By 1990s more than 50\% of Saudi work force was working in the public sector, whereas $90 \%$ of the private sector's workforce was dominated by foreign labour.

In general, the mentality of Saudi individuals involves a preoccupation with the idea of job security and a national aversion to "menial" jobs, and this offered major opportunities to foreign labour to enter the private labour market and, consequently, led to an increasing rate of unemployment among Saudi job seekers. The implications of the rentier state and the paternalistic role that the Saudi government adopted through providing a wide range of subsidies (in utilities, basic commodities, free education and health and the redistribution of incomes in many other forms of consumer channels), led to new forms of creativity- and innovation-free businesses in Saudi Arabia. Most of these businesses were risk-averse; for instance real estate, which was a very profitable sector, with mostly no risk (Allen, 1998), and some businesses which leveraged the open trade economy situation to import products and act as agents and distributors for big foreign companies (Kayed \& Hassan, 2011b).

In the business landscape manufacturing enterprises developed later and focused on light manufacturing or basic products without any potential to develop a more innovative attitude. The Dutch disease affected the Saudi rentier state (where unrealistic currency exchange rates caused high production costs and hindered Saudi industries' competitiveness in global markets and their position against imported products (Kayed \& Hassan, 2011b). This also impacted on the Saudization process (employing Saudi people), where the private sector failed to match their expectations, especially in terms of the levels of salaries and the skills required and, as a 
consequence, these businesses relied significantly on the cheaper foreign labour. The eighth and ninth development plans (2005-2014) were focused on improving education and human resource development (55.6\% of the expenditure was devoted to education and human resource development, whereas it was $20.5 \%$ in the first plan) as being very important factors for the transition to a knowledge-based economy. The government has been working on developing six industrial mega-cities, where knowledge production and innovation are to be considered important mechanisms and practices to develop a knowledge-based economy (Niblock, 2008). Today, the Saudi government has some promising initiatives and plans, and has mobilized national resources in an effort to deliver sustainable economic development (beyond oil).

As can be seen in Table 5-1, significant changes occurred between plan one and plan nine. The spending on human resources in plan one was $20.5 \%$ while by plan nine it had increased by about $150 \%$ to be $50.6 \%$ of total government expenditure. Expenditure on social and health services increased by about $90 \%$ to reach $19 \%$ of total government expenditure. Infrastructure dropped from $41.3 \%$ to $14.6 \%$, and the last sector, economic resources, decreased significantly from $27.9 \%$ to $15.8 \%$.

These changes highlight the government's orientation toward developing human resources (national human capital) and supporting social and health services. It should be noted that infrastructure is no longer perceived as a significant source for further investment, yet economic resources still rely on natural resources. 
Table 5-1: Distribution of Saudi government expenditures on different sectors.

\begin{tabular}{|l|c|c|c|c|c|} 
Development Plans & \multicolumn{2}{c|}{$\begin{array}{c}\text { Period } \\
\text { Infrastructure }\end{array}$} & $\begin{array}{c}\text { Ecomic } \\
\text { Resources }\end{array}$ & $\begin{array}{c}\text { Human } \\
\text { Resources }\end{array}$ & $\begin{array}{c}\text { Social \& } \\
\text { Health } \\
\text { 9ervices }\end{array}$ \\
\hline First plan & $1970-1974$ & $41.3 \%$ & $27.9 \%$ & $20.5 \%$ & $10.3 \%$ \\
\hline Second & $1975-1979$ & $49.3 \%$ & $28 \%$ & $14.7 \%$ & $8 \%$ \\
\hline Third & $1980-1984$ & $41.1 \%$ & $30.7 \%$ & $18.4 \%$ & $9.8 \%$ \\
\hline Forth & $1985-1989$ & $28.9 \%$ & $20.4 \%$ & $33 \%$ & $17.7 \%$ \\
\hline Fifth & $1990-1994$ & $21.8 \%$ & $10 \%$ & $48.3 \%$ & $19.9 \%$ \\
\hline Sixth & $1995-1999$ & $16.2 \%$ & $11.5 \%$ & $51.5 \%$ & $20.8 \%$ \\
\hline Seventh & $2000-2004$ & $12.6 \%$ & $11.2 \%$ & $57.1 \%$ & $19.1 \%$ \\
\hline Eight & $2005-2009$ & $14.1 \%$ & $12.2 \%$ & $55.6 \%$ & $18.1 \%$ \\
\hline Ninth & $2010-2014$ & $14.6 \%$ & $15.8 \%$ & $50.6 \%$ & $19 \%$ \\
\hline
\end{tabular}

Source: Saudi Ministry of Economy and Planning

Table 5-2: Structure of real GDP over the three sectors (governmemnt, private, Oil).

\begin{tabular}{|l|l|l|l|l|l|l|l|l|l|l|l|l|l|l|}
\multicolumn{4}{|c}{2005} & 2006 & 2007 & 2008 & 2009 & 2010 & 2011 & 2012 & 2013 & 2014 & 2015 \\
\hline Gov. \% & 16.48 & 16.05 & 15.1 & 13 & 18 & 17 & 14.8 & 15.1 & 15.8 & 16.6 & 22 \\
\hline PS \% & 33.5 & 33.1 & 34.45 & 31. & 41 & 38 & 33.9 & 34.4 & 37.6 & 40.7 & 49.7 \\
\hline Oil \% & 50.02 & 50.85 & 49.55 & 55.4 & 41 & 45 & 51.3 & 50.5 & 46.6 & 42.7 & 28.3 \\
\hline
\end{tabular}

Source: The Ministry of Finance (Saudi Arabia).

Table 5-2 shows that oil's contribution to real GDP increases and decreases with the oil price in international markets. It also shows that there is a steady growth in the private sector share in the real GDP. As a result, the government's initiatives to raise public sector efficiency and the shift to more private sector investment have resulted in the private sector increasing its share in real GDP.

\subsubsection{Entrepreneurial Attitude}

An entrepreneurial approach and attitude is one of the most important factors in the economic development of any nation. By following an entrepreneurship strategy more jobs, as well as new products and innovations that could create new industry sectors,

\footnotetext{
${ }^{6}$ Transport, telecommunication services, public works, housing, and municipal affairs

${ }^{7}$ Agriculture, water and electricity, petroleum \& mineral resources, and industrial services.

${ }^{8}$ General education, higher education, technical \& vocational training, science \& technology

${ }^{9}$ Health care, social, youth and information services, and cultural activities
} 
can be created. A new entrepreneurship-based model also has a social dimension in terms of direct involvement which can enhance human development - considered to be a good measure for economic development and diversification (Khan, 2013; Wim Naudé, 2011; Shane, 2007; Wennekers \& Thurik, 1999). As mentioned above, the rentier state and its implications resulted in governmental subsidies programmes, which have aggravated a consumeristic culture instead of promoting productivity among Saudis.

Generally speaking, Saudi individuals lack the entrepreneurial attitude and are riskaverse in their economic behaviour (Kayed \& Hassan, 2011b). According to Yates (1996), the rentier mentality is a result of rentier economy, where the people tend to practise non-productive activities. The above mentioned successive five-year development plans did not address this and failed to create national entrepreneurship in Saudi society, and this has resulted in persistent and chronic unemployment (Kayed \& Hassan, 2011b). Beside the traditional attitude toward manual work, the low quality of the education system has contributed to the low level of contribution by Saudi employees to economic development, whether through a lack of entrepreneurial attitude or the lack of the required skills and capabilities that match and fulfil the business market`s needs (Kayed \& Hassan, 2011b) .

Unlike physical infrastructure investment, human resource development is a long process and can consume a generation of time to yield real results; it is not a matter of quantity but of quality. Away from both western democracy and eastern discipline, Saudi governance developed a bureaucratic state and large public sector that actually hindered economic development (Kayed \& Hassan, 2011b). To address these inefficiencies in the economic system, the Saudi government adopted many steps and processes such as: strategies to reduce the role of the state in the economy; embarking on privatization processes to improve efficiency and competitiveness in the private sector, expand its role and involvement, and encourage entrepreneurship; raising efficiency in public institutions and relaxing the impeding regulations and procedures; developing human resources through building new world-class research universities 
and a travelling scholarship programme; developing mega industrial clusters, which will cost over $\$ 400$ billion in the next 20 years (Kayed \& Hassan, 2011b).

The data collected in this research uncovered quite similar information about the weak entrepreneurial attitude of Saudi people: is very important that this issue is properly addressed given the very important role played by entrepreneurship in transferring knowledge and investment in knowledge to create a knowledge-based economy.

\subsubsection{Enterprises in the KSA}

The term enterprise means creating a new business; a business itself or a collection of businesses (Sewell \& Pool, 2010). According to (Rae, 2007), enterprise involves many skills which are called "enterprise skills". These skills include "initiative, independence, creativity, problem solving, identifying and working on opportunities, leadership, acting resourcefully and responding to challenges". Others define enterprise as "the application of creative ideas and innovations to practical situations" (Education, 2012).

The main economic activities in Saudi Arabia can be broken down into seven categories, namely: natural resources (oil, gas, and minerals); manufacturing; agriculture; oil refining; trade and services; construction; utilities (electricity, gas, and water) (Saudi Arabia Monetary Agency, 2013).

The four biggest sectors (oil and minerals, trade and services, manufacture, and construction) are detailed below. The following chart illustrates their contribution to the Gross Domestic Product (GDP):

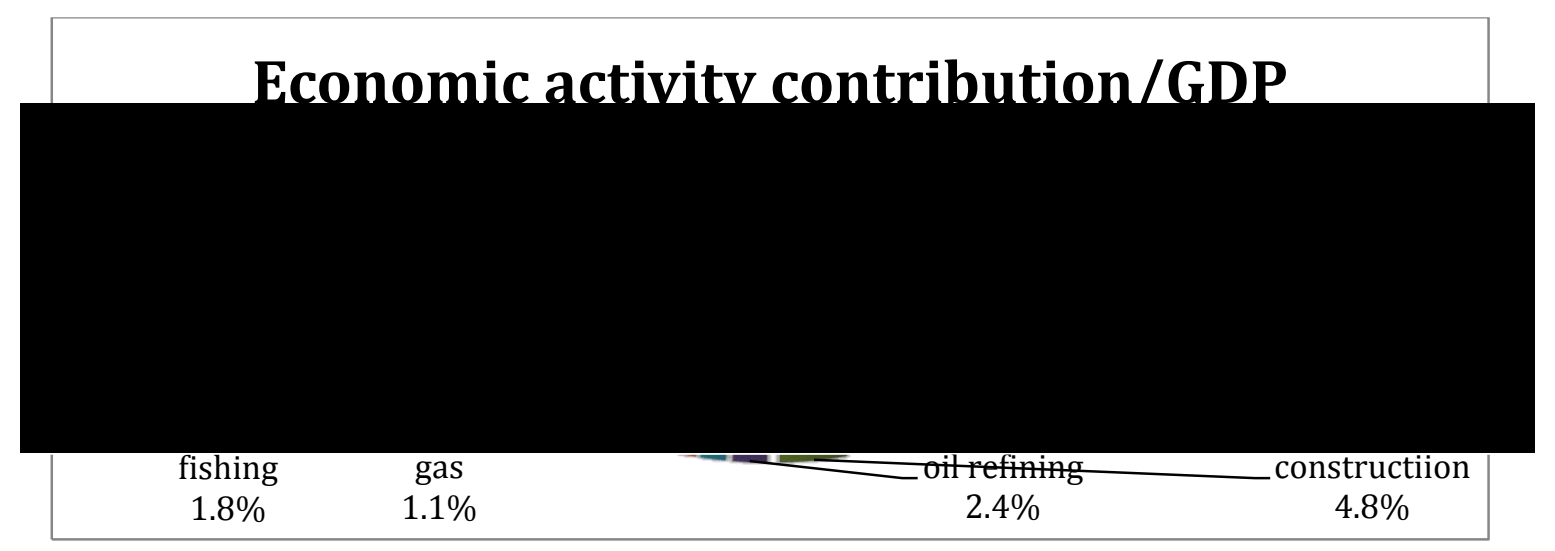

Figure 5-6: Economic activities distribution in Saudi economy (share of GDP).

Source: (Saudi Arabia Monetary Agency, 2013). 


\subsubsection{The Oil Sector}

The oil sector constitutes the main backbone of the economy and the majority of government revenues: in 2013 about $90 \%$ of government revenue came from crude oil production, which made up $78 \%$ of exports, and $44.8 \%$ of GDP (Barakat et al., 2014). Another important energy source is natural gas, of which Saudi Arabia produced 77.7 million tons in 2012. The government is developing many projects to raise the production of natural gas to 167 million tons by 2020. These projects are vital to match the huge growth rate in annual demand in the petrochemical industries (where it is used as a feed material) and other industries and services such as desalination stations and power generators, as well as domestic consumption. For instance, in 2013 the growth was 12.4\% and in 2012 11.5\% (Barakat et al., 2014).

In general, the Saudi government considers the energy sector to be one of the strategic sectors. Regarding this consideration it invests heavily in this sector; for example the government plans to run 16 nuclear reactors by 2032 for energy generation, and also has plans to be a solar energy exporter in the next 30-50 years (Barakat et al., 2014).

\subsubsection{The trade and services sector}

Saudi Arabia occupies a very significant geographic location between the East and the West and excellent port accessibility on the red sea and Arabic gulf; this location has facilitated trade transactions across the borders.

The Saudi population is relatively large. $47 \%$ of the population are aged between $15-$ 39 , and have quite a high rate of personal financial liquidity. All these factors have enhanced the significant growth of the retail sector. Saudi Arabia has been ranked eighth against the Retail Talent index. The retail sector (with non-grocery retail being about $60 \%$ of the retail sector) increased from $\$ 70$ billion in 2011 to $\$ 76$ in 2012, which represents about $42 \%$ of the GCC market size. The floor space/capita is still lower than other regional markets, which holds potential growth for this sector. Over the last five years, the sales per capita have increased by $9 \%$ annually. New style malls combining offices, hotels and leisure facilities have been developed and spread across the different provinces. Internet penetration in the society has enabled online retail shopping to increase exponentially (Alliance, 2014). 


\subsection{Franchise approach}

Franchise is a popular business model and is expected to grow annually in Saudi Arabia by $12-15 \%$. The large young segment of the population (57\% under 25 years) enhances this expectation, as well as the quite high per capita income. This kind of business model, which was estimated to be at the foundation of more than 500 concepts in Saudi Arabia, covers many sectors such as: retail; apparel; food and beverages; patisseries; laundry services; mail; hotels; cosmetics; education; health care, and automotive services. Recently, new franchise concepts entered the Saudi market: spas, health clubs, wellness and slimming clinics, and trendy fashion brands. $\$ 1.3$ Billion has been paid annually against fees and loyalties for the franchise businesses (Shujauddin, 2010)

Due to the lack of leisure activities within Saudi society, families usually spend most of their leisure time in the malls doing shopping, which helps to put Saudi people at the top globally in terms of per capita spend on, for example, cosmetics and fragrances. About 30 local franchise concepts have been developed and some of them have become international. The government, via the Centennial Fund, supports this business model and offers zero interest loans to encourage entrepreneurs to invest in new ideas (Shujauddin, 2010).

Unlike entrepreneurship and investment in new knowledge, this kind of approach is preferred for reasons such as lack of managerial expertise, lack of market information, promotion, the readiness level of the ideas (and the related tendency to avoid new ideas with higher risk), reduction in payroll cost, and also to overcome some resource scarcities (human, financial, information, and knowledge capital) (Sadi \& Henderson, 2011).

\subsection{Healthcare field}

In Saudi Arabia, healthcare is a thriving and promising business. With respect to the high population growth (the population in 2010 was 27.1 million, and expected to grow to about $39.8 \mathrm{~m}$ by 2025 , and to $54.7 \mathrm{~m}$ in 2050 ), and the growth of per capita income which increased from \$22,925 in 2007 to 24,726 (Almalki, Fitzgerald, \& Clark, 2011), in addition to new regulations that enforce the provision of health cover insurance by the private sector for its expatriate and Saudi citizen workforce, this sector holds huge potential opportunities (Yusuf, 2014). As shown in subsequent 
chapters, this potential is seen by the researcher as a strong factor that should motivate the government to invest in developing the LS sector and give the signal for other actors to become involved.

In 2006 government expenditure on the healthcare sector reached 13\% of the overall budget. The government operates 115 hospitals and 2037 primary health centres, while the private sector runs 125 hospitals and 2218 clinics and dispensaries. The private sector mostly focuses on cities and big towns. However, since government cannot afford to continue spending on the increasing healthcare financials dues, it exerts much effort and enacts regulations which can deliver the attractive conditions that will encourage the private sector to increase its investment and to leverage this profitable growing business (Group, 2007).

Current spending and investment focus only on providing health services, but, as it will be shown from the data, in order to develop a health sector that could treat local problems and present innovative solutions, investment in knowledge is a key requirement.

\subsubsection{Construction Sector}

The construction industry in Saudi Arabia constitutes $4.8 \%$ of the GDP. With many drivers, including government plans for economic diversification (the government is building six mega economic cities distributed over six provinces); fast urbanisation and the growth in the young population, and housing the two holy mosques in Mecca and Al Madinah and their relevance to religious tourism, Saudi market is one of the fastest growing in the Middle East. These drivers provide sustainable demand for construction across all sectors. The value of construction contracts has significantly increased, by $38.75 \%$ between 2008 (about $\$ 80$ bililon) and 2011 (about $\$ 111$ billion). The construction sector is distributed over many other sub-sectors, namely building, power and water, oil and gas, industrial, and infrastructure (Middle East, 2011).

Socio-economic factors are expected to support the growth in the construction industry.

The next PESTLE (political, economic, social, technological, legal and regulation, and environment) analysis can illustrate the situation relevant to every component:

Political: government plans for economic diversification and development of the infrastructure will enhance growth. 
Economic: inputs cost, low inflation, and schemes for affordable housing.

Social: the high growth in the young segment of the population will sustain demand.

Technological: the high growth in construction compared to other GCC countries attracts new technological and material solutions.

Legal and Regulation: relaxation of the procedures required to start a business and get a construction permit; allowing foreign investors to have $100 \%$ of ownership of their real estate properties and projects; releasing the mortgage law - all these factors should boost the real estate industry to grow by $20-30 \%$.

Environment: in spite of being at an early stage, government encourages green construction and smart buildings, and has allocated about $\$ 40$ billion to construct smart buildings that help in dioxide reduction and improved efficiency in electricity and water consumption (Middle east, 2011).

The following chart shows the distributed percentage across the construction sectors:

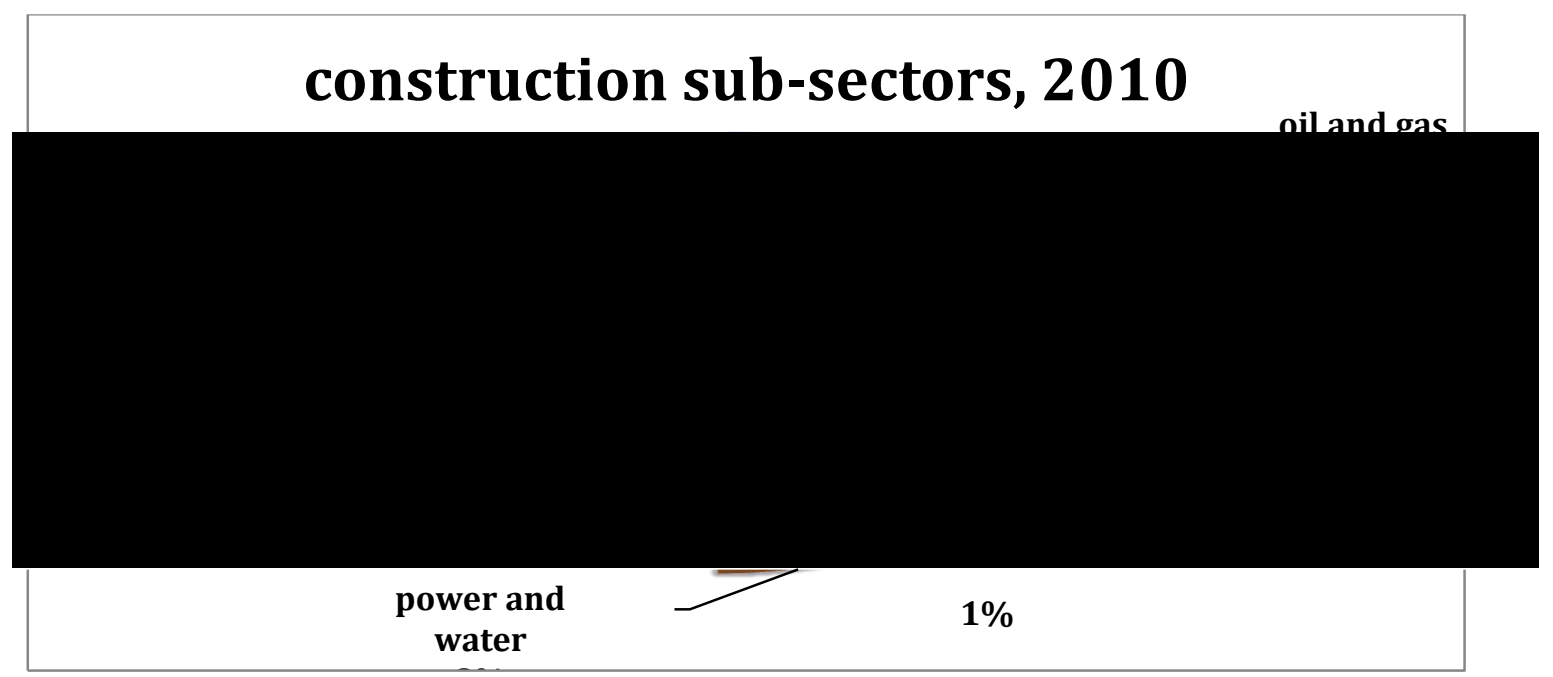

Figure 5-7: Construction sub-sectors. Source: Adapted to:(Middle East, 2011)

The government invests heavily in the oil, gas and refinery sectors; it allocated $\$ 100$ billion for exploration (of oil and gas) and building refineries over the period 20102015. ARAMCO will invest $\$ 120$ billion in the oil and petrochemical industry. The government`s target is to raise oil production to 15 million barrels/day, and expand the capacity of gas production to match the annual $7 \%$ gas growth demand. 
Regarding energy, consumption increased exponentially from 13,000 megawatts in 1991 to 40,000 megawatts in 2010, representing an average annual growth greater than $10 \%$, while the average annual production of water increases by $4.7 \%$. In terms of water production, and with respect to the scarcity of natural water resources, high population growth and large agricultural and industrial base, Saudi Arabia is one of the largest producers of desalinated water in the world. The government is adopting the privatization approach to satisfy the accelerated demand in power and water production, and to try to attract foreign investment in this fast-growing sector. One of the models that government uses is the BOT model (build, operation, transfer); through this model, the investor builds the project and commercially operates it for a specific agreed period, after which ownership transfers to the government. According to the statistics of power consumption distribution for 2009, the sectors' shares of consumption are: residential 54\%, industrial 19\%, government $13 \%$, commercial $12 \%$, agricultural $2 \%$. As a result of the growing demand for power and water, the government adopted plans to shift to solar power for producing electricity and water as well. The government has established a new organization in charge of developing the necessary means to develop nuclear projects and renewable energy sources. This organization is going to build 16 nuclear power stations, and collaborate with some local research centres to make renewable energy sources more economical, innovative and more efficient. The government has ambitious plans to be a big solar energy exporter (Middle East, 2011).

Saudi Arabia's desert climate poses big challenges for the provision of water - water is one of the most irritating issues for the Saudi government, and without innovative and creative solutions, the provision of water will be unsustainable. So, developing the LS sector is a strategic issue.

\subsubsection{Manufacture}

The manufacturing sector in Saudi Arabia contributes $10.1 \%$ to GDP. The government has adopted a new programme called the industrial clusters programme, working on developing clusters in automotive, construction materials, metals processing, plastics and consumer appliances. This cluster, besides the developing of the six-mega industrial cities, would enhance the growth of the construction industry. 
The growth in the number of operating factories over the period from 2008 to 2012 is as follows: 4807, 5053, 5233, 5549, 5991 units respectively (Planning, 2013).

The problem with the Saudi manufacturing sector is that, by undertaking only production processes, very few any knowledge activities take place, which affects the competitiveness of the sector against global players.

\subsubsection{Doing Business}

The Doing Business index gives indications about the business environment and measures to what extent it is easy to do business. This index contains 11 indicators, which extend over many areas relevant to the business life cycle: starting a business; dealing with construction permits; getting electricity; registering properties; getting credit; protecting investors; paying tax; trading across borders; enforcing contracts; resolving insolvency, and employing workers. The purpose of these indicators is to gauge the efficiency of issues such as: the complexity of regulation and the numbers of procedures required to start a business, register or transfer property; the time and cost needed for any procedure; legal protection; tax due to business operations, and employment regulations and their impact on business operations.

In spite of achieving only a low rank in some indicators, however, Saudi Arabia achieved a relatively good overall ranking among the world countries $\left(26^{\text {th }}\right.$ position in 2014). This position was achieved as a result of substantial government efforts and reforms aiming to improve the business environment to help in attracting foreign investment and enhancing the main government goal of diversifying the economy (World Bank, 2014).

From the data presented in the subsequent chapters, it transpires that the provision of regulations and a suitable environment to facilitate starting a business is very important to attracting FDI and encouraging entrepreneurs to build their businesses. However, there are still barriers to government staff, such as faculty members, building and owning their own businesses, and this impedes knowledge transfer.

\subsubsection{Competitiveness}

According to Michael Porter, "competitiveness depends on the productivity with which a nation uses its human, capital, and natural resources" (Porter, 2009). There 
are factors and determinants for competitiveness, some of which relate to the macroeconomic level, while the others are microeconomic components:

\subsection{Macroeconomic competitiveness}

Social Infrastructure and Political Institutions: this pillar consists of three components:

Basic Human Capacity:

- Basic education

- Health system

Political Institutions:

- Political freedom

- Voice and accountability

- Political stability

- Government effectiveness

- Centralization of economic policymaking

Rule of Law:

- Judicial independence

- Efficiency of legal framework

- Business costs of corruption

- Civil rights.

Macroeconomics policies: this pillar consists of two components:

Fiscal policy:

- Government surplus/deficit

- Government debt

Monetary policy:

- Inflation 
- Interest rate spread.

\subsection{Microeconomic competitiveness}

Three key areas pertain to microeconomic competitiveness: quality of business environment, cluster development status and sophistication of company operations and strategy:

Quality of business environment:

- Context of firm's strategy and rivalry: this factor concerns the rules and incentives that support productivity and investment such as protection of intellectual property, capital investment support, and performance-based compensation; and the existence of competition with local and foreign rivals.

- Demand conditions: the extent of customer sophistication creates high quality standards and enhances competitiveness to match the customers`expectations and needs.

- Availability of suppliers and supporting industries.

- Inputs conditions and availabilities; the elements of business inputs range over many components such as natural and human resources, physical and administrative infrastructures, scientific and technological support, availability of capital, and information and transparency (Porter, 2008).

Cluster development status:

According to Porter (2014), the features of clusters and their impact on the competitiveness range over many areas such as :

- Enhancing the productivity and the efficiency of operations

- Stimulating innovation

- Easing the creation of new enterprises

- Within clusters, the probabilities of improving innovation and productivity increase as a result of the linkages and spill over across firms and associated organizations.

Sophistication of company operations and strategy: 
This key area covers a range of activities such as production process, marketing, branding, distribution, and creativity in delivering new ideas and products (Schwab, 2014).

The Porter method was of interest to the researcher (from a systems perspective) but it did not offer a means of operationalisation and, to date there has been no suitable formal methodology to be applied to the specific case of KSA, hence the need for the particular approach that this study adopted (i.e., grounded theory), to originate a suitable index.

\subsubsection{KSA Competitiveness}

Against the foregoing indicators Saudi Arabia scored remarkable progress over the period from 2007 till 2014. For example, in 2014 the overall rank was 24 out of 148 countries; this ranking can be detailed as follow:

- The basic requirements rank is 15 ,

- The efficiency enhancers rank is 33 , and

- The innovation and sophistication factors rank is 32 (Schwab, 2014).

Competitiveness is very important to investors; therefore, improving and raising the competitiveness of Saudi Arabia is a very encouraging factor especially for FDI, as it enables funding to be channelled to Saudi Arabia, with positive impact on knowledge transfer.

\subsubsection{SMEs in the KSA}

The SME is considered to be a very important sector in all economies worldwide because of its usually high contribution to employment; the SME sector constitutes a substantial portion in terms of the number of businesses in any economy (Culkin \& Smith, 2000). The contribution of SMEs to Asian exports is around 35\%, while for the developed countries it matches $26 \%$ of the total exports (OECD, 2007).

In spite of some difficulties and challenges facing SMEs in Saudi Arabia, such as difficult access to financial support - in more recent times improved via the programme called "Kaphalah", which relies on the role of the Saudi Industrial Fund providing the relevant banks with financial guarantees to cover the loans requested by the applicants - Saudi SMEs are the subject of significant attention by the 
government. SMEs in Saudi Arabia constitute $80 \%$ of the workforce market, $92 \%$ of the number of businesses, however quite low a contribution to the GDP (35\% of GDP). The average life cycle of the Saudi SME is around seven years. This short life cycle can be attributed to reasons such as difficulties in getting loans, lack of highlevel managerial capabilities and skills and consequently defects in strategic vision, business planning and in innovative orientation, with most SMEs relying on imitating each other, often with an absence of any creative ideas or knowledge management strategy (Saleh, 2012). Kechrid argues that, because of the weakness of employers' capabilities, they lose the ability to develop, or recruit talent, or plan careers; at the same time, human resource management also scores low in their measures; this is reflected in the productivity and performance of the organizations (Kechrid, 2012). According to Ali \& Al-Aali (1997), global competition revolves around generating new ideas, developing competitive advantages and maintaining sustainable growth; Saudi SMEs should therefore recruit creative individuals and provide a professional environment for work. In this respect, the generation of a pool of creative individuals is necessary to sustain such a system.

The research uncovered some challenges facing Saudi SMEs, such as a lack of innovative enterprises, difficulty in accessing finance, and weak managerial capabilities, and barriers for faculty members to establish their businesses. All these challenges in the system inputs and policy have been addressed through the innovation system model proposed by this study.

\subsection{GCC (Gulf Cooperation Council)}

From a contextualisation of the reader, the researcher believes that proximity and proximal geography is an influence on knowledge transfer and the markets for products/services, and therefore this section will provide a review of the GCC countries. From an historical perspective, the Islamic revolution in Iran and the IraqIran war, besides the Soviet invasion of Afghanistan - all geopolitical unstable situations in the Arab Gulf area - created a necessary desire among the Arab Gulf States to prepare for any political consequences. Saudi Arabia was stimulated to lead the movement and pose the idea of the establishment of the Gulf Cooperation Council (GCC), which took place on May $25^{\text {th }} 1981$ and included all the Arabic Gulf States 
(Bahrain, Kuwait, Oman, Qatar, Saudi Arabia, and United Arab of Emirates). This political agglomeration was built to neutralize the Iraqi and Iranian threats and to maintain the security and economic resources of the members states (Kechichian, 1985).

\subsubsection{GCC Economic Structure}

The GCC countries, to a great extent, share the same economic structural features; for example, all of them have: a young fast-growing labour force; a strong reliance on oil income which makes a significant contribution to GDP, and labour markets dominated by expatriate labour. These characteristics have created challenges to transitioning the economy towards diversification and to enabling the private non-oil sector to contribute significantly to the economy.

Regarding the recent sizeable income that came as a result of the spiralling prices of oil and natural gas, which constitute the majority of the GCC income, the GCC is working on developing structural reforms that would help in developing a diversified economy. Bahrain and the UAE (United Arab Emirates) are in a better position in this regard (Sturm, Strasky, Adolf, \& Peschel, 2008), since their non-oil sector has grown rapidly and has overtaken oil sector growth. Despite the fact that the non-oil sector growth is related and supported by the governmental expenditures, which in turn is linked to the oil income, this also gives some indication of the development and potential of opportunities and growth in the sector. Governmental expenditure in the GCC countries positively affects economic growth and national income, according to Al-Faris (2002).

Neighbouring countries have influence in this context of the GCC. For instance, the Dubai model has inspired and encouraged the other gulf Arabic countries to think about developing their own - similar or different - models to tackle the shortcomings in their economies and deliver sustainable performance.

Saudi Arabia for example is developing six industrial mega clusters in different territories within the country (one of them is the life science cluster in Madinah), and has adopted a strategy to lead to diversification in the economy and provision of more job opportunities whilst stopping reliance on the depleted natural resources as its main source of income. 
The Saudi government is mainly focusing on the manufacturing sector, and has recently also started to develop the financial sector as it is now building King Abdullah Financial Centre in Riyadh. Bahrain, which is regarded as the main financial hub in the region, has focussed on the financial sector, and the UAE on tourism, reexporting, financial, shopping, and real estate (Sturm et al., 2008).

The GCC countries became big players in the global financial markets via reinvesting their cumulative oil revenues; also, most of the GCC managed to build a huge foreign assets base (ranging from \$1-1.5 trillion) to use their returns in support of the government budget at times of income decline.

Forty per cent of the oil reserves are located in the GCC countries (26\% in Saudi Arabia); this situation is the factor that gave the GCC countries their important position as influencers of demand and supply relationships, and as global investors, sources of energy, and key customers especially of the EU and East Asia (most of the imports being machinery, appliances, electrical equipment, vehicles and parts), as well as destinations for labour - especially Asian and Arabic. GCC countries are working to make the Arabian Gulf a regional trading hub by investing more in the infrastructure (airports, harbours, roads, railways, etc.) and making institutional reforms, in terms of reducing import and export costs via minimizing transaction documents and time.

Despite the improvement in this regard, they are still below the average level of the OECD, but they outperform other countries in the Middle East and North Africa. GCC nations have also made free trade agreements with different international partners (all GCC states are members of the WTO - World Trade Organization) (Sturm et al., 2008).

\subsubsection{GCC Integration}

"Regional Integration" (RI) is a worldwide phenomenon of territorial systems that increases the interactions between their components and creates new forms of organization, co-existing with traditional forms of state-led organization at the national level" (De Lombaerde \& Van Langenhove, 2005). This regional trade can leverage many benefits including: economies of scale, enhancing policies, gaining profits from inter-dependent trade, using this feature as bargaining power against 
other trade blocs, and accessing markets with preferable conditions. However, the intra-trade among GCC countries is still modest (Ravi, 2013).

Despite the establishment of the Customs Union, which took place in 2003, and of the Common Market, at the beginning of 2008 (allowing free movement of capital, and of citizen labourers), the rather similar exports of the GCC countries, which rely on hydrocarbons, limited the intra-trade among them to around $6 \%$ of the total trade, which can be considered low compared to other regional blocs such as the EU or NAFTA (North America Free Trade Agreement) (Sturm et al., 2008).

According to Sturm \& Siegfried (2005), the potential diversification plans in the economies' structures should lead to a rise in the intra-trade. However, if the trade excludes oil exports, the intra-trade within GCC countries amounts to $26.7 \%$ of the total exports (averaged over the period 1993-2004), which is comparable to other regional intra-trade scenarios, such as: ASEAN and MERCOSUR (MERCOSUR is a sub-regional bloc which includes Brazil, Argentina, Venezuela, Paraguay and Uruguay), which means that the intra-trade between GCC countries is quite intensive and, to some extent, integrated. Saudi Arabia is the only member which has borders with all the other GCC members, which enhances the increase of trade and reduces the trade cost compared to countries that do not have shared borders (Boughanmi, 2008).

Till now there has been no knowledge economy structure among the GCC, therefore the rise of a "role model" and the neighbouring effect would motivate the other countries to develop their own models, boost healthy competition and create a bigger market that the actors can move within. 


\subsection{Life Science (LS)}

The life science industry comprises mainly of three key sub-industries, namely: the biotechnology industry, pharmaceutical industry, and medical devices industry. Pharmaceutical companies develop products and services in fields such as pharmaceutical, food processing, waste management and agriculture. They collaborate with biotechnology firms to maintain their product pipelines, when the biotechnological firms develop new potential products and license their intellectual property to the big pharmaceutical companies (Valuation, 2013). Biotechnologybased industry has a low potential of failure in comparison with the conventional pharmaceutical industry (chemistry-based industry) (Pisano, 2006). The medical device industry has, similarly to the pharma industry, been in existence for a longer time than biotech; it is a highly multidisciplinary subsector that relies on engineering and the medical sciences (Loffler \& Stern 2008). The researcher focussed on the Biotech subsector, which is considered to be the most promising LS sector (Patzelt, 2012).

\subsubsection{Biotechnology as a Promising Sector}

According to Young (2000), biotechnology is defined as: "all innovative methods, processes, or products, which include the use of living organisms or their cellular compartments and draw on the results and knowledge generated from research in the fields of biochemistry, molecular biology, immunology, virology, microbiology, cell biology, or environmental and engineering sciences".

In contrast to the other knowledge-based industries, such as software and medical devices, developing biotechnology products is usually more time and capital intensive (Azoulay, Michigan, \& Sampat, 2007). However, according to Rader (2008) “criteria for what is biotechnology and biopharmaceutical are unfixed, subjective, adaptable to the needs of the moment, presumed to be continually evolving and rarely defined".

The biotechnology industry is a very promising sector in the twenty-first century, beginning its great rise with the advent of gene technology in 1976. With $\$ 200$ billion revenue in 2009 and average annual growth rate of 10.2\% over the period 2005-2009, 
the biotechnology industry has quite a large economic impact (Patzelt, 2012). The biotechnology industry overtook the traditional pharmaceutical industry twofold in terms of discovering new drugs, while only spending a quarter as much on research and development (Young, 2011). However, developing a new drug is a very costly and risky process; it costs, on average, $\$ 100$ million and requires 10 years of research and development (DiMasi, Hansen, \& Grabowski, 2003). Furthermore, only one out of 5000 initial candidate drugs reaches the market (Evans \& Varaiya, 2003).

These cost and risk downsides associated to knowledge activities within biotech are unfortunately the main factors preventing Saudi private sector from investing. Based on this perception it is seen as important and, indeed, crucial for the Saudi government to kick-start investment in the industry to give positive signals for academia, industry and other actors.

\subsubsection{Biotechnology Industry Dynamics}

Clustering is a key stimulating mechanism for the development of a biotechnology ecosystem that enables universities, investors, biotechnology ventures, incumbents firms, and other actors to interact, and work in conditions of proximity (Audretsch \& Stephan, 2002; Cooke, 2002; Patzelt, 2012; Stuart \& Sorenson, 2003; Zucker, Darby, $\&$ Brewer, 1998). The globalization and the nature of biotechnology industry has helped to ease access to the biotechnology ecosystem (patent lawyers, venture capital, unique testing capabilities organizations, manufacturers, and other start-up formation enablers) (Burill \& Co., 2007; Pisano, 2006).

The nature of the biotechnology industry makes it intrinsically linked to entrepreneurship, and consequently high-risk and strongly reliant on the innovation process (Patzelt, 2012). The mechanism of this industry usually depends on dedicated biotechnology firms (DBF) undertaking research activities and, when they have developed potential products, services, or processes, either licensing them out to manufacturing companies like pharmaceutical companies (who have powerful marketing arms), or manufacturing the products themselves. This mechanism currently is lacking in Saudi, as entrepreneurship in the universities is impeded by the culture and by the regulations. The weakness of research infrastructure and connection between industry and academia has also magnified this situation. 
Developing the biotechnology industry requires large public investment and high quality institutions to close the gap in technology (Niosi \& Reid, 2007). The industry is heavily dependent on public research centres. The establishment of new DBF spinoffs are a result of the collective efforts and collaborative work between venture capital firms, public funds, and research universities (Niosi, 2011b).

Both public and private institutions have a key role in developing dynamic innovation systems such as the biotechnological innovation systems. The institutional fabric works as the driving force that fosters commercial biotechnology (Niosi, 2011b). According to Freeman (1987) innovation systems are "The network of institutions in the public and private sector whose activities and interactions initiate, import, modify and diffuse new technologies", where the concept of an institution includes customs, norms, beliefs and values; organizations such as public research centres, universities and firms; laws, policies, and organizational routines (Nelson, 2005). This means that there are four kinds of institutions that constitute the innovation systems: organizations, routines, norms and customs, and laws and policies. The elements of these systems are under a continuous adaptation and change process. For example, 2030 years ago universities had no offices for technology transfer and intellectual property. Venture capitalists readily adapt to changing environments and contexts; many of those who previously worked in information technology started in the 1980s to work in biotechnology (Niosi, 2011b). Venture capital is one of the most important institutions playing a crucial role in biotechnology development; for example, the US has achieved leading status in biotechnology by virtue of a strong venture capitalist base (Ang, 2006; Niosi, 2011b). The implication of this work is that if any single element of the institutions listed above are absent, or insufficiently developed, then the system of innovation will fail to operate effectively and fail to achieve the KSA government intention to diversify the manufacturing base.

Most of the OECD countries have lagged behind US biotechnology because their institutions have not been set up to deal with such fast-changing and complex intensive knowledge-based sectors such as biotechnology (David, 1993, 1994; Henrekson \& Rosenberg, 2001; Orsenigo, 2001). According to Prevezer (2001) the US has some features that have enabled it to be ahead of European countries, such as: 
- More academic research financing sources in the US.

- The American faculty can build their start-ups and keep their academic posts.

- US start-ups have been focused on enterprises where commercial opportunity has high potential and better chances.

- The availability and conditions of financing tools and managerial expertise are better in the US.

- DBFs can find easy access to cooperation and alliances with big pharmaceutical companies.

In comparison with European and Asian countries, venture capitalists in the US have experienced better environments and situations by virtue of having more funding and more exit avenues (Haar, 2001). According to Bartholomew (1997), in the biotechnology institutional structure there are eight aspects or issues that should be respected as they effectively influence the stocks and flows of knowledge in research institutions and industry:

- Level and models of basic research national funding

- Networking with foreign research centres

- Market of venture capital

- Personnel mobility between academia and industry

- Commercial orientation of research centres

- Tradition of scientific education

- Technological accumulation in related sectors

- The government role in technology diffusion.

Biotechnology is a wide-ranging industry, thus presenting governments with the need to select which areas they are going to develop. This selection is determined with reference to the choices of the various actors too, be they pharmaceutical companies, DBFs, venture capitalists, universities etc. All these have their own priorities and options, so all the factors that might affect the selection process must be aligned and taken into account when government is working on developing a commercial biotechnology industry (Niosi, 2011b). This dynamic is compatible with the concept of system cohesion. In contrast, the Saudi system has no connection or collaboration 
among its sub-systems, which is reflected in the lack of cohesion and consequently the lack of ability to adapt to the changing environment.

\subsubsection{Challenges facing developing LS innovation system}

The mental models of the system members (for example a university faculty) could affect the adaptation process and resist any kind of proposed change in organization, routines and structure given that their pre-perception about their role is restricted to teaching and publishing papers only, and that would prevent any attempt at creating spin-offs, patenting technology or even licensing it.

The nature of this industry is characterized by risk, uncertainty and medium to longterm returns, and therefore it is to be expected that the government and private sector would invest cautiously. Policies in such innovation systems are evolutionary, complex, and path dependent, and require long period to apply, assess and modify in response to their effects (Niosi, 2011b). The lack of a collaborative culture is one of the potential challenges that would block the link between academia and industry (Sansom, 2004).

In Europe, where academics are civil servants/University employees there is no personnel mobility between academia and industry normally. These issues constitute institutional impediments and have a negative impact on probable linkages (Niosi, 2011b). However, entrepreneurship amongst life sciences faculty is widely recommended and encouraged in Europe as well as in developed countries (Haeussler \& Colyvas, 2009). With support from venture capital firms, start-up enterprises (whether they be University spin outs or not) are found to usually cluster around leading research university campuses (Finegold et al., 2004). It will be shown in subsequent chapters that locating close to University enhances proximity benefits for current and future products, and that the role of regulations in facilitating the whole entrepreneurial ecosystem is key in enabling knowledge transfer within the system.

\subsubsection{Fundamental Forces That Shape the LS Industry}

The main fundamental forces that constitute the biotechnology innovation systems are: universities, star scientists, venture capital, angel organizations, patenting offices, regulatory offices, pharmaceutical companies, public laboratories, consumers, 
farmers, mining corporations, health departments and science parks (Niosi, 2011b). In the US the public research institutes and small firms conduct basic research activities, and the small firms network within and across regions with all actors to form robust national linkages (Owen-Smith et al 2002).

In its plan to develop its biotechnology industry, Singapore adopted a foreign direct investment approach. It managed to attract leading multinational company investment through developing policies armed with financial incentives, world-class infrastructure, a high skilled workforce and business regulatory friendly environment. This industry was mainly driven by government participation and investment, once the need to develop world-class capabilities over the entire biotechnology value chain had been recognised; this ranged from basic research and clinical trials to manufacturing facilities (Finegold et al., 2004).

However, Kim et al. (2007) emphasize the role of the university as a key actor in innovation systems; for example, developing programmes in the life science and biotechnology fields helped the universities in Japan to create joint collaborations and ventures with industry (Kato \& Odagiri, 2012).

\subsection{Summary}

Saudi Arabia has a rentier economy that relies massively on oil income; this distorted economic structure faces many challenges, as the government has started to experience difficulties in compensating for fluctuations of the oil price.

Saudi Arabia has a growing LS sector with large potential revenue generation and fast-expanding governmental expenditure, particularly those relating to health and environmental issues; this situation has created extra financial burden for the government, whose finances are becoming distressed due to the growing expenditure. Saudi people also have a weak entrepreneurial culture that is not supported by benign regulations. Across the entire landscape of industries and business practices, the nature of enterprise also lacks innovation and the research infrastructure does not enable strong innovative activities. 
Life Science is one of the most complicated industries and requires substantial investment and ample time to provide returns on the investment made, which hints at the need to create a proximal, connected environment.

The next chapter, Chapter 6, will present the findings concerning the perceived current state of the Saudi system. Some intervention strategies that have the potential to be effective in addressing all the aforementioned challenges will be presented in Chapter (9). 


\section{Findings 1}

\subsection{Introduction}

The objective of this chapter is to answer the first question of the three research questions of this study which are stated below:

What knowledge economy (KE) framework could be developed in the life science sector (LS) that can practically deliver efficient outputs in the KSA?

This question will be addressed using the following three sub-questions:

\section{RQ1: What are the key requirements for developing KE in the LS in the KSA?}

RQ2: What are the challenges in developing KE in the LS in the KSA?

RQ3: What model of LS innovation system could fit the Saudi context?

To answer the first question, a grounded theory methodology has been applied, with the use of open coding and axial coding of the collected data. The data was collected through semi-structured, in-depth interviews of 31 highly qualified participants, constituting a sample of senior stakeholders and relevant people for the life science sector and knowledge economy.

The interview questions included five key questions and 20 secondary questions. The first four interviews (a pilot study) were conducted in Wales with two full professors in economic geography and regional development, a $\mathrm{PhD}$ in the life science and knowledge economy background, and one junior researcher in the area of knowledge economy; all of them purposively selected with respect to their background.

The other 27 participants were from the KSA and represent senior stakeholders in the Saudi life science sector and knowledge economy (see Table 4-3). All the interviews were conducted face to face and, to allow for more focus on discussion and building constructive mutual understanding without being distracted by any activity like 
writing; the interviews were conducted by using digital voice recording; later, the interviews were transcribed verbatim.

The open coding process resulted in 38 thematic codes, then an axial coding process was applied, resulting in developing five themes (categories). The themes and their properties represented the answer for question one, since they were the requirements that were proposed to develop the knowledge economy in the life science in the KSA.

\subsection{Open coding}

Through an open coding method, qualitative reduction for the data was performed through a constant comparison process for the incidents and concepts that emerged from the data. This interpretation process resulted in 38 codes. For more details about open coding process please see section 0 . The following table shows the open codes and their properties. 
Table 6-1: Open codes and their properties.

\begin{tabular}{|c|c|}
\hline Codes & Prop \\
\hline $\begin{array}{l}\text { The pool of } \\
\text { scientists is not so } \\
\text { big }\end{array}$ & $\begin{array}{l}\text { There is no critical mass of researchers that produce knowledge. The } \\
\text { discipline of life science is very difficult. There are no great motivations for } \\
\text { being involved in the research. }\end{array}$ \\
\hline $\begin{array}{l}\text { Shortage in } \\
\text { scientific } \\
\text { graduates }\end{array}$ & $\begin{array}{l}\text { The majority of students go to non-scientific disciplines. Even those go to } \\
\text { scientific disciplines usually work in different, irrelevant areas. }\end{array}$ \\
\hline $\begin{array}{l}\text { Lack of high } \\
\text { managerial and } \\
\text { technical skills }\end{array}$ & $\begin{array}{l}\text { There is a lack of highly qualified people (management and technicians) } \\
\text { whom can run highly sophisticated and complicated activities and businesses } \\
\text { like the life science industry. }\end{array}$ \\
\hline $\begin{array}{l}\text { Engaging more } \\
\text { medical doctors } \\
\text { (MD) in research }\end{array}$ & $\begin{array}{l}\text { The medical doctor is not interested in doing research. The number of medical } \\
\text { doctors in the health sector is not enough, so there is no space to engage in } \\
\text { research activities. }\end{array}$ \\
\hline $\begin{array}{l}\text { Exposing to } \\
\text { international } \\
\text { effects }\end{array}$ & $\begin{array}{l}\text { Building human capabilities by studying in high quality international } \\
\text { education and research institutes. Transferring knowledge by sending students } \\
\text { for study and work after finishing study. Updating local capabilities. Brain } \\
\text { circulation. Recruiting high quality foreign scientists, and high skilled people } \\
\text { in management (especially lawyers, high expertise in entrepreneurship and } \\
\text { building new start-ups, etc.). }\end{array}$ \\
\hline $\begin{array}{l}\text { Raising education } \\
\text { system quality }\end{array}$ & $\begin{array}{l}\text { Matching the local education system with international criteria. Graduate } \\
\text { quality does not meet industry requirements. }\end{array}$ \\
\hline $\begin{array}{l}\text { The research } \\
\text { capabilities are } \\
\text { weak }\end{array}$ & $\begin{array}{l}\text { Research quality does not produce promising technologies. Researcher's } \\
\text { attitude is poor. Research environment is lacking in motivation. }\end{array}$ \\
\hline $\begin{array}{l}\text { Research } \\
\text { infrastructure is } \\
\text { weak }\end{array}$ & $\begin{array}{l}\text { Lack of a complete life science ecosystem. No motivating research } \\
\text { environment. Lack of high quality logistic and delivery system. }\end{array}$ \\
\hline $\begin{array}{l}\text { Developing strong } \\
\text { research centres }\end{array}$ & $\begin{array}{l}\text { Low quality of current research centres. Weak specialization in research } \\
\text { centres. }\end{array}$ \\
\hline $\begin{array}{l}\text { Developing } \\
\text { clusters based on } \\
\text { strengths and } \\
\text { priorities }\end{array}$ & $\begin{array}{l}\text { Identifying priorities and advantages. Developing strategy. Building an } \\
\text { ecosystem within the cluster. Bringing in capabilities. Proximity easing } \\
\text { collaboration and interaction. Investing in knowledge activities. }\end{array}$ \\
\hline $\begin{array}{l}\text { Developing } \\
\text { research } \\
\text { entrepreneurial } \\
\text { universities }\end{array}$ & $\begin{array}{l}\text { Transferring knowledge. Investing in knowledge-based enterprises. Spreading } \\
\text { entrepreneurship culture among students and faculties. }\end{array}$ \\
\hline
\end{tabular}




\begin{tabular}{|c|c|}
\hline $\begin{array}{l}\text { Transferring } \\
\text { foreign knowledge }\end{array}$ & $\begin{array}{l}\text { Making joint ventures with multinational organizations. Making acquisitions } \\
\text { of foreign companies by Saudi companies. Licensing technologies from } \\
\text { international sources (universities, firms). Making partnerships with foreign } \\
\text { parties. }\end{array}$ \\
\hline $\begin{array}{l}\text { Developing } \\
\text { collaboration with } \\
\text { foreign parties }\end{array}$ & $\begin{array}{l}\text { Building bridges with international partners for continuous learning and } \\
\text { training. Developing joint research programme, especially for solving local } \\
\text { problems. }\end{array}$ \\
\hline $\begin{array}{l}\text { Developing an } \\
\text { online platform to } \\
\text { ease collaboration }\end{array}$ & $\begin{array}{l}\text { Building an online platform that has all the information about all actors and } \\
\text { capabilities working together like an online cluster to facilitate collaboration. }\end{array}$ \\
\hline $\begin{array}{l}\text { Developing LS } \\
\text { specialties }\end{array}$ & Building professional clinical trials, vivarium, special labs, etc. \\
\hline $\begin{array}{l}\text { Lack of } \\
\text { academia/private } \\
\text { sector strong } \\
\text { connection }\end{array}$ & $\begin{array}{l}\text { Every actor is working alone. Lack of motivation to collaborate. No strategy } \\
\text { to make innovation. }\end{array}$ \\
\hline $\begin{array}{l}\text { Lack of innovation } \\
\text { strategy and } \\
\text { attitude in the PS }\end{array}$ & $\begin{array}{l}\text { Believing that they can produce wealth without innovation. Lack of awareness } \\
\text { of the importance and role of innovation to the growth and sustainability of } \\
\text { businesses. No capabilities. }\end{array}$ \\
\hline $\begin{array}{l}\text { Developing } \\
\text { Academia/PS } \\
\text { strong connections }\end{array}$ & $\begin{array}{l}\text { Doing joint research and collaboration for developing new products, accessing } \\
\text { supply chains, and solving private sector problems. Labour mobility. }\end{array}$ \\
\hline $\begin{array}{l}\text { Funding } \\
\text { programme for } \\
\text { academia/PS } \\
\text { collaboration }\end{array}$ & Creating incentives and support for collaboration. Mitigating the risk. \\
\hline $\begin{array}{l}\text { Involving all } \\
\text { actors in } \\
\text { developing policy }\end{array}$ & $\begin{array}{l}\text { Collective development of policy creating motive and commitments for all } \\
\text { actors. Respecting all and common interests by developing suitable and fitting } \\
\text { policy. Optimizing policy quality with respect to collective work and different } \\
\text { opinions. Supporting collaboration. }\end{array}$ \\
\hline $\begin{array}{l}\text { Transferring } \\
\text { foreign knowledge } \\
\text { for PS }\end{array}$ & $\begin{array}{l}\text { Making joint ventures to raise competences of private sector. Making } \\
\text { acquisitions to fill manageable knowledge gaps in the firm. Licensing } \\
\text { technologies for desired and interesting technologies that cost too much for } \\
\text { internal development. }\end{array}$ \\
\hline $\begin{array}{l}\text { Raising } \\
\text { institutions' } \\
\text { quality }\end{array}$ & $\begin{array}{l}\text { Developing the abilities to identify strategic options. Developing the abilities } \\
\text { to wisely manage and implement strategies. Increasing efficiency. }\end{array}$ \\
\hline $\begin{array}{l}\text { Building social } \\
\text { capital }\end{array}$ & Building collaboration, trust, and networking culture. \\
\hline
\end{tabular}




\begin{tabular}{|c|c|}
\hline $\begin{array}{l}\text { Building on } \\
\text { strengths and } \\
\text { priorities }\end{array}$ & $\begin{array}{l}\text { Developing strategies based on local priorities (for example: local health or } \\
\text { environmental problems, or special endowments and advantages in the } \\
\text { country) }\end{array}$ \\
\hline $\begin{array}{l}\text { Supporting } \\
\text { entrepreneurship } \\
\text { culture }\end{array}$ & $\begin{array}{l}\text { Spreading entrepreneurship culture especially among researchers, students and } \\
\text { investors. Motivating and providing incentives for entrepreneurship. } \\
\text { Developing regulations and policy that mitigate the impact of failure socially } \\
\text { and economically. }\end{array}$ \\
\hline $\begin{array}{l}\text { Government-led } \\
\text { project }\end{array}$ & $\begin{array}{l}\text { Government should kick-start the process by leading this project (knowledge- } \\
\text { based economy) and investing hugely in infrastructure and enterprises. } \\
\text { Government should coordinate, motivate and incentivise all actors to engage } \\
\text { and invest in innovation. }\end{array}$ \\
\hline $\begin{array}{l}\text { Developing } \\
\text { enabling policy for } \\
\mathrm{R} \& \mathrm{D} \text {, and spin-off }\end{array}$ & $\begin{array}{l}\text { Developing policy and regulations that ease, reward and encourage spin off } \\
\text { from universities and research centres. Developing policy that allows actors } \\
\text { to benefit from government funded research centres (like for example the } \\
\text { Bay-Dole Act). Providing grants for R\&D. }\end{array}$ \\
\hline $\begin{array}{l}\text { Improving } \\
\text { industry } \\
\text { infrastructure and } \\
\text { business } \\
\text { environment }\end{array}$ & $\begin{array}{l}\text { Developing easy ways of doing business, and a friendly business } \\
\text { environment. Raising competitiveness,. Building a high quality logistic } \\
\text { system. }\end{array}$ \\
\hline $\begin{array}{l}\text { Improving living } \\
\text { standards and } \\
\text { relaxing cultural } \\
\text { barriers }\end{array}$ & $\begin{array}{l}\text { Improving education, and health quality, and accessibility for foreign people. } \\
\text { Improving entertainment quality. } \\
\text { Relaxing gender segregation regulations as much as possible. }\end{array}$ \\
\hline $\begin{array}{l}\text { Developing } \\
\text { supportive staffing } \\
\text { regulations }\end{array}$ & $\begin{array}{l}\text { Providing a flexible salary structure that helps in attracting highly skilled } \\
\text { people. }\end{array}$ \\
\hline $\begin{array}{l}\text { Developing KE } \\
\text { coordinating } \\
\text { organization }\end{array}$ & $\begin{array}{l}\text { A knowledge economy entity to help in developing enabling policy, } \\
\text { developing strategies and motivating and organizing interactions among } \\
\text { actors. }\end{array}$ \\
\hline $\begin{array}{l}\text { Risk-averse } \\
\text { financial system }\end{array}$ & Weak support for entrepreneurs and small and medium enterprises. \\
\hline $\begin{array}{l}\text { Scarcity of venture } \\
\text { capitalists (VCs) }\end{array}$ & $\begin{array}{l}\text { The industry of venture capital is at its very beginning. Investing is in low-risk } \\
\text { assets. }\end{array}$ \\
\hline
\end{tabular}




\begin{tabular}{|l|l|}
\hline $\begin{array}{l}\text { More funding for } \\
\text { R\&D }\end{array}$ & $\begin{array}{l}\text { The level of funding for R\&D significantly under the developed country } \\
\text { average. }\end{array}$ \\
\hline $\begin{array}{l}\text { Developing seed } \\
\text { funds }\end{array}$ & $\begin{array}{l}\text { Helping to transfer knowledge and build start-ups, and to overcome "death } \\
\text { valley". }\end{array}$ \\
\hline $\begin{array}{l}\text { Developing VC } \\
\text { funds }\end{array}$ & $\begin{array}{l}\text { Investing in middle stage ventures with moderate risk. } \\
\text { efficiency }\end{array}$ \\
\hline $\begin{array}{l}\text { Raising funding } \\
\text { Improving quality of funding on the more promising enterprises and activities. } \\
\text { Improving funding mechanisms. }\end{array}$ \\
\hline $\begin{array}{l}\text { Developing angel } \\
\text { investors }\end{array}$ & $\begin{array}{l}\text { Angel investors can help start-ups to survive at the early stages after } \\
\text { establishment. }\end{array}$ \\
\hline
\end{tabular}

Source: The researcher

The above codes represent concepts that informants suggested as intervention strategies that would help in developing the life science sector in the KSA. These concepts are distributed over some different areas such as human capital, research infrastructure, policy, financial system, and private sector.

The following table presents the codes against different perspectives (overall perspective: 31 participants; academia perspective: 13 participants, government perspective: 11 participants, and private sector perspective: 7 participants). The percentages are based on the attached number for every perspective. The codes are arranged in descending order of the overall perspective values. 
Table 6-2: Code distributions against different perspectives; except for the overall perspective, the percentages are based on number of participants for each respective sector, not the whole sample saize. $N=$ number of participants. Overall $N=31$, Academia $N=13$, government $N=11$, priate sector $\mathrm{N}=7$. The codes ordered in ascending order for the overall perspective values.

\begin{tabular}{|c|c|c|c|c|}
\hline Open codes & $\begin{array}{l}\text { Overall } \\
\mathrm{N}(\%)\end{array}$ & $\begin{array}{l}\text { Academia } \\
\mathrm{N}(\%)\end{array}$ & $\begin{array}{c}\text { Governme } \\
\text { nt } \\
\mathrm{N}(\%)\end{array}$ & $\begin{array}{l}\text { Private } \\
\mathrm{N}(\%)\end{array}$ \\
\hline $\begin{array}{l}\text { Funding programme for academia/PS } \\
\text { collaboration }\end{array}$ & $17(55)$ & $9(70)$ & $4(36)$ & $4(57)$ \\
\hline $\begin{array}{l}\text { Developing clusters based on strengths and } \\
\text { priorities }\end{array}$ & $16(52)$ & $10(77)$ & $5(45)$ & $1(14)$ \\
\hline $\begin{array}{l}\text { Developing enabling policy for } \mathrm{R} \& \mathrm{D} \text {, and } \\
\text { spin-off }\end{array}$ & $16(52)$ & $9(70)$ & $6(55)$ & $1(14)$ \\
\hline Supporting entrepreneurship culture & $15(50)$ & $7(54)$ & $7(64)$ & $1(14)$ \\
\hline $\begin{array}{l}\text { Lack of innovation strategy and attitude in } \\
\text { the PS }\end{array}$ & $15(45)$ & $5(38)$ & $6(55)$ & $4(57)$ \\
\hline Developing government-led project & $14(45)$ & $7(54)$ & $4(36)$ & $3(42)$ \\
\hline Developing LS specialties & $14(45)$ & $8(62)$ & $3(27)$ & $3(42)$ \\
\hline Exposing to international effects & $13(42)$ & $5(38)$ & $7(64)$ & $1(14)$ \\
\hline Raising education system's quality & $13(42)$ & $7(54)$ & $3(27)$ & $3(42)$ \\
\hline Raising funding efficiency & $13(42)$ & $8(62)$ & $3(27)$ & $2(28)$ \\
\hline Building on strengths and priorities & $12(38)$ & $7(54)$ & $4(36)$ & $1(14)$ \\
\hline $\begin{array}{l}\text { Improving industry infrastructure and } \\
\text { business environment }\end{array}$ & $12(38)$ & $7(54)$ & $2(18)$ & $3(42)$ \\
\hline $\begin{array}{l}\text { Developing academia/PS strong } \\
\text { connections }\end{array}$ & $12(38)$ & $8(62)$ & $4(36)$ & 0 \\
\hline Transferring foreign knowledge for PS & $12(38)$ & $7(54)$ & $3(27)$ & $2(29)$ \\
\hline More funding for R\&D & $12(38)$ & $5(38)$ & $4(36)$ & $3(42)$ \\
\hline Lack of academia/private collaboration & $11(35)$ & $6(46)$ & $3(27)$ & $2(29)$ \\
\hline $\begin{array}{l}\text { Developing research entrepreneurial } \\
\text { universities }\end{array}$ & $11(35)$ & $10(77)$ & 1(9) & 0 \\
\hline Raising institutions' qualities & $11(35)$ & $5(38)$ & $4(36)$ & $2(29)$ \\
\hline Weak research infrastructure & $10(33)$ & $4(31)$ & $4(36)$ & $2(29)$ \\
\hline Developing seed funds & $10(33)$ & $7(54)$ & $3(27)$ & 0 \\
\hline Developing VC funds & $10(33)$ & $8(62)$ & $2(18)$ & 0 \\
\hline
\end{tabular}




\begin{tabular}{|c|c|c|c|c|}
\hline Developing strong research centres & $10(33)$ & $7(54)$ & $3(27)$ & 0 \\
\hline Risk-averse financial system & $8(26)$ & $3(23)$ & $5(45)$ & 0 \\
\hline $\begin{array}{l}\text { Lack of high managerial and technical } \\
\text { skills }\end{array}$ & $8(26)$ & $4(31)$ & $2(18)$ & $2(29)$ \\
\hline Transferring foreign knowledge & $8(26)$ & $4(31)$ & $1(9)$ & $3(42)$ \\
\hline Building social capital & $8(26)$ & $4(31)$ & $4(36)$ & 0 \\
\hline The pool of scientists is not so big & $7(23)$ & $4(31)$ & $3(27)$ & 0 \\
\hline $\begin{array}{l}\text { Developing collaboration with foreign } \\
\text { parties }\end{array}$ & $7(23)$ & $3(23)$ & $4(36)$ & 0 \\
\hline Scarcity of VCs & $6(20)$ & $3(23)$ & $3(27)$ & 0 \\
\hline Research capabilities are weak & $6(20)$ & $1(8)$ & $2(18)$ & $3(42)$ \\
\hline Involving all actors in developing policy & $6(20)$ & $5(38)$ & $1(9)$ & 0 \\
\hline Developing KE coordinating organization & $5(16)$ & $4(31)$ & $1(9)$ & 0 \\
\hline $\begin{array}{l}\text { Developing online platform to ease } \\
\text { collaboration }\end{array}$ & $5(16)$ & $3(23)$ & $1(9)$ & $1(14)$ \\
\hline $\begin{array}{l}\text { Improving living standards and relaxing } \\
\text { cultural barriers }\end{array}$ & $4(13)$ & $2(15)$ & $1(9)$ & $1(14)$ \\
\hline Developing supportive staffing regulations & $4(13)$ & $2(15)$ & $2(18)$ & 0 \\
\hline Shortage of scientific graduates & $2(6)$ & 0 & $1(9)$ & $1(14)$ \\
\hline Developing angel investors & $2(6)$ & $2(15)$ & 0 & 0 \\
\hline Engaging more medical doctors in research & $1(3)$ & 0 & $1(9)$ & 0 \\
\hline
\end{tabular}

\section{Source: The researcher.}

The above table presents indicators against every code to show its prevalence and support among informants' opinions, its degree of support would represent the level of importance. In the following section, five charts show different perspectives associated with the codes (overall perspective, comparative perspective for all three sectors together, academia perspective, government perspective, and private sector perspective). 


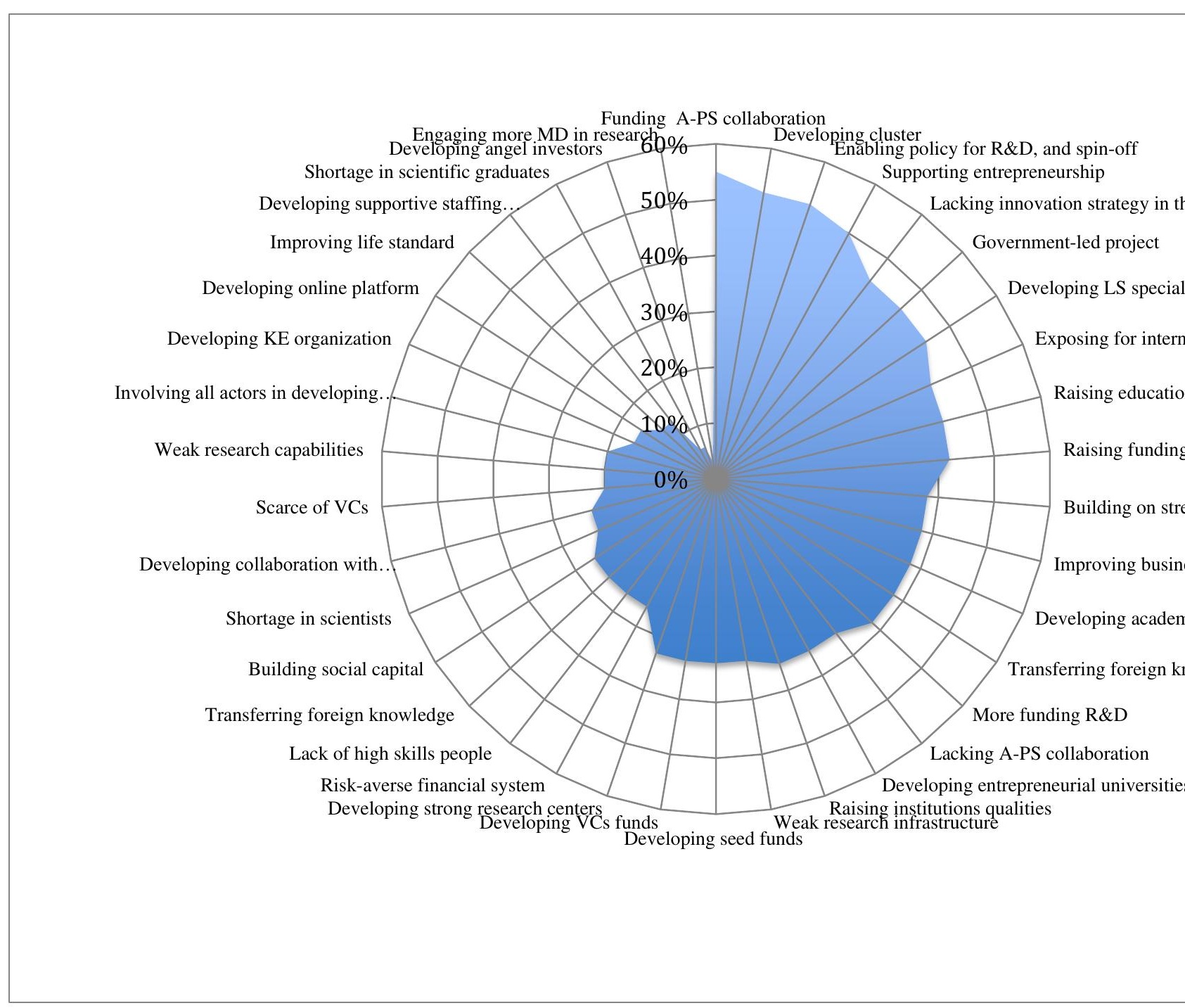

Figure 6-1: Overall perspective against codes (the numbers represent the percentage of informants in favour of th 


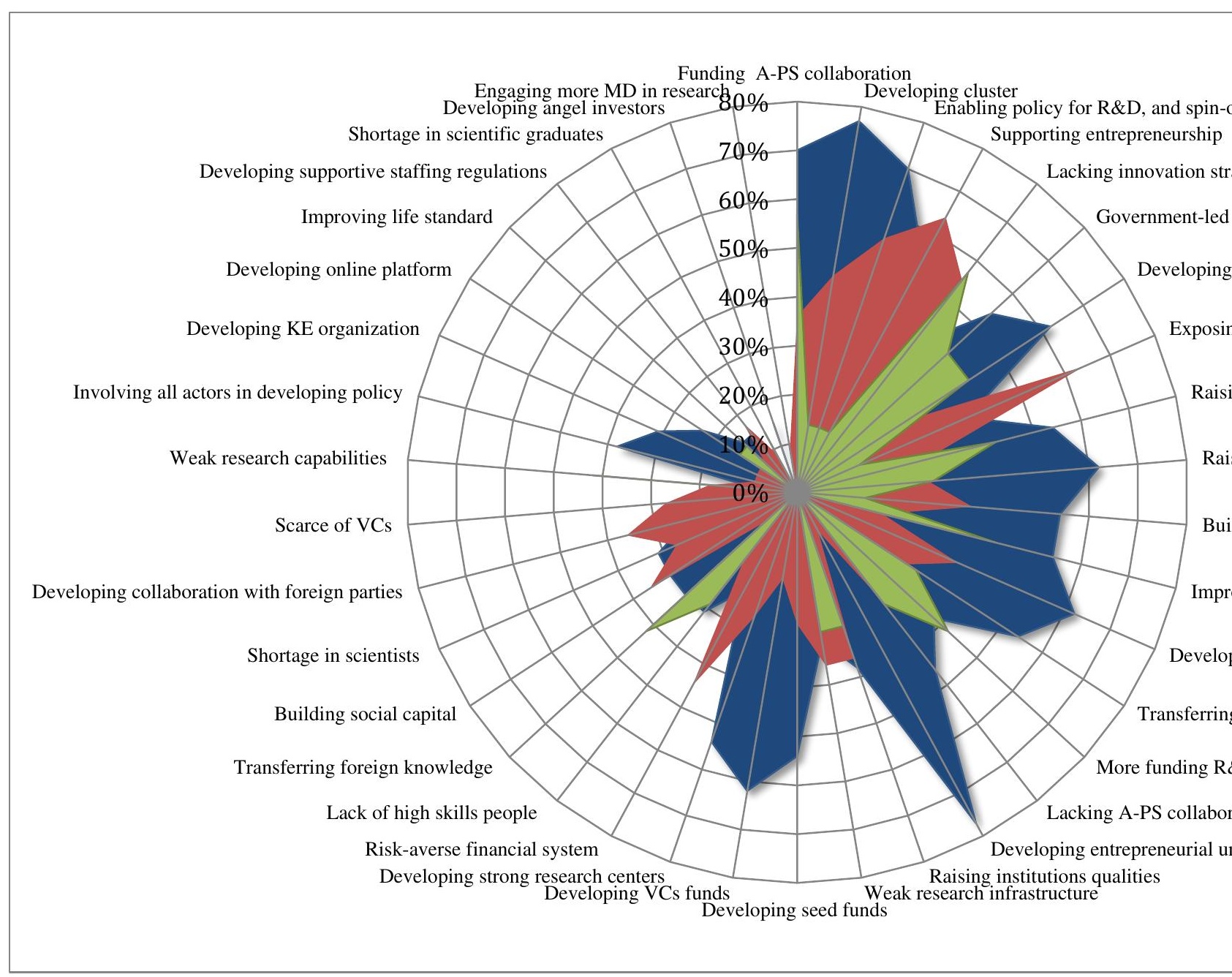

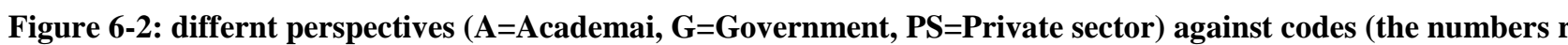
favour of the code). Source: The researcher. 


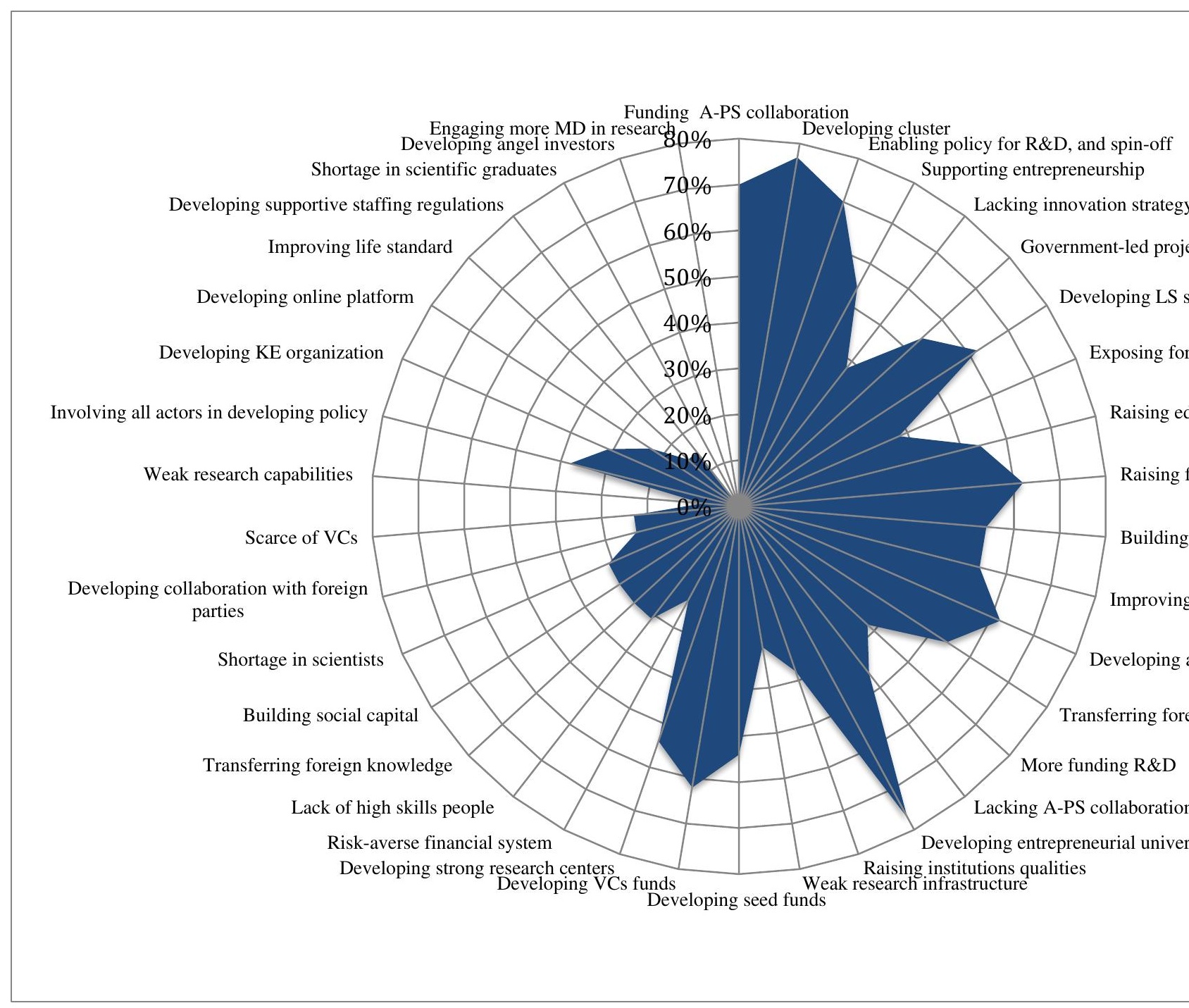

Figure 6-3: Figure 6 3: Academia perspective against codes (the number represents the percentage of inforn researcher. 


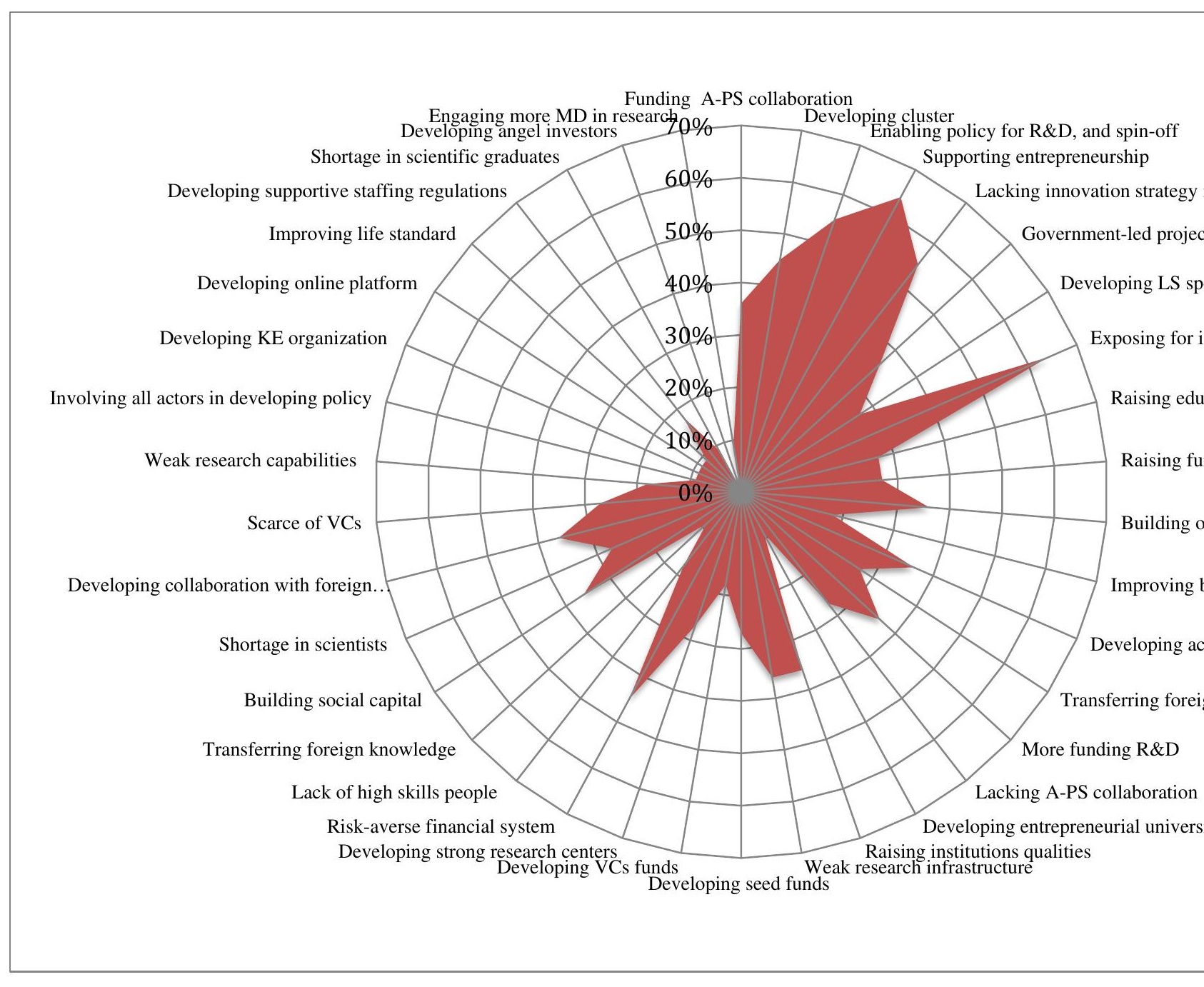

Figure 6-4: Governmemnt perspective against codes (the numbers represent the percentages of informants in fav 


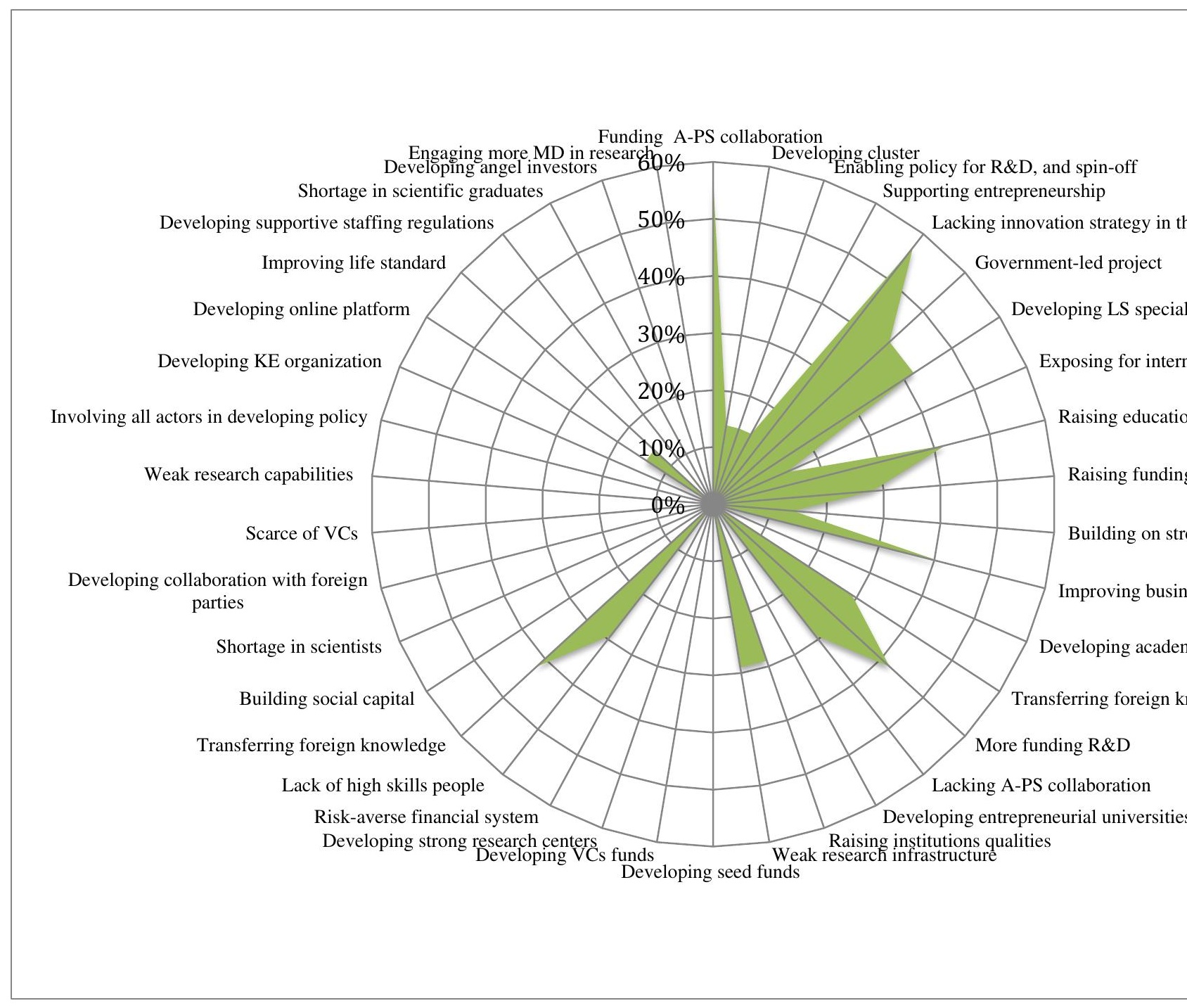

Figure 6-5: Private sector perspective against codes (the numbers represent the percentage of informants in favot 
The above figures (Figure 6-1, Figure 6-2, Figure 6-3, Figure 6-4, Figure 6-5) present different visual perspectives against all the codes, and this highlights the areas of interest that every actor (government, academia, private sector) see as relevant.

Notably, the participants' contribution from the private sector in identifying codes is modest, compared to the other participants from the other different sectors and despite the seniority of the private sector interviewees. This result seems to reflect the real and current private sector level of involvement, and supports the selection of "involving private sector" as a selective code, a selection which was undertaken using a structured approach.

\subsection{Axial Coding}

In the second stage an axial coding process was undertaken (for more details about axial coding process please see section 4.4.1.6.2 the research design chapter). Based on the paradigm shown below, a constant comparison process was undertaken with respect to the codes' properties to determine similarities and differences and to create groups; every group of open codes was categorized in upper level categories under an axial code (category or theme: used interchangeably); these sums of open codes represent the conditions and context, actions/strategies, and consequence of the phenomenon.

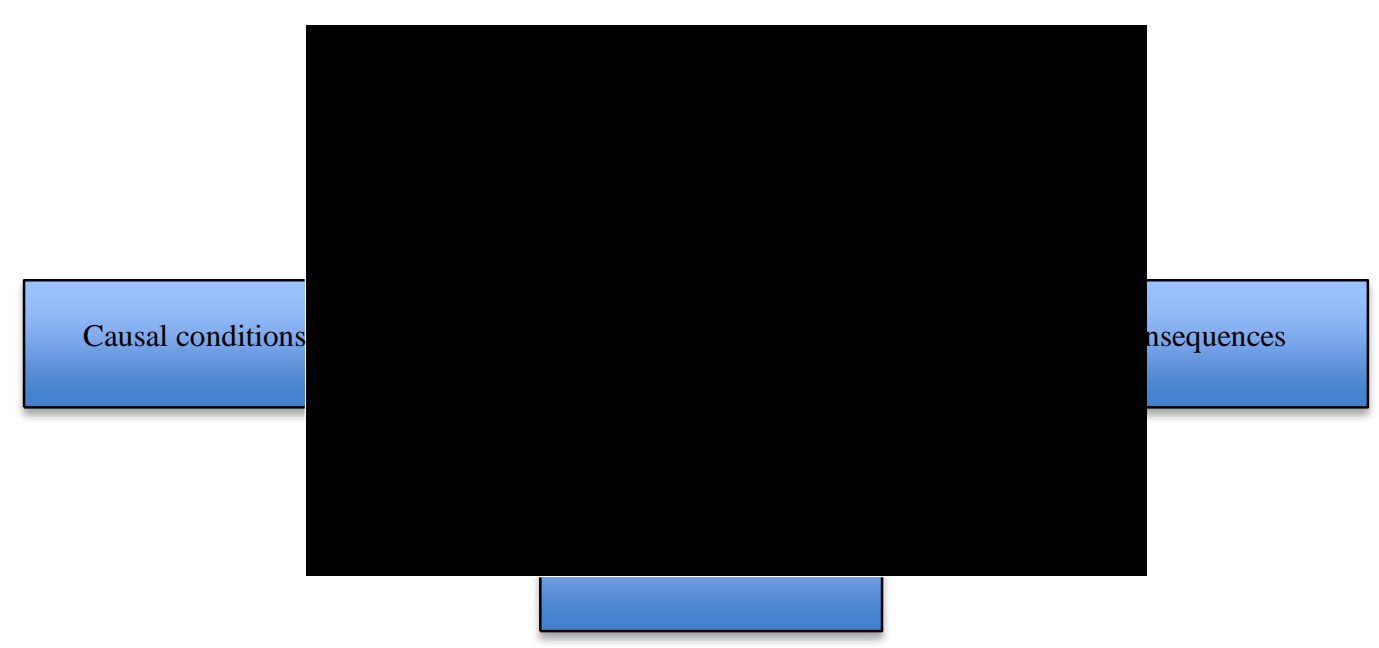

Figure 6-6: Axial coding paradigm. Source: Corbin \& Straus, 1990)

The following table shows axial codes and their properties (open codes). 
Table 6-3: Axial codes and their properties (open codes). Source: The researcher.

\begin{tabular}{|c|c|c|c|c|}
\hline Axial codes & Developing HC & $\begin{array}{l}\text { Developing World-class } \\
\text { research infrastructure }\end{array}$ & Involving (PS) & $\begin{array}{l}\text { Developi } \\
\text { regulation }\end{array}$ \\
\hline \multirow{10}{*}{ Open codes } & $\begin{array}{l}\text { The pool of scientists is not } \\
\text { so big }\end{array}$ & $\begin{array}{l}\text { The research capabilities are } \\
\text { weak }\end{array}$ & $\begin{array}{l}\text { Lacking Academia-PS strong } \\
\text { collaboration }\end{array}$ & $\begin{array}{l}\text { Raising inst } \\
\text { qualities }\end{array}$ \\
\hline & $\begin{array}{l}\text { Shortage of scientific } \\
\text { graduates }\end{array}$ & $\begin{array}{l}\text { Research infrastructure is } \\
\text { weak }\end{array}$ & $\begin{array}{l}\text { Lack of innovation strategy and } \\
\text { attitude in PS }\end{array}$ & Building so \\
\hline & $\begin{array}{l}\text { Lack of high managerial and } \\
\text { technical skills }\end{array}$ & $\begin{array}{l}\text { Developing strong research } \\
\text { centres }\end{array}$ & $\begin{array}{l}\text { Funding programme for academia- } \\
\text { PS collaboration }\end{array}$ & $\begin{array}{l}\text { Building on } \\
\text { priorities }\end{array}$ \\
\hline & $\begin{array}{l}\text { Engaging more medical } \\
\text { doctors in research }\end{array}$ & $\begin{array}{l}\text { Developing clusters based on } \\
\text { strengths and priorities }\end{array}$ & $\begin{array}{l}\text { Developing academia-PS strong } \\
\text { connections }\end{array}$ & $\begin{array}{l}\text { Supporting } \\
\text { entrepreneu }\end{array}$ \\
\hline & $\begin{array}{l}\text { Exposing to international } \\
\text { effects }\end{array}$ & $\begin{array}{l}\text { Developing research } \\
\text { entrepreneurial universities }\end{array}$ & $\begin{array}{l}\text { Involving all actors in developing } \\
\text { policy }\end{array}$ & Governmen \\
\hline & $\begin{array}{l}\text { Raising education system } \\
\text { quality }\end{array}$ & $\begin{array}{l}\text { Transferring foreign } \\
\text { knowledge }\end{array}$ & Transferring foreign knowledge & $\begin{array}{l}\text { Developing } \\
\text { for R\&D, a1 }\end{array}$ \\
\hline & & $\begin{array}{l}\text { Developing collaboration } \\
\text { with foreign parties }\end{array}$ & & $\begin{array}{l}\text { Improving } \\
\text { infrastructu } \\
\text { environmen }\end{array}$ \\
\hline & & $\begin{array}{l}\text { Developing online platform } \\
\text { to ease collaboration }\end{array}$ & & $\begin{array}{l}\text { Improving } 1 \\
\text { and relaxing } \\
\text { barriers }\end{array}$ \\
\hline & & Developing LS specialties & & $\begin{array}{l}\text { Developing } \\
\text { staffing reg }\end{array}$ \\
\hline & & & & $\begin{array}{l}\text { Developing } \\
\text { coordinatin }\end{array}$ \\
\hline
\end{tabular}




\subsection{Answer for Research Question 1}

\section{What are the key requirements for developing the LS sector in Saudi Arabia?}

This question is descriptive and explorative; it describes some of the current situational aspects of the life science sector in the KSA, and also explores the gaps which exist in the development of the life science sector. To answer this question, the data that was collected from the interview question (what are the key components for developing the LS sector in the KSA?) and the other interview questions where relevant (See the interview question Table 4-2), were open coded to result in 38 open codes, then those open codes underwent an axial coding process which came up with the following five variables as answers to the research question 1: developing human capital, developing World-Class infrastructure, involving PS, developing supportive regulations and enabling policy, and developing an entrepreneurial funding and financial system, which represented the key requirements for developing a $\mathrm{KE}$ in the LS in the KSA.

Through this section these five variables (requirements) and their properties are discussed.

\subsubsection{Developing Human Capital}

Under this category there are six codes that represent this category's properties:

\subsubsection{The pool of scientists is not so big (A 31\%, G 18\%, P 0\%)}

About $23 \%$ of participants describe the volume of scientists as insufficient and advocate increasing their number and scaling up research activities in the LS sector (G9, A13, G7, G3, A6, A8, A9) ${ }^{10}$. A participant who works as a vice president of a public research centre (PRC) and the ex-director of life science institute in the same PRC agreed that: "In terms of collaboration between researchers, I would say the majority of research is based on team work and multidiscipline model, and the pool of scientist is not so big (G9)"; a PhD manager from an Arabic country working in a Saudi university confirmed this point in a different way by proposing "if the KSA will

\footnotetext{
${ }^{10} \mathrm{G} 9=$ vice president for scientific research support in public research centre, $\mathrm{A} 13=\mathrm{PhD}$ director of technology \& prototyping centre, G7= MD researcher in tertiary public hospital, G3=director of biotechnology research centre, $\mathrm{A} 6=$ science park $\mathrm{CEO}, \mathrm{A} 8=$ manager of industry engagement office in private university, A9= Australian assistant professor in chemistry and biology in Saudi university.
} 
have ten universities like KAUST, you got it done, you produce quality PhDs, you have the ecosystem, and when those proposed universities coordinate with each other [the ecosystem] reaches a big scale (A8)" also he added, "In spite of that we're still a small university, we have about 135 faculty, some universities have 7000, 3000, and 4000 , but these very important efforts, if they would be duplicated in other locations, will lead to a large scale”. An assistant professor from Australia working in a Saudi university confirmed this idea: "And as you increase capacity so you grow your market, so without those things the market will not grow like this, so the market will remain small, and not much will be done in $L S$, and $L S$ will not be able to grow, all right, because capacity is limited, so you have to invest the resources to increase the capacity, once you increase the capacity you grow your [research-related] market, so that's what's missing here the most, in KSA (A9)"; he added also: "So, you need to duplicate this place [i.e., the university] twenty times at least, so, this is how you increase capacity, you really need to go to the foundation, that is the most important thing (A9)".

\subsubsection{Shortage of scientific graduates (A 0\%, G 9\%, P 14\%)}

This contextual condition was confirmed by more than $6 \%$ of the participants (G1, P6) ${ }^{11}$. A junior researcher from the Arabic gulf studying in Cardiff said, "The graduate numbers in scientific areas are quite small and at the same time the graduates mostly do not end up working in their area and apply for any job regardless of their subjects, they find more financial incentives in non-relevant areas (G1)"; a production manager in multinational pharmaceutical company working in Saudi Arabia confirms this view and stated "we have hundreds of colleges and professional institutes, but their graduates cannot run this kind of machines in the LS, the graduates do not match the requirements of this industry, so they should raise and improve their quality to fulfil the industry needs (P6)"

\footnotetext{
${ }^{11} \mathrm{G} 1=$ junior researcher in the knowledge economy from gulf Arabic country works in Cardiff, P6= production manager works in pharmaceutical multinational company in Saudi Arabia,
} 


\subsubsection{Lack of high managerial and technical skills (A 31\%, G 18\%, P 29\%)}

Managerial and technical skills availability is one of the contextual conditions of the Saudi LS sector, and participants defined such terms as the assistant jobs for research, and people running and managing organizations. $26 \%$ of the participants highlighted this contextual condition as important (P1, A13, G3, P6, A4, A6, G6, A12) ${ }^{12}$. A senior chief consultant in PRC in Saudi Arabia, who was CEO of a LS firm in the UK argues, "We need to develop highly skilled people in the management field, if there is a specific organization in charge of developing this ecosystem and managing the whole project of KE that would be good idea. Before we develop a cluster we need to think about how it will be managed and the mechanism that works. It is not a matter of infrastructure only (G6)". Another participant from an Arabic country working as a director of a VC owned by a Saudi university reinforced the issue of a lack of technical and managerial skills by saying, "for example if you're gonna bring a vaccine plant, you cannot hire Saudis that can run the plant today, because that would require so much training and so much time to make these folks ready to manage and operate vaccine plant, but what you need to start immediately is with a lot of foreign skilful workers, and that will be most impossible, because you need to be fulfil Saudization requirements (A4)". Another participant from an Arabic country working as a director of an innovation centre in a Saudi university mentioned that, "We have an IP licensing unit which includes a team specialized in negotiation; these are skills and careers we need to build in Saudi universities and PRCs, they know how to assess technology, they know how to negotiate a license, they know how to draft a license agreement (A12)"

\subsubsection{Engaging more medical doctors in research (A 0\%, G 9\%, P 0\%)}

A participant, the vice president of a public research centre (PRC) and the ex-director of life science institute in the same PRC (G9) ${ }^{13}$ suggested the strategy of integrating medical doctors was very important to meaningful progress in the KSA. The author

\footnotetext{
${ }^{12} \mathrm{P} 1=$ operation manager in venture capital company owned by $\mathrm{PRC}, \mathrm{A} 13=\mathrm{PhD}$, director of technology \& prototyping centre, G3= director of the national biotechnology centre, P6= production manager works in pharmaceutical multinational company in Saudi Arabia, A4= investment director in VC owned by Saudi university, A6= science park CEO, G6= MD, senior consultant in innovation and industrial development institute, $\mathrm{A} 12=$ science park director.

${ }^{13} \mathrm{G} 9=$ vice president for scientific research support in public research centre
} 
argues this idea is embedded in the majority of the opinions and suggestions of the participant, but would say this participant's statement was very specific, as he said, "first of all, developing the capabilities, choosing the distinctive researchers, training them on the used technologies, having MD-PhD: medical doctors in the scientific research, and because of the huge demand on medical doctors; we do not have many of these medical doctors researchers in the KSA, and this creates gap between the medical doctor and the researcher".

\subsubsection{Exposing for international effect (A 38\%, G 64\%, P 14\%)}

Another strategy concerning the development of human capital concerns international sources of influence and $42 \%$ of respondents expressed the importance of exposing relevant Saudi people to international influences and innovations to cultivate and raise their capabilities (A2, G9, G3, G4, A1, G7, A5, G10, A6, P1, A3, G1, G8) ${ }^{14}$. Here some examples of their arguments: a doctor from Swansea who used to work in a Saudi university said, "there is no need to restrict the teaching [staff] to Saudis, there is no need for not importing teachers and experts from any country (A1)". A director in biotechnology institute in Saudi Arabia expressed his view by saying: "I advocate post doctoral programs, the student when finishing his doctorate should move to other place to work as post doctorate for three or four years before coming back, to hone his skills (G3)". A operations manager in governmental venture capital confirms this concept by saying, "the main thing is developing high skilled people; and that should be through reforming the education system and raising its quality, secondly through the scholarship program and sending people abroad for specific mandate (P1)". These sentiments are easily defendable and understood if we connect them to the next concept concerning raising the quality of the KSA education system.

This strategy is very supported in the literature, for example, Singapore government implemented strategy to build human capital by recruiting high world-class scientists

\footnotetext{
${ }^{14}$ A2 = full professor in economic geography and regional development from Cardiff, G9= vice president for scientific research support in public research centre, G3= director of public biotechnology research centre, $\mathrm{G} 4=$ biotechnology incubator manager, $\mathrm{A} 1=\mathrm{PhD}$ in life science and knowledge economy from Swansea, G7= MD researcher in public tertiary hospital, A5= Dean of innovation and entrepreneurship institute in Saudi university, G10 $=$ a minister advisor, A6 $=$ science park CEO, P1= operation manager in venture capital company owned by PRC, A3= full professor in economic geography from Cardiff, G1=junior researcher in the knowledge economy from gulf Arabic country works in Cardiff, G8= planning and policy advisor in ministry of education.
} 
(some of them Nobel Prize Laureates), and sent students to attend high standard research institutions in the developed countries, and worked on raising the quality its education system as med-term strategy (Wong, 1999).

\subsubsection{Raising education system quality (A 46\%, G 18\%, P 42\%)}

A strategy to raise educational quality was supported by $42 \%$ of the participants (A3, A1, G4, A6, A8, P1, P6, P3, G11, A12, G6, A9, A7) ${ }^{15}$ who argue it should be implemented for human capital development. Examples of participants' words: a PhD holder from the Swansea LS ecosystem said, "I was teaching in the KSA - biology, English, and a bit of math, I wouldn't say ethics for study is high as I have seen in other countries. There is a culture that's allowing students to pass when they do not deserve it (A1)". Another professor from Cardiff added, "I think without a strong science base and very particular science base with very particular competences you are not going to go far (A3)'”. The CEO of a science park in a Saudi university argues that, "the technical colleges' outputs, and engineering colleges as well, graduate low quality students (A6)"

The next model shows the factors that influence the development of human capital, for developing human capital there is need for applying some strategies: raising education system quality, exposing for international effect (recruiting foreign scientists and skilled people, and sending Saudi people to study abroad), and engaging mode medical doctor in research. These strategies been suggested based on the shortage in quality and quantity of human resources.

\footnotetext{
${ }^{15} \mathrm{~A} 3=$ full professor in economic geography from Cardiff, $\mathrm{A} 1=\mathrm{PhD}$ in life science and knowledge economy from Swansea, G4= biotechnology incubator manager, A6= science park CEO, A8 $=\mathrm{PhD}$ works as industry engagement manager in Saudi private university, $\mathrm{P} 1=$ operation manager in venture capital company owned by PRC, P6= production manager works in pharmaceutical multinational company in Saudi Arabia, P3= business development manager in pharmaceutical company, G11= director assistant in a public fund for SMEs, A12= science park director, G6= MD, senior consultant in innovation and industrial development institute, A9= Australian assistant professor in chemistry and biology in Saudi university, A7= science park director in Saudi private university.
} 


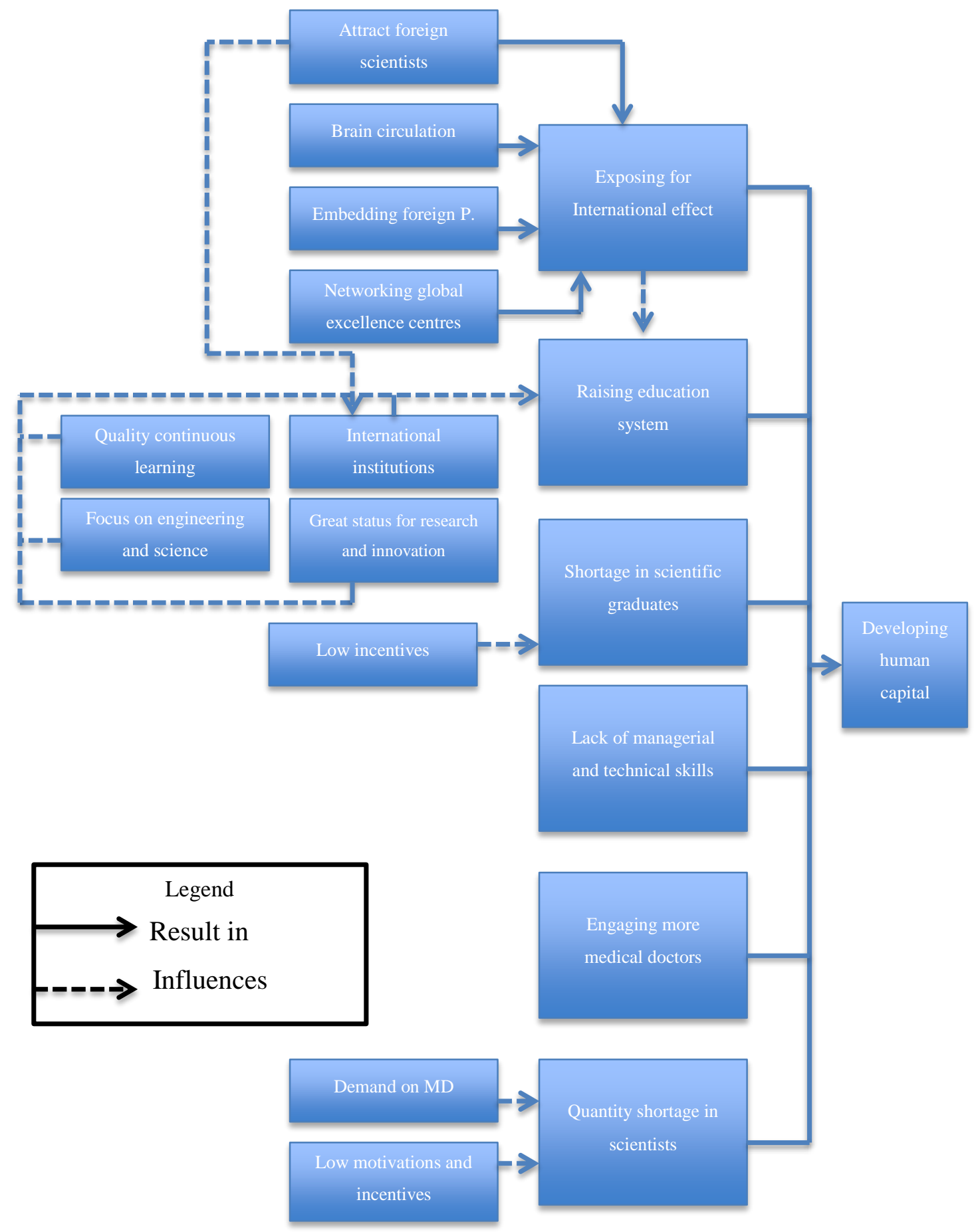

Figure 6-7: This model represents the "developing human capital" theme and its properties. Source: The researcher. 


\subsubsection{Summary}

This model explains the properties and enablers for human capital development resulting from the current shortage in the quantity and quality of high-qualified people to fulfil managerial, technicians, and researcher roles and a shortage of scientific graduates is also noted. It is found that the low level of incentives adversely influences the desire of students to take scientific subjects. The shortage in the number of scientists found is influenced by the lack of motivations and incentives as the graduates find more incentives to work in different and non-relevant areas. Also there is a substantial demand on medical doctors to work in hospitals, which negatively influences the engagement of medical doctors in research and consequently increases the shortage of scientists.

Strategies such as engaging more medical doctors in the research and innovation process is strongly detected amongst the informants and interventions to raise the quantity and quality of people exposed to international effects (through different mechanisms such as sending students abroad to attend high-quality education institutions and research centres and to stay there for more time after graduation) will increase their experience and the transfer knowledge to the KSA before they return to their home country to pursue their work (brain circulation). Also, the recruitment of high-calibre foreign 'innovation' staff in management and research, and other different areas is suggested, to deliver quality results and transfer knowledge. Another common informant agreement was the use of a strategy to improve the quality of the local education system, so that skills and attitude (outcomes) should match the industry requirements and result in greater industry-academia collaboration.

There are some factors - providing quality continuous learning; raising the local education institutes to international standard, in terms of having international faculties and also students and of applying international standards for education; focusing on engineering and science; giving great status to research and innovation - which have been found to positively influence the process of raising the quality of the education system. 


\subsubsection{Developing World-Class Research Infrastructure}

Under this category there are nine codes that describe the World Class research infrastructure and its properties.

\subsubsection{Research capabilities are weak (A 8\%, G 18\%, P 42\%)}

About $20 \%$ of the participants (G6, A6, P1, G9, P3, P4) asserted that research capabilities in the LS sector in Saudi Arabia are weak. A senior consultant in PRC in Saudi Arabia says: "as you know LS is a wide field and we cannot build capabilities and progress in all areas, so I think we need to search our advantages and look for unique things we have and build on (G6)". The CEO of a university science park also says, "at the level of the KSA, I would say there are two universities (King Fahd University for petroleum and minerals, and King Abdullah University for Science and Technology-KAUST) which can be considered as research universities as they have less students, more facilities and strengths in research capabilities (A6)". According to an operations manager in PRC-VC, "the other obstacle is the lack of capabilities and high skilled people (P1)". A vice president of PRC argues that, "Our policy is to develop our capabilities with national people; these are national institutes and labs that must be built on national capabilities (G9)". A business development manager in an LS firm says, "also I think there is a lack of incentives or motivations either from the university self-motivation [in the researcher], and lack of capabilities as well (P3)" Another participant working as a general manager in the support service sector in a pharmaceutical company stresses this point by saying, "Another point that hinders innovation and developing $L S$ is the lack of high quality researchers, and also low incentives for researching (P4)"

\subsubsection{Research infrastructure is weak (A 31\%, G 36\%, P 29\%)}

Regarding this point, about $33 \%$ of the participants confirmed the weak conditions of the research infrastructure (A1, G1, G3, G6, A6, A9, A12, G11, P2, P5). A PhD from the Swansea LS ecosystem says, "So, I think, in order to grow the capital, the human capital in Saudi, there'd have to be more incentives, whether it was higher economic pay, or promise of a better infrastructure to do research. (A1)". A director of biotechnology research centre in PRC confirms: "The most profitable and productive 
revenue is in the pharmaceutical sector, and I think currently, there is no one doing this in KSA, because the infrastructure and ecosystem are not yet ready (G3)". A senior consultant in PRC says: "I do believe that there is no problem with building infrastructure, we can do it very quickly, I think the problem is how to manage these infrastructure and run them, how to develop the required ecosystem (G6)". A dean of innovation and entrepreneurship in a Saudi university says, "I would say there are many obstacles to do that, because there is a lack of distinctive scientific research infrastructure, so some researchers try to collaborate with foreign researchers, especially those they were together with when studying abroad. For example in the summer they go there to have collaboration or get research leave for a specific period of time, because building high quality research infrastructure takes a long time and a big budget and the process goes through very complicated bureaucratic procedures (A5)". A CEO of a university Science Park says, "In general there is no complete ecosystem in the KSA, so we try to perform multi roles (A6)". He adds, "We need, besides the scholarship programme and post-doctoral programmes, to build a strong facilities and scientific research infrastructure in the universities". A CEO of pharmaceutical company argued in support and stated, "We do not have professional clinical trial centres for commercial drugs approval, which require specific standards and requirements. Academia does not have experience about the industry practices and requirements (P5)".

\subsubsection{Developing strong research centres (A 54\%, G 27\%, P 0\%)}

More than $33 \%$ of the interviewees emphasized the importance of research centre development to create a world-class research infrastructure (A1, A3, A11, A12, G7, G3, G4, A6, A9, A8). An incubator manager from Saudi Arabia mentioned, "We need to develop high quality research centres (G4)". A director of a biotechnology centre confirms the need for "...enhancing the quality of research centres (G3)". A PhD from Swansea argues, "but you need to build strong research centres equipped with skilled people and high standard labs (A1)". A CEO of a university science park confirms, "We need, besides the scholarship programme and post-doctoral programmes, to build a strong facilities and scientific infrastructure in the universities (A6)". 
This suggestion for developing strong research centre is similar to what Singaporean government has done, it invested significantly in building the research infrastructure (Finegold, Wong \& Cheah, 2004).

\subsubsection{Developing cluster based on strengths and priorities (A 77\%, G 45\%, $P$ $14 \%)$}

Approximately 52\% of participants (A1, A2, A3, G4, A5, A6, A9, A10, A7, G9, G10, A13, A12, P1, A8, G6) confirmed the benefits of developing a cluster which supports the positive view held by academic authors. A CEO of a university science park says, "Actually our role here is to facilitate knowledge transfer and running the cluster, so we try to attract foreign firms with their research centres into this cluster and local firms, and we will try to create the suitable environment that helps in developing collaboration and eases spill over, so I am an advocate of cluster one hundred per cent (A6)". Another director for university science park argues that, "it is very important, but you need to be careful, you should bring in the ones who collaborate with each other not compete against each other; if you bring them from the same sector in the same area they might will not collaborate but compete, so you need to design the cluster very carefully (A7)". An Australian assistant professor in a Saudi university confirms the benefits of working in cluster as a supportive environment: "Because you cannot work in a vacuum, if I am a biotechnology company I would like multiple LS companies around me where I can collaborate with them, buy things from them, interchange with them, so kind of nice to have them in fairly close atmosphere, and for people to know each other more and more, I think it is very nice idea I like it a lot." (A9).

According to (Porter, 2000) cluster is important for knowledge spill over and collaboration among cluster members, it is works like regional innovation ecosystem.

\subsubsection{Developing research entrepreneurial universities (A 77\%, G 9\%, P 0\%)}

More than $35 \%$ of the participants see this strategy as being very important (A1, A2, A4, G6, A5, A6, A7, A8, A9, A10, A11). A doctor in the knowledge economy in the life science sector from Swansea's life science ecosystem supported this strategy by giving the example of Swansea university: "So, the European Union funding through 
the European Regional Development fund, fund the Welsh European Funding Office to run a programme called A 4 B. Academics for Business, so, essentially they had projects within the university that supported businesses and were mostly helping with knowledge transfer, helping with, you know, discovering new things, to transmit that knowledge to businesses, helping them to commercialise it (A1)"; he added: "so the whole mechanism is to support the early stages $R \& D$ in the university and spinning out into a company, I have no idea how easy it is to start a company in KSA, I do not know how to license technology from a university and have no idea what the venture capital or grant funding situation, but if all were in place and increased I am sure that would assist in spin off culture, but again I do not know how much money would be devoted". A full professor from Cardiff confirms that idea by saying, "so okay you create knowledge in research labs and university but you also you create knowledge by having links to consumers (A2)" he also added, "one is taught in university in terms of relation to entrepreneurship, enterprise and what the university actually does itself, so in other words how created knowledge is transferred, that kind of stuff"; another full professor argues, "think about the US, they probably had some successes in high tech in silicon valley because of Stanford University, but what they were doing all that time, they were educated to become entrepreneurs, making entrepreneurship to be a good thing, making innovation to be a good thing (A3)”. He added, “you know this is where the key human capital is generated and this is where the key human capital lives within the university, because they spin out companies. You need a university to generate spin out and these spun outs need to be run by people [who are] entrepreneurs." A senior consultant in PRC in Saudi Arabia argues, "here actually we have a problem where the researchers especially in the universities don't think about the market; they do research for research's sake, they do not have an entrepreneurial attitude, they do not have incentives to make spin-offs because the ownership will go to the university (G6)"; A CEO of a university science park argues: "the solution is like what is going on in Malaysia, they developed about 10 research universities with limited numbers of students (from 10,000 up to 20,000), this low load of students give the faculty the time to be involved seriously in the research process. Also even the other universities with high student number can be entrepreneurial universities and be engaged in the research process (A6)" 
Developing entrepreneurial universities become one of the most important element for the innovation system, this concept enables universities to doing entrepreneurship and commercialising knowledge (Leydesdorff, 1995; Leydesdorff \& Etzkowitz, 1998).

\subsubsection{Transferring foreign knowledge (A 31\%, G 9\%, P 42\%)}

For the purpose of developing a world-class research infrastructure, which implies 'transferring foreign knowledge', $26 \%$ of the participants see that making JV, acquisition, and licensing technologies are helpful strategies (G1, A6, A8, A9, P1, A12, P3, P5). A junior researcher in the knowledge economy field from Cardiff University says, "consultation no longer works, so I guess memoranda of understanding, joint programmes (for example to do research on local problems or local advantages with the sharing of local researchers); exchanging students. You need to look for mutual benefits, so the others can be attracted to work with you (G1)". A CEO of a university science park recommends that, "I think it is very helpful, but before we go there and have such kinds of investment, we should consider two determinants; first, it should be in the area of our interest; second, our researcher must have the capacity to assimilate that technology otherwise the benefits would not be sustainable, because we are not looking for profit only (A6)". Also a manager of an industry engagement department in a Saudi private university argues: "I think there are many ways, but in my opinion licensing from foreign entities as step one, and I think in some cases building start-ups around small products. It is three things that should be done: licensing technologies, building start-ups, entering into joint ventures; all these approaches work well (A8)" An Australian professor in a Saudi private university confirms the same idea and proposes this strategy to shorten development time and save efforts and resources: "I think it is the best we can do right now, I think it is the fastest way; no need to reinvent the wheel, no need to go to build the entire technology yourself, everybody around the world, these companies are mobile they can move around, you just need to give them incentives (A9)"

\subsubsection{Developing collaboration with foreign parties (A $15 \%, G 36 \%, P 0 \%)$}

The academic and government community supports the development of international collaboration and localisation of international businesses which is also a view 
supported in the literature. This strategy was proposed by about $23 \%$ of participants (A2, G1, G2, G3, G6, A12). A full professor from Cardiff says, "yeah possibly, but what I mean really by connecting is by people, it is easy to move the know-how, it easy to move the brains and easy to move the know-how into the brains, and that starts the way to think about it really, so by connection I mean people moving (A2)"; he means collaboration with foreign individuals not necessary with firms or universities. An Arabic Gulf junior researcher from Cardiff argues, "consultation no longer works, so I guess memoranda of understanding, joint programmes (for example to do research on local problems or local advantages with the sharing of local researchers); exchanging students. You need to look for mutual benefits, so the others can be attracted to work with you (G1)". A senior consultant of Saudi PRC says, "The researchers in the universities know who to target, for example some researchers get scientific leave for three months and go there for training, if the professors from both countries exchange their students and have a collaboration this will have a good effect (G6)", he added, "Also I recommend to develop joint ventures not only with foreign companies, but between Saudi universities and their counterparts especially western ones to develop specific projects, by this our researchers and staff can enhance their capabilities and get benefit from the foreign experience". A deputy director in Saudi LS institute says, "so to mitigate this obstacle we established a company (VC) to invest in the produced knowledge from our labs and also to develop partnership with foreign partners (G2)"

This concept is widely supported by literature. For example, Indian companies practiced this strategy and managed to transfer foreign knowledge and enhance their capabilities (Capron et al., 1998; Gubbi et al., 2010; Krishnan \& Jha, 2011). 


\subsubsection{Developing an online platform (A 23\%, G 9\%, P 14\%)}

Approximately $16 \%$ of the participants suggested developing an online platform containing all the data about Saudi research capabilities and information which could help in easing collaboration (G6, A6, A10, A13, P5). A senior consultant suggests, "Also we need a database and online platform that includes all information about research society (G6)".

A director of a prototype centre says, "you cannot find a common website that give you the right information about the existing devices and tools in the KSA, later, our university developed system (database) that enable you to know everything about the existing devices and tools in the university. Also we do not know the research capabilities in the KSA, so, we need to develop a common database that provides information about all research capabilities, human resources, and devices in the KSA, that helps, encourages and facilitates the collaboration and contact among our research society; sometimes you are looking for a specific thing abroad, but without knowing it is available locally (A13)". A CEO of a university science park argues, "actually this is one of our problems: there is not a specific organization that we can feedback to or collaborate with, to develop suitable regulations and policy; also this organization should build a database that could help research within industry (A6)". A dean of applied medical science and director of an innovation centre in personalized medicine argues, "I think if there is a database centre about the researchers and the capabilities that exist in the KSA, I think this might help in facilitating the collaboration, and avoid replication (A10)" A CEO of pharmaceutical company says, "We need to develop a data centre about human capabilities or those related to devices and labs and to facilitate the benefits provided by these capabilities, and collaboration (P5)".

South Korea applied a similar strategy by building an organisation as a conduit to link different actors together and facilitate collaboration between them (Lee et al., 2010). 


\subsubsection{Developing LS specialties (A 62\%, G 27\%, P 42\%)}

The development of LS specialities for differentiation and a distinct LS offering is a sub-category and refers to clinical trials and other service providers. More than $45 \%$ of the participants mentioned this by speaking about a complete ecosystem, or by embedding it under infrastructure (A1, P1, A11, A10, P5, G3, A12, A6, A13, P7, G8, G6, A7, A8) A director of a biotechnology research centre complains, "we have problems in logistics - things like for example delivering chemicals can take a few months while in the USA they are delivered overnight (G3)". An Australian assistant professor working in a Saudi university also confirms, "so for the LS, you need infrastructure to support $L S$, and that infrastructure is much better to come from private sector rather than universities, so all I say the best thing to do is you have to establish service providing companies in KSA, companies that work with genotyping, phenotype of various diseases, bio-banking of samples producing the key reagents, some of the key techniques that are used by everybody, so you need those heavy service providers companies in the country (A9)". A CEO of a big Saudi pharmaceutical company also supports this proposal: "we do not have infrastructure for research, for example we send one milligram of EPO (protein) to labs in Germany for analysis, we put this amount in $20 \mathrm{Kg}$ of dry ice to keep it viable; we need also clinical trial centres", he added, "and also developing all ecosystem elements, clinical trials, non-clinical trial centres, capabilities, etc. (P5)"

These kinds of activities are a very significant part of the entire innovation ecosystem (Miles et al., 1995; Muller, 1999). For example, in Singapore the government built all these units to provide services to innovation system members (Kong, 2003). 
Figure 6-8: This model shows "developing world-class research infrastructure" theme and its properties. Source: the researcher

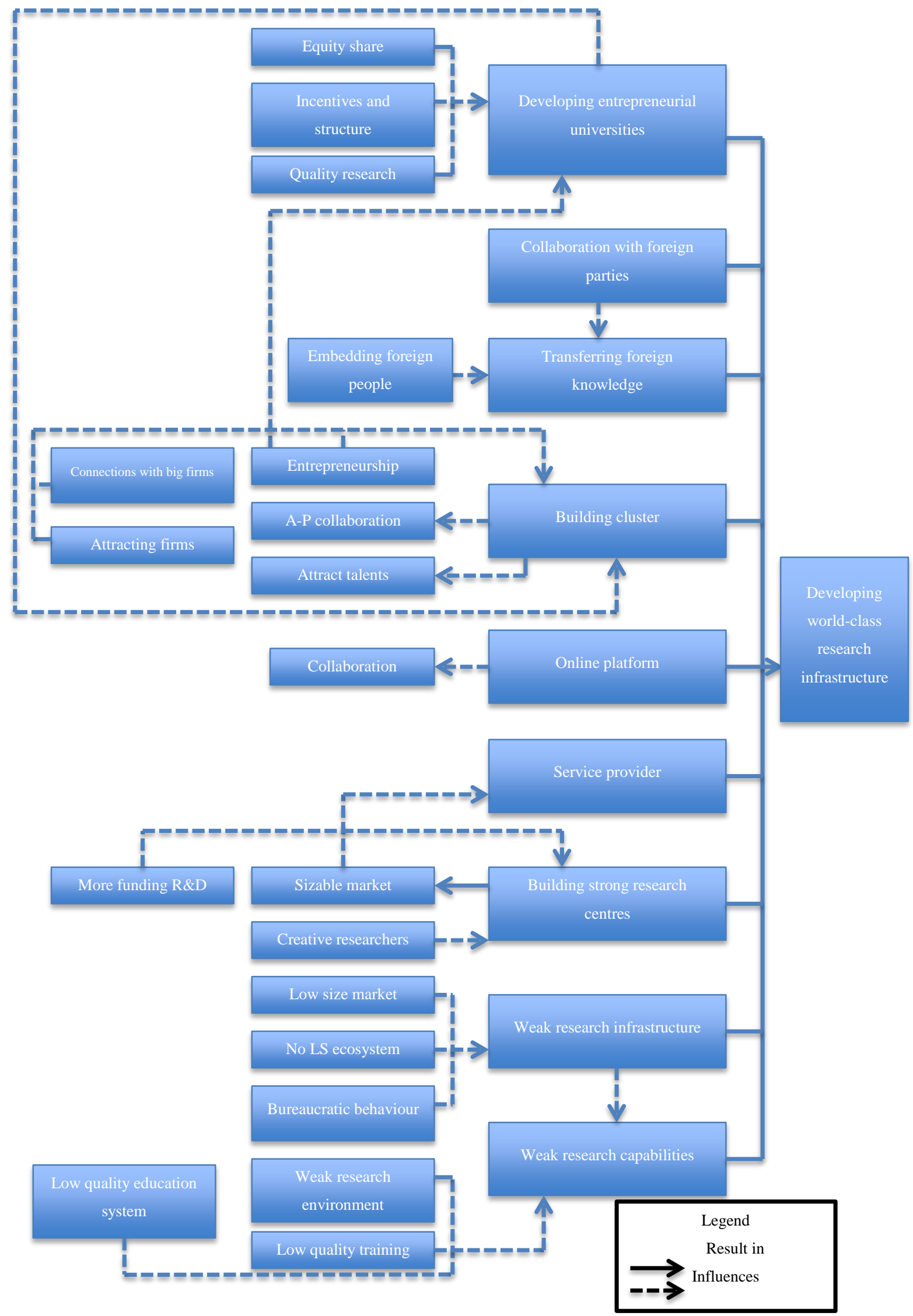




\subsubsection{Summary}

The research infrastructure in life science in the KSA is, from these findings, suffering from significant perceived weaknesses and lack of effective research capabilities. There are some factors that influence this weakness of research infrastructure: there is no complete LS ecosystem - for example there are no professional clinical trials; there are no special labs for making special tests; there are no service providers for delivering special chemical materials and those materials requested from abroad take one or two months for delivery; the logistics system is also weak. Another factor is the size of the KSA market (a market demand for such services), as there is no critical mass of people that are involved in research.

The last factor that influences the weakness of research infrastructure is bureaucratic behaviour, in terms of funding procedures, the decision-making process, identifying priorities and strategies, and facilitating collaboration.

The weak research capabilities were found to be influenced by three factors: weak research environment, low quality training for researchers and technical and managerial staff, and the low quality of the education system where the output does not match the market needs and criteria.

For developing world-class research infrastructure as a key requirement for developing a knowledge economy in the life science sector, some strategies were proposed by the interviewees:

1) Building strong research centres in terms of equipment and human capabilities. To develop strong research centres there is a need for more funding for $R \& D$, so that these research centres will then lead to increasing the size of the research-related market and consequently will influence positively the research infrastructure. Also there is a need for recruiting creative researchers to build strong research centres.

2) Another variable for developing world-class research infrastructure is service providers: the growth of the market helps in attracting service providers. 
3) Building an online platform containing a database about all relevant information would improve collaboration and contribute to developing a world-class research infrastructure.

4) Developing research and entrepreneurial universities helps in transferring and investing in knowledge and spin offs, and creates connections beyond their boundaries with industry, society and government. There are some variables helping to influence the development of entrepreneurial universities: developing incentives and supportive structures that encourage faculties and students to engage in research, innovation and enterprise; doing quality research that must be orientated to the market; supporting entrepreneurship and providing equity share for entrepreneurs.

5) Entrepreneurial universities were also found to positively influence the development of clusters, where clusters were found to be a key variable in developing world-class research infrastructure by providing proximity for actors to collaborate. and benefiting spill overs. However, such clusters must consider the local advantages and priorities as references for development strategies. For building clusters, there are three influencing factors: supporting entrepreneurship, connecting with big firms, and attracting firms. In turn, building clusters helps in developing academia-private sector collaboration, and attracting talents.

6) The last variable in developing world-class research infrastructure is the work of transferring foreign knowledge by different ways; joint ventures, acquisition, collaboration and partnership with universities and companies and key global actors in the field, FDI, or licensing technologies. It was found also that for transferring foreign knowledge it is important to embed foreign people and to develop good relations with them.

In the next section the theme: developing an entrepreneurial and efficient funding system is detailed. 


\subsubsection{Developing Entrepreneurial and Efficient Funding System}

The category concerning funding and entrepreneurship has seven codes that describe its properties. The Saudi financial system has been described as risk-averse and lacking in efficiency. The following seven open codes show properties of this phenomenon.

\subsubsection{Risk-averse financial system (A 23\%, G 45\%, P 0\%)}

More than $26 \%$ of the participants (G4, G5, G6, A6, G8, A12, A13, G11) see that there is a problem with the attitude of the financial system towards the sector and its development, which has been described as risk-averse. A university science park director argues, "We invest in physical assets, real estate but we do not invest in ideas, we have a risk-averse financial system, (A12)". He also added, "We have a problem with entrepreneurship on all levels; family level, and financial system level; all risk averse". A director of a technology advancement and prototyping centre says, "The investment in technology-based products is very risky, but our financial system does not support this orientation. We need to understand that the investment in risky ventures has a very high potential of return when successful, which means high-risk high profit (A13)". He added, "The stages start by basic research funding, seed funding, angel funding, and then venture capitalist, respectively. All funds here do not encourage risk taking". An assistant director of a SME government fund confirms that the risk-averse behaviour comes from both sides: the innovators (entrepreneurs) and the funding organizations, "we have troubles or obstacles to fund this kind of enterprises, we tried to contact the organizations that incubate and develop these innovative enterprises, but the people or entrepreneurs do not like to take risks and apply for loans, they want partnership; they are looking for equity not debt (G11)". A senior consultant in PRC confirms the core idea of a risk-averse funding system and stresses the early stage of technology transfer and setting up enterprise, "the problem with financial systems; they are looking for zero risk, they should develop VCs that enable them to fund the knowledge society, getting a share of the potential projects, and also providing seed funds. Some of them actually do, but unfortunately it is like individual initiatives, we need that the concept and the tools are realized and spread (G6)" 


\subsubsection{Scarcity of VCs (A 23\%, G 27\%, P 0\%)}

According to participants (20\%), VCs struggle, due to both low numbers and overly conservative behaviour in terms of investment risks with the KSA sector (G3, A4, A5, G9, A12, G11). A university science park director says, "There is a lot of money in the KSA, but the culture of venture investment does not exist" (A12). He added, "Yes we have (their own VC); we completed the cycle, but this VC firm cannot cover everything. There is a study done by KAUST which has been submitted to government about the venture capital market and the relevant regulations, but I do not have more information about it".

A Biotechnology centre director gave an example of a large governmental VC fund: "Also the government has a big VC called Tagniah, which invests in the inventions with high potential for developing products, and making acquisition as well (G3)”.

An assistant director in a government SME fund confirms that there is a lack of VCs and he criticises the existing ones: "We try to redesign our products to be close to the model of VC (G11)" he also added, "of course I advocate that (to develop VC model), but it is not easy. The country lacks real VC tools, all the existent VCs still struggle and they are very careful". A vice president of PRC sees that the problem is not in the number of VCs but in the scarcity of promising technologies that attract this investment: "to some extent there are fair numbers of VCs in all levels whether institutions, government, or the private sector. But it should be new ideas that deserve the investment (G9)"

\subsubsection{More funding for $R \& D(A 38 \%, G 36 \%, P 42 \%)$}

More than $38 \%$ of participants mentioned the need for money for R\&D, some of them stress the funding efficiency more than the availability of money (A1, A3, G3, A8, A10, P1, G9, A12, G8, G10, P4, P5). A ministerial advisor says, "The government spends more than $80 \%$ of the spent money on $R \& D$ and supporting entrepreneurship; it is at the top rank in the Arabic list in terms of rates with respect to GDP. But still the rate is low when compared to developed countries (G10)". A researcher in the Wales LS ecosystem confirms the importance of allocating more money for doing research, "So, I think, in order to grow the capital, the human capital in Saudi, there'd have to be more incentives, whether it was higher economic pay, or promise 
of a better infrastructure to do research, or more funding to actually do the research, based out of Saudi (A1)." The same idea has been confirmed by a director of a technology advancement and prototyping centre, "And also research funding is a key component for developing the KE (A13)". An Australian assistant professor in chemistry and biology in a Saudi private university argues, "I think it is very generous what the government doing here, I do not think many places fund research like this, so, I would say in terms of funding it is quite good (A9)". A dean of faculty of applied medical science/director of an innovation centre in personalized medicine assures, "we need financial support (A10)", much the same being said by a planning and policy advisor in the ministry of education, "logistic and financial support (G8)". An operations manager in a government VC says, "The other thing supporting the SMEs is funding and relaxing business regulations (P1)". A general manager in a pharmaceutical company said about the cost for doing $R \& D$ in the private sector: "they need a big budget (P4)". A CEO of a pharmaceutical company confirms the need for government support for doing R\&D, "the government do not fund or support research in the private sector (P5)"; he added, "Secondly, the companies' size and capital cannot afford them to develop new drugs". He also added, "if the government offer me a developing partnership (70\% government, and 30\% our company) to build an $R \& D$ centre, to mitigate the risk and mix the experience of researchers, and do research oriented to the market, and that would be managed regarding private sector mentality, I will accept". A vice president of PRC argues and tries to explain, "see, developing drugs come over three stages: firstly, lab stage; and at this stage not too much money is required, and there is enough funding for this stage. Secondly, clinical trials stage; at this stage there is need for a significant amount of funding, and in our plan we're going to hook up the partners with investors to make sure that there are interested people to progress the development of the potential drug (G9)", he added, "The government support and funding for $R \& D$ in $L S$ is at a very high level, because we do believe that there is a big opportunity".

A CEO of a university science park argues, "There is a programme allowing doctors to have one year abroad to do their research, and this is a good idea, but unfortunately the financial incentives are not enough (A6)". A manager of the industry engagement office in a Saudi private university confirms, "I think KSA is following the right way, but if they are going to be on the same rate [as developed 
countries] that would take a very long time, not less than thirty or forty years, the start is good but needs more coordination and more funding, they must spend more on $R \& D$, with the emphasis on $L S(\mathrm{~A} 8)$ ".

Funding R\&D is a key-enabling factor for supporting innovation (Godin, 2008b), for example, in Malaysia the government, to develop the life science sector, allocated a huge money to fund the R\&D over the entire stages of research (Ahn \& York, 2011).

\subsubsection{Developing seed funds (A 54\%, G 27\%, P 0)}

About 33\% of the participants (A1, G3, G4, G6, A5, A6, A7, A9, A12, A13) confirm the importance of developing seed funds to help in the process of technology transfer potentially as a means of stimulating new products and creating an offering to entice VCs to invest. A director of a technology advancement and prototyping centre says: "This one comes [he means Saudi VC called Taqniah] at a late stage, only in the very developed stage of a venture, we need seed and angel investment; we do not have angel investors in the KSA”(A13).

A dean of an innovation and entrepreneurship institute says, "But at the stage of developing prototypes and real production line they do not find funding, the university cannot afford to do that, the VCs do not enter in this stage, they're looking for startups or spin-offs at late stages with lower risk, and we can't blame them because they are private sector, so, we need public-private partnership; the government funds should take the risk and fill the gap of the 'valley of death' (A5)'. A CEO of a university science park argues, "at the early stages there are enough funds for $R \& D$ from the government and also some big companies give donations to finance research, but when it comes to the scale-up stage we have a problem, because VCs enter at a late stage, the problem lies at the stage of transferring [from a] prototype to a product (A6)". An incubator manager says, "Our clients find difficulties to hook up with investors because they are risk-averse, they can apply for loans from financial funds, but they prefer to have partners instead of taking risks alone and applying for loans (G4)". However, a science park director in a private university (KAUST) argues, "I think there is no problem in terms of money; the problem is lack of promising technologies, not lack of money (A7)". 
Seed funding is one of the important strategies that Singapore country implemented to support knowledge transfer and building new start-ups (Finegold et al., 2004).

\subsubsection{Developing VC funds (A 62\%, G 18\%, P 0\%)}

About $33 \%$ of the participants see the need for developing new VC funds (A1, A3, A4, G6, A5, A8, A9, A12, A13, G11). A director of a technology advancement and prototyping centre says, "We need VCs to transfer the knowledge to real products and invest in the new technology (A13)"; he added, "We need a national organization fund for venture capital". An assistant director in a SME government fund confirms, "It must be a big VC, it is very necessary, but it mustn't be governmental, it must be based on partnership between the government and the private sector. If it would be purely governmental it will fail, because the people here do not care about paying off the government, and should be managed by the private sector mentality (G11)”. An investment director in a university VC assures the need for developing more VCs and engaging private sector in this industry, "And we start to show other private investors that what we are doing can be done at a larger scale in the private setting as well, so when they see a government-owned VC firm is successful and what is likely they would try to duplicate it by bringing more private money into VC investment (A4)". A senior consultant in PRC confirms, "I do believe that VCs are a must to develop the KE but they need highly experienced people to manage them, and to facilitate things and to filter a lot of deals, it is not easy. And I think we have high liquidity either in the government or the private sector and they should invest locally instead of hedge funds and other investment channels abroad, and developing VCs to help in knowledge transfer from local or overseas sources (G6)". A dean of an innovation and entrepreneurship institute argues, "At the same time we have limited VCs, but I think some of the big companies start to develop their own VC (A5)". An Australian assistant professor of chemistry and biology confirms the importance of developing more VCs: "You need universities, you need VCs, infrastructure, government lining up with them, you need initiatives like KAUST, and more of these (A9)".

Venture capital and angel funding are one of the fundamental forces that significantly influence the innovation system (Niosi, 2011a). In the USA, venture capital is 
considered a key driver behind the significant development and progress of the biotechnology industry (Thomas \& Carl, 2001).

\subsubsection{Raising funding efficiency (A 62\%, G 27\%, P 28\%)}

About $42 \%$ of the participants see the need for raising funding efficiency as part of developing a funding system (A2, G5, A5, A6, A7, A8, A9, A10, P1, G9, G10, A11, P5). A manager of an industry engagement office in a Saudi private university sees the need for coordination among financial systems: "Another thing that is lacking in KSA is the coordination amongst various government entities dealing with funding, for example how KAUST, ARAMCO, and others work together to benefit the Saudi economy, this case is the same in most middle east if not all (A8)". An Australian assistant professor in chemistry and biology in a Saudi private university argues that there is no matching between input and output and the need for raising funding efficiency, "I really feel that the government is very genuine, they are really putting in quite big resources, they are quite big into funding, all right, but at the same time the infrastructure is not matching (A9)". A vice president of PRC confirms the idea of raising efficiency by saying, "I think we do not have a real problem in funding, we have some delay in funding research in the universities (G9)". A CEO of a pharmaceutical company argues, "the government do not fund or support research in the private sector (P5)". A faculty dean of applied medical science/director of an innovation centre in personalized medicine sees that there is a shortage in terms of motivations and incentives: "we have been chosen among all centres in the KSA as an excellence innovation centre in personalized medicine, we competed with all others, and we won, but we had no financial incentives or rewards (A10)". A ministerial advisor, when he mentioned a previous (now abandoned) strategy, says, "there is part of the strategy relevant to improving the financial system to fulfil the requirements of $K E$ (G10)". A full professor of information networks at the college of engineering complained about the procedures that funding goes through, "but the researchers who are supported and funded by the university might face some kind of bureaucracy to apply for anything like new tools, or equipment (A11)". He added, "in terms of the budget that is dedicated to research, it is really huge; but actually we need an integrated financial system to identify the distribution of funding needs; for example we spend on basic research without potential for application. We need to spend on 
research with high potential for commercialization so it can contribute to economic growth." A CEO of a university Science Park confirms, "I think there are enough sources of money but the problem is in the way of funding (A6)". An operation manager in a government VC also mentioned the low efficiency of funding, "The issue of funding $R \& D$ has been discussed a few years ago; and the comments stress the poor output of research and poor impact on industry and economy (P1)"

Up to the knowledge of the researcher, there is no specific and direct support for this concept (raising funding efficiency) in the literature, but in general, raising the efficiency of financial system is important to identify the promising options that would have a potential for better return (Gerschenkron, 1962).

\subsubsection{Developing angel investors (A 15\%, G 0\%, P 0\%)}

More than $6 \%$ of the participants confirm the need for developing angel investment (A8, A13). A director of a technology advancement and prototyping centre says, "This one [he means a Saudi VC called Taqniah] come at a late stage only in a very developed stage of the venture. We need seed and angel investment; we do not have angel investors in the KSA (A13)". The manager of an industry engagement office in a Saudi private university confirms, "In order to come up with knowledge-based economy there are various contributors: angel investors, or any type of investors; to build a start-up, you need an ecosystem that includes all components to transfer oilbased economy to knowledge-based economy or LS-based economy (A8)”. 


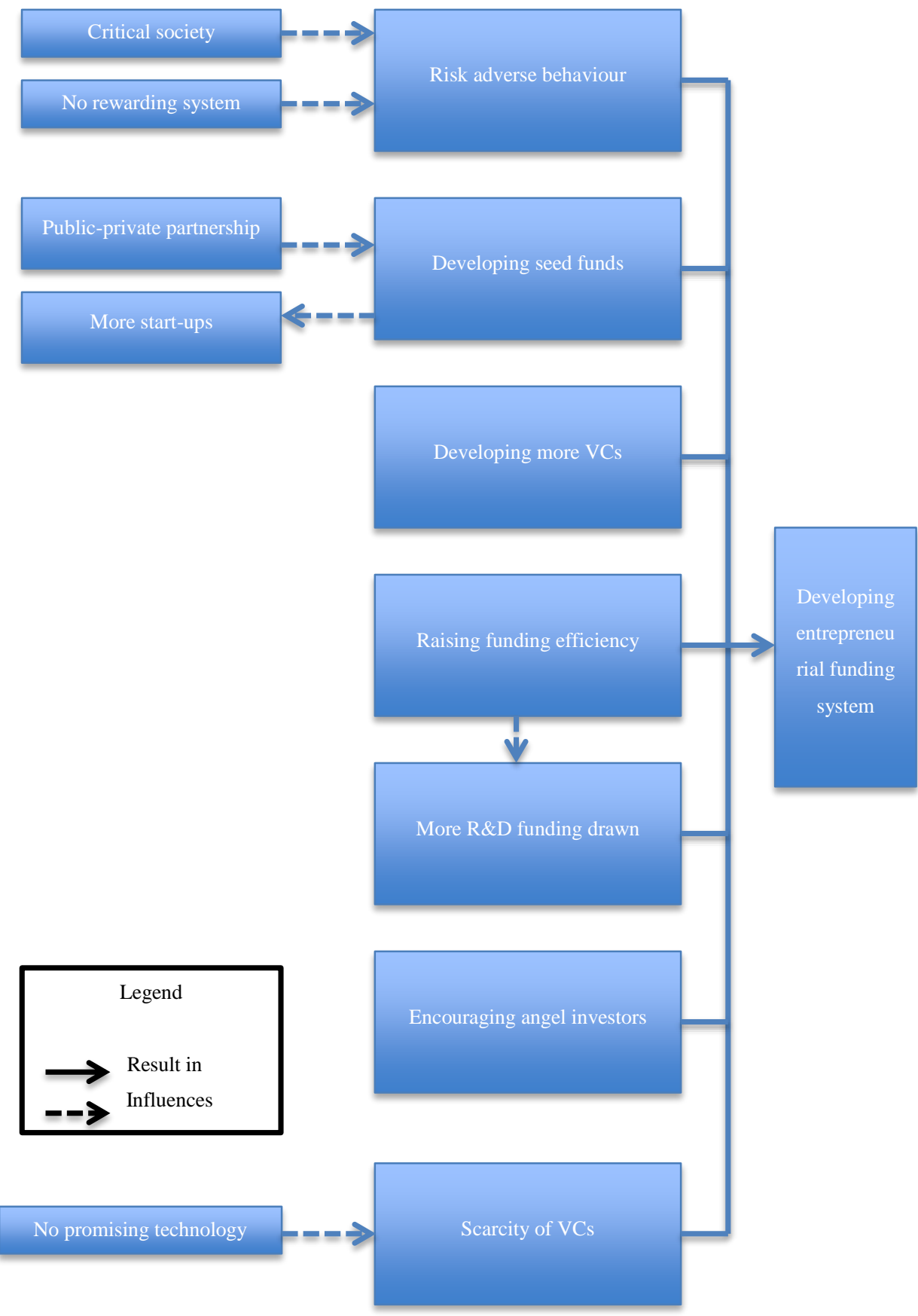

Figure 6-9: model represents the "entreprenurial funding and effecient system" theme and its properties. Source: The researcher. 


\subsubsection{Summary}

This model shows the key themes that influence the development of an entrepreneurial and efficient financial systems that support the ecosystem of innovation by providing in inputs to it. From the properties of this theme it is clear that the financial system is risk-averse and has a scarcity of venture capital firms. Also no mechanisms seem to be present or being established that would reduce these fears - such as government indications that this is a priority sector for investment in line with the future strategy for the nation.

It is found that there are two factors influencing the existence of this risk adverse behaviour: firstly, the society is critical, so that it does not encourage risk taking. Also, there is no rewarding system that encourages or supports the practice of taking risk whether in funding or business.

The scarcity of VCs was also, interestingly, found to be influenced by the lack of promising technologies that could encourage venture capitalists to seek these technologies and invest in them.

From the findings, it is suggested that the financial system develop seed funds, which would lead to developing more start-ups and transferring knowledge. The partnership between public and private actors helps in developing the seed funds.

Four other factors were found to help in developing entrepreneurial funding and efficient system: encouraging angel investors, more funding for $R \& D$, raising funding efficiency (which in turn helps in providing more funding for R\&D), and developing more VCs. 


\subsubsection{Developing Supportive Regulations and Enabling Policy}

This category has ten properties represented in the following codes

\subsubsection{Raising the quality of institutions (A 38\%, G 36\%, P 29\%)}

More than $35 \%$ of participants asserted the importance and relevance of raising institutions' quality and efficiency (A3, A10, G10, A9, G7, A11, A7, P6, G6, P1, G8). A planning and policy advisor in a ministry says, with regards to raising institutions quality and efficiency, "recruit foreign experts in all government institutions and be careful about applying nationalization everywhere (G8)". An operation manager in a public VC says, "In all the government institutions; no one is motivated to try new technology, they look for famed brands, they think if they would apply local technology, some problem might emerge, they will be blamed, the governmental procurement does not support the local technology and investment (P1)". He added, "Also, as you know there is excess staff in most of the governmental institutions and governmental companies". A ministerial advisor, when talking about a previous (now abandoned) strategy, confirms, "The strategy included long-term reform plans to reform the organization and structure of the economy and relevant institutions, and there are other short-term programmes dedicated to make reforms and adjustment to some institutions and systems to help in developing a knowledge-based economy (G10)". He added, "In general raising the efficiency of government institutes is one of the key objectives". A full professor of information networks at a college of engineering stresses the idea of raising institutions' quality, “....we need development in all institutions; we need to apply quality management and reengineering processes for all procedures, automating procedures and works. Some staff do not fit with this era, they are undevelopable, so after some years we will have a knowledge generation and staff that can be up skilled (A11)".

Raising institutions quality is important to identify promising options and mobilising resources (Gerschenkron, 1962). 


\subsubsection{Building social capital (A 31\%, G 36\%, P 0\%)}

About $26 \%$ of the participants see building social capital as an important factor, as values such as trust, collaboration and networking help the innovation process (A1, G1, A7, A10, A5, G4, G3, G11). An assistant director in a SME fund considers building social capital to be one of the challenges for developing a KE in Saudi Arabia, "social capital for collaboration, networking, and trust (G11)". A researcher in the knowledge economy, from Cardiff University, emphasizes the importance of building social capital, "We do not have the culture of sharing information. We need to enhance the social capital that includes trust, networking, collaboration, so clusters can be effective. Anyway with the new technology the geography is no longer a big problem and collaboration can even be with people overseas. If we would invest in networking, interactions will be more effective than just developing clusters (G1)"; she added, about international collaboration, "consultation no longer works, so I guess memoranda of understanding, joint programmes (for example to do research on local problems or local advantages with the sharing of local researchers); exchanging students. You need to look for mutual benefits, so the others can be attracted to work with you." A researcher in the Swansea LS ecosystem emphasizes the role of collaboration in changing culture, "I think a lot of the cultural change comes from collaboration and changes in thoughts (A1)". A director of a biotechnology centre in PRC confirms the low level of domestic collaboration, "we have a problem with local collaboration and I think this is a chronic problem, but at the international level it is fine (G3)"

According to Cooke, social capital plays significant role in developing network and building collaboration (Cooke, 2004).

\subsubsection{Building on strengths and priorities (A 54\%, G 36\%, P 14\%)}

More than $38 \%$ of the participants confirm that it is difficult to succeed without building on the priorities, strengths and advantages that Saudi Arabia already has (A1, A2, A3, G1, A11, G9, P2, G3, A4, G6, A6, A12). A full professor from Cardiff, with a background in knowledge economy says, when he was talking about sector-based policy: "I mean you know you could build on existing strengths (A2)", he added with example: "I mean it has been a problem, even at the UK level, every region wants to 
develop a LS sector and create an industry sector, maybe you think about what strengths you do have that might relate to that sector but you cannot create it. You know, obviously you get Swansea university, you get Cardiff university to invest a lot of money, it is easy investing a lot of money, they build new innovation centres, they obviously attract people, so I mean yeah there have been some successes in attracting these people, but there is a difference between having university-based expertise and having industry-based expertise". Another full professor from Cardiff, in developing competitive geographies, highlights a similar concept, "you know KSA has the highest level of diabetics, which must say something about LS, the importance of health (A3)". He was asked (do you mean we should start by identifying the priorities?) and added, "and find solutions, maybe, maybe that would be one direction, when you look at these countries, very well developed LS, they take very seriously the wellbeing of the people, so where you have high levels of wellbeing you have attained high levels of bioscience and LS activities, because they go hand in hand in a way, and when you have low levels of wellbeing such as in parts of your country, parts of my country, you attain less activities because it is seen as a lower priority, so it's like entrepreneurship, prioritizing entrepreneurship; you need prioritizing wellbeing as well, and if you are looking to develop a solution maybe that some way you can start to develop a LS system".

Developing strategies based on natural advantages and priorities are important for the success of an innovation system, for example, Malaysia benefited from the cultivation of its native palm tree farms to create palm derived products that could be supplied to the life science sector as an ingredient (Ahn \& York, 2011).

\subsubsection{Supporting entrepreneurship culture (A 54\%, G 55\%, P 14\%)}

About $50 \%$ of the participants see supporting entrepreneurship culture as an important policy that would help in developing KE in Saudi Arabia (A1, A2, A3, G1, A12, P1, G3, G4, A13, G6, G9, G8, A8, A5, G11).

An assistant of a direct manager in a public VC confirms the need for supporting entrepreneurship, "We need to encourage risk taking and might enact regulations that reward the risk-takers (P1)". He added, "I would suggest the government enact regulations to allow an employee who has an idea of enterprise to be released for a 
period of time, let me say two years, to try implanting his idea; and if he fails can go back to his ex-job. Also labour mobility is important for knowledge transfer. We need a regulation that encourages and motivates entrepreneurship, and does not blame or take account of failure". An assistant director in a SME fund considers the lack of entrepreneurship culture to be one of the challenges that should be met, "I would say entrepreneurship culture, innovation culture, social capital for collaboration, networking, and trust, and finally the strong research infrastructure that provides all needs for research and innovation (G11)". A researcher in the Swansea LS ecosystem endorses supporting entrepreneurship saying, "So you have to encourage people to take risks then give them safety in case of failure (A1)". A full professor from Cardiff with a background in developing competitive geographies and the knowledge economy also confirms that, "Think about the US, they probably had some successes in high tech in silicon valley because of Stanford university, but what they were doing all that time that made sure they were successful: within the education system they were educated to become entrepreneurs, making entrepreneurship to be a good thing, making innovation to be a good thing (A3)". A manager of the industry engagement office in a Saudi private university argues, "You need investors, you need entrepreneurial spirit; the entrepreneurial attitude does not come with birth, you need to learn it, we have an entrepreneurial centre here in the university to help people to think in an entrepreneurial way, you need people to help the inventors realize the economic value in their inventions; usually the researcher, even if the Nobel prize laureate, is interested in the knowledge, but may be not aware of the potential business in his inventions (A8)". An operations manager stresses the role of education and regulation that could support entrepreneurship, "This can be through developing motivating regulations, reforms in the education system, and spreading this culture (P1)". A director of a biotechnology centre in PRC also emphasises the idea of focusing on Saudi priorities, "anyway we need $R \& D$ in the universities and $P R C$ and we need to focus on local chronic diseases as priority (G3)". A senior consultant in PRC confirms the same concept: "The researchers who go there [meaning study abroad] they should go based on pre-planning, interested in our priorities and topics or technology instead of doing their research on behalf of them [the host universities], we should not just focus on getting the certificate (G6)" he added, "as you know LS is a wide field and we cannot build capabilities and progress in all areas, so I think we need to search out our advantages and look for the unique things we have and build 
on these, or even if we do not have a specific thing we can focus on a specific area and develop the sector in that regard".

Entrepreneurship plays significant role in generating jobs and developing innovation (Wim Naudé, 2011), it is strongly linked to biotechnology (Patzelt, 2012).

\subsubsection{Developing government-led project (A 54\%, G 36\%, P 42\%)}

More than $45 \%$ of the participants believe that such a project, like developing the knowledge economy in the LS sector in Saudi Arabia, should be firstly kicked off by the government, as the private sector would need time to do this and to change attitudes (A2, A3, G1, G2, G3, A4, A5, A7, A12, P5, G6, P7, A8, P3). A full professor from Cardiff researching competitive geographies and the knowledge economy says, "and people in Singapore, they do like what you are doing actually, they go around the world trying to find the best knowledge possible of who is doing what, what would be the best initiatives for the government to fund, constantly, and I guess as for what we need anywhere is to make sure there is a strong strategy in place, and commitment from the government for long periods of time, and I think without that it will be a key challenge to develop this sector (A3)". Another full professor from Cardiff, with background in developing clusters, says, "Most of the Middle East does not get a significant contribution from the private sector, so by default it has to be by public sector (A2)". A dean of an innovation and entrepreneurship institute in a Saudi university says: "but what is important here is that the government has commitment and plans to do that (A5)". He added, "The government funds should take the risk and fill the gap of the 'valley of death". The manager of an industry engagement office in a Saudi private university confirms the idea, "It should be kick-started by the government, but the government cannot do everything, for example VCs, and angel investors that I have mentioned in the ecosystem are private entities, but the project should start by the government developing a special funding institution to prepare the environment for the private firms, either local or foreign, to invest in research in KSA, but these firms will not come if they do not expect good research outcomes (A8)". A science park director in a Saudi university confirms the role of government in developing the LS sector, "The third thing; we need to create infrastructure for $R \& D$ translation, for example here in the university we push innovation, but the country needs many prototyping and $3 D$ 
printing centres; the government should invest in these, to encourage creativity, encourage product development and design (A12)". A business development manager in a pharmaceutical company argues, "Also the government can support the research centres in the universities and PRC to develop research based on local diseases and at the same time build collaboration with government-owned pharmaceutical companies to adopt the research outputs and produce potential products. The government should fund the research; and research centres can do the entire process from invention to production, and focus on the KSA's priorities in terms of prevalent diseases; this step would increase the trust in the research centres and raise their credibility, and consequently that achievement would create collaboration with the private sector either local or foreign. The people need the first step to build trust (P3)". A business development director in a LS cluster says, "we believe that the government is the one which is supposed to invest in knowledge; it can be patient to invest in long-term investment (P7)".

Providing enabling and initial factors and developing infrastructure are crucial for developing life science industry (Ahn \& York, 2011). The Singaporean government invested massively to build the research infrastructure and developing the biotechnology sector (Finegold et al., 2004).

\subsubsection{Developing enabling policy for $R \& D$, and spin-off (A 70\%, G 55\%, $P$ 14\%)}

About $52 \%$ of the participants see the need for developing new policies that support R\&D and encourage spin-offs (A1, A2, G1, G6, A10, G4, G3, A6, A7, A9, A4, G2, G7, A5, P2, A12). A researcher of the Wales LS ecosystem talks about policy in general, "The government should develop the policy and environment and be enabling, and people create entrepreneurship themselves (A1)"; he added, "I think we need to look for evidence through other governments and see which country does one thing or many things and see how much they spend in proportion to GDP in supporting innovation, entrepreneurship, infrastructure, tax incentives and then see what actually grows, and develop a model to measure the effect of spending on the output. Policy is very subjective and hard to measure; many countries have come up with a lot of policy and strategy in the last ten, fifteen years. I would say look for evidence and see the quantitative measure for success in this area". He also added, "I 
have seen many successes through technology spin-outs of the university, [...], quite a few from the engineering school working well with large companies and small companies, so I said it is possible and just a matter of how we write a policy and how we can write support mechanisms; so Swansea University has a very straightforward licenses mechanism, if you create something then the university gets a third, the college gets a third and the venture gets a third, so you understand how much ownership you have, or you can license technology from the university for a fixed amount of equity, and start a spin-off company, because it is very straightforward, it is easy to start a company or as easy to license technology - very straightforward and the process to raise capital too, so if you have a good idea engaging with venture capital is very straightforward, or having a government system for grants, so the whole mechanism is to support the early stages of $R \& D$ in a university and then spinning out into a company". An Arabic gulf junior researcher in the knowledge economy from Cardiff says, "The FDI will enhance the work culture, we have problems in the work culture in terms of creativity, enthusiasm and discipline, and also entrepreneurship (G1)". She added, "You need to instil the concepts of entrepreneurship, self-employment, creativity in the minds of students in schools, universities, and other society segments by staging for example prestigious competitions and having a judgment panel from different sectors (private, government, education system). I don't think promotions, advertisements work very well. And the rewards should be valuable". A senior consultant in PRC sees that there is a need for enabling policy for spin-offs, "Every university has its own IP policy, and the problem is the researchers are government employees and they legally do not have the right to own an enterprise or even a share of the enterprise, and this obstacle must be tackled, the concept of spin-offs is still absent (G6)". A full professor of information networks in a Saudi university mentioned research activity, "the first thing is the researcher; when we say Research University that means at least third of the students are postgraduate; master and PhD. We do not have that (A11)". He added, "Actually there is a gap between the research and the science park, there (in the professor's university) is no contact between the two activities". A faculty dean of applied medical science/director of an innovation centre in personalized medicine complains, saying, "unfortunately, the centre, the regulations do not permit the researcher to spin off and build his business, and this is a big and disappointing problem. Sometimes I feel devastated; I do research and come up with innovative 
ideas, but the major benefits go to other parties (A10)". A vice president of PRC says, "I mean the R\&D must be connected to the regulations and policies of the Saudi FDA (Food and Drug Authority), also integration with universities to link research with the country's priorities and needs, so at the end these potential products would be used by the ministry of health and licensed easily from the Saudi FDA (G9)"

The government needs to develop enabling policy that help in developing innovation policy, funding technical innovation, supporting technical and academic research and education (Niosi, 2011a).

\subsubsection{Improving industry infrastructure and business environment (A 54\%, G $18 \%, P 42 \%)$}

More than $38 \%$ of the participants suggest improving industry infrastructure and the business environment; they think that would help in growing business, especially new start-ups, and attracting FDI (A1, A2, P6, A5, P1, A9, G4, A4, A7, P2, A12, G8). A researcher in the Wales LS ecosystem suggests lowering the risk as a motivating factor for doing business, "I think it is about economics, people do their best to find ways to make money, and are averse to taking risks. So, the risk should be lowered. If the risk is lowered then more innovation would happen, so risk can be decreased by social care, health care system, funding, grants, to ensure that even if someone does fail he can survive (A1)". A biotechnology incubator manager says, "we started in 2010, and we have growth in our activities, but actually we need support from the government in terms of easing the procedures related to doing business; especially for high technology enterprises, it takes a long time (G4)". An investment director in a university VC complains about labour regulations, "but what you need to start immediately is a lot of foreign skilful workers, and that will be most impossible, because you need to fulfil Saudization requirements (A4)". A science park director in a private Saudi university highlights the need for improving business regulations, "first of all, the regulations, like regulations on doing business, and Visa regulations (A7)", he added, "Visa regulations, and friendly regulations on doing business are important in developing an ecosystem". An Australian professor in chemistry and biology, working in a Saudi private university, asserts the need for improving infrastructure, "I think Saudis are very good at that, I mean clearly they have a very 
strong economy, they are very good in business, they know how to communicate, they know how to build business very well, they have good economy that means they know how to do it, I think what they need is the infrastructure, I keep going back to the infrastructure (A9)". A production manager in a big multinational pharmaceutical company also confirms the idea of improving the business environment and industry infrastructure, "attracting the FDI is important component, and to do that, the government should more and more improve on the environment for doing business, and improving the infrastructure for this industry (P6)".

Singapore managed to attract MNOs by developing strong infrastructure and friendly business environment (Wong, 2001).

\subsubsection{Improving living standards and relaxing cultural barriers (A 15\%, G 11\%, P 14\%)}

About $13 \%$ of the participants (A1, G3, A9, P7) mentioned the importance of improving living standards and relaxing cultural barriers that would help in attracting foreign expertise and FDI to work in Saudi Arabia. "I mean the visa situation is very strict in Saudi, maybe not for teachers, but it is very strict for, especially for women and, yeah, it is more difficult to, to come to Saudi and to find a work permit. They take away your passport, so it is very restrictive. Yes, I say, I would say it is difficult. I believe that if it was a priority of Saudi to open up the country a bit more, to make it less restrictive for, these bringers of knowledge, but it's a difficult situation between the Western, the clash of the Western culture and, and, I guess, Saudi customs. So, it is possible, I think some things would have to be changed a little bit (A1)"; he added,

" Well, I mean I think, I think you mentioned before that, that KAUST is allowing women and men to be taught together in the same classrooms, and I think those kinds of compromises will need to be made, both women teaching men, men teaching women, men and women teaching and learning together". A director of a biotechnology centre in PRC argues, "actually, when you try to recruit western people you face some problems because of the difference in the cultures and living standards, but in spite of that we have good collaboration with Chinese scientists and they work with our scientists in the city (PRC) and are doing well (G3)". An Australian professor in chemistry and biology in a Saudi private university argues, "So, you need 
to go really aggressively to grow it like this, this the only way, it has to be international, it has to be open, and it has to let people come in and compete (A9)". For developing human capital he said, "you have to create a good atmosphere; the only way science can work is if it is international, it doesn't work otherwise, so all over the world science is international, so here it has to be international; in the sense that you have to open it up to professors from all the world, and give them the right atmosphere to come here, and you have to have rotation; fresh blood coming in, the students need to communicate and need international students next to them, you need to create that competitive edge". A business development director in a LS cluster also corroborates the idea, "Another important component; tank thinkers, good living standards (housing, education, transportation, health facilities, entertainments, etc.) and a good ecosystem to work in (P7)".

Relaxing cultural barriers and embedding foreign people enhances knowledge transfer (Markusen \& Venables, 1999).

\subsubsection{Developing supportive staffing regulations (A 15\%, G 18\%, P 0\%)}

More than $13 \%$ of the participants complain about employment regulations and restrictive terms in the government institutes (academia and public) (G3, A10, G8, A12). A director of a biotechnology centre in PRC says, "unfortunately we have, the problem is we are in competition with other sectors that offer very high salaries and incentives, and we are restricted by the governmental rules. We do not have flexible regulations (G3)". A science park director in a Saudi university also complains, "for the link with industry we have here about 10 to 12 employees; they have capabilities to negotiate collaborative $R \& D$ in terms of technical and legal aspects, they can do market research for any technology, market assessment, identify technology readiness, and what are the next possible levels; these took a long time and hard work to be built, but we have a problem; we are not sustainable, because their salary and incentives are under the public system (governmental staff), but they are worth better in the market, so they can quit us at any time (A12)". He added, "So, the system of civil service (public employees) should improve to fulfil the required incentives for these people, because they are important for research". A faculty dean of applied medical sciences/director of a innovation centre in personalized medicine says, "But 
we have deficiencies in two things; the employment structure and lack of incentives do not motivate the people to join us or stay with us, we train fresh graduates and they work for a specific time then they move to more secure jobs anywhere (A10)". He added, "we have enough technicians and researchers, but as I said above, there is a problem in the incentives and employment system, in terms of salaries and job security”.

The literature does not support this concept specifically, but in general supports developing supportive regulations and policy that facilitates the performance of innovation system and knowledge commercialisation (Niosi, 2011a).

\subsubsection{Developing a KE coordinating organization (A 31\%, G 9\%, P 0\%)}

More than $16 \%$ of the participants (G6, A5, A6, A8, A12) think that establishing a specific organization for organizing relevant activities, developing plans and enacting enabling policy would be more helpful. A senior consultant in PRC argues saying, "The problem is that there are many disparate and fragmented activities but without an umbrella that embeds all these; this organization should keep away from the governmental style and the bureaucracy but at the same time have a high mandate, power, authorities that enable it to develop and impose policies and deal with different entities. For example China has a similar idea, they have an organization mandated to organize and develop policies with respect to this orientation (G6)". A CEO of a science park in a Saudi university confirms, "I think the government should establish an organization in charge of organizing all dynamics and activities that all stakeholders are engaged in (A6)". A manager of the industry engagement office in a Saudi private university says, "Another thing that is lacking in KSA is the coordination amongst various government entities dealing with funding (A8)"; he added: "As I said before we need to coordinate together at the national level in order to produce collectively". A Science park director in a Saudi university corroborates, "Another point; there are many institutions here trying to support SMEs, but it is fragmented and scattered, I would suggest there should be one entity in charge of mastering all those efforts through developing a master plan (A12)." 


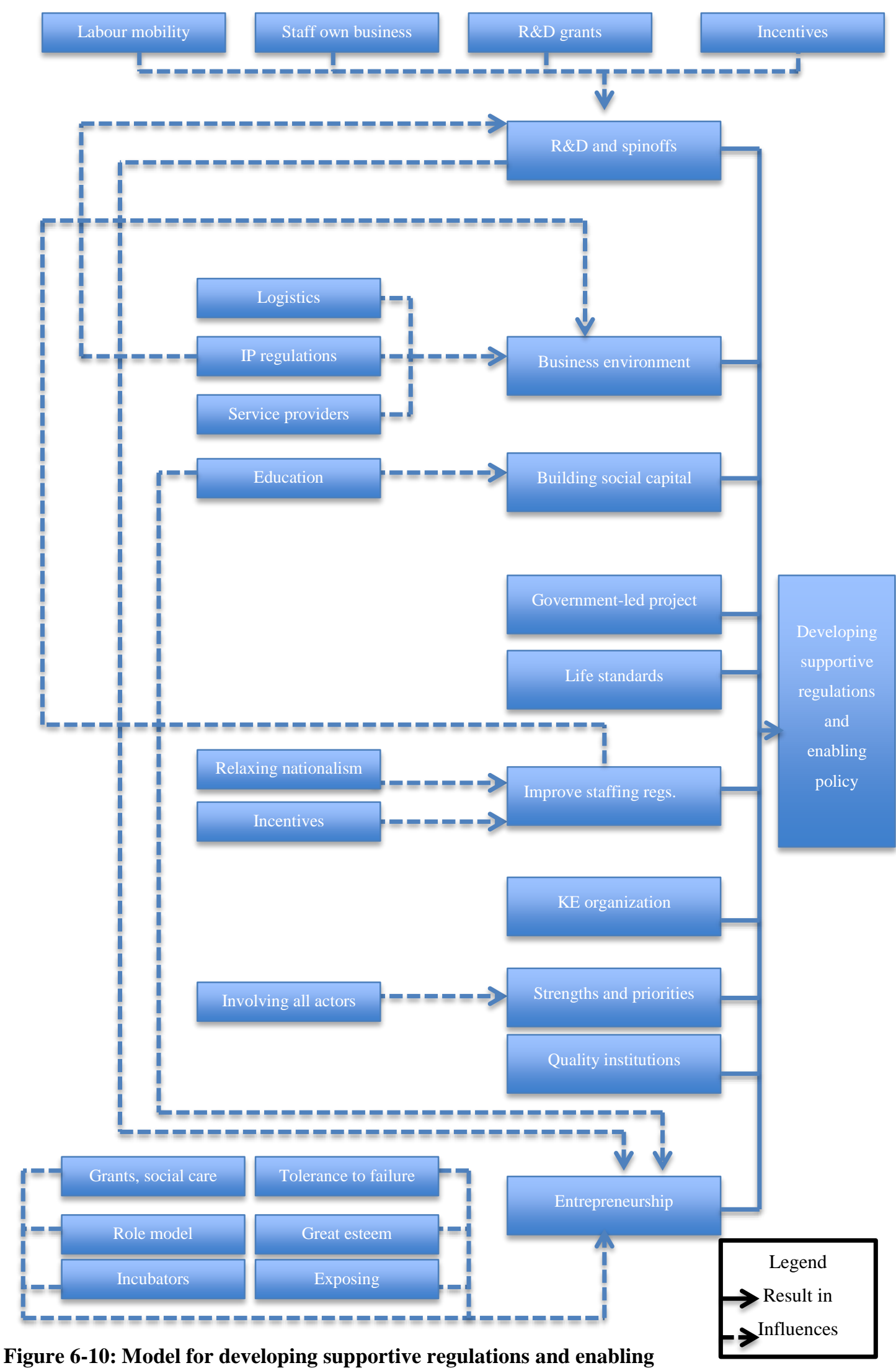

Figure 6-10: Model for developing supportive regulations and enabling policy.Source: the researcher. 


\subsubsection{Summary}

This model above describes the properties and factors that support the development of supportive regulations and enabling policies as a key requirement for developing a knowledge economy from the life science sector in the KSA.

It is perceived that there is gap in the regulations and policy that support the development of the life science sector in Saudi Arabia. Ten strategic interventions (variables) have been identified and proposed to treat this gap and shortcomings.

Developing enabling policy for $R \& D$ and spinoffs: there are several factors which have been found to influence this strategy. These include providing incentives to exploit through spinoffs the outcomes of research; providing R\&D grants to encourage research and mitigate the risk; enabling faculty and staff to own their businesses, so the researchers find encouragement to direct their research to the market, and allowing labour mobility, which helps in transferring knowledge between actors such as academia and private sector or public institutions. Enacting strong IP regulations is also a factor in developing enabling policy for $R \& D$ and spinoffs.

Relaxing nationalism regulations (i.e. lowering the percentage of Saudis who must be employed in any firm) and providing incentives for high skilled people will help in developing supportive staffing regulations, the latter in turn contributes to improving the business environment.

In its turn, the business environment was found to be affected positively by improving logistics, improving IP regulations and having service providers.

The variable (enabling policy for $R \& D$ and spinoffs) helps in supporting entrepreneurship culture. Other factors for supporting entrepreneurship culture include: providing grants and social care (to reduce the risk); being tolerant of failure; giving great esteem for entrepreneurs; presenting role models; exposing people to places with entrepreneurial attitude; building incubators to contain and support entrepreneurs, and education. This last has also been found to help build the social capital that encourages the culture of networking, trust and collaboration. 
The variable of a Government-led project was proposed as a key element to help in developing supportive regulations and enabling policy. The life science sector in the KSA needs huge investment and risk taking, but the private sector is not ready yet to take the lead on this, so it is very important for the government to start and lead the project.

To do that, and directly linked to the previously described variable, it is proposed to build a knowledge economy organization to involve all actors (public, academia, private) to develop strategies based on existing strengths and priorities.

At the same time the government needs to raise its institutions' quality to be able to manage this kind of project, to implement the strategies properly, and to work efficiently.

The last variable in this model is improving living standards, in terms of quality of life, health, education, entertainment, and relaxing cultural barriers, so that foreign people can find a suitable environment to work in Saudi Arabia and become embedded. 


\subsubsection{Involving the private sector (PS)}

Six codes constitute the properties of this category; the following sections will break down all these codes in detail.

\subsubsection{Lack of academia-PS collaboration (A 46\%, G 27\%, P 29\%)}

More than $35 \%$ of participants mentioned the weak collaboration with PS (G2, G3, G11, A4, A9, A10, P6, A11, A12, A14, P5). The deputy director of a national centre for environmental technology says, "There is no private partner especially for SMEs so, when they are looking for solutions for their problems, they look abroad, they do not trust the local researcher (G2)". A Director of a biotechnology centre complains about a lack of collaboration, "Also the problem is working alone, there is a gap between industry and academia (G3)". An investment director in VC argues, "so this type of collaboration will benefit both sides, I mean you get the students and faculty real time exposure to commercial problems and technological problems that affect commercial opportunities while at the same time this gives the opportunity to the company to tackle those problems from an academic perspective, so this type of interaction is very fruitful, but unfortunately very few companies and few universities have this type of relationship in Saudi (A4)". An Australian professor in a Saudi private university displays a different reason for no collaboration: "I did not try because none of these companies work on what I am trying to do, so my collaboration is international; so I have tried out my first patent, and we are trying to adapt it more toward something that is marketable, and at that point will start up a company; there is no biotech in the country entirely (A9)". A faculty dean of applied medical sciences/director of an innovation centre in personalized medicine confirms the lack of trust and credibility as reasons for no collaboration, "no we do not [collaborate with PS], we need to build our credibility and then let the private sector trust us and deal with us (A10)".

The collaboration in Singapore context not very strong and that been considered a shortcoming and hindering in the Singaporean national system (Finegold et al., 2004; Stein, 2003). 


\subsubsection{Lack of innovation strategy and attitude in the PS (A 38\%, G 55\%, P 57\%)}

More than $45 \%$ of the participants identified the strategic behaviour and attitudinal mentality of the PS as a key factor for the lack of PS involvement (G6, P4, G2, P3, P5, A6, A7, G1, P2, G7, G3, G10, A4, A12, A13). A CEO of a university science park says, "And basically the companies here are mostly not involved in the innovation or $R \& D$ activities (A6)". A medical doctor researcher in a tertiary public hospital confirms that, "The private sector is not working on developing new products, which leads to no need for collaboration (G7)”. A ministerial advisor says, "Another challenge concerns the contribution of the private sector in $R \& D$; their contribution is very weak (G10)" he added, "So, the private sector should increase its involvement and contribution to $R \& D$ and entrepreneurship, and innovation". Here a deputy of a national centre for environmental technology mentioned the strategic behaviour towards innovation, "There is no desire or strategy for innovation among private companies, except big companies from other sectors (G2)". This idea was confirmed by a CEO of a big pharmaceutical company, "Actually, we do not have a strategy for $R \& D$ for developing new products and I think all Arabic firms are the same (P5)". A junior researcher in the knowledge economy from Cardiff talks about the gap between academic research and market needs, and the low research capabilities and absorptive capacity of the PS, "One of the problems is that research is not orientated to the market. Also the private sector is not ready or qualified to absorb the research output (G1)”. A science park director in a Saudi university argues to confirm the lack of innovative practice of the PS, "The model of industry here was established on intensive labour, cheap labour, and low skills; this model has to be changed. The other model is called micro-enterprises (MEs), which constitutes the majority and covers many definitions of business. According to Battelle $R \& D$ reports, the [prevailing] business attitude in the KSA does not encourage entrepreneurship (A12)".

For sustainable growth, firms should feature entrepreneurial attitude and developing innovation(Gray, 2006). Saudi people mostly lack an innovative and entrepreneurial attitude in managing their businesses (Kayed \& Hassan, 2011a). 


\subsubsection{Developing academia-PS strong connections (A 62\%, G 36\%, P 0\%)}

More than $38 \%$ proposed developing academia-PS connections as a strategy for involving the PS. More than a third of the participants (A2, G1, G2, G3, A4, A7, A9, A10, G9, A11, A12, A13) see this strategy as important for knowledge transfer and the innovation process.

A full professor from Cardiff researching the development of competitive geographies says, "But I do think the key factor with any science emerging cluster or area around biotech or LS is to have good connections with large bio pharmaceutical companies (A2)". Also a junior researcher at Cardiff University, from an Arabic gulf country, interested in the field of the knowledge economy, stresses the role of the PS, "the private sector is a key partner and cannot be substituted (G1)". A science park director in a Saudi private university explains that PS is key part of the LS ecosystem, "Yes it is one of the key areas, here in the research and technology park (we) try to attract big firms, SMEs, start-ups, spin-in, spin-off, and service providers, so we work on developing an ecosystem that includes all needed elements (A7)". A full professor of information networks at the college of engineering in a Saudi university discusses the importance of involving the PS for the aim of knowledge transfer, "There are enabling factors but the outputs as products are quite limited, so we need to support and provide tools for increasing the outputs by motivating private sector investment and involvement in the innovation process (A11)". An Australian professor in a Saudi private university hopes that the PS can invest in service provider companies so it can help create a LS ecosystem and contribute to the innovation process, "Alright, so for the LS, you need infrastructure to support LS, and that infrastructure is much better to come from the private sector rather than universities, so all I say (is) the best thing to do is you have to establish service providing companies in KSA (A9)". "The big companies and organizations in the world mostly have all kinds of knowledge activities that help in developing and practising their key activities and products or services (A13)"; he added: "the big companies and banks should contribute in funding $R \& D$, and to have collaboration with innovation centres or researchers and develop together their need for technologies. They should invest in the service and supportive industries that present technical solutions to the industry (A13)". An investment director in VC mentions the benefit of academia-PS collaboration, "That is like private-public partnership, a very nice thing to start with (A4)"; he added: "so 
this type of collaboration will benefit both sides, I mean you get the students and faculty real time exposure to commercial problems and technological problems that affect commercial opportunities while at the same time this gives an opportunity to the company to tackle those problems from an academic perspective, so this type of interaction is very fruitful". An Australian professor working in a Saudi private university explains, "So, usually you make the discovery, you secure your patent and then go to those in the private sector. Or via other mechanisms when collaboration starts from the beginning based on a common interest or a problem which needs to be sorted out (A9)". A faculty dean of applied medical sciences asserts the need for the PS to complete the cycle of a LS ecosystem, "You know we cannot do everything, we need the private sector to collaborate and deal with us, but unfortunately, they do not trust our work, they do not trust Saudi universities (A10)". A full professor in a Saudi university gives a typical example of the relations between universities and the PS, "Current universities in the world are integrated universities; they have science parks (SP), and cover all aspects; they have contacts and relations with the Private Sector (PS), the best example I have seen is in Hong Kong University (A11)".

The connection and collaboration between industry and academia is one of the most important strategy that supports the progress and flourish of innovation system (Cooke \& Wills, 1999; Keizer et al., 2002).

\subsubsection{Funding programmes for academia-PS collaboration (A 70\%, G 36\%, $P$ $57 \%)$}

About $55 \%$ of the participants identified the need for developing funding programmes to facilitate collaboration between academia and the PS (A1, A2, A3, G1, G3, G4, A4, G6, A5, A6, A10, A11, A12, P2, P3, P5, P6). A director of a biotechnology centre suggests that the PS should contribute to funding a programme to make collaborations and partnerships with academia: "I would suggest the industry supports and funds certain $R \& D$ in the universities. \{Do you think the problem is in the fund?\} No actually the partnership and logistic things are very important (G3)", he added: "we need initiatives to bridge the gap, we need to develop an ecosystem, I mean everything that makes that kind of industry-academia overlap flourish, establish postgraduate programmes funded by industry and then they go back work in industry, this sort of 
thing moves the stagnant medium". A Biotechnology incubator manager also asks the PS to contribute to funding and supporting research: "actually there is a gap [academia-PS], and I think the private sector should develop their social responsibility and support researchers (G4)". An investment director of a university VC sees the importance of involving the PS to develop VCs and investment in technology, "and we try to show other private investors that what we are doing can be done on a larger scale in the private setting as well, so they can see a governmentowned VC firm is successful and what is likely is they would try to duplicate it, by bringing more private money into VC investment. This will allow the diversification of economic growth (A4)". He added, "there is a need to have more incentives given to both the private investor and the foreign investor to allow money and skills to come to this country as well as technology". A director of a university science park says, "We need partnership between the public and private sector to facilitate and provide funding (A5)". At the other end, the CEO of a big pharmaceutical company argues, "The government does not fund or support research in the private sector (P5)"; he added, "if the government offers me to develop a partnership (70\% government, and $30 \%$ our company), to build a R\&D centre, to mitigate the risk and mix the experience of researchers, and do research orientated to the market, and it could be managed with regard to the private sector mentality, I will accept". He also added, "The problem of the universities' research centres and researchers is always to look at the companies as a source of funding because of their income; we need collaboration to be from all sides, we need the government to support and fund this collaboration". Another Saudi working as production manager in a MNC proposes another approach: "We need highly sophisticated research centres with partnership with the private sector (P6)".

This strategy contributed significantly in developing innovation among SMEs in the USA, and helped in building new start-ups, the USA government developed programme called, Business Innovation Research Programme (SBIR) to support collaboration between SMEs (Audretsch, 2003). 


\subsubsection{Involving all actors in developing policy (A 38\%, G 9\%, P 0\%)}

About $20 \%$ of the participants see that there is a need to involve all relevant parties while developing enabling policy to make sure that every party is aware of their role and can fulfil the common targets (G1, A8, A9, A10, A11, A12). A full professor in information networks in a Saudi university says, "The collaboration with the private sector is absent. And I think we need an initiative to gather all parties, this initiative can be proposed from the third sector, professional organizations. In general, we fail to work together even here [the university] among different departments. The problem of the private sector is in its dependence on foreign companies, and foreign companies dependence on foreign universities. So, we need to bring all parties together to develop a collaboration (A11)". The same concept is suggested by an Australian professor working in a Saudi private university: "Top-down, this has to come from many places to do it simultaneously, and involving government. It has to be a big design, it has to be a master plan; but several places, several universities, industry, and several governmental agencies must come together to define the big problems (A9)". An industry engagement office manager in a Saudi private university says, "we are at the beginning, currently there is collaboration but we hope to develop better collaboration. As I said before we need to coordinate at the national level in order to work collectively (A8)". A junior researcher at Cardiff University from an Arabic gulf country, interested in the knowledge economy, stresses the role of culture and the lack of awareness of the need for involving all relevant parties in developing collaboration and enabling policy: "It is culture, it is all about culture; the awareness is very important, when government is developing policy the other partners (private sector, academia) must be involved (G1)".

The government should involve all actors in developing policy related to innovation system to align actors interests and secure the collective benefits (Niosi, 2010).

\subsubsection{Transferring foreign knowledge (A 54\%, G 27\%, P 29\%)}

More than $38 \%$ of the participants suggest using joint ventures, acquisition and licensing technology as practical mechanisms that local PS firms can adopt to transfer foreign knowledge and raise the quality of their knowledge activities (A2, A3, G1, A4, G6, A6, A8, A9, G10, A12, P5, P6). 
A CEO of a large Saudi pharmaceutical company says, "Even us, if we would think about developing a new technology I will look for a foreign company with a high reputation, to make joint ventures with them, and I think this is a good way to bring technology here (P5)". A manager of the industry engagement office in a Saudi private university endorses the same idea, "If I were in position of decision maker, I will go to see some big world biotechnology companies, and take a license for how to make biologics, this requires a human capital but at the same time you will learn a lot:- from a regulations perspective; GMP (good manufacturing practices); quality control, etc. when you gain this knowledge coupled with good research, you can do the same process on a new biologic (A8)". An Australian professor working in a Saudi private university also argues that such strategy will accelerate the development process and shorten the time required for this, "I think it is the best way we can do it right now, I think it is the fastest way; no need to reinvent the wheel, no need to go to build the entire technology yourself, everybody around the world, these companies are mobile they can move around, you just need to give them incentives (A9)". Another participant working as an investment director in a university VC suggests developing incentives to attract foreign investment and technology, "There is a need for more incentives to be given to both the local private investor and foreign investor to allow money and skills to come to this country as well as technology (A4)"; he added, "We might have strengths, because we have a population that is mixing, or we might have better samples here to study disease than anywhere else, we can start to build networks from different directions on a large scale, and then you can bring big companies (A9)". Another participant working as a production manager in a MNC in Saudi Arabia asserts, however, that Saudi has attractive incentives: "the government offers very attractive investment environment such as nominal land fees, cheap utilities fees, zero custom fees on raw materials and machines, and we have a big and growing market. The government does this for the sake of many things: supporting industry and diversifying the economic base, heath security, job creation, and technology transfer (P6)". A full professor from Cardiff sees that without embedding foreign people and entities, local industry would not benefit from foreign knowledge transfer: "If you basically import in high skilled people for a short period and they are not embedded - you know living in separate area and totally separate life - and they go, has any of the knowledge been transferred to the host country? Probably, not very much (A2)". He added, "if you want to attract companies where there are 
incentives, you need also to have a strategy in place where we can embed skills and learn from these companies in high positions in the value chain; otherwise they come and say thank you very much for the subsidies and they go, so think how to embed and how you get knowledge transfer, there is link for SMEs, you can link between knowledge transfer and you get in spin out companies but if these companies are not embedded then you will not get that" . A junior researcher at Cardiff University, from an Arabic gulf country, interested in the knowledge economy, mentions other benefits of FDI: "The FDI will enhance the work culture, we have a problem in work culture in terms of creativity, enthusiasm and discipline, and also entrepreneurship (G1)”.

This strategy is strongly supported in the literature, and it is believed as vital in filling the gap in human and research capabilities, for example, Indian companies adopted this strategy by making joint venture, acquisition and alliance with companies from developed countries (Krishnan \& Jha, 2011). 
Figure 6-11: Model represents the theme "involving the private sector". Source: The researcher.

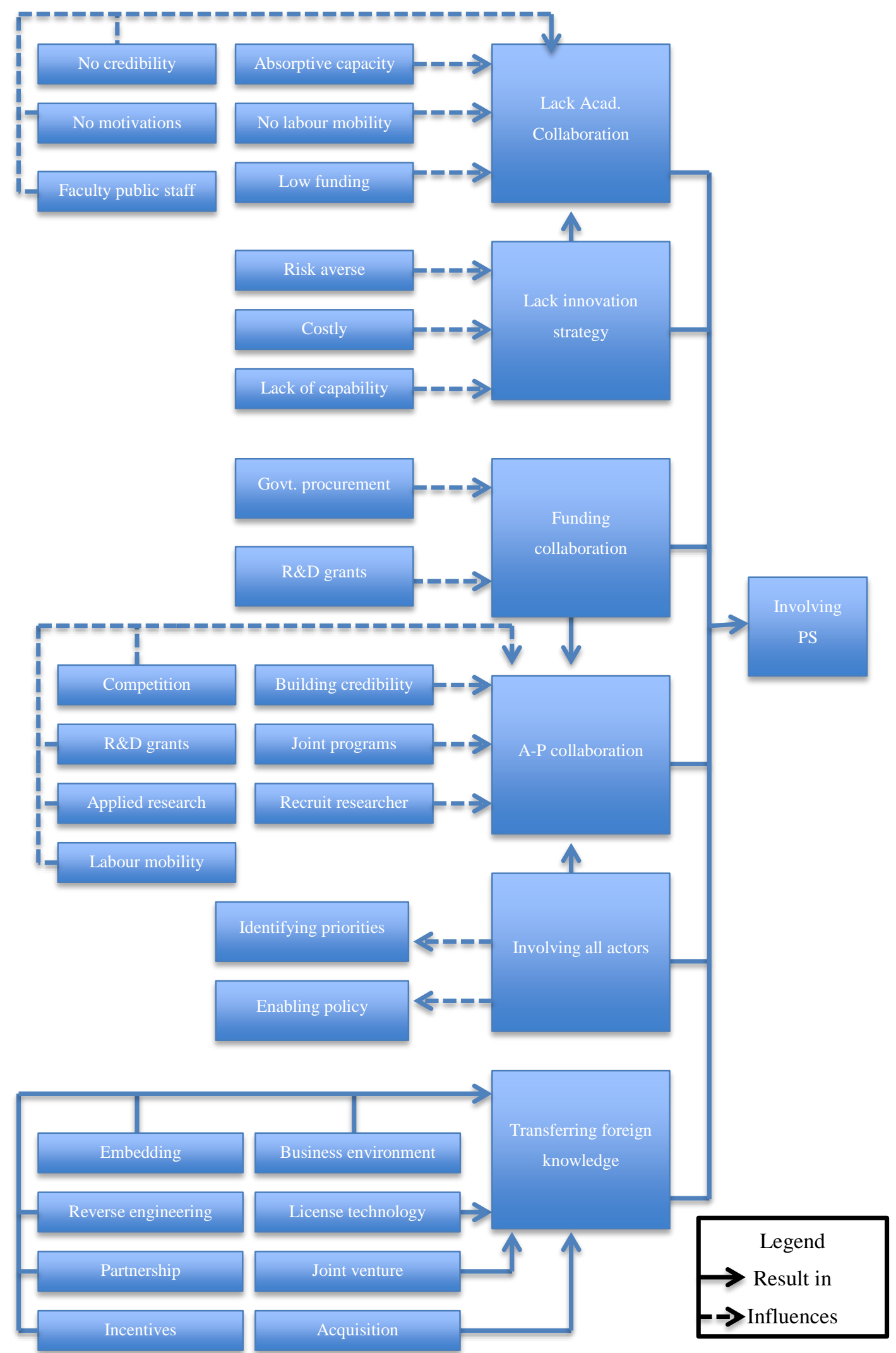




\subsubsection{Summary}

This is the last key requirement that has been proposed to develop a knowledge economy in life science in the KSA. Involving the private sector in the innovation process is one of the big issues in the Middle East and developing countries as the ordinal practices rely on imitation and lack of innovation. So, there is no significant contribution that can be attributed to the private sector in innovative activities.

Generally speaking, there is no innovation strategy or attitude to encourage engagement in the innovation process. According to this investigation, there are several factors behind this behaviour: risk-averse behaviour, innovation perceived as very costly, a lack of capabilities to develop strategies and to conduct strong research.

Another condition found in this study is that there is no collaboration between the PS and academia for the purpose of transferring knowledge or solving problems. This condition has also been affected by many factors: lack of any innovation strategy; lack of absorptive capacity in the private sector to collaborate and to conduct knowledge activities; no motivation for this collaboration between academia and private sector, no credibility for academia in terms of doing good research that convinces and encourages the private sector to engage in collaboration with them; lack of funding for this kind of collaboration; no labour mobility, preventing collaboration and knowledge transfer; and, finally, the public employment status of faculty, with regulations not allowing or encouraging them to commercialise faculty's research outcomes.

Based on the above conditions, four strategic interventions were developed to help involve the private sector in the innovation process and these are presented in the conclusions of this thesis.

Firstly, the funding of academia-private sector collaboration; this can happen through two mechanisms: government procurement, and providing R\&D grants.

Secondly, the development of strong connections between academia and private sector: many factors can help in achieving this - funding collaboration; building 
credibility (if academia produces strong research, that would encourage PS to collaborate); creating a competitive environment in industry, that would motivate PS to seek innovation in order to create differentiation; doing applied research; allowing labour mobility between academia and the PS; developing joint programmes; recruiting talented researchers in PS, and involving all actors.

In turn, and thirdly, involving all actors also helps in developing an enabling policy and identifying priorities and advantages.

The last important intervention to involve the private sector is the transfer of foreign knowledge. This variable needs eight factors to be implemented: improving the business environment; providing incentives; embedding foreign workers; doing reverse engineering; developing partnerships; developing joint ventures; making acquisitions, and licensing technologies. These eight strategies help in developing PS capabilities and then in involving the PS in the innovation process. 


\section{Findings 2}

\subsection{Introduction}

This chapter is dedicated to answering the second research question (What are the challenges in developing $K E$ in $L S$ in the $K S A$ ?). To answer this question data was extracted from the interview transcripts and displayed with context rich participant quotes. A quantitative perspective is also presented to give a clear picture of the challenges that were expected in developing the KSA life sciences sector.

The answer to research question 2 will be extracted and draws from interview question responses: what are the challenges/barriers to progressing LS in the KSA? And also from responses to the other linked questions that were designed to allow insight into these challenges.

\subsection{The challenges}

The answer to this question lies in the following 17 codes we have come across before (see Section 6.4):

No attractive standard of living and restrictive cultural barriers (1), no leadership (2), lack of innovation strategy and entrepreneurial attitude (3), weak entrepreneurship culture (4), lack of connections and academia-PS collaboration (5), weak research infrastructure (6), lack of credibility (7), risk-averse behaviour (8), weak supportive staffing regulations (9), weak institutional quality (10), shortage of medical doctor researchers (11), no collective work in developing policy (12), lack of awareness of the requirements for a KE (13), weak collaboration and networking culture (14), gaps in enabling policy for R\&D and spin-offs (15), lack of high managerial and technical skills (16), and weak business environment (17).

With respect to the author's observation, this many challenges should be expected for a developing country that is just starting to consider building a KE ecosystem. Interestingly, some of the following challenges have been mentioned by other 
participants but without identifying them as challenges; hence, those were not accounted for in the findings relating to the following codes.

\subsubsection{No attractive life standard, and restrictive cultural barriers $(16 \%)^{16}$}

This challenge was suggested by $16 \%$ of the participants (A1, A3, G1, G3, A9). To recruit high-qualified foreign people, especially western people, there are cultural barriers that would make Saudi Arabia, a conservative society where there is gender segregation and some special local customs, not a preferred and competitive destination (A1, G3). Foreign workers should be embedded in order to benefit knowledge transfer (A3, G1). Universities and the entire ecosystem must be international and open to people from everywhere to come and compete, otherwise it will be difficult to develop a KE (A9).

\subsubsection{No leadership (10\%)}

It will be a big challenge to develop a $\mathrm{KE}$ in the LS sector without constant funding and the commitment of the government (A3, P5, P6).

Developing life science industry needs high investment to build infrastructure and attracting key actors to involve in the innovation system, without a leading role from the government the sector would not be developed (Ahn \& York, 2011).

\subsubsection{Lack of credibility $(23 \%)$}

About $23 \%$ of the participants mentioned the credibility issue with regards to different aspects. Because of their modest record in knowledge activities, the local research centres have built no credibility, so local companies do not collaborate with them $(\mathrm{G} 2$, G3, A10, A11). The problem of local research centres is that they are not specialized in very specific areas of knowledge, so that could hinder collaboration with the PS (P3) whenever they are looking for in-depth expertise in well-defined areas. Saudi customers do not trust Saudi products because of this lack of credibility (G4). There is also a lack of promising technologies (A7).

\footnotetext{
16 The percentage based on the entire sample size (31)
} 
The researcher did not find support for this concept in the literature.

\subsubsection{Risk-averse behaviour $(20 \%)$}

This is another challenge hindering the developing of a KE in the LS sector in the KSA. This variable was suggested by many of the participants, but $20 \%$ of them see it as a challenge. The problem with the financial system is that they are looking for zero risk investment (G6), since in the KSA the financial system is risk-averse (G5, A12). The PS is risk averse because it does not have knowledge and background to navigate and manage risk (A4). There is a problem in funding the "Death Valley" stage (seed fund) (A6, G9). Venture investment is a big problem, and it is crucial for developing a KE (A12).

\subsubsection{Lack of innovation strategy and attitude in PS (39\%)}

About $39 \%$ of the participants see the lack of innovation and entrepreneurial attitude in the PS as a big challenge for progressing LS. The PS does not have significant research capabilities (A3, G1, A12) and it doesn't carry out any research activities (A12). There is no strategy for innovating in the PS (G2, P5). The PS is risk averse because it does not have the knowledge to navigate and manage risk (A4). The lack of an innovation strategy has led to no need for collaboration with academia (G7). The private sector does not contribute in doing R\&D and innovation (G10, A11, A12). Many people are not driven by science, technology, innovation, and technology entrepreneurship (A12). A knowledge project needs a lot of things in order to be built, and will take a long time, however, the PS would not consider these factors (P7). Increasing capacity in terms of the number of people doing research is a big challenge and it is necessary for building a LS ecosystem (scaling up current limited knowledge activities) (A9, G9, A12).

\subsubsection{Lack of connections and Academia-PS collaboration (42\%)}

About $42 \%$ of the participants identified the lack of collaboration between academia and PS as a barrier for developing LS. There is no private partner so there is no collaboration (G2, G3, G4, A6, A10, A11). Universities see the PS as a source of money only (P5, P6). Research in the universities does not match commercial needs 
(G1, A4, P5). The PS does not work on developing new products so there is no need for collaboration (G7). There is a gap between the pharmaceutical industry and the academia (P5).

\subsubsection{Weak entrepreneurial culture $(20 \%)$}

About $20 \%$ of the participants see that there is problem with entrepreneurship at all levels: family level, government, PS, financial system level, and the education system. These are all seen to be risk averse: it is a cultural issue (A12). As a result of relying on oil income for long time, people have a cultural problem with entrepreneurship (A3, G11). There is a problem in the work culture in terms of creativity, enthusiasm and discipline, and also entrepreneurship (G1). Researchers do not have an entrepreneurial attitude (G2, A10).

Because of the rentier economy that has was been for long time in the KSA, and the low quality of education system the Saudi people do not tend to take risk in business, and developing this skills take long time, may be generation (Kayed \& Hassan, 2011b).

\subsubsection{Weak research infrastructure $(39 \%)$}

About $39 \%$ of the participants complain about weak research infrastructure and consider it as a serious challenge. Delivering chemicals and reagents or any materials takes a long time because the logistics system is poor (G3, G4, A9, A10). There are no advanced high quality specialized manufacturing centres (prototyping) (G11). The problem of local research centres is that they are not specialized in a very specific area of knowledge (A3). The lack of promising technologies also proved an issue to the informants of this study (A7, A8). There are many technology-based products and ventures, but all are related to the oil and petrochemical industry (A13) and this overdominance of the economy on oil is reflected in the allocation of research assets with few institutions diversifying in line with government strategy. In addition, there is no professional clinical trial centre for commercial drugs approval, which requires specific standards and requirements (P5). Making clinical trials is also quite difficult; people do not agree to do it (P5). An unsuitable LS ecosystem makes Saudi 
researchers, graduated from very reputable international universities, lose their ability and desire to conduct quality research (A8). Increasing capacity in terms of the number of people doing research is a big challenge; however it is necessary for building a LS ecosystem (scaling up current limited knowledge activities) (A9, G9, A12).

Building world-class research infrastructure in Singapore attracted multinational companies to invest and be involved in innovation system. (Finegold, Wong \& Cheah, 2004; Wong, 2005).

\subsubsection{Strict staffing regulation $(16 \%)$}

More than $16 \%$ of the interviewees highlighted the fact that public employment regulations do not offer enough incentives for technicians working in laboratories or for highly skilled managerial jobs, so people are constantly looking for better income and secure jobs (G3, A10, A12). There is no official job title of "researcher" in the government system; the fact that we do not have research jobs, or technology specialists, does not help (A13, P2).

The literature supports the development of enabling policy, but noting special about strict staffing regulations.

\subsubsection{Weak institutional quality $(16 \%)$}

More than $16 \%$ of the interviewees complain about the low efficiency and effectiveness of government institutes and see this as a big challenge. The customs, the Saudi FDA and other relevant institutes are bureaucratic (A10). There is too much bureaucracy in the government institutions $(\mathrm{G} 7)$, there is no systematic organization of work in government institutions (G8). Raising the Saudi FDA's quality and efficiency is a big challenge (P5).

\subsubsection{Shortage of medical doctor researchers $(3 \%)$}

Only one of the participants sees engaging more medical doctors in the research as a serious challenge (G9). 
The literature didn't refer specifically to medical doctors, but scientists in general.

\subsubsection{Weak business environment (16\%)}

More than $16 \%$ of the participants see that there is a challenge in improving the business environment. Visas and regulations for doing business are hindering the development of LS and industry in general (A7, A9, P6). Visa regulations do not have a category for researcher in the workers' system (P2). Non-Saudis are not allowed to own any assets in the two holy cities, Makah and Medina (P7).

According to (Porter, 2014) quality of business environment reflect on the competitiveness of a country, and countries work on improving it to attract investment especially foreign investment.

\subsubsection{No collective work in developing policy (3\%)}

One of the participants sees there are difficulties in developing integration or links between relevant actors (universities, ministry of health, industry, Saudi FDA) for developing R\&D policy (G9).

For most studies, the government has been the leader in establishing a system to allow to other actors to engage but this form of relationship and mutual support is not reflected in the views of the informants. Instead the system is portrayed as disjointed and without a common purpose.

\subsubsection{Lack of awareness of the requirements for a KE (10\%)}

About $10 \%$ consider a lack of awareness as constituting a challenge to developing a $\mathrm{KE}$ (G10). There is poor awareness in terms of knowledge values for society and the economy (A11) the relevant entities fail to understand the requirements to move to a KE (A13).

The reviewed literature did not mention directly to this concept. 


\subsubsection{Weak collaboration and networking culture (13\%)}

With respect to people culture, about $13 \%$ of the participants see building social capital as a serious challenge. There is a lack of collaboration between researchers (G4). Also universities do not have research collaboration (A10). Developing collaboration, networking and trust values are challenges for developing LS (G11). Lack of local collaboration is a chronic problem (G3).

Such relationships were seen to be key to other academic studies, which consider collaboration as significant tool in the innovation process (Rothaermel \& Deeds, 2006) Firms in Singapore have shortcomings in collaboration, and this been considered a hindering factor in Singapore industry (Stein, 2003).

\subsubsection{Lack of enabling policy for R\&D and spin-offs (10\%)}

About $10 \%$ of the interviewees see that there are difficulties in developing enabling policy for R\&D and spin-offs. Public employees cannot own a business and so cannot spin off firms (A10). Regulations and policy do not support private research centres (P2). The government does not fund or support research in the private sector or programme for collaboration with academia (P5).

\subsubsection{Lack of high managerial and technical skills (13\%)}

About $13 \%$ of the participants see the lack of high managerial and technical skills as a big challenge facing the development of a knowledge economy in the life science sector in the KSA. Developing human resources is one of the most critical challenges (A12, P2). The main challenge is in providing the human capital to do high quality research, and also running factories (P6). There is the problem of how to manage the infrastructure and run it, and how to develop the required ecosystem (G6).

To develop its life science sector, Singaporean government developed a specific strategy to build its human capital: by recruiting foreign scientists, sending students to study in developed country and by improving the quality of its education system (Finegold et al., 2004). 


\subsubsection{Summary}

All the seventeen challenges were implicitly addressed in the answer to the first question, which was investigating the requirements for developing LS in the KSA. Developing policy and strategies is a relatively easy task for any country, however implementing those strategies usually presents many difficulties and challenges distributed over different areas extending from government and society to industry.

In this study, the interpretation of collected data originated 17 points perceived by the participants as serious challenges for developing the life science sector in the KSA and requiring special awareness, considerations and attention. Those challenges have different aspects and dimensions including political, economic, social, and financial.

No attractive standard of living and restrictive cultural barriers: this was perceived as an area that must be seriously considered for improvement. Creating an attractive area in terms of a quality education system, quality health system, leisure and also relaxing some cultural barriers in terms of gender segregation, especially for foreign people, is important: the point is to create an international environment instead of being very local.

No leadership: this is a serious challenge; developing LS requires huge investment and patience, and the private sector will not kick off such a project, so it is inevitable that government leadership needs to be developed, especially at the beginning.

Lack of innovation strategy and attitude in private sector: this is a mentality and cultural issue, which means that it needs a long time for adaptation to a new way of thinking. It is concerned with the philosophy of how to create wealth; the prevailing attitude is dominated by traditional thinking which portrays the PS as being able to create wealth without taking risks. Hence it would be a difficult mission to change people's attitude and to convince them to behave innovatively in their strategies and practices.

Weak entrepreneurship culture: a rentier country producing rentier people - this is another social and cultural matter as people are usually looking for security in jobs. 
There is a need to change the attitude toward entrepreneurship and also enact regulations to support entrepreneurship.

A lack of Academia-Industry connections: there are only very modest innovative practices; there are no connections between academia and industry. There is also the cultural issue of the absence of collaboration for the purpose of innovation.

Weak research infrastructure: huge investment is needed for building a strong research infrastructure, creating research capabilities and providing a complete and interactive life science ecosystem; who will fund it and also how it will be managed are issues not yet addressed.

A lack of credibility: this is quite an interesting point; Saudi people are used to buying quality and expensive health items and they are quite "picky", so local products without a previous history and record of credibility may face serious challenges, especially at the beginning.

Risk-averse behaviour: this is the dominating behaviour of the financial system. Developing an entrepreneurial funding system is a really hard endeavour, needing a change in management attitudes and also requiring specific regulations and acts, and government intervention.

Restricted staffing regulations: the public staffing regulation is fixed and does not respond to differentiation in capabilities in terms of salaries and evaluation, so it must be made more flexible to provide the matching salaries which will help in recruiting distinctive people.

Weak public institutional quality: the government system has, for a long time, been very heavy and bureaucratic, so streamlining this system and raising its efficiency and quality is a very tough task and will require a long time, but it is crucial for developing and managing sophisticated strategies and identifying strategic options. 
The shortage of researcher medical doctors: there is a huge shortage in medical doctors in hospitals, so involving them in research is not an easy job in terms of provision and attitude.

Lack of collective planning: the traditional way of developing policy in the KSA has been via a government mission. Changing this behaviour takes a long time, potentially a generational shift.

Lack of awareness of the requirements for a knowledge economy: without raising awareness it would be difficult for society and the relevant actors to understand and support any new orientation, or policies.

Weak collaboration: innovation is no longer a closed model. Collaboration, networking and exchanging trust with other people inside or outside of the boundaries are very important factors. This is a social and cultural issue of the kind that usually requires a long time to change.

The gap in enabling policy for R\&D and spinoffs: the rate of spending on R\&D is still significantly lower than that of developed countries. Providing more spending cannot be assured, especially with fluctuating oil prices, also enacting policy for spinoffs is still delimited by long-standing and obstructive public regulations.

Finally: a lack of managerial and technical skills. Life science is one of the more difficult and complex sciences, and developing promising technologies in this field requires, beside many other things, a huge investment in human capital for extended periods of time to create a critical mass of scientists and highly skilled people.

The following chart presents a quantitative perspective for the challenges of progressing LS in the KSA. Each challenge shown in the horizontal axis corresponds to the value of its number of participants in the vertical axis. 


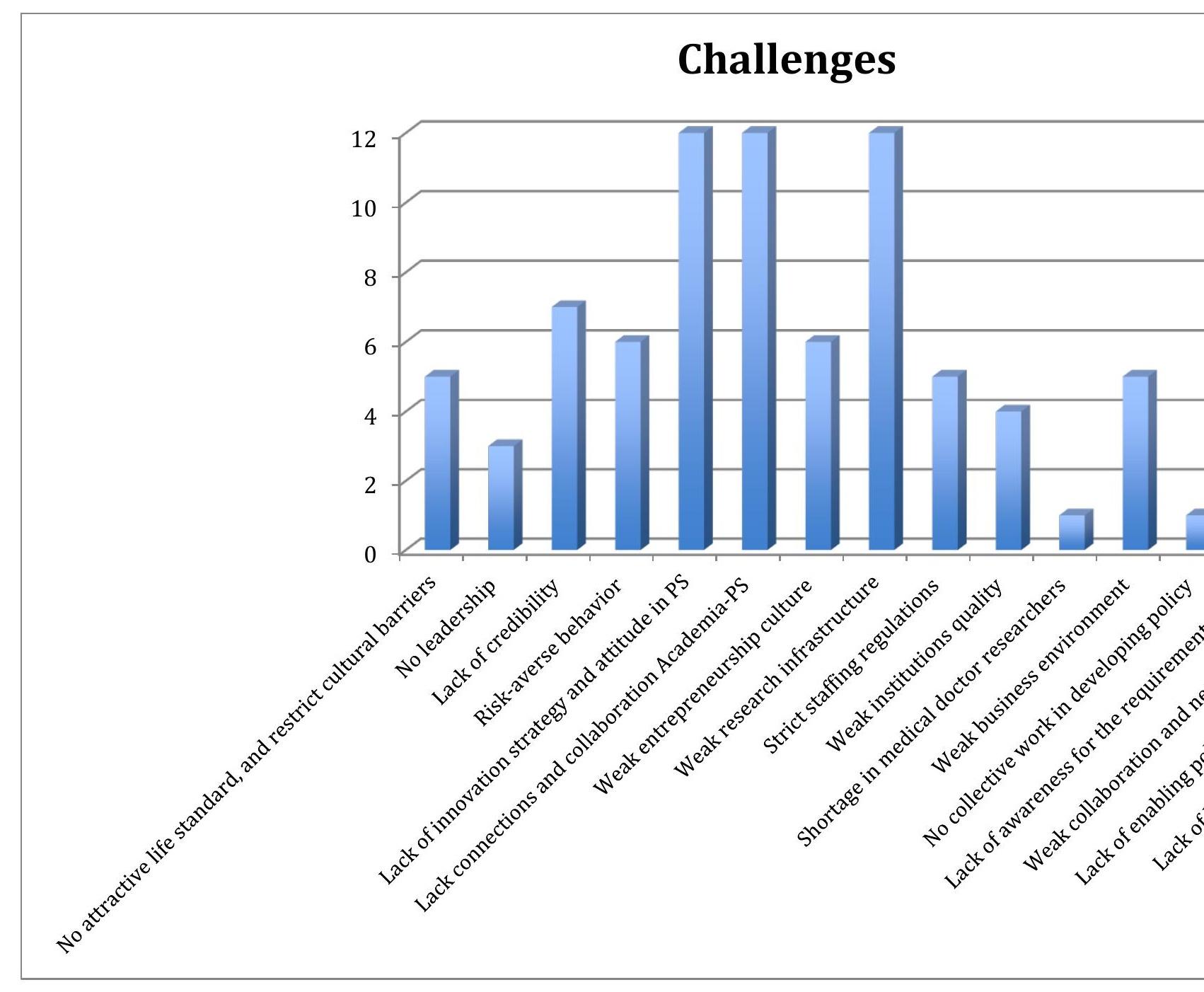

Figure 7-1: Quantitative perspective for challenges facing progressing LS in the KSA. Vertical axis represents pal represents challenges. Source: The researcher. 


\section{Findings 3}

\subsection{Introduction}

Based on the requirements and challenges that have been addressed in Chapters 6 and 7, the researcher systematically developed a LS innovation system model, in order to answer research question 3 (What model of LS innovation system could fit the Saudi context?).

For confirmative purpose a questionnaire was developed to make use of the selective coding theme (involving the private sector) which resulted from a series of SWOT analyses covering the 4 subsectors of the private sector (Multinationals, Large Local companies, SMEs and start-ups).

As first step, for answering research question3 a selective coding process was approached.

\subsection{Selective Coding}

Through the selective coding process the core category ("involving the PS") was selected as a selective code among the axial codes (categories) - developing human capital, developing world-class research infrastructure, developing supportive regulations and enabling policy, and developing entrepreneurial funding and an efficient financial system.

By going through the collected data, this category emerged as the main phenomenon where all other categories connected and interacted; this category captures the story of the data.

For enhancing the rightness of the choice and mitigating the effect of any bias or subjective perspective in identifying the selective code, a quantitative process for the keywords was developed. The flow chart shows to what extent the selective code prevailed within the collected data among other categories. By examining the transcripts, the keywords and relevant meanings were counted with respect to each 
theme. In the following table, the label numbers represent the frequencies of keywords $(\mathrm{KW})$ with respect to each category; this table is followed by a chart portraying the intensity of each theme. 
Table 8-1: Quantitative perspectives for categories` relevant keywords. This table details and compares the relevant keywords appended to each of the five themes (categories) to show which theme has the more prominence and importance in the collected data. About 9 keywords were found related to the private sector, and their frequency achieved 592 times, overcaming all the other keywords related to the other categories (developing human capital, developing world-class research infrastructure, developing supportive regulations and enabling policy, and developing entrepreneurial funding and financial system).

\begin{tabular}{|c|c|c|c|c|c|c|c|c|c|}
\hline \multicolumn{2}{|c|}{ Involving PS } & \multicolumn{2}{|c|}{ Developing HC } & \multicolumn{2}{|c|}{$\begin{array}{l}\text { Developing world } \\
\text { class research } \\
\text { infrastructure }\end{array}$} & \multicolumn{2}{|c|}{$\begin{array}{l}\text { Developing } \\
\text { supportive } \\
\text { regulations and } \\
\text { enabling policy }\end{array}$} & \multicolumn{2}{|c|}{$\begin{array}{l}\text { Developing } \\
\text { entrepreneurial } \\
\text { funding and } \\
\text { financial } \\
\text { system }\end{array}$} \\
\hline $\begin{array}{l}\text { KW } \\
\text { number }\end{array}$ & I & KW / number & & KW / number & & KW / numb & & $\mathrm{KW} / \mathrm{nu}$ & \\
\hline PS & 70 & $\begin{array}{l}\text { Human } \\
\text { (capital, } \\
\text { resources) }\end{array}$ & 42 & Infrastructure & 54 & Regulation & 60 & Fund & 172 \\
\hline Company & 76 & $\begin{array}{l}\text { Gradate, } \\
\text { postgraduate }\end{array}$ & 33 & $\begin{array}{l}\text { Research } \\
\text { centre }\end{array}$ & 44 & Policy & 45 & Money & 43 \\
\hline Firm & 140 & Researcher & 96 & Ecosystem & 41 & Plan & 63 & Finance & 15 \\
\hline Industry & 129 & Student & 102 & Logistic & 10 & Strategy & 47 & $\mathrm{VC}$ & 36 \\
\hline SMEs & 29 & Capabilities & 42 & & & Rules & 4 & $\begin{array}{l}\text { Death } \\
\text { valley }\end{array}$ & 2 \\
\hline Start-up & 48 & $\begin{array}{l}\text { Expert, } \\
\text { expertise }\end{array}$ & 16 & & & Law & 3 & & \\
\hline FDI & 9 & Doctor & 15 & & & System & 85 & & \\
\hline $\mathrm{MNCs}$ & 3 & $\mathrm{PhD}$ & 14 & & & Initiative & 17 & & \\
\hline $\begin{array}{l}\text { Service } \\
\text { providers }\end{array}$ & 8 & & & & & & & & \\
\hline Total & 592 & & 360 & & 149 & & 378 & & 268 \\
\hline
\end{tabular}

Source: The researcher.

${ }^{17} \mathrm{KW}=$ keywords 


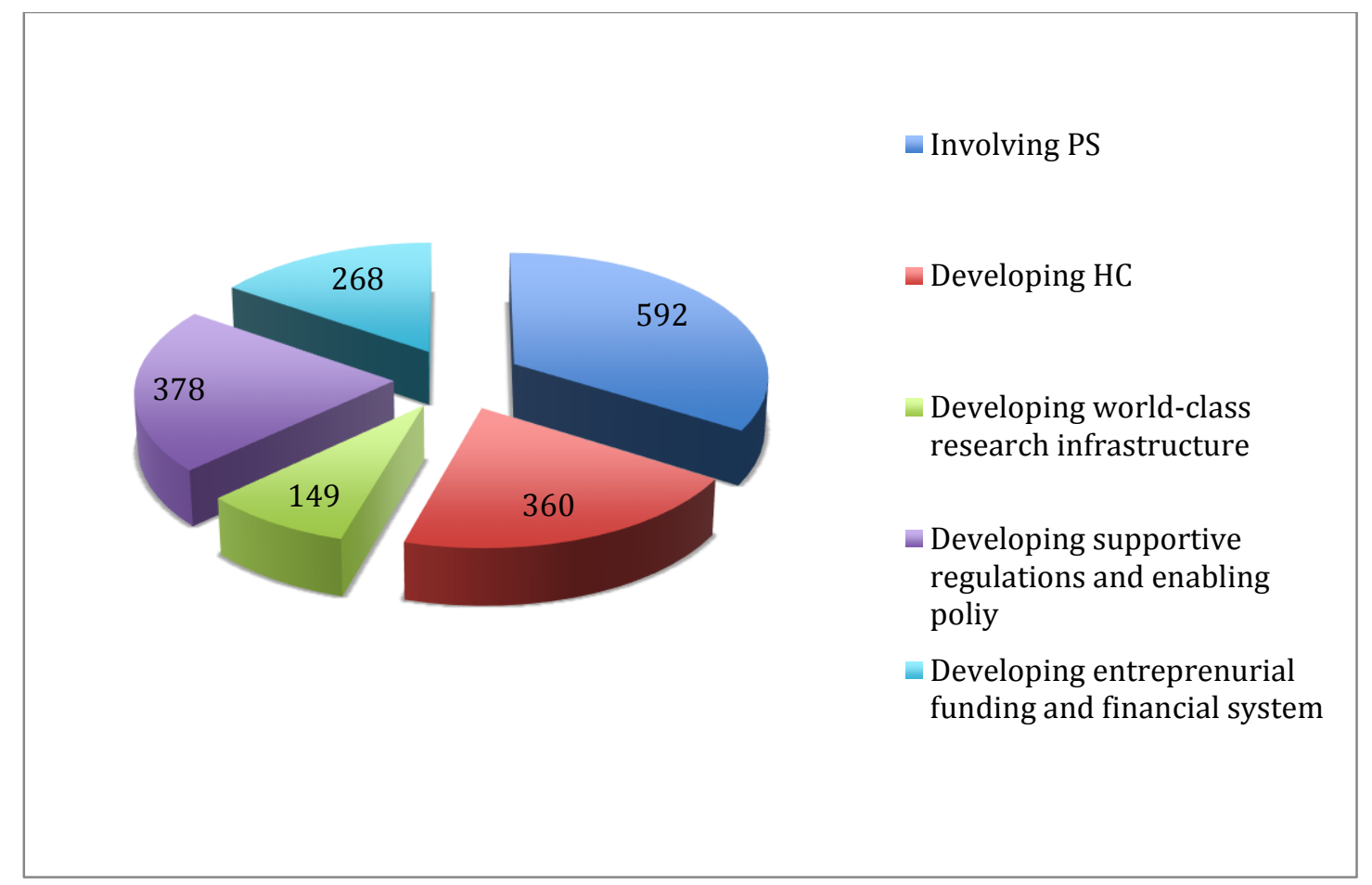

Figure 8-1: Quantitative perspectives for catgories`relevant keywords. Source: The researcher.

The selective code is considered the pivot and the centre that collected data revolve around. It dominates the story of the data and the phenomena embedded in the data. The prevalence of meanings in the data that refer to a specific theme increase its probability to be selective code, solving the phenomena problem relies significantly in addressing the role of selective code (Corbin \& Strauss, 1990; Corbin \& Strauss, 1994; Glaser \& Holton, 2005).

The following table shows all themes and codes resulting from the three coding processes (open coding, axial coding and selective coding). 
Table 8-2: Properties of categories as identified by their related open codes. The selective code is highlighted in co

\begin{tabular}{|c|c|c|c|c|}
\hline Axial codes & Developing $\mathrm{HC}$ & $\begin{array}{l}\text { Developing World-class } \\
\text { research infrastructure }\end{array}$ & $\begin{array}{l}\text { Involving (PS) } \\
\text { (Selective code) }\end{array}$ & $\begin{array}{l}\text { Developi } \\
\text { regulatiol } \\
\text { policy }\end{array}$ \\
\hline \multirow{10}{*}{ Open codes } & $\begin{array}{l}\text { The pool of scientists not so } \\
\text { big }\end{array}$ & $\begin{array}{l}\text { The research capabilities are } \\
\text { weak }\end{array}$ & $\begin{array}{l}\text { Lack of Academia-PS strong } \\
\text { collaboration }\end{array}$ & Raising in \\
\hline & $\begin{array}{l}\text { Shortage of scientific } \\
\text { graduates }\end{array}$ & $\begin{array}{l}\text { Research infrastructure is } \\
\text { weak }\end{array}$ & $\begin{array}{l}\text { Lack of innovation strategy and } \\
\text { entrepreneurial attitude in PS }\end{array}$ & Building : \\
\hline & $\begin{array}{l}\text { Lack of high managerial and } \\
\text { technical skills }\end{array}$ & $\begin{array}{l}\text { Developing strong research } \\
\text { centres }\end{array}$ & $\begin{array}{l}\text { Funding programme for academia- } \\
\text { PS collaboration }\end{array}$ & $\begin{array}{l}\text { Building } \\
\text { priorities }\end{array}$ \\
\hline & $\begin{array}{l}\text { Engaging more medical } \\
\text { doctors in research }\end{array}$ & $\begin{array}{l}\text { Developing clusters based on } \\
\text { strengths and priorities }\end{array}$ & $\begin{array}{l}\text { Developing academia-PS strong } \\
\text { connections }\end{array}$ & $\begin{array}{l}\text { Supportin } \\
\text { culture }\end{array}$ \\
\hline & $\begin{array}{l}\text { Exposing to international } \\
\text { effects }\end{array}$ & $\begin{array}{l}\text { Developing research } \\
\text { entrepreneurial universities }\end{array}$ & $\begin{array}{l}\text { Involving all actors in developing } \\
\text { policy }\end{array}$ & Governme \\
\hline & $\begin{array}{l}\text { Raising education system's } \\
\text { quality }\end{array}$ & $\begin{array}{l}\text { Transferring foreign } \\
\text { knowledge }\end{array}$ & Transferring foreign knowledge & $\begin{array}{l}\text { Developir } \\
\text { for } R \& D\end{array}$ \\
\hline & & $\begin{array}{l}\text { Developing collaboration } \\
\text { with foreign parties }\end{array}$ & & $\begin{array}{l}\text { Improvin } \\
\text { infrastruct } \\
\text { environm }\end{array}$ \\
\hline & & $\begin{array}{l}\text { Developing online platform } \\
\text { to ease collaboration }\end{array}$ & & $\begin{array}{l}\text { Improving } \\
\text { and relaxi } \\
\text { barriers }\end{array}$ \\
\hline & & Developing LS specialties & & $\begin{array}{l}\text { Developir } \\
\text { staffing re }\end{array}$ \\
\hline & & & & $\begin{array}{l}\text { Developir } \\
\text { coordinati }\end{array}$ \\
\hline
\end{tabular}


The results in the above table come with key categories (themes) and their properties (under every category). Generally speaking these categories strongly supported by the literature. For example, the World Bank developed four pillars for building knowledge economy (human capital, innovation system, regime and regulation, ICT) see section 3.5.1, and there are considerable similarities with these categories. However, every context has its own conditions which might entail different strategies and management.

\subsection{Answer for Research Question 3}

\section{What model of LS innovation system could fit Saudi context?}

A National Innovation System "is a set of institutions whose interactions determine the innovative performance of national firms" (Nelson, 1993). Nelson also emphasizes that the core of an innovation system is the policies for science, technology and innovation that support R\&D. Lundvall considers a National Innovation System as "constituted by elements and relationships, which interact in production, diffusion and use of new, and economically useful knowledge" The mentioned elements include institutions such as universities and public research centres, firms, government regulatory systems, the educational system and financial institutions (Benoît Godin, 2009). Although the national scope covers regional and sectorial systems, some industries and regions have their own specific characteristics and conditions which should be taken into account when developing policies (Malerba \& Nelson, 2011; Niosi, 2011a).

\subsubsection{LS model (themes level)}

The theoretical coding process that was applied over the coding process resulted in a basic social process where the theme "developing supportive and enabling policy" feeds into the themes: developing human capital, developing research infrastructure, involving the private sector, and developing an entrepreneurial funding financial system. All these five influences result in the development of a LS innovation system that is constantly changing and evolving over time. 
"As s/he constantly compares incidents and concepts s/he will generate many codes, while being alert to the one or two that are core. S/he is constantly looking for the "main theme", for what -in his or her view-is the main concern or problem for the people in the setting; for that which sums up, in a pattern of behaviour, the substance of what is going on in the data, for what is the essence of relevance reflected in the data, for categories (gerunds) which bring out process and change (two properties of BSPs [Basic social processes])” (B. G. Glaser \& Holton, 2005).

This model has been considered the first stage in developing the final Viable Saudi LS innovation system (VSLSIS) (Figure 8-8), it gives the foundation and draws the first image about the final model and shows aspects of the relationships between themes. 


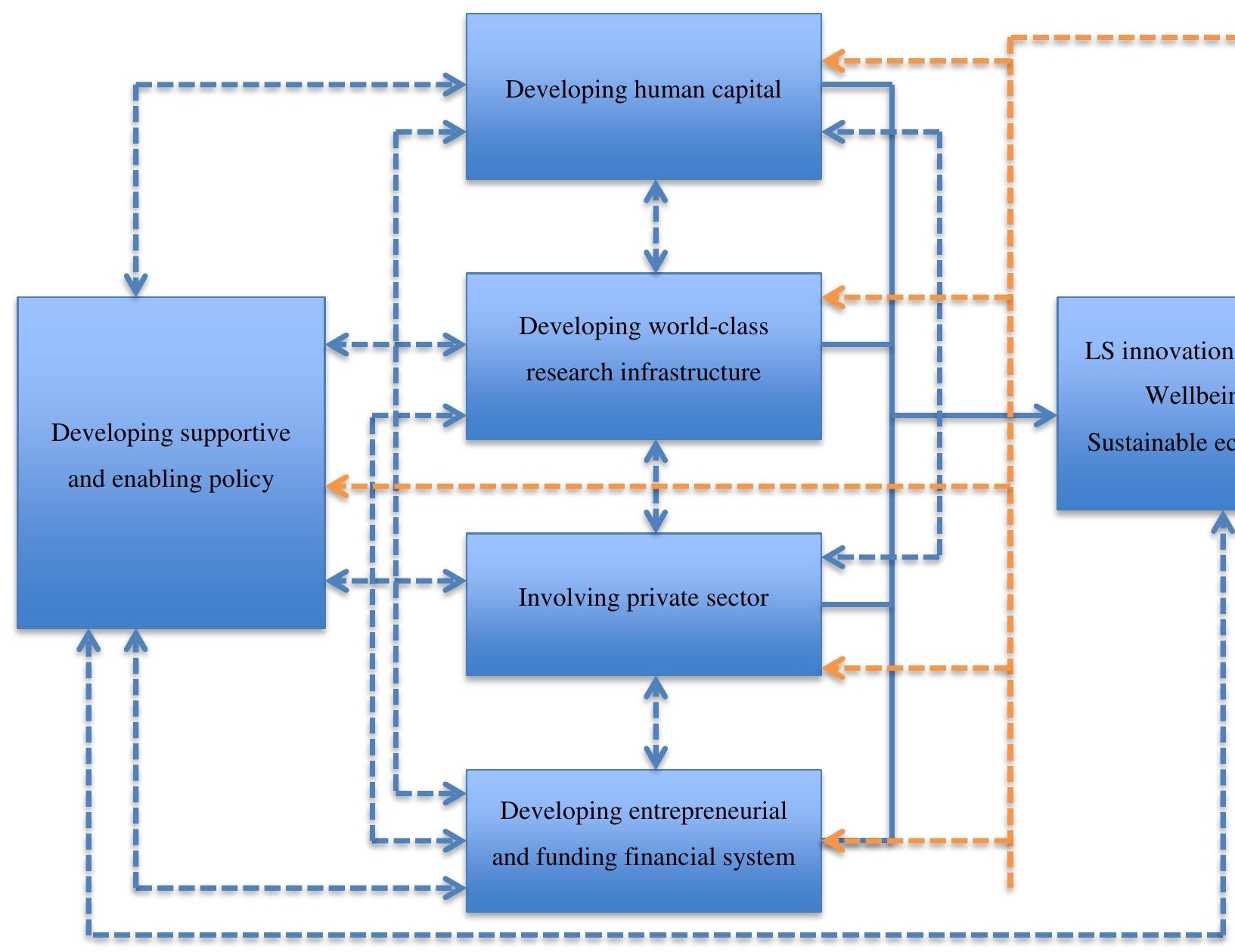

Figure 8-2: The themes model. Developing supportive and enabling policy is viewed as the initial step that influen themes. Source: the researcher. 


\subsubsection{The themes model summary}

The above figure represents the life science innovation model (themes model); this model shows the relationships of variables at the higher level of analysis (themes level).

This model was developed to serve as a foundation to the detailed model (codes model) shown in Figure 8-4.

The Saudi LS sector is relatively big compared to that of the Middle East region. There are special advantages, for example, religious visitors to the two holy mosques (Makkah, and Madinah), currently more than 10 million annual visitors with plans to accommodate more than 30 million by 2030 ; people from very different ethnicities and with good financial capacity.

Currently, knowledge activities in the LS sector are very limited, and collaboration culture with the aim of creating innovation is very weak. From the above developed themes model (Figure 8-2), we can understand how the model works; for developing life science sector in the KSA, the model assumes the beginning should be through developing supportive and enabling policy (Figure 6-10), with this category connecting, in a reciprocal manner, with the other four categories (developing human capital, developing world-class research infrastructure, involving the private sector, and developing entrepreneurial and funding financial system) (see Figure 6-7, Figure 6-8, Figure 6-9, Figure 6-10 and Figure 6-11 respectively). All five categories interact and influence each other in a complex manner and all of them collectively result in a life science innovation system, local wellbeing, and contributing and helping to develop a sustainable economy.

The local LS innovation system also connects with key actors in the global landscape to keep updating and improving by transferring best practice, so this connection with the global context enables the local life science innovation system to feedback the category "developing supportive and enabling policy", whereby policy can be updated 
and fitting strategies developed, to keep it constantly responsive and adaptive to the changing global environment.

The global context also directly influences these processes: developing HC, developing policy, developing research infrastructure, developing an entrepreneurial funding system, and involving the PS, and helps with developing capabilities, learning best practices, and transferring knowledge.

The model starts by developing policy for all relevant areas as identified by the four categories (human capital, research infrastructure, involving the private sector, and funding system). The project should be led by the government, and this policy should be developed by a special organization involving all actors (academia, government, private sector) in developing policy based on the priorities and advantages that already exist. Raising the quality of the institutions involved in the development of the policy is very important, otherwise the quality of the policy and strategies for development and management would be questioned.

As a result of the shortage in scientific graduates, a small pool of high quality researchers and scientists, and the lack of high managerial and technical capabilities in academia, the PS, and even the public sector, the policy must work by tackling these issues linked to human capital, through the development of strategies and policy that help in building human capital through raising the quality of the education system, by exposure to international effects through sending students abroad to study in high-quality research and education institutes and through the recruitment of highly qualified foreign people. Development in policy is also needed to help support entrepreneurship, and to build social capital. The relationship is reciprocal, so the development of human capital will impact later on improving the policy quality by engaging highly knowledgeable people; also, the policy is path dependent ${ }^{18}$ and the feedback cycle between the two elements (developing policy and developing human capital or any other element in the model) is a continuing process.

\footnotetext{
${ }^{18}$ Which means that ongoing development of the policy will depend on the consequent experience and outputs.
} 
The second strand of policy concerns developing world-class research infrastructure. Because of the weak research infrastructure, weak research capabilities, and lack of LS specialties, this requires the development of strong research centres and research entrepreneurial universities; the improvement of the business environment; supportive staffing regulations, living standards and more relaxed cultural barriers; clusters based on strengths and advantages; providing LS specialties; developing an online platform to ease local collaboration, and developing partnerships and collaborations with foreign partners to feedback and update local innovation system quality, and to sustain potential growth.

The third strand of policy concerns developing an entrepreneurial funding and financial system; the current situation is a risk-averse financial system and there is a scarcity of Venture Capitalist firms (VCs), so there is a need for developing R\&D funding grants; more VCs; seed funding; raising funding efficiency, and attracting and motivating angel investors.

The fourth strand of policy concerns developing strategies to help in involving the private sector in the innovation process; one of the main problems of the innovation system in the LS sector is the low research capabilities and capacity of private sector organizations; the lack of innovation strategy and attitude, working in silos, with consequent isolation of the innovation process; these conditions make the knowledge transfer (KT) process and survival of any potential innovative project or process difficult, whereas involving the PS would increase the research output and complete the cycle of innovation.

There are no linkages between academia and PS and government for the sake of developing innovation systems. Therefore, the model pays great attention to the development of strong connections between academia and the PS; the funding of programmes for collaboration between academia and the PS; the involvement of all actors in developing relevant policy; the raising of PS absorptive capacity; the embedding of foreign workers to facilitate the KT; the transfer of foreign knowledge by the means of acquisition, JV, and licensing technologies, where FDI helps also in interaction with global value chain and improving work culture in term of discipline, collaboration, creativity, and entrepreneurship. 
All the innovation system and themes connect the global actors so that they may keep updated and transfer best practice which can enable the local actors and the entire innovation system to be adaptive and responsive to any changes that might take place in the global landscape.

Before presenting the detailed model at the codes level, the following Figure 8-3 shows the intensity of all concepts that constitute the codes model in Figure 8-4. 


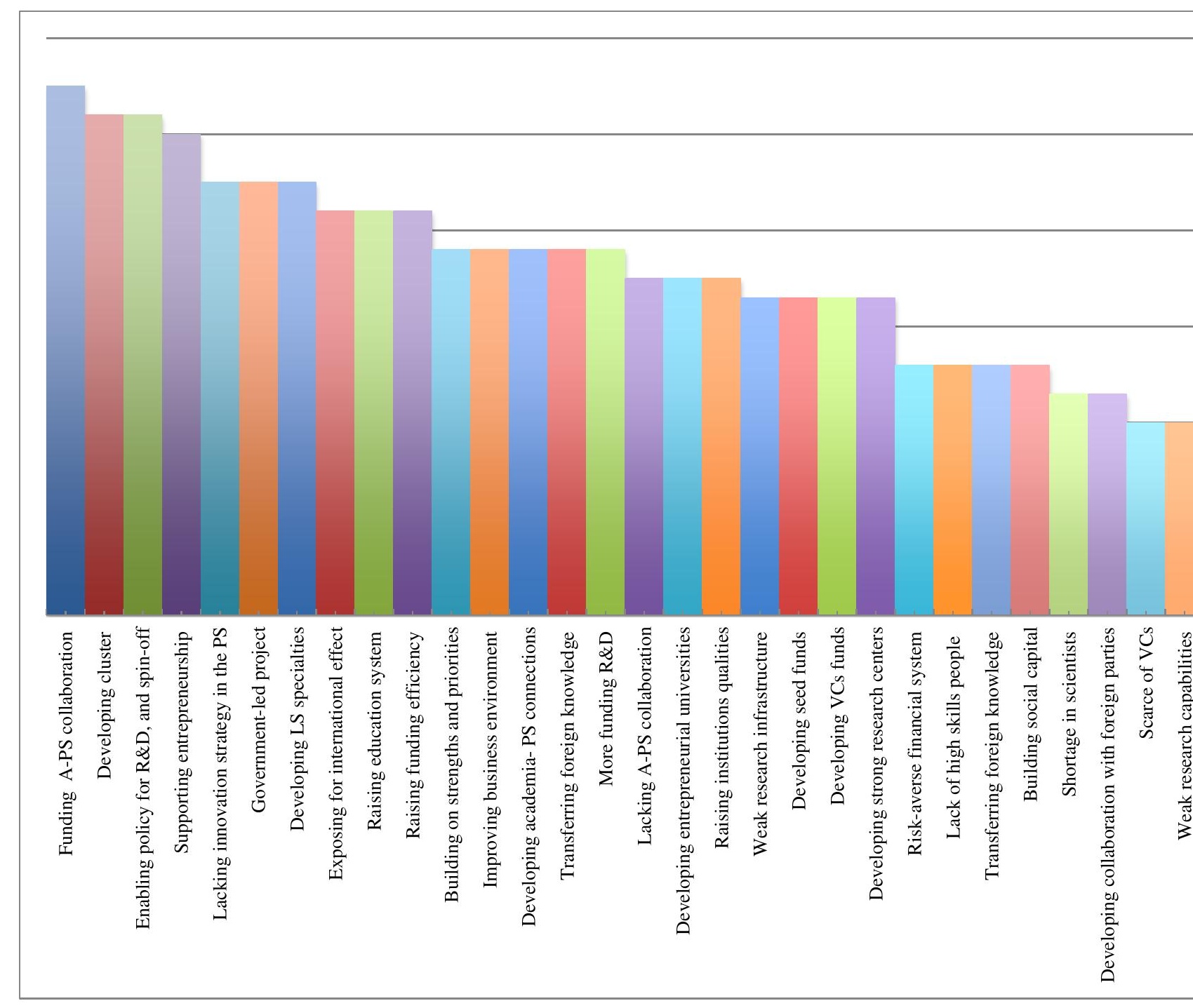

Figure 8-3: All the codes (Vertical Axil) ordered in intensity descending order according to participants' point of 


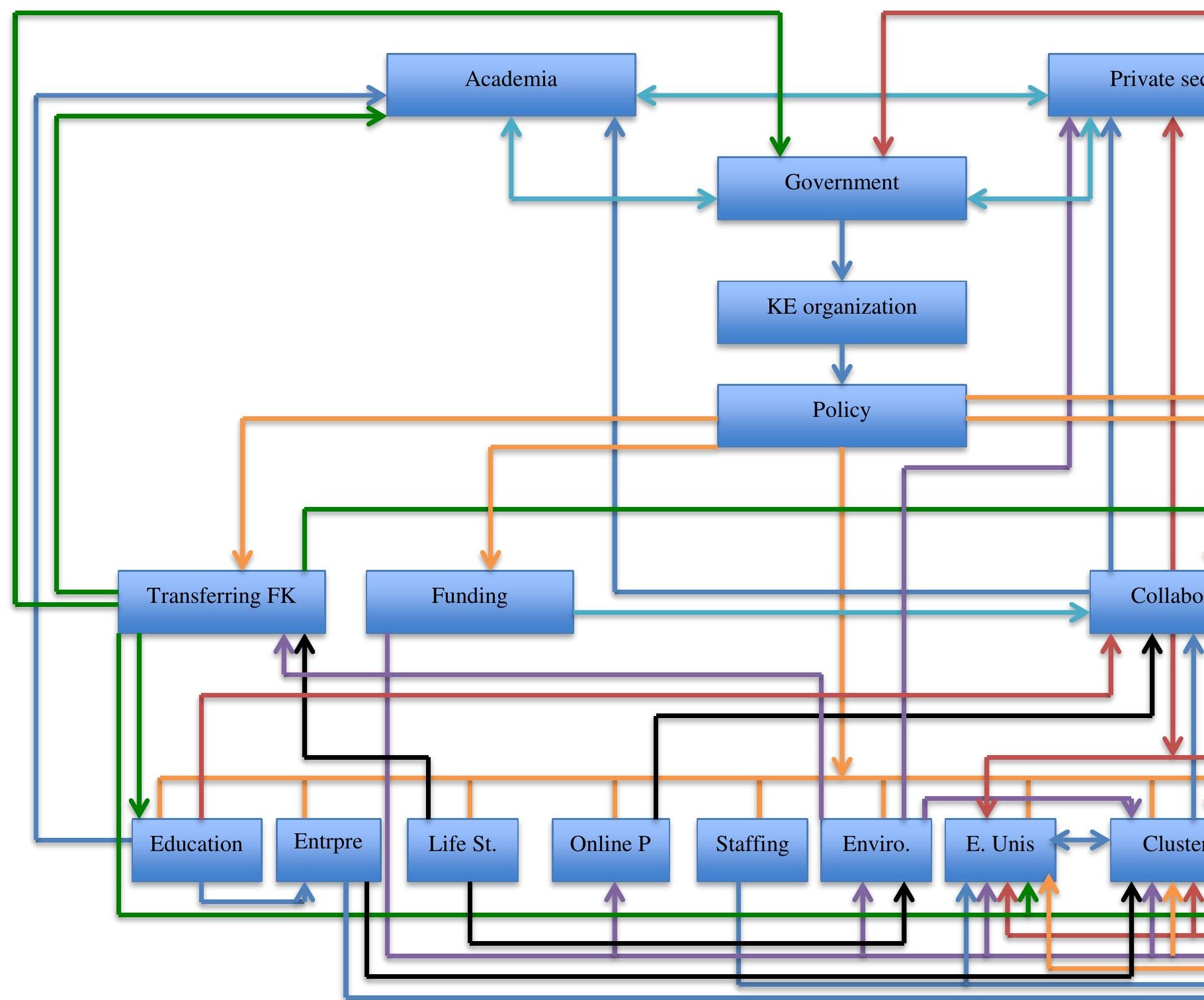

Figure 8-4: The codes model. This model shows the prominent relationships among the key codes). Source: The $r$ 


\subsubsection{The code model summary}

The model in Figure 8-4 outlines in detail the complex relationships between the concepts (codes). The different colours have been used to help identify and distinguish the various relationships and are not indicative of any specific property.

The model tackles the current shortcomings and weaknesses, and identifies and organically introduces interventions in order to build an effective life science innovation system.

Currently the research infrastructure is weak and there is no quality research. There are shortages in both the quality and quantity of capabilities, and there is no enabling policy supporting entrepreneurship and engagement in knowledge activities. The funding system is risk-averse, and the private sector has no strategy or desire to conduct research and create innovation.

The project of building the KE in KSA has currently no effective, invested leadership drive; there is also no system through which all actors should work to develop policy and share decision-making.

The code model of suggests that all actors (government, academia, and private sector) should work together through the Knowledge Economy Organization (KEO). The KEO should develop the relevant policies.

The policies cover many areas and activities:

- Raising institutional quality and efficiency, which would reflect on the performance of government institutions.

- Funding R\&D, collaboration between academia and private sector, and other infrastructure investments such as an online platform, the business environment and logistics, entrepreneurial universities, LS clusters, research centres (RCs), and specialties.

○ Developing policy that concerns the transfer of foreign knowledge to academia and the PS and RCs, building clusters' capabilities; and from academia to the PS to solve problems and build spinoffs.

- Developing policy that concerns raising the quality of the education system; supporting entrepreneurship; improving living standards; building online 
platforms to ease collaboration; improving the business environment; relaxing staffing regulations; building entrepreneurial universities; building LS clusters; building strong research centres; developing specialties, like clinical trials and labs etc., and developing strategies based on local priorities and advantages.

○ In turn, the education system would work on spreading an entrepreneurial culture and collaboration and developing human capabilities.

- Improving living standards would help with the transfer of knowledge by providing an attractive environment for businesses and talents to come to Saudi Arabia.

- Relaxing staffing regulations would help to encourage knowledge transfer from universities, research centres, and clusters by attracting talent.

- Improving the business environment would influence the transfer of knowledge from foreign sources, the PS, entrepreneurial universities, and the planned clusters.

○ Developing entrepreneurial universities would influence the development of clusters and would develop reciprocal relationships to help transfer knowledge and labour mobility.

○ Building LS clusters would positively influence the collaboration between PS members, with academia, and with public RCs.

○ Developing specialties would help universities, clusters and RCs by providing some specialist, value-added and distinctive services.

- Finally, identifying the priorities and advantages will inform the relevant parties such as cluster members, academia and RCs with information and plans about the areas of interest.

It is believed that it will be worthwhile to present different aspects and perspectives for the Saudi LS innovation model, which will hopefully at the end draw a clear and complete picture that reflects and interprets the data relevant to the proposed innovation system.

The results presented different concepts (codes) and mostly all these concepts are supported by the literature, however every context has its own dynamics that shape the relations between all concepts. 
The next figure presents the model in a way that clarifies the relationships between all key actors in the Saudi LS Innovation Model (SALSIM). 
Figure 8-5: Relationships among the actors model.

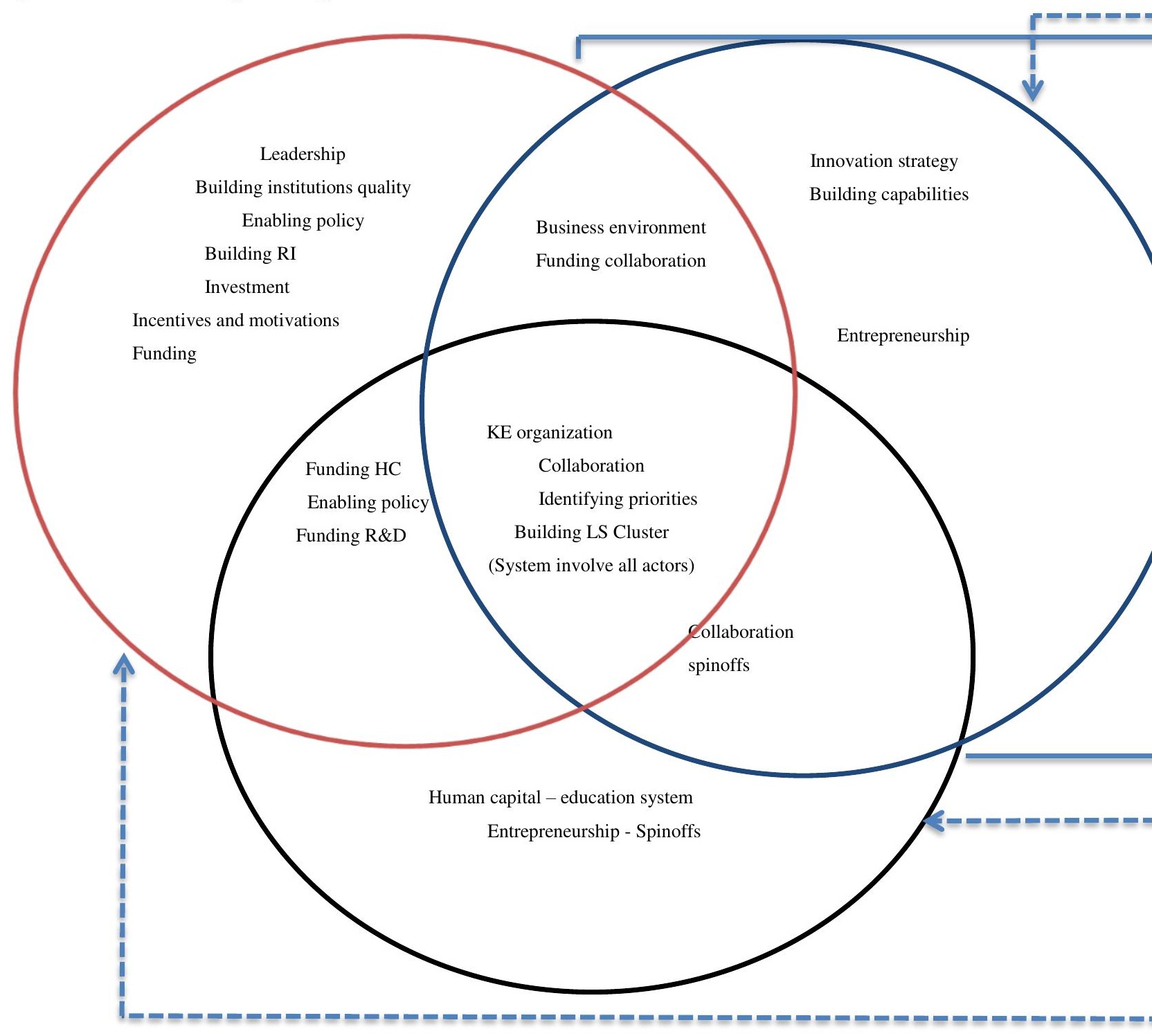




\subsection{Relationships between Actors Model}

Figure 8-5 shows the relationships in the developed Saudi LS innovation model between the key actors: government; industry, and academia.

In this model, the government is responsible for:

- Assuming the leadership for this system; otherwise no other actor would take any significant initiative,

- Building a strong research infrastructure,

- Raising its institutions' quality to be able to develop and manage enabling policy; raising efficiency and performance, and adapting any kind of change in the global landscape,

- Developing enabling policy that helps in transferring knowledge and supporting entrepreneurship, and improving the business environment,

- Providing incentives and motivations, and

- Providing funding.

All these activities meet with the interests with other actors. For example, industry will be involved and benefit from these activities through improvements in the business environment, and funding being made available for collaboration between academia and industry.

At the same time industry should be involved in the innovation system by adopting some strategies such as:

- Developing innovation strategies

- Building capabilities that can undertake high-quality research and manage innovative projects, and

- Developing entrepreneurial attitudes.

These strategies would also intersect with academic interests, which would benefit from government funding and support for building collaborations for common projects, having labour mobility, problem solving and developing spinoffs.

Furthermore, academia will benefit from government funding and enabling policy for:

- Building human capital,

- Raising the quality of the education system, 
- Entrepreneurship and creating spinoffs.

This intertwining and overlapping between the key actors should result in a system which is an organic unity. To this end it is suggested that the system should have a platform constituted as an entity (a knowledge economy organisation, or KEO) which works in a systematic way and is in charge of involving all actors in developing the enabling policy; identifying priorities and advantages; supporting and funding collaboration, and building a life science cluster representing a physical life science innovation project (i.e. similar to a science park). The initial development of this should be kick-started by government investment to create the critical mass of investment in knowledge activities.

This innovation system should interact within the global context to transfer knowledge and learning from best practice developed around the world; it should adapt to any changes which might take place and affect local systems.

Individually, all actors should be concerned with transfer of foreign knowledge, developing partnerships and collaborations with foreign partners.

The next figure shows the empirically derived model, developed based on the raw grounded theory research, which represents the actual practice of innovation in the Kingdom of Saudi Arabia (the LS Innovation System). 
Figure 8-6: Saudi Life Science Innovation Model (SALSIM). The figure shows the emperically derived model th theory research, which represents the actual practice of innovation in the Kingdom of Saudi Arabia (the LS Inno)

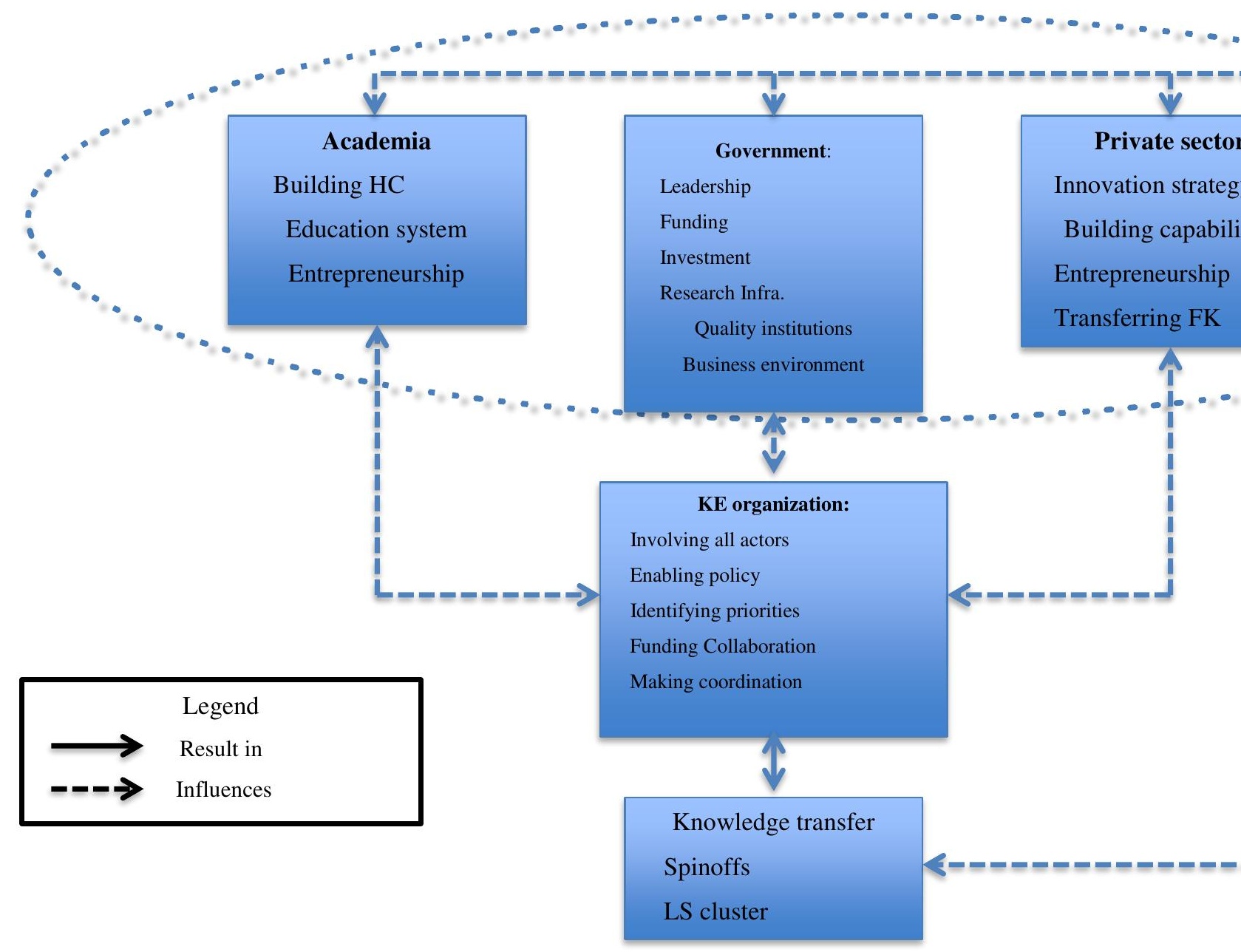




\subsubsection{Saudi LS Innovation Model (SALSIM)}

The above Figure 8-6 the empirically derived model, the Saudi LS Innovation Model (SALSIM). The three key actors represent the inputs to the system. The Knowledge Economy Organization processes activities such as: involving all actors; developing enabling policy; identifying priorities; promoting coordination, and funding collaboration. It is envisaged that the outputs of this system will result in knowledge transfer from academia and research centres to industry and vice versa, spinoffs, and life science clusters.

The proposed system interacts within the global context through activities aimed at transferring knowledge; these activities are: building joint ventures; making acquisitions; licensing technologies, and developing partnerships. At the same time, and individually, all the local key actors interact with the global context to develop capabilities, learning best practice and keeping updated with any changes.

\subsection{Questionnaire}

In order to increase the robustness of the investigation this questionnaire (quantitative approach) has been used as a triangulation method (descriptive and exploratory study) to help further identify the variables that would help to involve private sector.

Survey monkey has been used and the questionnaire has been sent out to 36 senior stakeholders of the LS sector, four of them in the UK and the rest from Saudi Arabia. About $80 \%$ (29 respondents) of them answered the questionnaire, three of the respondents were from the UK and the rest were from the KSA. Questionnaire form and coding book are included in the appendices $(12.5,12.6$.) respectively.

\subsubsection{Objective of questionnaire}

The objective of this part of the study was to identify the strategies or factors that would encourage/discourage or hinder the different private sub-sectors, namely: Multinational enterprises (MNEs), local large companies (LLCs), small to medium enterprises (SMEs), and knowledge-based start-ups (KB-SUs) being involved in undertaking knowledge activities. 
Beside the usual demographic questions (gender, sector, position, nationality, country where the informant lives, age) the questionnaire included 88 ordinal variables distributed unequally into 17 questions (four questions for every sub-sector) and one question about the private sector in general: to what extent it is important to involve the private sector in developing the knowledge economy (KE) in the life science (LS) sector in the KSA?

All these variables and strategies were mostly derived from the first study findings in the qualitative part of this research, and from literature.

\subsubsection{Frame of analysis}

Regarding the types of questions (ordinal questions) and the objective of investigation please see (4.4.2.2.3), descriptive statistics were implemented to measure the central tendency of the participants by applying a mode test to identify any internal or external variables that would encourage or hinder the involvement of the private subsector in the knowledge activities in the LS sector in the KSA. Furthermore, frequencies for the demographic questions were also extracted.

\section{Measurement code}

Table 8-3: Measurement codes.

\begin{tabular}{|l|l|}
\hline Measurement & Value \\
\hline Strongly agree & 5 \\
\hline Agree & 4 \\
\hline Uncertain & 3 \\
\hline Disagree & 2 \\
\hline Strongly disagree & 1 \\
\hline
\end{tabular}

Source: The researcher 


\subsubsection{The findings}

This section includes six sub-sections: demographic statistics, the private sector (life science), multinational enterprises (MNEs), local large companies (LLCs), small and medium enterprises (SMEs), and knowledge-based start-ups (KB-SUs).

\subsubsection{Demographic statistics}

\section{Gender details}

Table 8-4: Gender details.

\begin{tabular}{|l|l|l|l|}
\hline Gender & Frequency & Per cent & Cumulative Per cent \\
\hline Male & 27 & 93.1 & 93.1 \\
\hline Female & 2 & 6.9 & 100.0 \\
\hline Total & 29 & 100.0 & \\
\hline
\end{tabular}

Source: The researcher

\section{Sectors details}

Table 8-5: Sectors details.

\begin{tabular}{|l|l|l|l|}
\hline \multicolumn{1}{|c|}{ Sector } & Frequency & \multicolumn{1}{c|}{ Per cent } & \multicolumn{1}{c|}{ Cumulative Per cent } \\
\hline Government & 5 & 17.2 & 17.2 \\
\hline Private sector & 10 & 34.5 & 51.7 \\
\hline Academic (Public) & 10 & 34.5 & 86.2 \\
\hline Academic private & 3 & 10.3 & 96.6 \\
\hline $\begin{array}{l}\text { Public research } \\
\text { centre }\end{array}$ & 1 & 3.4 & 100.0 \\
\hline Total & 29 & 100.0 & \\
\hline
\end{tabular}

Source: The researcher 
Job details

Table 8-6: Job details.

\begin{tabular}{|c|c|c|}
\hline Main Occupation & Frequency & Per cent \\
\hline Research & 9 & 31.0 \\
\hline Management & 18 & 62.1 \\
\hline Consultancy & 2 & 6.9 \\
\hline Total & 29 & 100.0 \\
\hline
\end{tabular}

Source: The researcher

\section{Nationality details}

Table 8-7: Nationality details.

\begin{tabular}{|l|l|l|l|}
\hline Nationality & Frequency & Per cent & Cumulative Per cent \\
\hline Saudi & 20 & 69.0 & 69.0 \\
\hline Other Arabic & 5 & 17.2 & 86.2 \\
\hline Other & 4 & 13.8 & 100.0 \\
\hline Total & 29 & 100.0 & \\
\hline
\end{tabular}

Source: The researcher

\section{Country of residence details}

Table 8-8: Country of residence details.

\begin{tabular}{|l|l|l|l|} 
Living country & Frequency & Per cent & Cumulative Per cent \\
\hline Saudi Arabia & 26 & 86.2 & 86.2 \\
\hline Other country & 3 & 13.8 & 100.0 \\
\hline Total & 29 & 100.0 & \\
\hline
\end{tabular}

Source: The researcher 


\section{Age details}

Table 8-9: Age details.

\begin{tabular}{|l|l|l|l|}
\hline Age & \multicolumn{2}{l}{ Frequency } & \multicolumn{2}{l|}{ Per cent } & \multicolumn{1}{l|}{ Cumulative Present } \\
\hline $26-35$ & 2 & 6.9 & 6.9 \\
\hline $36-45$ & 12 & 41.4 & 48.3 \\
\hline $46-55$ & 11 & 37.9 & 86.2 \\
\hline $56-65$ & 2 & 6.9 & 93.1 \\
\hline Above 65 & 2 & 6.9 & 100.0 \\
\hline & 29 & 100.0 & \\
\hline
\end{tabular}

Source: The researcher

\subsubsection{Frequency analysis (Mode Test)}

\subsection{Private sector-life science (PS-LS)}

The following analysis is a frequency test (mode) to measure the tendency of responses toward the importance of involving private sector in developing knowledge economy in Saudi Arabia.

\section{Frequencies (mode)}

Table 8-10: PS mode.

\begin{tabular}{|l|l|} 
Item & Result \\
\hline Valid responses & 29 \\
\hline Missing responses & 0 \\
\hline Mode & $\mathbf{5}$ \\
\hline Total Sum & 144 \\
\hline
\end{tabular}

Source: The researcher 
Table 8-11: PS frequencies result details.

\begin{tabular}{|l|l|l|l|l|}
\multicolumn{2}{|c}{ Frequency } & \multicolumn{2}{c}{$\begin{array}{c}\text { Per } \\
\text { cent }\end{array}$} & \multicolumn{2}{c|}{$\begin{array}{l}\text { Valid Per } \\
\text { cent }\end{array}$} & $\begin{array}{l}\text { Cumulative Per } \\
\text { cent }\end{array}$ \\
\hline Agree & 1 & 3.4 & 3.4 & 3.4 \\
\hline $\begin{array}{l}\text { Strongly } \\
\text { agree }\end{array}$ & 28 & 96.6 & 96.6 & 100.0 \\
\hline Total & 29 & 100.0 & 100.0 & \\
\hline
\end{tabular}

Source: The researcher

Regarding the results in the above two tables, it is very clear that responses to the question: "In order to develop the knowledge economy in the life science sector, to what extent do you agree or disagree that involving the private sector is needed?" strongly support the idea that involving the private sector in order to develop a knowledge economy in the life science sector is very important and needed. The mode result is 5 which corresponds to strongly agree, this is a very high indication that represents the highest score and consequently represents a response of the highest degree of agreement toward involving the private sector.

\subsection{Multinational enterprises (MNEs)}

The relevant data in this section has been descriptively analysed by extracting frequency statistics (mode) to measure the central tendency of responses toward the proposed strategies and variables. The questions were divided into four groups: the first group of questions concerns the internal encouraging variables (internal means within the boundaries of the organization) that encourage MNEs to invest in knowledge-based activities in Saudi Arabia, the second group concerns the internal discouraging variables, the third group concerns external encouraging variables, and the fourth group the external hindering variables. The same procedure was applied to the other sub-sectors (LLCs, SMEs, KB-SUs). 
MNEs`internal encouraging variables

Table 8-12: MNEs` internal encouraging variables modes.

\begin{tabular}{|l|l|l|l|l|}
\hline Variable & 29 & 0 & $\mathbf{5}$ & 126 \\
\hline MNEs have know how & 29 & 0 & $\mathbf{4}$ & 116 \\
\hline MNEs have highly innovative practices & 29 & 0 & $\mathbf{4}$ & 108 \\
\hline $\begin{array}{l}\text { MNEs have highly sophisticated } \\
\text { management and manufacturing } \\
\text { systems }\end{array}$ & 29 & & & \\
\hline MNEs have strong networks & 29 & 0 & $\mathbf{4}$ & 116 \\
\hline $\begin{array}{l}\text { MNEs have highly competitive } \\
\text { capabilities }\end{array}$ & 29 & 0 & $\mathbf{4}$ & 110 \\
\hline MNEs are financially strong & 29 & 0 & $\mathbf{4}$ & 108 \\
\hline $\begin{array}{l}\text { MNEs have strong marketing and } \\
\text { distribution capability }\end{array}$ & 29 & 0 & $\mathbf{4}$ & 114 \\
\hline MNEs have strong brands & 29 & 0 & $\mathbf{4}$ & 119 \\
\hline
\end{tabular}

Source: The researcher

The mode results were 4 for all variables except on variable (MNEs have know-how) scored 5. These results show a positive tendency and agreement toward the role of variables in the above table in encouraging MNEs to invest in knowledge activities in Saudi Arabia.

MNEs` internal discouraging variables

Table 8-13: MNEs`internal discouraging variables mode.

\begin{tabular}{|l|l|l|l|l|}
\hline Variable & Valid & Missing & Mode & \multicolumn{1}{c|}{ Sum } \\
\hline $\begin{array}{l}\text { MNEs have a lack of information } \\
\text { regarding new market dynamics }\end{array}$ & 29 & 0 & $\mathbf{4}$ & 97 \\
\hline
\end{tabular}

Source: The researcher 
Table 8-14: Breakdown of MNEs` internal discouraging variables frequencies.

\begin{tabular}{|c|c|c|c|c|}
\hline & Frequency & Per cent & $\begin{array}{l}\text { Valid Per } \\
\text { cent }\end{array}$ & $\begin{array}{l}\text { Cumulative Per } \\
\text { cent }\end{array}$ \\
\hline $\begin{array}{l}\text { Strongly } \\
\text { disagree }\end{array}$ & 1 & 3.4 & 3.4 & 3.4 \\
\hline Disagree & 8 & 27.6 & 27.6 & 31.0 \\
\hline Uncertain & 5 & 17.2 & 17.2 & 48.3 \\
\hline Agree & 10 & 34.5 & 34.5 & 82.8 \\
\hline $\begin{array}{l}\text { Strongly } \\
\text { agree }\end{array}$ & 5 & 17.2 & 17.2 & 100.0 \\
\hline Total & 29 & 100.0 & 100.0 & \\
\hline
\end{tabular}

Source: The researcher

This question has only one variable, and the participants' central tendency scored 4, which mean they agree that this factor or variable has an effect on the MNEs with regard to undertaking knowledge activities in Saudi Arabia.

\section{MNEs`external encouraging variables}

Table 8-15: MNEs` external encouraging variables modes.

\begin{tabular}{|l|l|l|l|l|}
\hline Variable & \multicolumn{2}{c}{ Valid } & Missing & \multicolumn{1}{l|}{ Mode } \\
\hline High growth Saudi sizeable new market & 29 & 0 & $\mathbf{4}$ & 121 \\
\hline Accessibility to middle east market & 29 & 0 & $\mathbf{4}$ & 110 \\
\hline Making joint ventures with key Saudi partners & 29 & 0 & $\mathbf{5}$ & 120 \\
\hline $\begin{array}{l}\text { Ongoing economic reforms, which support } \\
\text { FDI }\end{array}$ & 29 & 0 & $\mathbf{4}$ & 120 \\
\hline Financial incentives & 29 & 0 & $\mathbf{4}$ & 123 \\
\hline $\begin{array}{l}\text { Special comparative advantages (religious } \\
\text { tourism) }\end{array}$ & 29 & 0 & $\mathbf{3}$ & 98 \\
\hline
\end{tabular}

Source: The researcher

This third aspect concerns the external factors that would or would not encourage MNEs to invest in knowledge activities in Saudi Arabia. Four variables scored 
"agree" (mode 4), one scored "strongly agree" (mode 5), while "special comparative advantages (religious tourism)" scored "uncertain".

\section{MNEs` external hindering variables}

Table 8-16:MNEs` external hindering variables modes.

\begin{tabular}{|c|c|c|c|c|}
\hline Variable & Valid & Missing & Mode & Sum \\
\hline Unstable geopolitical area & 29 & 0 & 4 & 101 \\
\hline Lack of high qualified labour market & 29 & 0 & 4 & 120 \\
\hline Cultural barriers & 29 & 0 & 4 & 105 \\
\hline $\begin{array}{l}\text { Lower standards of living than developed } \\
\text { countries }\end{array}$ & 29 & 0 & 2 & 88 \\
\hline Weak life science ecosystem & 29 & 0 & 5 & 133 \\
\hline Economic vulnerability & 29 & 0 & 3 & 91 \\
\hline Barriers in doing business & 29 & 0 & 4 & 116 \\
\hline $\begin{array}{l}\text { Weak research infrastructure in Saudi } \\
\text { Arabia }\end{array}$ & 29 & 0 & 5 & 133 \\
\hline Lack of developed life science cluster & 29 & 0 & 4 & 119 \\
\hline
\end{tabular}

Source: The researcher

The participants' central tendency about the external hindering factors scored differently with respect to the different variables. "Lower standards of living than developed countries" scored 2, which means that they disagreed about its role as a hindering factor. Also "economic vulnerability" scored as "uncertain". The rest exhibited a positive tendency between "strongly agree" and "agree". 


\subsection{Large local companies (LLCs)}

For this sub-sector the frequencies test (mode) covered four sets of variables/factors (internal encouraging factors, internal hindering factors, external encouraging factors, and external hindering factors) to provide an answer to the following question: "The following encourage (discourage) local large companies (LLCs) to invest in knowledge-based activities in Saudi Arabia. To what extent do you agree or disagree?".

\section{LLCs` internal encouraging variables}

Table 8-17: LLCs` internal encouraging variables modes.

\begin{tabular}{|l|l|l|l|l|}
\hline Variable & Valid & Missing & Mode & Sum \\
\hline LLCs have good financial strength & 29 & 0 & $\mathbf{4}$ & 109 \\
\hline $\begin{array}{l}\text { LLCs have a significant share in the } \\
\text { Saudi market and the neighbouring } \\
\text { countries }\end{array}$ & 29 & 0 & $\mathbf{4}$ & 106 \\
\hline $\begin{array}{l}\text { LLCs have easy access to the financial } \\
\text { system }\end{array}$ & 29 & 0 & $\mathbf{3}$ & 105 \\
\hline
\end{tabular}

Source: The researcher

Only one variable "LLCs have easy access to the financial system" returned a mode 3

- "uncertain" as an internal encouraging factor; the other two scored "agreed" (mode

4) as encouraging variables to making investment in the knowledge activities in Saudi Arabia. 


\section{LLCs`internal hindering variables}

Table 8-18: LLCs` internal hindering variables modes.

\begin{tabular}{|l|l|l|l|l|}
\hline Variable & Valid & Missing & Mode & \multicolumn{1}{l|}{ Sum } \\
\hline $\begin{array}{l}\text { LLCs have a lack of competitive } \\
\text { capabilities }\end{array}$ & 29 & 0 & $\mathbf{4}$ & 113 \\
\hline $\begin{array}{l}\text { LLCs have a lack of collaboration } \\
\text { and networking culture }\end{array}$ & 29 & 0 & $\mathbf{4}$ & 113 \\
\hline $\begin{array}{l}\text { LLCs have no interaction with the } \\
\text { global value chain }\end{array}$ & 29 & 0 & $\mathbf{3}$ & 94 \\
\hline $\begin{array}{l}\text { LLCs have no previous expertise in } \\
\text { knowledge activities }\end{array}$ & 29 & 0 & $\mathbf{4}$ & 122 \\
\hline $\begin{array}{l}\text { LLCs have a lack of entrepreneurial } \\
\text { strategy and attitude }\end{array}$ & 29 & 0 & $\mathbf{4}$ & 128 \\
\hline
\end{tabular}

Source: The researcher.

These variables were proposed as hindering factors that would create obstacles for LLCs to invest in knowledge activities. Four of them were supported and recorded a mode of 4 (agree), while one of them, namely "LLCs have no interaction with global value chain" scored 3 (uncertain) as a hindering factor. 


\section{LLCs` external encouraging variables}

Table 8-19: LLCs` external encouraging variables modes.

\begin{tabular}{|l|l|l|l|l|}
\hline Variable & Valid & Missing & Mode & Sum \\
\hline $\begin{array}{l}\text { Raising capabilities through JV, } \\
\text { acquisition, or licensing technology }\end{array}$ & 29 & 0 & $\mathbf{4}$ & 119 \\
\hline $\begin{array}{l}\text { Developing alliances and partnership } \\
\text { with MNEs, and research centres }\end{array}$ & 29 & 0 & $\mathbf{4}$ & 121 \\
\hline $\begin{array}{l}\text { Leveraging Saudi government orientation } \\
\text { for building KE }\end{array}$ & 29 & 0 & $\mathbf{4}$ & 118 \\
\hline $\begin{array}{l}\text { Tapping into local Saudi priorities } \\
\text { Tapping into local Saudi advantages }\end{array}$ & 29 & 0 & $\mathbf{5}$ & 121 \\
\hline Government procurement support & 29 & 0 & $\mathbf{4}$ & 118 \\
\hline Targeting niche market ignored by rivals & 29 & 0 & $\mathbf{4}$ & 112 \\
\hline
\end{tabular}

Source: The researcher

All variables have scored a mode of at least 4, with the notable exception of "Tapping into local Saudi priorities", which exhibited a mode of 5.

\section{LLCs` external hindering variables}

Table 8-20: LLCs` external hindering variables modes.

\begin{tabular}{|l|l|l|l|l|}
\hline Variable & Valid & Missing & Mode & Sum \\
\hline $\begin{array}{l}\text { New foreign multinational enterprise } \\
\text { entrants }\end{array}$ & 29 & 0 & $\mathbf{2}$ & 90 \\
\hline Weak LS ecosystem & 29 & 0 & $\mathbf{5}$ & 129 \\
\hline Weak national research infrastructure & 29 & 0 & $\mathbf{5}$ & 129 \\
\hline Lack of developed LS cluster & 29 & 0 & $\mathbf{4}$ & 115 \\
\hline $\begin{array}{l}\text { Weak culture of external collaboration } \\
\text { Lack of high qualified Saudi labour } \\
\text { market }\end{array}$ & 29 & 0 & $\mathbf{4}$ & 111 \\
\hline
\end{tabular}

Source: The researcher 
The central tendency of the participants shows that the "new foreign multinational entrants" is not perceived as a hindering factor; however, all the remaining factors were considered as hindering factors (mode 4 and 5).

\subsection{Small and Medium Enterprises (SMEs)}

In the following section frequency tests were implemented over the four areas of investigation (internal encouraging factors, internal hindering factors, external encouraging factors, and external hindering factors), and applied to the Small to Medium Enterprise (SMEs) sector. A total number of variables equal to 22 were considered across the four areas. See appendix (12.6)

\section{SMEs`internal encouraging variables}

Table 8-21: SMEs` internal encouraging variables modes.

\begin{tabular}{|l|l|l|l|l|}
\hline Variable & Valid & Missing & Mode & Sum \\
\hline $\begin{array}{l}\text { SMEs have high awareness and } \\
\text { expertise in the local Saudi market }\end{array}$ & 29 & 0 & $\mathbf{3}$ & 89 \\
\hline $\begin{array}{l}\text { SMEs have more flexibility to respond } \\
\text { to market change }\end{array}$ & 29 & 0 & $\mathbf{4}$ & 102 \\
\hline
\end{tabular}

Source: the researcher.

Two factors were suggested as internal encouraging factors, but as we see in the above table the results show that one of them scored 3 (uncertain), and the other was supported and scored 4 (agree). 


\section{SMEs` internal hindering variables}

Table 8-22: SMEs` internal hindering variables modes.

\begin{tabular}{|l|l|l|l|l|}
\hline Variable & Valid & Missing & Mode & Sum \\
\hline SMEs have low competitive capabilities & 29 & 0 & $\mathbf{4}$ & 120 \\
\hline $\begin{array}{l}\text { SMEs have a lack of entrepreneurial } \\
\text { strategy and attitude }\end{array}$ & 29 & 0 & $\mathbf{5}$ & 129 \\
\hline SMEs have low financial strength & 29 & 0 & $\mathbf{4}$ & 118 \\
\hline $\begin{array}{l}\text { SMEs have difficult access to financial } \\
\text { system }\end{array}$ & 29 & 0 & $\mathbf{4}$ & 120 \\
\hline $\begin{array}{l}\text { SMEs have a lack of collaboration and } \\
\text { networking culture }\end{array}$ & 29 & 0 & $\mathbf{4}$ & 108 \\
\hline SMEs have weak marketing capabilities & 29 & 0 & $\mathbf{4}$ & 106 \\
\hline
\end{tabular}

Source: The researcher.

All the above six factors were supported as hindering factors to different levels; five factors scored 4 (agree), while one scored 5 (strongly agree).

\section{SMEs` external encouraging variables}

Table 8-23: SMEs` external encouraging variables modes.

\begin{tabular}{|l|l|l|l|l|}
\hline Variable & Valid & Missing & Mode & Sum \\
\hline $\begin{array}{l}\text { Government strategy for building a KE that } \\
\text { would have special support for SMEs }\end{array}$ & 29 & 0 & $\mathbf{4}$ & 117 \\
\hline Government procurement support & 29 & 0 & $\mathbf{4}$ & 109 \\
\hline High growth local sizeable market & 29 & 0 & $\mathbf{4}$ & 114 \\
\hline Leveraging national priorities & 29 & 0 & $\mathbf{4}$ & 113 \\
\hline $\begin{array}{l}\text { Leveraging national endowments and } \\
\text { advantages }\end{array}$ & 29 & 0 & $\mathbf{4}$ & 107 \\
\hline
\end{tabular}

Source: The researcher.

All the external encouraging factors returned the same mode of 4 . 


\section{SMEs` external hindering variables}

Table 8-24: SMEs` external hindering variables modes.

\begin{tabular}{|l|l|l|l|l|}
\hline Variable & \multicolumn{3}{|l}{ Valid Missing } & \multicolumn{1}{l|}{ Mode Sum } \\
\hline Weak culture of external collaboration & 29 & 0 & $\mathbf{4}$ & 121 \\
\hline $\begin{array}{l}\text { Uncertainty caused by developing a new } \\
\text { product/s that could be displaced by competitors }\end{array}$ & 29 & 0 & $\mathbf{4}$ & 110 \\
\hline Weak research infrastructure in Saudi Arabia & 29 & 0 & $\mathbf{5}$ & 134 \\
\hline Risk-averse financial system & 29 & 0 & $\mathbf{5}$ & 133 \\
\hline Fledgling venture capital market & 29 & 0 & $\mathbf{5}$ & 124 \\
\hline Lack of developed LS cluster & 29 & 0 & $\mathbf{4}$ & 120 \\
\hline $\begin{array}{l}\text { Local customers do not trust local innovative } \\
\text { products }\end{array}$ & 29 & 0 & $\mathbf{4}$ & 109 \\
\hline Weak national LS ecosystem & & & & 134 \\
\hline Lack of high qualified Saudi labour market & 29 & 0 & $\mathbf{5}$ & 128 \\
\hline
\end{tabular}

Source: The researcher.

Nine factors were proposed as external hindering factors; all these factors were supported, with five of them scoring 5 (strongly agree), and the rest scoring 4 (agree).

\subsection{Knowledge-based start-ups (KB-SUs)}

In this fourth and final sub-sector of the life science private sector considered, the same mode test was executed for the four aforementioned aspects (internal encouraging factors, internal hindering factors, external encouraging factors, and external hindering factors). Results are shown in the following four sections (KBSUs` internal encouraging variables, KB-SUs` internal hindering variables, KB-SUs` external encouraging variables, and KB-SUs` external hindering variables). 


\section{KB-SUs`internal encouraging variables}

Table 8-25: KB-SUs` internal encouraging variables modes.

\begin{tabular}{|l|l|l|l|l|}
\hline Variable & Valid & Missing & Mode & \multicolumn{1}{l|}{ Sum } \\
\hline $\begin{array}{l}\text { KB-SUs have pipeline of opportunities } \\
\text { from research centres }\end{array}$ & 29 & 0 & 4 & 95 \\
\hline $\begin{array}{l}\text { KB-SUs have benefit of public funding } \\
\text { for R\&D }\end{array}$ & 29 & 9 & 4 & 112 \\
\hline $\begin{array}{l}\text { KB-SUs founders or entrepreneurs } \\
\text { usually have scientific background } \\
\text { (linked to knowledge activities) }\end{array}$ & 29 & 0 & $\mathbf{4}$ & 107 \\
\hline
\end{tabular}

Source: The researcher

All three identified factors scored the same central tendency of support and returned a mode of 4 (agree). 


\section{KB-SUs`internal hindering variables}

Table 8-26: KB-SUs`internal hindering variables modes.

\begin{tabular}{|l|l|l|l|l|}
\hline \multicolumn{2}{|l|}{ Variable } & & & \\
\hline $\begin{array}{l}\text { KB-SUs have a lack of high promising } \\
\text { technology }\end{array}$ & 29 & 0 & $\mathbf{4}$ & 105 \\
\hline $\begin{array}{l}\text { KB-SUs have weak connection with } \\
\text { supply chain }\end{array}$ & 29 & 0 & $\mathbf{4}$ & 120 \\
\hline $\begin{array}{l}\text { KB-SUs have a lack of collaboration } \\
\text { among private sector }\end{array}$ & 29 & 0 & $\mathbf{4}$ & 122 \\
\hline $\begin{array}{l}\text { KB-SUs have a lack of collaboration } \\
\text { with health sector and stakeholders }\end{array}$ & 29 & 0 & $\mathbf{4}$ & 125 \\
\hline $\begin{array}{l}\text { KB-SUs have a lack of capital (seed } \\
\text { fund) }\end{array}$ & 29 & 0 & $\mathbf{5}$ & 124 \\
\hline $\begin{array}{l}\text { KB-SUs have low expertise in the } \\
\text { market and developing new knowledge- } \\
\text { based start-ups }\end{array}$ & 29 & 0 & $\mathbf{4}$ & 117 \\
\hline $\begin{array}{l}\text { KB-SUs have low entrepreneurial } \\
\text { attitude }\end{array}$ & 29 & 0 & $\mathbf{4}$ & 108 \\
\hline
\end{tabular}

Source: The researcher.

Internal hindering factors scored the same score of 4 (agree), except for the factor "KB-SUs have a lack of capital (seed fund)" which scored the highest agreement level - score 5 (strongly agree). 
KB-SUs`external encouraging variables

Table 8-27: KB-SUs` external encouraging variables.

\begin{tabular}{|l|l|l|l|l|}
\hline Variable & & & & \\
\hline $\begin{array}{l}\text { Leveraging national advantages (e.g. Religious } \\
\text { tourism) }\end{array}$ & 29 & 0 & $\mathbf{4}$ & 106 \\
\hline $\begin{array}{l}\text { Tapping into specific local priorities (e.g. } \\
\text { Diabetes) }\end{array}$ & 29 & 0 & $\mathbf{4}$ & 117 \\
\hline Targeting niche market ignored by rivals & 29 & 0 & $\mathbf{4}$ & 116 \\
\hline Leveraging government procurement support & 29 & 0 & $\mathbf{3}$ & 108 \\
\hline $\begin{array}{l}\text { Leveraging government strategy and orientation } \\
\text { for building KE }\end{array}$ & 29 & 0 & $\mathbf{4}$ & 122 \\
\hline
\end{tabular}

Source: The researcher.

Five factors were suggested as external variables; only one, namely "leveraging government procurement support” scored 3 (uncertain), the other four factors scored 4 (agree).

\section{KB-SUs` external hindering variables}

Table 8-28: KB-SUs` external hindering variables modes.

\begin{tabular}{|c|c|c|c|c|}
\hline Variable & Valid & Missing & Mode & Sum \\
\hline $\begin{array}{l}\text { Barriers for researchers to own their own } \\
\text { business }\end{array}$ & 29 & 0 & 5 & 133 \\
\hline Weak national LS ecosystem & 29 & 0 & 5 & 134 \\
\hline Insufficient national research infrastructure & 29 & 0 & 5 & 131 \\
\hline Lack of seed fund organizations & 29 & 0 & 4 & 124 \\
\hline Lack of developed LS cluster & 29 & 0 & 4 & 122 \\
\hline Fledgling venture capital market & 29 & 0 & 4 & 117 \\
\hline Weak culture of external collaboration & 29 & 0 & 4 & 119 \\
\hline Lack of high qualified Saudi labour market & 29 & 0 & 4 & 120 \\
\hline Barriers in doing business & 29 & 0 & 4 & 122 \\
\hline
\end{tabular}

Source: The researcher. 
Quite a large number of potential external hindering factors were proposed; all of them were perceived by respondents to be hindering factors; three scored the highest level of agreement 5 (strongly agree), whereas the remaining six factors scored 4 (agree).

\subsubsection{Summary}

The main purpose of the survey was to add robustness to the methodology and to further explore the findings of the first stage of research but to adopt a private sector perspective. As such it serves as a form of quality assurance and feedback to validate the previous feedback from stakeholders.

The questionnaire comprised 6 questions for demographic statistics, followed by 17 questions concerning the exploration of the internal and external variables (through the implementation of SWOT analysis) that could encourage or hinder the private sector from engaging in investment in knowledge activities.

The private sector is a key sector in any innovation system; however, the private sector in Saudi Arabia has no significant role or serious involvement in knowledge activities. This questionnaire was therefore primarily designed in order to understand this challenge with a view of exploring potential avenues for private sector involvement.

Twenty-nine participants were selected, all of whom were senior stakeholders from different sectors in life sciences - see section (8.5.3.1) demographic statistics. The majority (22) of participants involved in this survey phase were also informants in the first research phase (in depth interviews). This approach was adopted, as explained above, primarily to enable the feedback of the research to the informants-stakeholders (continuous stakeholder engagement. In addition, the difficulty of accessing an extended pool of expert interviewees/survey participants within an embryonic KSA life science ecosystem directly influenced the shaping of the research design for phase two - questionnaire (in particular the sampling methodology) corroborating the conscious choice made of retaining a subset of phase 1 interviewees to serve as the participants in phase 2, thus enabling stakeholder engagement throughout the research as well as confirmation of phase 1 results. 
The following figure was developed from the findings of this study and presents the systems approach and holistic view of the current system failings in the Innovation Systems of the KSA. The model clearly indicates a causal relationship between a lack of strategy to unite the system (its purpose) and the resultant poor cohesion, poor investments and lack of learning and meaningful progress in the sector.

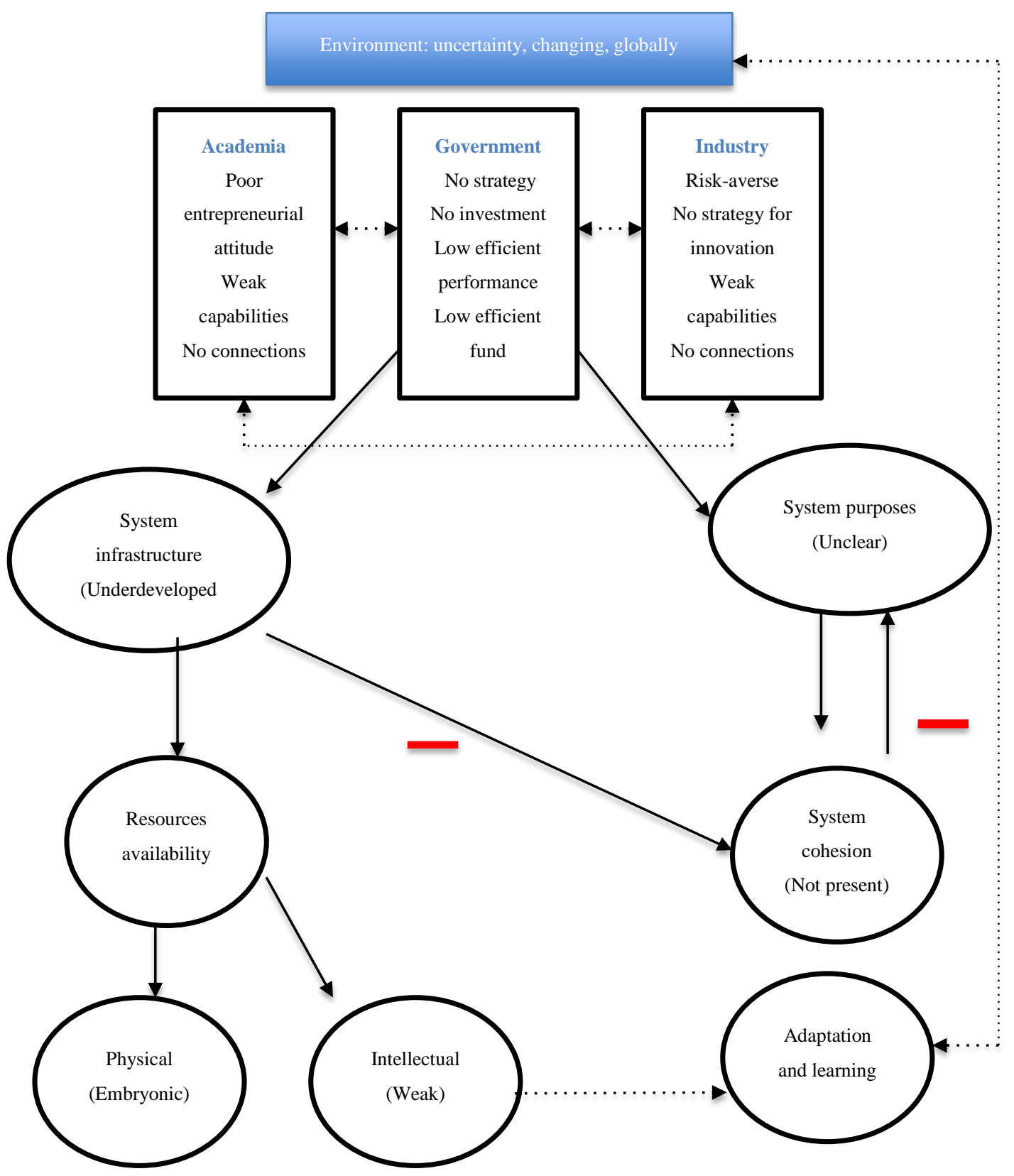

Figure 8-7: Current Saudi innovation system (Dotted lines mean the relationships are very weak or do not exist). 
The model in Figure 8-8 has been developed by the researcher to demonstrate how to create a viable system from the current state of the KSA, using a systems approach to the necessary fundamental building blocks. This figure contributes directly to the academic understanding of innovation in the context of the Kingdom of Saudi Arabia and to academic studies more generally. It shows clearly how the catalysts identified by previous studies are not present in this context and how this has hindered the system viability and adaptability (learning) needed for business relationships to flourish.

The figure also shows how there is a break in the input-process-output cycle of the innovation system (as well as deficiencies in the feed forward planning of the sector) where inputs (academic and other resources as well as commercial businesses and university collaborations) are not available in sufficient quantity and/or quality. Without such investments and a direction to the system (to signal the need for investment by universities and private business) the system is likely to remain dormant and unable to generate outputs which can satisfy the need for the economy to diversify.

The figure is presented here to assist the reader when reviewing the next chapter, so that the discussion and answers to this study can be clearly identified using the systems theory approach to studying innovation systems and processes. 


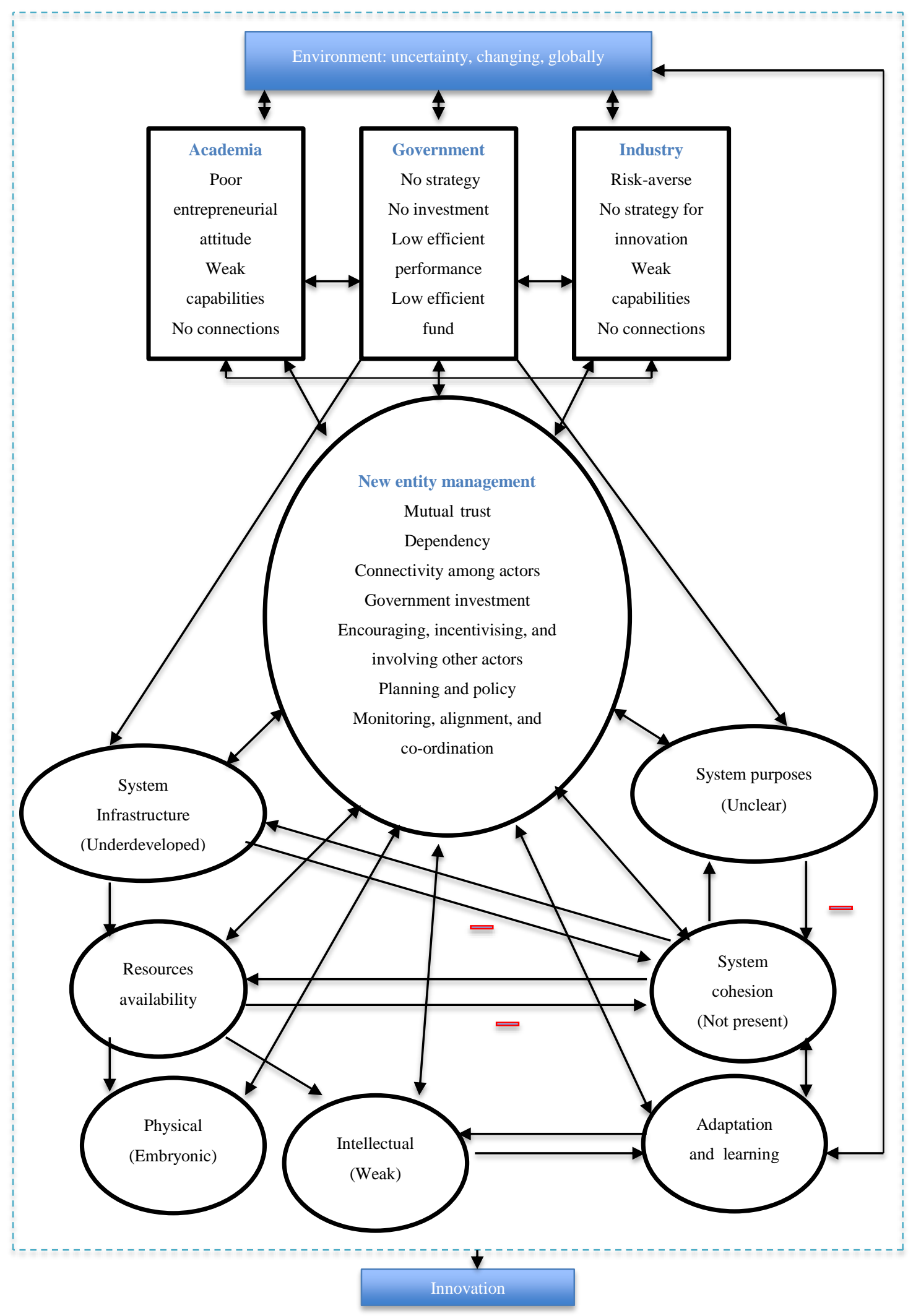

Figure 8-8: current innovation system, and the proposed Viable Saudi LS Innovation System (VSLSIS) 


\section{Discussion}

\subsection{Introduction}

In this chapter, the findings presented in Chapters $(6,7,8)$ will be discussed and analysed with reference to the research questions and in contrast to the literature review. It must be noted that the literature review stage of a grounded theory research study is conducted after field research has taken place, during a process whereby the student is 'making sense' of his experience in the field. As such this chapter will show how the study contributes to both background theory and focal literature.

The research questions for this study were designed to explore a gap in the body of knowledge (innovation in a developing economy migrating from a rentier economy) using the life science sector of the Kingdom of Saudi Arabia as the context of the study. So, the ultimate aim of this study is to inform national policy concerning the LS innovation "system" in the KSA. This chapter will focus on the analysis of "the system" and the features that are driving or inhibiting progress.

The answer to the main guiding research question of this study is addressed through the three sub-questions below:

Research question 1: What are the key requirements for developing knowledge economy in the life science sector in the KSA?

Research question 2: What are the challenges in developing a LS sector in the KSA?

Research question3: What model of LS innovation system would best fit the Saudi context?

This chapter is divided into a number of sections. The first section will present the findings and analysis in the light of the background theories (system theory, human capital theory, innovation diffusion theory). In the second section, the findings will be 
analysed in the light of the focal literature review. Finally, in the conclusions chapter, the overall analysis of this chapter will be used to identify and suggest future research work and practical interventions needed to support the development of an innovation system in the KSA.

\subsection{Findings in the Light of Theories}

This section analyses the findings in the light of three relevant theories, namely system theory, human capital theory, and innovation diffusion theory.

\subsubsection{The findings and system theory}

The findings uncovered many challenges and aspects that considered the Saudi innovation system to be an unviable system: the government has no strategy for developing a life science innovation system (lack of purposiveness); the government does not invest significantly in the infrastructure (IPO) or in knowledge activities; the private sector also has no strategy (recursive viability) or will to be positively involved in innovation practices, and does not have high skilled people (inputs) to conduct significant research or manage highly sophisticated knowledge-based enterprises (capability). Academia has no regulations that encourage them to develop new start-ups, and does not have high capabilities to produce promising technologies, or an entrepreneurial attitude to business. No collaboration and connection between the key actors exists (dependency recognition and process of exploitation), and the financial system is risk-averse with insufficient venture money (input). The lack of government signalling to direct investments in the private and the public sector means that the environment for universities and business remains uncertain - without such signalling by the government (which has underpinned all other studies of transition) the non-government actors will seek less risky activities or locations in the world. As such, government as 'first mover' to create a true system has yet to appear or to reach a critical stage where academia and business engage to resource and exploit knowledge and innovation systems.

All these conditions have created an unviable innovation system, where the system purposes are unclear, there is no cohesive system, and no adaptive system (learning 
and engagement). In comparison, the researcher found that Singapore had adopted a strategy to develop the biotechnology sector as a very important and key sector that would enhance their economic growth, and developed their strategy as early as the 1980s (Finegold et al., 2004). This initiative toward developing the biotechnology sector, with its embedded significant investment, entailed a strong governmental intervention to work as the driver for the initiative (Finegold et al., 2004). Such 'strategic direction and drive' is absent from the KSA landscape.

The Singaporean government has embarked on many steps and actions for this purpose, for instance supporting basic research and building technological infrastructure. Some examples of their endeavour include:

○ Establishing the Institute of Molecular and Cellular Biology in 1987 at the National University of Singapore;

- Establishing other research institutes (Bioinformatics Centre, the Genome Institute of Singapore, the Bioprocessing Technology Centre, and the Institute of Bioengineering and Nanotechnology); all these were created between 1996 and 2002.

- In order to support basic research, the government developed financial grant schemes (project grants as seed funding, programme grants for more extensive research, grants for strengthening the core capabilities and competencies of the research centres).

From the data, it is evident that the government's strategy, investment and intervention in the context of the KSA is very important, as these would be seen as tools to mitigate the risk and decrease the environmental uncertainty, and as encouraging signals to attract and involve academia and the private sector to invest in knowledge-based businesses. The government should play a central role in studying the system to provide feed-forward mechanisms that enable all actors to overcome any barriers, unite the system and encourage investment.

According to Kast \& Rosenzweig (1985), a system is "an entity in its own right, with unique properties understandable only in terms of the whole, especially in the face of a more traditional reductionist or mechanistic focus on the separate parts and a more simplistic notion of how these parts fit". The life science innovation system is an open 
system with inputs such as universities, banking, government strategies and interventions, firms etc., and processes are represented through undertaking collaborative research and innovations, developing feed-forward planning, learning and adaptation, feedback; outputs appear as new products or services, as well as firms. The system comprises many organizations and entities connected by common aims and virtual boundaries. This open system interacts with its environment locally and globally in order to sustain growth and success. As previously articulated in the Background Theory section of Chapter 2, building a system that sustains success and growth is not solely about bringing perfect components together: for a perfect open system there must be perfect interactions and synergies among all components so that emergent properties and outcomes can be significantly optimized by virtue of organic unity. Such organic unity involves high inter-dependency between actors and their exchanges and a recursive viability (every actor derives value from interaction).

For making a system viable two key mechanisms should be in place and activated. The first one is to secure the cohesion among organization members where the individuals and collective interests are not necessarily the same but aligned; the second is to provide the capacity for organizational systems and units to be adaptable and have the capacity to respond to significant environmental changes, so that organizations are updated and flexible regarding the changes which may take place around them and which may disturb the dependency relations between actors (Raul Espejo, 1990). These mechanisms of cohesion do not exist and have not been detected by previous studies, as no previous studies have taken a systems approach.

The findings suggest that some interventions would help develop cohesion in the proposed innovation system, and that 'involving all actors in developing policy' (20\%); 'developing a knowledge economy coordinating organizations' (16\%); 'raising institutional quality' (35\%), and 'developing strong academia-private sector connections' (38\%) (these codes and associated figures were derived from the researcher's open coding of interviews) would all help to build adaptive and continuous learning processes. 'Exposing all actors to international learning would enhance knowledge and understanding' (42\%); 'developing collaboration with foreign parties' $(23 \%)$ would also allow greater inflow of innovation, and 'transferring 
foreign knowledge' would support this too. Again this evidence is taken from the systematic coding of the data and is reflected in the systems model of the KSA presented in the previous chapter (Figure 8-6).

A continued connection with the global context would provide the local system with the continuous updates and learning that would enable it to adapt to any change in the industrial environment. The latter implies the development and sharing of learning processes and mechanisms to transmit "best practice" to all users/actors of the innovation system.

Viability - the most important of all system concepts - means the capacity for a system and subsystems to solve problems and be adaptable, to have the capacity to respond to and survive any significant changes which occur in the environment, and to sustain growth (Beer, 1989). For the KSA there is no profit motivation or effective knowledge exploitation cycle to ensure viability; critical mass is also small. It would appear that business and academia cannot establish a system and it is only the government, as a first mover, that can create this scenario. The latter confirms the findings of previous studies in more advanced economies.

From the findings, and contained in the model in Figure 8-6, the proposed Saudi LS innovation system has three key inputs: Academia, Government, Private sector; under each key input there are further inputs:

Academia:

- Building human capital and providing continuous learning by connecting with environment and global partners (adaptation)

- Raising the quality of the education system (adaptation)

- Encouraging and undertaking entrepreneurship (investment).

Government:

- Leading the development of the life science sector project, and building a new entity to be in charge of managing the project, by involving the key actors, 
developing enabling policy and regulations (feed-forward), providing feedback and performance assessment

- Providing funding (inputs)

- Investing in knowledge activities (IPO)

- Building research infrastructure (IPO)

- Raising public institutions quality and efficiency (adaptation)

- Improving business environment (feed-forward, IPO).

Private sector:

- Developing innovation strategy (recursive viability)

- Building capabilities and connecting the environment and global partners (adaptation)

- Developing entrepreneurial attitude and investing in knowledge-based businesses (IPO)

- Transferring foreign knowledge and building collaboration with local and foreign partners (investment and adaptation).

These constituent components create an infrastructure and inputs to the system but one of the more important issues to review - from the perspective of a systems theorist - is the relationships that exist amongst the actors and the processes that are at their foundation.

\section{Processes:}

In the developed model (Figure 8-6) there are processes that take place within the boundaries of the knowledge economy organization and extend to other stakeholders. These include:

- Involving all actors to work in a systemic way and enabling a cohesive performance that would sustain the purposes of the system and alignment among its members, thus achieving both collective and the individual aims.

- Developing enabling policy (feed-forward)

- Identifying priorities and advantages

- Funding collaboration (IPO)

- Bringing about coordination (cohesion). 
The outputs:

- Knowledge transfer

- Spinoffs

- Life science cluster.

The above elements in the proposed Saudi innovation system are very important to the building of viable innovation systems that generate cohesion and adaptation, and are compatible with the essence of system theory.

According to Buchanan and Huczynski (2013), Input-process-output (IPO) is the very essence of a system, where the system members work as a team to produce value. Feed-forward processes are important to understand local policies for a system and its relations with the wider innovation environment dynamics and to be able to adjust accordingly Brown (1996; 2007). Broader system relationships \& the Internal Fit are the study of the organization as a system, and should include various stakeholder perspectives (Delery and Gupta, 2016). Feedback measurement processes show the importance of assessing the measures adopted and provide feedback of performance to staff (Kast \& Rosenzweig, 1985). Learning processes and capabilities, the mechanisms engaged by employers to support staff to learn and improve, are a critical aspect of this study, as are the adaptive capabilities of organizations and their staff (performance management) Senge $(1990 ; 1992)$. 
Table 9-1: System concepts in the findings and their implications.

\begin{tabular}{|c|c|c|}
\hline $\begin{array}{l}\text { Systems } \\
\text { Concept }\end{array}$ & Findings & Implications \\
\hline IPO & $\begin{array}{l}\text { No such evidence about the current situation, } \\
\text { but from the data a number of interventions are } \\
\text { suggested: } \\
\text { - } \quad \text { Government should develop strategy } \\
\text { - } \quad \text { Government should build infrastructure } \\
\text { and invest in knowledge. } \\
\text { - } \text { Development of the venture capital market } \\
\text { - Academia and industry should develop } \\
\text { their strategies and build their capabilities. } \\
\text { The output would be new businesses and } \\
\text { innovations }\end{array}$ & $\begin{array}{l}\text { - The Saudi government, by } \\
\text { developing strategy, } \\
\text { building infrastructure, and } \\
\text { investing in knowledge- } \\
\text { based enterprises can } \\
\text { reduce uncertainty and give } \\
\text { positive signals for other } \\
\text { actors to develop their } \\
\text { capabilities and invest in } \\
\text { knowledge. } \\
\text { Venture money helps in } \\
\text { knowledge transfer and } \\
\text { building new businesses. }\end{array}$ \\
\hline $\begin{array}{l}\text { Feedforward } \\
\text { Planning }\end{array}$ & $\begin{array}{l}\text { Low for each actor. But the findings suggest } \\
\text { that: } \\
\text { - Government should develop enabling } \\
\text { policy and regulations through involving } \\
\text { other actors. } \\
\text { - Advantages and priorities for investment } \\
\text { should be identified and capabilities based } \\
\text { on these developed. }\end{array}$ & $\begin{array}{l}\text { - Usually government-led to } \\
\text { develop key sectors } \\
\text { Exploring opportunities and } \\
\text { uncertainty in environment } \\
\text { to develop relevant } \\
\text { strategies, helps system to } \\
\text { survive and compete. }\end{array}$ \\
\hline $\begin{array}{l}\text { Broader system } \\
\text { relationships \& } \\
\text { the Internal Fit }\end{array}$ & $\begin{array}{l}\text { No viable system. But the findings suggested } \\
\text { to: } \\
\text { - Building collaboration between actors. } \\
\text { - Creating alignment between individuals' } \\
\text { purposes and collective purposes. }\end{array}$ & $\begin{array}{l}\text { Development of a cohesive } \\
\text { system that is necessary to build } \\
\text { a viable system. }\end{array}$ \\
\hline $\begin{array}{l}\text { Feedback } \\
\text { measurement } \\
\text { processes }\end{array}$ & $\begin{array}{l}\text { The current system has no evidence for this } \\
\text { process. But the findings present some } \\
\text { suggestions: } \\
\text { - Government should assess performance } \\
\text { and provide feedback. } \\
\text { - The other actors should feed back the }\end{array}$ & $\begin{array}{l}\text { Feedback process keeps } \\
\text { involved people aware of any } \\
\text { deviations, helps improve } \\
\text { performance, and informs } \\
\text { strategies via real inputs. }\end{array}$ \\
\hline
\end{tabular}




\begin{tabular}{|c|c|c|}
\hline & $\begin{array}{l}\text { developed policy and regulations to } \\
\text { overcome any hindering factors }\end{array}$ & \\
\hline $\begin{array}{l}\text { Learning } \\
\text { processes and } \\
\text { capabilities }\end{array}$ & $\begin{array}{l}\text { Current capabilities are weak in quality and } \\
\text { small in quantity. But the findings suggest: } \\
\text { - Developing human capital } \\
\text { - } \quad \text { Providing continuous learning to update } \\
\text { staff on any new additions or changes to } \\
\text { the environment. }\end{array}$ & $\begin{array}{l}\text { - Investment in human } \\
\text { capital is a key factor in } \\
\text { developing any country's } \\
\text { economic growth. } \\
\text { - Continuous learning } \\
\text { provides an adaptive system } \\
\text { that can respond to } \\
\text { disruption in the } \\
\text { environment. }\end{array}$ \\
\hline
\end{tabular}

Source: The researcher

Overall, when contrasted with the extant literature, it can be seen that the Saudi system lacks many of the features of a viable system. The main deviance with the existing literature is the role of the government and its ability to influence the university and business sectors through investments and the signalling of investment intentions.

The next section will review the Saudi Innovation System from the perspective of improved human capital.

\subsubsection{The findings and human capital theory}

The findings show significant shortages and weakness in general human resources and a lack of highly-skilled people in terms of entrepreneurial attitudes, management and technical fields. The void of skills crosses academia, government, and industry and this shortage poses serious challenges because the human capital subsystems must be effectively built and conditioned so as to produce valuable knowledge (to manage knowledge-based businesses) and to exploit this knowledge effectively. To transfer knowledge it is very necessary to build national absorptive capacity to understand and assimilate existing knowledge and then to produce new knowledge (the input-processoutput cycle). This is exactly what countries like Singapore, India, and South Korea have done in making the transition to a knowledge-based economy. However, such investment is more confused in Saudi Arabia and, while any actor could invest in these skills, collectively they choose not to. 
To build the required highly-skilled people Singapore developed three plans: shortterm, medium-term, and long-term, for their national transition. To build immediate credibility as a nascent player in the biotechnology field, Singapore offered financial incentives for international scientist "stars" to bring their labs and research to Singapore (investment in capability) and to work also as a magnet for other young scientists. Given the modest experience of Singaporean companies in this new field, the government encouraged them to recruit scientific and managerial leaders from foreign firms to help in developing new start-ups (knowledge as an input). Through the short/medium term plans the government sent talented students to study at top foreign research universities and then to come back to Singapore (investments returning from knowledge-rich systems). Lastly, via its long-term strategy, the government encouraged the local universities to build alliances with top international research universities and encourage them to have a presence in Singapore, which would help in developing the required skills for the future (Finegold et al., 2004).

Investment in human capital is very lucrative and strongly supported by human capital theorists, as exemplified by the following three quotes from notable scholars in the field:

"Human capital is the knowledge and skills that people acquire through education and training being a form of capital, and that this capital is a product of deliberate investment that yields returns" (Theodore W Schultz, 1961). "Human capital is a form of investment by individuals in education up to the point where the returns in extra income are equal to the costs of participating in education. Returns are both private to the individual in the form of additional income, and to the general society in the form of greater productivity provided by the educated" (Becker, 1962, 1975). And from the later economists, "human capital is acquired human capabilities that are durable traits yielding some positive effects upon performance in socially valued activities" (David \& Lopez, 2001).

These embedded principles, which treat human capabilities as assets with values like other conventional production inputs, invest in new emergent capital and fuel economic growth. Without these investments, then, value cannot be generated and exploited nor can knowledge be adapted and improved for next generation 
innovations. These human assets should be effectively employed to impact profitably individuals, organizations and society (Schultz, 1961) and should result in three positive outcomes: people assimilate the accumulation of knowledge created by past generations; people build on that knowledge to develop new knowledge and ideas in the form of products, services or production methods and, finally, people should be prompted to generate totally new knowledge in form of ideas, products, services or processes (Babalola, 2003).

According to the findings, the study supports the extant human capital literature, with $42 \%$ of the participants asking for improvements in the quality of the education system, and agreeing that exposing staff to international effects and best practices was beneficial (42\%), as was recruiting talented foreign staff to fill human resource shortages and to transfer knowledge. There was also significant evidence to show that sending Saudi students and scientists abroad to study and work in high quality centres of excellence around the world was another favoured option. This investment in human resources was significantly supported in the findings for developing a life science innovation system in the KSA and this reflects the same process as that of the Singaporean government model. However, even in Singapore there remains a level of risk aversion and less-than-full-engagement of private businesses in the process of investing for the national future.

In the next section the findings are discussed against the innovation diffusion theory.

\subsubsection{The findings and the innovation diffusion theory}

Academia and industry in Saudi Arabia lack an innovation strategy, have a weak entrepreneurial attitude and weak innovative capabilities. These are fundamental prerequisites for a viable system and for innovation diffusion. Collaboration between actors is very poor and the lack of collaboration with foreign partners (to help reduce uncertainty associated with commercialisation processes and innovation transfers) has yet to be addressed. Also the government does not offer and entice businesses with incentives, or enact encouraging regulations for knowledge transfer or collaboration by business and academia at this current stage of system development (despite research evidence to show that other nations use their governments as the "first 
mover' to establish innovation systems). These conditions are identified as barriers for knowledge transfer and innovation diffusion in the KSA.

Knowledge transfer is the key issue and it lies at the heart of any innovation system according to the extant literature. Knowledge transfer is also the starting point for any innovation process. In developed or developing countries, knowledge transfer activities are seen as one of the government's responsibilities, yet in the KSA this capability has yet to be developed, despite calls from industry to do so and supporting evidence from the informants of this study. Knowledge transfer concerns $R \& D$ activities and transferring the output to the end users (Rogers, 2007) and, in some countries, such as emerging economies, where there is a lack of local capability to withstand global competitiveness, local firms look to acquire such capabilities and valuable skills through diffusion from MNCs and transferring knowledge through developing joint ventures locally (Lane et al., 2001; Tsang, 2002). There is no lack of MNCs in the Saudi context and the life sciences sector, but transferring knowledge by developing strategic alliances or building joint ventures (Powell et al., 1996) - is absent and has yet to be catalysed. Also making acquisitions and mergers helps in filling the knowledge gap - another form of transfer that has been identified in the literature as an enabler for success (Haunschild \& Miner, 1997). This study finds that, whilst corporations exist, they do so in isolation, and follow local corporate strategies rather than engaging with the other actors of the system to truly innovate and diffuse the ideas that will eventually lead to higher value-added employment for the KSA. Without such systems, and with the skills vacuum created and identified by the innovation system, investments in human capital will also be lacking and other mechanisms for knowledge transfer such as training, communication, and labour mobility (Argote et al., 2000) will remain underdeveloped.

In the findings $45 \%$ of participants think that developing the sector should be government-led through key projects, by 'building strong research centres' (33\%); 'developing research entrepreneurial universities' (35\%); 'more funding for R\&D' (38\%); developing enabling policy for R\&D, and spinoffs (52\%). The knowledge transfer concept was represented very clearly in the findings, as $38 \%$ of participants see a serious need to transfer knowledge from foreign partners to local firms through developing joint ventures, making acquisitions or licensing technologies. Also, 38\% 
suggest that exposure to international effects through recruiting foreign scientists and sending Saudi students and scientists to study or work abroad in developed countries would help to build and transfer knowledge, develop networks and keep up to date regarding the global context.

Networking comes in three types: within an organization, between organizations in either different or the same geographies (strategic alliances), or within a cluster. However, there are some factors that effectively influence knowledge transfer within networks; those factors and conditions include mainly absorptive capacity and social capital. Such absorptive capacity helps recipients to assimilate and effectively understand transferred knowledge and build on it to eventually "commercialise" such information. Social capital provides motivations and increased willingness for collaboration and transfer of knowledge (Reagans \& McEvily, 2003).

Social aspects (which unlock the potential of technological and inter-actor value added) play a significant role in transferring knowledge, especially in turbulent markets and developing economic environments (Martin \& Salomon, 2003; Minbaeva et al., 2003). Social aspects are supposed to intertwine with commercial activities to help develop relational embeddedness (Uzzi \& Lancaster, 2003). The findings of this study reinforce similar concepts - as captured within the coded categories of "building social capital" (26\%) and "improving living standards and relaxing cultural barriers" (13\%). These two strategies help to develop networking, collaboration, trust, and the embedding of foreign staff, effectively forming an innovation system.

For building absorptive capacity, the findings derived from the study suggest increasing the funding for $R \& D$, and this is supported by Cohen \& Levinthal (1990), who contend that $R \& D$ helps significantly in building absorptive capacity. Hence, this study shows that relational embeddedness and absorptive capacity are linked and identified both as critically important to the development of an innovation system, although no sequential evidence was found to suggest which should come first. Such a finding supports the validity of the final model (Figure 8-8) developed by the researcher as these 'concepts', derived from grounded research, have received little or no attention in the extant literature. 
A successful knowledge transfer system should, according to studies, be initiated following from an understanding of users` needs, as opposed to the researchers` needs. It should have a clear goal for knowledge transfer, created by a development policy, which supports users by guiding research orientation, investments in research and feedback to the research system. A high degree of customer contact should also be maintained according to Rogers (2007), which would also reinforce the relational aspects of the system in KSA that need to be developed. This has consistency with Rogers (2010) and his identification of innovation characteristics, such as: relative advantage - "the degree to which an innovation is perceived as being better than the idea it supersedes"- one of the most influential characteristics of those concerning the rate of adoption and including innovation aspects, cost, and social status (Sahin, 2006); and compatibility - "the degree to which an innovation is perceived as consistent with the existing values, past experiences, and needs of potential adopters" - with increased compatibility, the rate of adoption increases. The latter characteristic explores context in terms of needs and values to see if potential innovations meet these determinants. This study would suggest that appealing to social status and similar features are important to the development of the KSA, as well as a systems theory signalling device to the actors, from an innovation perspective; such government actions are means of focusing investment to generate new value (and value from an indigenous population that are by nature conscious of social status).

So, in developing policy and strategies for innovation, the implication is that all Saudi Arabian actors should consider the advantages of local endowments and the strengths they have whilst, in parallel, develop strong connections between academia and industry to understand the market needs and dynamics. Without this engagement, the emerging sector will face challenges to gaining investment in applied research in a way that yields benefits for business and allows the translation of research into knowledge produced for greater societal benefit.

The following findings strongly support the theory and its core concepts of networking, collaboration, and knowledge transfer leading to innovation diffusion: categories such as: "developing funding programmes for academic-private sector collaboration" (55\%) - to allow the development of a common policy based on their 
needs, interests, and problems; "developing clusters based on strengths and advantages" (52\%); "supporting entrepreneurship culture" (50\%), “developing an online platform" (16\%) for facilitating collaboration and networking; and "involving all actors in developing policy" (20\%) to identify priorities, "developing supportive regulations and policy", and maintaining the collective interests (feed-forward processes to mitigate uncertainty). This study supports the general literature and theories concerning innovation at the national level - both in terms of perceived enablers and current inhibitors. The overall reflection, by the researcher, is that the KSA context has similar issues to the rest of the world (from grounded research) yet, as a country in true transition, offers new insights of high value to countries that are attempting to move from rentier or manufacturing bases as opposed to existing models that reflect economies that have made such a transition (e.g. Singapore). In this manner, the KSA context has originated a new model that reflects these conditions.

\subsubsection{The Saudi LS Innovation Model against other models in the literature}

Some countries that have that have been compared by the researcher in this study were presented to identify differences of applied approach. For two key reasons, namely the location (Asia) and also the recent experiences, countries such as Singapore, India, Taiwan, and South Korea were chosen for comparison. Table 9-2 contains the key aspects of different innovation models from said Asian countries; all these models have different conditions and strategies.

The Eastern Asian countries (South Korea, Taiwan, Singapore) had similar common factors: heavy investment in developing human capital, stability in their political situation, wise macroeconomic policies, export-orientation, and high saving rate government supportive policies (Page \& Campos, 1993). However, these three new industrialized economies (NIEs) have different technological capability development strategies (L. Kim, 1997). They adopted three different national innovation systems models, which embedded different mixes of firms' strategies, government intervention roles, and innovation network structure. The three countries also applied three different models: for example, Korea relied on the agglomeration of large firms, while Taiwan depended on the SMEs to achieve their strategies in the catch up 
process, and Singapore heavily relied on the MNCs. As such, no existing model in the literature adequately captures the essence of the KSA system - a new model has therefore been developed by the researcher.

NIEs did not engage directly in developing innovations of new products or services as in the developed economies; instead they relied firstly on developing process and production capabilities, and then involved their activities within the global value chains to move their targets and expand the activities within the value chain ladder (P.-K. Wong, 1999). This was not found in the study of the KSA. In their study of the Taiwanese biotechnology industry, Su et al. (2009) confirm the concept of advantage and its relation to local comparative strengths; the Taiwanese biotechnology industry managed to outperform the others and excel by the virtue of its advantage in chipmaking and precision engineering, which in turn enabled it to excel in the diagnostic kit area. Again this was not found in the KSA study.

The Saudi context presents another model on the continuum of how best to develop innovation systems and must therefore look for special endowments and advantages and start with identifying the priority fields that have a potential of success, and that should be developed based on a collaborative approach between the actors. This would help the understanding of opportunities and market dynamics to reduce uncertainty in the environment. As such, the researcher believes each innovation system is contingent upon a number of actors and factors; his model is therefore another contribution which is sympathetic to the KSA context - in these circumstances the researcher does not believe that a universal or general model is possible and can be defended, as such innovation systems must be localised and 'fitted' to the local environment and stage of sector development (including capacities and relationality of actors). Some contingencies include the fact that Saudi Arabia, over the years, has received millions of visitors to its holy places; it is believed that this holds an opportunity that deserves to be investigated for investment. Also, Saudi society does not discourage marriage between relatives, which has resulted in many genetic diseases among the people; this problem should be scientifically and medically investigated, which would generate a new industry for the life science sector. 
Table 9-2: Aspects of different innovation models from different countries with compared with Saudi context. Sol

\begin{tabular}{|c|c|c|c|c|}
\hline Item & Singapore & India & Taiwan & South Korea \\
\hline Location & Asia & Asia & Asia & Asia \\
\hline Funding & Huge government fund & No significant fund & Government fund & Government fi \\
\hline $\begin{array}{l}\text { Approach and } \\
\text { strategy }\end{array}$ & $\begin{array}{l}\text { World-class infrastructure. } \\
\text { Attracts FDI. } \\
\text { Attracts talents. } \\
\text { Developing VCs working abroad }\end{array}$ & $\begin{array}{l}\text { Acquisitions attained by } \\
\text { doing local research. } \\
\text { Market-pull strategy. }\end{array}$ & $\begin{array}{l}\text { Started by manufacturing bespoke } \\
\text { inputs for global value chains, then } \\
\text { making innovation. }\end{array}$ & $\begin{array}{l}\text { Started by mar } \\
\text { bespoke inputs } \\
\text { value chains, } \mathrm{t} \\
\text { innovation. } \\
\text { Relies on Kore } \\
\text { capabilities. }\end{array}$ \\
\hline Market & Export orientation & Local market orientation & Export orientation & Export orienta \\
\hline Driver sector & MNCs & Big local companies & SMEs & $\begin{array}{l}\text { Agglomeration } \\
\text { firms }\end{array}$ \\
\hline Advantages & $\begin{array}{l}\text { Friendly business environment. } \\
\text { Attractive infrastructure. }\end{array}$ & $\begin{array}{l}\text { No guidelines } 19 \\
\text { Affordability }\end{array}$ & $\begin{array}{l}\text { High standards of manufacturing and } \\
\text { production practices. }\end{array}$ & $\begin{array}{l}\text { High standards } \\
\text { manufacturing } \\
\text { production pra }\end{array}$ \\
\hline Weaknesses & $\begin{array}{l}\text { Weak entrepreneurial attitude. } \\
\text { Government companies-focused } \\
\text { support. }\end{array}$ & Low funding & & \\
\hline
\end{tabular}

${ }^{19}$ Indian firms exploited the weak guidelines for drugs production to build their capabilities at the expense of qu advantages. 
The nation of Singapore has adopted a strategy to develop biotechnology as a critically important sector for a national competitive advantage, and a key sector that would enhance its economic growth, having developed its strategy in the 1980s (Finegold et al., 2004). By developing a "business friendly" environment, world-class infrastructure, financial incentives and high quality human capital, Singapore had a good and successful experience with foreign direct investment in ICT, electronics, and petrochemicals (Wong, 2002; Wong, 2001). In this regard, Singapore has relied on its local contingencies of multinational companies (MNCs) and an approach which includes these corporations as key contributors and drivers for developing the biotechnology sector. This initiative toward developing the biotechnology sector and the embedded huge investment entailed strong governmental intervention working as a driver for the initiative (Finegold et al., 2004). Again this model supports the primary role of the government in establishing the innovation system.

As a conclusion, such investment is really what the Saudi government needs to provide. It must signal to academia, industry and any other actors by investing significantly in infrastructure and knowledge-based businesses; this step would attract and encourage the other actors to become involved seriously in the innovation system.

The Singaporean government has embarked on many steps and actions for the purpose of infrastructure development, for instance, supporting basic research and building a technological infrastructure:

- Establishing the Institute of Molecular and Cellular Biology in 1987 at the National University of Singapore;

- Establishing other research institutes (the Bioinformatics Centre, the Genome Institute of Singapore, the Bioprocessing Technology Centre, and the Institute of Bioengineering and Nanotechnology), all these were created between 1996 and 2002.

○ Supporting the basic research through government-developed financial grants (project grants as seed funding, programme grants for more extensive research, and grants for strengthening the core capabilities and competencies of the research centres). 
Other key steps for Singapore to attract foreign investment were taken via developing venture capital offices in the US, which aimed to invest in US-dedicated biotechnology firms to provide a foothold globally, to build networks among American firms and promote Singapore among American venture capitalists. Later those venture capitalists brought some of their investment to Singapore and start-up alliances took place.

Saudi Arabia, on the other hand, has a weak venture capital sector (again a reflection of poor signalling in the system), which is reflected in the shortage of investments in new innovative businesses. The Singaporean experience of building venture capital is an inspiring model and could help to overcome funding challenges in other contexts such as Saudi Arabia. To ensure sustainability for this initiative and assure the impetus of programmes to stimulate change, the Singapore government allotted $\$ 1$ billion to build three bioscience funds, namely: Life Science Investment, Pharm Bio Growth Fund, and Singapore Bio Innovations, which enabled the building of new start-ups in Singapore and joint ventures based in Singapore, and the attracting of MNCs to invest in Singapore via financial incentives.

World-class research centres attracted by MNCs were also drawn to Singapore (Saywell, 2001). As mentioned above in section 9.2.2, these organisations were necessary to provide the required highly-skilled people, and as seen in the aforementioned section, Singapore developed three plans: short-term, medium-term, and long-term, in order to address the issue. To build immediate credibility as a nascent player in the biotechnology field, Singapore offered financial incentives for international scientist stars to bring their laboratories and research to Singapore and to work also as a magnet for other young scientists. Given the modest experience of Singaporean companies in this new field, the government encouraged them to recruit scientific and managerial leaders from foreign firms to help in developing new startups. Through the short/medium term plan the government sent talented students to study in top foreign research universities and then to come back to Singapore. Lastly, via its long-term strategy, the government encouraged local universities to build alliances with top international research universities and encourage them to have a 
presence in Singapore which would help in developing the skills required for the future (Finegold et al., 2004).

To provide a supportive and attractive life science ecosystem and meet the needs of the biotechnology industry, Singapore developed Tusa Biomedical Park and Biopolis near the National University of Singapore; this park included a vivarium to accommodate the mice for pre-clinical trials. Such an investment created capacity and relational advantages through proximity and co-location of actors. Beside the high quality of living requirements of the country, the government developed necessary services (such as lawyers' patent offices) to enhance relationships and "close" working. To develop the relevant requirements in the areas of legal, ethical and social issues, the government also established the Bioethics Advisory Committee (Kong, 2003), in effect building a system and creating the preconditions necessary for value exploitation.

In contrast to the quite weak status of Intellectual Property protection systems (IP) in some of the Asian countries (China, India), Singapore has a strong IP system. In this context Singapore established an Exploit Technologies body to commercialize the intellectual output of national research institutes. However, its method of managing IP was to centralize and work counter to the USA model, which works according to the Bayh-Dole Act and gives universities more freedom to commercialize government-funded research. Such centralization stifled creativity and enhanced government involvement (Vig, 2003) which is a paradox in that this study finds that the government should be the 'first mover', yet over-centralisation seems to limit the extent of innovation.

In terms of risk taking, the Singaporean model lacks the entrepreneurial attitude which is considered vitally important by other authors and this lack of attitude impedes entrepreneurship and its growth (Finegold et al., 2004). This lack is also reflected in the behaviour of research scientists who do not have enough enthusiasm and willingness to evolve research into "spin out" commercial businesses to exploit their own research outputs. Such an entrepreneurial academic base is also absent in the KSA, with the added problem constituted by the fact that academics (employed by the Government) are prohibited from developing any such private ventures. It would 
appear from the experience of other countries that this actor (academia) needs to play a role in the commercialization process and that this is a worthy output of research (to allow academics to profit from their labours).

Given the status of a government-driven and top-down approach, the companies that work in this context find difficulties in accessing financial resources, unlike their governmental counterparts. Collaborations within companies, research centres and industry (and between companies) suffer, and all these constitute impediments and shortcomings in the Singaporean context (Stein, 2003). These voids (in terms of weak entrepreneurial attitude and governmental businesses-centred funding) should be avoided in developing the innovation system in Saudi Arabia, so that all actors receive the same treatment and are involved in the innovation system.

Indian biotechnology companies have managed to compete with the multinational companies (MNEs) in the local market, despite the MNEs' strengths in terms of financial and technological resources. The Indian firms displayed high quality performance in exploiting and exploring practices, managing to leverage external knowledge resources while at the same time developing internal innovation activities. They worked on linking their innovation strategies to their competitive strategies (Krishnan \& Jha, 2011). Biocon, as a big Indian pharmaceutical company, worked on a strategy that relies on exploiting its core competences and exploring the areas that require special capabilities and potential opportunity, and then developing collaboration with external partners to transfer knowledge, taking on a joint venture and then a complete acquisition of the joint venture. The company also follows a partnership strategy with key actors in the global industry and small biotechnology firms "spun out" from universities, and has relied heavily on external sources, either by acquisition or alliance, to enhance its internal capabilities.

External sourced knowledge was a key strategy for Indian firms; to access international tangible and intangible resources, and fill the gap in their capabilities that are difficult to be traded through the normal market transactions, Indian firms practiced an acquisition strategy (Gubbi et al., 2010). This strategic choice was emphasized by many authors (Capron et al., 1998; Ethiraj \& Levinthal, 2004), is an enabling tool for emerging companies, with the complementary assets required for 
achieving strategic objectives and solving complex problems, to interact within a global scenario. Internally generated growth is time consuming and path dependent, whilst corporate acquisitions can shorten the time needed to develop high quality capabilities for strategic renewal (Nelson, 2005).

A knowledge transfer strategy is crucial to enable companies from developing countries to catch up with industry in developed countries, and to shorten the catch-up process time. However, building the necessary internal absorptive capacity and capabilities are key requirements for transferring knowledge from external sources (investment in human capital, adaptation). The Saudi private sector is not involved in innovation and that represents a serious challenge for developing the Saudi innovation system, which entails the government developing supportive policy and regulations, and "kicking off" the investment in knowledge and infrastructure to encourage other actors.

Affordability is an aspect that has been emphasized in the literature and taken into account to ensure, for example, that people have the ability to access drugs (outputs of an effective innovation system) without excessive financial burdens. In relation to the Indian market conditions and the market demands on, for example, diabetes drugs, Biocon adopted a market-pull strategy and product innovation. In addition to adding research and manufacturing facilities, the company also expanded its position within the value chain by setting up facilities for clinical trials activities (Krishnan \& Jha, 2011).

The Saudi market is quite different to the Indian market. Saudi citizens have high expectations of the market and they can afford to pay more to make sure they will get high quality medical products; they usually do not compromise on quality, especially in medical products. This situation challenges Saudi firms on the quality front to compete with key players in the global LS industry.

Based on the researcher's findings, the sentiment concerning funding from the Saudi government is that of a moderate level; weak research infrastructure and FDI do not enable knowledge transfer activities (without actual research or a structure to do so), so the Singaporean model cannot be imposed or applied. The country has high 
expectations from its customer base, but no national entrepreneurial attitude, no industry involvement, weak capabilities and strict guidelines, so it cannot apply the Indian model either. The KSA has a weak manufacturing sector and no private role in $\mathrm{R} \& \mathrm{D}$, which would make it difficult to apply the Taiwanese or South Korean models, too. It is believed that these different innovation systems (each with unique national conditions and endowments) would not work very well in the Saudi context. This led the researcher to adopt a grounded theory approach to developing an innovation system grounded on data instead of testing the extant innovation models. The researcher favours a contingent approach and has therefore built a model that reflects, through actual grounded theory, differences in economic, political and other key features. The current Saudi situation and its many challenges and different political, social, and economic conditions differentiate the country from its Asian peers.

Currently there is no system, no collaboration, weak research infrastructure (no cohesive behaviour), weak human capabilities (inputs), no private sector interested in $\mathrm{R} \& \mathrm{D}$, and no culture or supportive policy for entrepreneurship in academia in the KSA. The government has not given any serious signal to encourage the development of an LS innovation system, and the other actors (academia, PS) will not move before seeing a serious initiative and movement, incentives, motives, investment and strategy from the government. The research infrastructure and the policy do not encourage the various actors to engage in knowledge activities. Indeed, the actors are working separately and there is no entity or initiative bringing them together to collaborate and work for developing the LS sector.

Grounded in the collected data and having regard to the Saudi conditions and challenges, a LS Innovation Model, Figure 8-6, has been developed to fit the Saudi context and work efficiently. This LS Innovation Saudi Model of Figure 8-6, is led mainly by the Government and relies on government's role and investment, at least at the beginning, by raising public institutions' quality through interacting with the global context; improving the business environment; building a research infrastructure; investing in knowledge; providing funding, incentives and motives; establishing a Saudi Knowledge Economy Organization (SKEO) to be in charge of involving all actors and developing enabling policy; supporting entrepreneurship; 
providing coordination; identifying priorities; funding collaboration between academia and industry without any discrimination for the sake of the public companies, and building a LS cluster.

The key role of the Saudi Knowledge Economy Organization (SKEO) should involve all actors in planning and decision-making, and coordinate the relevant activities for every actor. There is a need to develop a LS cluster, invested in by the government and involving and motivating other parties to contribute to its development. The intention is that it will work as a physical magnet for investment and talents, and for the progress of the LS sector.

In turn, the private sector should develop an entrepreneurial attitude and an innovation strategy; transfer foreign knowledge - through building joint ventures, making acquisitions, and partnerships - and develop the capabilities for conducting good research and managing innovative projects.

Academia should work on raising the quality of the education system; developing human capital; engaging in entrepreneurship and spin-off technologies; developing collaborations and partnerships with foreign institutions to build capabilities, learning and knowledge transfer, and collaborating with industry and research centres.

In addition, all actors in the innovation system should interact with the global context to undertake knowledge transfer (through joint ventures, acquisitions, licensing technologies, and building partnerships), learn from best practice, and adapt to any potential change.

As has been argued, it is obvious that the Viable Saudi Life Science Innovation System (VSLSIS) (Figure 8-8) has its own conditions and context which have been found to differ from the models of Singapore, Taiwan, India, and South Korea, which have been discussed in this section. This difference is to be expected, given the different social, economic, and political differences between the countries. According to Cooke (2004), there is no "one-size-fits all" model, and the context will determine the innovation system which is suitable and can work efficiently and effectively. 
Building an innovation system must take into account the context, which means that "copying and pasting" other policies would not work well and would not result in innovation and economic development. National scope covers regional and sectoral systems, but some industries and regions have their own specific characteristics and conditions which should be taken into account when developing overall policies (Malerba \& Nelson, 2011; Niosi, 2011a). In essence the researcher would support the view that additional local contingencies should be respected even though a national innovation system is established by the government in its role as 'first mover'.

\subsection{Findings in the Light of Focal Literature}

In this section, discussion will be presented to show similarities and differences between the findings of this study against the World Bank's knowledge economy framework (World Bank, 2012).

\subsubsection{Knowledge economy requirements}

The World Bank, through its World Bank Institution`s "knowledge for development" programme, developed the Knowledge Economy Framework, comprising of four "pillars" (economic and institutional regime, human capital, ICT infrastructure, and innovation systems) as key components that can illustrate the status of a country against the knowledge economy, firstly measuring the knowledge accumulation in a country, and then using this information to develop the appropriate policy that will help build the country's capacity to assimilate, create, share and use knowledge that will lead to a transition to the knowledge economy (World Bank, 2012).

The framework does not suggest or explain any kind of relationship among these four pillars, but it provides guidelines to utilise them as key blocks for building a knowledge economy. The findings of this study against the backdrop of the knowledge economy framework are discussed below with specific reference to its four constituent pillars.

\subsubsection{Economic and institutional regime}

Generally, the economic and institutional regime concerns the provision of suitable regulations and incentives that have the potential to enable effective use of existing 
knowledge and the creation of new knowledge. These regulations or incentives are intended to enhance the economy's capacity to respond and adapt to competitive and changing business conditions and to address opportunities. They should also produce the ability to increase productivity and develop new innovative activities. The economic and institutional regime is a central factor in the economy's ability to use knowledge effectively, and also it is critical to the functioning of the other three pillars of the knowledge economy. The influence of this pillar covers many areas of the economy such as the product market, financial market, labour market, knowledge market, industrial restructuring and entrepreneurship. According to Thomas \& Carl (2001) the incentives and regulations introduced should lead to support and facilitation of practices and matters such as: competition between foreign and domestic firms; customer protection; corporate governance; venture capital and equity; transparency; labour mobility; intellectual property rights; valuation of intangible assets; entrepreneurship and SMEs.

The current situation in the Saudi innovation system lacks effective deployment of venture capital money, and suffers from a weak attitude toward entrepreneurship. The regulations do not support entrepreneurship or labour mobility. This gap in regulations has a negative impact on the building of new start-ups and on knowledge transfer. However, the findings of this study suggest some interventions and strategies that are believed to be capable of challenging these barriers in the regulations: raising institutional quality $(35 \%)^{20}$; supporting an entrepreneurship culture (50\%); developing enabling policy for R\&D and spinoffs $(52 \%)$; improving industry infrastructure and the business environment (38\%); developing supportive staffing regulations (13\%); developing VCs funds (33\%); developing seed funds $(33 \%)$; encouraging angel investors (16\%), and raising funding efficiency (42\%).

The economic regime that builds barriers to entry for business, impedes competition, does not protect intellectual property rights, increases tax burdens and impedes entrepreneurship will hamper innovation and the development of new products and services (Kaminski \& Kaminski, 2013). Some of these conditions were found to be present in the embryonic state of the KSA Life Sciences sector.

\footnotetext{
${ }^{20}$ The percentage represents the number of participants supporting the themes.
} 


\subsubsection{Human capital}

Human capital has been defined as the collection of investments in education, training, health, and migration which enhance the productivity of individuals in the labour market (Becker, 1962; Kiker, 1966). Another definition describes human capital as the collection of knowledge, skills and innate abilities that people gain and develop through their lifetime (Laroche, Mérette, \& Ruggeri, 1999). The shift to a knowledge-based economy, which was driven by technological change and market globalization, put human capital and other related themes at the centre of policymakers`attention and concerns, with human capital contributing significantly in raising the level of income in developing countries through the capacity of absorption and imitation of international technology (Laroche, Mérette, \& Ruggeri, 1999). Human capital has five aspects (Douglass, 2010):

- cultural capital - concerned with cultural background and family traits such as language, community, neighbourhoods, and ethnic ties;

- economic and educational capital - correlates educational attainment to the economic status of the family;

- social capital - the behavioural knowledge that concerns understanding of a society's manners and institutions, the best use of opportunities, and dealing effectively with and navigating growing bureaucracies;

- cognitive capital, which concerns the different intelligence forms and their distribution

- aspiration capital, which emphasizes that ambition plays a major part in shaping both social capital and cognitive capital, and is itself influenced by environmental factors and personal traits.

The development of these is significantly influenced by the national education system, which affects the individual's path to higher education. Developing educated and entrepreneurial people who are able to create, share, and make effective use of knowledge and skills is a key element in developing a knowledge economy (Thomas $\&$ Carl, 2001). For a developing knowledge economy, education and training is vital for human resources development, enhancing abilities for research and knowledge generation, and up-skilling worker competencies (Peters, 2001). According to Drucker, "In the knowledge society into which we are moving, individuals are central. 
Knowledge is not impersonal like money. Knowledge does not reside in a book, a databank, or software program. Knowledge is always embodied in person; carried by a person; created, augmented, or improved by a person; applied by a person; taught and passed on by a person; used or misused by a person. The shift to the knowledge society therefore puts the person in the centre" (Drucker, 1994). All the above literature confirms the extreme importance of developing human capital as a key requirement for developing knowledge economy.

In Saudi Arabia, there is a clear void in human resources able to produce new technologies and manage high standard and complex businesses. The shortage lies in all sectors (government, academia, industry), and the education system suffers from weaknesses in its outputs. There is also a weak collaboration culture and entrepreneurship. All these conditions contribute to the lack of producing promising technologies and investment in new knowledge-based businesses. These conditions have also created barriers to transfer knowledge either locally or from international sources because of the lack of absorptive capacity and ability to undertake significant R\&D.

In the grounded study findings there is overwhelming support for the idea of developing human capital: $42 \%$ of the participants confirm the need for raising education system to the level where its outputs satisfy market needs in the private and public sectors or academia so they can produce, exchange, and use knowledge for the sake of the economy; $42 \%$ of the participants recommend exposing people to international effects (through attracting talented foreign people, sending people to study abroad (and to stay long enough to gain and transfer knowledge), and making the education system international in terms of faculty and students; $26 \%$ of participants see building social capital as an important strategy and that policy contributes in developing some aspects of human capital such as collaboration, trust, and networking; supporting entrepreneurship culture (50\%) is seen as a key factor in developing entrepreneurs to transfer knowledge and engage in innovation. This all demonstrates a close match between the literature and findings with regard to developing human capital for building a knowledge economy. 


\subsubsection{ICT infrastructure}

The purpose of providing an information and communication infrastructure is to enable effective means and tools for communication, knowledge dissemination, and information processing (Thomas \& Carl, 2001). Globalization has implications for economic and social networks, which require effective information and communication technologies infrastructure to ease and facilitate the accessibility of information or knowledge. This provision of effective accessibility and its effect on knowledge production helps in bridging the knowledge gap which exists between nations. The knowledge produced has the potential to develop into innovation, which has become an imperative for surviving global competitiveness and open markets (Lor \& Britz, 2007). According to Polder et al. (2009) at the firm level, ICTs in services sectors are key drivers for all kinds of innovation (product, process, and organizational innovation), while it is less important in the manufacturing sector, in spite of the positive effects that it may have. In many studies within different contexts (Black \& Lynch, 2001; Bresnahan et al., 1999; Bugamelli \& Pagano*, 2004; Castiglione, 2010) a positive relation was found between ICTs investment and increased productivity at the firm level. However, the ICTs would not work effectively alone, requiring interaction with some complementarities such as human capital, R\&D, and organizational innovation (Black \& Lynch, 2001; Bresnahan et al., 1999).

In the findings there is no direct mention of ICT as a key requirement for developing a knowledge economy from the grounded theory and coding; however some participants stressed the need for improving industry infrastructure and the business environment (38\%) and this concept can be perceived to include ICT. Another explanation may be that the participants feel that the current ICT infrastructure is adequate and satisfies at least the bottom line of knowledge economy needs.

\subsubsection{Innovation systems}

The key contribution of this study is to inform Saudi policy in developing a LS innovation system for the Kingdom of Saudi Arabia.

In his seminal book Japan: a New National Innovation System, Christopher Freeman was the first to mention the concept of a National Innovation System (Niosi, 2010, 
2011a). The emergence of National Innovation systems began in the mid-1980s over the debates on the European Industrial Policy (Freeman, 1987). The OECD was one of the first International organizations that started to use the NIS concept as an integral part of its analytical perspective (Lundvall et al. 2002). According to Nelson (1993), A National Innovation System "is a set of institutions whose interactions determine the innovative performance of national firms. "Nelson also emphasizes that the core of an innovation system is the policy of science, technology, and innovation that supports R\&D. Lundvall (1992), furthermore, defines the National Innovation System as "constituted by elements and relationships, which interact in production, diffusion and use of new and economically useful knowledge." The elements mentioned include institutions such as universities and public research centres, firms, government regulatory systems, the educational system and financial institutions (Benoît Godin, 2009). Smith (1995), the author of the OECD methodological manual on measuring innovation, also emphasizes the concept of interactions between actors: "the overall innovation performance of an economy depends not so much on how specific formal institutions (firms, research institutions, universities, etc.) perform, but on how they interact with each other". Freeman (1987) also defines the national innovation system as "the network of institutions in the public and private sectors whose activities and interactions initiate, import, modify and diffuse new technologies". According to Metcalfe (1995), a national innovation system is "the set of distinct institutions which jointly and individually contribute to the development and diffusion of new technologies and which provides the framework within which governments form and implement policies to influence the innovation process. As such it is a system of interconnected institutions to create, store and transfer the knowledge, skills and artefacts, which define new technologies". More recently, the World Bank Institute, which developed the knowledge economy framework and its embedded four pillars and considered the NIS as one of these four pillars, defines NIS: "An efficient innovation system comprising firms, science and research centres, universities, think tanks, consultants and other organizations that can interact and tap into the growing stock of global knowledge; assimilate and adapt it to local needs; and use it to create new knowledge and technology" (Bank, 2012).

The production and innovation processes within a knowledge economy need effective linkages; effective use of resources; motivating incentive structures, which can 
respond and come up with changes in technology, market and social conditions, and involve complementarity among the innovation systems actors (Thomas \& Carl, 2001). However, what can be concluded is that the concept of a National Innovation System pays great attention to the interactions between all actors and the innovative output of these interactions and collaborations (OECD, 1997). National scope covers the regional and sectoral systems, but some industries and regions have their own specific characteristics and conditions which should be taken into account when developing policies (Malerba \& Nelson, 2011; Niosi, 2011a, 2011b).

The findings of this study generated many themes and concepts that match the literature conceptual frameworks, but do not entirely support any existing model. For example, the theme 'developing supportive regulations and enabling policy': under this theme the findings presented concepts including: building social capital (26\%) to help in developing collaboration, trust, and networking among actors and relevant people; supporting entrepreneurship culture (50\%) to help in developing businesses and transferring knowledge; government led projects (45\%) - which is specifically supported by the literature. Niosi (2011a) argued that, in spite of the markets having an important role in creating innovative situations for private sector organizations and routines, the role of the government was key (in providing and developing innovation policy, funding technical innovation, supporting technical and academic research and education).

In the findings also are developing enabling policy for $R \& D$ and spinoffs (52\%), funding programmes for academia and private sector collaboration (55\%), involving all actors in developing policy (20\%), developing strong research centres (33\%), developing clusters based on strengths and priorities (52\%) and developing research entrepreneurial universities (35\%). In the literature there is clear demonstration of the importance of knowledge clusters and entrepreneurial universities in developing and progressing innovation systems; the cluster is a geographic area containing a group of firms and institutions in a specific field, interacting and connected in a complementary and collaborative manner (Porter 1998), also: “A knowledge cluster is a local innovation system organized around universities, research institutions and firms which intend to drive innovations and create new industries" (Evers, 2008). Within the context of the Knowledge Economy, scientific knowledge constitutes a 
key input in the innovation and production theory (Mansfield \& Lee, 1996; Mowery \& Sampat, 2005), thus becoming a third mission for universities in addition to the previous traditional two (education and research). The third mission has shaped the new role of the universities in commercializing the knowledge produced, in knowledge transfer, and in engagement with society's activities and the business sector (Etzkowitz, 1998; Hansen et al., 2008; Lehmann et al., 2009; B. R. Martin, 2003). The emerging of disciplines like biotechnology, and the effect of funding reductions, globalization and the nature of innovation has been seen as a process which involves different actors, all of which has helped the emergence of what has been called the entrepreneurial university (Rasmussen et al., 2006).

\subsubsection{Questionnaire findings against first study findings}

The findings of the questionnaire showed strong confirmation for the findings of the first study, and this confirmation enhances the validity of the whole study (the ultimate aim of the triangulation method in confirming and strengthening the study findings). Besides the demographic questions, the questionnaire has 17 questions, one to measure the importance of involving the private sector in the innovation process, and the other 16 to explore the encouraging and hindering factors that influence the four sub-sectors of the private sector (MNEs, LLCs, SMEs, and KB-SUs).

The 16 questions explored the influence of 91 variables (see Table 8-12 to Table 8-28) (also see the appendices (12.5, and12.6) and the role of those variables in encouraging the private sector to, or hindering it from engaging in the innovation system. The results supported the first study findings; the sub-sectors have many common hindering and encouraging variables, which constitute the variables that affect the involvement of the private sector in the innovation system. The next four tables detail the results based on categorizing the variables in two groups (encouraging variables and hindering variables) for each sub-sector. 
Table 9-3: Encouraging and hindering variables for MNEs. Source: The researcher.

\begin{tabular}{|l|l|}
\multicolumn{1}{|c|}{ Encouraging variables } & \multicolumn{1}{c|}{ MNEs } \\
\hline \multicolumn{1}{|c|}{ Hindering variables } \\
\hline Existence of capabilities & Lack of system \\
\hline Existence of funding & Cultural barriers \\
\hline Networking & Weak labour market capabilities \\
\hline Improving business environment & Weak research infrastructure \\
\hline Government reforms & Business environment \\
\hline Market size & Lack of cluster \\
\hline Priorities and advantages & Unstable geopolitical area \\
\hline Making JV, acquisition, licensing technologies, & Lack of cluster \\
partnership. & \\
\hline Location & \\
\hline Providing incentives and motivations & \\
\hline
\end{tabular}

Table 9-4: Encouraging and hindering variables for LLCs. Source: The researcher

\begin{tabular}{|c|c|}
\hline \multicolumn{2}{|c|}{ LLCs } \\
\hline Encouraging variables & Hindering variables \\
\hline Accessing funding & Lack of capabilities \\
\hline Market & Lack of collaboration \\
\hline $\begin{array}{l}\text { Making JV, acquisition, or licensing } \\
\text { technologies }\end{array}$ & Having no strategy \\
\hline Partnership & No entrepreneurial attitude \\
\hline Providing incentives & No system \\
\hline Government reforms and orientation & Weak research infrastructure \\
\hline Local advantages and priorities & Weak labour market capabilities \\
\hline
\end{tabular}


Table 9-5: Encouraging and hindering variables for SMEs. Source: The researcher.

\begin{tabular}{|c|c|}
\hline \multicolumn{2}{|c|}{ SMEs } \\
\hline Encouraging variables & Hindering variables \\
\hline Size effect & Lack of funding \\
\hline Government reforms and orientation & No strategy \\
\hline Providing incentives & No entrepreneurial attitude \\
\hline Market size & No collaboration culture \\
\hline \multirow[t]{7}{*}{ Local advantages and priorities } & Weak capabilities \\
\hline & Weak research infrastructure \\
\hline & Fledgling VC market \\
\hline & No system \\
\hline & Weak labour market capabilities \\
\hline & No credibility \\
\hline & Lack of cluster \\
\hline
\end{tabular}

Table 9-6: Encouraging and hindering variables for KB-SUs. Source: The researcher.

\begin{tabular}{|l|l|}
\multicolumn{1}{|c|}{ Encouraging variables } & \multicolumn{1}{c|}{ Hindering variables } \\
\hline Government funding for public research & Lack of quality research \\
\hline Knowledgeable people & No connection or collaboration with other actors \\
\hline Local advantages and priorities & Lack of seed funding \\
\hline Government reforms & Weak managerial capabilities \\
\hline & Weak entrepreneurial attitude \\
\hline & Regulation barriers for entrepreneurship \\
\hline & No system \\
\hline & Weak research infrastructure \\
\hline & No developed cluster \\
\hline & Fledgling VC market \\
\hline & Barriers in doing business \\
\hline & Weak labour market capabilities \\
\hline
\end{tabular}


To sum up these four tables, belonging to the four private sub-sectors, the following table contains all the encouraging and hindering variables which concern the private sector.

Table 9-7: Encouraging and hindering variables for the private sector. Source: The researcher.

\begin{tabular}{|c|c|}
\hline Encouraging variables & Hindering variables \\
\hline Providing incentives and motivations & Lack of system \\
\hline Improving business environment & Weak capabilities \\
\hline Government reforms and orientation & Weak research infrastructure \\
\hline Market size & Funding \\
\hline Priorities and advantages & Policy gap \\
\hline $\begin{array}{l}\text { Making JV, acquisition, licensing technologies, } \\
\text { partnership }\end{array}$ & Lack of strategy \\
\hline \multirow[t]{5}{*}{ Location of Saudi Arabia } & Weak entrepreneurial attitude \\
\hline & Lack of collaboration and connection \\
\hline & Lack of quality research \\
\hline & Lack of credibility \\
\hline & Lack of developed cluster \\
\hline
\end{tabular}

As has been seen from the results of question number 7 in the survey ("In order to develop the knowledge economy in the life science sector, to what extent do you agree or disagree that involving the private sector is needed?") (See appendix 12.5), it was found that involving the private sector in the innovation system is inevitable and that the system would not work without the private sector as a key actor.

The findings of the questionnaire regarding the involvement of the private sector in the innovation process also found that the key variables for business were included, embedded and relevant to all of the other actors (government and academia). Going back to the developed LS Innovation Model, Figure 8-6, and Figure 8-8, all the findings that have been displayed in Table 9-7 were represented here and support the developed model, thus confirming their importance in progressing the life science sector in Saudi Arabia. Therefore, to involve the private sector in the innovation system and in progressing the life science sector, the findings of the questionnaire 
suggest overcoming the hindering variables and at the same time satisfying the encouraging variables.

\subsection{Summary}

The findings uncovered three very worrying areas of weakness in the Saudi context:

- the lack of systematic behaviour: there is a gap between the key actors; there is no significant connection between them; there are no strategies that identify the aims or that plans to achieve those aims and to involve them in the innovation process

- the lack of human capabilities that can produce or use knowledge to add economic value, and

- the gap in the innovation cycle in the Saudi system.

These conditions entailed the researcher focusing on those areas in the analysis and adopting a systems approach as a unique method to develop the Saudi LS innovation system. This approach is embodied in the Viable Life Science Saudi Innovation System model in Figure 8-8. 


\section{Conclusion}

This chapter will present the research reflections, recommendations for future research, government policy and teaching.

\subsection{Research Journey}

Over the course of this study many points of reflection have arisen. The more exciting stage of the study was data collection. The main concern was how to access a real and representative sample for the study, to ensure its reliability and to provide rich and relevant data. This was a successful journey that has yielded very interesting results. Generally speaking, Saudi people culturally do not have a great respect for time, and this was one of the concerns when accessing very senior people. In Saudi Arabia, access and time factors prolonged my data collection time by more than six months. An adequate number of experts in the UK and Saudi Arabia were accessed. Data collection took more than six months travelling from city to city, and more than 7 thousand kilometres were driven. However it was worth the effort, and the assistance of Saudi government sponsorship was gratefully appreciated.

Grounded theory presents a special journey for the researcher, as it is unusual for $\mathrm{PhD}$ students. If the study were to be conducted again, a greater amount of time would have been allowed between interviews to allow the researcher to codify and write up the interview content before entering into another one. Due to the compressed timeline for data gathering, the researcher had to work long hours to ensure that all interviews were coded effectively in a relatively short time span. The protocol adopted and the good practice followed allowed the findings to be effectively archived and later analysed without any loss of data.

There is no such thing as a perfect doctoral study. As such if the researcher were to begin the process again a number of changes would be made to the data collection strategy. In particular, greater strides would be made to ensure that the three sectors government, academia, and the private sector - would be balanced in equal sizes as opposed to the current skew in this study. However, it must be noted that the main 
reason for the imbalance is to be attributed to the difficulty to retrieve suitable representatives from the private sector, that had both the required seniority and specialism, primarily due to the relatively smaller size of the sector, compared to government and academia. Furthermore, it must be noted that private sector in general was not particularly collaborative or interested in the innovation aspects of the study.

\subsection{Contribution}

The first contribution of this study is methodological. The systematic literature review, conducted after the grounded fieldwork, reveals that this is the first study of its type in the life sciences and innovation fields of study and offers a good method for generating contextually-rich insights into complex systems. Many previous studies have simply adopted existing scales and applied them in countries where business practices are fundamentally different to those of the established Western and capitalist economies.

It was always the intention of this study to offer practical insight into how best to manage the Saudi Arabian system. Therefore contributions are made to the development of government policy and the necessary infrastructure that is needed to exploit a knowledge-based economy. Previous studies have tended towards the application of existing models, particularly those drawn from Singapore, Taiwan, or India. These countries have different contextual, political, economic, social and cultural conditions. As such this research methodology was of limited use when studying the rich context of the Kingdom of Saudi Arabia. The main contribution is to identify key gaps in the Saudi Arabian model of innovation interaction. This research finds that the country has a weak infrastructure where the government has not taken a lead role in investing, developing structures and enhancing relationships between actors - the latter features are typical of previous studies in this field of innovation driven economies.

This study is the first of its kind, in the English or Arabic language, to address the state of the system in a GCC country. The major contribution offered is a new and fundamentally different system design than those that are currently investigated, from western, Indian, Taiwanese and Singaporean backgrounds. The Kingdom of Saudi 
Arabia is an immature system and at the moment is embryonic in its approach to the development of the knowledge economy. This study therefore finds that the model operated by the Kingdom of Saudi Arabia is qualitatively different from that of any other country. Under this model the government has not yet taken the lead in driving the innovation agenda. At this current juncture both industry and academia are in a vacuum, where they are waiting for the government to lead on innovation policy and signal to the other system actors that this is a good, worthwhile, and highly investable area.

This study has contributed to a growing body of international research concerning Life Sciences. The major literature in this field is dominated by non-Life Sciences studies. This research therefore adds a new dimension within the context-rich country of the Kingdom of Saudi Arabia.

The main contribution of this work, however, is the presentation of the model (see Section 3.12). Initially this was a theoretical model drawn from analysing the literature after the field research had been conducted (the grounded approach). The resultant model is a new addition to the body of knowledge, one that has not been presented by any other author. This study therefore offers the first insight into a modern economy that is in transition from a rentier economy to a modern knowledgebased economy. All other studies in this field have addressed issues of minor national transformations from economies with an existing but embryonic manufacturing sector (building upon this base) towards a high-value and knowledge-based business (beyond the manufacturing sector to include additional services to support the ecosystem).

The next figure shows the research-derived Viable Saudi LS Innovation System Model (VSLSIS). The model is a "combined model" and is based upon the analysed data collected and literature review stages of this thesis. 


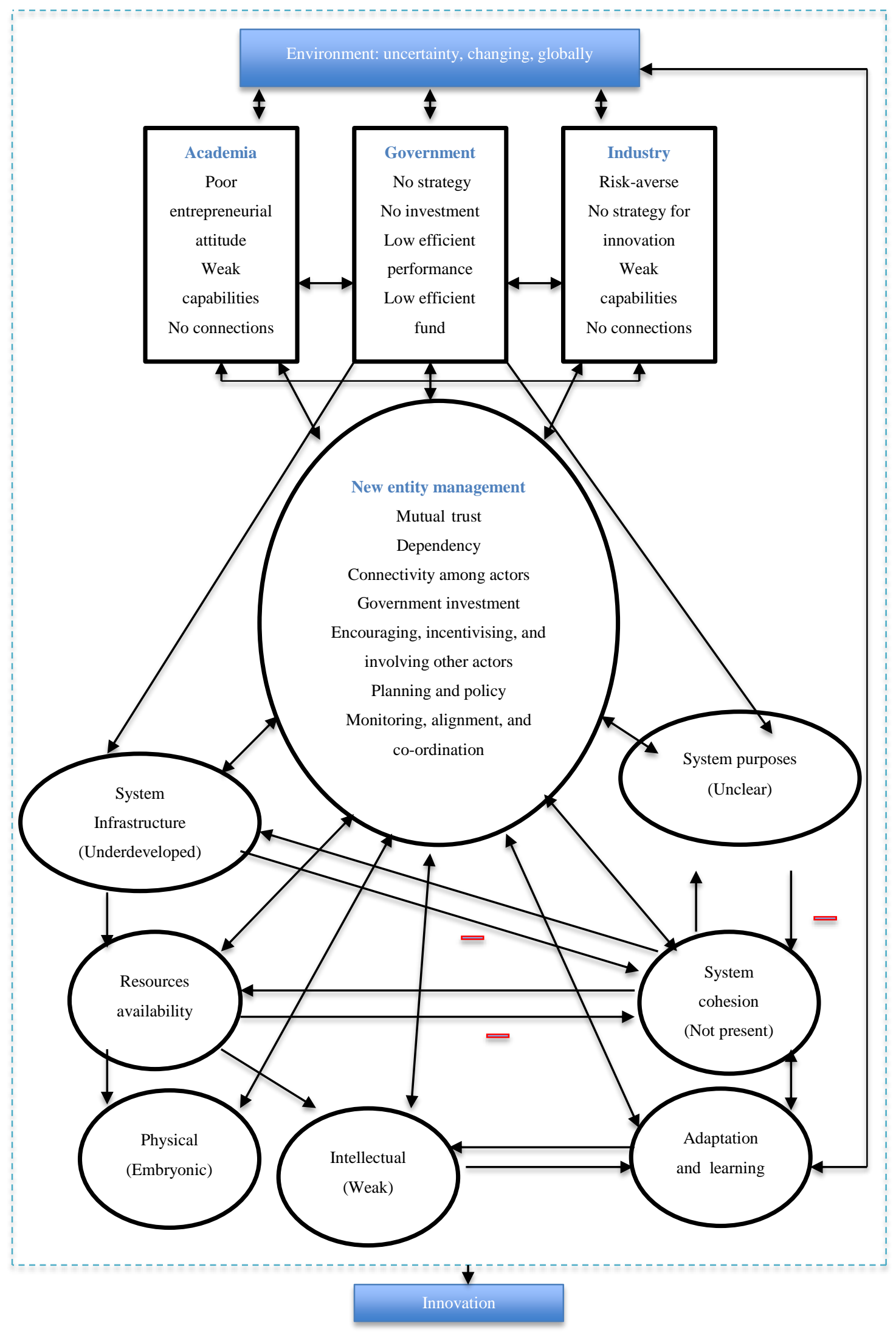

Figure 10-1: current innovation system, and the proposed Viable Saudi LS Innovation System (VSLSIS) 


\subsection{Implications}

This section presents the implications for teaching, government policy and future research, together with recommendations.

\subsubsection{Implication on teaching}

The first implication of this research work concerns the teaching of the subject of innovation at schools and universities. This study clearly shows that the innovation ecosystem is important when developing relationships between national actors. These actors include the government, industry, and academia as well as organizations of different sizes and nationalities. Given such a rich context and the way in which the environment shapes actor behaviour, this thesis supports the view that all teaching should contain an element of context, which cannot be taught using standard lectures. Instead students must be shown what the impact of different contexts is on a particular organization and its relationships. Therefore, the research supports a case-based approach to teaching. Under the case-based approach students should examine practices as they are adopted and developed by a company in the context of the national system within which it operates. Hence the researcher would support the view that this research shows the context and the social actors that are involved in innovation and knowledge transfer. Students of a particularly Saudi Arabian approach to innovation management would therefore benefit from understanding the perspectives of many different actors rather than the more limited approach undertaken by current studies which focus mainly on industry or the government.

\subsubsection{Government policy}

The Saudi government has a Vision 2030 document (Mckinsy global, 2015); among its many areas of interest is the knowledge economy. This study matches the general objectives of Vision 2030, but also contains strategic directions within, as well as specific outputs concerning the development of the LS innovation model. This model can be utilized by the government to shorten the time needed to investigate the life science sector. Therefore, the study needs to be presented to the government for discussion of the way that it can best be employed. The researcher is already sponsored by the government and works in the Saudi Ministry of Industry, so he will 
be very involved and close to the dynamics relevant to the Vision 2030 implementation and management.

This study clearly shows that government policy needs to be aligned with the interests of academia and the interests of the private sector. Only by signalling to the market that Life Sciences is a legitimate and growing area of the economy would that sector attract bankers and private equity funds. In sending such a signal government policy should join up the systems of Saudi Arabian knowledge extraction and knowledge exploitation. In this manner, the researcher firmly supports the view that the government must take a proactive lead in establishing these relationships and formalizing its relationships with industry and academia. The researcher would also firmly support the development of clusters in the key areas of the country, with active government support for each cluster, by moving knowledge between academia and the local groups. The government should also look to develop national companies, including government-owned companies, and to attract foreign direct investment (FDI) into the new system.

\subsubsection{Future research}

The first and natural research study that emerges from this research is the conduction of a longitudinal study of how Saudi Arabian actors evolve over time and accommodate the 2030 Vision document (Mckinsey global, 2015). This study will look at how the system changes and what relationships are built that successfully exploit Saudi Arabian knowledge and turn it into value for the country.

The second study is a comparison of this Saudi Arabian system with others in the GCC community and beyond. This study will seek to test whether national cultures influence working practices.

The third piece of future research is the creation of a typology of different systems at different stages of evolution. This will help position any country system within the wider global context. As such the researcher will identify common characteristics of systems at different stages and in different forms. In this manner, a government led and wholly managed system would be given a label (government unitarism) and the 
characteristics of how this form of system changes over time would allow any country to find its position in that lifecycle. Other labels may be 'industry-led' system or Research Focussed Innovation (university-led).

The final area of study is to compare what has been found in this study under the life sciences sector with other high-value added sectors. This would include looking at other sectors and how they interface with governments to establish whether more formal structures exist in other high-value adding sectors and whether forms of government support may be different to those detected in this study. This final study will test to see whether the importance of relationship management to innovation and knowledge transfer has resulted in a poorer performance in the life sciences sector than in certain other government ministry areas.

\subsubsection{Recommendations}

The recommendations arising from the study and the creation of the model include:

○ The LS innovation model should be promoted to the private sector and other actors.

- A specific organization or programme should be developed to co-ordinate all relevant activities.

- An exemplar cluster (government-led project) should be created, consisting of universities, firms, government investment and management, service providers and ventures capital. Investment should be encouraged by developing financial incentives. The location for the cluster should be chosen carefully.

- Particular attention should be paid to developing human capital by raising the quality of the education system, attracting talent and developing potential Saudi staff through study abroad in developed countries. 


\subsection{Final words}

This thesis has been a long journey of discovery. It represents many hundreds of hours of work. It was a great experience and I hope future researchers will find it useful. It comes at very important and critical time when the government of the KSA is starting to reform the economy and its structure and is paying a great attention to developing a knowledge economy as a key strategy for future economic prosperity.

I hope this work will help shape and contribute to this important national mission and will hopefully have a positive effect on the national economy and the wellbeing of Saudi citizens. 


\section{References}

Abramovitz, M. (1994). Catch-up and convergence in the postwar growth boom and after. Convergence of Productivity: Cross-National Studies and Historical Evidence, 86-125.

Acemoglu, D., Robinson, J. A., \& Woren, D. (2012). Why nations fail: the origins of power, prosperity and poverty (Vol. 4). SciELO Chile.

Acs, Z. J. (1992). Small business economics: A global perspective. Challenge, 38-44.

Administration, small business. (1998). The new American evolution: The role and impact of small firms.

Afzal, I., Naser, M., \& Lawrey, R. (2012). A Measurement Framework for Knowledge-Based Economy (KBE) Efficiency in ASEAN: A Data Envelopment (DEA) Window Approach. International Journal of Business \& Management, $7(18)$.

Afzal, M. N. I., \& Lawrey, R. (2012a). Evaluating the comparative performance of knowledge-based economies (KBEs) in ASEAN: a data envelopment analysis (DEA) application of additive efficiency. Research Journal of Social Science \& Management, 2(6), 48-55.

Afzal, M. N. I., \& Lawrey, R. (2012b). Investigating World Bank knowledge assessment methodology (KAM) using data envelopment analysis (DEA): a study in the ASEAN region. Research Journal of Science \& IT Management, l(12), 33-38.

Ahn, M. J., \& York, A. S. (2011). Resource-based and institution-based approaches to biotechnology industry development in Malaysia. Asia Pacific Journal of Management, 28(2), 257-275.

Al-Faris, A. F. (2002). Public expenditure and economic growth in the Gulf Cooperation Council countries. Applied Economics, 34(9), 1187-1193.

Alfaro, L. (2014). Foreign Direct Investment: Effects, Complementarities, and Promotion.

Alfaro, L., Chanda, A., Kalemli-Ozcan, S., \& Sayek, S. (2004). FDI and economic growth: the role of local financial markets. Journal of International Economics, 
64(1), 89-112.

Alfaro, L., \& Chen, M. X. (2013). Market reallocation and knowledge spillover: The gains from multinational production. Harvard Business School BGIE Unit Working Paper No. 12, 111.

Alfaro, L., Kalemli-Ozcan, S., \& Sayek, S. (2008). Foreign Direct Investment, Productivity, and Financial Development: An Empirical Analysis of Complementarities and Channels. The World Economy.

Ali, A. J., \& Al-Aali, A. (1997). A Lack of Creativity: Executives Speak Out. Management Research News, 20(6), 1-8.

Allen, R. (1998). Sinking oil price rattles Saudi Arabia's shaky structures. Financial Times, 3.

Alliance, S. (2014). Saudi Arabia : Retail Sector Overview OUR STRATEGIES, YOUR SOLUTIONS Investor Document - Saudi Arabia Retail Sector.

Almalki, M., Fitzgerald, G., \& Clark, M. (2011). Health care system in Saudi Arabia: an overview. Eastern Mediterranean Health Journal, 17(10).

Alshahrani, M. S. A., \& Alsadiq, M. A. J. (2014). Economic Growth and Government Spending in Saudi Arabia: an Empirical Investigation. International Monetary Fund.

Alshumaimri, A., Aldridge, T., \& Audretsch, D. B. (2010). The university technology transfer revolution in Saudi Arabia. The Journal of Technology Transfer, 35(6), $585-596$.

Amsden, A. H. (2011). Firm Ownership and Entrepreneurship. Entrepreneurship, Innovation, and Economic Development, 65.

Andreff, W., \& Balcet, G. (2013). Emerging countries' multinational companies investing in developed countries: at odds with the HOS paradigm? The European Journal of Comparative Economics, 10(1), 3-26.

Ang, S. H. (2006). Country-of-origin effect of VC investment in biotechnology companies. Journal of Commercial Biotechnology, 13(1), 12-19.

Ardic, O. P., Mylenko, N., \& Saltane, V. (2011). Small and medium enterprises: A cross-country analysis with a new data set.

Argote, L. (2012). Organizational learning: Creating, retaining and transferring knowledge. Springer Science \& Business Media.

Argote, L., \& Ingram, P. (2000). Knowledge transfer: A basis for competitive advantage in firms. Organizational Behavior and Human Decision Processes, 
82(1), 150-169.

Argote, L., Ingram, P., Levine, J. M., \& Moreland, R. L. (2000). Knowledge transfer in organizations: Learning from the experience of others. Organizational Behavior and Human Decision Processes, 82(1), 1-8.

Ashby, W. R. (1956). An introduction to cybernetics. An Introduction to Cybernetics.

Ashby, W. R. (1991). Requisite variety and its implications for the control of complex systems. In Facets of Systems Science (pp. 405-417). Springer.

Audretsch, D. B. (2003). Standing on the shoulders of midgets: The US Small Business Innovation Research program (SBIR). Small Business Economics, 20(2), 129-135.

Audretsch, D. B., \& Stephan, P. E. (2002). Knowledge spillovers in biotechnology: sources and incentives. In Economic Evolution, Learning, and Complexity (pp. 127-137). Springer.

Audretsch, D. B., \& Thurik, A. R. (1998). The Knowledge Society, Entrepreneurship, and Unemployment.

Augier, M., \& Teece, D. J. (2007). Dynamic capabilities and multinational enterprise: Penrosean insights and omissions. Management International Review, 47(2), $175-192$.

Augier, M., \& Teece, D. J. (2009). Dynamic capabilities and the role of managers in business strategy and economic performance. Organization Science, 20(2), 410421.

Avlonitis, G. J., \& Salavou, H. E. (2007). Entrepreneurial orientation of SMEs, product innovativeness, and performance. Journal of Business Research, 60(5), $566-575$.

Azariadis, C., \& Drazen, A. (1990). Threshold externalities in economic development. The Quarterly Journal of Economics, 501-526.

Azoulay, P., Michigan, R., \& Sampat, B. N. (2007). The anatomy of medical school patenting. New England Journal of Medicine, 357(20), 2049-2056.

Babalola, J. B. (2003). Budget preparation and expenditure control in education. Basic Text in Educational Planning. Ibadan Awemark Industrial Printers.

Baker, W. E., \& Sinkula, J. M. (2009). The Complementary Effects of Market Orientation and Entrepreneurial Orientation on Profitability in Small Businesses*. Journal of Small Business Management, 47(4), 443-464.

Balasubramanyam, V. N., Salisu, M., \& Sapsford, D. (1996). Foreign direct 
investment and growth in EP and IS countries. The Economic Journal, 92-105.

Bank, W. (2012). world bank insritute.

Bao, G., \& Chen, M. X. (2013). When Foreign Rivals are Coming to Town: Firm Responses to Multinational Investment News. WORK.

Barakat, M. S., Naayem, J. H., Baba, S. S., Kanso, F. A. K., Borgi, S. F., \& Arabian, J. H. (2014). SAUDI ARABIA ECONOMIC REPORT FUNDAMENTAL STRENGTHS OUTPACING KEY CHALLENGES FOR THE KINGDOM'S ECONOMY.

Bartholomew, S. (1997). National systems of biotechnology innovation: complex interdependence in the global system. Journal of International Business Studies, 241-266.

Bass, F. M. (1969). A new product growth for model consumer durables. Management Science, 15(5), 215-227.

Bathelt, H., Malmberg, A., \& Maskell, P. (2004). Clusters and knowledge: local buzz, global pipelines and the process of knowledge creation. Progress in Human Geography, 28(1), 31-56.

Baum, J. A. C., Calabrese, T., \& Silverman, B. S. (2000). Don’t go it alone: Alliance network composition and startups' performance in Canadian biotechnology. Strategic Management Journal, 21(3), 267-294.

Baumol, W. J., Litan, R. E., \& Schramm, C. J. (2007). Good capitalism, bad capitalism, and the economics of growth and prosperity. Bad Capitalism, and the Economics of Growth and Prosperity.

Beblawi, H. (1987). The rentier state in the Arab world. Arab Studies Quarterly, 383398.

Becker, G. S. (1962). Investment in human capital: A theoretical analysis. The Journal of Political Economy, 9-49.

Becker, G. S. (1975). Human capital: a theoretical and empirical analysis, with special refence to education.

Becker, G. S. (1993). Nobel lecture: The economic way of looking at behavior. Journal of Political Economy, 101(3), 385-409.

Becker, G. S. (1994). Human capital revisited. In Human Capital: A Theoretical and Empirical Analysis with Special Reference to Education (3rd Edition) (pp. 1528). The University of Chicago Press.

Beer, S. (1984). The viable system model: Its provenance, development, methodology 
and pathology. Journal of the Operational Research Society, 35(1), 7-25.

Beer, S. (1985). Diagnosing the system for organizations. John Wiley \& Sons Inc.

Beer, S. (1989). The viable system model. Viable Systems Model, Wiley, Chicester.

Bellows, T. J. (1985). Bureaucracy and development in Singapore. Asian Journal of Public Administration, 7(1), 55-69.

Bercovitz, J. E. L., \& Feldman, M. P. (2007). Fishing upstream: Firm innovation strategy and university research alliances. Research Policy, 36(7), 930-948.

Berk, G., \& Galvan, D. (2009). How people experience and change institutions: a field guide to creative syncretism. Theory and Society, 38(6), 543-580.

Bhaskar, R. (2010). Reclaiming reality: A critical introduction to contemporary philosophy. Taylor \& Francis.

Birchall, D.W., Chanaron, J.J., Soderquist, K. (1996). NoArticle: Managing innovation in SMEs: a comparison of companies in the UK, France and Portugal Title. InternationalJournalofTechnologyManagement, 12, 291-305.

Black, S. E., \& Lynch, L. M. (2001). How to compete: the impact of workplace practices and information technology on productivity. Review of Economics and Statistics, 83(3), 434-445.

Blaikie, N. (2003). Analyzing quantitative data: From description to explanation. Sage.

Blalock, G., \& Gertler, P. J. (2008). Welfare gains from foreign direct investment through technology transfer to local suppliers. Journal of International Economics, 74(2), 402-421.

Blalock, G., \& Gertler, P. J. (2009). How firm capabilities affect who benefits from foreign technology. Journal of Development Economics, 90(2), 192-199.

Bligh, A. (1985). The Saudi Religious elite (ulama) as participant in the political system of the kingdom. International Journal of Middle East Studies, 17(1), 3750 .

Bocquet, R., \& Mothe, C. (2010). Knowledge governance within clusters: the case of small firms. Knowledge Management Research \& Practice, 8(3), 229-239.

Böhm, A. (2004). Theoretical coding: text analysis in grounded theory. Dans U. Flick, E. von Kardorff, \& I. Steinke (Éds), A companion to qualitative research (pp. 270-275). London: Sage.

Borensztein, E., De Gregorio, J., \& Lee, J.-W. (1998). How does foreign direct investment affect economic growth? Journal of International Economics, 45(1), 
$115-135$.

Boughanmi, H. (2008). The trade potential of the Arab Gulf Cooperation Countries

(GCC): a gravity model approach. Journal of Economic Integration, 23(1), 4256.

Braczyk, H.-J., Cooke, P. N., \& Heidenreich, M. (1998). Regional innovation systems: the role of governances in a globalized world. Psychology Press.

Brem, A., \& Voigt, K.-I. (2009). Integration of market pull and technology push in the corporate front end and innovation management-Insights from the German software industry. Technovation, 29(5), 351-367.

Bresnahan, T. F., Brynjolfsson, E., \& Hitt, L. M. (1999). Information technology, workplace organization and the demand for skilled labor: firm-level evidence. National bureau of economic research.

Bresnahan, T. F., \& Trajtenberg, M. (1995). General purpose technologies 'Engines of growth'? Journal of Econometrics, 65(1), 83-108.

Brock, W. A., \& Evans, D. S. (1989). Small business economics. Small Business Economics, 1(1), 7-20.

Bronchi, C. (2003). The effectiveness of public expenditure in Portugal.

Bruno, R., \& Campos, N. F. (2013). Reexamining the conditional effect of foreign direct investment. IZA Discussion Paper.

Bryman, A. (2012). Social Research Methods. Oxford University Press.

Bryman, A. (2015). Social research methods. Oxford university press.

Bugamelli, M., \& Pagano*, P. (2004). Barriers to Investment in ICT. Applied Economics, 36(20), 2275-2286.

Burns, P. (2007). Entrepreneurship and Small Business. New York: Palgrave Macmillan.

Burns, T. E., \& Stalker, G. M. (1961). The management of innovation. University of Illinois at Urbana-Champaign's Academy for Entrepreneurial Leadership Historical Research Reference in Entrepreneurship.

Capron, L., Dussauge, P., \& Mitchell, W. (1998). Resource redeployment following horizontal acquisitions in Europe and North America, 1988-1992. Strategic Management Journal, 19(7), 631-661.

Cardona, M., Kretschmer, T., \& Strobel, T. (2013). ICT and productivity: conclusions from the empirical literature. Information Economics and Policy, 25(3), 109125. 
Carkovic, M. V, \& Levine, R. (2002). Does foreign direct investment accelerate economic growth? U of Minnesota Department of Finance Working Paper.

Carlsson, B. (1992). The rise of small business: causes and consequences. Industrial Institute for Economic and Social Research.

Carlsson, B. (1999). Small business, entrepreneurship, and industrial dynamics. In Are Small Firms Important? Their Role and Impact (pp. 99-110). Springer.

Carson, D., Gilmore, A., Perry, C., \& Gronhaug, K. (2001). Qualitative marketing research. Sage.

Carter, S. M., \& Little, M. (2007). Justifying knowledge, justifying method, taking action: Epistemologies, methodologies, and methods in qualitative research. Qualitative Health Research, 17(10), 1316-1328.

Castiglione, C. (2010). ICT investment and firm technical efficiency. EWEPA.

Charles, D. (2006). Universities as key knowledge infrastructures in regional innovation systems. Innovation: The European Journal of Social Science Research, 19(1), 117-130.

Charmaz, K. (2006). Constructing grounded theory: A practical guide through qualitative research. London: Sage.

Chatterton, P., \& Goddard, J. (2000). The response of higher education institutions to regional needs. European Journal of Education, 35(4), 475-496.

Checkland, P. (2000). Soft systems methodology: a thirty year retrospective. Systems Research and Behavioral Science, 17(S1), S11.

Checkland, P., \& Poulter, J. (2010). Soft systems methodology. In Systems approaches to managing change: A practical guide (pp. 191-242). Springer.

Chen, D. H. C., Dahlman, C. J., \& Dc, W. (2005). The Knowledge Economy, the KAM Methodology and World Bank Operations.

Chesbrough, H. W. (2003). Open innovation: The new imperative for creating and profiting from technology. Harvard Business Press.

Christensen, J. F., \& Maskell, P. (2003). The industrial dynamics of the new digital economy. Edward Elgar Publishing.

Christiansen, C. (1997). The innovator's dilemma. Harvard Business School Press, Boston.

Clandinin, D. J., \& Connelly, F. M. (2000). Narrative inquiry: Experience and story in qualitative research.

Clarke, T. (2001). The knowledge economy. Education+ Training, 43(4/5), 189-196. 
Clegg, F. (1982). Simple statistics: A course book for the social sciences. Cambridge University Press.

Co., B. \&. (2007). Life Sciences: A Global Transformation. Burrill Life Sciences Venture Capital Group San Francisco, CA.

Cobb, C. W., \& Douglas, P. H. (1928). A theory of production. The American Economic Review, 139-165.

Coffey, A., \& Atkinson, P. (1996). Making sense of qualitative data: Complementary research strategies. Sage Publications, Inc.

Cohen, W. M., \& Levinthal, D. A. (1990). Absorptive Capacity: A New Perspective on Learning and Innovation. Administrative Science Quarterly, 35(1), 128. https://doi.org/10.2307/2393553

Colombo, M. G., Croce, A., \& Grilli, L. (2013). ICT services and small businesses' productivity gains: An analysis of the adoption of broadband Internet technology. Information Economics and Policy, 25(3), 171-189.

Communities, commission of the E. (2008). GREEN PAPER Copyright in the Knowledge Economy. Brussels, COM(2008) 466/3.

Cooke, P. (2002). Regional innovation systems: general findings and some new evidence from biotechnology clusters. The Journal of Technology Transfer, 27(1), 133-145.

Cooke, P. (2004). The role of research in regional innovation systems: new models meeting knowledge economy demands. International Journal of Technology Management, 28(3), 507-533.

Cooke, P., \& Wills, D. (1999). Small firms, social capital and the enhancement of business performance through innovation programmes. Small Business Economics, 13(3), 219-234.

Cooper, A. C., Markman, G. D., \& Niss, G. (2000). The evolution of the field of entrepreneurship. Entrepreneurship as Strategy, 115-133.

Cooper, H. M. (1988a). Organizing knowledge syntheses: A taxonomy of literature reviews. Knowledge in Society, 1(1), 104-126.

Cooper, H. M. (1988b). The integrative research review: a systematic aproach. Sage Publications.

Corbin, J. M., \& Strauss, A. (1990). Grounded theory research: Procedures, canons, and evaluative criteria. Qualitative Sociology, 13(1), 3-21.

Corbin, J., \& Strauss, A. (1994). Grounded theory methodology. Handbook of 
Qualitative Research, 273-285.

Corbin, J., \& Strauss, A. (2008). Basics of qualitative research: Techniques and procedures for developing grounded theory. Sage.

Corrocher, N., \& Cusmano, L. (2014). The 'KIBS engine' of regional innovation systems: empirical evidence from European regions. Regional Studies, 48(7), $1212-1226$.

Cortright, J. (2006). Making sense of clusters: Regional competitiveness and economic development. Brookings Institution, Metropolitan Policy Program.

Crepaz, M. M. L., \& Moser, A. W. (2004). The impact of collective and competitive veto points on public expenditures in the global age. Comparative Political Studies, 37(3), 259-285.

Creswell, J. W. (1998). Qualitative inquiry and research design: Choosing among five traditions Sage. Thousand Oaks, CA.

Creswell, J. W. (2007). Qualitative enquiry and research design: Choosing among five approaches. US: Sage Publications Ltd.

Creswell, J. W. (2013). Qualitative inquiry and research design: Choosing among five approaches. Sage.

Creswell, J. W., Hanson, W. E., Plano, V. L. C., \& Morales, A. (2007). Qualitative research designs selection and implementation. The Counseling Psychologist, 35(2), 236-264.

Creswell, J. W., \& Miller, D. L. (2000). Determining validity in qualitative inquiry. Theory into Practice, 39(3), 124-130.

Culkin, N., \& Smith, D. (2000). An emotional business: a guide to understanding the motivations of small business decision takers. Qualitative Market Research: An International Journal, 3(3), 145-157.

Darr, E. D., \& Kurtzberg, T. R. (2000). An investigation of partner similarity dimensions on knowledge transfer. Organizational Behavior and Human Decision Processes, 82(1), 28-44.

David, P. A. (1993). Intellectual property institutions and the panda's thumb: patents, copyrights, and trade secrets in economic theory and history. Global Dimensions of Intellectual Property Rights in Science and Technology, 19, 29.

David, P. A. (1994). Why are institutions the 'carriers of history'?: Path dependence and the evolution of conventions, organizations and institutions. Structural Change and Economic Dynamics, 5(2), 205-220. 
David, P., \& Lopez, J. (2001). Knowledge, capabilities and human capital formation in economic growth. Unpublished Research Report Prepared for the New Zealand Treasury.

De Lombaerde, P., \& Van Langenhove, L. (2005). Indicators of regional integration: Methodological issues. Institute for International Integration Studies, Discussion Paper, (64).

de Oslo, M. (1992). Proposed guidelines for collecting and interpreting technological innovation data. Manual Oslo", Eurostat.

De Tarde, G. (1903). The laws of imitation. H. Holt.

Delery, J., \& Gupta, N. (2016). Human resource management practices and organizational effectiveness: internal fit matters. Journal of Organizational Effectiveness: People and Performance, 3(2), 139-163.

Delgado, M., Porter, M. E., \& Stern, S. (2010). Clusters and entrepreneurship. Journal of Economic Geography, 10(4), 495-518.

Desai, M. A. (2009). The decentering of the global firm. The World Economy, 32(9), 1271-1290.

Development, O. for E. C. and. (2001). OECD Science, Technology and Industry Scoreboard 2001: Towards a Knowledge-based Economy. Organisation for Economic Co-operation and Development.

Dewan, S., \& Kraemer, K. L. (2000). Information technology and productivity: evidence from country-level data. Management Science, 46(4), 548-562.

Dhanaraj, C., Lyles, M. A., Steensma, H. K., \& Tihanyi, L. (2004). Managing tacit and explicit knowledge transfer in IJVs: the role of relational embeddedness and the impact on performance. Journal of International Business Studies, 35(5), $428-442$.

Dillman, D. A. (2007). Mail and Internet Surveys: The Tailored Design Method., 2nd edn.(John-Wiley \& Sons: New York.).

DiMasi, J. A., Hansen, R. W., \& Grabowski, H. G. (2003). The price of innovation: new estimates of drug development costs. Journal of Health Economics, 22(2), $151-185$.

Docter, H. J., \& Stokman, C. T. M. (1988). Innovation in SMEs (Innovatie in het industriële midden en kleinbedrijf). In Paper for symposium on TechnologyEconomics (Vol. 31).

Dooley, K. E. (1999). Towards a holistic model for the diffusion of educational 
technologies: An integrative review of educational innovation studies. Educational Technology \& Society, 2(4), 35-45.

Dosi, G. (1982). Technological paradigms and technological trajectories: a suggested interpretation of the determinants and directions of technical change. Research Policy, 11(3), 147-162.

Douglass, J. A. (2010). Creating a Culture of Aspiration: Higher Education, Human Capital and Social Change. Procedia - Social and Behavioral Sciences, 2(5), 6981-6995. https://doi.org/10.1016/j.sbspro.2010.05.050

Draucker, C. B., Martsolf, D. S., Ross, R., \& Rusk, T. B. (2007). Theoretical sampling and category development in grounded theory. Qualitative Health Research, 17(8), 1137-1148.

Drejer, I., \& Vinding, A. L. (2007). Searching near and far: determinants of innovative firms' propensity to collaborate across geographical distance. Industry and Innovation, 14(3), 259-275.

Drucker, P. F., \& Drucker, P. F. (1994). Post-capitalist society. Routledge.

Dunlop John, T. (1958). Industrial Relations Systems. New York, Henry Holt and Co.

Dunning, J. H., \& Rugman, A. M. (1985). The influence of Hymer's dissertation on the theory of foreign direct investment. The American Economic Review, 228232.

Dynamics, T. L. (2013). The Global Innovation Index 2013.

Education, T. Q. A. A. for H. (2012). Enterprise and entrepreneurship education :

EIM. (1997). The European Observatory for SMEs. Zoetermeer.

Emery, J. C. (1969). Organizational planning and control systems: theory and technology. Macmillan New York.

Enkel, E., Gassmann, O., \& Chesbrough, H. (2009). Open R\&D and open innovation: exploring the phenomenon. $R \& D$ Management, 39(4), 311-316. https://doi.org/10.1111/j.1467-9310.2009.00570.x

Espejo, R. (1990). The viable system model. Systems Practice, 3(3), 219-221.

Espejo, R. (2003). The viable system model, a briefing about organizational structure. Aston Science Park, Birmingham: SYNCHO Limited.

Espejo, R., \& Gill, A. (1997). The viable system model as a framework for understanding organizations. Phrontis Limited \& SYNCHO Limited.

Ethiraj, S. K., \& Levinthal, D. (2004). Bounded rationality and the search for organizational architecture: An evolutionary perspective on the design of 
organizations and their evolvability. Administrative Science Quarterly, 49(3), 404-437.

Etzkowitz, H. (1998). The norms of entrepreneurial science: cognitive effects of the new university-industry linkages. Research Policy, 27(8), 823-833. https://doi.org/10.1016/S0048-7333(98)00093-6

Etzkowitz, H. (2003). Innovation in Innovation: The Triple Helix of UniversityIndustry-Government Relations. Social Science Information, 42(3), 293-337. https://doi.org/10.1177/05390184030423002

Etzkowitz, H., Webster, A., Gebhardt, C., \& Terra, B. R. C. (2000). The future of the university and the university of the future: evolution of ivory tower to entrepreneurial paradigm. Research Policy, 29(2), 313-330.

European, \& Commission. (2005). The new SME definition, User guide and model declaration.

Evans, A. G., \& Varaiya, N. P. (2003). Anne Evans: assessment of a biotechnology market opportunity. Entrepreneurship Theory and Practice, 28(1), 87-105.

Evers, H.-D. (2008). Knowledge hubs and knowledge clusters: Designing a knowledge architecture for development. ZEF Working Paper Series.

Fagerberg, J., \& Srholec, M. (2008). National innovation systems, capabilities and economic development. Research Policy, 37(9), 1417-1435.

Fägerlind, I., \& Saha, L. J. (2016). Education and national development: A comparative perspective. Elsevier.

Farmer, R. N. (1959). Local Entrepreneurship in Saudi Arabia. Business History Review, 33(01), 73-86.

Ferraro, F., \& Borroi, M. (1998). The emerging landscape of research: dynamics of trust and cooperation. In documento presentado en la conferencia "A Triple Helix of University, Industry, Government Relations, The New Location of Research", Nueva York, enero.

Finegold, D., Wong, P., \& Cheah, T. (2004). Adapting a foreign direct investment strategy to the knowledge economy: the case of Singapore's emerging biotechnology cluster. European Planning Studies, 12(7), 921-941.

Fleming, L., \& Marx, M. (2006). Managing creativity in small worlds. California Management Review, 48(4), 6.

Foddy, W. (1994). Constructing questions for interviews and questionnaires: Theory and practice in social research. Cambridge university press. 
Forman, C., \& Zeebroeck, N. van. (2012). From wires to partners: How the Internet has fostered R\&D collaborations within firms. Management Science, 58(8), $1549-1568$.

Forrest, J. E. (1990). Strategic Alliances and the Small Technology-Based Firm. Journal of Small Business Management, 28(3), 37.

Forrester, J. W. (1994). System dynamics, systems thinking, and soft OR. System Dynamics Review, 10(2-3), 245-256.

Foss, N. J., \& Santos, J. F. P. dos J. (2011). A knowledge system approach to the multinational company: conceptual grounding and implications for research (Vol. 24). Emerald Group Publishing Limited.

Fram, S. M. (2013). The constant comparative analysis method outside of grounded theory. The Qualitative Report, 18(1), 1.

Freel, M. S. (1999). Where are the skills gaps in innovative small firms? International Journal of Entrepreneurial Behaviour \& Research, 5(3), 144-154.

Freeman, C. (1987). Technology and economic performance. Lessons from Japan.

Gallarotti, G. M. (2013). Smart Development : Saudi Arabia's Quest for a Knowledge Economy.

Garnsey, E., \& Heffernan, P. (2005). High-technology clustering through spin-out and attraction: The Cambridge case. Regional Studies, 39(8), 1127-1144.

Gerschenkron, A. (1962). Economic backwardness in historical perspective. Economic Backwardness in Historical Perspective.

Gilfillan, S. (1935). The Sociology of Invention Cambride. MIT Press.

Gill, J., \& Johnson, P. (2010). Research methods for managers. Sage.

Gittell, R., \& Sohl, J. (2005). Technology centres during the economic downturn: what have we learned? Entrepreneurship \& Regional Development, 17(4), 293312.

Glaeser, E. L., Kallal, H. D., Scheinkman, J. A., \& Shleifer, A. (1991). Growth in cities. National Bureau of Economic Research.

Glaser, B. G. (1992). Emergence vs forcing: Basics of grounded theory analysis. Sociology Press.

Glaser, B. G., \& Holton, J. (2005). Basic social processes. Grounded Theory Review, $4(3), 1-29$.

Glaser, B., \& Strauss, A. (1967). The Discovery Grounded Theory: Strategies for 
Qualitative Inquiry. Observations. London: Wiedenfeld and Nicholson.

Goddard, J. B., \& Chatterton, P. (1999). Regional Development Agencies and the knowledge economy: harnessing the potential of universities. Environment and Planning C, 17, 685-700.

Godin, B. (2008a). In the shadow of Schumpeter: W. Rupert Maclaurin and the study of technological innovation. Minerva, 46(3), 343-360.

Godin, B. (2008b). Innovation: the History of a Category. Project on the Intellectual History of Innovation Working Paper, (1).

Godin, B. (2009). National innovation system: The system approach in historical perspective. Science, Technology \& Human Values.

Godin, B. (2013). Measurement and Statistics on Science and Technology: 1920 to the Present. Routledge.

Goedhuys, M., \& Veugelers, R. (2012). Innovation strategies, process and product innovations and growth: Firm-level evidence from Brazil. Structural Change and Economic Dynamics, 23(4), 516-529.

Goldfarb, B., \& Henrekson, M. (2003). Bottom-up versus top-down policies towards the commercialization of university intellectual property. Research Policy, 32(4), 639-658.

Görg, H., Hanley, A., \& Strobl, E. (2011). Creating backward linkages from multinationals: Is there a role for financial incentives? Review of International Economics, 19(2), 245-259.

Govindarajan, V., \& Ramamurti, R. (2011). Reverse innovation, emerging markets, and global strategy. Global Strategy Journal, 1(3-4), 191-205.

Gray, C. (2002). Enterprise and culture. Routledge.

Gray, C. (2006). Absorptive capacity, knowledge management and innovation in entrepreneurial small firms. International Journal of Entrepreneurial Behaviour \& Research, 12(6), 345-360.

Greffe, X. (2016). The Economic Impact of the Louvre. The Journal of Arts Management, Law, and Society, 4l(2), 121-137. https://doi.org/10.1080/10632921.2011.573323

Group, O. B. (2007). The Report: Emerging Saudi Arabia 2007. Oxford Business Group.

Gu, S. (1999). Implications of National Innovation Systems for Developing Countries: 
managing change and complexity in economic development. United Nations University-INTECH.

Guba, E. G., \& Lincoln, Y. S. (1982). Epistemological and methodological bases of naturalistic inquiry. ECTJ, 30(4), 233-252.

Guba, E. G., \& Lincoln, Y. S. (1994). Competing paradigms in qualitative research. Handbook of Qualitative Research, 2(163-194), 105.

Gubbi, S. R., Aulakh, P. S., Ray, S., Sarkar, M. B., \& Chittoor, R. (2010). Do international acquisitions by emerging-economy firms create shareholder value\&quest; The case of Indian firms. Journal of International Business Studies, 41(3), 397-418.

Guest, G., Bunce, A., \& Johnson, L. (2006). How many interviews are enough? An experiment with data saturation and variability. Field Methods, 18(1), 59-82.

Gulati, R. (1999). Network location and learning: The influence of network resources and firm capabilities on alliance formation. Strategic Management Journal, 20(5), 397-420.

Haar, B. (2001). Venture capital funding for biotech pharmaceutical companies in an integrated financial services market: regulatory diversity within the EC. European Business Organization Law Review, 2(3-4), 585-602.

Haeussler, C., \& Colyvas, J. (2009). Breaking the Ivory Tower : Academic Entrepreneurship in the Life Sciences in UK and Germany, 1-58.

Hamel, G. (1996). Competing for the Future. Harvard Business Press.

Hansen, J. A., Dirckinck-Holmfeld, L., Gregersen, B., \& Lehmann, M. (2008). Universities as innovation hubs for sustainable development. Ministry of Foreign Affairs of Denmark Udenrigsministeriet.

Harding, R., Hart, M., Jones-Evans, D., \& Levie, J. (2002). Global Entrepreneurship Monitor. London: London Business School.

Hargreaves, D. (2000). Knowledge management in the learning society. In Proceeding of Forum of OECD Education Ministers.

Harrison, A., \& Rodríguez-Clare, A. (2009). Trade, foreign investment, and industrial policy for developing countries. National Bureau of Economic Research.

Harrison, R. T., Cooper, S. Y., \& Mason, C. M. (2004). Entrepreneurial activity and the dynamics of technology-based cluster development: the case of Ottawa. Urban Studies, 41(5-6), 1045-1070.

Haunschild, P. R., \& Miner, A. S. (1997). Modes of interorganizational imitation: The 
effects of outcome salience and uncertainty. Administrative Science Quarterly, $472-500$.

Hausmann, R., Hidalgo, C. A., Bustos, S., Coscia, M., Simoes, A., \& Yildirim, M. A. (2014). The atlas of economic complexity: Mapping paths to prosperity.

Henrekson, M., \& Rosenberg, N. (2001). Designing efficient institutions for sciencebased entrepreneurship: Lesson from the US and Sweden. The Journal of Technology Transfer, 26(3), 207-231.

Hertog, S. (2010). Defying the resource curse: explaining successful state-owned enterprises in rentier states. World Politics, 62(2), 261-301.

Hobohm, S. (2000). small and medium-sized enterprises in economic development: unido experience. In industry for growth in mellennium (pp. 1-42).

Hoffman, K., Parejo, M., Bessant, J., \& Perren, L. (1998). Small firms, R\&D, technology and innovation in the UK: a literature review. Technovation, 18(1), $39-55$.

Holland, J. L. (1997). Making vocational choices: A theory of vocational personalities and work environments . Psychological Assessment Resources.

Holton, J. A. (2010). The Coding Process and Its Challenges. Grounded Theory Review, 9(1).

Hoskisson, R. E., \& Busenitz, L. W. (2002). Market uncertainty and learning distance in corporate entrepreneurship entry mode choice. Strategic Entrepreneurship: Creating a New Mindset, 151-172.

Hoverstadt, P., \& Bowling, D. (2002). Modelling organisations using the viable system model. In Royal academy of Engineering Systems Engineering Workshop, May (Vol. 23).

Howitt, P. A.-P. (1992). A Model of Growth through Creative Destruction, in «Econometrica». LX.

Huang, S. K., \& Wang, Y.-L. (2011). Entrepreneurial orientation, learning orientation, and innovation in small and medium enterprises. Procedia-Social and Behavioral Sciences, 24, 563-570.

Huberman, A. M., \& Miles, M. B. (1994). Data management and analysis methods.

Huczynski, A., \& Buchanan, D. A. (2010). Organizational behaviour. Financial Times Prentice Hall.

Hudson, L. A., \& Ozanne, J. L. (1988). Alternative ways of seeking knowledge in consumer research. Journal of Consumer Research, 14(4), 508-521. 
Huggins, R. (2008). The evolution of knowledge clusters: progress and policy. Economic Development Quarterly.

Hunterl, K., Hariz, S., Egbu, C., \& Kelly, J. (2011). Grounded Theory: Its Diversification and Application Through two Examples from Research Studies on Knowledge and Value Management. Leading Issues in Business Research Methods, 1, 22.

Huntington, S. P. (1996). The clash of civilizations and the remaking of world order. Penguin Books India.

Hymer, S. (1976). The international operations of national firms: A study of direct foreign investment (Vol. 14). MIT press Cambridge, MA.

Ilie, V. (2014). Developing Entrepreneurial Competencies in Students through Constructivist Education. Education Journal, 3(5), 293-302.

Inkpen, A. C., \& Tsang, E. W. K. (2005). Social capital, networks, and knowledge transfer. Academy of Management Review, 30(1), 146-165.

Innovation, U. S. P. on I. and, Charpie, R. A., \& De Simone, D. V. (1967). Technological innovation: Its environment and management. US Department of Commerce.

Institute, world bank. (2011a). KI and KEI indexes. Retrieved from http://web.worldbank.org/WBSITE/EXTERNAL/WBI/WBIPROGRAMS/KFDL P/EXTUNIKAM/0,,contentMDK:20584278 menuPK:1433216 pagePK:641684 45 piPK:64168309 theSitePK:1414721,00.html

Institute, world bank. (2011b). Knowledge Assessment Methodology 2012.

Ireland, R. D., Covin, J. G., \& Kuratko, D. F. (2009). Conceptualizing corporate entrepreneurship strategy. Entrepreneurship Theory and Practice, 33(1), 19-46.

Jacobs, J. (1986). Cities and the Wealth of Nations. Penguin Harmondsworth,, UK.

Jacobs, W., van Rietbergen, T., Atzema, O., van Grunsven, L., \& van Dongen, F. (2014). The Impact of Multinational Enterprises (MNEs) on KnowledgeIntensive Business Services (KIBS) Start-ups: Empirical Evidence from the Dutch Randstad. Regional Studies, (ahead-of-print), 1-16.

Jakob, A. (2001). On the Triangulation of Quantitative and Qualitative Data in Typological Social Research: Reflections on a Typology of Conceptualizing" Uncertainty" in the Context of Employment Biographies. In Forum Qualitative Sozialforschung/Forum: Qualitative Social Research (Vol. 2).

Jamieson, S. (2004). Likert scales: how to (ab) use them. Medical Education, 38(12), 
$1217-1218$.

Javorcik, B. S. (2004). Does foreign direct investment increase the productivity of domestic firms? In search of spillovers through backward linkages. American Economic Review, 605-627.

Javorcik, B. S., \& Spatareanu, M. (2011). Does it matter where you come from? Vertical spillovers from foreign direct investment and the origin of investors. Journal of Development Economics, 96(1), 126-138.

Jones, G. (1996). The evolution of international business: An introduction. Routledge. Jones, O., \& Tilley, F. (2003). Competitive advantage in SMEs: organising for innovation and change. Wiley.

Jorgenson, D., \& Fraumeni, B. M. (1989). The accumulation of human and nonhuman capital, 1948-84. In The measurement of saving, investment, and wealth (pp. 227-286). University of Chicago Press, 1989.

Jovanovic, B., \& Rousseau, P. L. (2005). General purpose technologies. Handbook of Economic Growth, 1, 1181-1224.

Kaminski, A. Z., \& Kaminski, B. (2013). Economic Regime and Innovative Entrepreneurship: Compatibility Index.

Kast, F. E., \& Rosenzweig, J. E. (1985). Organization and Management: A Systems and Contingency Analysis. New York: McGraw-Hill.

Kato, M., \& Odagiri, H. (2012). Development of university life-science programs and university-industry joint research in Japan. Research Policy. https://doi.org/10.1016/j.respol.2012.02.011

Kayed, R. N., \& Hassan, M. K. (2011a). Saudi Arabia's economic development: entrepreneurship as a strategy. International Journal of Islamic and Middle Eastern Finance and Management, 4(1), 52-73. https://doi.org/10.1108/17538391111122203

Kayed, R. N., \& Hassan, M. K. (2011b). Saudi Arabia's economic development: entrepreneurship as a strategy. International Journal of Islamic and Middle Eastern Finance and Management, 4(1), 52-73.

Kechichian, J. A. (1985). The gulf cooperation council: Search for security. Third World Quarterly, 7(4), 853-881.

Kechichian, J. A. (1986). The Role of the Ulama in the Politics of an Islamic State: The Case of Saudi Arabia. International Journal of Middle East Studies, 18(01), 53-71. 
Kechrid, D. (2012). Management Practices and Productivity: the Case of Saudi Arabia. Stanford University.

Keizer, J. A., Dijkstra, L., \& Halman, J. I. M. (2002). Explaining innovative efforts of SMEs.: An exploratory survey among SMEs in the mechanical and electrical engineering sector in The Netherlands. Technovation, 22(1), 1-13.

Keskin, H. (2006). Market orientation, learning orientation, and innovation capabilities in SMEs: An extended model. European Journal of Innovation Management, 9(4), 396-417.

Khan, M. R. (2013). Mapping entrepreneurship ecosystem of Saudi Arabia. World Journal of Entrepreneurship, Management and Sustainable Development, 9(1), $28-54$.

Khursani, S. A., Bazuhair, O. S., \& Khan, M. R. (2011). Strategy for rapid transformation of Saudi Arabia by leveraging intellectual capital and knowledge management. Saudi Aramco Journal of Technology, 4, 1-13.

Kiker, B. F. (1966). The historical roots of the concept of human capital. The Journal of Political Economy, 481-499.

Kim, K., ByungHwan, H., \& Chung, S. (2007). US National Innovation System for Biotechnology: From the Korean Perspectives. In Management of Engineering and Technology, Portland International Center for (pp. 369-381). IEEE.

Kim, L. (1997). Imitation to innovation: The dynamics of Korea's technological learning. Harvard Business Press.

Kizner, I. M. (1973). Competition and Entrepreneurship. Chicago: University of Chicago Press.

Klofsten, M., \& Jones-Evans, D. (2000). Comparing academic entrepreneurship in Europe-the case of Sweden and Ireland. Small Business Economics, 14(4), 299309.

Kluger, A. N., \& Nir, D. (2010). The feedforward interview. Human Resource Management Review, 20(3), 235-246.

Knapp, T. R. (1990). Treating ordinal scales as interval scales: an attempt to resolve the controversy. Nursing Research, 39(2), 121-123.

Koike, K. (1996). Globalization, competitiveness and workers'skills. In Proceedings of the Regional Meeting of the Asian Industrial Relations Association.

Kong, H. L. (2003). Personal interview with authors. Singapore Biomedical Research Council, January. 
Kostiner, J. (1985). On instruments and their designers: the Ikhwan of Najd and the emergence of the Saudi state. Middle Eastern Studies, 21(3), 298-323.

Kothari, T., Kotabe, M., \& Murphy, P. (2013). Rules of the game for emerging market multinational companies from China and India. Journal of International Management, 19(3), 276-299.

Krishnan, R. T., \& Jha, S. K. (2011). Innovation strategies in emerging markets: what can we learn from Indian market leaders. ASCI Journal of Management, 41(1), $21-45$.

Kugler, M. (2006). Spillovers from foreign direct investment: within or between industries? Journal of Development Economics, 80(2), 444-477.

Lall, S. (1992). Technological capabilities and industrialization. World Development, 20(2), 165-186.

Lane, P. J., Salk, J. E., \& Lyles, M. A. (2001). Absorptive capacity, learning, and performance in international joint ventures. Strategic Management Journal, 22(12), 1139-1161.

Laroche, M., Mérette, M., \& Ruggeri, G. C. (1999). On the concept and dimensions of human capital in a knowledge-based economy context. Canadian Public Policy/Analyse de Politiques, 87-100.

Laroche, M., Mérette, M., Ruggeri, G. C., \& Merette, M. (1999). On the Concept and Dimensions of Human Capital in a Knowledge-Based Economy Context. Canadian Public Policy / Analyse de Politiques, 25(1), 87. https://doi.org/10.2307/3551403

Larson, E. W., Gobeli, D. H., \& Gray, C. F. (1991). Application of project management by small businesses to develop new products and services. Journal of Small Business Management, 29(2), 30-41.

Lassen, A. H., Gertsen, F., \& Riis, J. O. (2006). The nexus of corporate entrepreneurship and radical innovation. Creativity and Innovation Management, 15(4), 359-372.

Lawson, C., \& Lorenz, E. (1999). Collective learning, tacit knowledge and regional innovative capacity. Regional Studies, 33(4), 305-317.

LeBlanc, L. J., Nash, R., Gallagher, D., Gonda, K., \& Kakizaki, F. (1997). A comparison of US and Japanese technology management and innovation. International Journal of Technology Management, 13(5), 601-614.

Lee, S., Park, G., Yoon, B., \& Park, J. (2010). Open innovation in SMEs-An 
intermediated network model. Research Policy, 39(2), 290-300.

Leedy, P. D., \& Ormrod, J. E. (2005). Practical research. Planning and Design, 8.

Lehmann, M., Christensen, P., Thrane, M., \& Jørgensen, T. H. (2009). University engagement and regional sustainability initiatives: some Danish experiences. Journal of Cleaner Production, 17(12), 1067-1074.

Leonard-Barton, D. (1992). Core capabilities and core rigidities: a paradox in managing new product development. Strategic Management Journal, 13(S1), 111-125.

Levine, R. (2005). Finance and growth: theory and evidence. Handbook of Economic Growth, 1, 865-934.

Levinthal, D. A., \& March, J. G. (1993). The myopia of learning. Strategic Management Journal, 14(S2), 95-112.

Leydesdorff, L. A. (1995). Universities and the global knowledge economy: a triple helix of university-industry-government relations.

Leydesdorff, L., \& Etzkowitz, H. (1998). The triple helix as a model for innovation studies. Science and Public Policy, 25(3), 195-203.

Likert, R. (1932). A technique for the measurement of attitudes. Archives of Psychology.

Lincoln, Y. S., \& Guba, E. G. (1985). Naturalistic inquiry (Vol. 75). Sage.

Lindlof, T. R., \& Taylor, B. C. (2010). Qualitative communication research methods. Sage.

Loffler, A., \& Stern, S. (n.d.). The future of the biomedical industry in a era of globlalization.[online]. 2008. 76.[Date of reference: 2015-09-30]. Available at: Available at: Http://Www. Kellogg. Northwestern. Edu/Biotech/Faculty/Articles/Future_biomedical_industry. Pdf [Links].

Lor, P. J., \& Britz, J. J. (2007). Is a knowledge societypossible without freedom of access to information? Journal of Information Science.

Lucas, R. E. (1998). On the mechanics of economic development. ECONOMETRIC SOCIETY MONOGRAPHS, 29, 61-70.

Lumpkin, G. T., \& Dess, G. G. (1996). Clarifying the entrepreneurial orientation construct and linking it to performance. Academy of Management Review, 21(1), $135-172$.

Lund, L. (1986). Locating corporate R\&D facilities. Conference Board. 
Lundvall, B.-A. (1992). National innovation system: towards a theory of innovation and interactive learning. Pinter, London.

Lundvall, B.- $\AA$. (2002). The learning economy: challenges to economic theory and policy. Chapters.

Lundvall, B.-Å., Johnson, B., Andersen, E. S., \& Dalum, B. (2002). National systems of production, innovation and competence building. Research Policy, 31(2), 213-231.

Maclaurin, W. R. (1953). The sequence from invention to innovation and its relation to economic growth. The Quarterly Journal of Economics, 67(1), 97-111.

Mahajan, V., Muller, E., \& Srivastava, R. K. (1990). Determination of adopter categories by using innovation diffusion models. Journal of Marketing Research, $37-50$.

Mahdavy, H. (1970). The patterns and problems of economic development in rentier states: the case of Iran. Life, 1000, 1.

Mahdjoubi, D. (1997). Schumpeterian Economics and the Trilogy of 'InventionInnovation-Diffusion'. UT School of Information, Available at: Http://Www. Ischool. Utexas. Edu/ Darius/17-Schumpeter-Innovation. Pdf.

Malerba, F., \& Nelson, R. (2011). Learning and catching up in different sectoral systems: evidence from six industries. Industrial and Corporate Change, 20(6), $1645-1675$.

Mansfield, E., \& Lee, J.-Y. (1996). The modern university: contributor to industrial innovation and recipient of industrial R\&D support. Research Policy, 25(7), 1047-1058.

Markusen, J. R., \& Venables, A. J. (1999). Foreign direct investment as a catalyst for industrial development. European Economic Review, 43(2), 335-356.

Marshall, A. (2009). Principles of economics: unabridged eighth edition. Cosimo, Inc.

Martin, B. R. (2003). The changing social contract for science and the evolution of the university. Science and Innovation: Rethinking the Rationales for Funding and Governance. Edward Elgar, Cheltenham, 7-29.

Martin, X., \& Salomon, R. (2003). Knowledge transfer capacity and its implications for the theory of the multinational corporation. Journal of International Business Studies, 34(4), 356-373.

Mathews, J., \& Cho, D. S. (1996). Tiger Chips: The Emergence of East Asian Forces 
in Global Semiconductor Industry. mimeo, Institute of Industrial Policy Studies, Seoul, Korea.

McGee, D. (1995). Making up mind: The early sociology of invention. Technology and Culture, 773-801.

Mckinsy global. (2015). SAUDI ARABIA BEYOND OIL : THE INVESTMENT AND PRODUCTIVITY TRANSFORMATION.

McVoy, E. C. (1940). Patterns of diffusion in the United States. American Sociological Review, 5(2), 219-227.

Mees, C. E. K. (1920). The organization of industrial scientific research. McGrawHill Book Company, Incorporated.

Melitz, M., Helpman, E., \& Yeaple, S. (2004). Export versus FDI with heterogeneous firms. American Economic Review, 94.

Melitz, M. J. (2003). The impact of trade on intra-industry reallocations and aggregate industry productivity. Econometrica, 71(6), 1695-1725.

Merriam, S. B. (2002). Qualitative research in practice: Examples for discussion and analysis. Jossey-Bass Inc Pub.

Metcalfe, S. (1995). The economic foundations of technology policy: equilibrium and evolutionary perspectives. Handbook of the Economics of Innovation and Technological Change, 446.

Meyer, K. E., \& Sinani, E. (2009). When and where does foreign direct investment generate positive spillovers\&quest; A meta-analysis. Journal of International Business Studies, 40(7), 1075-1094.

Middle east, V. (2011). The Saudi Construction Industry.

Miles, I., Kastrinos, N., Flanagan, K., Bilderbeek, R., Den Hertog, P., Huntink, W., \& Bouman, M. (1995). Knowledge-intensive business services. EIMS Publication, (15).

Miles, M. B., \& Huberman, A. M. (1994). Qualitative data analysis: Grounded theory procedures and techniques. Sage Publications, London.

Miles, M. B., Huberman, A. M., \& Saldana, J. (2013). Qualitative data analysis. Sage.

Miller, D. (1983). The correlates of entrepreneurship in three types of firms. Management Science, 29(7), 770-791.

Minbaeva, D., Pedersen, T., Björkman, I., Fey, C. F., \& Park, H. J. (2003). MNC 
knowledge transfer, subsidiary absorptive capacity, and HRM. Journal of International Business Studies, 34(6), 586-599.

Moaddel, M. (2010). Religious regimes and prospects for liberal politics: Futures of Iran, Iraq and Saudi Arabia. Futures, 42(6), 532-544. https://doi.org/10.1016/j.futures.2010.01.004

Moher, D., Liberati, A., Tetzlaff, J., Altman, D. G., \& Group, P. (2009). Preferred reporting items for systematic reviews and meta-analyses: the PRISMA statement. PLoS Medicine, 6(7), e1000097.

Mojab, F., Zaefarian, R., \& Azizi, A. H. D. (2011). Applying Competency based Approach for Entrepreneurship education. Procedia - Social and Behavioral Sciences, 12, 436-447. https://doi.org/10.1016/j.sbspro.2011.02.054

Moran, T. H., Alfaro, L., \& Javorcik, B. S. (2007). How to Investigate the Impact of Foreign Direct Investment on Development and Use the Results to Guide Policy [with Comments and Discussion]. In Brookings Trade Forum (pp. 1-60). JSTOR.

Morris, M., Kuratko, D., \& Covin, J. (2010). Corporate entrepreneurship \& innovation. Cengage Learning.

Moustakas, C. (1994). Phenomenological research methods. Sage.

Mowery, D. C., \& Sampat, B. N. (2005). The Bayh-Dole act of 1980 and universityindustry technology transfer: a model for other OECD governments? In Essays in honor of Edwin Mansfield (pp. 233-245). Springer.

Muller, E. (1999). Innovation interactions between knowledge-intensive business services and small and medium-sized enterprises: an analysis in terms of evolution, knowledge and territories. Strasbourg 1.

Muller, E., \& Doloreux, D. (2007). The key dimensions of knowledge-intensive business services (KIBS) analysis: a decade of evolution. Arbeitspapiere Unternehmen und Region.

Muller, E., \& Zenker, A. (2001). Business services as actors of knowledge transformation and diffusion : some empirical findings on the role of KIBS in regional and national innovation systems.

Naranjo-Valencia, J. C., Jiménez-Jiménez, D., \& Sanz-Valle, R. (2011). Innovation or imitation? The role of organizational culture. Management Decision, 49(1), 5572.

Naudé, W. (2008). Entrepreneurship in economic development. Research Paper, 
UNU-WIDER, United Nations University (UNU).

Naudé, W. (2011). Entrepreneurship and Economic Development. Palgrave Macmillan. Retrieved

from http://books.google.co.uk/books?id=EAq8bwAACAAJ

Naudé, W. (2011). Entrepreneurship is not a binding constraint on growth and development in the poorest countries. World Development, 39(1), 33-44.

Naudé, W. A. (2013). Entrepreneurship and economic development: Theory, evidence and policy. IZA Discussion Paper.

Nelson, R. R. (1993). National innovation systems: a comparative analysis. University of Illinois at Urbana-Champaign's Academy for Entrepreneurial Leadership Historical Research Reference in Entrepreneurship.

Nelson, R. R. (2004). The market economy, and the scientific commons. Research Policy, 33(3), 455-471.

Nelson, R. R. (2005). Technology, institutions, and economic growth. Harvard University Press.

Niblock, T. (2008). Saudi Arabia's Economic Development: Ambitious Visions, Difficult Dilemmas. Journal of Middle Eastern and Islamic Studies (in Asia).

Niosi, J. (2010). Building national and regional innovation systems: institutions for economic development. Edward Elgar Publishing.

Niosi, J. (2011a). Building innovation systems: an introduction to the special section. Industrial and Corporate Change, 20(6), 1637-1643.

Niosi, J. (2011b). Complexity and path dependence in biotechnology innovation systems. Industrial and Corporate Change, 20(6), 1795-1826.

Niosi, J., \& Reid, S. E. (2007). Biotechnology and nanotechnology: Science-based enabling technologies as windows of opportunity for LDCs? World Development, 35(3), 426-438.

OCED. (2005). OECD Studies on SMEs and Entrepreneurship. Paris.

OECD. (1996). the knowledge economy. Paris.

OECD. (1997). national innovation systems.

OECD. (2007). OECD Annual Report 2007.

Oerlemans, L. A. G., Meeus, M. T. H., \& Boekema, F. W. M. (1998). Do networks matter for innovation? The usefulness of the economic network approach in analysing innovation. Tijdschrift Voor Economische En Sociale Geografie, 89(3), 298-309. 
Ogburn, W. F. (1922). Social change with respect to culture and original nature. BW Huebsch, Incorporated.

Olaniyan, D. A., \& Okemakinde, T. (2008). Human capital theory: Implications for educational development. Pakistan Journal of Social Sciences, 5(5), 479-483.

Onstenk, J. (2003). Entrepreneurship and vocational education. Symposium Journals.

Orsenigo, L. (2001). The (failed) development of a biotechnology cluster: The case of Lombardy. Small Business Economics, 17(1-2), 77-92.

Owen-Smith, J., Riccaboni, M., Pammolli, F., \& Powell, W. W. (2002). A comparison of US and European university-industry relations in the life sciences. Management Science, 48(1), 24-43.

Page, J., \& Campos, E. (1993). The East Asian miracle: economic growth and public policy. World Bank, Washington DC.

Paper, W., Analysis, C., \& Set, N. D. (2011). Small and Medium Enterprises A CrossCountry Analysis with a New Data Set, (January).

Parisot, A. H. (1997). Distance education as a catalyst for changing teaching in the community college: Implications for institutional policy. New Directions for Community Colleges, 1997(99), 5-13.

Patton, M. Q. (1990). Qualitative evaluation and research methods. SAGE Publications, inc.

Patzelt, H. (2012). Biotechnology Entrepreneurship (Vol. 8). https://doi.org/10.1561/0300000041

Pavitt, K. (2003). Specialization and systems integration: where manufacture and services still meet. The Business of Systems Integration, 78-91.

Peeters, C., \& de la Potterie, B. van P. (2006). Innovation strategy and the patenting behavior of firms. Journal of Evolutionary Economics, 16(1-2), 109-135.

Pessoa, A. (2007). FDI and Host country productivity: a review. Universidade do Porto, Faculdade de Economia do Porto.

Peter, D. (1969). The age of discontinuity: guidelines to our changing society. Harper and Row, NY.

Peters, M. (2001). National education policy constructions of the " knowledge economy ': towards a critique, 2(1), 1-22.

Peterson, R. A. (1973). A note on optimal adopter category determination. Journal of Marketing Research, 10(3), 325-329.

PhRMA. (2011). The U.S. Biopharmaceuticals sector: Economic Contribution to the 
Nation. Battelle, (July).

Pigou, A. C. (1924). The economics of welfare. Transaction Publishers.

Piore, M. J. (1984). The second industrial divide: possibilities for prosperity. Basic books.

Pisano, G. (2006). Can science be a business? Harvard Business Review, 10, 1-12.

Pitelis, C. N., \& Teece, D. J. (2010). Cross-border market co-creation, dynamic capabilities and the entrepreneurial theory of the multinational enterprise. Industrial and Corporate Change, 19(4), 1247-1270.

Planning, M. of E. and. (2013). The Saudi Economy in Figures.

Polder, M., Leeuwen, G. van, Mohnen, P., \& Raymond, W. (2009). Productivity effects of innovation modes.

Porter, M. (1998). On Competition (A Harvard Business Review Book). Boston: Harvard Business School Publishing.

Porter, M. E. (1990). The competitive advantage of notions. Harvard Business Review.

Porter, M. E. (1998). Clusters and the new economics of competition (Vol. 76). Harvard Business Review Boston.

Porter, M. E. (2000). Location, competition, and economic development: Local clusters in a global economy. Economic Development Quarterly, 14(1), 15-34.

Porter, M. E. (2008). Competitiveness as an engine for economic growth: implications for Saudi Arabia. In The Global Competiveness Forum.

Porter, M. E. (2009). Competitiveness and the State of Entrepreneurship p p p in Saudi Arabia.

Porter, M. E. (2011). Competitive advantage of nations: creating and sustaining superior performance. Simon and Schuster.

Porter, M. E. (2014). Clusters and Shared Value: Drivers of Competitiveness (p. 21).

Powell, W. W., Koput, K. W., \& Smith-Doerr, L. (1996). Interorganizational collaboration and the locus of innovation: Networks of learning in biotechnology. Administrative Science Quarterly, 116-145.

Prevezer, M. (2001). Ingredients in the early development of the US biotechnology industry. Small Business Economics, 17(1-2), 17-29.

Punch, K. F., \& Oancea, A. (2014). Introduction to research methods in education. Sage.

Quah, D. (2002). Technological dissemination and economic growth: Some lessons 
for the New Economy. LSE Economics Department, January.

Radas, S., \& Božić, L. (2009). The antecedents of SME innovativeness in an emerging transition economy. Technovation, 29(6), 438-450.

Rader, R. A. (2008). (Re) defining biopharmaceutical. Nature Biotechnology, 26(7), $743-751$.

Rae, D. (2007). Connecting enterprise and graduate employability: challenges to the higher education culture and curriculum? Education+ Training, 49(8/9), 605619.

Ram, P. (2014). New Strategic Initiatives-A Case Study of the Saudi Health Ministry. International Journal of Academic Research in Economics and Management Sciences, 3(1), 236.

Ramamurti, R. (2012). What is really different about emerging market multinationals? Global Strategy Journal, 2(1), 41-47.

Rasmussen, E., Moen, Ø., \& Gulbrandsen, M. (2006). Initiatives to promote commercialization of university knowledge. Technovation, 26(4), 518-533.

Rauch, J. E., \& Evans, P. B. (2000). Bureaucratic structure and bureaucratic performance in less developed countries. Journal of Public Economics, 75(1), $49-71$.

Ravi, N. (2013). INTRA REGIONAL TRADE AMONG GULF CO-OPERATION COUNCIL.

Reagans, R., \& McEvily, B. (2003). Network structure and knowledge transfer: The effects of cohesion and range. Administrative Science Quarterly, 48(2), 240-267.

Reich, R. (1991). The work of nations: Preparing ourselves for twenty-first century capitalism. New York: Alfred Knopf.

Remenyi, D., Williams, B., Money, A., \& Swartz, E. (1998). Research in business and management. London: Sage. Remmen, D.(2003). Performance Pays off. Strategic Finance, 84(9), 24-31.

Rentier State and Shi ' a Islam in the Iranian Revolution Author ( s ): Theda Skocpol. (2014), 11(3), 265-283.

Richard, L. (2004). The rise of the creative class: and how it's transforming work, leisure, community and everyday life. Basic Books.

Richard, L. (2005). The Flight of the Creative Class: The new global competition for talent. HarperCollinsBusiness.

Robinson, J. (1938). The classification of inventions. The Review of Economic 
Studies, 5(2), 139-142.

Robson, C. (2002). Real world research. 2nd. Edition. Blackwell Publishing. Malden.

Rocha, H. O. (2004). Entrepreneurship and development: The role of clusters. Small Business Economics, 23(5), 363-400.

Rodriguez-Clare, A. (1996). Multinationals, linkages, and economic development. The American Economic Review, 852-873.

Roelandt, T. J. A., \& den Hertog, P. (1998). Cluster Analysis \& Cluster Based Policy in OECD Countries. Hague Utrecht. OECD Focus Group.

Rogers, E. M. (1983). Diffusion of innovations.

Rogers, E. M. (2007). Models of knowledge transfer: critical perspectives. Knowledge and Innovation Management, 23.

Rogers, E. M. (2010). Diffusion of innovations. Simon and Schuster.

Romer, P. M. (1986). Increasing returns and long-run growth. The Journal of Political Economy, 1002-1037.

Romer, P. M. (1990). Endogenous technological change.

Rorida, R. (1999). Engine or infrastructure? The university role in economic development.

Rosenberg, N. (1976). Marx as a Student of Technology. Monthly Review, 28(3), 5677.

Ross, M. L. (2001). Does oil hinder democracy? World Politics, 53(3), 325-361.

Rothaermel, F. T., \& Deeds, D. L. (2006). Alliance type, alliance experience and alliance management capability in high-technology ventures. Journal of Business Venturing, 21(4), 429-460.

Sadi, M. A., \& Henderson, J. C. (2011). Franchising and small medium-sized enterprises (SMEs) in industrializing economies: A Saudi Arabian perspective. Journal of Management Development, 30(4), 402-412.

Sahin, I. (2006). Detailed review of Rogers' diffusion of innovations theory and educational technology-related studies based on Rogers' theory. TOJET: The Turkish Online Journal of Educational Technology, 5(2).

Saleh, A. Al. (2012). Exploring Strategies for Small and Medium Enterprises in Saudi Arabia. In RIBM Doctoral Symposium.

Sánchez, J. C. (2011). University training for entrepreneurial competencies: Its impact on intention of venture creation. International Entrepreneurship and Management Journal, 7(2), 239-254. 
Sandhu, M. S., \& Ching, P. W. (2014). Relationship between Individual Cultural Values and Knowledge Sharing in Selected Multinational Companies in Malaysia. International Journal of Business and Economics, 13(1), 1-24.

Sansom, C. (2004). EU expansion: enlarged horizons or false dawn? Nature Biotechnology, 22(5), 501-504.

Sathe, V., \& Drucker, P. F. (2003). Corporate entrepreneurship: Top managers and new business creation. Cambridge University Press Cambridge.

saudi arabia monetory agency. (2013). Ninth, Forty Report, Annual Developments, Latest Economic.

Saunders, M. N. K., Saunders, M., Lewis, P., \& Thornhill, A. (2011). Research methods for business students, 5/e. Pearson Education India.

Saywell, T. (2001). Medicine for the Economy. Far Eastern Economic Review, 15.

Schmitt-Rodermund, E. (2004). Pathways to successful entrepreneurship: Parenting, personality, early entrepreneurial competence, and interests. Journal of Vocational Behavior, 65(3), 498-518. https://doi.org/10.1016/j.jvb.2003.10.007

Schmookler, J. (1966). Invention and economic growth.

Schultz, T. W. (1961). Education and economic growth.

Schultz, T. W. (1961). Investment in human capital. The American Economic Review, $1-17$.

Schumpeter, J. (1911). The theory of economic development. Cambridge, MA: Harvard University Press.

Schumpeter, J. (1928). The instability of capitalism. The Economic Journal, 361-386.

Schumpeter, J. A. (1947). The creative response in economic history. The Journal of Economic History, 7(02), 149-159.

Schumpeter, J. A. (1961). The Theory of Economic Development: An Inquiry into Profits, Capital, Credit, Interest, and the Business Cycle. Translated by Redvers Opie. Oxford University Press.

Schumpeter, J. A. (2013). Capitalism, socialism and democracy. Routledge.

Schwab, K. (2014). The Global Competitiveness Report.

Schwandt, T. A. (1997). Qualitative inquiry: A dictionary of terms. Sage Publications, Inc.

Science, P., \& York, N. (2014). Rentier Wealth , Unruly Law, and the Rise of Opposition The Political Economy of Oil States, 31(3), 295-315.

Senge, P. (1990). The fifth discipline: The art and science of the learning 
organization. New York: Currency Doubleday.

Senge, P. M. (2006). The fifth discipline: The art and practice of the learning organization. Broadway Business.

Sewell, P., \& Pool, L. D. (2010). Moving from conceptual ambiguity to operational clarity: employability, enterprise and entrepreneurship in higher education. Education+ Training, 52(1), 89-94.

Sexenian, A. (1994). Regional advantage: culture and competition in Silicon Valley and Route 128. Harvard University Press, Cambridge, MA.

Shan, W., Walker, G., \& Kogut, B. (1994). Interfirm cooperation and startup innovation in the biotechnology industry. Strategic Management Journal, 15(5), 387-394.

Shane, S. A. (2007). Economic Development Through Entrepreneurship: Government, University and Business Linkages. Edward Elgar Publishing, Incorporated.

Shaw, J. K., \& Allison, J. (1999). The intersection of the learning region and local and regional economic development: Analysing the role of higher education. Regional Studies, 33(9), 896-902.

Shenton, A. K. (2004). Strategies for ensuring trustworthiness in qualitative research projects. Education for Information, 22(2), 63-75.

Sherry, L. (1997). The boulder valley internet project: Lessons learned. THE Journal (Technological Horizons In Education), 25(2), 68.

Sherry, L., \& Gibson, D. (2002). The path to teacher leadership in educational technology. Contemporary Issues in Technology and Teacher Education, 2(2), 178-203.

Shin, J.-S. (1996). The Economics of the Latecomers: Catching-up. Technology Transfer and Institutions in Germany, Japan and South Korea, London and New York: Routledge.

Shujauddin, M. (2010). Saudi Arabia : Franchising Market Update. Washington Dc.

Singley, M. K., \& Anderson, J. R. (1989). The transfer of cognitive skill. Harvard University Press.

Smith, D. K., \& Alexander, R. C. (1988). Fumbling the Future: How Xerox Invented. Then Ignored, the First Personal Computer (William Morrow, New York, 1988) ISBN, 1-58348.

Smith, K. (1995). Interactions in knowledge systems: foundations, policy implications 
and empirical methods. The STEP Group, Studies in technology, innovation and economic policy.

Solow, R. M. (1956). A contribution to the theory of economic growth. The Quarterly Journal of Economics, 65-94.

Sorenson, O., Rivkin, J. W., \& Fleming, L. (2006). Complexity, networks and knowledge flow. Research Policy, 35(7), 994-1017.

Spradley, J. P. (1980). Doing participant observation. JP Spradley, Participant Observation, 53-84.

Spradley, J. P. (2016). Participant observation. Waveland Press.

Stake, R. E. (1995). The art of case study research. Sage.

Stein, S. (2003). Consultant for Singapore start-ups. Personal Interview with Researchers.

Stiglitz, J. (1999). Public policy for a knowledge economy. Remarks at the Department for Trade and Industry and Center for Economic Policy Research, 27.

Stoneman, P. (1995). Handbook of the economics of innovation and technological change.

Stuart, T., \& Sorenson, O. (2003). The geography of opportunity: spatial heterogeneity in founding rates and the performance of biotechnology firms. Research Policy, 32(2), 229-253.

Stuart, W. D. (2000). Influence of sources of communication, user characteristics and innovation characteristics on adoption of a communication technology. University of Kansas, Communication Studies Kansas.

Sturgeon, T. J. (2001). How do we define value chains and production networks? IDS Bulletin, 32(3), 9-18.

Sturm, M., \& Siegfried, N. (2005). Regional monetary integration in the member states of the Gulf Cooperation Council. ECB Occasional Paper, (31).

Sturm, M., Strasky, J., Adolf, P., \& Peschel, D. (2008). The Gulf Cooperation Council Countries-Economic Structures, Recent Developments and Role in the Global Economy. ECB Occasional Paper, (92).

Su, Y.-S., Tsang, E. W. K., \& Peng, M. W. (2009). How do internal capabilities and external partnerships affect innovativeness? Asia Pacific Journal of Management, 26(2), 309-331.

Sullivan, G. M., \& Artino Jr, A. R. (2013). Analyzing and interpreting data from 
Likert-type scales. Journal of Graduate Medical Education, 5(4), 541-542.

Szirmai, A., Naudé, W. A., \& Goedhuys, M. (2011). Entrepreneurship, innovation, and economic development. Oxford University Press.

Szulanski, G. (1996). Exploring internal stickiness: Impediments to the transfer of best practice within the firm. Strategic Management Journal, 17(S2), 27-43.

Tapscott, D. (1996). The digital economy: Promise and peril in the age of networked intelligence (Vol. 1). McGraw-Hill New York.

Teece, D. J. (1977). Technology transfer by multinational firms: the resource cost of transferring technological know-how. The Economic Journal, 242-261.

Teece, D. J. (1986). Profiting from technological innovation: Implications for integration, collaboration, licensing and public policy. Research Policy, 15(6), $285-305$.

Teece, D. J. (2007). Explicating dynamic capabilities: the nature and microfoundations of (sustainable) enterprise performance. Strategic Management Journal, 28(13), 1319-1350.

Teece, D. J. (2014). A dynamic capabilities-based entrepreneurial theory of the multinational enterprise. Journal of International Business Studies, 45(1), 8-37.

the world bank. (2014). Economy Profile: Saudi Arabia. https://doi.org/10.1596/978-0-8213-9615-5.

the world bank. (2016). knowledge for development.

Thomas, A., \& Carl, D. (2001). Korea and the Knowledge-based Economy Making the Transition: Making the Transition. OECD Publishing.

Thornberry, N. (2001). Corporate entrepreneurship:: antidote or oxymoron? European Management Journal, 19(5), 526-533.

Thurik, R., \& Wennekers, S. (2004). Entrepreneurship, small business and economic growth. Journal of Small Business and Enterprise Development, 11(1), 140-149.

Toma, S.-G., Grigore, A.-M., \& Marinescu, P. (2014). Economic Development and Entrepreneurship. Procedia Economics and Finance, 8, 436-443.

Tsang, E. W. K. (2002). Acquiring knowledge by foreign partners from international joint ventures in a transition economy: learning-by-doing and learning myopia. Strategic Management Journal, 23(9), 835-854.

Umpleby, S. A. (2007). Viable system model. In International encyclopedia of organization studies. Volume 4 (pp. 1616-1617). Sage. 
Uzzi, B., \& Lancaster, R. (2003). Relational embeddedness and learning: The case of bank loan managers and their clients. Management Science, 49(4), 383-399.

Valuation, K. \& V. (2013). Site Selection for Life Sciences Companies European Life Sciences Cluster. switzerland.

Van de Vrande, V., De Jong, J. P. J., Vanhaverbeke, W., \& De Rochemont, M. (2009). Open innovation in SMEs: Trends, motives and management challenges. Technovation, 29(6), 423-437.

Velasquez, M. G., \& Velazquez, M. (2002). Business ethics: Concepts and cases. Prentice Hall Upper Saddle River, NJ.

Vig, P. (2003). Consultant for Singapore start-ups. Personal Interview with Researchers.

Vu, K. M. (2011). ICT as a source of economic growth in the information age: Empirical evidence from the 1996-2005 period. Telecommunications Policy, $35(4), 357-372$.

Walshok, M. L. (2012). Reinventing Continuing Higher Education, 76, 38-53.

Wennekers, S., \& Thurik, R. (1999). Linking Entrepreneurship and Economic Growth, 27-55.

Weston, M. (2011). Prophets and princes: Saudi Arabia from Muhammad to the present. Wiley. com.

Wiseman, A. W., \& Anderson, E. (2012). ICT-integrated education and national innovation systems in the Gulf Cooperation Council (GCC) countries. Computers \& Education, 59(2), 607-618.

Witmer, D. F., Colman, R. W., \& Katzman, S. L. (1999). „From paper and pen to screen and keyboard. Towards a methodology for survey research on the Internet" in S. Jones (ed) Doing Internet Research. Thousand Oaks, CA: Sage.

Wong, P.-K. (1999). National innovation systems for rapid technological catch-up: An analytical framework and a comparative analysis of Korea, Taiwan, and Singapore. In DRUID Summer Conference held in Rebild.

Wong, P.-K. (2002). Globalisation of US, European and Japanese production networks and the growth of Singapore's electronics industry. International Journal of Networking and Virtual Organisations, 1(1), 55-81.

Wong, P.-K., Ho, Y.-P., \& Singh, A. (2007). Towards an "entrepreneurial university" model to support knowledge-based economic development: The case of the National University of Singapore. World Development, 35(6), 941-958. 
Wong, P. K. (2001). Leveraging multinational corporations, fostering technopreneurship: the changing role of S\&T policy in Singapore. International Journal of Technology Management, 22(5), 539-567.

Wong, P. K. (2005). Singapore as an innovative city in East Asia: an explorative study of the perspectives of innovative industries (Vol. 3568). World Bank Publications.

Woollacott, L. C. (2009). Taxonomies of engineering competencies and quality assurance in engineering education. In Engineering education quality assurance (pp. 257-295). Springer.

$\mathrm{Xu}$, B. (2000). Multinational enterprises, technology diffusion, and host country productivity growth. Journal of Development Economics, 62(2), 477-493.

Yates, D. A. (1996). The rentier state in Africa: Oil rent dependency and neocolonialism in the Republic of Gabon. Africa World Press.

Yeasmin, S., \& Rahman, K. F. (2012). Triangulation'research method as the tool of social science research. Bup Journal, 1(1), 154-163.

Young, E. and. (2000). Grüunderzeit - Deutscher Biotechnologie-Report 2000. Mannheim, Germany.

Young, E. and. (2011). beyond border-global biotechnology report 2011. Cambridge, UK.

Yusuf, N. (2014). Private and public healthcare in Saudi Arabia: future challenges.

Zahra, S. A., \& George, G. (2002). Absorptive capacity: A review, reconceptualization, and extension. Academy of Management Review, 27(2), 185-203.

Zucker, L. G., Darby, M. R., \& Brewer, M. B. (1998). Intellectual Human Capital and the Birth of US Biotechnology Enterprises. American Economic Review, 290306. 


\section{Appendices}

\subsection{Appendix 1: Interview questions (Pilot study)}

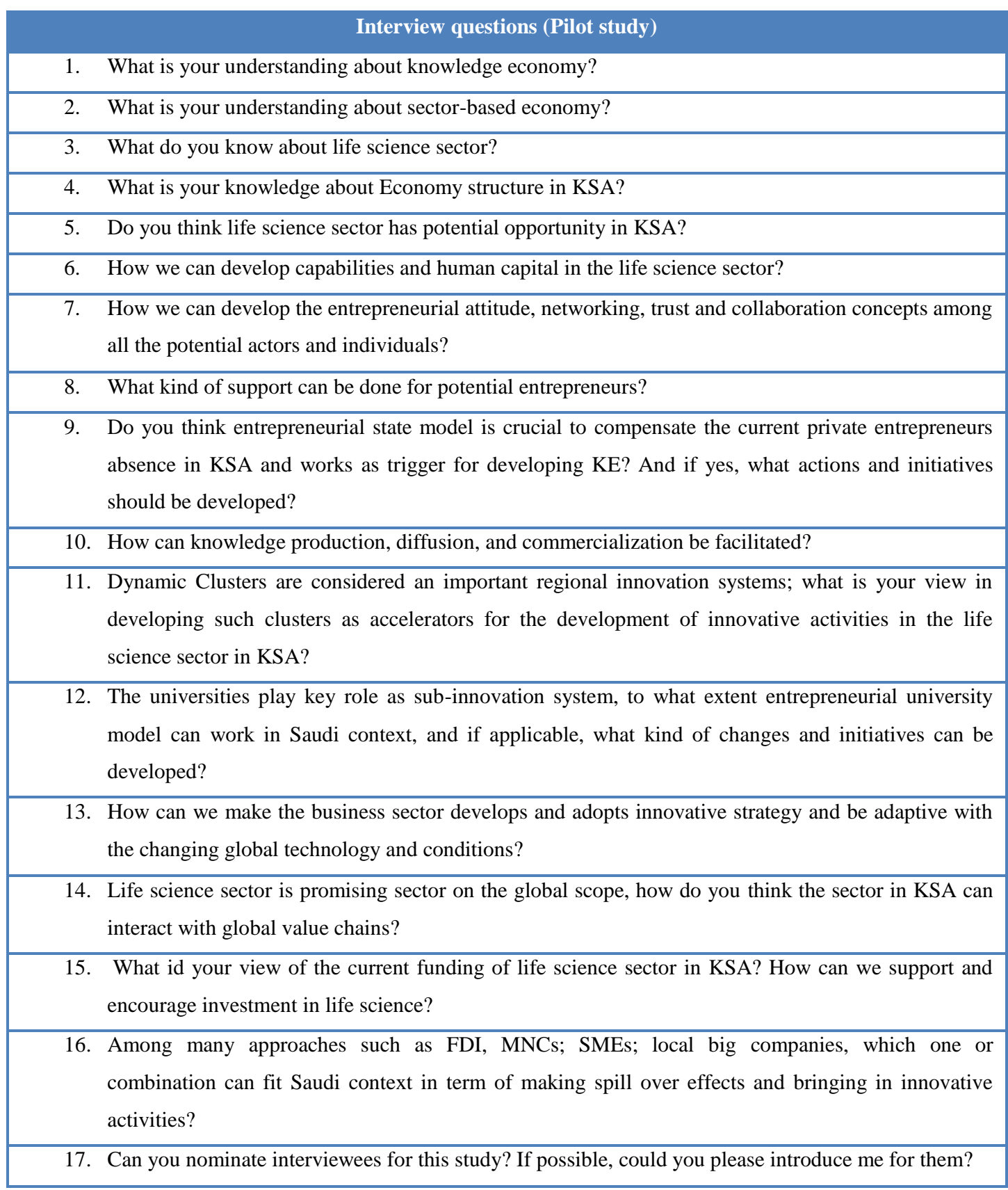




\subsection{Appendix 2: Interview questions (Main Sample)}

\begin{tabular}{|c|c|c|c|c|c|}
\hline R.Q. & Key themes & Sub-themes & Master questions & & \\
\hline $\begin{array}{l}\text { What are the key } \\
\text { requirements of } \mathrm{LS} \\
\text { sector in KSA? }\end{array}$ & $\begin{array}{l}\text { Infrastructure } \\
\text { High skilled people } \\
\text { Funding }\end{array}$ & $\begin{array}{l}\text { General infrastructure } \\
\text { Scientific infrastructure } \\
\text { High life standard } \\
\text { ecosystem } \\
\text { Scientific capabilities } \\
\text { Managerial capabilities } \\
\text { Brain recycling } \\
\text { Education system } \\
\text { Venture capital market } \\
\text { Seed funding }\end{array}$ & $\begin{array}{l}\text { 1) Could u tell me a little bit } \\
\text { about your role in relation } \\
\text { to LS and GGC? } \\
\text { 2) What is your knowledge } \\
\text { about Economy structure } \\
\text { in KSA? } \\
\text { 3) What are the key } \\
\text { components to develop } \\
\text { LS sector in KSA? }\end{array}$ & $\begin{array}{l}\text { 1) } \\
\text { 2) } \\
\text { 3) } \\
\text { 4) } \\
\text { 5) } \\
\text { 6) } \\
\text { 7) } \\
\text { 8) } \\
\text { 9) }\end{array}$ & $\begin{array}{l}\text { What is your un } \\
\text { What is your un } \\
\text { What do you kn } \\
\text { What is your kn } \\
\text { To what extent } \\
\text { in KSA? } \\
\text { How can we de } \\
\text { How can we cre } \\
\text { What kind of } \\
\text { sector? } \\
\text { To what exten } \\
\text { innovative ecosy }\end{array}$ \\
\hline $\begin{array}{l}\text { What are the } \\
\text { challenges in } \\
\text { developing KE in } \\
\text { LS in KSA? }\end{array}$ & $\begin{array}{l}\text { Culture aspects } \\
\text { Social capital } \\
\text { Priorities } \\
\text { identification } \\
\text { Global vision } \\
\text { Government } \\
\text { commitment }\end{array}$ & $\begin{array}{l}\text { Entrepreneurship } \\
\text { Attitude and values } \\
\text { Collaboration } \\
\text { Networking } \\
\text { Global value chains } \\
\text { interaction }\end{array}$ & $\begin{array}{l}\text { 4) What are the challenges/ } \\
\text { barriers to progressing LS } \\
\text { in KSA? }\end{array}$ & $\begin{array}{l}\text { 10) } \\
\text { 11) } \\
\text { 12) } \\
\text { 13) } \\
\text { 14) }\end{array}$ & $\begin{array}{l}\text { How can we } \\
\text { entrepreneurial } \\
\text { How can we sup } \\
\text { To what ext } \\
\text { involvement wo } \\
\text { To what extent } \\
\text { success in LS se } \\
\text { How can we inte }\end{array}$ \\
\hline $\begin{array}{l}\text { What model of } \\
\text { innovation system } \\
\text { best fit the Saudi } \\
\text { context? }\end{array}$ & $\begin{array}{l}\text { University role } \\
\text { Knowledge transfer } \\
\text { Commercial } \\
\text { orientation-based } \\
\text { researches } \\
\text { Industry innovative } \\
\text { strategy } \\
\text { Government role }\end{array}$ & $\begin{array}{l}\text { Entrepreneurial university } \\
\text { Science parks } \\
\text { Incubators } \\
\text { Spin-offs } \\
\text { Entrepreneurial state } \\
\text { Clusters } \\
\text { Friendly Business } \\
\text { regulations } \\
\text { Incentives }\end{array}$ & $\begin{array}{l}\text { 5) what extent can } \\
\text { clusters perform } \\
\text { accelerator role for } \\
\text { developing LS sector? }\end{array}$ & $\begin{array}{l}\text { 15) } \\
\text { 16) } \\
\text { 17) } \\
\text { 18) } \\
\text { 19) } \\
\text { 20) }\end{array}$ & $\begin{array}{l}\text { What is the pote } \\
\text { centres in devel } \\
\text { How can we fac } \\
\text { To what extent } \\
\text { developing LS s } \\
\text { How can we inc } \\
\text { How can we } \\
\text { universities and } \\
\text { What initiatives } \\
\text { knowledge exch }\end{array}$ \\
\hline
\end{tabular}




\subsection{Appendix 3: interviews` informants}

\begin{tabular}{|c|c|c|c|c|}
\hline Informant 21 & Location & Position & Subsector & Interaction \\
\hline $\mathrm{A} 1^{22}$ & Swansea & $\mathrm{PhD}$ & Academia & $\begin{array}{l}\text { Face to face } \\
\text { Interview }\end{array}$ \\
\hline $\mathrm{A} 2$ & Cardiff & Full Professor & Academia & $\begin{array}{l}\text { Face to face } \\
\text { Interview }\end{array}$ \\
\hline $\mathrm{A} 3$ & Cardiff & Full professor & Academia & $\begin{array}{l}\text { Face to face } \\
\text { Interview }\end{array}$ \\
\hline G1 & Cardiff & PhD student & $\begin{array}{l}\text { Arabic gulf } \\
\text { government }\end{array}$ & $\begin{array}{l}\text { Face to face } \\
\text { Interview }\end{array}$ \\
\hline G2 & Saudi Arabia & $\begin{array}{l}\text { Deputy director-research } \\
\text { professor }\end{array}$ & PRC & $\begin{array}{l}\text { Face to face } \\
\text { Interview }\end{array}$ \\
\hline G3 & Saudi Arabia & Director-PhD & PRC & $\begin{array}{l}\text { Face to face } \\
\text { Interview }\end{array}$ \\
\hline G4 & Saudi Arabia & Manager & PRC-incubator & $\begin{array}{l}\text { Face to face } \\
\text { Interview }\end{array}$ \\
\hline A4 & Saudi Arabia & Director-PhD & Academia (VC) & $\begin{array}{l}\text { Face to face } \\
\text { Interview }\end{array}$ \\
\hline G5 & Saudi Arabia & Assistant manger & Government (fund) & $\begin{array}{l}\text { Face to face } \\
\text { Interview }\end{array}$ \\
\hline G6 & Saudi Arabia & Senior chief consultant-MD & PRC & $\begin{array}{l}\text { Face to face } \\
\text { Interview }\end{array}$ \\
\hline A5 & Saudi Arabia & Dean & Academia & $\begin{array}{l}\text { Face to face } \\
\text { Interview }\end{array}$ \\
\hline A6 & Saudi Arabia & CEO- PhD & $\begin{array}{l}\text { Academia- science } \\
\text { park }\end{array}$ & $\begin{array}{l}\text { Face to face } \\
\text { Interview }\end{array}$ \\
\hline A7 & Saudi Arabia & Director of science park & Academia-private & $\begin{array}{l}\text { Face to face } \\
\text { Interview }\end{array}$ \\
\hline A8 & Saudi Arabia & Manager- PhD & Academia-private & $\begin{array}{l}\text { Face to face } \\
\text { Interview }\end{array}$ \\
\hline A9 & Saudi Arabia & Assistant professor-researcher & Academia-private & $\begin{array}{l}\text { Face to face } \\
\text { Interview }\end{array}$ \\
\hline A10 & Saudi Arabia & Full Prof. Dean-researcher & Academia & $\begin{array}{l}\text { Face to face } \\
\text { Interview }\end{array}$ \\
\hline G7 & Saudi Arabia & Researcher-MD & Ministry of Health & $\begin{array}{l}\text { Face to face } \\
\text { Interview }\end{array}$ \\
\hline
\end{tabular}

21 A=Academia, $\mathrm{G}=$ Government, $\mathrm{P}=$ Private sector.

22 The first four informants represent the interview sample in the pilot study. 


\begin{tabular}{|c|c|c|c|c|}
\hline G8 & Saudi Arabia & $\begin{array}{l}\text { Planning and policy advisor- } \\
\mathrm{PhD}\end{array}$ & Government & $\begin{array}{l}\text { Face to face } \\
\text { Interview }\end{array}$ \\
\hline G9 & Saudi Arabia & Operation manager & PRC-VC & $\begin{array}{l}\text { Face to face } \\
\text { Interview }\end{array}$ \\
\hline G10 & Saudi Arabia & Vice president & PRC & $\begin{array}{l}\text { Face to face } \\
\text { Interview }\end{array}$ \\
\hline G11 & Saudi Arabia & Minister advisor-PhD & Government & $\begin{array}{l}\text { Face to face } \\
\text { Interview }\end{array}$ \\
\hline A11 & Saudi Arabia & Full professor-Researcher & Academia & $\begin{array}{l}\text { Face to face } \\
\text { Interview }\end{array}$ \\
\hline $\mathrm{A} 12$ & Saudi Arabia & $\begin{array}{l}\text { Director of innovation centre- } \\
\mathrm{PhD}\end{array}$ & Academia & $\begin{array}{l}\text { Face to face } \\
\text { Interview }\end{array}$ \\
\hline A13 & Saudi Arabia & Director prototyping centre-PhD & Academia & $\begin{array}{l}\text { Face to face } \\
\text { Interview }\end{array}$ \\
\hline G12 & Saudi Arabia & Deputy director & Government-fund & $\begin{array}{l}\text { Face to face } \\
\text { Interview }\end{array}$ \\
\hline P1 & Saudi Arabia & CEO & Private & $\begin{array}{l}\text { Face to face } \\
\text { Interview }\end{array}$ \\
\hline $\mathrm{P} 2$ & Saudi Arabia & Business development manager & Private & $\begin{array}{l}\text { Face to face } \\
\text { Interview }\end{array}$ \\
\hline P3 & Saudi Arabia & General manger & Private & $\begin{array}{l}\text { Face to face } \\
\text { Interview }\end{array}$ \\
\hline $\mathrm{P} 4$ & Saudi Arabia & CEO & Private & $\begin{array}{l}\text { Face to face } \\
\text { Interview }\end{array}$ \\
\hline P5 & Saudi Arabia & Production manager in MNO. & Private & $\begin{array}{l}\text { Face to face } \\
\text { Interview }\end{array}$ \\
\hline P6 & Saudi Arabia & CEO & Private & $\begin{array}{l}\text { Face to face } \\
\text { Interview }\end{array}$ \\
\hline
\end{tabular}




\subsection{Appendix 4: Transcript sample}

My topic is about developing knowledge economy (KE) framework for life science sector in Saudi Arabia.

Q: to what extent do you think LS sector has potential opportunity in KSA?

A: Uh, yeah, I think that the life science sector has opportunity in any geography, so, it could be here in Wales, it could be in the UK or Europe, the US, or Saudi. Um, I think Saudi Arabia probably has, uh, a lot more catching up to do versus other, uh, countries, because, uh, I don't think they have focused much on science in the past. So, uh, there's been a lot of investment in both Europe and North America, and, and in Asia as well, um, but yeah, I think there's a lot of opportunity for, uh, Saudi Arabia to, to grow in the life sciences.

Q: What is you knowledge about economic structure in KSA?

A: I mean, I've lived in Saudi Arabia for about three months, so, not too long, not really long enough to really understand the economy. Um, I've, I worked in a university, I was very sheltered from, I guess, uh, the way that companies work their structure and how investments happen, so, uh, I would say very little, um, maybe a little bit more than some, but I wouldn't say that I fully understand, uh, how things work in Saudi.

Q: How can we develop capabilities and human capital in LS sector in KSA?

A: Ok. Um, not very sure, so like, I think, I think there are a lot of cultural barriers, um, and it kind like if you look into the World Bank, the economic and, uh, I guess, uh, the current regime that's in power, so I think there's a lot of, uh, there are a lot of things working against Saudi in terms of people wanting to move there, so like, I think in terms of Western culture, or even Eastern culture, ah, I don't think, um, I don't think people have a desire to move to Saudi unless there's an economic incentive, or a desire to , ah, make very big changes, either academic through academic research, or through industry. So, um. I think, in order to grow the capital, the human capital in Saudi, um, there'd have to be more incentives, whether it was higher economic pay, 
or promise of a better infrastructure to do research, or more funding to actually do the research, uh, based out of Saudi.

Q: For indigenous human capital, is there specific plan?

A: I think its possible, I think there would need to be higher levels of, of, uh, I guess, skills and, uh, education, in terms of, um, focus on stem, stem types of subject, so, um, science, technology and engineering and mathematics. Uh, I was teaching in Saudi and, um, I was teaching biology and English, er, and a bit of maths, tutoring in the university and I wouldn't say, er... I've had experience tutoring and teaching in other countries as well, and I wouldn't say the Saudis' ethic for studying is as high as I've seen in other countries, so I think there's definitely a need for, uh, a bit of a cultural change in terms of the desire to learn. I didn't, uh, some students were very keen, some weren't, and, uh, I think on average there wasn't the greatest performance of the students based on the incentives. So, because there is no strict regime of marking or holding students accountable. And it might just be my personal experience within one university, but I've talked to a lot of teachers in Saudi, and its pretty much the same across, no matter what: students are paying, so they will be passed, whereas, um, in a lot of Western and, I guess, more prodigious schools, they're not, they don't, they're not scared to fail students. So I think, ah, I think that the universities need to be more strict in terms of..., and its starts with lower schools as well, which I wasn't exposed to, um, but there was a culture of, ah, allowing students to pass when they didn't deserve. So, ah, yes, building more human capital and, and working capital, I think, if you want to train indigenous Saudis to, ah, have those skills, then the institutions that are teaching those skills need to be very strict in terms of the standards they hold themselves to.

Q: do you think developing the high skill capabilities can be in KSA or with foreign partners?

A: Um, I think anything is possible; it just depends on how much resource is being willing to be allocated to it. So I mean they could have Saudi teachers teach Saudi students the, the things necessary, I mean I'm sure there's plenty of engineers and scientists, uh, but, I mean, uh, there's no need to, to restrict the teaching, ah, only 
from Saudis, so like, there's no reason, I mean every country, uh, that is strong in the life sciences I'm sure imports expertise from different countries. I was teaching in San Diego. Most of my teachers were German, Japanese, uh, from around the world. So there's, uh, I wouldn't see the need to restrict, uh, the people that are providing the knowledge. I mean the visa situation is very strict in Saudi, uh, maybe not for teachers, but it is very strict for, especially for women and, uh, yeah, its more difficult to, to come to Saudi and to find a work permit. They take away your passport, so it is very restrictive. Uh, yes, I say, I would say it is difficult. Um, I believe that if it was a priority of Saudi to open up the country a bit more, to make it less restrictive for, uh, these bringers of knowledge, but it's a difficult situation between the Western, the clash of the Western culture and, and, I guess, uh, Saudi customs. So, uh, it is possible, I think some things would have to be changed a little bit.

$Q:$ Compromise traditional values?

A: Well, I mean I think, I think you mentioned before that, uh, that caste is allowing women and men to be taught together in the same classrooms, and I think those kind of compromises will need to be made, both women teaching men, men teaching women, uh, men and women teaching and learning together.

\section{Q: Do you think it's a matter?}

A: I don't think it's required, but I think it would, uh, be more synergistic or helpful if there weren't as many restrictions, but anything can be done, even with restrictions. So, so again it depends on how much money and how much effort and, ah, yeah, how much willingness there is to, to promote something like that.

Q: How we can develop the entrepreneurial attitude, networking, trust and collaboration concepts among all the potential actors and individuals?

A: Well, I mean there's a lot of, uh, things, that you can learn from other regions that have done something similar, so, uh, if you look at the European Union, they've supported Wales. So you can look at Wales as a, as a case study, but, uh, over the last, I don't know, fourteen years, uh, Wales has received European structural funds. So, that includes a European Regional Development fund and the European Social 
fund. So, the European Social fund has, is, aimed at promoting things such as entrepreneurship, networking skills, uh, you know, business skills, uh, confidence, uh, raising capital, things to that nature, so, uh, the, investing in the human capital side of things, so, focussing on the individual and the way that they see things and the way that they do things. Uh, the European Regional Development fund, that was aimed more towards infrastructure and helping companies. So, the Institute of Life Science One building, this building, um, a lot of other buildings within Wales have been built using European Regional Development funds, as well as a lot of, uh, programmes. So, the European Union funding through the European Regional Development fund, fund the Welsh European Funding Office to run a programme called A for B, Academic for Business, so, essentially they had projects within the university that supported businesses and was mostly helping with knowledge transfer, helping with, uh, you know, discovering new things, to transmit that knowledge to businesses, helping them to commercialising it. And then a lot of projects that ran out of that, so, uh, Mark's whole team, Mark Clément's whole team, was essentially based out of the IMF to assist companies with, um, either raising finance to have new products developed and new patents, new everything. There, there is a mechanism set up to help companies succeed, so, um, I think if you look at what the European Union has done, as well as the US, the US has done very similar things to fund small businesses. Um, I would look at legislation, I would look at funding, I would look at support mechanisms, and infrastructure. Uh, and if Saudi can somehow clone a lot of them, which I think they've started with the universities, uh, they might have a chance at being successful. So, I think it will be a mixture, so, I mean, like, again going back to the European Social funding, the European Regional Development fund, that was led by the European, the funding was from the European Union, the Welsh government set up the programmes, but it was a collaboration between universities, companies and governments, the typical triple helix. So, uh, I'm sure it will take other players as well, including individuals and third sector, and, uh professionals pulling their weight. If you're talking more specifically about life sciences and health care, then I guess Saudi has, does Saudi have public health care? Yeah, so, similar to the NHS, yeah, they would have to be involved as well. But it would have to, I think a lot of the cultural change comes from collaboration and changes in thoughts, but funding can 
really change the way people think, so in Wales, I would say, there's definitely been a change in mind sets. Uh, even though our European funding is done, our minds are still very much sets in helping companies develop products and succeed, even though we're not funded to do that anymore. So, there, I, I would say, definitely look at the funding programmes. 


\subsection{Appendix 5: questionnaire form}

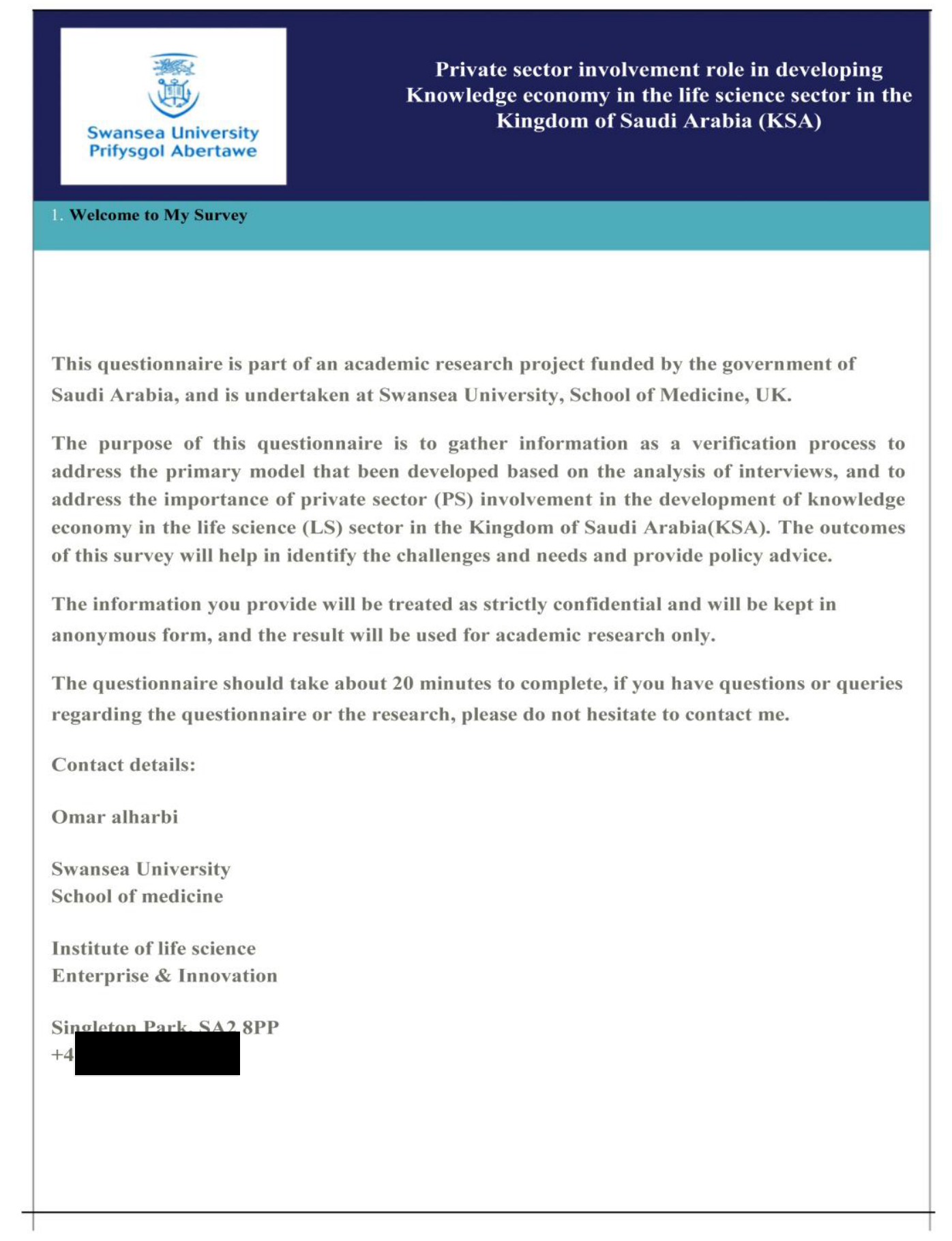


Private sector involvement role in developing Knowledge economy in the life science sector in the

Swansea University Prifysgol Abertawe Kingdom of Saudi Arabia (KSA)

2. Personal information (PI)

1. PI 1

Please indicate your gender

Male

Pemale

I prefer not to say

2. PI 2

Predominantly, which sector do you belong to?

Government

Private sector

Academic (public)

Academic (private)

Public research centers

Third sector

3. PI 3

What is your role in the organisation?

$\square$ Research

Education

Management

$\square$ Consultancy

Manufacturing 


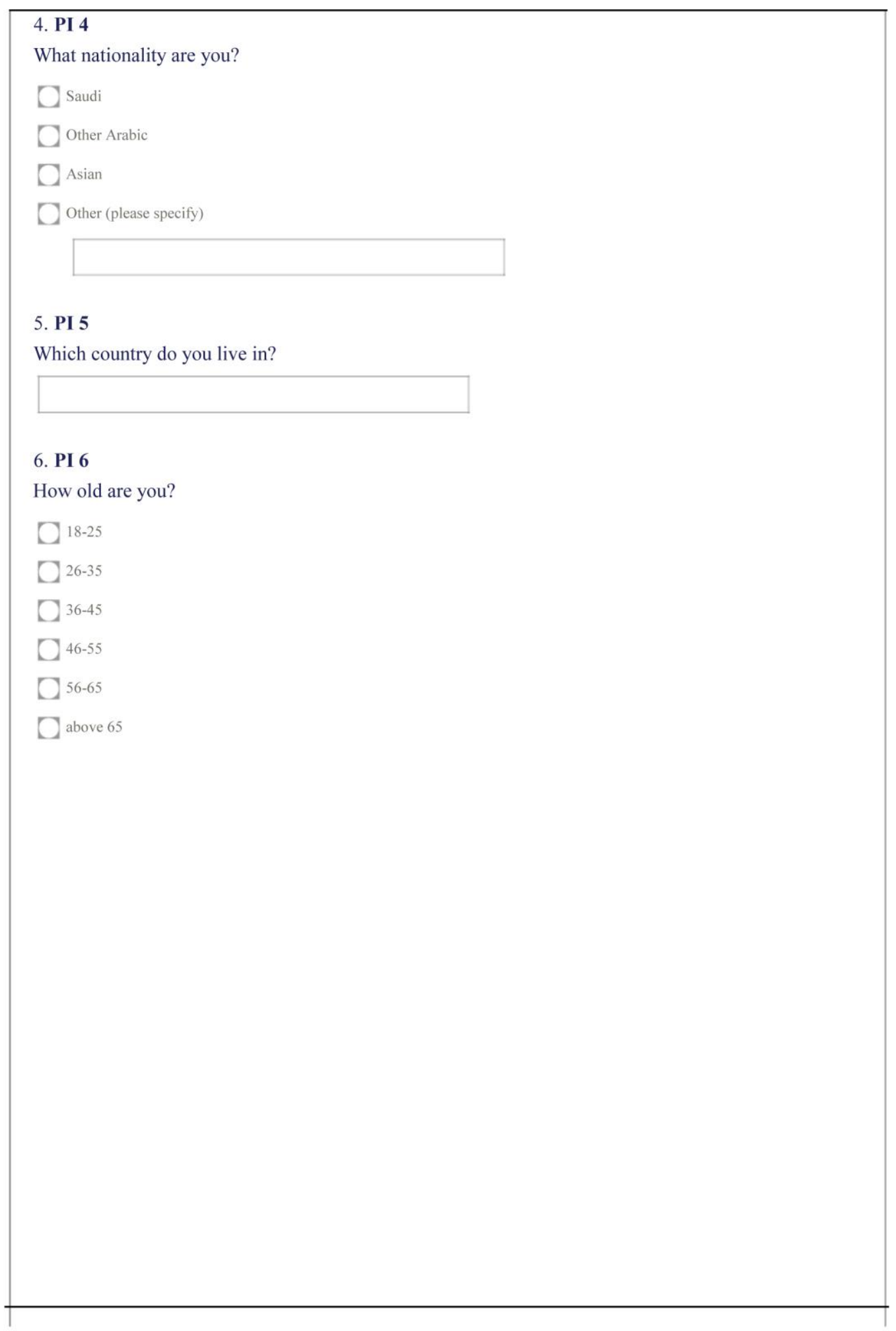




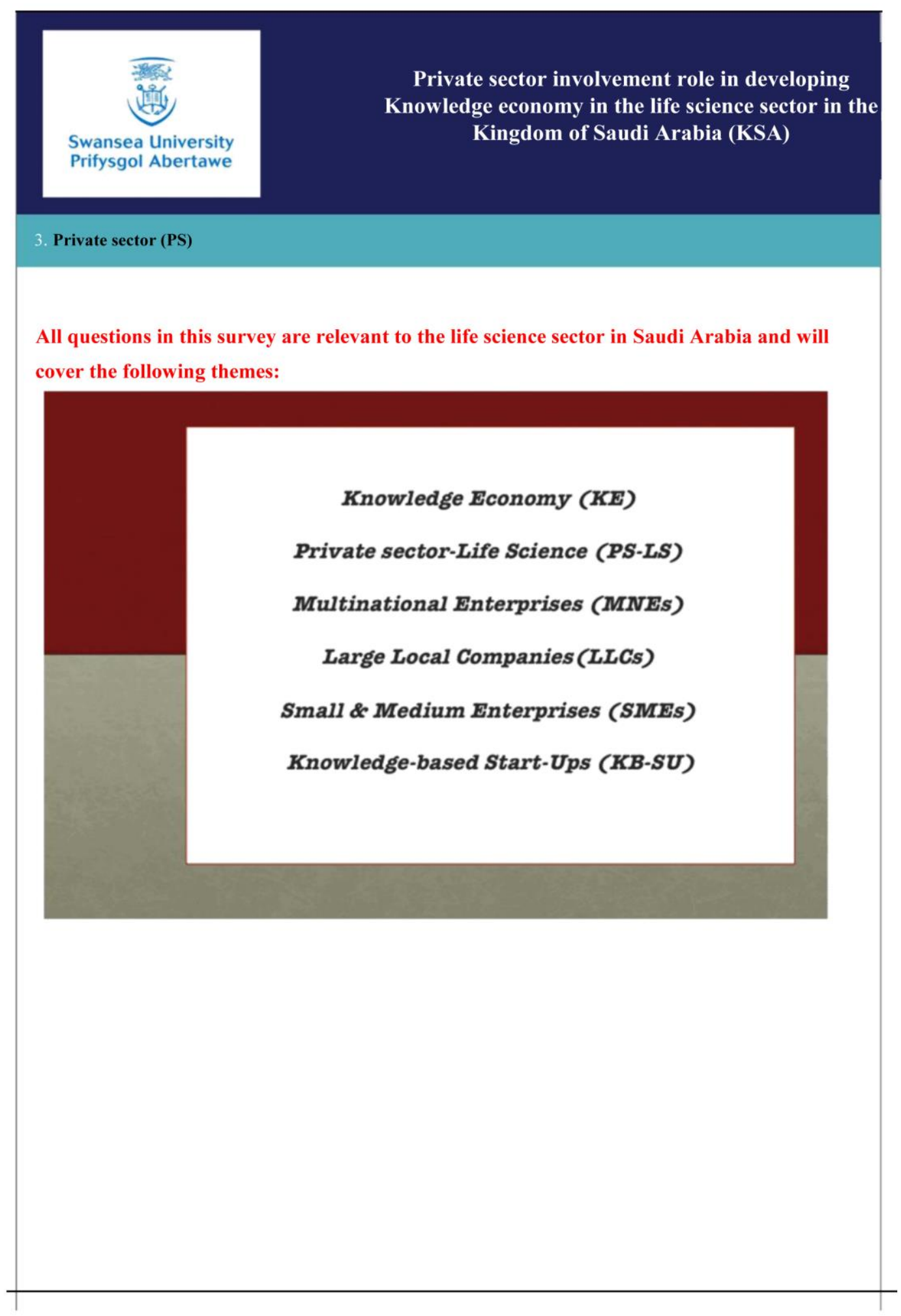




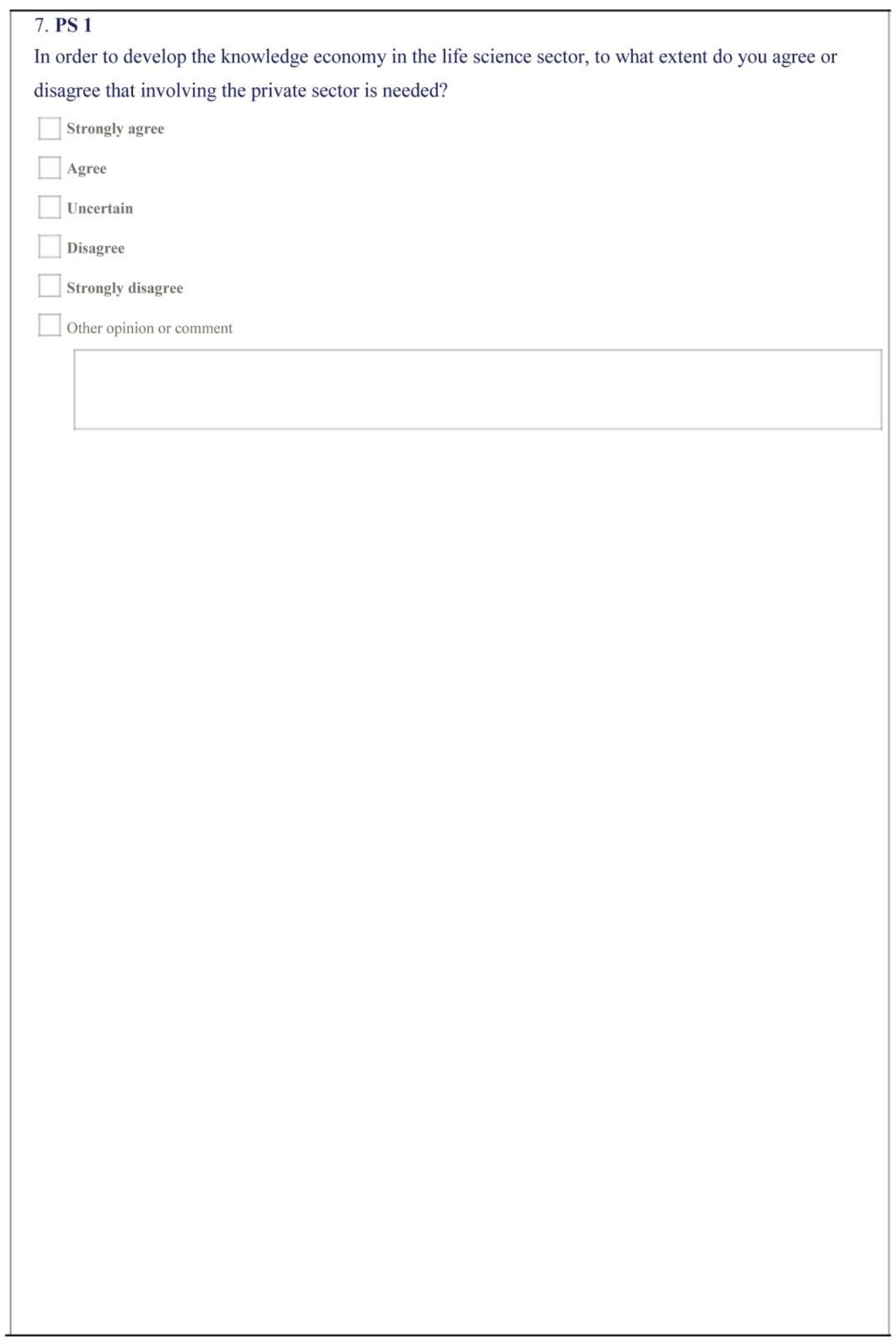


Private sector involvement role in developing Knowledge economy in the life science sector in the Kingdom of Saudi Arabia (KSA)

Swansea University Prifysgol Abertawe

4. The following questions concern Multinational enterprises (MNEs) in the life science sector

\section{MNEs 1 (Internal factors)}

The following encourage multinational enterprises (MNEs) to invest in knowledge-based activities in Saudi Arabia.

To what extent do you agree or disagree?

MNEs have know-how
MNEs have highly
innovative practices
MNEs have highly
sophisticated
management and
manufacturing systems
MNEs have
strong networks
MNEs have highly
competitive capabilities
MNEs are
financially strong
MNEs Strong
marketing and
distribution capability
MNEs have
strong brands




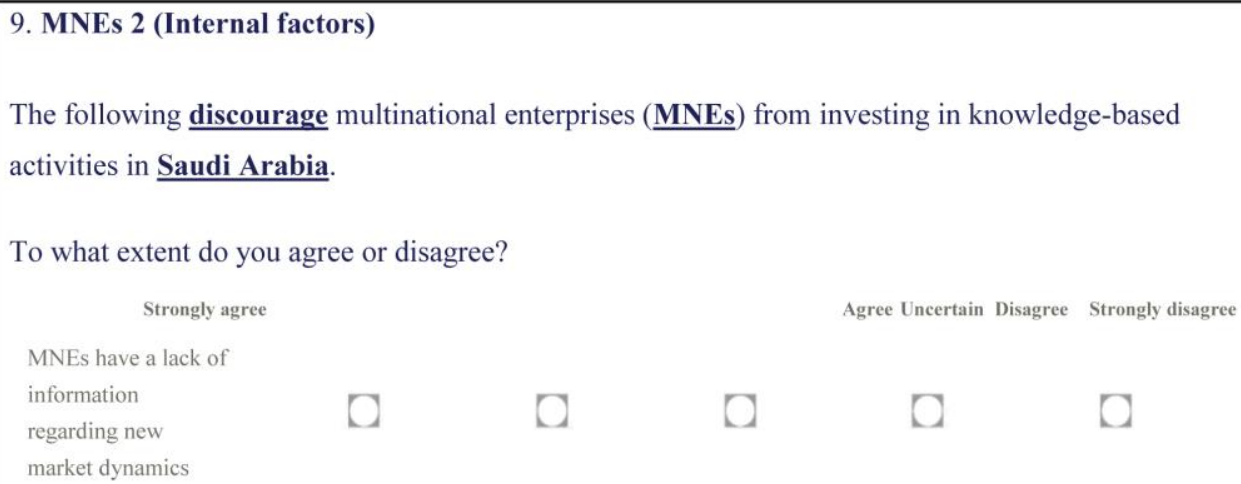

The following may encourage multinational enterprises (MNEs) to invest in knowledge-based activities in Saudi Arabia.

To what extent do you agree or disagree?

Sigh growth Saudi
sizable new market
to the MNEs
Accessibility to
Middle East market
Making joint ventures
with key Saudi partners
Ongoing economic
reforms, which
support foreign direct
investment
Financial incentives
Special comparative
advantages
(religious tourists)




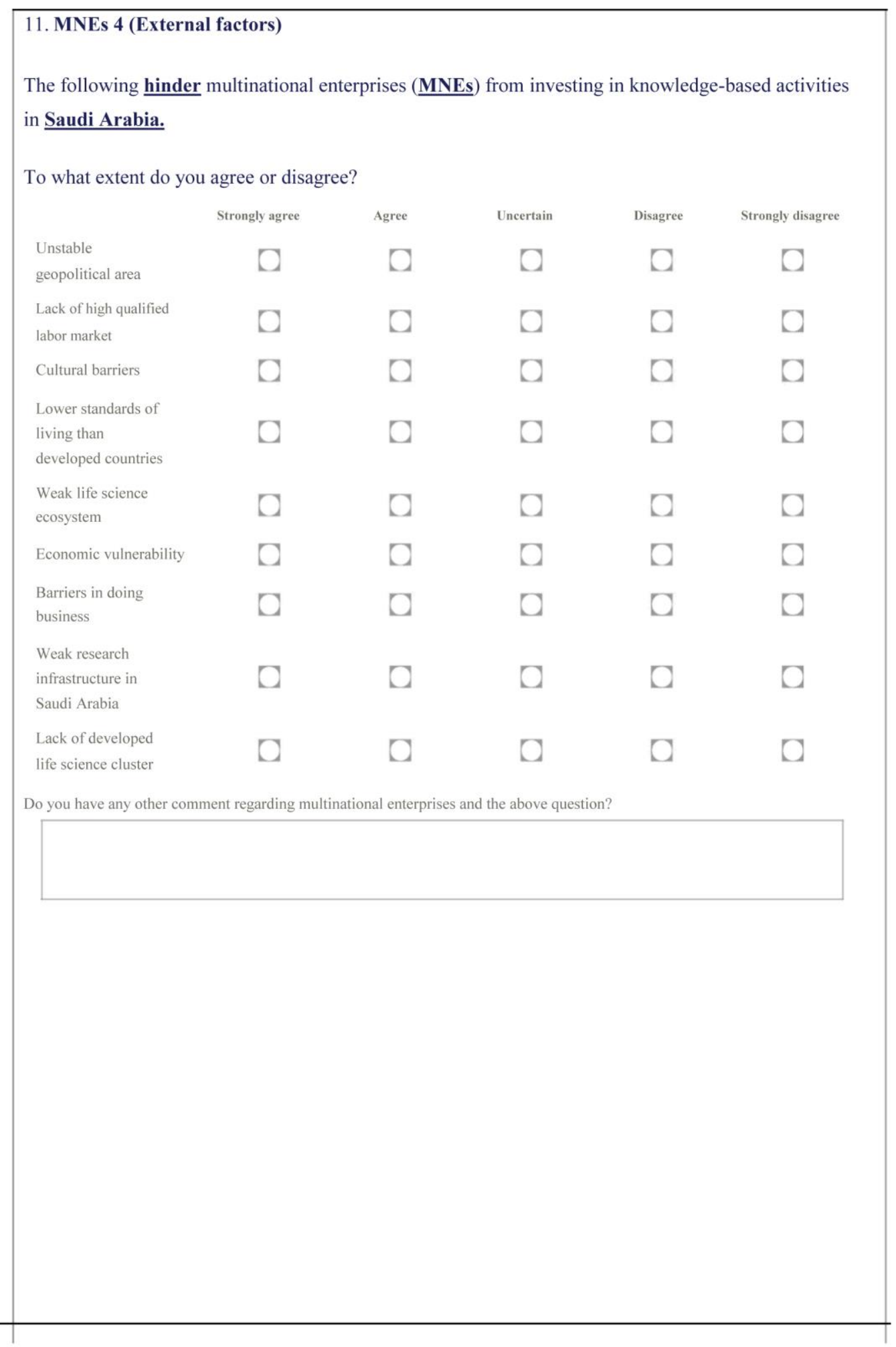


Private sector involvement role in developing Knowledge economy in the life science sector in the

Swansea University Prifysgol Abertawe Kingdom of Saudi Arabia (KSA)

5. The following questions concern local large companies in the life science sector in Saudi Arabia

\section{LLCs 1 (Internal factors)}

The following encourage local large companies ( $\underline{\text { LLCs }}$ ) to invest in knowledge-based activities in $\underline{\text { Saudi Arabia }}$

To what extent do you agree or disagree?

LLCs have
good financial strength
LLCs have
significant share in the
Saudi market and the
neighbouring countries
LLCs have easy access
to the financial system


13. LLCs 2 (Internal factors)

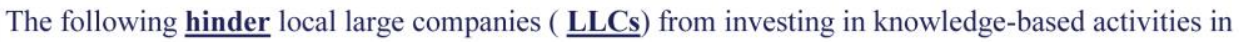
Saudi Arabia.

To what extent do you agree or disagree?

LLCs have a lack of
competitive capabilities
LLCS have a lack
of collaboration and
networking culture
LLCs have no
interaction with
global value chain
LLCs $\quad$ have
previous expertise in
knowledge activities
LLCs have lack of
entrepreneurial
strategy and attitude




\begin{tabular}{|c|c|c|c|c|c|}
\hline \multicolumn{6}{|c|}{ 14. LLCs 3 (External factors) } \\
\hline \multicolumn{6}{|c|}{$\begin{array}{l}\text { The following encourage local large companies ( } \underline{\mathbf{L L C s}} \text { ) to invest in knowledge-based activities } \\
\text { in Saudi Arabia. }\end{array}$} \\
\hline \multicolumn{6}{|c|}{ To what extent do you agree or disagree? } \\
\hline & Strongly agree & Agree & Uncertain & Disagree & Strongly disagree \\
\hline $\begin{array}{l}\text { Raising capabilities } \\
\text { through Joint venture, } \\
\text { acquisition, or } \\
\text { licensing technology }\end{array}$ & $\mathrm{Q}$ & Q & D & Q & D \\
\hline $\begin{array}{l}\text { Developing alliances } \\
\text { and partnership with } \\
\text { multinational } \\
\text { enterprises, and } \\
\text { research centers }\end{array}$ & D & D & D & D & D \\
\hline $\begin{array}{l}\text { Leveraging Saudi } \\
\text { government orientation } \\
\text { for building knowledge } \\
\text { economy }\end{array}$ & $\mathrm{O}$ & O & D & O & D \\
\hline $\begin{array}{l}\text { Tapping into local } \\
\text { Saudi priorities }\end{array}$ & $\mathrm{O}$ & D & D & D & $\mathrm{O}$ \\
\hline $\begin{array}{l}\text { Tapping into local } \\
\text { Saudi advantages }\end{array}$ & $\mathrm{O}$ & Q & D & D & D \\
\hline $\begin{array}{l}\text { Government } \\
\text { procurement support }\end{array}$ & $a$ & D & D & D & D \\
\hline $\begin{array}{l}\text { Targeting niche market } \\
\text { ignored by rivals }\end{array}$ & $\mathrm{D}$ & $\mathrm{D}$ & Q & Q & $\mathrm{O}$ \\
\hline
\end{tabular}




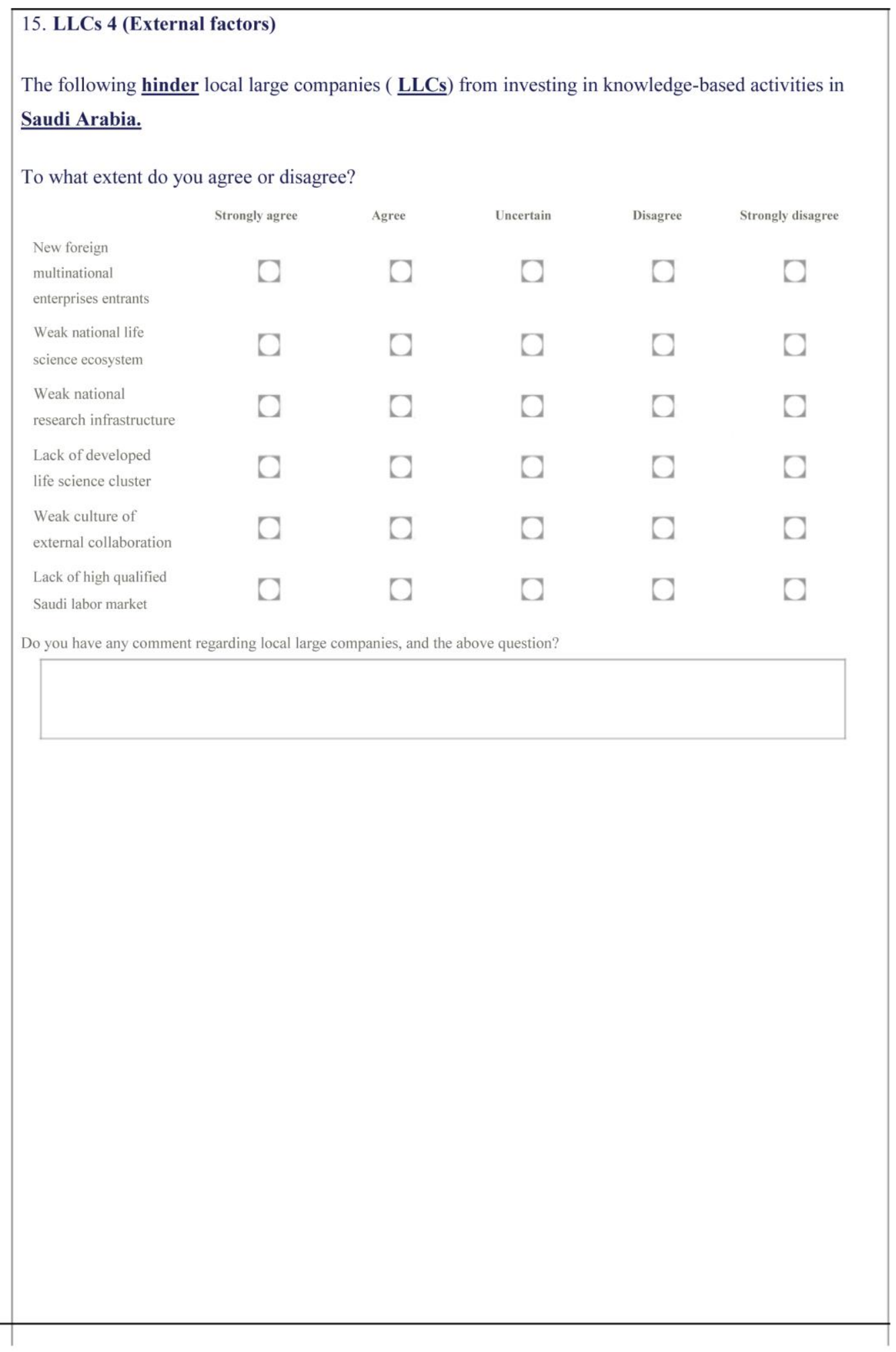


Private sector involvement role in developing Knowledge economy in the life science sector in the

Swansea University Kingdom of Saudi Arabia (KSA)

6. The following questions concern small \& medium enterprise in the life science sector in Saudi Arabia

\section{SMEs 1 (Internal factors)}

The following encourage small \& medium enterprises ( $\underline{\text { SMEs) }}$ ) to invest in knowledge-based activities

in Saudi Arabia.

To what extent do you agree or disagree?

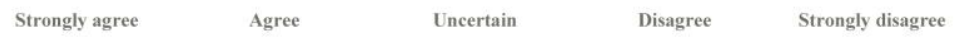

SMEs have high

awareness and

expertise in the local

Strongly agree

Agree

Uncertain

Strongly disagree

Saudi market

SMEs have more

flexibility to respond to

market change

O

D

D

O

O

O

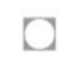

O 


\begin{tabular}{|c|c|c|c|c|c|}
\hline \multicolumn{6}{|c|}{ 17. SMEs 2 (Internal factors) } \\
\hline \multicolumn{6}{|c|}{ 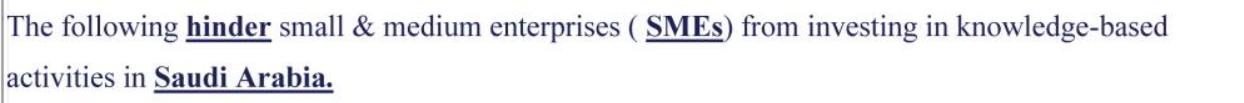 } \\
\hline \multicolumn{6}{|c|}{ To what extent do you agree or disagree? } \\
\hline & Strongly agree & Agree & Uncertain & Disagree & Strongly disagree \\
\hline $\begin{array}{l}\text { SMEs have low } \\
\text { competitive capabilities }\end{array}$ & $\mathrm{D}$ & $\mathrm{D}$ & $\mathrm{D}$ & $\mathrm{D}$ & $\mathrm{D}$ \\
\hline $\begin{array}{l}\text { SMEs have a lack of } \\
\text { entrepreneurial } \\
\text { strategy and attitude }\end{array}$ & Q & Q & D & $\mathrm{D}$ & Q \\
\hline $\begin{array}{l}\text { SMEs have low } \\
\text { financial strength }\end{array}$ & $\mathrm{O}$ & $\mathrm{D}$ & $\mathrm{D}$ & $\mathrm{D}$ & $\mathrm{D}$ \\
\hline $\begin{array}{l}\text { SMEs have } \\
\text { difficult access to } \\
\text { financial system }\end{array}$ & D & $\mathrm{D}$ & $D$ & $\mathrm{D}$ & $\mathrm{D}$ \\
\hline $\begin{array}{l}\text { SMEs have a lack } \\
\text { of collaboration and } \\
\text { networking culture }\end{array}$ & Q & $\mathrm{D}$ & Q & $\mathrm{D}$ & D \\
\hline $\begin{array}{l}\text { SMEs have weak } \\
\text { marketing capabilities }\end{array}$ & Q & $\mathrm{D}$ & Q & Q & $\mathrm{D}$ \\
\hline \multicolumn{6}{|c|}{ 18. SMEs 3 (External factors) } \\
\hline \multicolumn{6}{|c|}{$\begin{array}{l}\text { The following encourage small \& medium enterprises (SMEs) to invest in knowledge-based activities } \\
\text { in } \underline{\text { Saudi Arabia. }}\end{array}$} \\
\hline \multicolumn{6}{|c|}{ To what extent do you agree or disagree? } \\
\hline & Strongly agree & Agree & Uncertain & Disagree & Strongly disagree \\
\hline $\begin{array}{l}\text { Government strategy for } \\
\text { building knowledge } \\
\text { economy that has special } \\
\text { support for small \& } \\
\text { medium enterprises }\end{array}$ & Q & Q & Q & $\mathrm{Q}$ & $\mathrm{Q}$ \\
\hline $\begin{array}{l}\text { Government } \\
\text { procurement support }\end{array}$ & Q & $\mathrm{D}$ & Q & $\mathrm{Q}$ & $\mathrm{Q}$ \\
\hline $\begin{array}{l}\text { High growth } \\
\text { local sizeable market }\end{array}$ & $\mathrm{D}$ & $\mathrm{D}$ & $\mathrm{D}$ & $\mathrm{O}$ & $\mathrm{D}$ \\
\hline $\begin{array}{l}\text { Leveraging } \\
\text { national priorities }\end{array}$ & Q & $\mathrm{O}$ & D & $\mathrm{D}$ & $\mathrm{D}$ \\
\hline $\begin{array}{l}\text { Leveraging national } \\
\text { endowments and } \\
\text { advantages }\end{array}$ & Q & $\mathrm{D}$ & Q & Q & Q \\
\hline
\end{tabular}




\begin{tabular}{|c|c|c|c|c|c|}
\hline \multicolumn{6}{|c|}{ 19. SMEs 4 (External factors) } \\
\hline \multicolumn{6}{|c|}{$\begin{array}{l}\text { The following hinder small \& medium enterprises ( } \underline{\text { SMEs }} \text { ) from investing in knowledge-based } \\
\text { activities in } \underline{\text { Saudi Arabia. }}\end{array}$} \\
\hline \multicolumn{6}{|c|}{ To what extent do you agree or disagree? } \\
\hline & Strongly agree & Agree & Uncertain & Disagree & Strongly disagree \\
\hline $\begin{array}{l}\text { Weak culture of external } \\
\text { collaboration }\end{array}$ & 0 & $D$ & 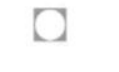 & 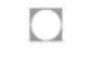 & $D$ \\
\hline $\begin{array}{l}\text { Uncertainty caused } \\
\text { by developing new } \\
\text { products that could be } \\
\text { displaced by } \\
\text { competitors }\end{array}$ & 0 & 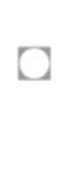 & 0 & 0 & 0 \\
\hline $\begin{array}{l}\text { Weak research } \\
\text { infrastructure in } \\
\text { Saudi Arabia }\end{array}$ & $\mathrm{D}$ & $a$ & $\mathrm{O}$ & 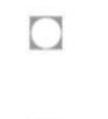 & 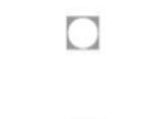 \\
\hline $\begin{array}{l}\text { Risk-averse } \\
\text { financial system }\end{array}$ & 0 & 0 & 0 & 0 & 0 \\
\hline $\begin{array}{l}\text { Fledgeling venture } \\
\text { capital market }\end{array}$ & 0 & 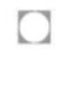 & 0 & 0 & 0 \\
\hline $\begin{array}{l}\text { Lack of developed } \\
\text { life science cluster }\end{array}$ & $\mathrm{O}$ & 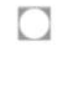 & Q & Q & Q \\
\hline $\begin{array}{l}\text { Local customers do } \\
\text { not trust local } \\
\text { innovative products }\end{array}$ & $\mathrm{O}$ & 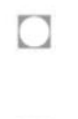 & $\mathrm{O}$ & 0 & 0 \\
\hline $\begin{array}{l}\text { Weak national life } \\
\text { science ecosystem }\end{array}$ & $\mathrm{O}$ & 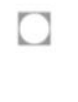 & 0 & 0 & 0 \\
\hline $\begin{array}{l}\text { Lack of high } \\
\text { skilled Saudi labour } \\
\text { market }\end{array}$ & Q & $D$ & O & 0 & 0 \\
\hline Do you have any comme & regarding smal & um enter & the above q & & \\
\hline
\end{tabular}




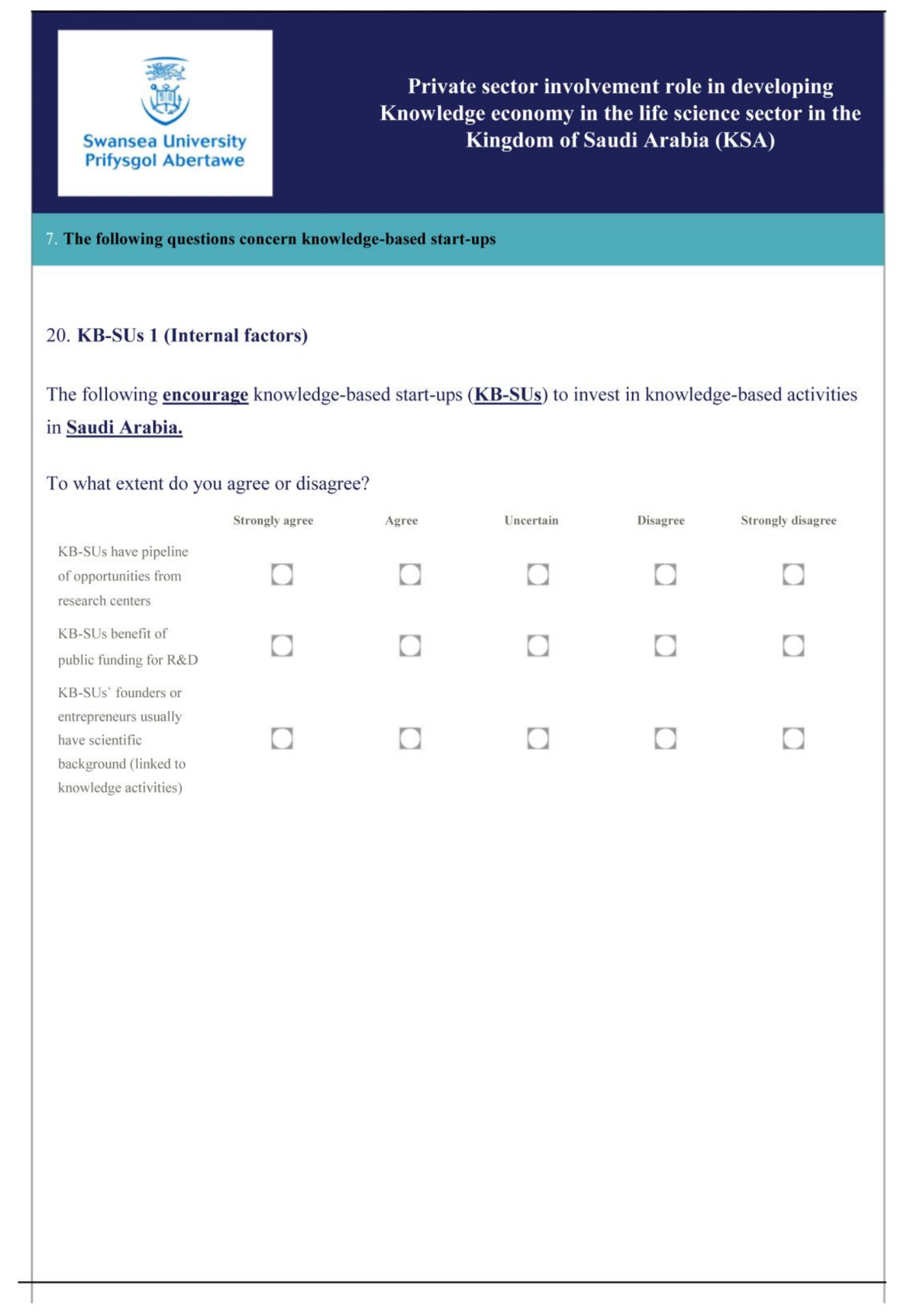




\begin{tabular}{|c|c|c|c|c|c|}
\hline \multicolumn{6}{|c|}{ 21. KB-SUs 2 (Internal factors) } \\
\hline \multicolumn{6}{|c|}{$\begin{array}{l}\text { The following hinder knowledge-based start-ups ( } \underline{\text { KB-SUs }} \text { ) from investing in knowledge-based } \\
\text { activities in } \underline{\text { Saudi Arabia. }}\end{array}$} \\
\hline \multicolumn{6}{|c|}{ To what extent do you agree or disagree? } \\
\hline & Strongly agree & Agree & Uncertain & Disagree & Strongly disagree \\
\hline $\begin{array}{l}\text { KB-SUs have a lack } \\
\text { of high promising } \\
\text { technologies }\end{array}$ & $\mathrm{D}$ & $\mathrm{D}$ & D & $\mathrm{Q}$ & $\mathrm{D}$ \\
\hline $\begin{array}{l}\text { KB-SUs have weak } \\
\text { connection with } \\
\text { supply chain }\end{array}$ & D & $\mathrm{O}$ & D & $\mathrm{O}$ & $\mathrm{D}$ \\
\hline $\begin{array}{l}\text { KB-SUs have a lack } \\
\text { of collaboration } \\
\text { among private sector }\end{array}$ & D & $\mathrm{D}$ & $\mathrm{D}$ & $\mathrm{Q}$ & $\mathrm{D}$ \\
\hline $\begin{array}{l}\text { KB-SUs have a lack } \\
\text { of collaboration with } \\
\text { health sector and other } \\
\text { stakeholders }\end{array}$ & D & $\mathrm{O}$ & $\mathrm{D}$ & $\mathrm{O}$ & $\mathrm{D}$ \\
\hline $\begin{array}{l}\text { KB-SUs have a lack of } \\
\text { capital (seed fund) }\end{array}$ & $\mathrm{D}$ & Q & D & D & $\mathrm{D}$ \\
\hline $\begin{array}{l}\text { KB-SUs have low } \\
\text { expertise in the market } \\
\text { and developing new } \\
\text { knowledge-based } \\
\text { start-ups }\end{array}$ & $\mathrm{D}$ & $\mathrm{O}$ & D & $\mathrm{D}$ & $\mathrm{Q}$ \\
\hline $\begin{array}{l}\text { KB-SUs have low } \\
\text { entrepreneurial attitude }\end{array}$ & $\mathrm{D}$ & $\mathrm{D}$ & D & $\mathrm{D}$ & $\mathrm{D}$ \\
\hline
\end{tabular}




\section{KB-SUs 3 (External factors)}

The following encourage knowledge-based start-ups ( $\underline{\text { KB-SUs })}$ to invest in knowledge activities in Saudi Arabia.

To what extent do you agree or disagree?

Severaging national
advantages
Tapping into
specific local priorities
Targeting niche market
ignored by rivals
$\begin{aligned} & \text { Leveraging government } \\ & \text { procurement support } \\ & \text { Leveraging government } \\ & \text { strategy and orientation } \\ & \text { for building knowledge }\end{aligned}$

economy 


\section{KB-SUs 4 (External factors)}

The following hinder knowledge-based start-ups ( $\underline{\text { KB-SUs) }}$ from investing in knowledge activities in Saudi Arabia.

To what extent do you agree or disagree?

\begin{tabular}{|c|c|c|c|c|c|}
\hline & Strongly agree & Agree & Uncertain & Disagree & Strongly disagre \\
\hline $\begin{array}{l}\text { Barriers for researchers } \\
\text { or innovators to do } \\
\text { their own business }\end{array}$ & D & 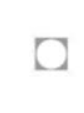 & Q & $D$ & O \\
\hline $\begin{array}{l}\text { Weak national life } \\
\text { science ecosystem }\end{array}$ & 0 & $O$ & 0 & 0 & 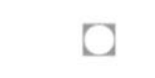 \\
\hline $\begin{array}{l}\text { Insufficient national } \\
\text { research infrastructure }\end{array}$ & 0 & 0 & O & 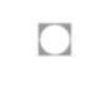 & 0 \\
\hline $\begin{array}{l}\text { Lack of seed fund } \\
\text { organisations }\end{array}$ & O & 0 & 0 & $O$ & O \\
\hline $\begin{array}{l}\text { Lack of developed } \\
\text { life science cluster }\end{array}$ & O & $D$ & O & 0 & Q \\
\hline $\begin{array}{l}\text { Fledgeling venture } \\
\text { capital market }\end{array}$ & O & 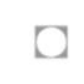 & O & 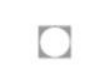 & O \\
\hline $\begin{array}{l}\text { Weak culture of } \\
\text { external collaboration }\end{array}$ & O & 0 & O & 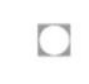 & O \\
\hline $\begin{array}{l}\text { Lack of high qualified } \\
\text { Saudi labour market }\end{array}$ & 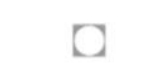 & 0 & 0 & $\mathrm{~T}$ & 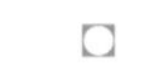 \\
\hline Barriers in doing business & 0 & 0 & 0 & 0 & O \\
\hline
\end{tabular}

Do you have any comment regarding knowledge-based start-ups and the above question, or any thing in the questionnaire? 


\subsection{Appendix 6: Code Book}

\begin{tabular}{|c|c|c|c|c|}
\hline No. & Variable name & Label & Values & Source \\
\hline 1 & PA & Participant & None & The researcher \\
\hline 2 & Gender & Gender & $\begin{array}{l}0=\text { male } \\
1=\text { female } \\
2=I \text { prefer not } \\
\text { to say }\end{array}$ & The researcher \\
\hline 3 & Sector & $\begin{array}{l}\text { Where participant } \\
\text { works }\end{array}$ & $\begin{array}{l}1=\text { government } \\
2=\text { private sector } \\
3=\text { academic } \\
\text { (public) } \\
4=\text { academic } \\
\text { (private) } \\
5=\text { public } \\
\text { research center } \\
6=\text { third sector }\end{array}$ & The researcher \\
\hline 4 & Job & Job nature & $\begin{array}{l}\text { 1=research } \\
2=\text { education } \\
3=\text { management } \\
\text { 4=consultancy } \\
5=\text { manufacturin } \\
\text { g }\end{array}$ & The researcher \\
\hline 5 & Nationality & Nationality & $\begin{array}{l}\text { 1=Saudi } \\
2=\text { other Arabic } \\
3=\text { Asian } \\
\text { 4=other }\end{array}$ & The researcher \\
\hline 6 & Living country & Living country & $\begin{array}{l}1=\text { Saudi Arabia } \\
2=\text { other }\end{array}$ & The researcher \\
\hline 7 & Age & Age in years & $\begin{array}{l}1=18-25 \\
2=26-35 \\
3=36-45 \\
4=46-55 \\
5=56-65 \\
6=\text { above } 65\end{array}$ & The researcher \\
\hline
\end{tabular}




\begin{tabular}{|c|c|c|c|c|}
\hline 8 & PS_involvement & $\begin{array}{l}\text { Private sector } \\
\text { involvement }\end{array}$ & $\begin{array}{l}\text { 1=strongly } \\
\text { disagree } \\
2=\text { disagree } \\
3=\text { uncertain } \\
4=\text { agree } \\
\text { 5=strongly } \\
\text { agree }\end{array}$ & Interviews`Transcript \\
\hline 9 & MNE_KH_I_ENC & $\begin{array}{l}\text { Multi national } \\
\text { enterprise know how, } \\
\text { internal factor, } \\
\text { encouragement }\end{array}$ & The same above & (Hymer, 1976) \\
\hline 10 & $\begin{array}{l}\text { MNE_INPR_I_EN } \\
\mathrm{C}\end{array}$ & $\begin{array}{l}\text { MNE innovative } \\
\text { practices, internal } \\
\text { factor, encouragement }\end{array}$ & The same above & (Hymer, 1976) \\
\hline 11 & $\begin{array}{l}\text { MNE_SOSY_I_E } \\
\mathrm{NC}\end{array}$ & $\begin{array}{l}\text { MNE sophisticated } \\
\text { systems, internal } \\
\text { factor, encouragement }\end{array}$ & The same above & (Hymer, 1976) \\
\hline 12 & $\begin{array}{l}\text { MNE_STNW_I_E } \\
\text { NC }\end{array}$ & $\begin{array}{l}\text { MNE strong } \\
\text { networks, internal } \\
\text { factor, encouragement }\end{array}$ & The same above & Interviews`Transcript \\
\hline 13 & $\begin{array}{l}\text { MNE_COCA_I_E } \\
\mathrm{NC}\end{array}$ & $\begin{array}{l}\text { MNE competitive } \\
\text { capabilities, internal } \\
\text { factor, encouragement }\end{array}$ & The same above & (Hymer, 1976) \\
\hline 14 & MNE_FST_I_ENC & $\begin{array}{l}\text { MNE financially } \\
\text { strong, internal factor, } \\
\text { encouragement }\end{array}$ & The same above & Interviews`Transcript \\
\hline 15 & $\begin{array}{l}\text { MNE_STM_I_EN } \\
\text { C }\end{array}$ & $\begin{array}{l}\text { MNE strong } \\
\text { marketing, internal } \\
\text { factor, encouragement }\end{array}$ & The same above & Interviews`Transcript \\
\hline 16 & MNE_STP_I_ENC & $\begin{array}{l}\text { MNE strong brands, } \\
\text { internal factor, } \\
\text { encouragement }\end{array}$ & The same above & Interviews`Transcript \\
\hline 17 & MNE_LINF_I_DIS & $\begin{array}{l}\text { MNE lack of } \\
\text { information, external } \\
\text { factor, } \\
\text { discouragement }\end{array}$ & The same above & Interviews`Transcript \\
\hline
\end{tabular}




\begin{tabular}{|c|c|c|c|c|}
\hline 18 & $\begin{array}{l}\text { MNE_GM_E_EN } \\
\mathrm{C}\end{array}$ & $\begin{array}{l}\text { MNE growth market, } \\
\text { external factor, } \\
\text { encouragement }\end{array}$ & The same above & (Desai, 2009) \\
\hline 19 & $\begin{array}{l}\text { MNE_MEM_E_E } \\
\text { NC }\end{array}$ & $\begin{array}{l}\text { MNE middle east } \\
\text { market, external } \\
\text { factor, encouragement }\end{array}$ & The same above & (Desai, 2009) \\
\hline 20 & MNE_JV_E_ENC & $\begin{array}{l}\text { MNE joint venture, } \\
\text { external factor, } \\
\text { encouragement }\end{array}$ & The same above & $\begin{array}{l}\text { (Pitelis \& Teece, 2010), } \\
\text { (Rodriguez-Clare, 1996) }\end{array}$ \\
\hline 21 & MNE_ER_E_ENC & $\begin{array}{l}\text { MNE economic } \\
\text { reforms, external } \\
\text { factor, encouragement }\end{array}$ & The same above & (Moran et al., 2007) \\
\hline 22 & MNE_FI_E_ENC & $\begin{array}{l}\text { MNE financial } \\
\text { incentives, external } \\
\text { factor, encouragement }\end{array}$ & The same above & $\begin{array}{l}\text { (Alfaro, 2014), (Thomas } \\
\text { \& Carl, 2001) }\end{array}$ \\
\hline 23 & MNE_LA_E_ENC & $\begin{array}{l}\text { MNE local } \\
\text { advantages, external } \\
\text { factor, encouragement }\end{array}$ & The same above & (Moran et al., 2007) \\
\hline 24 & MNE_GEO_E_H & $\begin{array}{l}\text { MNE geopolitical, } \\
\text { external factor, hinder }\end{array}$ & The same above & Interviews`Transcript \\
\hline 25 & MNE_LM_E_H & $\begin{array}{l}\text { MNE labour market, } \\
\text { external factor, hinder }\end{array}$ & The same above & $\begin{array}{l}\text { (Desai, 2009), (Koike, } \\
\text { 1996). }\end{array}$ \\
\hline 26 & MNE_CB_E_H & $\begin{array}{l}\text { MNE cultural barriers, } \\
\text { external factor, hinder }\end{array}$ & The same above & Interviews` Transcript \\
\hline 27 & MNE_SL_E_H & $\begin{array}{l}\text { MNE standards of } \\
\text { living, external factor, } \\
\text { hinder }\end{array}$ & The same above & Interviews` Transcript \\
\hline 28 & MNE_ECO_E_H & $\begin{array}{l}\text { MNE ecosystem, } \\
\text { external factor, hinder }\end{array}$ & The same above & (Pitelis \& Teece, 2010) \\
\hline 29 & MNE_EV_E_H & $\begin{array}{l}\text { MNE economic } \\
\text { vulnerability, external } \\
\text { factor, hinder }\end{array}$ & The same above & (Moran et al., 2007) \\
\hline 30 & MNE_BB_E_H & $\begin{array}{l}\text { MNE business } \\
\text { barriers, external } \\
\text { factor, hinder }\end{array}$ & The same above & $\begin{array}{l}\text { Interviews`Transcript } \\
\text { (A. Harrison \& } \\
\text { Rodríguez-Clare, 2009) }\end{array}$ \\
\hline
\end{tabular}




\begin{tabular}{|c|c|c|c|c|}
\hline 31 & MNE_RI_E_H & $\begin{array}{l}\text { MNE research } \\
\text { infrastructure, } \\
\text { external factor, hinder }\end{array}$ & The same above & (Pitelis \& Teece, 2010) \\
\hline 32 & MNE_CL_E_H & $\begin{array}{l}\text { MNE cluster, external } \\
\text { factor, hinder }\end{array}$ & The same above & $\begin{array}{l}\text { Interviews`Transcript } \\
\text { (Kong, 2003), (M. E. } \\
\text { Porter, 2000, 2014). }\end{array}$ \\
\hline 33 & $\overline{\text { LLC_FS_I_ENC }}$ & $\begin{array}{l}\text { Local large company, } \\
\text { financial strength, } \\
\text { internal factor, } \\
\text { encouragement }\end{array}$ & The same above & Interviews` Transcript \\
\hline 34 & LLC_MS_I_ENC & $\begin{array}{l}\text { LLC market share, } \\
\text { internal factor, } \\
\text { encouragement }\end{array}$ & The same above & Interviews` Transcript \\
\hline 35 & LLC_FSY_I_ENC & $\begin{array}{l}\text { LLC access to } \\
\text { financial system, } \\
\text { internal factor, } \\
\text { encouragement }\end{array}$ & The same above & Interviews`Transcript \\
\hline 36 & LLC_COCA_I_H & $\begin{array}{l}\text { LLC competitive } \\
\text { capabilities, internal } \\
\text { factor, hinder }\end{array}$ & The same above & $\begin{array}{l}\text { Interviews`Transcript } \\
\text { (Ireland et al., 2009), } \\
\text { (Clarke, 2001; Drucker } \\
\text { \& Drucker, 1994; } \\
\text { Laroche, Mérette, \& } \\
\text { Ruggeri, 1999; Peters, } \\
\text { 2001). }\end{array}$ \\
\hline 37 & LLC_CC_I_H & $\begin{array}{l}\text { LLC collaboration } \\
\text { culture, internal } \\
\text { factor, hinder }\end{array}$ & The same above & $\begin{array}{l}\text { Interviews`Transcript, } \\
\text { (Cooke, 2004), (Keizer } \\
\text { et al., 2002; OECD, } \\
\text { 1997). }\end{array}$ \\
\hline 38 & LLC_GC_I_H & $\begin{array}{l}\text { LLC global value } \\
\text { chain, internal factor, } \\
\text { hinder }\end{array}$ & The same above & Interviews` Transcript \\
\hline 39 & LLC_KA_I_H & $\begin{array}{l}\text { LLC knowledge } \\
\text { activities expertise, } \\
\text { internal, hinder }\end{array}$ & The same above & (Ireland et al., 2009) \\
\hline
\end{tabular}




\begin{tabular}{|c|c|c|c|c|}
\hline 40 & LLC_ENT_I_H & $\begin{array}{l}\text { LLC entrepreneurial } \\
\text { strategy, internal } \\
\text { factor, hinder }\end{array}$ & The same above & $\begin{array}{l}\text { (A. C. Cooper et al., } \\
\text { 2000; Hoskisson \& } \\
\text { Busenitz, 2002; Morris } \\
\text { et al., 2010; Thornberry, } \\
\text { 2001) }\end{array}$ \\
\hline 41 & $\begin{array}{l}\text { LLLC_RC_E_EN } \\
\mathrm{C}\end{array}$ & $\begin{array}{l}\text { LLC raising } \\
\text { capabilities, external } \\
\text { factor, encouragement }\end{array}$ & The same above & $\begin{array}{l}\text { Interviews`Transcript, } \\
\text { (Hoskisson \& Busenitz, } \\
\text { 2002), (Gray, 2006). }\end{array}$ \\
\hline 42 & LLC_AP_E_ENC & $\begin{array}{l}\text { LLC alliances and } \\
\text { partnership, external } \\
\text { factor, encouragement }\end{array}$ & The same above & Interviews` Transcript, \\
\hline 43 & LLC_GO_E_ENC & $\begin{array}{l}\text { LLC government } \\
\text { orientation, external } \\
\text { factor, encouragement }\end{array}$ & The same above & $\begin{array}{l}\text { Interviews` Transcript, } \\
\text { (Toma et al., 2014). }\end{array}$ \\
\hline 44 & LLC_LP_E_ENC & $\begin{array}{l}\text { LLC local priorities, } \\
\text { external factor, } \\
\text { encouragement }\end{array}$ & The same above & $\begin{array}{l}\text { Interviews` Transcript, } \\
\text { (E M Rogers, 1983) }\end{array}$ \\
\hline 45 & LLC_LA_E_ENC & $\begin{array}{l}\text { LLC local advantages, } \\
\text { external factor, } \\
\text { encouragement }\end{array}$ & The same above & $\begin{array}{l}\text { Interviews` Transcript, } \\
\text { (E M Rogers, 1983) }\end{array}$ \\
\hline 46 & LLC_GP_E_ENC & $\begin{array}{l}\text { LLC government } \\
\text { procurement, external } \\
\text { factor, encouragement }\end{array}$ & The same above & $\begin{array}{l}\text { Interviews` Transcript, } \\
\text { (Toma et al., 2014). }\end{array}$ \\
\hline 47 & LLC_NM_E_ENC & $\begin{array}{l}\text { LLC niche market, } \\
\text { external factor, } \\
\text { encouragement }\end{array}$ & The same above & Interviews`Transcript \\
\hline 48 & LLC_NE_E_H & $\begin{array}{l}\text { LLC new entrant, } \\
\text { external factor, hinder }\end{array}$ & The same above & $\begin{array}{l}\text { Interviews` Transcript, } \\
\text { (Markusen \& Venables, } \\
\text { 1999). }\end{array}$ \\
\hline 49 & $\overline{\text { LLC_ECO_E_H }}$ & $\begin{array}{l}\text { LLC ecosystem, } \\
\text { external factor, hinder }\end{array}$ & The same above & $\begin{array}{l}\text { Interviews` Transcript } \\
\text { (Fagerberg \& Srholec, } \\
\text { 2008), (L. Kim, 1997) }\end{array}$ \\
\hline 50 & LLC_RI_E_H & $\begin{array}{l}\text { LLC research } \\
\text { infrastructure, } \\
\text { external factor, hinder }\end{array}$ & The same above & $\begin{array}{l}\text { Interviews` Transcript } \\
\text { (Fagerberg \& Srholec, } \\
\text { 2008), (L. Kim, 1997), } \\
\text { (Toma et al., 2014). }\end{array}$ \\
\hline
\end{tabular}




\begin{tabular}{|c|c|c|c|c|}
\hline 51 & $\overline{\text { LLC_CL_E_H }}$ & $\begin{array}{l}\text { LLC cluster, external } \\
\text { factor, hinder }\end{array}$ & The same above & $\begin{array}{l}\text { Interviews`Transcript } \\
\text { (Kong, 2003), (M. E. } \\
\text { Porter, 2000, 2014). }\end{array}$ \\
\hline 52 & LLC_COL_E_H & $\begin{array}{l}\text { LLC collaboration } \\
\text { culture, external } \\
\text { factor, hinder }\end{array}$ & The same above & Interviews`Transcript \\
\hline 53 & LLC_LM_E_H & $\begin{array}{l}\text { LLC labour market, } \\
\text { external factor, hinder }\end{array}$ & The same above & $\begin{array}{l}\text { Interviews`Transcript, } \\
\text { (A. Harrison \& } \\
\text { Rodríguez-Clare, 2009) }\end{array}$ \\
\hline 54 & SME_EXP_I_ENC & $\begin{array}{l}\text { Small Medium } \\
\text { Enterprise market } \\
\text { expertise, internal } \\
\text { factor, encouragement }\end{array}$ & The same above & Interview`s Transcript \\
\hline 55 & $\begin{array}{l}\text { SME_FLEX_I_EN } \\
\text { C }\end{array}$ & $\begin{array}{l}\text { SME flexibility, } \\
\text { internal factor, } \\
\text { encouragement }\end{array}$ & The same above & $\begin{array}{l}\text { (Hamel, 1996; Sathe \& } \\
\text { Drucker, 2003) }\end{array}$ \\
\hline 56 & SME_COCA_I_H & $\begin{array}{l}\text { SME lack of } \\
\text { competitive } \\
\text { capabilities, internal } \\
\text { factor, hinder }\end{array}$ & The same above & $\begin{array}{l}\text { Interviews`Transcript } \\
\text { (Gray, 2006). } \\
\text { (Ireland et al., 2009), } \\
\text { (Clarke, 2001; Drucker } \\
\text { \& Drucker, 1994; } \\
\text { Laroche, Mérette, \& } \\
\text { Ruggeri, 1999; Peters, } \\
\text { 2001). }\end{array}$ \\
\hline 57 & SME_ENTR_I_H & $\begin{array}{l}\text { SME entrepreneurial } \\
\text { strategy, internal } \\
\text { factor, hinder }\end{array}$ & The same above & $\begin{array}{l}\text { Interviews`Transcript, } \\
\text { (Cohen \& Levinthal, } \\
\text { 1990), (Birchall, D.W., } \\
\text { Chanaron, J.J., } \\
\text { Soderquist, 1996; Docter } \\
\text { \& Stokman, 1988) }\end{array}$ \\
\hline 58 & SME_FS_I_H & $\begin{array}{l}\text { SME lack of financial } \\
\text { strength, internal } \\
\text { factor, hinder }\end{array}$ & The same above & $\begin{array}{l}\text { Interviews`Transcript, } \\
\text { (Keskin, 2006). }\end{array}$ \\
\hline 59 & SME_FSY_I_H & $\begin{array}{l}\text { SME difficult access } \\
\text { to financial system, } \\
\text { internal factor, hinder }\end{array}$ & The same above & $\begin{array}{l}\text { Interviews`Transcript, } \\
\text { (Keizer et al., 2002) }\end{array}$ \\
\hline
\end{tabular}




\begin{tabular}{|c|c|c|c|c|}
\hline 60 & SME_COL_I_H & $\begin{array}{l}\text { SME collaboration, } \\
\text { internal factor, hinder }\end{array}$ & The same above & Interviews`Transcript \\
\hline 61 & SME_M_I_H & $\begin{array}{l}\text { SME weak marketing } \\
\text { capabilities, internal } \\
\text { factor, hinder }\end{array}$ & The same above & $\begin{array}{l}\text { Interviews` Transcript, } \\
\text { (Hoffman et al., 1998; } \\
\text { LeBlanc et al., 1997) }\end{array}$ \\
\hline 62 & SME_GS_E_ENC & $\begin{array}{l}\text { SME government } \\
\text { strategy, external } \\
\text { factor, encouragement }\end{array}$ & The same above & $\begin{array}{l}\text { Interviews`Transcript, } \\
\text { (Toma et al., 2014). }\end{array}$ \\
\hline 63 & SME_GP_E_ENC & $\begin{array}{l}\text { SME government } \\
\text { procurement, external } \\
\text { factor, encouragement }\end{array}$ & The same above & $\begin{array}{l}\text { Interviews`Transcript, } \\
\text { (Toma et al., 2014). }\end{array}$ \\
\hline 64 & SME_GM_E_ENC & $\begin{array}{l}\text { SME growing market, } \\
\text { external factor, } \\
\text { encouragement }\end{array}$ & The same above & Interviews`Transcript \\
\hline 65 & SME_P_E_ENC & $\begin{array}{l}\text { SME priorities, } \\
\text { external factor, } \\
\text { encouragement }\end{array}$ & The same above & $\begin{array}{l}\text { Interviews`Transcript, } \\
\text { (E M Rogers, 1983) }\end{array}$ \\
\hline 66 & SME_AD_E_ENC & $\begin{array}{l}\text { SME, local } \\
\text { advantages, external } \\
\text { factor, encouragement }\end{array}$ & The same above & $\begin{array}{l}\text { Interviews`Transcript, } \\
\text { (E M Rogers, 1983) }\end{array}$ \\
\hline 67 & SME_COL_E_H & $\begin{array}{l}\text { SME, lack of } \\
\text { collaboration, external } \\
\text { factor, hinder }\end{array}$ & The same above & $\begin{array}{l}\text { Interviews`Transcript, } \\
\text { (Cooke, 2004; Cooke \& } \\
\text { Wills, 1999; Forrest, } \\
\text { 1990; Keizer et al., } \\
\text { 2002). }\end{array}$ \\
\hline 68 & SME_SUB_E_H & $\begin{array}{l}\text { SME, substitute } \\
\text { products, external } \\
\text { factor, hinder }\end{array}$ & The same above & $\begin{array}{l}\text { Interviews`Transcript, } \\
\text { (Keskin, 2006). }\end{array}$ \\
\hline 69 & SME_RI_E_H & $\begin{array}{l}\text { SME, weak research } \\
\text { infrastructure, } \\
\text { external factor, hinder }\end{array}$ & The same above & $\begin{array}{l}\text { Interviews`Transcript } \\
\text { (Fagerberg \& Srholec, } \\
\text { 2008), (L. Kim, 1997), } \\
\text { (Toma et al., 2014). }\end{array}$ \\
\hline 70 & SME_FSY_E_H & $\begin{array}{l}\text { SME, risk-averse } \\
\text { financial system, } \\
\text { external factor, hinder }\end{array}$ & The same above & Interviews`Transcript, \\
\hline
\end{tabular}




\begin{tabular}{|c|c|c|c|c|}
\hline 71 & SME_VC_E_H & $\begin{array}{l}\text { SME, nascent venture } \\
\text { capital market, } \\
\text { external factor, hider }\end{array}$ & The same above & $\begin{array}{l}\text { Interviews`Transcript, } \\
\text { (Stiglitz, 1999; Thomas } \\
\text { \& Carl, 2001) }\end{array}$ \\
\hline 72 & SME_CL_E_H & $\begin{array}{l}\text { SME, lack of } \\
\text { developed life science } \\
\text { cluster, external } \\
\text { factor, hinder }\end{array}$ & The same above & $\begin{array}{l}\text { Interviews`Transcript, } \\
\text { (Kong, 2003), (M. E. } \\
\text { Porter, 2000, 2014). }\end{array}$ \\
\hline 73 & SME_CT_E_H & $\begin{array}{l}\text { SME, lack of } \\
\text { customer trust, } \\
\text { external factor, hinder }\end{array}$ & The same above & Interviews`Transcript \\
\hline 74 & SME_ECO_E_H & $\begin{array}{l}\text { SME, weak life } \\
\text { science ecosystem, } \\
\text { external factor, hinder }\end{array}$ & The same above & $\begin{array}{l}\text { Interviews` Transcript } \\
\text { (Fagerberg \& Srholec, } \\
\text { 2008), (L. Kim, 1997). }\end{array}$ \\
\hline 75 & SME_LM_E_H & $\begin{array}{l}\text { SME, lack of high } \\
\text { qualified labour } \\
\text { market, external } \\
\text { factor, hinder }\end{array}$ & The same above & $\begin{array}{l}\text { Interviews`Transcript, } \\
\text { (A. Harrison \& } \\
\text { Rodríguez-Clare, 2009). }\end{array}$ \\
\hline 76 & SU_PO_I_ENC & $\begin{array}{l}\text { Startup, pipeline } \\
\text { opportunities, internal } \\
\text { factor, } \\
\text { encouragement. }\end{array}$ & The same above & Interviews`Transcript \\
\hline 77 & SU_FUND_I_ENC & $\begin{array}{l}\text { Startup, benefit of } \\
\text { public funding for } \\
\text { R\&D, internal factor, } \\
\text { encouragement }\end{array}$ & The same above & $\begin{array}{l}\text { Interviews` Transcript. } \\
\text { (Keizer et al., 2002) }\end{array}$ \\
\hline 78 & SU_ENT_I_ENC & $\begin{array}{l}\text { Startup, founders } \\
\text { usually have scientific } \\
\text { background, internal } \\
\text { factor, encouragement }\end{array}$ & The same above & $\begin{array}{l}\text { Interviews` Transcript, } \\
\text { (Hoffman et al., 1998; } \\
\text { LeBlanc et al., 1997). }\end{array}$ \\
\hline 79 & SU_PT_I_H & $\begin{array}{l}\text { Startup, lack of } \\
\text { promising technology, } \\
\text { internal factor, hinder }\end{array}$ & The same above & Interviews`Transcript \\
\hline 80 & SU_SC_I_H & $\begin{array}{l}\text { Startup, weak } \\
\text { connection with } \\
\text { supply chain, internal } \\
\text { factor, hinder }\end{array}$ & The same above & Interviews' Transcript, \\
\hline
\end{tabular}




\begin{tabular}{|c|c|c|c|c|}
\hline 81 & SU_COL_I_H & $\begin{array}{l}\text { Startup, lack of } \\
\text { collaboration among } \\
\text { private sector, internal } \\
\text { factor, hinder }\end{array}$ & The same above & $\begin{array}{l}\text { Interviews' Transcript, } \\
\text { (Cooke, 2004). }\end{array}$ \\
\hline 82 & SU_COLH_I_H & $\begin{array}{l}\text { Startup, lack of } \\
\text { collaboration with } \\
\text { health sector, internal } \\
\text { factor, hinder }\end{array}$ & The same above & Interviews' Transcript \\
\hline 83 & SU_C_I_H & $\begin{array}{l}\text { Startup, lack of capital } \\
\text { (seed fund), internal } \\
\text { factor, hinder }\end{array}$ & The same above & $\begin{array}{l}\text { Interviews' Transcript, } \\
\text { (Ardic, Mylenko, \& } \\
\text { Saltane, 2011). }\end{array}$ \\
\hline 84 & SU_DEV_I_H & $\begin{array}{l}\text { Startup, low expertise } \\
\text { in developing new } \\
\text { enterprise, internal } \\
\text { factor, hinder }\end{array}$ & The same above & Interviews' Transcript \\
\hline 85 & SU_ENT_I_H & $\begin{array}{l}\text { Startup, low } \\
\text { entrepreneurial } \\
\text { attitude, internal } \\
\text { factor, hinder }\end{array}$ & The same above & $\begin{array}{l}\text { Interviews' Transcript, } \\
\text { (Cohen \& Levinthal, } \\
\text { 1990), (Birchall, D.W., } \\
\text { Chanaron, J.J., } \\
\text { Soderquist, 1996; Docter } \\
\text { \& Stokman, 1988). }\end{array}$ \\
\hline 86 & SU_AD_E_ENC & $\begin{array}{l}\text { Startup, leveraging } \\
\text { national advantage, } \\
\text { internal factor, } \\
\text { encouragement }\end{array}$ & The same above & $\begin{array}{l}\text { Interviews`Transcript, } \\
\text { (E M Rogers, 1983) }\end{array}$ \\
\hline 87 & SU_P_E_ENC & $\begin{array}{l}\text { Startup, taping into } \\
\text { local priorities, } \\
\text { external factor, } \\
\text { encouragement }\end{array}$ & The same above & $\begin{array}{l}\text { Interviews`Transcript, } \\
\text { (E M Rogers, 1983). }\end{array}$ \\
\hline 88 & SU_NM_E_ENC & $\begin{array}{l}\text { Startup, targeting } \\
\text { niche market, external } \\
\text { factor, encouragement }\end{array}$ & The same above & $\begin{array}{l}\text { Interviews' Transcript, } \\
\text { (Ardic et al., 2011). }\end{array}$ \\
\hline 89 & SU_GP_E_ENC & $\begin{array}{l}\text { Startup, leveraging } \\
\text { government } \\
\text { procurement, external } \\
\text { factor, encouragement }\end{array}$ & The same above & $\begin{array}{l}\text { Interviews`Transcript, } \\
\text { (Toma et al., 2014). }\end{array}$ \\
\hline
\end{tabular}




\begin{tabular}{|c|c|c|c|c|}
\hline 90 & SU_GS_E_ENC & $\begin{array}{l}\text { Startup, leveraging } \\
\text { government strategy } \\
\text { and orientation for } \\
\text { building knowledge } \\
\text { economy, external } \\
\text { factor, encouragement }\end{array}$ & The same above & $\begin{array}{l}\text { Interviews`Transcript, } \\
\text { (Toma et al., 2014). }\end{array}$ \\
\hline 91 & SU_BB_E_H & $\begin{array}{l}\text { Startup, barriers for } \\
\text { researchers or } \\
\text { innovators to } \\
\text { developing their own } \\
\text { businesses, external } \\
\text { factor, hinder }\end{array}$ & The same above & Interviews` Transcript. \\
\hline 92 & SU_ECO_E_H & $\begin{array}{l}\text { Startup, weak national } \\
\text { life science } \\
\text { ecosystem, external } \\
\text { factor, hinder }\end{array}$ & The same above & $\begin{array}{l}\text { Interviews` Transcript } \\
\text { (Fagerberg \& Srholec, } \\
\text { 2008), (L. Kim, 1997). }\end{array}$ \\
\hline 93 & SU_RI_E_H & $\begin{array}{l}\text { Startup, insufficient } \\
\text { research } \\
\text { infrastructure, } \\
\text { external factor, hinder }\end{array}$ & The same above & $\begin{array}{l}\text { Interviews`Transcript } \\
\text { (Fagerberg \& Srholec, } \\
\text { 2008), (L. Kim, 1997), } \\
\text { (Toma et al., 2014). }\end{array}$ \\
\hline 94 & SU_SEED_E_H & $\begin{array}{l}\text { Startup, lack of seed } \\
\text { fund organisations, } \\
\text { external factor, hinder }\end{array}$ & The same above & Interviews`Transcript \\
\hline 95 & SU_CL_E_H & $\begin{array}{l}\text { Startup, lack of } \\
\text { developed life science } \\
\text { cluster, external } \\
\text { factor, hinder }\end{array}$ & The same above & $\begin{array}{l}\text { Interviews` Transcript, } \\
\text { (Kong, 2003), (M. E. } \\
\text { Porter, 2000, 2014). }\end{array}$ \\
\hline 96 & SU_VC_E_H & $\begin{array}{l}\text { Startup, nascent } \\
\text { venture capital } \\
\text { market, external } \\
\text { factor, hinder }\end{array}$ & The same above & $\begin{array}{l}\text { Interviews`Transcript, } \\
\text { (Stiglitz, 1999; Thomas } \\
\text { \& Carl, 2001). }\end{array}$ \\
\hline 97 & SU_COL_E_H & $\begin{array}{l}\text { Startup, weak culture } \\
\text { of external } \\
\text { collaboration, } \\
\text { external, hinder }\end{array}$ & The same above & $\begin{array}{l}\text { Interviews`Transcript, } \\
\text { (Cooke, 2004; Cooke \& } \\
\text { Wills, 1999; Forrest, } \\
\text { 1990; Keizer et al., } \\
\text { 2002). }\end{array}$ \\
\hline
\end{tabular}




\begin{tabular}{|l|l|l|l|l|}
\hline 98 & SU_LM_E_H & $\begin{array}{l}\text { Startup, lack of high } \\
\text { qualified Saudi labour } \\
\text { market, external, } \\
\text { hinder }\end{array}$ & The same above & $\begin{array}{l}\text { Interviews` Transcript, } \\
\text { (A. Harrison \& } \\
\text { Rodríguez-Clare, 2009). }\end{array}$ \\
\hline 99 & SU_DB_E_H & $\begin{array}{l}\text { Startup, barriers for } \\
\text { doing business, } \\
\text { external, hinder }\end{array}$ & The same above & $\begin{array}{l}\text { Interviews`Transcript, } \\
\text { (A. Harrison \& } \\
\text { Rodríguez-Clare, 2009) }\end{array}$ \\
\hline
\end{tabular}

